NATIONAL LABORATORY

MANAGED BY UT-BATTELLE

FOR THE DEPARTMENT OF ENERGY

\title{
Isotopic Details of the Spent Catawba-1 MOX Fuel Rods at ORNL
}

\section{April 10, 2015}

\section{Prepared by}

Ronald J. Ellis

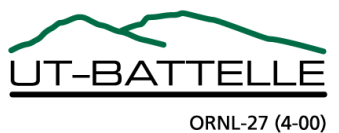




\section{DOCUMENT AVAILABILITY}

Reports produced after January 1, 1996, are generally available free via US Department of Energy (DOE) SciTech Connect.

Website http://www.osti.gov/scitech/

Reports produced before January 1, 1996, may be purchased by members of the public from the following source:

National Technical Information Service

5285 Port Royal Road

Springfield, VA 22161

Telephone 703-605-6000 (1-800-553-6847)

TDD 703-487-4639

Fax 703-605-6900

E-mail info@ntis.gov

Website http://www.ntis.gov/support/ordernowabout.htm

Reports are available to DOE employees, DOE contractors, Energy Technology Data Exchange representatives, and International Nuclear Information System representatives from the following source:

Office of Scientific and Technical Information

PO Box 62

Oak Ridge, TN 37831

Telephone 865-576-8401

Fax 865-576-5728

E-mail reports@osti.gov

Website http://www.osti.gov/contact.html

This report was prepared as an account of work sponsored by an agency of the United States Government. Neither the United States Government nor any agency thereof, nor any of their employees, makes any warranty, express or implied, or assumes any legal liability or responsibility for the accuracy, completeness, or usefulness of any information, apparatus, product, or process disclosed, or represents that its use would not infringe privately owned rights. Reference herein to any specific commercial product, process, or service by trade name, trademark, manufacturer, or otherwise, does not necessarily constitute or imply its endorsement, recommendation, or favoring by the United States Government or any agency thereof. The views and opinions of authors expressed herein do not necessarily state or reflect those of the United States Government or any agency thereof. 


\title{
ISOTOPIC DETAILS OF THE SPENT CATAWBA-1 MOX FUEL RODS AT ORNL
}

\author{
Ronald J. Ellis
}

Date Published: April 2015

\author{
Prepared by \\ OAK RIDGE NATIONAL LABORATORY \\ Oak Ridge, Tennessee 37831-6283 \\ managed by \\ UT-BATTELLE, LLC \\ for the \\ US DEPARTMENT OF ENERGY \\ under contract DE-AC05-00OR22725
}





\section{CONTENTS}

Page

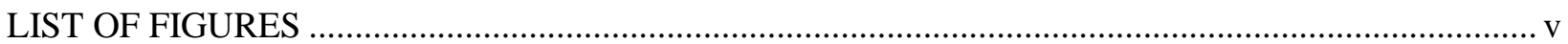

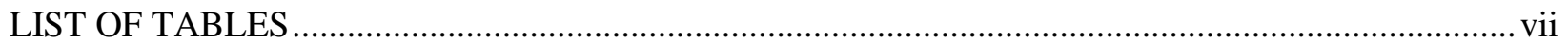

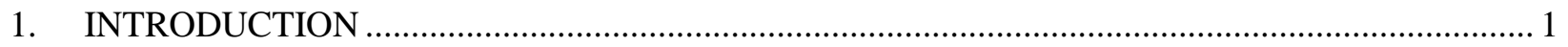

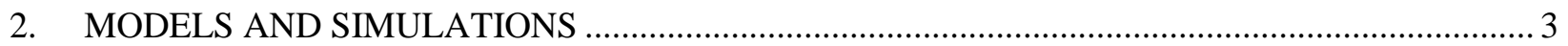

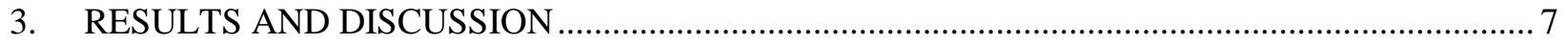

3.1 TABLES OF THE CALCULATED MOX FUEL ROD COMPOSITION RESULTS ............. 7

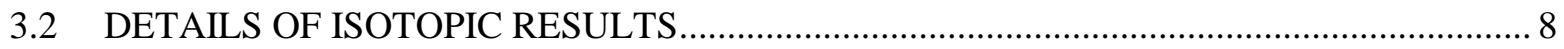

4. RECOMMENDATIONS FOR FUTURE MODELING OF THE MOX FUEL RODS..................... 15

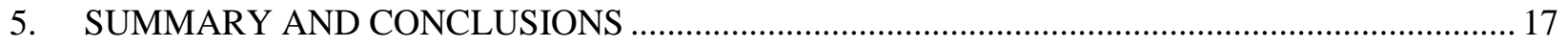

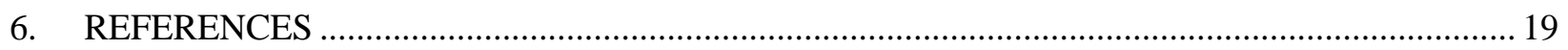

APPENDIX A. SAMPLE TRITON IRRADIATION/DEPLETION CASE MOXB4X.INP FOR

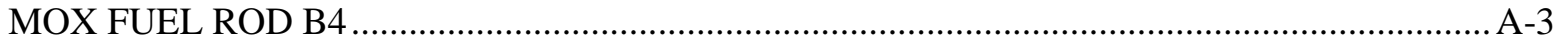

APPENDIX B. ORIGEN RESULTS FOR COOLING MOX FUEL RODS …................................. B-3

B.1 ORIGEN RESULTS FOR COOLING MOX FUEL ROD B4 ….....................................

B.2 ORIGEN RESULTS FOR COOLING MOX FUEL ROD A1 …................................. B-18

B.3 ORIGEN RESULTS FOR COOLING MOX FUEL ROD C1 …......................................

B.4 ORIGEN RESULTS FOR COOLING MOX FUEL ROD B14 ...................................... B-48

B.5 ORIGEN RESULTS FOR COOLING MOX FUEL ROD K5 ….................................... B-63

APPENDIX C. MOX FUEL ROD NUCLIDE WEIGHT FRACTIONS AND ACTIVITIES................. -3 



\section{LIST OF FIGURES}

Figure

Fig. 1. Location of the MOX fuel rods in the MX03 LTA irradiated in Catawba-1............................... 4

Fig. 2. Representative quarter-assembly (LTA based on the AREVA Mark-BW/MOX1 17×17 fuel assembly) view of the TRITON model for the MOX Fuel Rod irradiation/depletion calculations. Legend identifies the major components. ............................................................ 5

Fig. 3. Decay heat for the MOX fuel rods as function of cooling time.................................................. 9

Fig. 4. Decay heat for the MOX fuel rods......................................................................................

Fig. 5. Decay heat (total, actinide, fission product) for the higher burnup MOX fuel rod B4................. 10

Fig. 6. Activity of the MOX fuel rods as function of cooling time.................................................... 11

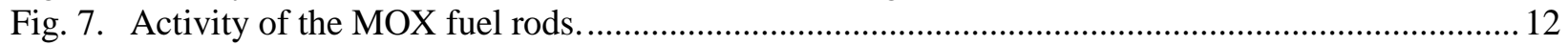

Fig. 8. Activity (total, actinide, fission product) for the higher burnup MOX fuel rod B4..................... 12 



\section{LIST OF TABLES}

Table

Page

Table 1. Summary of the MOX Fuel Rods that came to ORNL ................................................... 1

Table 2. Total decay heat (W per MTIHM) for Irradiated MOX Fuel Rods as function of cooling time...... . .8

Table 3. Activity (Ci per MTIHM) for Irradiated MOX Fuel Rods as function of cooling time........11

Table 4. Dose Equivalent Rates (rem/hr) at one-foot from a one-gram sample of the oxide fuel .....13

Table C-1. MOX Fuel Rod B4 (high Pu): Nuclide mass in grams per MTIHM..................................3

Table C-2. MOX Fuel Rod Al (low Pu): Nuclide mass in grams per MTIHM ..................................

Table C-3. MOX Fuel Rod Cl (medium Pu): Nuclide mass in grams per MTIHM............................31

Table C-4. MOX Fuel Rod B14 (high Pu): Nuclide mass in grams per MTIHM..............................45

Table C-5. MOX Fuel Rod K5 (high Pu): Nuclide mass in grams per MTIHM ..............................59

Table C-6. Activity in Fuel Rod B4 (high Pu loading) in Ci per MTIHM........................................... C-74

Table C-7. Activity in Fuel Rod Al (low Pu loading) in Ci per MTIHM .......................................... C-84

Table C-8. Activity in Fuel Rod Cl (medium Pu loading) in Ci per MTIHM .....................................94

Table C-9. Activity in Fuel Rod B14 (high Pu loading) in Ci per MTIHM..................................... C-104

Table C-10. Activity in Fuel Rod K5 (high Pu loading) in Ci per MTIHM .................................... C-114 


\section{INTRODUCTION}

The United States Department of Energy funded Shaw/AREVA MOX Services LLC to fabricate four MOX Lead Test Assemblies (LTA) from weapons-grade plutonium. A total of four MOX LTAs (including MX03) were irradiated in the Catawba Nuclear Station (Unit 1) "Catawba-1" PWR which operated at a total thermal power of $3411 \mathrm{MW}_{\mathrm{t}}$ and had a core with 193 total fuel assemblies. The MOX LTAs were irradiated along with Duke Energy's irradiation of eight Westinghouse Next Generation Fuel (NGF) LEU LTAs (ref.1) and the remaining 181 LEU fuel assemblies. The MX03 LTA was irradiated in the Catawba-1 PWR core (refs.2,3) during cycles C-16 and C-17. C-16 began on June 5, 2005, and ended on November 11, 2006, after 499 effective full power days (EFPDs). C-17 started on December 29, 2006, (after a shutdown of 48 days) and continued for 485 EFPDs. The MX03 and three other MOX LTAs (and other fuel assemblies) were discharged at the end of C-17 on May 3, 2008. The design of the MOX LTAs was based on the (Framatome ANP, Inc.) Mark-BW/MOX1 17×17 fuel assembly design (refs. 4,5,6) for use in Westinghouse PWRs, but with MOX fuel rods with three $\mathrm{Pu}$ loading ranges: the nominal $\mathrm{Pu}$ loadings are $4.94 \mathrm{wt} \%, 3.30 \mathrm{wt} \%$, and $2.40 \mathrm{wt} \%$, respectively, for high, medium, and low Pu content. The Mark-BW/MOX1 (MOX LTA) fuel assembly design is the same as the Advanced Mark-BW fuel assembly design but with the LEU fuel rods replaced by MOX fuel rods (ref. 5). The fabrication of the fuel pellets and fuel rods for the MOX LTAs was performed at the Cadarache facility in France, with the fabrication of the LTAs performed at the MELOX facility, also in France.

Five MOX fuel rods were selected from MX03 LTA and sent to Oak Ridge National Laboratory, arriving on January 22, 2009. These MOX fuel rods encompassed fuel rods B4, B14, and K5 (all high Pu loading), fuel rod $\mathrm{C} 1$ (medium Pu loading), and fuel rod A1 (low Pu loading). Table 1 presents details and parameters of the five MOX fuel rods that were transported to ORNL after irradiation in the MX03 LTA and is a summary of important initial and operational details of the five MOX fuel rods. The discharge burnups for each MOX fuel rod were not made public by Duke-Energy or AREVA, but the range in burnup levels was documented as shown in the last column (ref. 5,6). The exact as-built details (from Melox) of the fuel rods were used in the models and burnup simultations considered in this work.

Table 1. Summary of the MOX Fuel Rods that came to ORNL

\begin{tabular}{|c|c|c|c|c|}
\hline MOX Fuel Rod & $\begin{array}{c}\text { Pu Loading } \\
\text { Category }\end{array}$ & $\begin{array}{c}\text { Nominal Amount of Pu } \\
(\mathrm{Pu}+\mathrm{Am}) / \mathrm{HM}\end{array}$ & $\begin{array}{c}\text { Mass of initial } \\
\text { heavy metal (IHM) } \\
(\mathrm{g})\end{array}$ & $\begin{array}{l}\text { Exit Burnup } \\
\text { GWd/MTIHM }\end{array}$ \\
\hline B4 & high & $4.94 \mathrm{wt} \%$ & 1772.37 & \multirow{5}{*}{ Range of 39.7 to 47.3} \\
\hline A1 & low & $2.40 \mathrm{wt} \%$ & 1773.90 & \\
\hline C1 & Medium & $3.30 \mathrm{wt} \%$ & 1760.50 & \\
\hline B14 & High & $4.94 \mathrm{wt} \%$ & 1765.39 & \\
\hline K5 & High & $4.94 \mathrm{wt} \%$ & 1763.11 & \\
\hline
\end{tabular}

The MX03 LTA underwent two cycles of irradiation (C-16 and C-17) in the Catawba-1 PWR core. The fuel assembly average burnup for MX03 LTA for the total of cycles C-16 and C-17 was published by AREVA to be $41.8 \mathrm{GWd} / \mathrm{MTIHM}$, with the average burnup in cycle $\mathrm{C}-16$ for the MOX LTA was 22.0 GWd/MTIHM, and for C-17, it was $19.8 \mathrm{GWd} / \mathrm{MTIHM}$. The burnup of the five individual selected MOX fuel rods range from 39.7 to $47.3 \mathrm{GWd} / \mathrm{MTIHM}$, in the given discharge burnup determinations (ref. 5,6). All five MOX fuel rods were examined nondestructively, with four chosen for destructive examination; MOX fuel rod B14 (similar to B4) was not subjected to destructive examination (ref. 6). This report tabulates the nuclides in the calculated isotopic compositions of the irradiated and depleted fuel rods following the irradiation in Catawba-1 and the subsequent cooling (decay) time since discharge. 


\section{MODELS AND SIMULATIONS}

The depletion calculations were performed with the SCALE 6.1 (ref.7) scientific code package (using TRITON in t-depl mode, and ORIGEN, both as part of the TRITON sequence, and in stand-alone operation). The models used were based on existing Westinghouse W17 $\times 17$ PWR fuel assembly models updated with the as-built (at the MELOX facility - AREVA/Cogema) MOX LTA and fuel rod initial compositions, and any new dimensions and other details appropriate for the FCF (AREVA) (Framatome ANP, Inc.) Mark-BW/MOX1 17×17 fuel assembly design (ref. 5,6,7) used for the MOX LTAs, in conjunction with actual assembly operational data for the Catawba-1 irradiation in cycles C-16 and C-17.

As mentioned above, the Catawba-1 PWR core during cycles C-16 and C-17 had four MOX LTAs (including MX03 from which the five MOX fuel rods were selected) in addition to the remaining LEU fuel assemblies, but information about the neighboring LEU assemblies was not available to allow for a core model simulation. Consequently, the simulations in this work involved performing TRITON t-depl calculations for a single MOX LTA with appropriate boundary conditions, with the burnup and power history for the case normalized to the given burnup level and operational history information for each of the five MOX fuel rods provided by AREVA/Duke Energy. Five individual cases were completed, each one focused on maintaining the given power history for one of the selected MOX fuel rods.

Appendix A lists the input file used with SCALE 6.1.3/TRITON for fuel rod B4. The proprietary data for this fuel rod composition is not shown. The other four selected MOX fuel rods are modeled with similar TRITON input files, with the actual input initial fuel composition, the fuel rod location in the LTA, and the burnup history of the selected fuel rod being different for each case. The fuel rods in the MOX LTAs had cladding made from M5 represented by an approximate composition specified in the Appendix A input case. The guide tube and instrument tube dimensions and materials are used in the TRITON input cases for the MOX LTAs.

Figure 1 (ref. 6) indicates the location of the five chosen MOX fuel rods, using the AREVA numbering system, for the MX03 LTA rod position in the matrix schematic diagram. The positions of the guide thimbles and instrument tubes are shown, in addition to the three classes (Pu loading) of fuel rods, as well as the specific locations of the selected fuel rods. The high Pu content fuel rods are loaded into the central region of the MOX LTA. The low Pu content fuel rods are positioned at the corners of the LTA. The medium $\mathrm{Pu}$ loaded fuel rods are positioned along the outer sides of the assembly.

The initial five TRITON cases simulated the power history of each of the selected MOX fuel rods during the two Catawba-1 PWR cycles (C-16 and C-17) with the appropriate cycle full power days and the downtime between cycles. Furthermore, the cooling time (decay) for the discharged MOX fuel rods from the end of C-17 until shipment to ORNL was modeled. The material information and fuel compositions for the MOX fuel rods are stored in the SCALE-created ft71 output binary file, organized by material number and burnup/decay steps. The details of the MOX fuel rod compositions presented in Appendix C are obtained in ORIGEN calculations that start with the appropriate data in the $\mathrm{ft} 71 \mathrm{files}$, and further decay the compositions to the necessary times and dates. The raw output results of these five SCALE/ORIGEN cases are listed in Appendix B in subsections B1-B5. These results were then normalized in terms of a basis of 1 MTIHM, to determine the data to put into the tables in this report. 


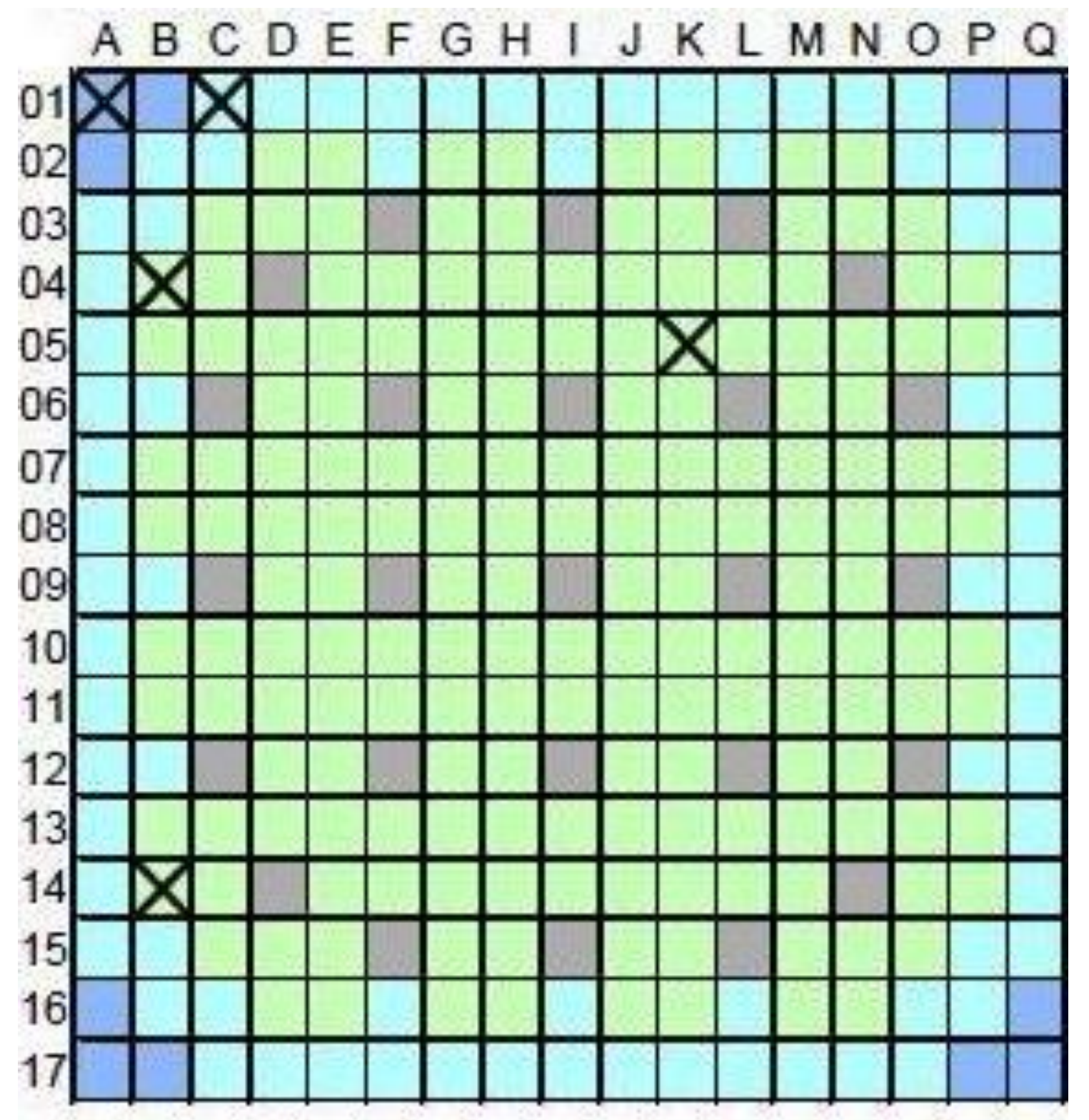

$$
\begin{aligned}
& \bigotimes=\text { Rod selected for hot cell examination } \\
& \text { = Guide thimble or instrument tube location } \\
& =\text { High-Pu fuel rod location (4.94\%) } \\
& =\text { Medium-Pu fuel rod location }(3.30 \%) \\
& =\text { Low }- \text { Pu fuel rod location ( } 2.40 \% \text { ) }
\end{aligned}
$$

Fig. 1. Location of the MOX fuel rods in the MX03 LTA irradiated in Catawba-1. 
Figure 2 is a depiction of the SCALE/TRITON model representing the MX03 LTA. The diagram is a quarter-view of the MOX LTA. Please see the legend in Figure 1 which identifies the various features and fuel rods. Fuel Rod A1 is specifically indicated as yellow within a yellow square at the upper right of the diagram; (it is a low-Pu content rod). The rest of the high $\mathrm{Pu}$, medium $\mathrm{Pu}$, and low $\mathrm{Pu} \mathrm{MOX}$ fuel rods are indicated. The specific selected MOX fuel pin placements are indicated in Figure 1.
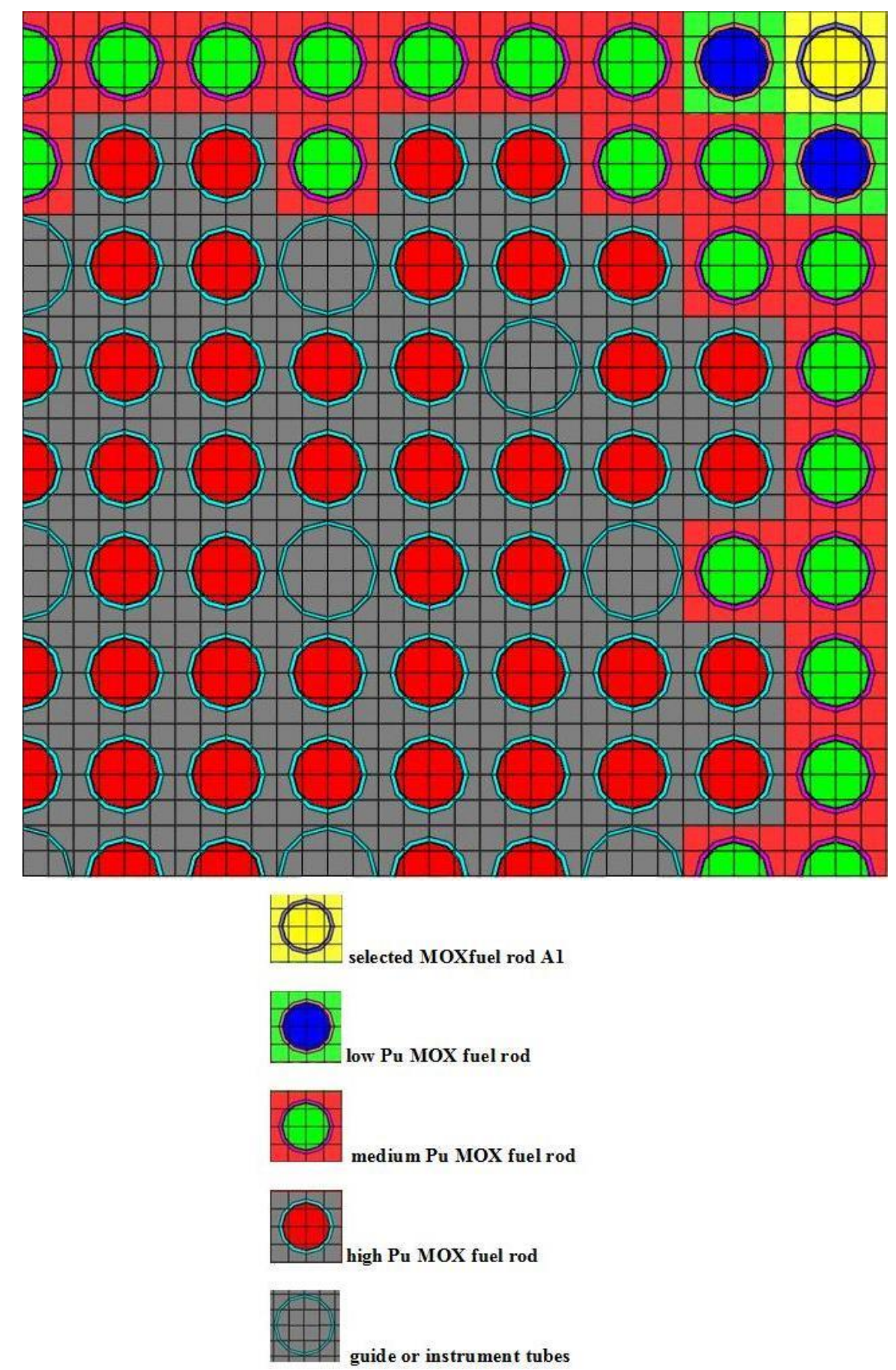

Fig. 2. Representative quarter-assembly (LTA based on the AREVA Mark-BW/MOX1 17×17 fuel assembly) view of the TRITON model for the MOX Fuel Rod irradiation/depletion calculations. Legend identifies the major components. 


\section{RESULTS AND DISCUSSION}

In this report, calculated the isotopic compositions for each of the spent MOX fuel rods sent to ORNL are tabulated. The results presented in this report are shown in terms of mass ( $\mathrm{g}$ ), activities (Ci), and decay heat (W) normalized per 1 MT of initial heavy metal (MTIHM) for the individual MOX fuel rods. The various tables in this report show details of the fuel compositions for at least five representative dates. As mentioned above, the five selected MX03 LTA fuel rods were removed from the Catawba-1 reactor core on May 3, 2008. These fuel rods were shipped to ORNL arriving on January 22, 2009. This date corresponds to the first column of data in all of the tables in this report. The other columns of results in the tables represent the MOX fuel rod compositions on October 21, 2013, the date of the first draft version of this report, and on the January 22 anniversary in each of the years 2014, 2015, and 2016.

The isotopic compositions for the five MOX fuel rods depend on the initial fuel composition (Pu loading, nuclide weight fractions, total mass, location of the rod in the LTA, neighboring rods and regions, etc), the location of the fuel rod in the assembly (assembly central zone, on the periphery, near a corner, next to a guide tube, etc.), the discharge burnup level for the rods (and the associated power history), and the cooling time following removal from irradiation in the Catawba-1 core. The range in discharge burnup levels is about $20 \%$ according to the given data on burnup from Duke Energy/AREVA.

The main results for the fuel composition (weight fractions) and activities, by nuclide, are presented in Tables C1-C5, and Tables C6-C10, respectively. The data are tabulated by relevant dates. The fuel rods were discharged from Catawba-1 on May 3, 2008, and shipped to ORNL, arriving on January 22, 2009; this was 264 days (of cooling) after discharge. The other dates for which the data are listed are October 21, 2013, which is 1997 days after discharge, and on the January 22 anniversary for each of 2014, 2015, and 2016 (2090, 2455, and 2820 days of cooling after discharge). As seen in the output listings for each MOX fuel rod listed in Appendix B, the SCALE isotopic results were calculated for many additional time steps, starting with the compositions at 240 days of cooling (from the TRITON case output material libraries), for each fuel rod, designated "initial" in the output tables in Appendix B.

For convenience and usefulness for the readers, the weight fractions for each non-negligible nuclide is given in terms of grams per metric ton of initial heavy metal in each of the fuel rods. The fuel nuclide libraries in SCALE (with over 2300 nuclides) were available in the TRITON and ORIGEN calculations; about $530+$ active and non-active nuclides were non-negligible $\left(>1.0 \times 10^{-20} \mathrm{~g} / \mathrm{MTIHM}\right)$ for inclusion in these mass-fraction tables. For the tables of calculated activities for the radionuclides in the fuel materials, about $350+$ non-negligible $\left(>1.0 \times 10^{-32} \mathrm{Ci} / \mathrm{MTIHM}\right)$ radioactive nuclides are listed for each MOX fuel rod. The active nuclides are listed in terms of $\mathrm{Ci}$ for each nuclide based on a total normalization of 1 metric ton of initial heavy metal, for each fuel rod. To consider these activities in terms of SI units, the following conversion would be used: $1 \mathrm{Ci}=3.7 \times 10^{10}$ Becquerel (disintegrations per second).

\subsection{TABLES OF THE CALCULATED MOX FUEL ROD COMPOSITION RESULTS}

Appendix B includes files added to this report that present the raw output of ORIGEN calculations for the subsequent decay of the irradiated MOX fuel rod compositions, spanning a total of about eight years of cooling time. These ORIGEN case outputs, in sections B1 to B5 in Appendix B, used the output nuclide libraries (files ft71f001) from the appropriate TRITON t-depl case runs (see Appendix A) and performed the decay for the specified cooling time duration arranged to tabulate data for specific dates of interest. Appendix $\mathrm{C}$ includes 10 large tables that present the weight fractions for a large list of nuclides (in grams per MTIHM) for the compositions of the MOX fuel rods B4, A1, C1, B14, and K5 (Tables C1-C5 
respectively). Nuclide activities (in Ci per MTIHM) are presented for the MOX fuel rods in Tables C6C10. These 10 tables represent the main calculated results for the isotopics of the five decayed and cooled MOX fuel rods that were received at ORNL from Catawba-1.

\subsection{DETAILS OF ISOTOPIC RESULTS}

Spreadsheets containing the information presented in Tables C1-C10 were prepared and distributed to ORNL and other staff involved in the handling and disposition of the MOX fuel rods.

Table 2 below presents the decay heat (in W per MTIHM) for the five MOX fuel rods as a function of the time after removal (cooling time) from irradiation in the Catawba-1 PWR core. The total decay heat is the sum of the heat energy rate from all $\alpha, \beta$, and $\gamma$ decay emissions. The results are listed in the Appendix B output files under the three groupings of the nuclides: light elements (LE) [activation products], actinides (Act), and fission products (FP).

Table 2 presents the actinide and fission product contributions separately as well as the total decay heat (the decay heat from the light element activation products is very small). Note that the total decay heat is an upper limit to what is manifested in the spent fuel rod material: a sizeable portion of the gamma ray emissions can escape the material sample and containers and thus not contribute to the heating, but all the decay heat from the $\alpha$ - and $\beta$-radiation is captured because of the short-range of these particles.

Table 2. Total decay heat (W per MTIHM) for Irradiated MOX Fuel Rods as function of cooling time

\begin{tabular}{|c|c|c|c|c|c|c|}
\hline \multicolumn{2}{|c|}{ Date: } & $1 / 22 / 2009$ & $10 / 21 / 2013$ & $1 / 22 / 2014$ & $1 / 22 / 2015$ & 1/22/2016 \\
\hline \multicolumn{2}{|c|}{ Days after removal on 5/03/2008: } & 264 & 1997 & 2090 & 2455 & 2820 \\
\hline \multirow{3}{*}{$\begin{array}{c}\text { B4 } \\
\text { (high Pu) }\end{array}$} & Act & $3.391 \mathrm{E}+03$ & $7.661 \mathrm{E}+02$ & $7.675 \mathrm{E}+02$ & $7.741 \mathrm{E}+02$ & 7.807E+02 \\
\hline & $\mathrm{FP}$ & $1.855 \mathrm{E}+04$ & $1.931 \mathrm{E}+03$ & $1.819 \mathrm{E}+03$ & $1.501 \mathrm{E}+03$ & $1.304 \mathrm{E}+03$ \\
\hline & Total & 2.194E+04 & $2.697 \mathrm{E}+03$ & $2.586 \mathrm{E}+03$ & $2.275 \mathrm{E}+03$ & $2.085 E+03$ \\
\hline \multirow{3}{*}{$\begin{array}{c}\text { A1 } \\
\text { (low Pu) }\end{array}$} & Act & $2.880 \mathrm{E}+03$ & $7.712 \mathrm{E}+02$ & $7.698 \mathrm{E}+02$ & $7.652 \mathrm{E}+02$ & 7.612E+02 \\
\hline & FP & $1.505 \mathrm{E}+04$ & $1.634 \mathrm{E}+03$ & $1.540 \mathrm{E}+03$ & $1.273 \mathrm{E}+03$ & $1.106 \mathrm{E}+03$ \\
\hline & Total & $1.793 E+04$ & $2.405 E+03$ & $2.310 \mathrm{E}+03$ & 2.039E+03 & $1.867 \mathrm{E}+03$ \\
\hline \multirow{3}{*}{$\begin{array}{c}\text { C1 } \\
\text { (medium Pu) }\end{array}$} & Act & $3.075 \mathrm{E}+03$ & $7.511 \mathrm{E}+02$ & $7.511 \mathrm{E}+02$ & $7.511 \mathrm{E}+02$ & $7.518 \mathrm{E}+02$ \\
\hline & FP & $1.619 \mathrm{E}+04$ & $1.725 \mathrm{E}+03$ & $1.626 \mathrm{E}+03$ & $1.343 \mathrm{E}+03$ & $1.166 \mathrm{E}+03$ \\
\hline & Total & $1.926 \mathrm{E}+04$ & $2.476 \mathrm{E}+03$ & $2.377 \mathrm{E}+03$ & $2.094 \mathrm{E}+03$ & $1.918 E+03$ \\
\hline \multirow{3}{*}{$\begin{array}{c}\text { B14 } \\
\text { (high Pu) }\end{array}$} & Act & $3.403 \mathrm{E}+03$ & $7.695 \mathrm{E}+02$ & $7.708 \mathrm{E}+02$ & $7.768 \mathrm{E}+02$ & $7.827 \mathrm{E}+02$ \\
\hline & $\mathrm{FP}$ & $1.855 \mathrm{E}+04$ & $1.931 \mathrm{E}+03$ & $1.820 \mathrm{E}+03$ & $1.502 \mathrm{E}+03$ & $1.305 \mathrm{E}+03$ \\
\hline & Total & $2.195 \mathrm{E}+04$ & $2.701 \mathrm{E}+03$ & $2.591 \mathrm{E}+03$ & $2.279 \mathrm{E}+03$ & $2.088 \mathrm{E}+03$ \\
\hline \multirow{3}{*}{$\begin{array}{c}\text { K5 } \\
\text { (high Pu) }\end{array}$} & Act & $2.709 \mathrm{E}+03$ & $5.940 \mathrm{E}+02$ & $5.966 \mathrm{E}+02$ & $6.074 \mathrm{E}+02$ & $6.182 \mathrm{E}+02$ \\
\hline & FP & $1.643 \mathrm{E}+04$ & $1.635 \mathrm{E}+03$ & $1.540 \mathrm{E}+03$ & $1.271 \mathrm{E}+03$ & $1.106 \mathrm{E}+03$ \\
\hline & Total & $1.914 \mathrm{E}+04$ & $2.229 \mathrm{E}+03$ & $2.136 \mathrm{E}+03$ & $1.878 \mathrm{E}+03$ & $1.725 \mathrm{E}+03$ \\
\hline
\end{tabular}

The decay heat contributions presented in Table 2 and plotted in detail in Figures 3 and 4 show that the total decay heat drops off quickly at shorter cooling times then declines steadily with time. Figure 3 shows the time behavior of the decay heat (W/MTIHM) for the five MOX fuel rods from the time the rods were transported to ORNL until January 2016. 


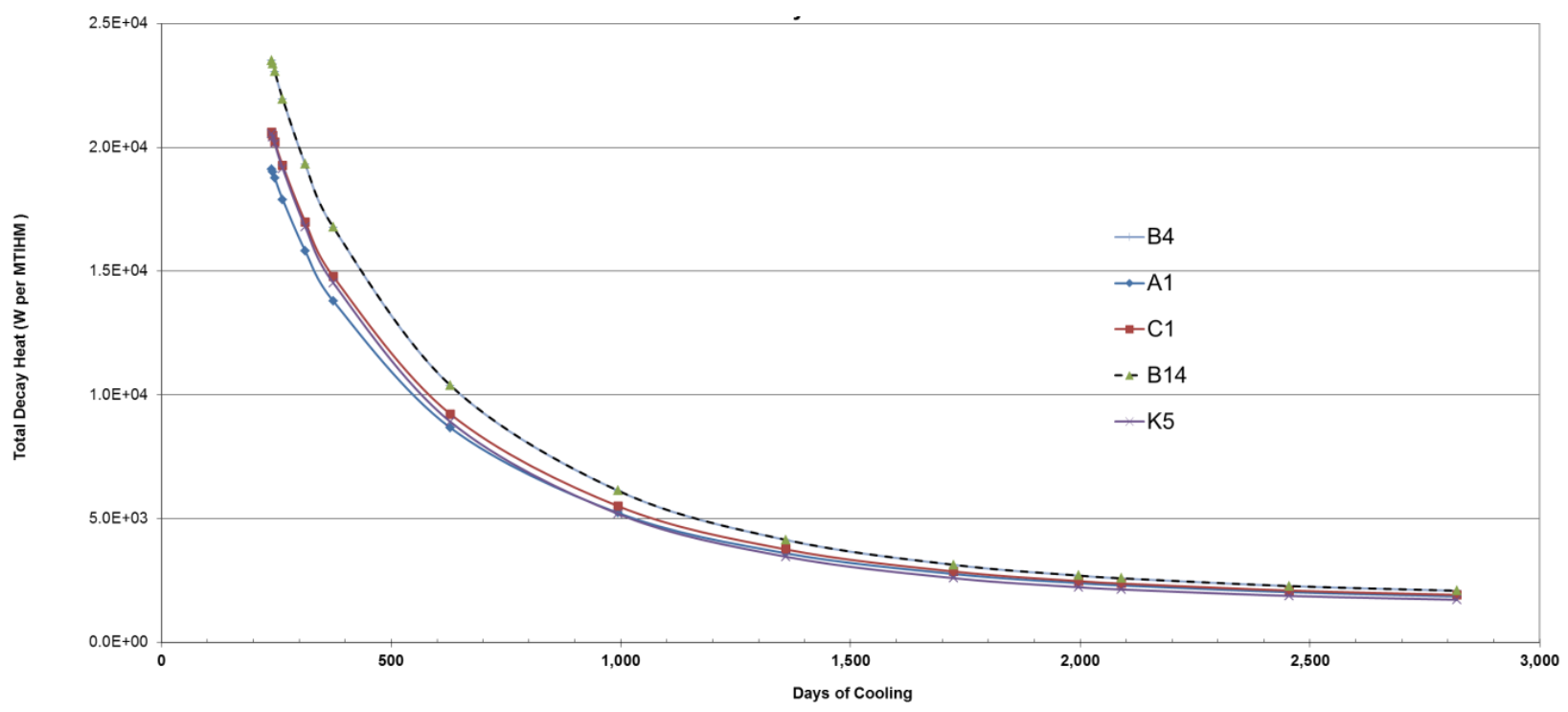

Fig. 3. Decay heat for the MOX fuel rods as function of cooling time.

Figure 4 show the time dependence of the decay power for the MOX fuel rods for recent and future dates when the curves are flatter. Figure 4 concentrates on the shallower declining stage of the decay heat curves from about the start of 2012 until January 2016. The trend is that the higher the initial Pu content in the MOX fuel rods, the greater the total decay heat at each time step; however, the location of the fuel rod in the LTA has a bearing on the decay heat behavior.

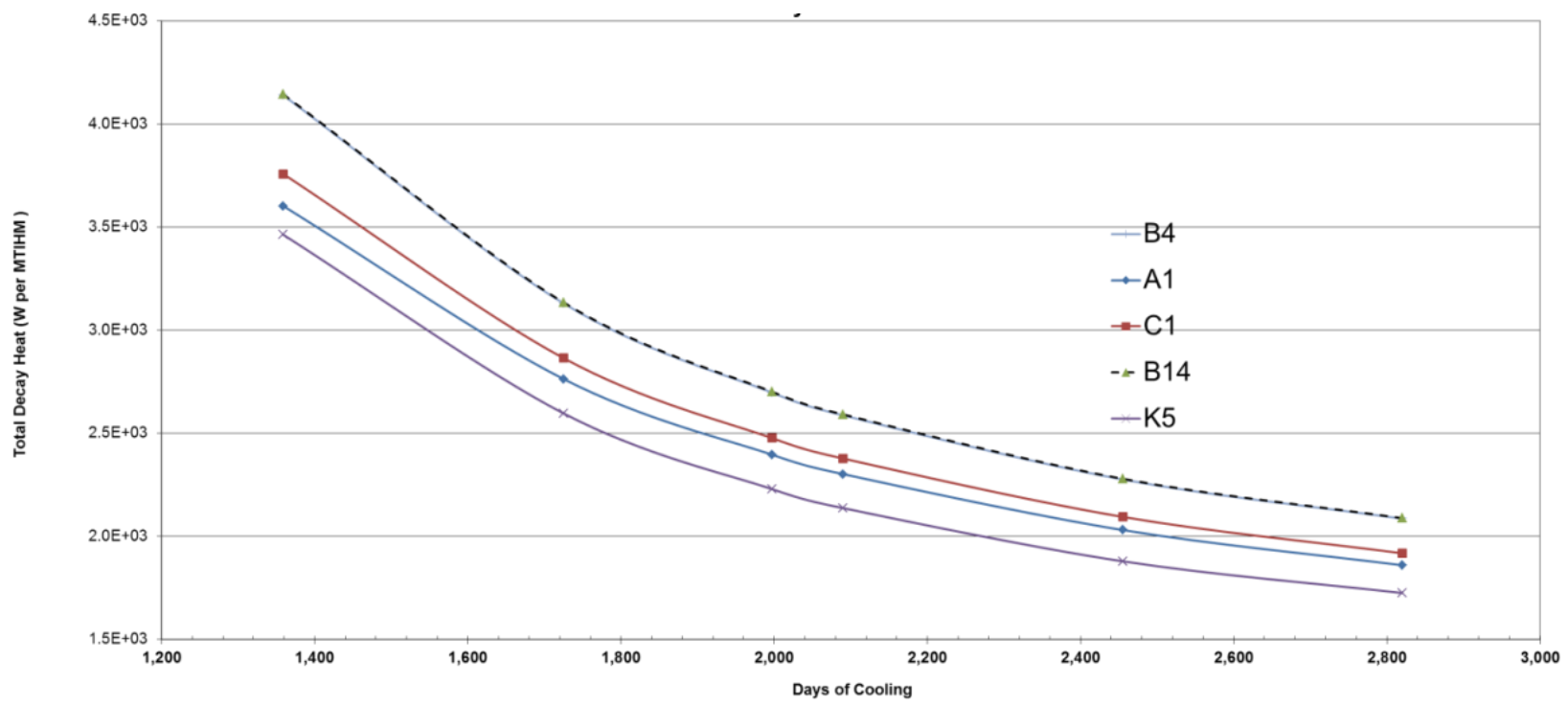

Fig. 4. Decay heat for the MOX fuel rods.

Figure 5 shows the decay heat for the high-Pu MOX fuel rod B4 which had one of the highest reported burnup levels at discharge. The total decay heat is shown as well as the contributions from fission products (initially quite large) and the contributions from actinide nuclides. 


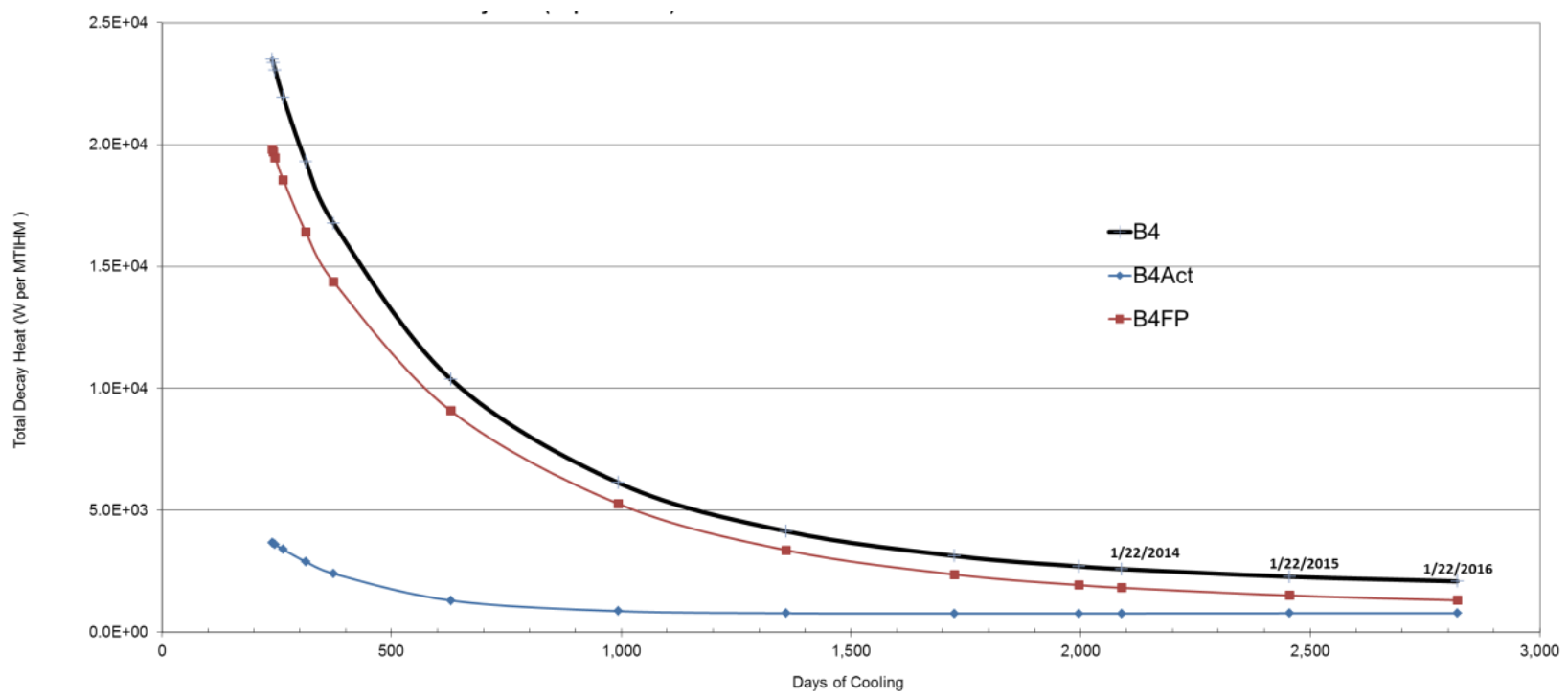

Fig. 5. Decay heat (total, actinide, fission product) for the higher burnup MOX fuel rod B4.

Figure 5 shows the decay heat curves from actinide and fission product nuclides, in high Pu content, high burnup, MOX fuel rod B4, in addition to the total decay heat curve. The initial fission product contribution to the decay heat is considerably greater than the actinide component, with the two components nearing in magnitude by 2016 and should be equal in 2017 or 2018, after which the actinide decay heat will generally dominate.

Table 3 presents details of the activity of the MOX fuel rods for a range of milestone dates, from January 2009 until January 2016. The very high activities in the eight months from discharge (May 2008) until they arrived at ORNL, are not shown in Table 3. However, for example, at the time of discharge, the activity of the material in MOX fuel rod B4 is high: the actinide component is $49.45 \mathrm{MCi} / \mathrm{MTIHM}$ and the fission product component is 203.24 MCi/MTIHM, for a total activity of 252.27 MCi/MTIHM. By the time MOX fuel rod B4 arrived at ORNL, the total activity had dropped substantially, by a factor of nearly 52. On January 22, 2009 (after a cooling time of 264 days), the total activity was 4.863 $\mathrm{MCi} / \mathrm{MTIHM}$, with the actinide component being $0.635 \mathrm{MCi} / \mathrm{MTIHM}$ and the fission product component being 4.228 MCi/MTIHM. 
Table 3. Activity (Ci per MTIHM) for Irradiated MOX Fuel Rods as function of cooling time

\begin{tabular}{|c|c|c|c|c|c|c|}
\hline \multicolumn{2}{|c|}{ Date: } & 1/22/2009 & $10 / 21 / 2013$ & $1 / 22 / 2014$ & $1 / 22 / 2015$ & $1 / 22 / 2016$ \\
\hline \multicolumn{2}{|c|}{ Days after removal on $5 / 03 / 2008$ : } & 264 & 1997 & 2090 & 2455 & 2820 \\
\hline \multirow{3}{*}{$\begin{array}{c}\text { B4 } \\
\text { (high Pu) }\end{array}$} & Act & $6.347 \mathrm{E}+05$ & $4.525 \mathrm{E}+05$ & $4.473 \mathrm{E}+05$ & 4.275E+05 & $4.085 \mathrm{E}+05$ \\
\hline & FP & $4.228 \mathrm{E}+06$ & $5.625 \mathrm{E}+05$ & $5.385 \mathrm{E}+05$ & $4.690 \mathrm{E}+05$ & $4.246 \mathrm{E}+05$ \\
\hline & Total & $4.863 \mathrm{E}+06$ & $1.015 E+06$ & $9.858 \mathrm{E}+05$ & $8.964 \mathrm{E}+05$ & $8.331 \mathrm{E}+05$ \\
\hline \multirow{3}{*}{$\begin{array}{c}\mathrm{A1} \\
(\text { low Pu) }\end{array}$} & Act & $4.451 \mathrm{E}+05$ & $3.129 \mathrm{E}+05$ & $3.093 \mathrm{E}+05$ & $2.957 \mathrm{E}+05$ & $2.826 \mathrm{E}+05$ \\
\hline & FP & $3.406 \mathrm{E}+06$ & $4.683 \mathrm{E}+05$ & $4.486 \mathrm{E}+05$ & $3.918 \mathrm{E}+05$ & $3.553 \mathrm{E}+05$ \\
\hline & Total & $3.851 E+06$ & $7.812 \mathrm{E}+05$ & $7.580 \mathrm{E}+05$ & $6.875 \mathrm{E}+05$ & $6.379 \mathrm{E}+05$ \\
\hline \multirow{3}{*}{$\begin{array}{c}\mathrm{C1} \\
\text { (medium Pu) }\end{array}$} & Act & $5.163 \mathrm{E}+05$ & $3.648 \mathrm{E}+05$ & $3.606 \mathrm{E}+05$ & $3.446 \mathrm{E}+05$ & $3.294 \mathrm{E}+05$ \\
\hline & FP & $3.669 \mathrm{E}+06$ & $4.971 \mathrm{E}+05$ & $4.761 \mathrm{E}+05$ & $4.153 \mathrm{E}+05$ & $3.764 \mathrm{E}+05$ \\
\hline & Total & $4.186 \mathrm{E}+06$ & $8.619 E+05$ & $8.367 \mathrm{E}+05$ & $7.599 \mathrm{E}+05$ & $7.057 \mathrm{E}+05$ \\
\hline \multirow{3}{*}{$\begin{array}{c}\text { B14 } \\
\text { (high Pu) }\end{array}$} & Act & $6.331 \mathrm{E}+05$ & $4.511 \mathrm{E}+05$ & $4.458 \mathrm{E}+05$ & $4.260 \mathrm{E}+05$ & $4.072 \mathrm{E}+05$ \\
\hline & FP & $4.227 \mathrm{E}+06$ & $5.624 \mathrm{E}+05$ & $5.383 \mathrm{E}+05$ & $4.688 \mathrm{E}+05$ & $4.244 \mathrm{E}+05$ \\
\hline & Total & $4.860 \mathrm{E}+06$ & $1.013 E+06$ & $9.842 \mathrm{E}+05$ & $8.949 \mathrm{E}+05$ & $8.316 \mathrm{E}+05$ \\
\hline \multirow{3}{*}{$\begin{array}{c}\text { K5 } \\
\text { (high Pu) }\end{array}$} & Act & $5.892 \mathrm{E}+05$ & $4.264 \mathrm{E}+05$ & 4.215E+05 & $4.027 \mathrm{E}+05$ & $3.848 \mathrm{E}+05$ \\
\hline & FP & $3.789 \mathrm{E}+06$ & $4.883 \mathrm{E}+05$ & $4.671 \mathrm{E}+05$ & $4.061 \mathrm{E}+05$ & $3.673 \mathrm{E}+05$ \\
\hline & Total & $4.378 \mathrm{E}+06$ & $9.147 E+05$ & $8.886 \mathrm{E}+05$ & $8.088 E+05$ & $7.521 \mathrm{E}+05$ \\
\hline
\end{tabular}

Figure 6 presents the curves of the time-dependence of activity for the five MOX fuel rods from early 2009 until 2016. The large spikes in activity at discharge and in the early cooling stages are not shown in the table above nor shown in the curves, but are discussed in the next section.

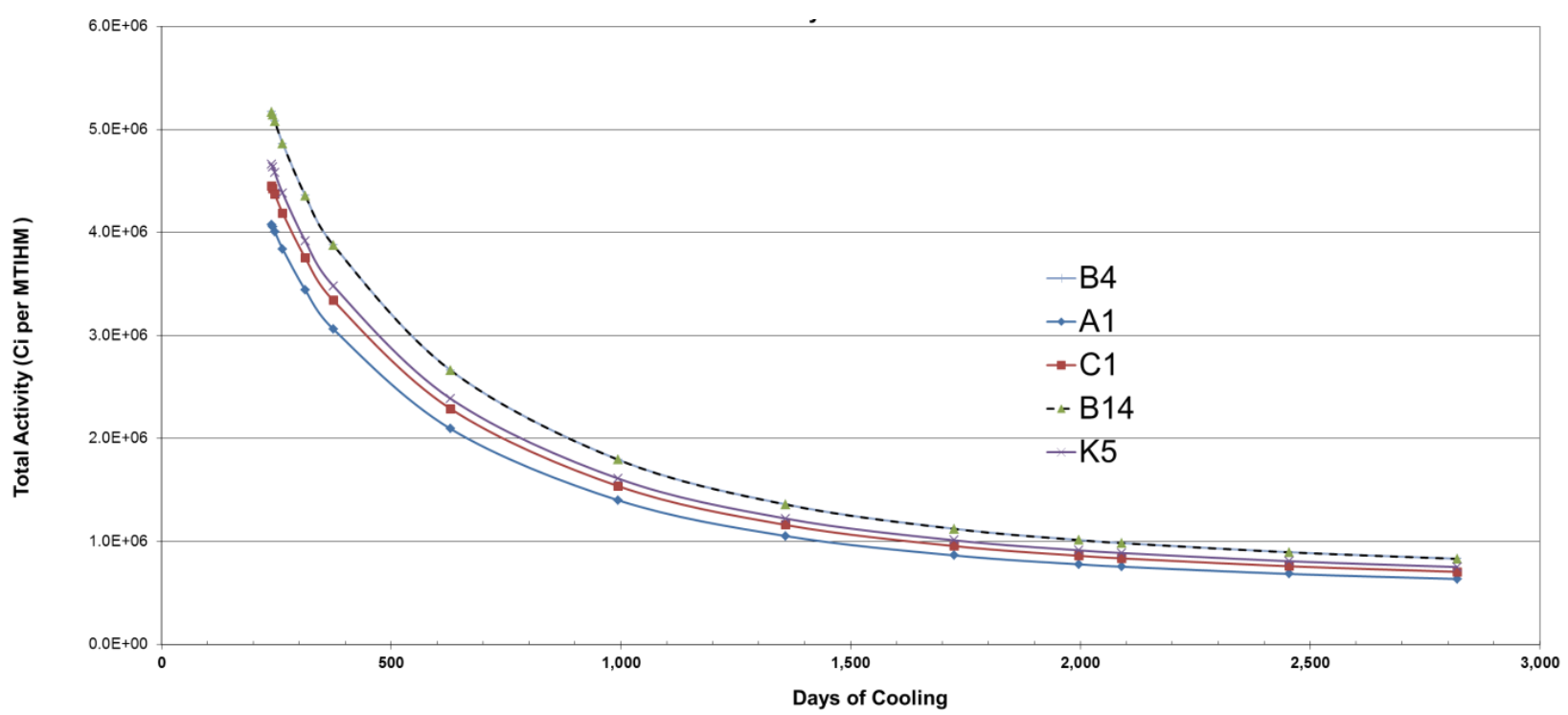

Fig. 6. Activity of the MOX fuel rods as function of cooling time.

Figure 7 shows the total activity curves for the five MOX fuel rods for the last few years from early 2012 until January 2016. 


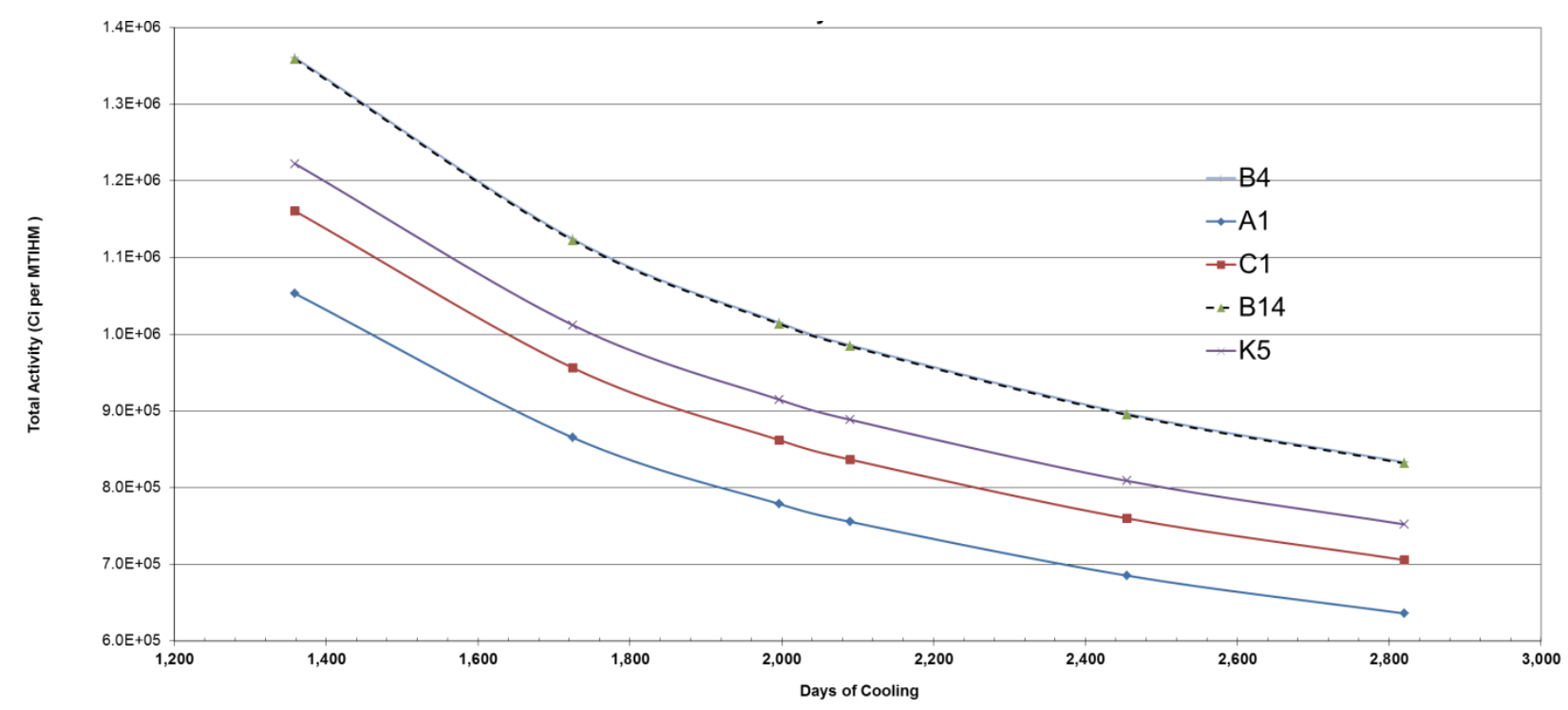

Fig. 7. Activity of the MOX fuel rods.

Table 3 and Figures 6 and 7 present the data and curves for the activity (in Ci per MTIHM) for the five MOX fuel rods. The time behavior and dependencies of the radioactivities is similar to that of the decay heat. The fission product activity comes very close to equaling the actinide activity by 2016 . Figure 7 , like Figure 5 for decay heat, shows the time behavior of the activity from 2012 until early 2016. As with the decay heat curves, the activity curves are similar in shape for the various fuel rods, with the relative magnitude of the curves ranging from the highest (high-Pu content B4 and B14) to the lowest (low-Pu A1).

Figure 8 compares the fission product and actinide activities for the high activity fuel rod B4 (very similar to B14), with the total activity also shown

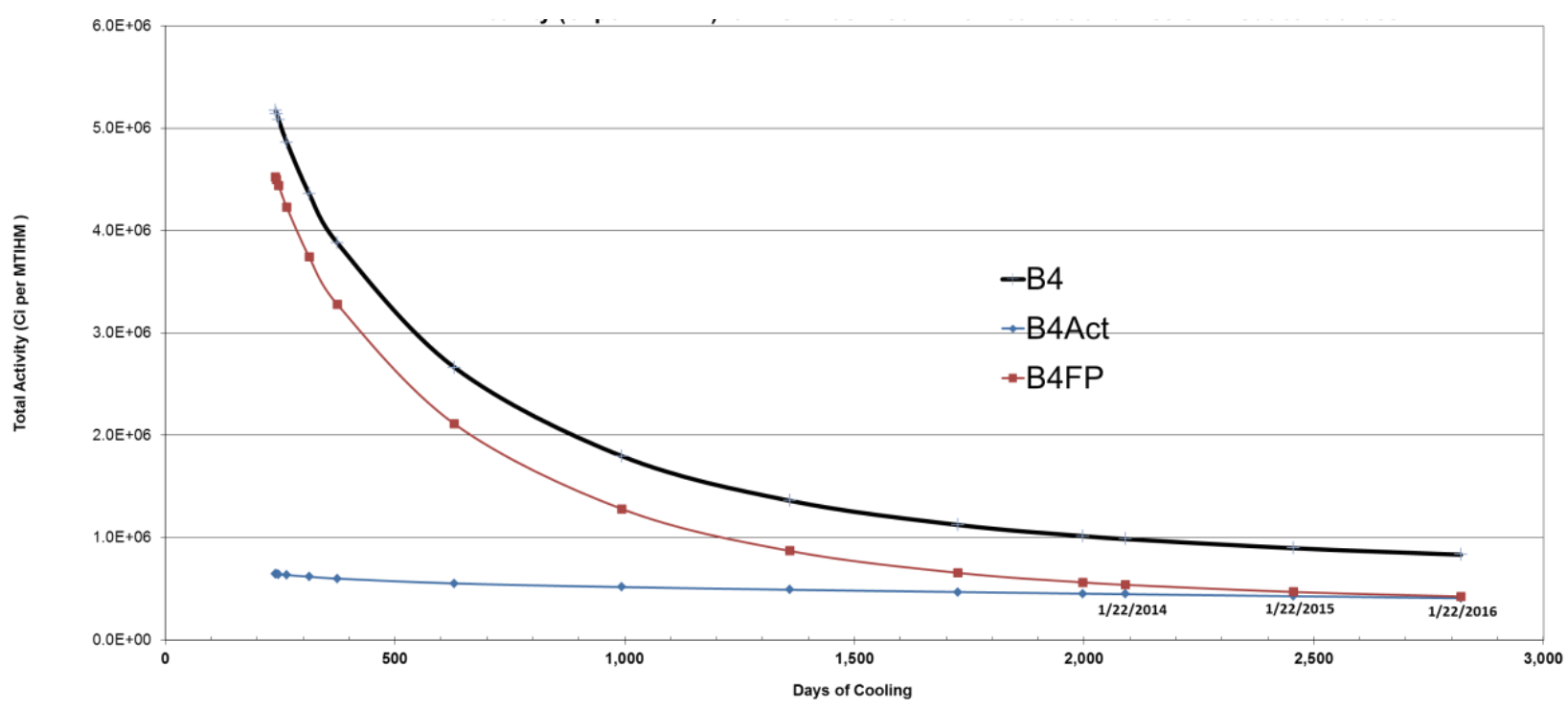

Fig. 8. Activity (total, actinide, fission product) for the higher burnup MOX fuel rod B4. 
As evident in this figure, the fission product and actinide activities are very similar in magnitude by 2016. The activities due to the fission product radionuclides and due to the actinide nuclides are shown, as well as the total activity. Some of the current and latter important milestone dates are shown on the curves.

Table 4 presents the dose equivalent rates (rem/hr) for the five MOX fuel rods, which span a range in initial Pu loading and in discharge burnup levels. The dose equivalent values were determined using the SCALE/MAVRIC code with the gamma source information from the appropriate ORIGEN case output results. For convenience, the specific dose equivalent rates in rem/hr per gram of discharged fuel material are tabulated.

Table 4. Dose Equivalent Rates (rem/hr) at one-foot from a one-gram sample of the oxide fuel

\begin{tabular}{|c|c|c|c|}
\hline Cooling Days: & 2090 & 2455 & 2820 \\
\hline Date: & $1 / 22 / 2014$ & $1 / 22 / 2015$ & $1 / 22 / 2016$ \\
\hline $\begin{array}{c}\text { MOX Fuel Rod B4 } \\
\text { (high Pu loading) }\end{array}$ & $\mathbf{1 . 2 4 2}$ & $\mathbf{1 . 0 4 2}$ & $\mathbf{0 . 9 0 9}$ \\
\hline $\begin{array}{c}\text { MOX Fuel Rod A1 } \\
\text { (low Pu loading) }\end{array}$ & $\mathbf{1 . 0 5 0}$ & $\mathbf{0 . 8 8 0}$ & $\mathbf{0 . 7 7 0}$ \\
\hline $\begin{array}{c}\text { MOX Fuel Rod C1 } \\
\text { (medium Pu loading) }\end{array}$ & $\mathbf{1 . 1 0 8}$ & $\mathbf{0 . 9 3 0}$ & $\mathbf{0 . 8 1 1}$ \\
\hline $\begin{array}{c}\text { MOX Fuel Rod B14 } \\
\text { (high Pu loading) }\end{array}$ & $\mathbf{1 . 2 3 7}$ & $\mathbf{1 . 0 3 8}$ & $\mathbf{0 . 9 0 6}$ \\
\hline $\begin{array}{c}\text { MOX Fuel Rod K5 } \\
\text { (high Pu loading) }\end{array}$ & $\mathbf{1 . 0 3 0}$ & $\mathbf{0 . 8 6 5}$ & $\mathbf{0 . 7 5 5}$ \\
\hline
\end{tabular}

The discharge burnup levels for MOX fuel rods B14 and B4, both high Pu-loaded rods, are greater than for A1, a low Pu-loaded fuel rod, but they are also higher than the other high Pu-loaded fuel rod K5. The specific dose equivalent rates are shown for convenient milestone dates for the MOX fuel rod compositions. The MOX fuel rods were removed from irradiation in the Catawba-1 core in May 2008 and have been cooling since then; the fuel rods arrived at ORNL on January 22, 2009. Of interest in regards to comparison to the dose equivalent rates presented in Table 4, at the time of discharge from Catawba-1 in May 2008, after a simulated 1-second of cooling, the dose equivalent rate at a distance of 1 foot from a sample was calculated to have been $1322.3 \mathrm{rem} / \mathrm{hr}$ per gram of fuel material from MOX fuel rod B4. This high specific 1-foot dose equivalent rate has thus dropped by a factor of nearly 1200 from the time of discharge until mid-2014. As of mid-2014, the dose equivalent rate at 1-foot distance for the five irradiated and cooled MOX fuel rods range from about 1.10 to $0.90 \mathrm{rem} / \mathrm{hr}$ per gram of the discharged MOX fuel material. 


\section{RECOMMENDATIONS FOR FUTURE MODELING OF THE MOX FUEL RODS}

As discussed above, the five MOX fuel rods analyzed in this work were modeled as components of a TRITON t-depl model representing the MX03 LTA fuel assembly. The fuel rods were individually modeled, in the environment of the full MOX LTA assembly normalized to the MOX pin power and burnup history based on given AREVA/Duke Energy exit rod burnup values.

An independent assessment and calculation of the burnup compositions for the five MOX fuel rods would require details and specifics of the neighboring LEU fuel assemblies in the Catawba-1 reactor core, and added details for any burnable posons and other aspects of the MOX LTAs. It is likely that the surrounding LEU assemblies were specialized in terms of WABA or IFBA burnable poisons, especially in the LEU assembly peripheries, such that the neutron flux in the MOX to LEU assembly interface regions is controlled so that the MOX fuel rods in the outer row of the LTA are not over-powered.

The selected MOX fuel rods, especially $\mathrm{A} 1$ (low $\mathrm{Pu}$ ) and $\mathrm{C} 1$ (medium $\mathrm{Pu}$ ), may have attained higher burnup levels than estimated in the given data. Preliminary assay measurements of the MOX fuel rod compositions at ORNL suggest that there are trends and biases in the measured results not reflected in the calculations discussed in this report, that was based on the available modeling, material, and power history data. 


\section{SUMMARY AND CONCLUSIONS}

This report presents the results calculated for the nuclide compositions of the MOX fuel rods (B4, A1, C1, B14, and K5) from MX03 LTA irradiated in Catawba-1 PWR core, discharged in May 2008 and shipped to ORNL on January 22, 2009. The calculations were performed with the SCALE 6.1.3 scientific code package with models based on as much information about the MX03 LTA design and the Catawba-1 core details that were available, and the simulated power histories used in the calculations were contingent on considering the discharge burnup levels provided by AREVA/Duke Energy.

This report presents details on the nuclide weight fraction and activity (by radionuclide) for a range of useful time steps (dates) for the five selected MOX fuel rods. In addition, results are provided for the decay heat and dose equivalent rates for the discharged MOX fuel rod material.

MOX fuel rods B4, B14, and K5 were all high initial Pu content, and were located in the interior of the MX03 LTA. MOX fuel rods $\mathrm{C} 1$ (medium $\mathrm{Pu}$ ) and $\mathrm{A} 1$ (low Pu) were irradiated on the outer periphery of the LTA, and with fuel rod A1 located at a vertex location. These two fuel rods are most susceptible to the thermal neutron flux and influence of the neighboring LEU fuel assemblies in the Catawba-1 core, for cycles $\mathrm{C}-16$ and $\mathrm{C}-17$. To determine accurately the compositions of these pins, in particular, details and information about the nearby LEU assemblies, the inter-assembly gaps, burnable poisons in the LEU assemblies and in the MX03 LTA are required. These would allow for fuel rod burnup levels to be determined based on a detailed core model. Preliminary assay measurements conducted at ORNL for the MOX fuel rods suggest that there are differences in isotopics and estimated burnup levels.

The MOX fuel rods were removed from irradiation in the Catawba-1 core in May 2008 and have been cooling since then; the fuel rods arrived at ORNL on January 22, 2009. The ultimate fuel burnup and sample dose equivalent rates for the various MOX fuel rods are dependent on the initial Pu loading of the initial rod fuel material and on the location of the fuel rod within the MOX LTA (MX03). 


\section{REFERENCES}

1. NRC, Supplement No. 3 to safety evaluation by the Office of Nuclear Reactor Regulation and the Office of Nuclear Security and Incident Response related to Amendment No. 220 to renewed Facility Operating License NPF-35 and Amendment No. 215 to renewed Facility Operating License NPF-52 Duke Energy Corporation, et al. Catawba Nuclear Station, Units 1 and 2 Docket Nos. 50-413 and 50-414, March 3, 2005. Available online. URL: http://pbadupws.nrc.gov/docs/ML0423/ML042320059.pdf

2. Duke-Energy website for Catawba Nuclear Station, available online. URL: http://www.dukeenergy.com/power-plants/nuclear/catawba.asp

3. United States Nuclear Regulatory Commission webpage for Catawba Nuclear Station, Unit 1, available online. URL: http://www.nrc.gov/info-finder/reactor/cat1.html

4. Ronald J. Ellis, System Definition Document: Reactor Data Necessary for Modeling Plutonium Disposition in Catawba Nuclear Station Units 1 and 2, ORNL/TM-1999/255, Oak Ridge National Laboratory, Oak Ridge, Tennessee, September 2000.

5. MOX Fuel Design Report, BAW-10238(NP), Revision 1, 43-10238NP-01, NRC ADAMS Accession No. ML031550349, Framatome ANP, Inc., Lynchburg, VA, May 2003.

6. Kevin McCoy, Patrick Blanpain, Robert Morris, Bruce Bevard, "Hot Cell Examination of Weapons-Grade MOX Fuel”, Proceedings of the 2010 LWR Fuel Performance/TopFuel/WRFPM Conference, Orlando, Florida, September 26-29, 2010.

7. SCALE: A Comprehensive Modeling and Simulation Suite for Nuclear Safety Analysis and Design, ORNL/TM-2005/39, Version 6.1, Oak Ridge National Laboratory, Oak Ridge, Tennessee, June 2011. Available from Radiation Safety Information Computational Center as CCC-785. 
APPENDIX A.

SAMPLE TRITON IRRADIATION/DEPLETION CASE

MOXB4X.INP FOR MOX FUEL ROD B4 



\section{APPENDIX A. SAMPLE TRITON IRRADIATION/DEPLETION CASE MOXB4X.INP FOR MOX FUEL ROD B4}

The following is a sample SCALE 6.1.3/TRITON input case used in t-depl mode to model the irradiation of the B4 fuel rod in the MX03 LTA, during cycles C-16 and C-17 in the Catawba-1 PWR core. In the following input file listing, proprietary and non-published details and material specifications are redacted for protection. The simulation calculates the depletion and generation of the fuel composition nuclides during the two cycles of operation, with appropriate down times, and some post-discharge decay time. The materials (for this fuel rod, and the other four) are further decayed with ORIGEN in cases discussed in Appendix B.

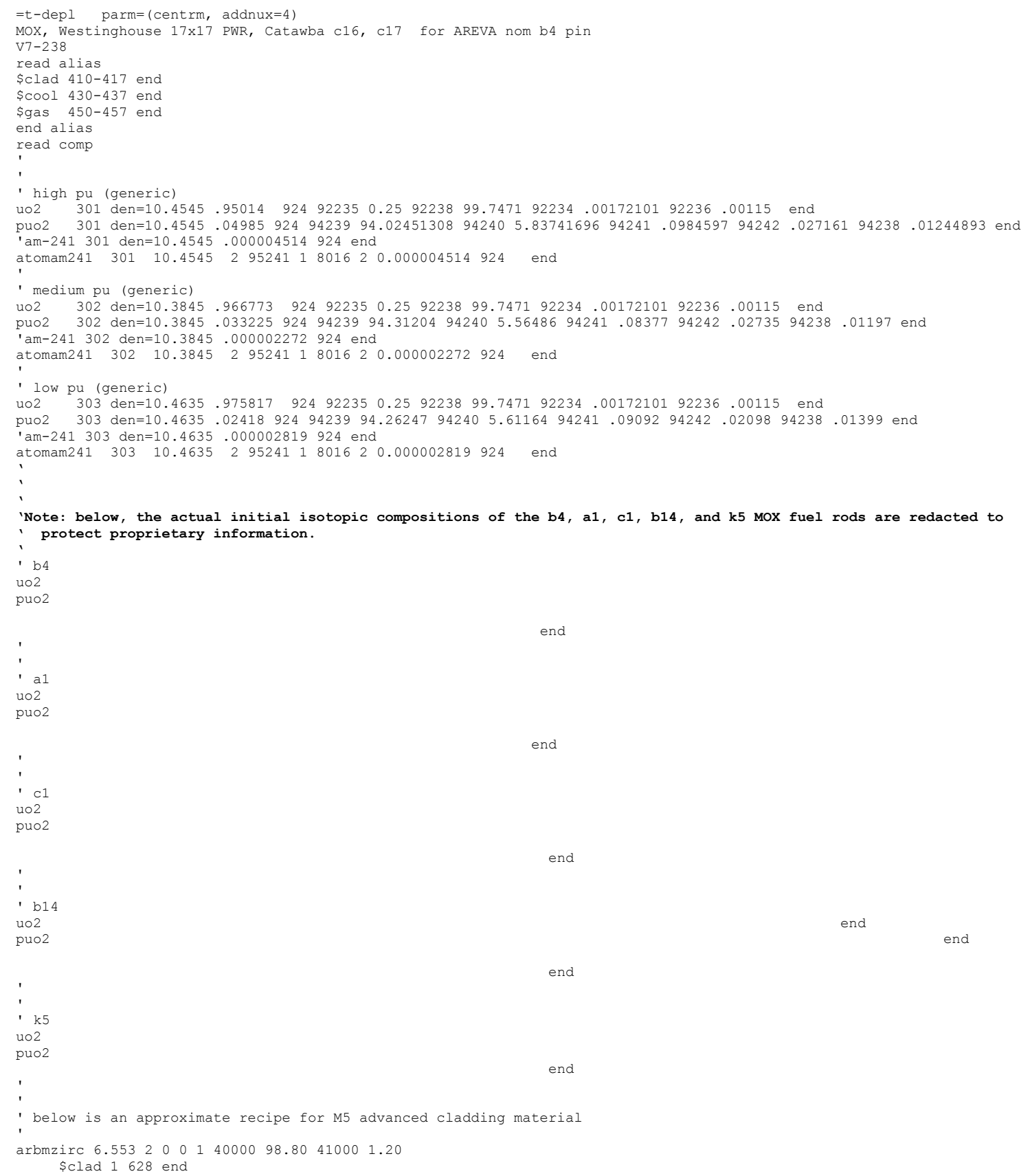




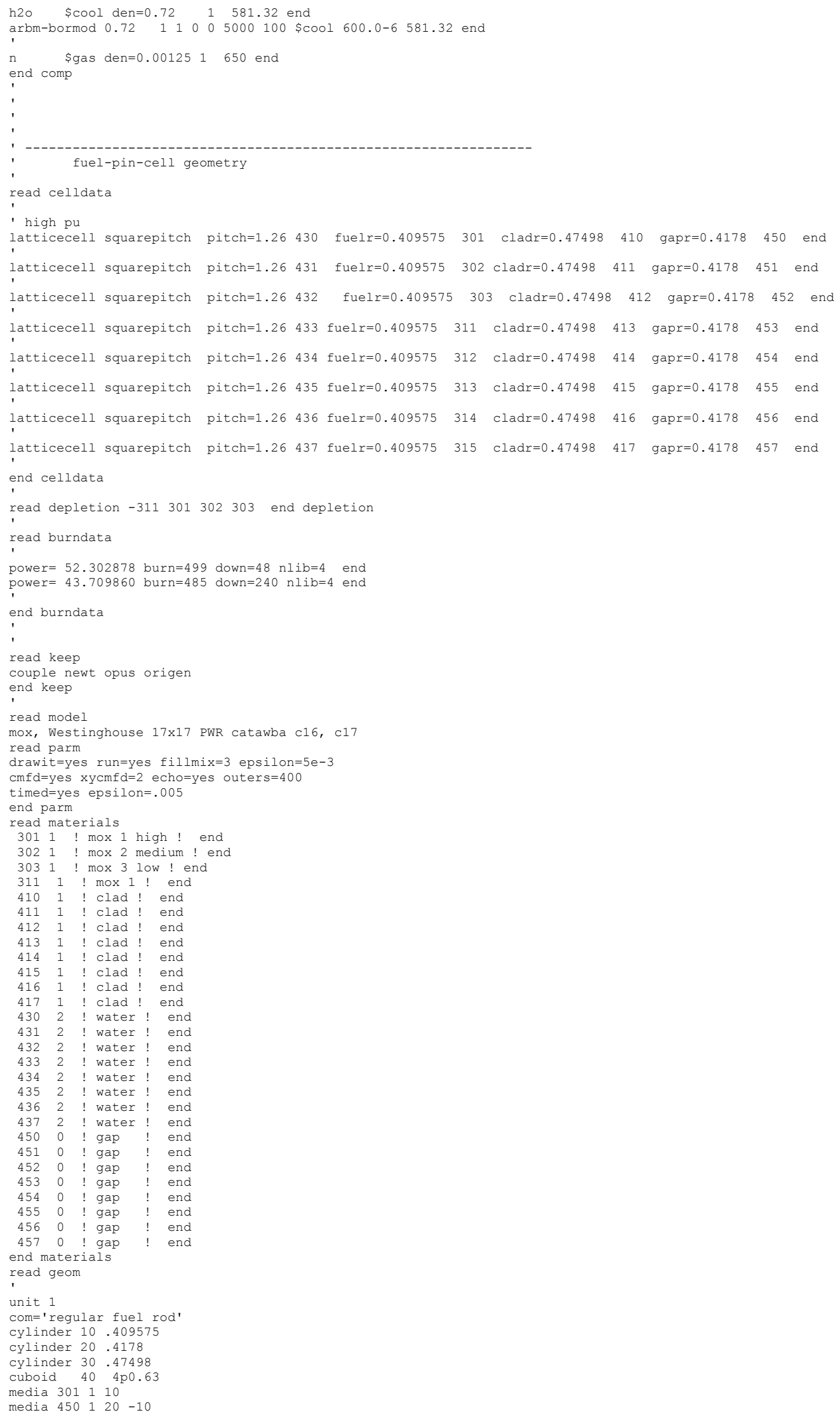


$\begin{array}{lllll}\text { media } & 410 & 1 & 30 & -20\end{array}$

media $430 \quad 1 \quad 40-30$

boundary $40 \quad 4 \quad 4$

unit 2

$\mathrm{com}=$ 'regular fuel rod'

cylinder 10.409575

cylinder 20.4178

cylinder 30.47498

cuboid $40 \quad 4 \mathrm{p} 0.63$

media 302110

media $451 \quad 1 \quad 20 \quad-10$

media $411 \quad 1 \quad 30 \quad-20$

media $431 \quad 1 \quad 40-30$

boundary $40 \quad 4 \quad 4$

unit 3

com='regular fuel rod'

cylinder $10 \quad .40957$

cylinder 20.4178

cylinder 30.47498

cuboid $40 \quad 4 \mathrm{p} 0.63$

media 303110

media $452 \quad 1 \quad 20-10$

media $412 \quad 1 \quad 30-20$

media $432 \quad 1 \quad 40 \quad-30$

boundary 4044

unit 311

com='regular fuel rod'

cylinder 10.409575

cylinder 20.4178

cylinder 30.47498

cuboid $40 \quad 4 \mathrm{p} 0.63$

media 311110

media $454 \quad 1 \quad 20 \quad-10$

media $414 \quad 1 \quad 30 \quad-20$

media $434 \quad 1 \quad 40 \quad-30$

boundary $40 \quad 4 \quad 4$

unit 5

com='guide tube'

cylinder $10 \quad .56135$

cylinder 20.6050

cuboid $40 \quad 4 \mathrm{p} 0.63$

media 430110

media $410 \quad 1 \quad 20-10$

media $430 \quad 1 \quad 40 \quad-20$

boundary $40 \quad 4 \quad 4$

unit 11

com='right half of high fuel rod'

cylinder 10.409575 chord $+x=0$

cylinder 20.4178 chord $+x=0$

cylinder 30.47498 chord $+x=0$

$\begin{array}{lllll}\text { cuboid } 40 & 0.63 & 0.0 & 2 \mathrm{p} 0.63\end{array}$

media 301110

media $450 \quad 1 \quad 20 \quad-10$

media $410 \quad 1 \quad 30-20$

media 430140

boundary $40 \quad 2 \quad 4$

com='right half of medium fuel rod'

cylinder 10.409575 chord $+x=0$

cylinder 20.4178 chord $+x=0$

cylinder 30.47498 chord $+x=0$

$\begin{array}{lllll}\text { cuboid } 40 & 0.63 & 0.0 & 2 \mathrm{p} 0.63\end{array}$

media 302110

media $4 \begin{array}{llll}51 & 1 & 20 & -10\end{array}$

media $411 \quad 1 \quad 30$

media 41

boundary $402 \quad 4$

unit 12

com='top half of high fuel rod

cylinder 10.409575 chord $+\mathrm{y}=0$

cylinder 20.4178 chord $+y=0$

cylinder 30.47498 chord $+y=0$

$\begin{array}{llllll}\text { cuboid } 40 & 2 \mathrm{p} 0.63 & 0.63 & 0.0\end{array}$

media 301110

media $450 \quad 1 \quad 20 \quad-10$

$\begin{array}{lllll}\text { media } 410 & 1 & 30 & -20\end{array}$

media 4301

media 430

unit 22

com='top half of medium fuel rod'

cylinder 10.409575 chord $+y=0$

cylinder 20.4178 chord $+y=0$

cylinder 30.47498 chord $+y=0$

cuboid $40 \quad 2 \mathrm{p} 0.63 \quad 0.63 \quad 0.0$

media 302110

media $\begin{array}{lllll}451 & 1 & 20 & -10\end{array}$ 
media $411 \quad 1 \quad 30 \quad-20$

media $431 \quad 1 \quad 40-30$

boundary 4042

unit 51

com='right half of guide tube'

cylinder $10 \quad .56135$ chord $+x=0$

cylinder 20.6050 chord $+x=0$

$\begin{array}{lllll}\text { cuboid } 40 & 0.63 & 0.0 & 2 \mathrm{p} 0.63\end{array}$

media 430110

media $4 \begin{array}{llll}410 & 1 & 20 & -10\end{array}$

media $430 \quad 1 \quad 40 \quad-20$

boundary 4024

unit 52

com='top half of guide tube'

cylinder 10.56135 chord $+y=0$

cylinder 20.6050 chord $+y=0$

$\begin{array}{lllll}\text { cuboid } 40 & 2 \mathrm{p} 0.63 & 0.63 & 0.0\end{array}$

media $430 \quad 110$

media $4 \begin{array}{llll}10 & 1 & 20 & -10\end{array}$

media $430 \quad 1 \quad 40 \quad-20$

boundary $40 \quad 4 \quad 2$

unit 53

com=' $1 / 4$ instrument tube'

cylinder 10.56135 chord $+x=0$ chord $+y=0$

cylinder 20.6050 chord $+x=0$ chord $+y=0$

$\begin{array}{lllllll}\text { cuboid } 40 & 0.63 & 0.0 & 0.63 & 0.0\end{array}$

media 430110

media $4 \begin{array}{llll}410 & 1 & 20 & -10\end{array}$

media $430 \quad 1 \quad 40 \quad-20$

boundary 4022

global unit 10

cuboid $\begin{array}{llllll}10 & 10.71 & 0.0 & 10.71 & 0.0\end{array}$

array $1 \quad 10$ place 11100

media 430110

boundary $10 \quad 36 \quad 36$

end geom

read array

ara=1 nux=9 nuy=9 typ=cuboidal fill

$\begin{array}{lllllllll}53 & 12 & 12 & 52 & 12 & 12 & 52 & 22 & 22\end{array}$

$\begin{array}{lllllllll}11 & 1 & 1 & 1 & 1 & 1 & 1 & 1 & 2\end{array}$

$\begin{array}{lllllllll}11 & 1 & 1 & 1 & 1 & 1 & 1 & 1 & \end{array}$

$\begin{array}{lllllllll}51 & 1 & 1 & 5 & 1 & 1 & 5 & 2 & 2 \\ 11 & 1 & 1 & 1 & 1 & 1 & 1 & 1 & 2\end{array}$

$\begin{array}{lllllllll}11 & 1 & 1 & 1 & 1 & 5 & 1 & 311 & 2\end{array}$

$\begin{array}{lllllllll}51 & 1 & 1 & 5 & 1 & 1 & 1 & 2 & 2 \\ 21 & 1 & 1 & 2 & 1 & 1 & 2 & 2 & 3\end{array}$

$\begin{array}{llllllllll}21 & 2 & 2 & 2 & 2 & 2 & 2 & 3 & 3\end{array}$ end fill

end array

read bounds all=refl end bounds

end model

end

cp ft71f001 \$RTNDIR/ft71_mox_b4x

end 
APPENDIX B.

ORIGEN DECAY CASES FOR THE FIVE SELECTED MOX FUEL RODS 



\section{APPENDIX B. ORIGEN RESULTS FOR COOLING MOX FUEL RODS}

\section{B.1 ORIGEN RESULTS FOR COOLING MOX FUEL ROD B4}

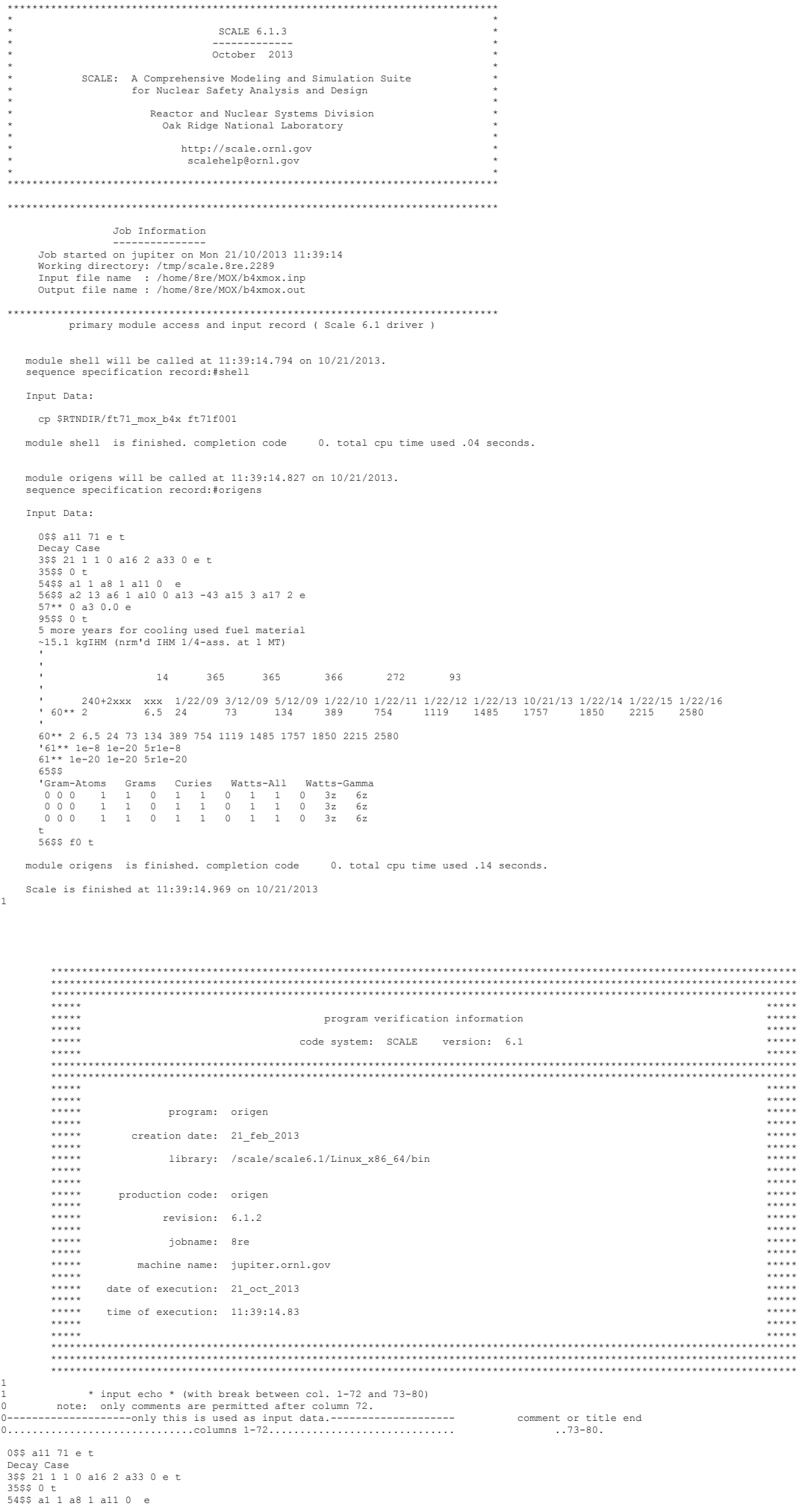

B-3 
$565 \$$ a2 13 a 6 r a 100 a13 -43 a15 3 a17 2 e
$57 * \star 00$ a 30.0 e

5 more years for cooling used fuel material
15.1 kgIHM (nrm'd IHM $1 / 4$-ass. at 1 MT)

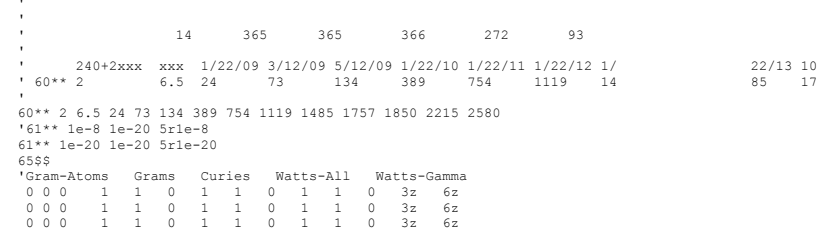

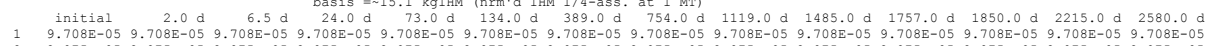

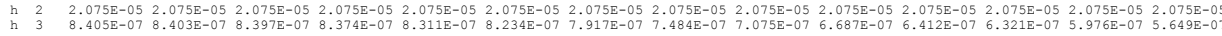

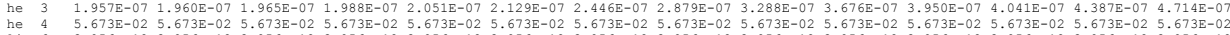

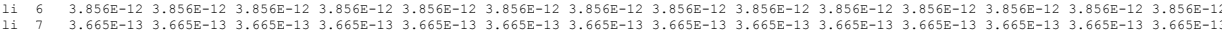

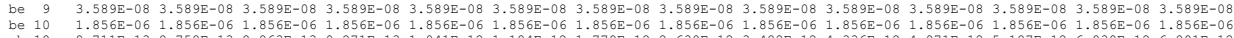

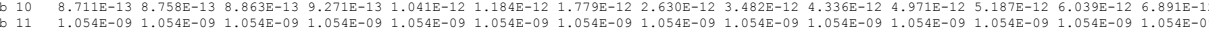

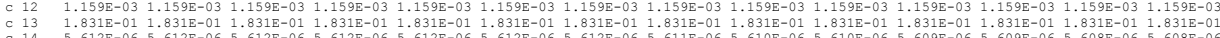

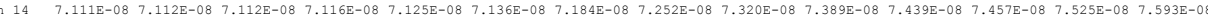

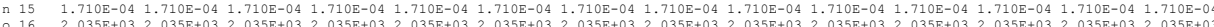

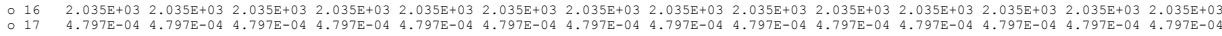

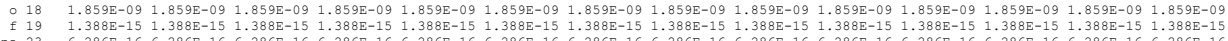

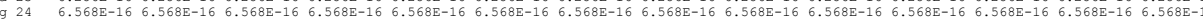

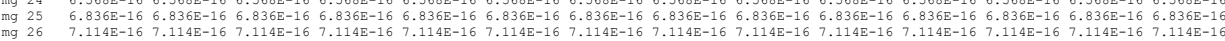

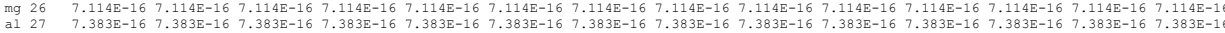

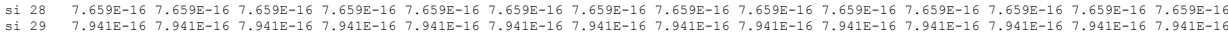

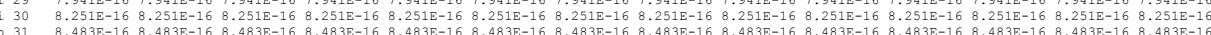

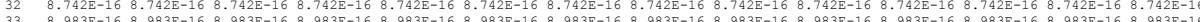

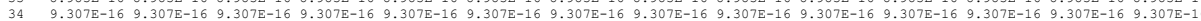
$36 \quad 9.844 \mathrm{E}-16 \quad 9.844 \mathrm{E}-16 \quad 9.844 \mathrm{E}-16 \quad 9.844 \mathrm{E}-16 \quad 9.844 \mathrm{E}-16 \quad 9.844 \mathrm{E}-16 \quad 9.844 \mathrm{E}-16 \quad 9.844 \mathrm{E}-16 \quad 9.844 \mathrm{E}-16 \quad 9.844 \mathrm{E}-16 \quad 9.844 \mathrm{E}-16 \quad 9.844 \mathrm{E}-16 \quad 9.844 \mathrm{E}-16 \quad 9.844 \mathrm{E}-16$

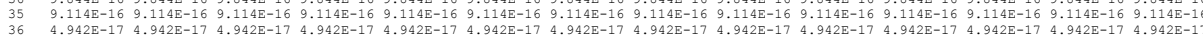

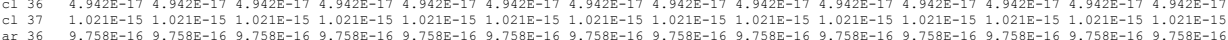

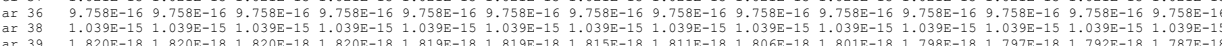

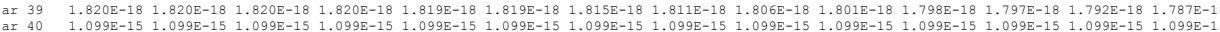

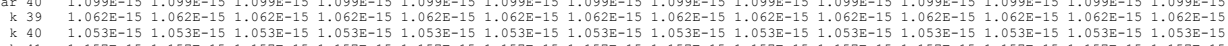

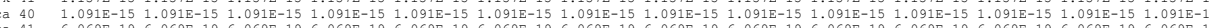

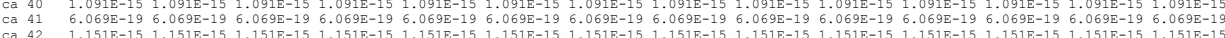

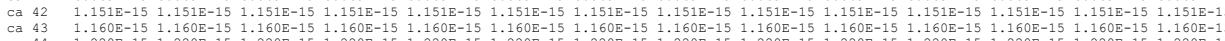

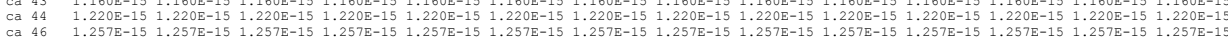

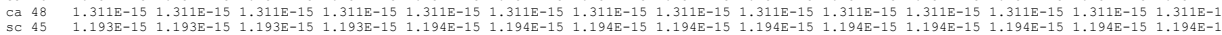




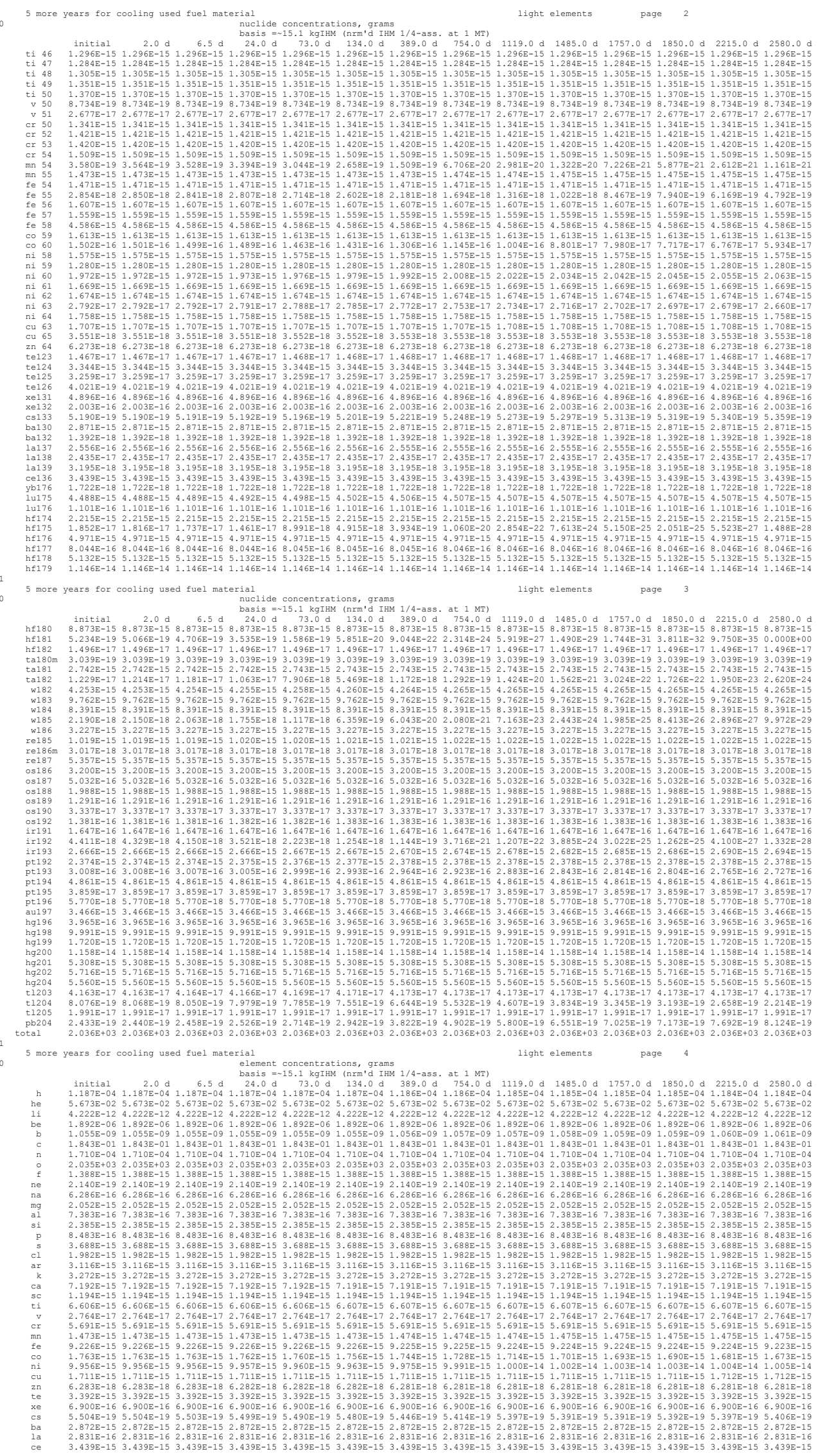

B-5 


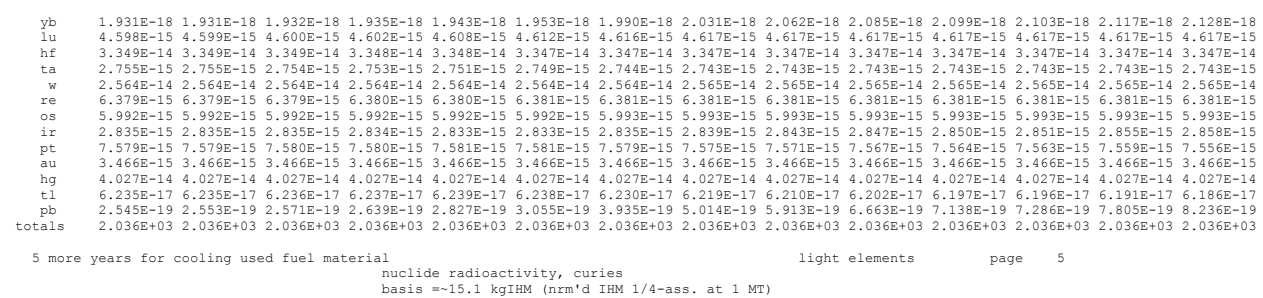

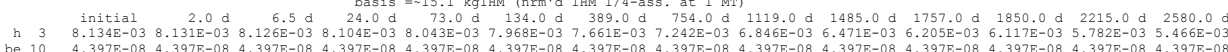

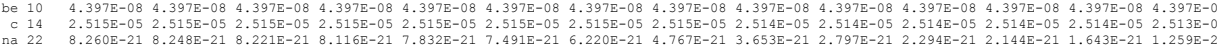

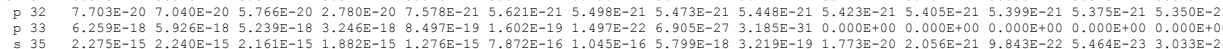

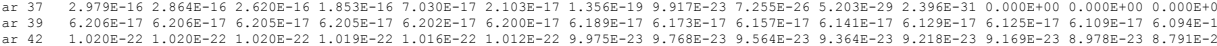

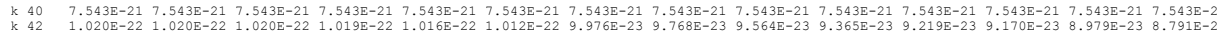

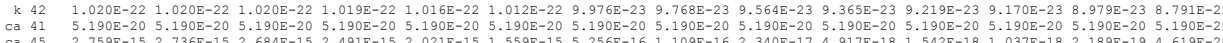

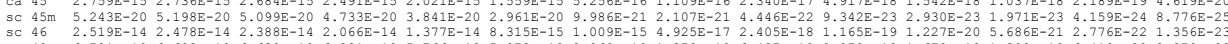

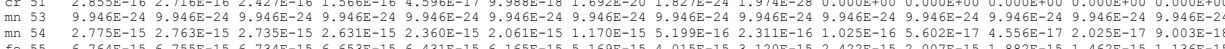

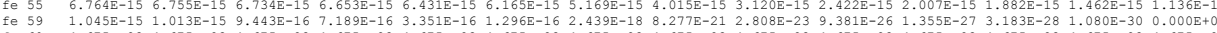

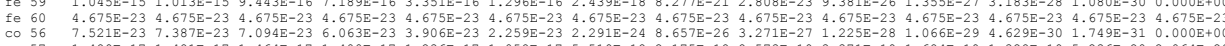

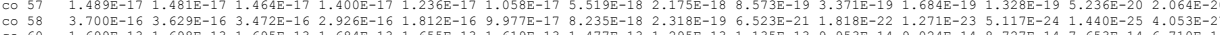

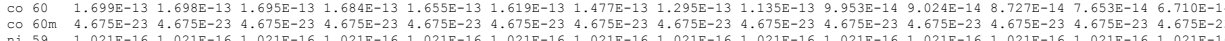

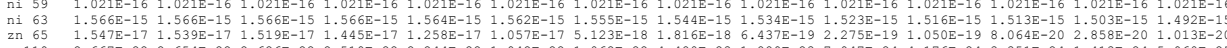

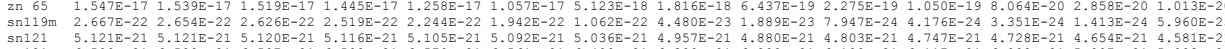

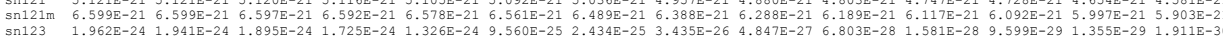

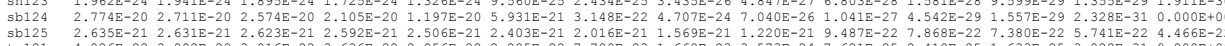

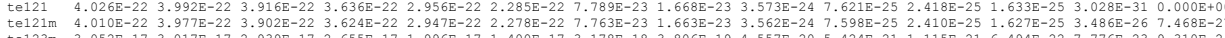

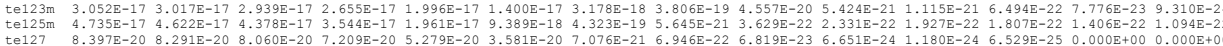

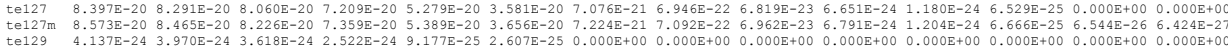

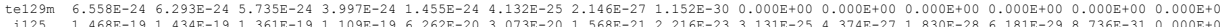

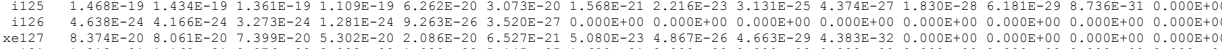

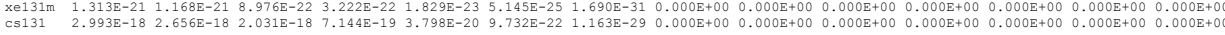

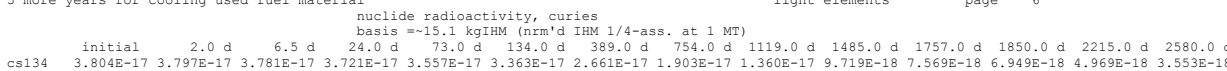

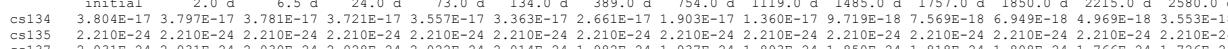

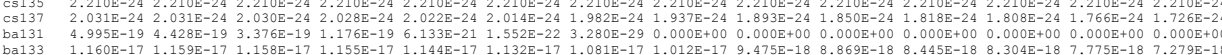

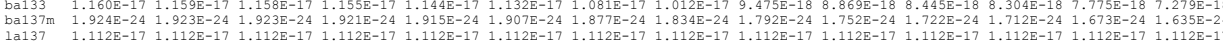

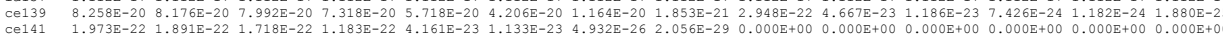

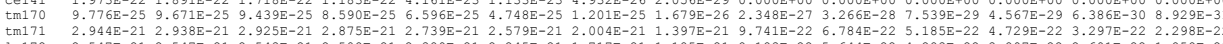

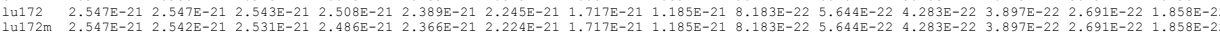

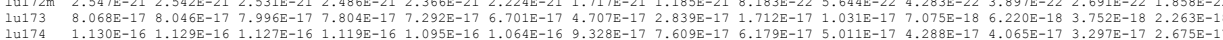

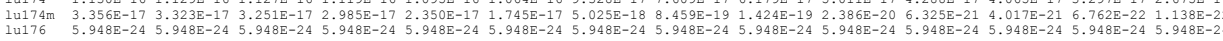

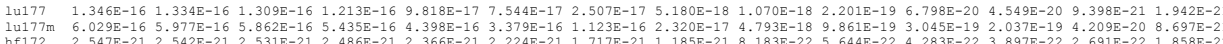

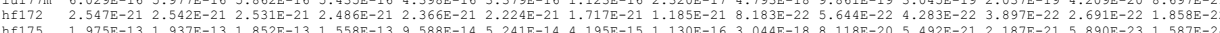

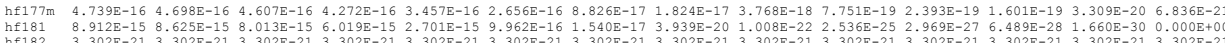

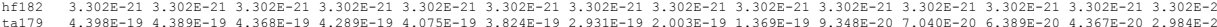

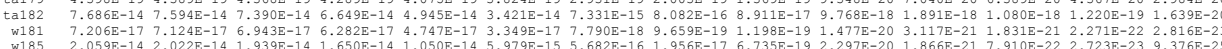

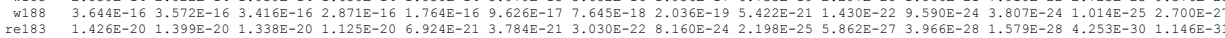

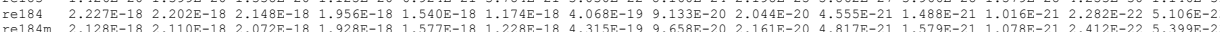

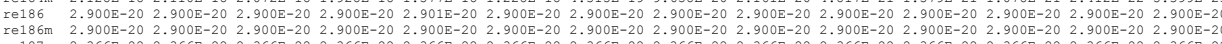

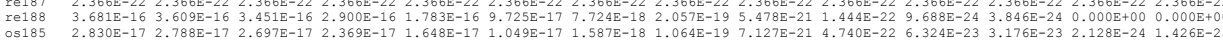

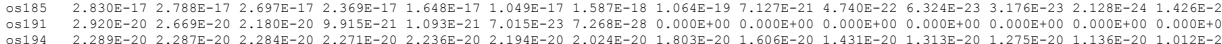

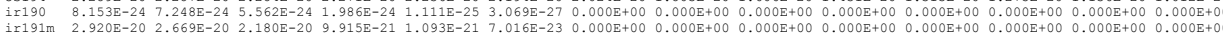

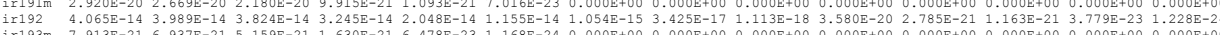

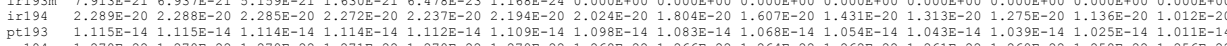

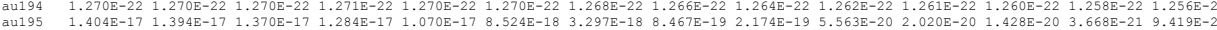

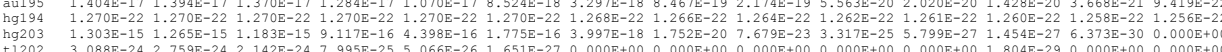

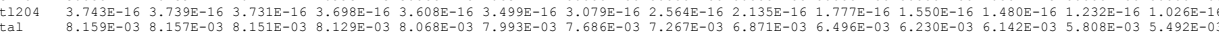
5 more years for cooling used fuel material

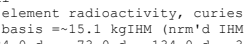
light elements page

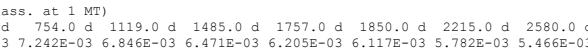

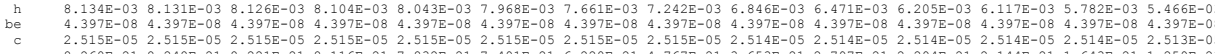

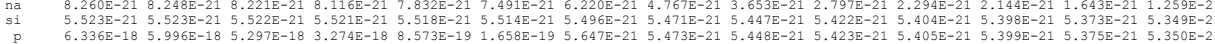

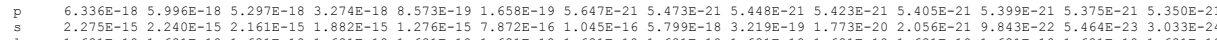

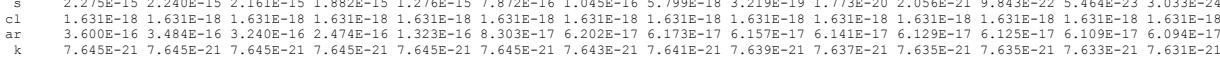

B-6 


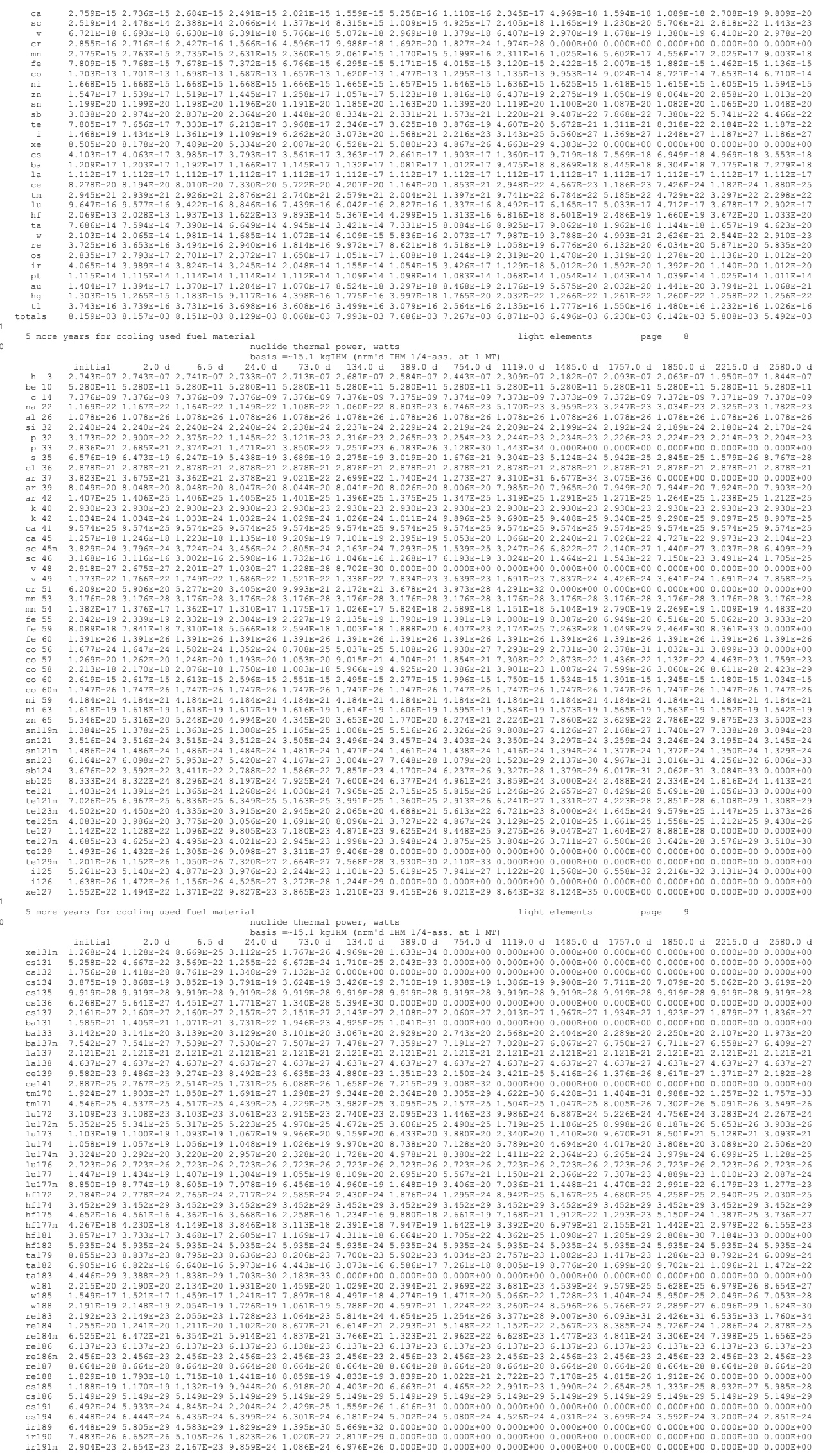

B-7 


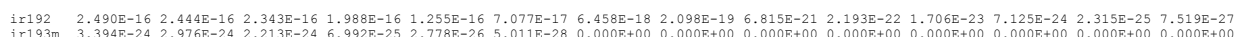

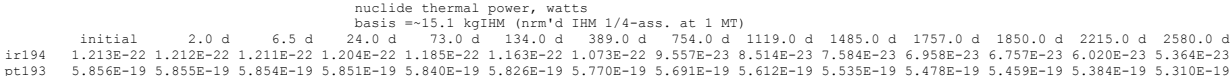

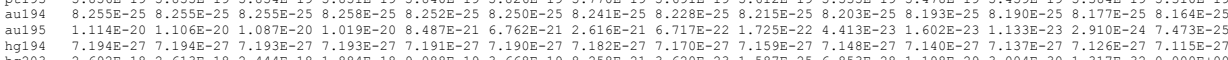

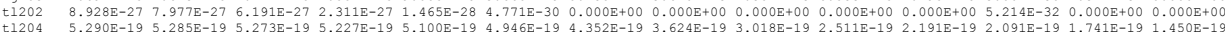

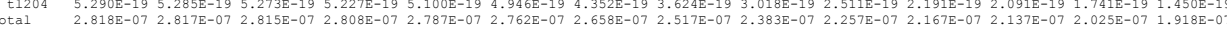

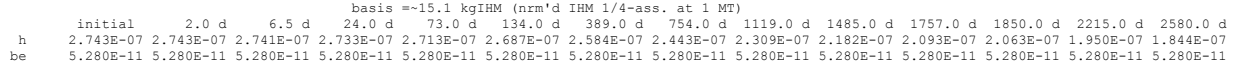

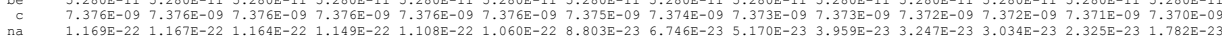

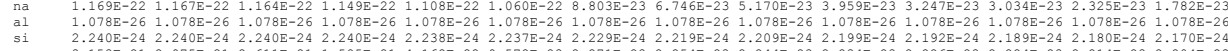

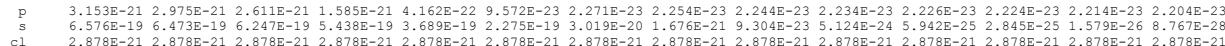

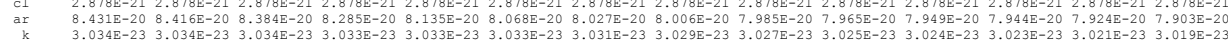

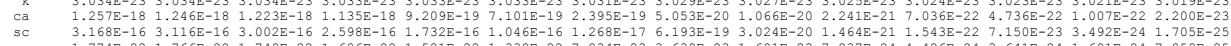

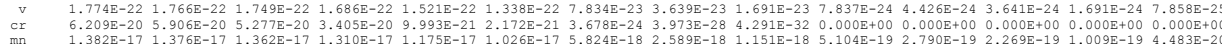

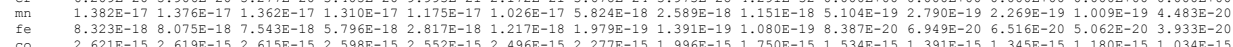

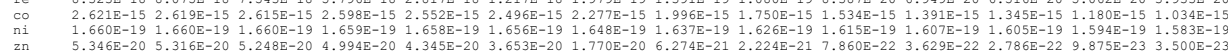

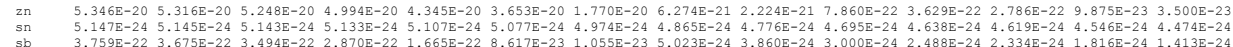

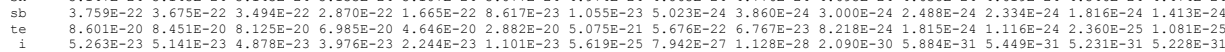

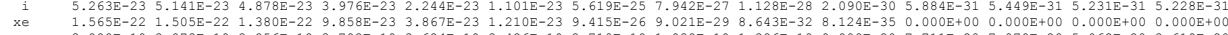

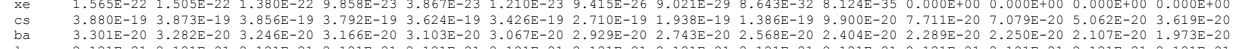

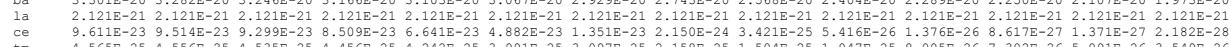

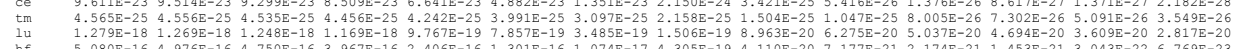

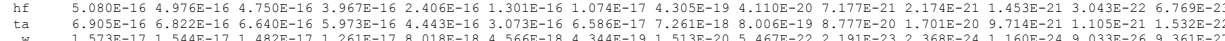

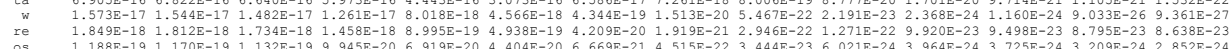

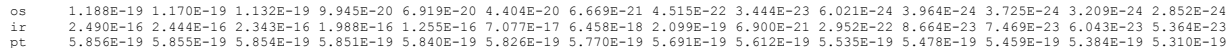

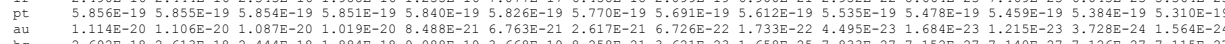

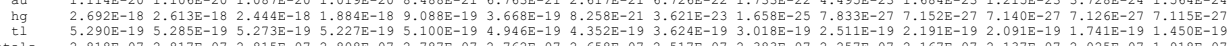
totals $2.818 \mathrm{E}-072.817 \mathrm{E}-072.815 \mathrm{E}-07 \quad 2.80$ nuclide concentrations, grams
basis $=\sim 15.1 \mathrm{kgIHM}$ (nrm'd IHM $1 / 4-$ a actinides page 12

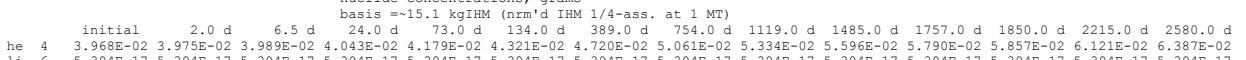

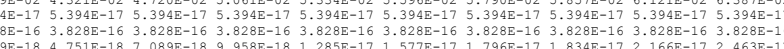

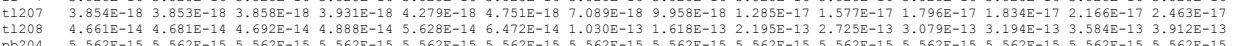

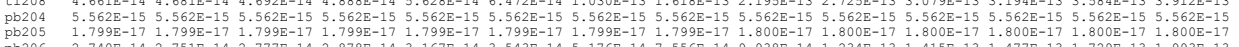

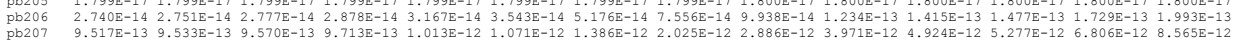

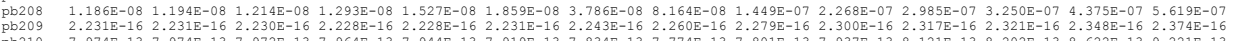

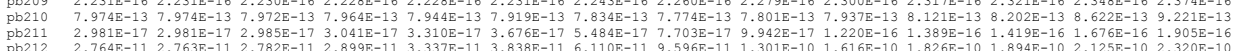

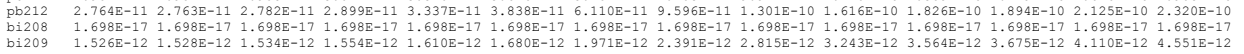

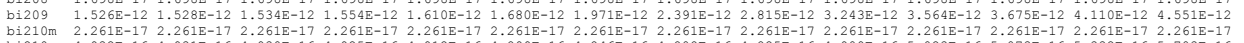

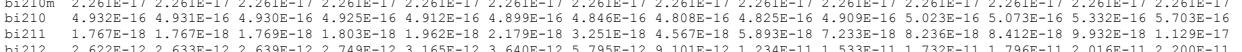

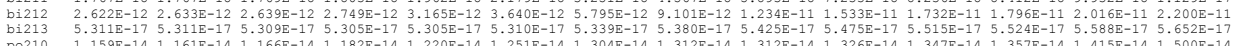

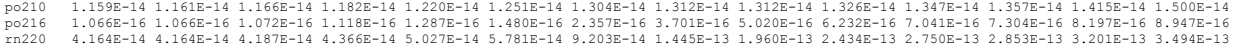

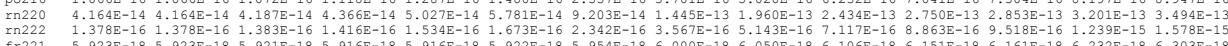

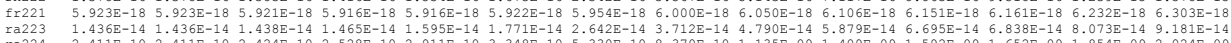

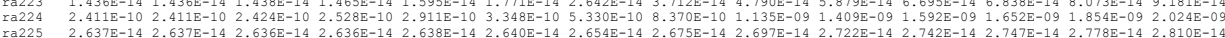

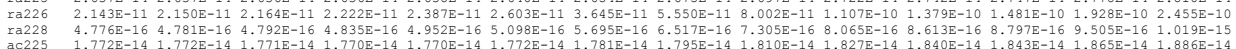
$\begin{array}{llllllllllllllll}\text { ac225 } & 1.772 \mathrm{E}-14 & 1.772 \mathrm{E}-14 & 1.771 \mathrm{E}-14 & 1.770 \mathrm{E}-14 & 1.770 \mathrm{E}-14 & 1.772 \mathrm{E}-14 & 1.781 \mathrm{E}-14 & 1.795 \mathrm{E}-14 & 1.810 \mathrm{E}-14 & 1.827 \mathrm{E}-14 & 1.840 \mathrm{E}-14 & 1.843 \mathrm{E}-14 & 1.865 \mathrm{E}-14 & 1.886 \mathrm{E}-14 \\ \text { ac227 } & 1.068 \mathrm{E}-11 & 1 & 072 \mathrm{E}-11 & 1.081 \mathrm{E}-11 & 1.117 \mathrm{E}-11 & 1.217 \mathrm{E}-11 & 1.343 \mathrm{E}-11 & 1.868 \mathrm{E}-11 & 2.625 \mathrm{E}-11 & 3.387 \mathrm{E}-11 & 4.156 \mathrm{E}-11 & 4.731 \mathrm{E}-11 & 4.929 \mathrm{E}-11 & 5.706 \mathrm{E}-11 & 6.489 \mathrm{E}-11\end{array}$

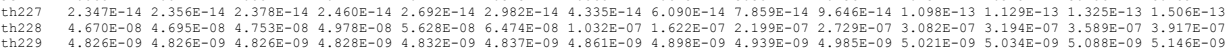

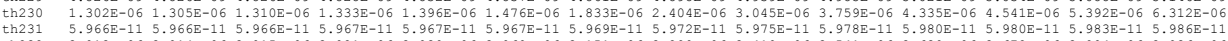

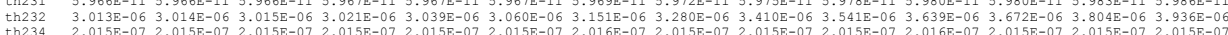

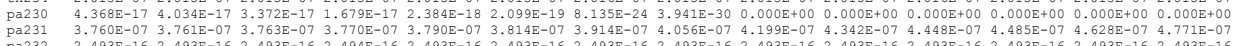

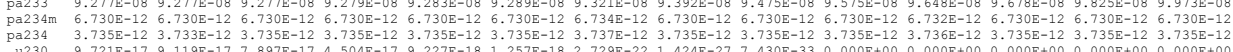

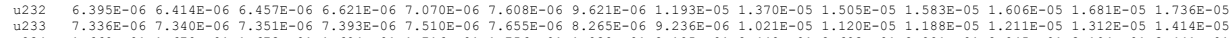

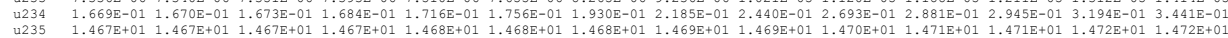

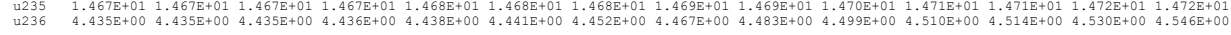
5 more years for cooling used fuel material $\quad$ actinides page 13

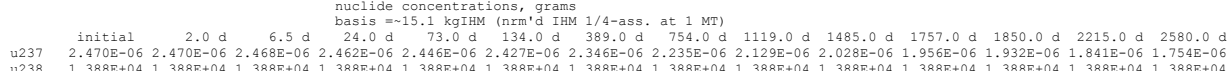

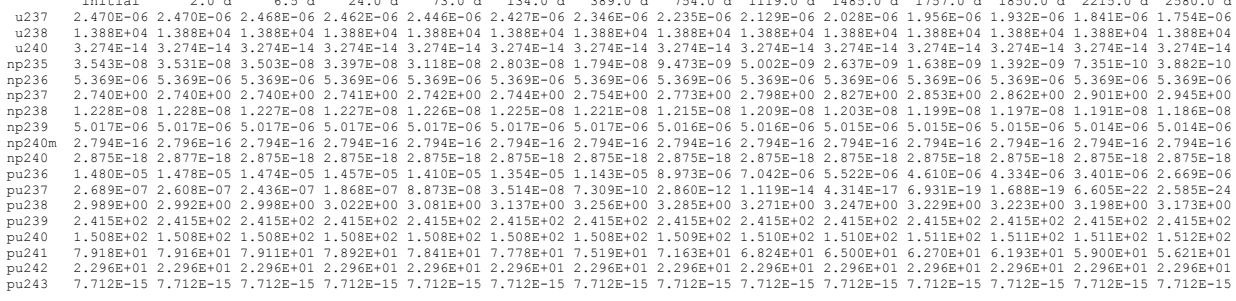




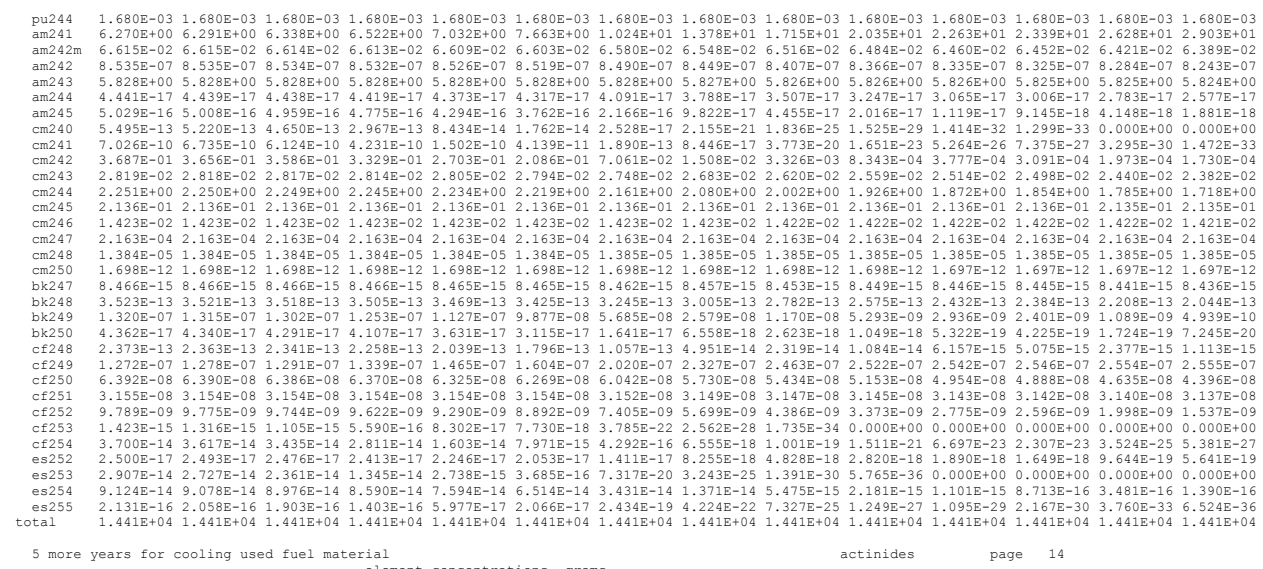

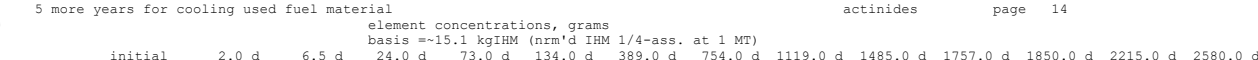

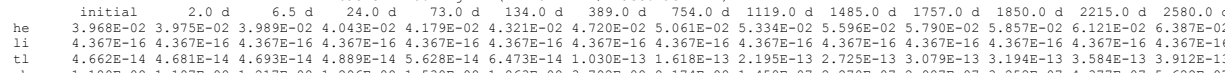

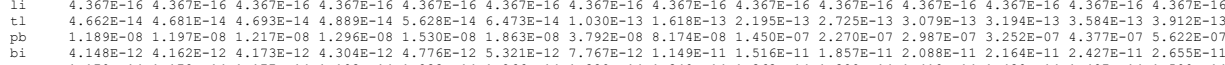

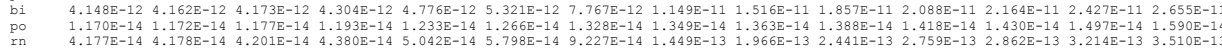

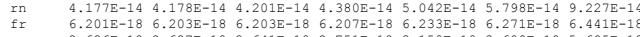

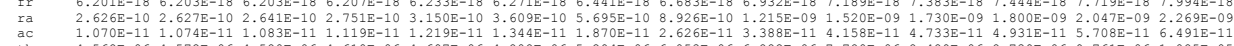

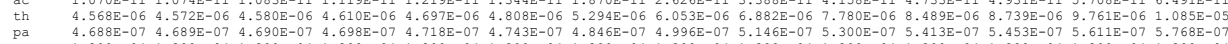

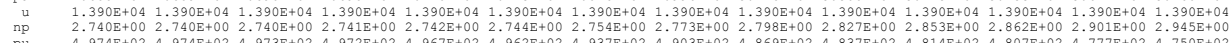

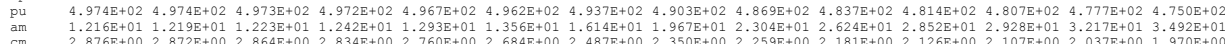

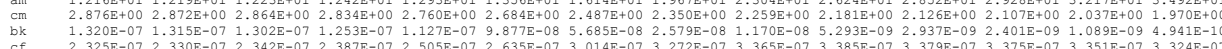

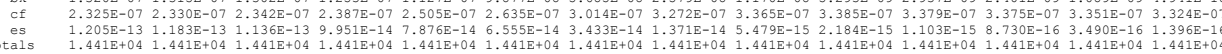

5 more years for cooling used fuel materiat

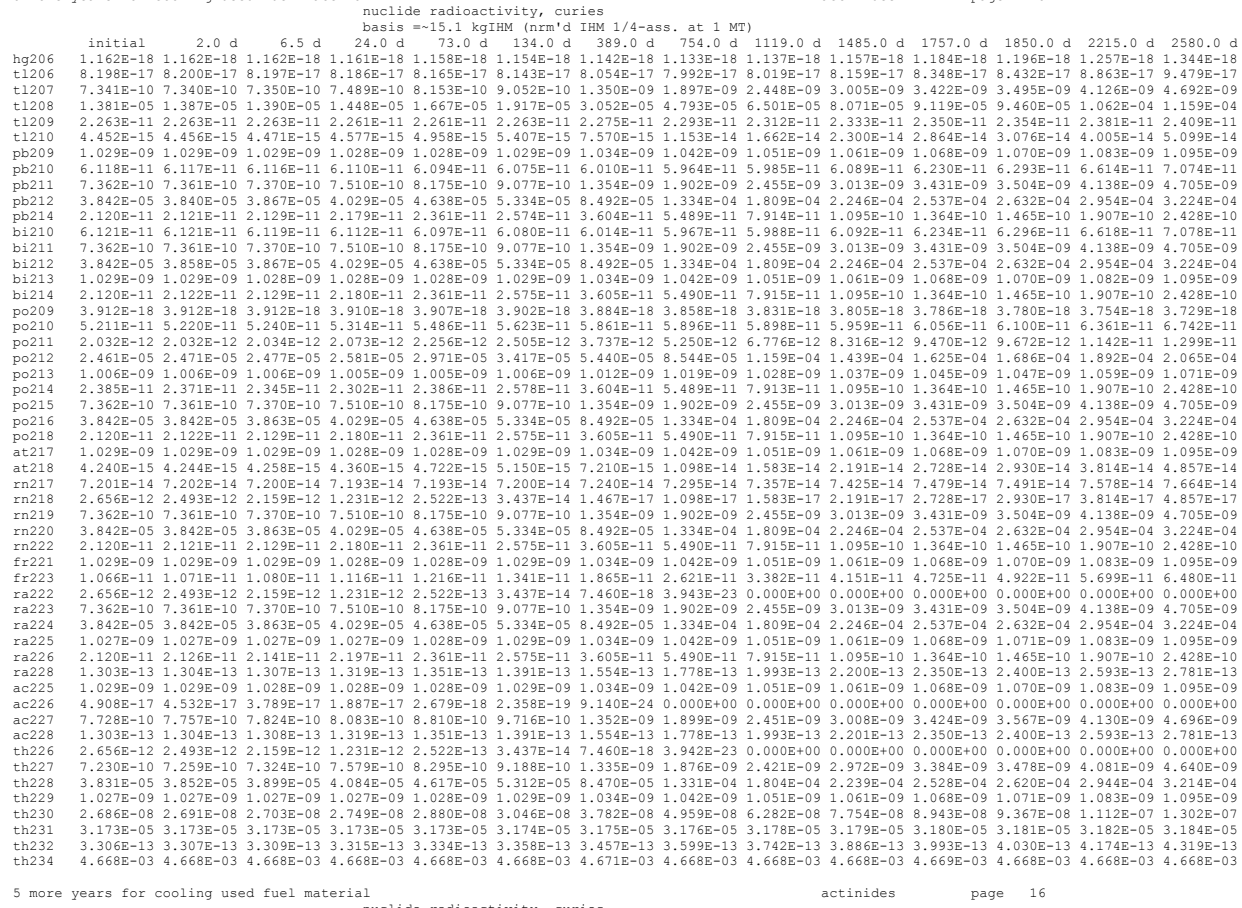

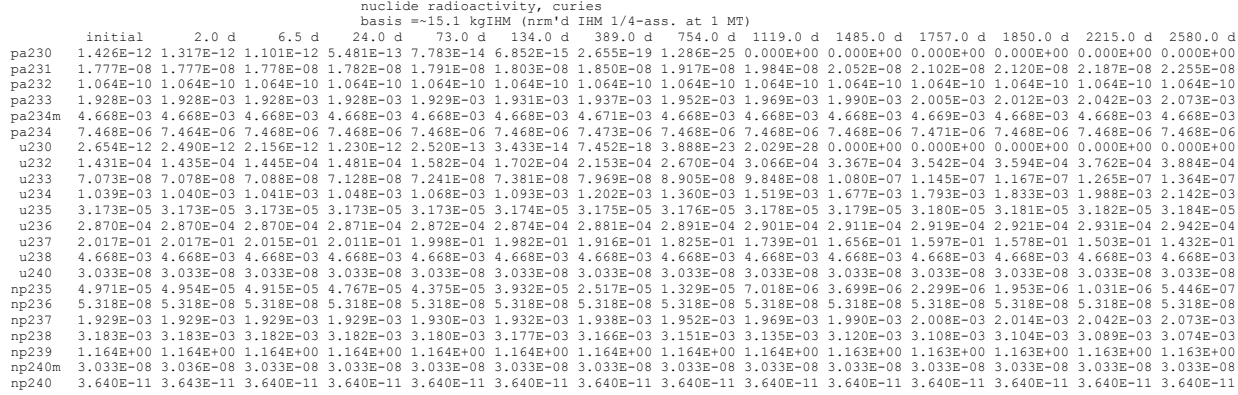

B-9 


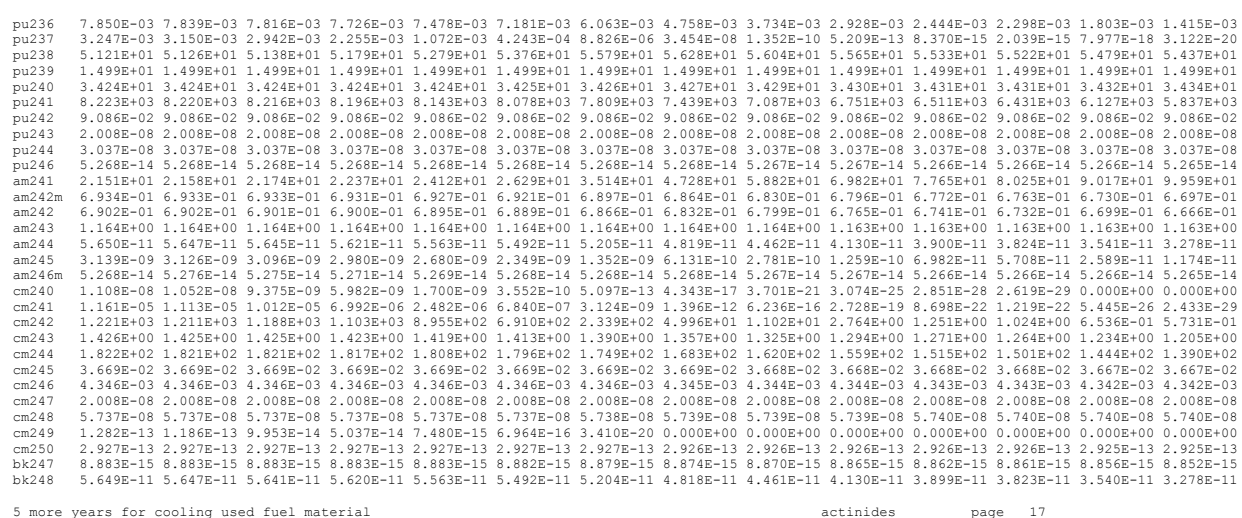
5 more years for cooling used fuel material

$$
\begin{aligned}
& \text { actinides page } 17 \\
& \text { nuclide radioactivity, curies } \\
& \text { basis }
\end{aligned}
$$

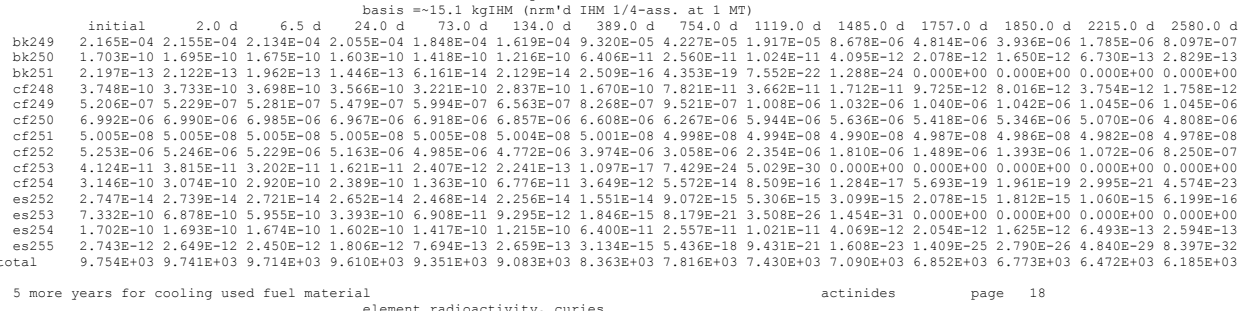

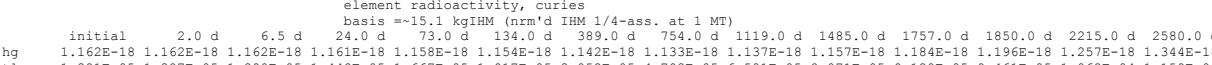

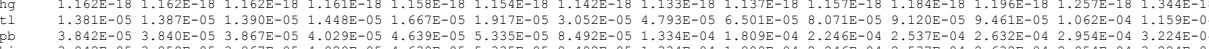

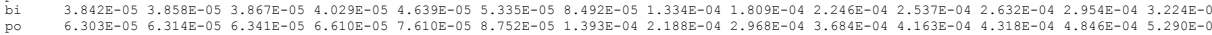

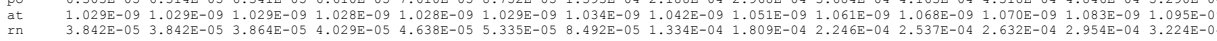

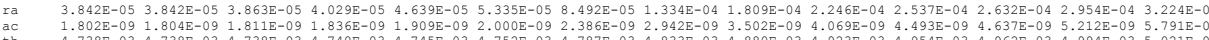

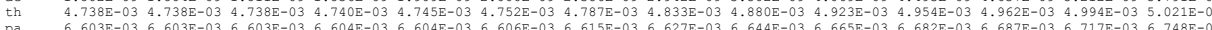

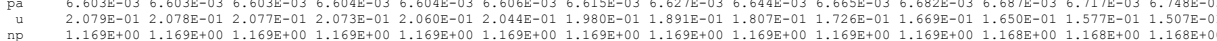

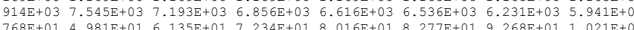

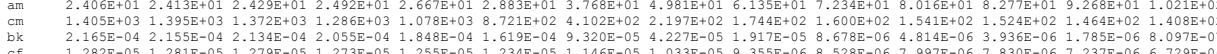

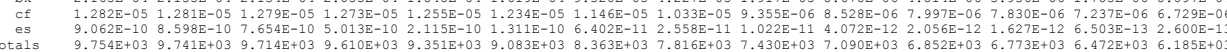

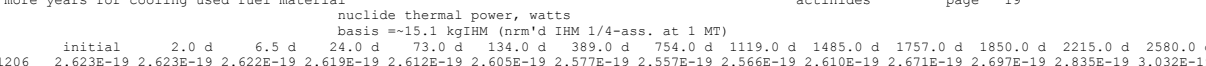
actinides page 19

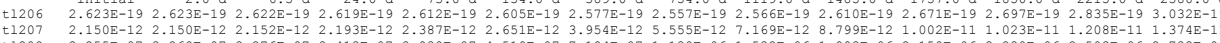

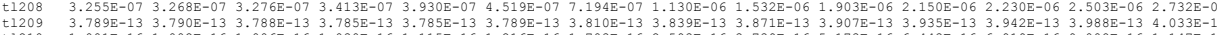

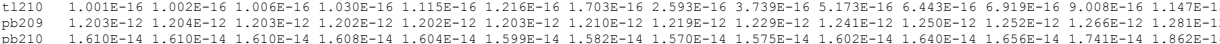

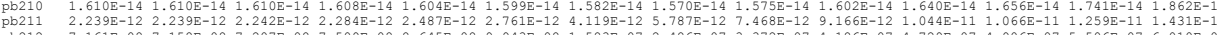
$\begin{array}{llllllllllllllll}\mathrm{pb} 212 & 7.161 \mathrm{E}-08 & 7.158 \mathrm{E}-08 & 7.207 \mathrm{E}-08 & 7.509 \mathrm{E}-08 & 8.645 \mathrm{E}-08 & 9.943 \mathrm{E}-08 & 1.583 \mathrm{E}-07 & 2.486 \mathrm{E}-07 & 3.372 \mathrm{E}-07 & 4.186 \mathrm{E}-07 & 4.729 \mathrm{E}-07 & 4.906 \mathrm{E}-07 & 5.506 \mathrm{E}-07 & 6.010 \mathrm{E}-0 \\ \mathrm{pb} 214 & 6.607 \mathrm{E}-14 & 6.613 \mathrm{E}-14 & 6.635 \mathrm{E}-14 & 6.793 \mathrm{E}-14 & 7.358 \mathrm{E}-14 & 8.025 \mathrm{E}-14 & 1.123 \mathrm{E}-13 & 1.711 \mathrm{E}-13 & 2.467 \mathrm{E}-13 & 3.413 \mathrm{E}-13 & 4.251 \mathrm{E}-13 & 4.565 \mathrm{E}-13 & 5.943 \mathrm{E}-13 & 7.568 \mathrm{E}-1\end{array}$

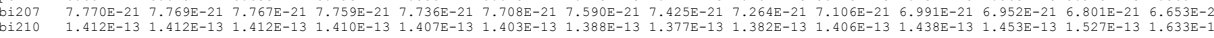

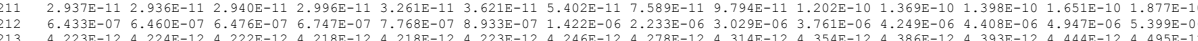

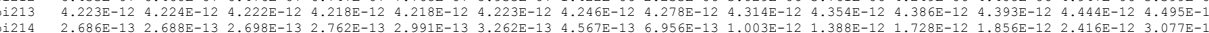

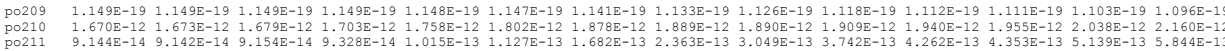

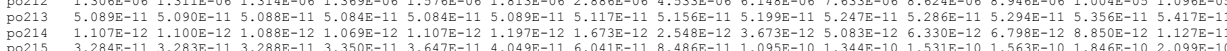
$\begin{array}{llllllllll} & \end{array}$

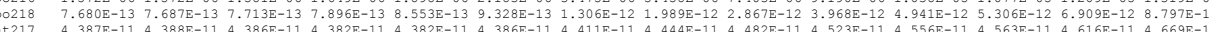
(15 $3.501 \mathrm{E}-15 \quad 3.541 \mathrm{E}-15 \quad 3.582 \mathrm{E}-15$

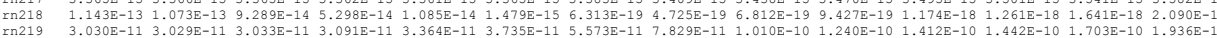

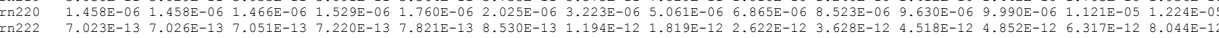

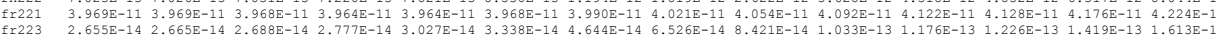

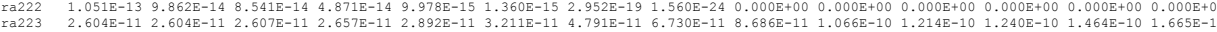

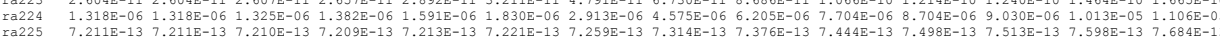

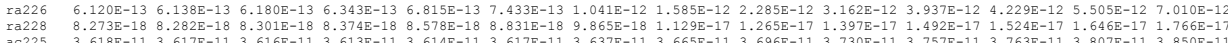

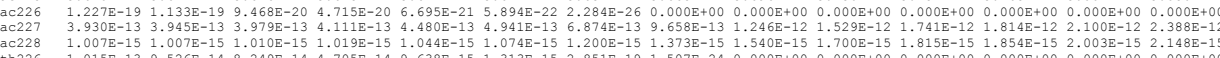
$\begin{array}{lllllllllllllllll}n & 2.668 \mathrm{E}-11 & 2.679 \mathrm{E}-11 & 2.703 \mathrm{E}-11 & 2.797 \mathrm{E}-11 & 3.061 \mathrm{E}-11 & 3.390 \mathrm{E}-11 & 4.928 \mathrm{E}-11 & 6.923 \mathrm{E}-11 & 8.935 \mathrm{E}-11 & 1.097 \mathrm{E}-10 & 1.249 \mathrm{E}-10 & 1.284 \mathrm{E}-10 & 1.506 \mathrm{E}-10 & 1.712 \mathrm{E}-10\end{array}$ $\begin{array}{lllllllllllllll}\operatorname{th} 228 & 1.254 \mathrm{E}-06 & 1.261 \mathrm{E}-06 & 1.276 \mathrm{E}-06 & 1.337 \mathrm{E}-06 & 1.511 \mathrm{E}-06 & 1.739 \mathrm{E}-06 & 2.773 \mathrm{E}-06 & 4.355 \mathrm{E}-06 & 5.905 \mathrm{E}-06 & 7.329 \mathrm{E}-06 & 8.276 \mathrm{E}-06 & 8.576 \mathrm{E}-06 & 9.638 \mathrm{E}-06 & 1.052 \mathrm{E}-0 \\ \mathrm{th} 229 & 3.198 \mathrm{E}-11 & 3.198 \mathrm{E}-11 & 3.198 \mathrm{E}-11 & 3.199 \mathrm{E}-11 & 3.202 \mathrm{E}-11 & 3.206 \mathrm{E}-11 & 3.221 \mathrm{E}-11 & 3.246 \mathrm{E}-11 & 3.273 \mathrm{E}-11 & 3.303 \mathrm{E}-11 & 3.328 \mathrm{E}-11 & 3.336 \mathrm{E}-11 & 3.372 \mathrm{E}-11 & 3.410 \mathrm{E}-1\end{array}$

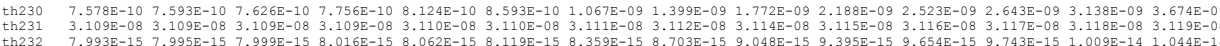

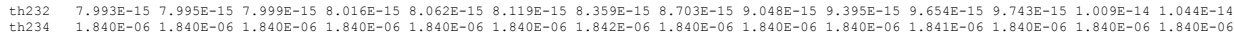

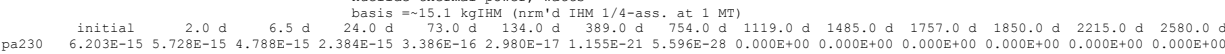

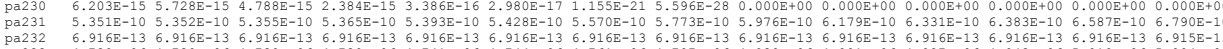

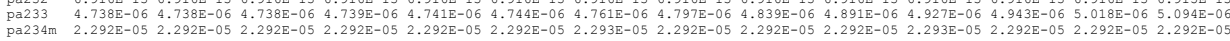



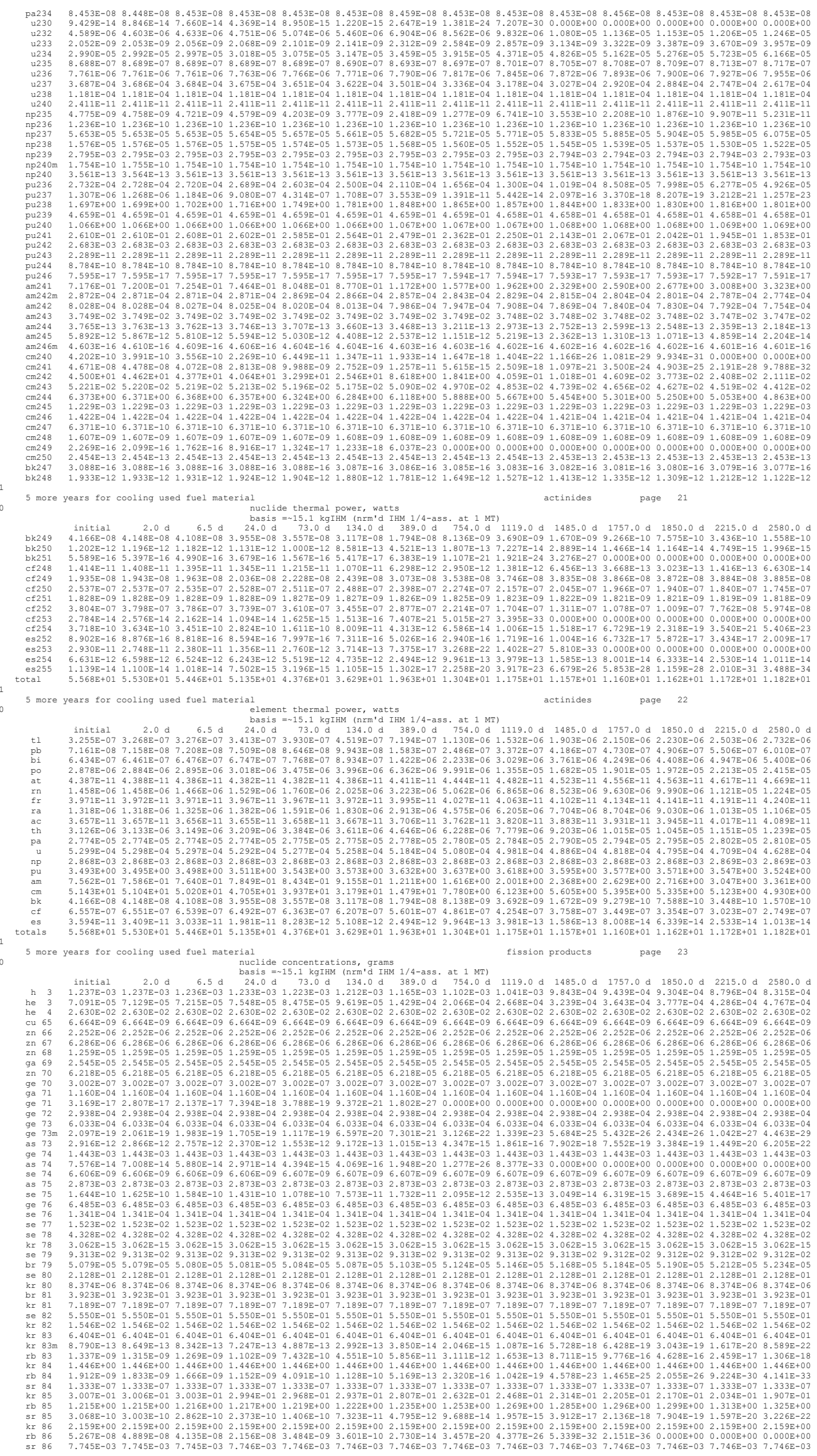

B-11 


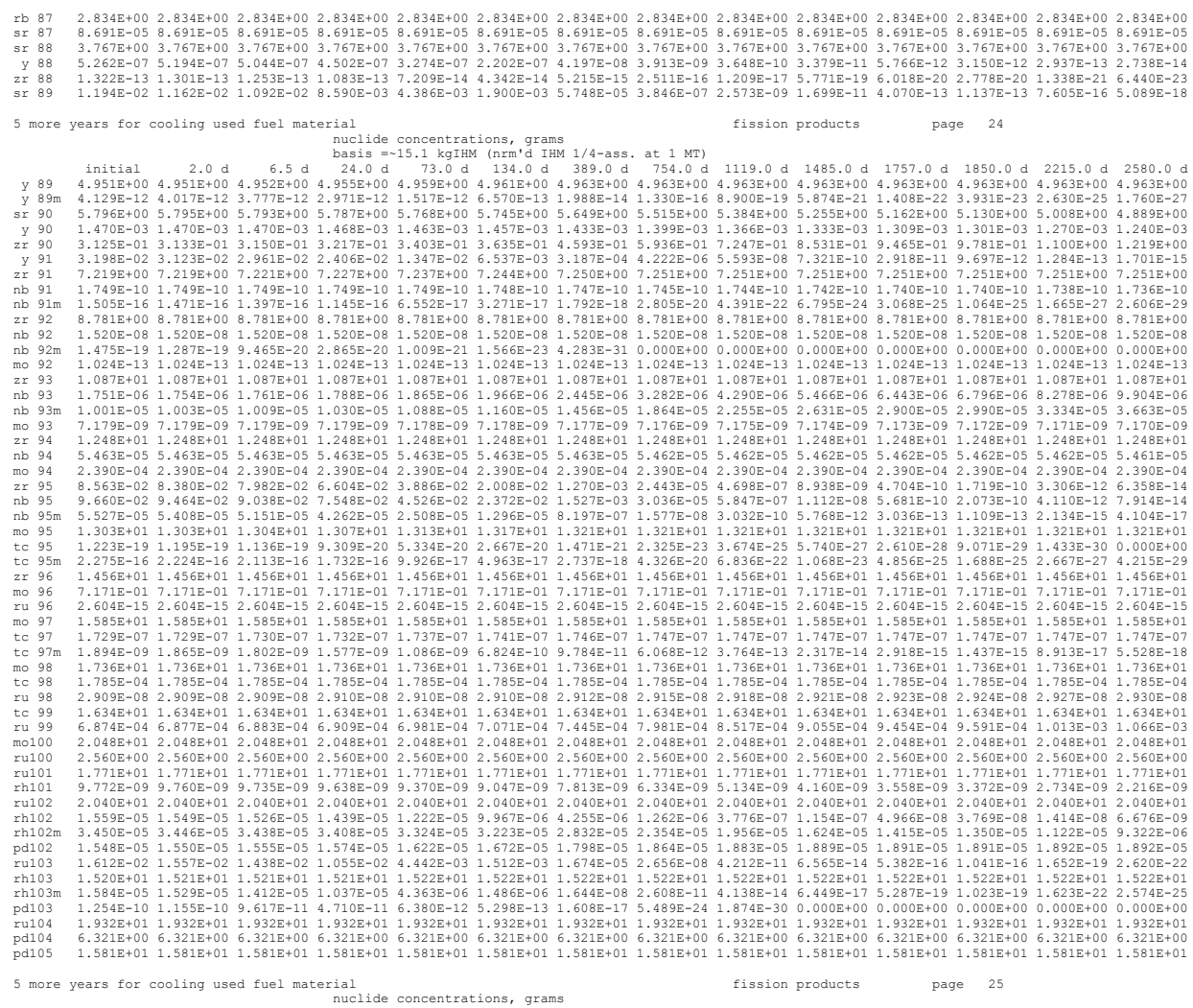

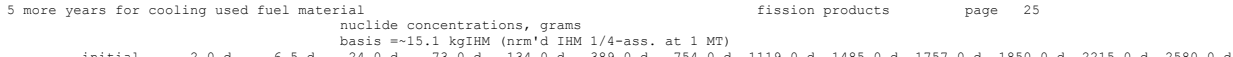

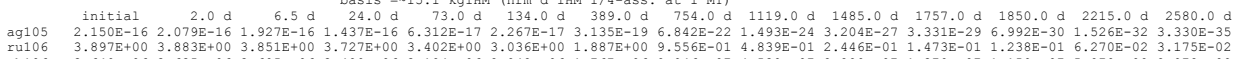

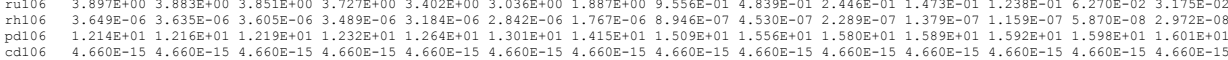

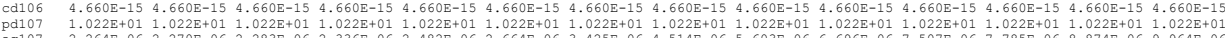

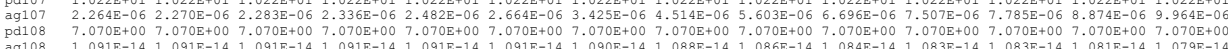

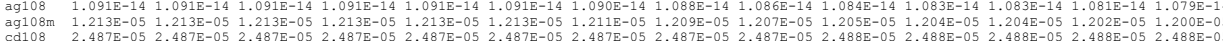

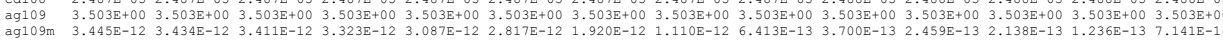

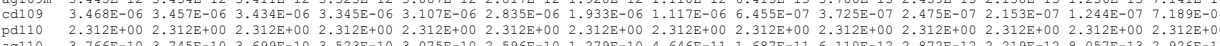

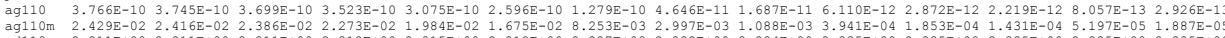

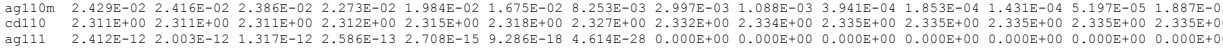

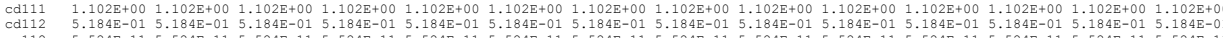

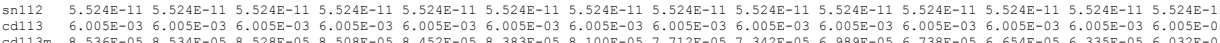

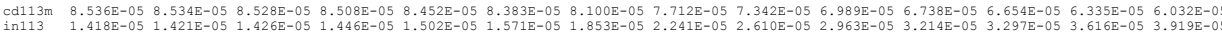

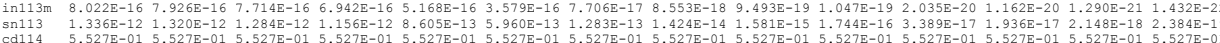

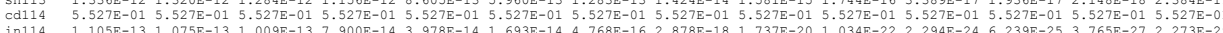

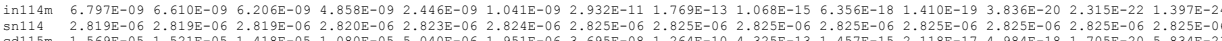

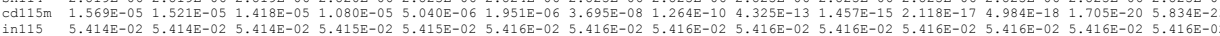

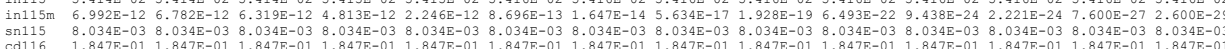

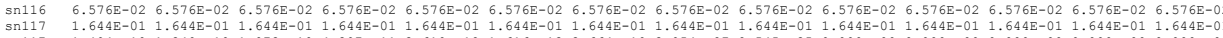

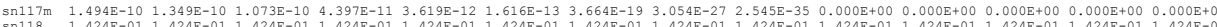

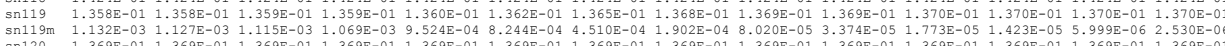

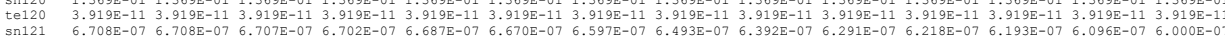

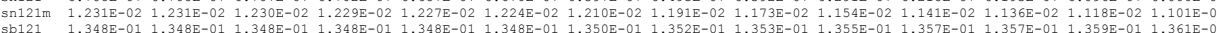

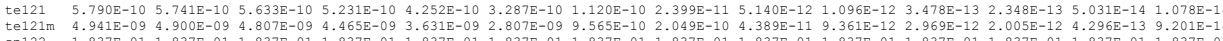

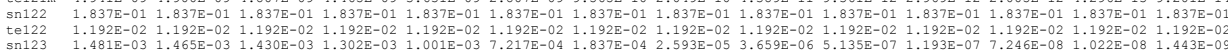

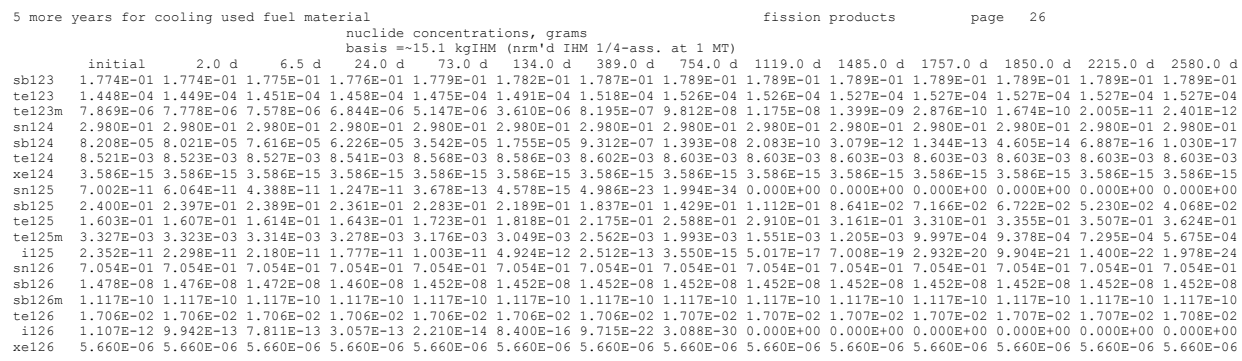

B-12 


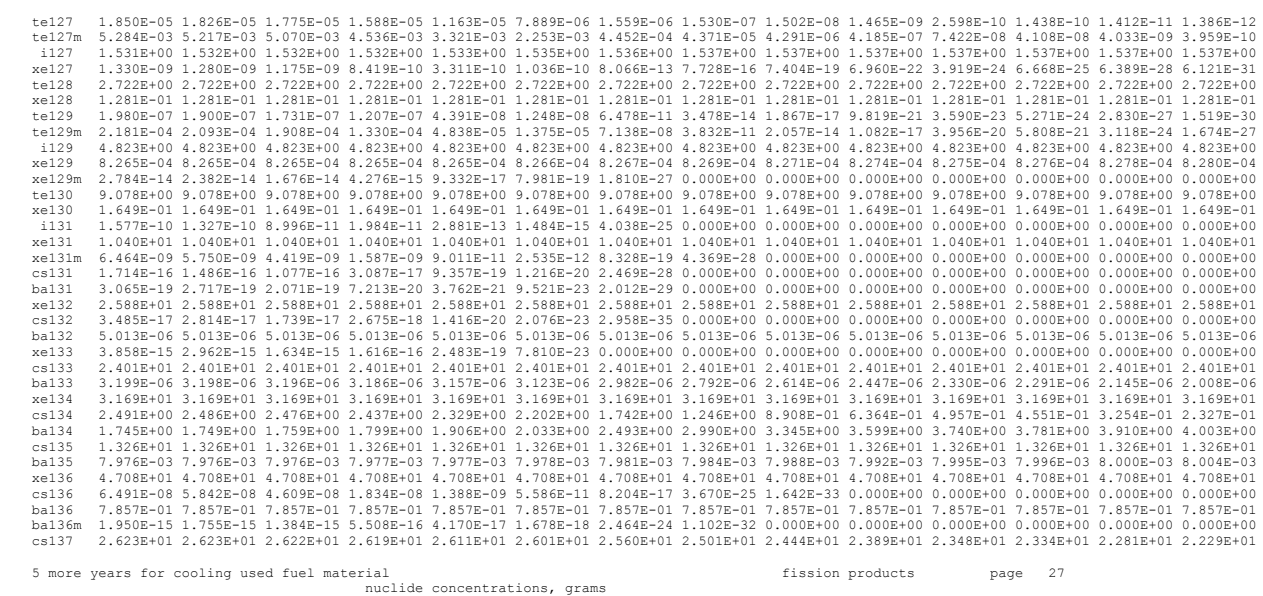
5 more years for cooling used fuel materia

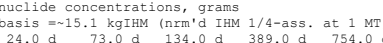
fission products page 27
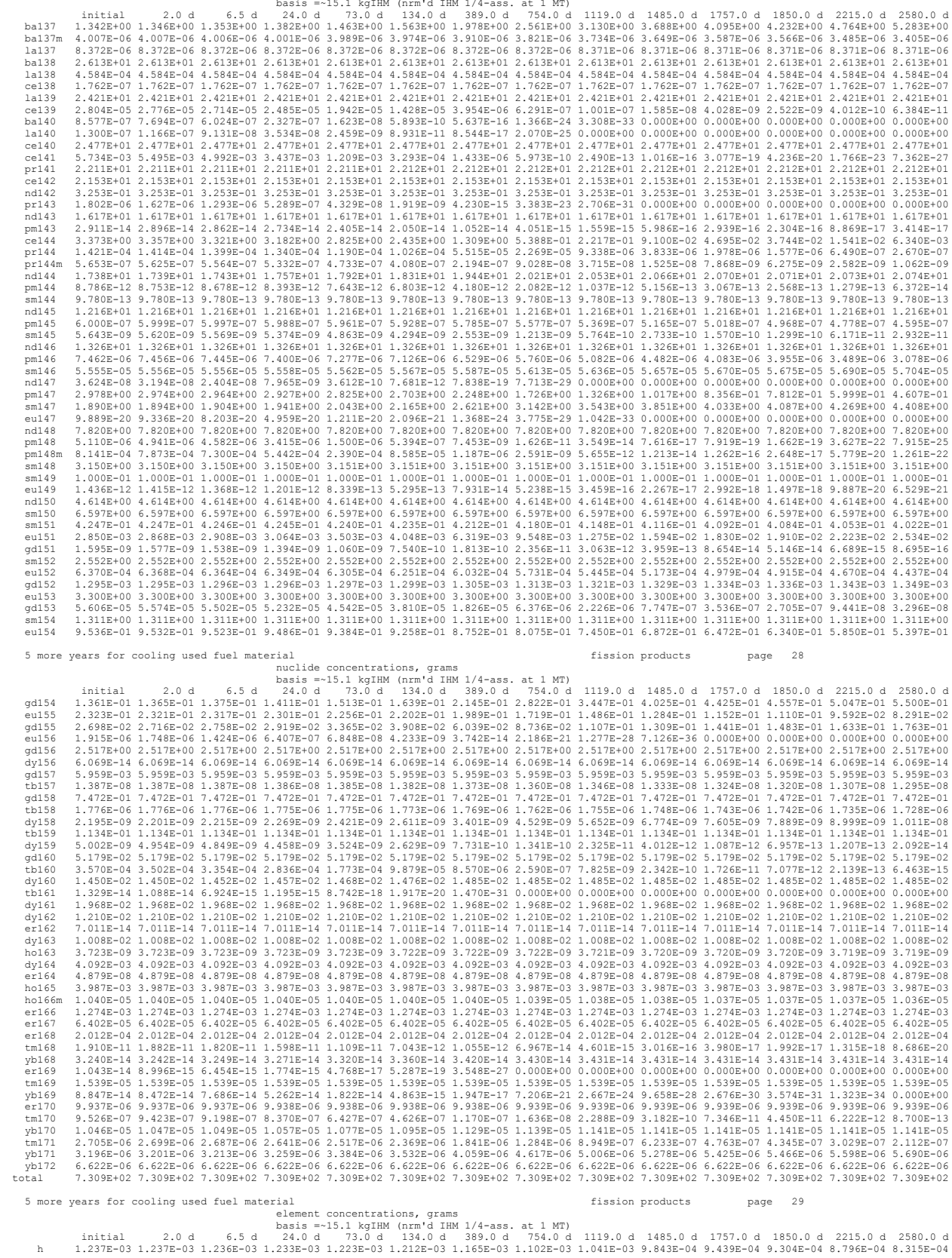

B-13 


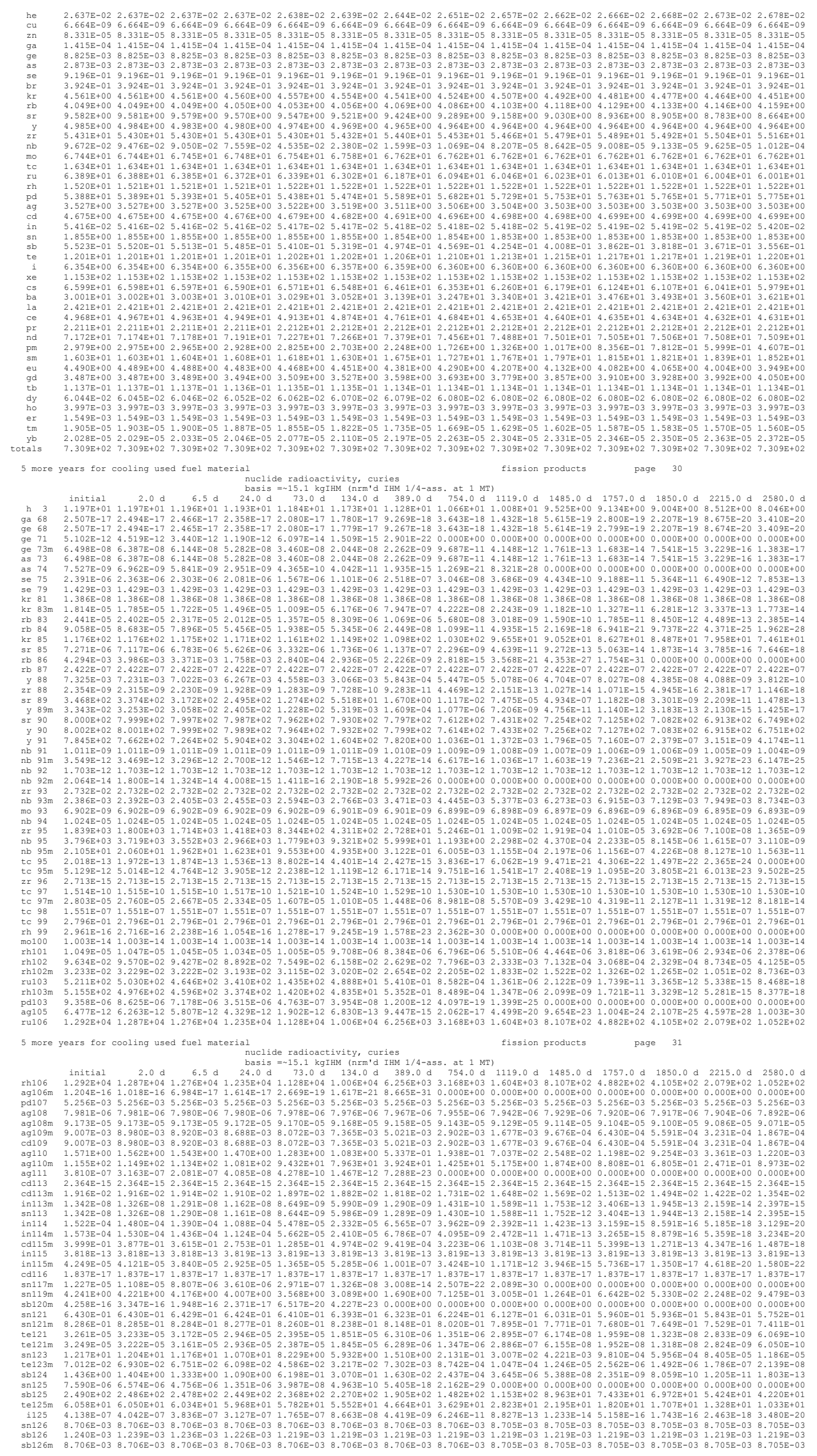

B-14 


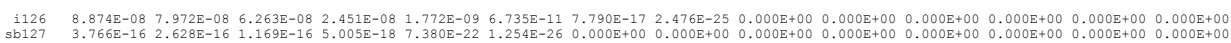

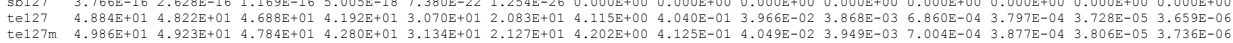

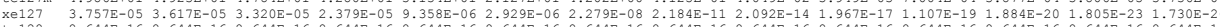

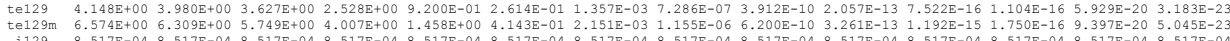
$\begin{array}{llllllllllllllllll}x e 129 m & 3.175 \mathrm{E}-09 & 2.716 \mathrm{E}-09 & 1.912 \mathrm{E}-09 & 4.877 \mathrm{E}-10 & 1.064 \mathrm{E}-11 & 9.102 \mathrm{E}-14 & 2.064 \mathrm{E}-22 & 0.000 \mathrm{E}+00 & 0.000 \mathrm{E}+00 & 0.000 \mathrm{E}+00 & 0.000 \mathrm{E}+00 & 0.000 \mathrm{E}+00 & 0.000 \mathrm{E}+00 & 0.000 \mathrm{E}+0\end{array}$

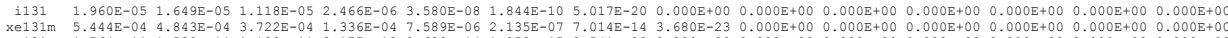

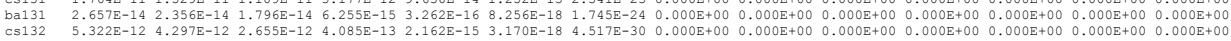
5 more years for cooling used fuel material

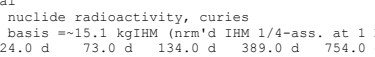

$$
\text { fission products page } 32
$$

1119.0 d 1485.0 d 1757.0 d 1850.0 d 2215.0 d 2580.0 d

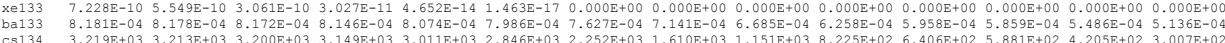

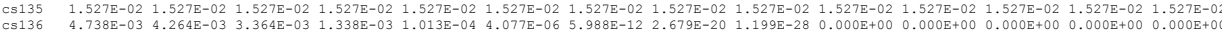

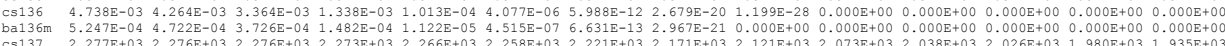

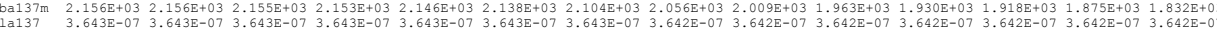

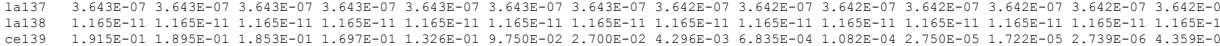
ba140 6.276E-02 5.629E-02 $4.408 \mathrm{E}-02 \quad 1.703 \mathrm{E}-02 \quad 1.187 \mathrm{E}-03 \quad 4.311 \mathrm{E}-05 \quad 4.125 \mathrm{E}-11 \quad 9.991 \mathrm{E}-20 \quad 2.420 \mathrm{E}-28 \quad 0.000 \mathrm{E}+00 \quad 0.000 \mathrm{E}+00 \quad 0.000 \mathrm{E}+00 \quad 0.000 \mathrm{E}+00 \quad 0.000 \mathrm{E}+0$

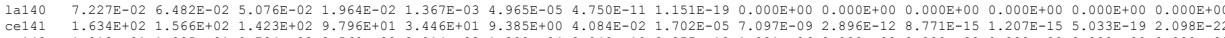

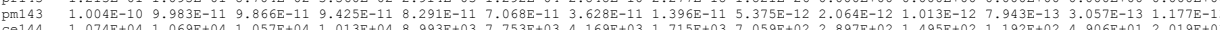

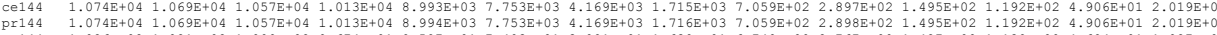

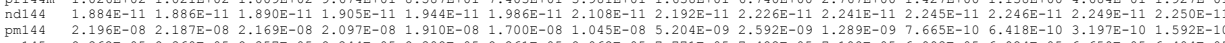

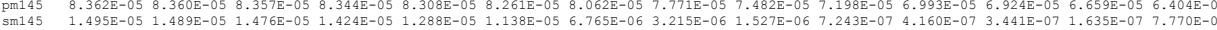

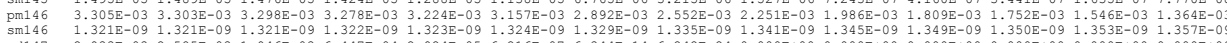

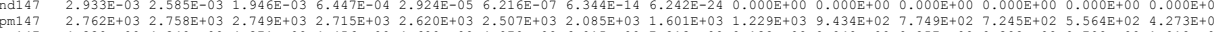
\begin{tabular}{lllllllllll} 
& \\
\hline
\end{tabular}

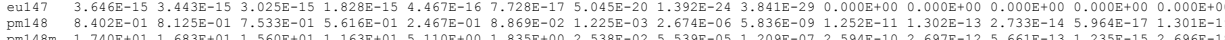

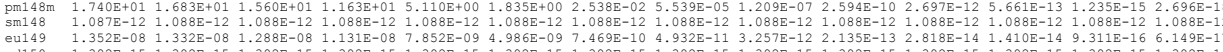

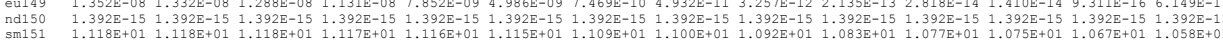

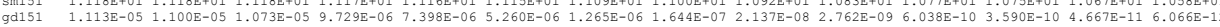

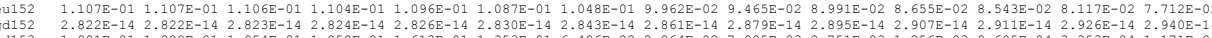

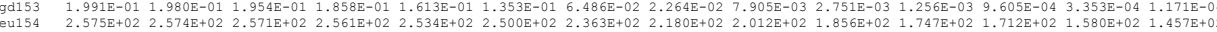

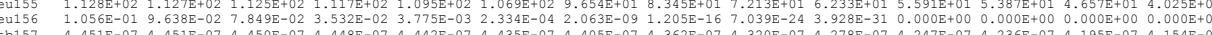

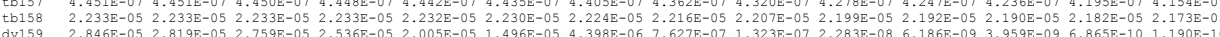

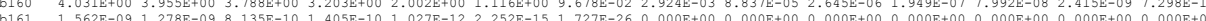

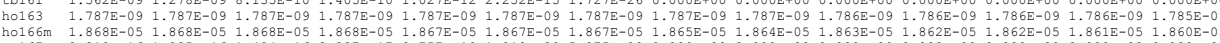

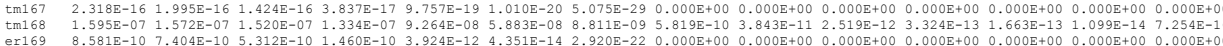
5 more years for

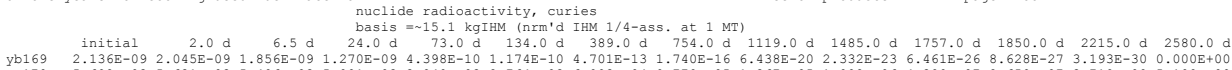

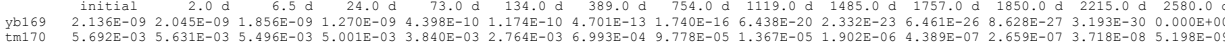

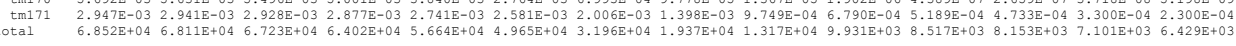
5 more years for cooling used fuel materia

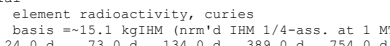

fission products page 34

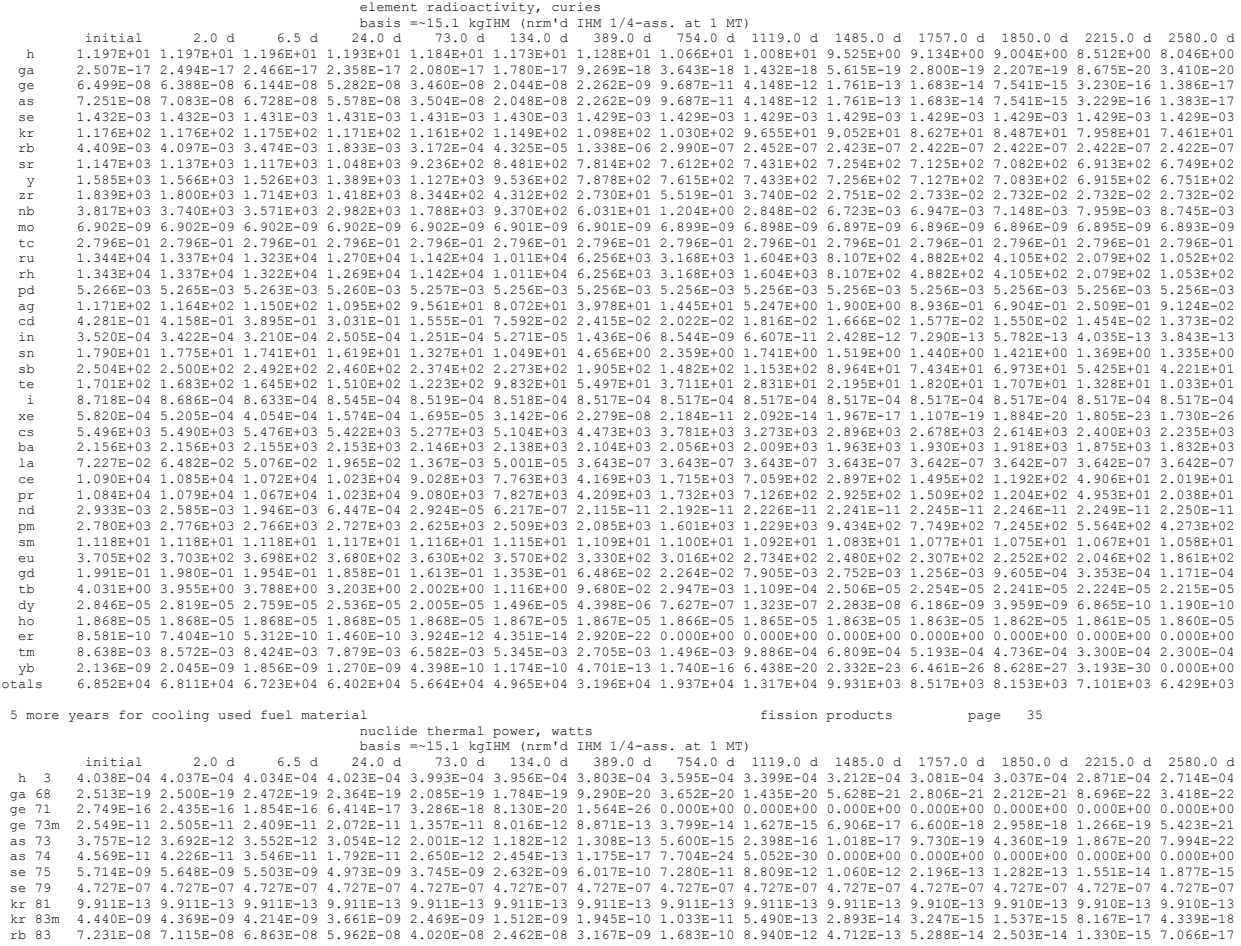

B-15 


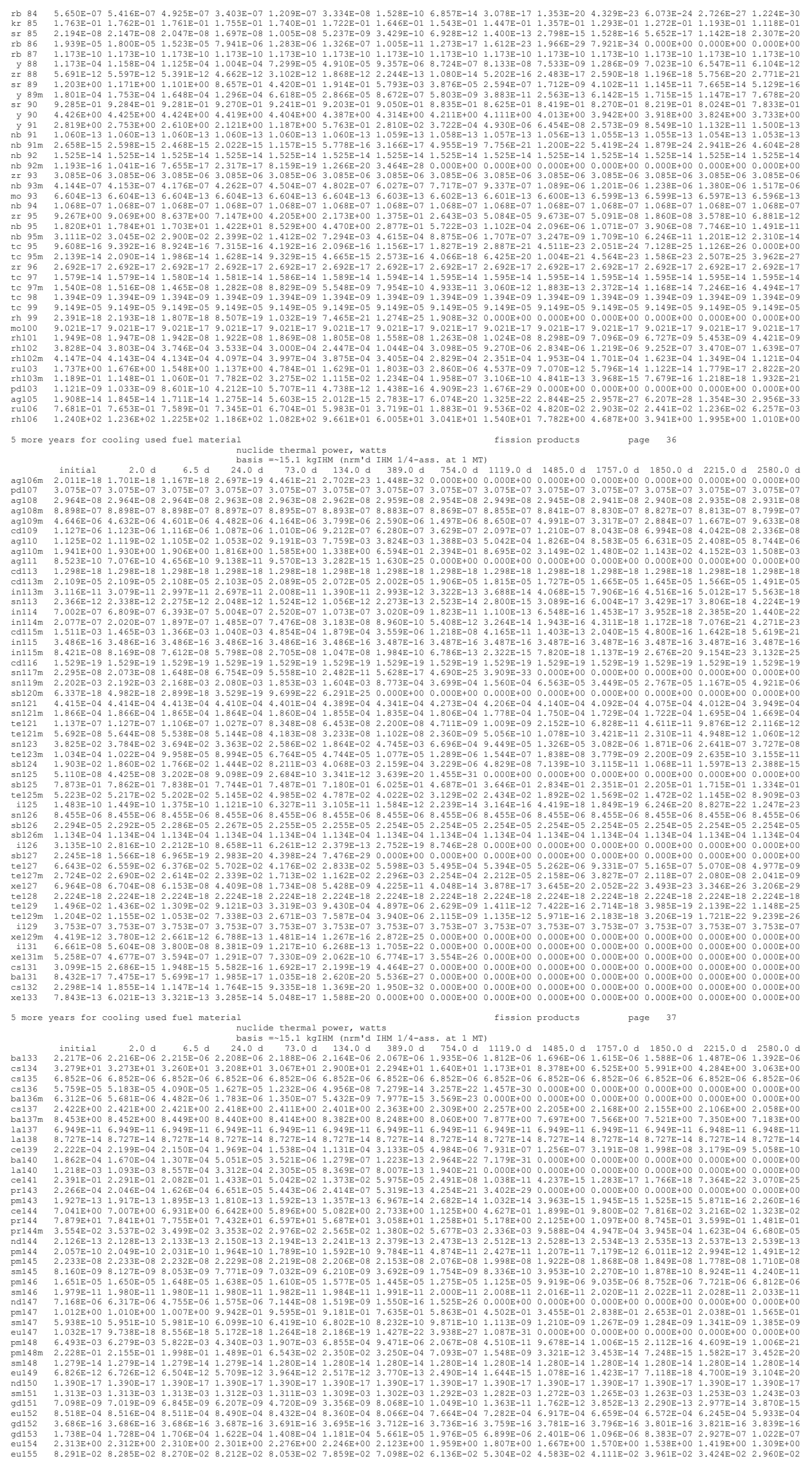

B-16 


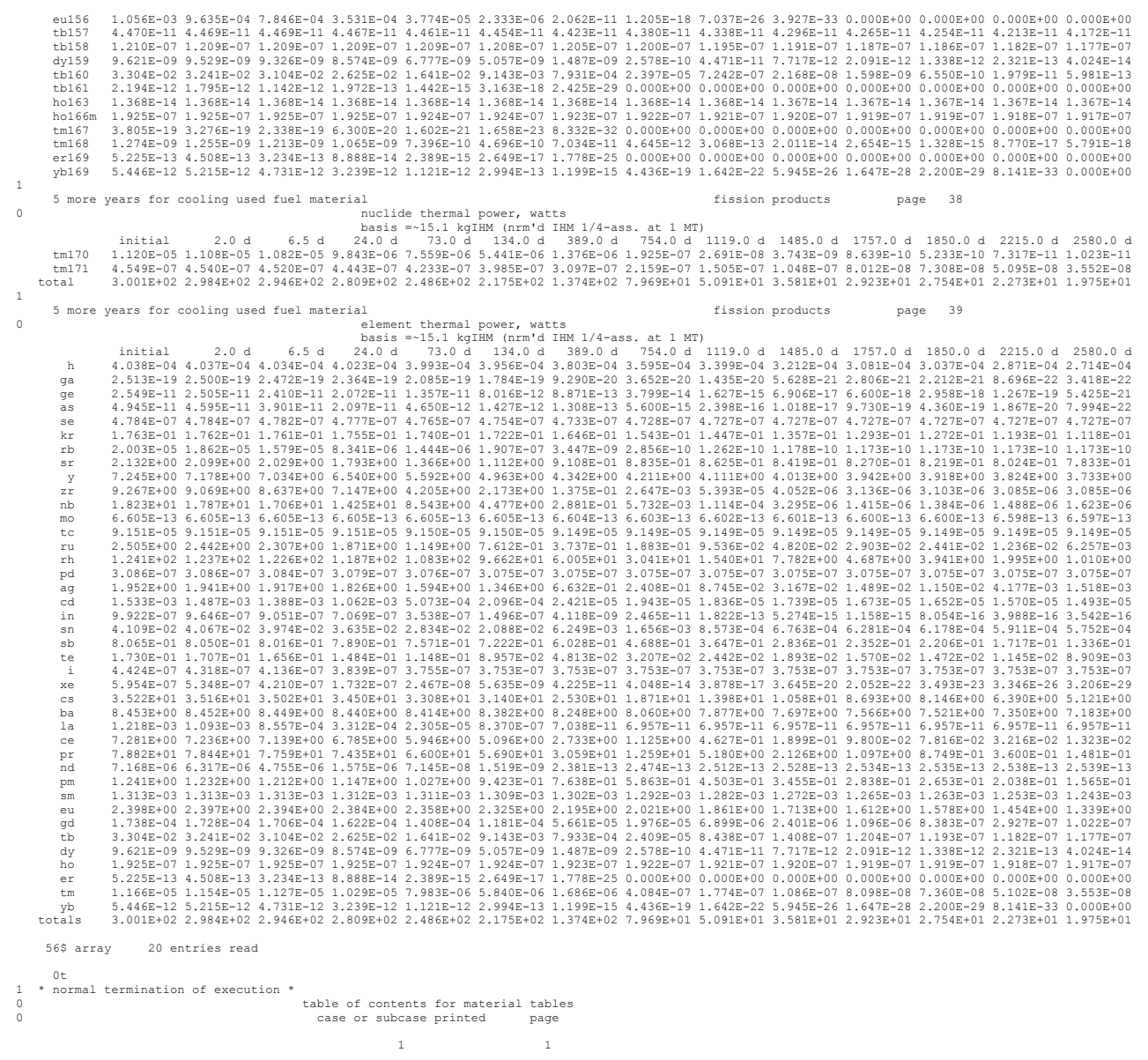

B-17 


\section{B.2 ORIGEN RESULTS FOR COOLING MOX FUEL ROD A1}
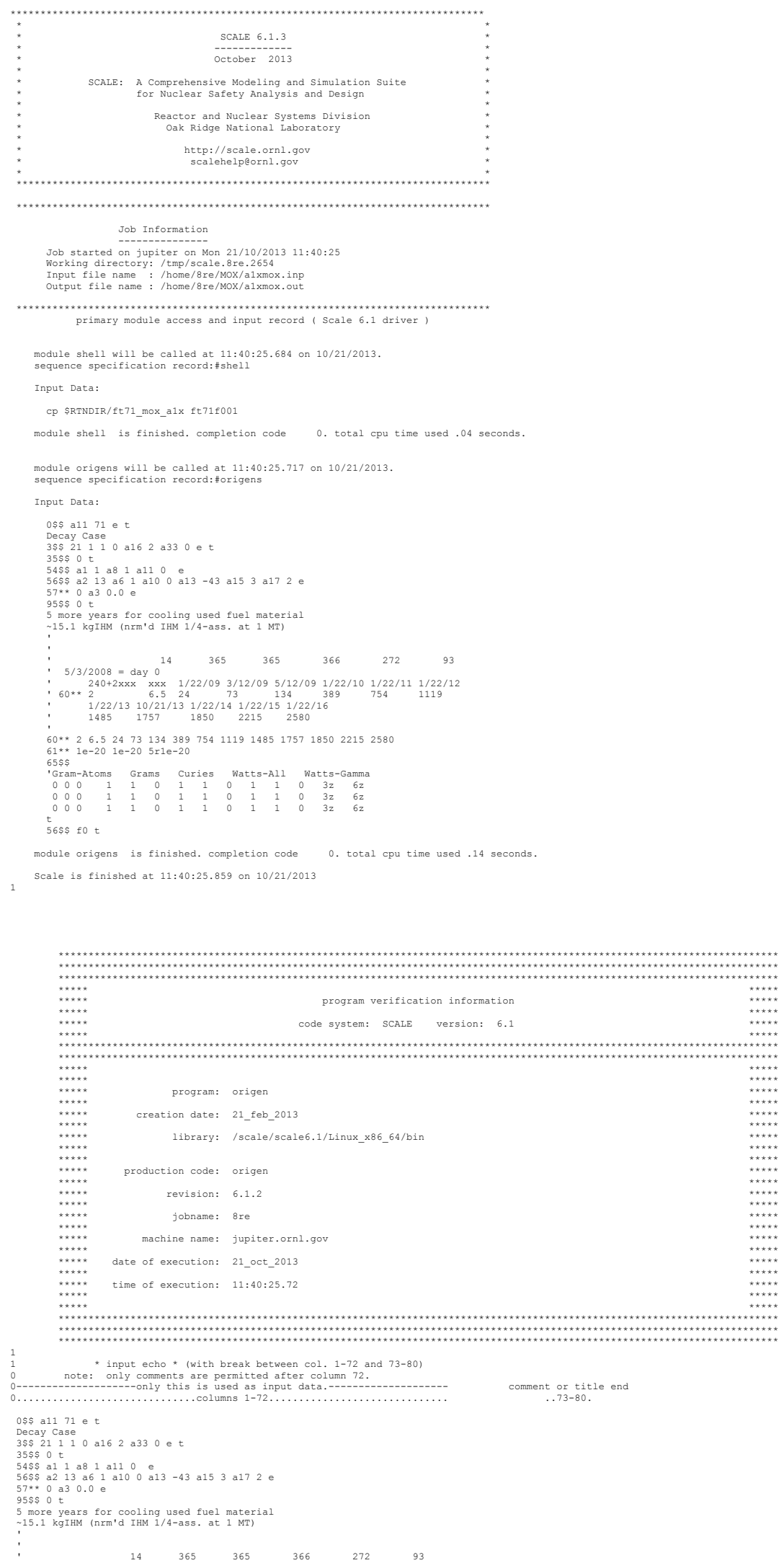

B-18 


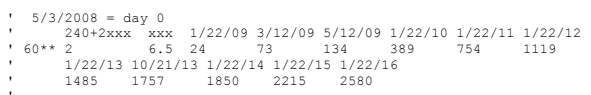

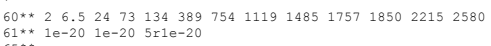

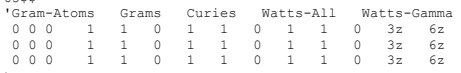

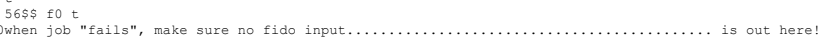

0 array $\quad 12$ entries read
ot
3 array $\quad 33$ entries read

ot

$$
\begin{aligned}
& \text { cross-section data taken from position number } 1 \text { of library on unit } 21 \text {. }
\end{aligned}
$$

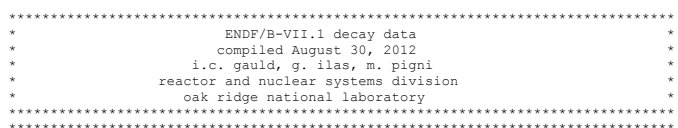

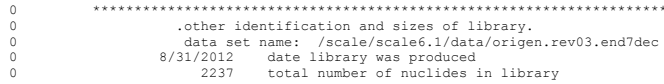

$$
\begin{aligned}
& \begin{array}{l}
2237 \\
910 \text { total number of nuclides in library } \\
176
\end{array} \\
& 176 \text { number of actinide nuclides } \\
& \begin{array}{lll}
1151 & \begin{array}{l}
\text { number of fission product nuclides } \\
\text { number of nonzero off-diagonal matrix elements }
\end{array}
\end{array}
\end{aligned}
$$

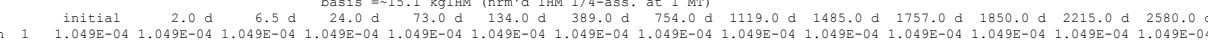

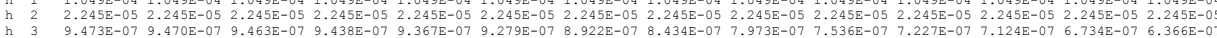

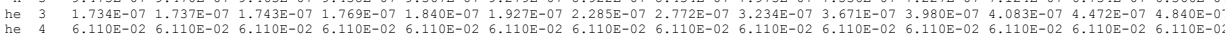

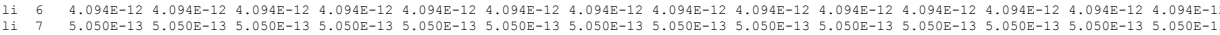

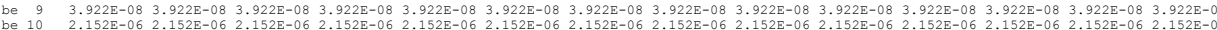

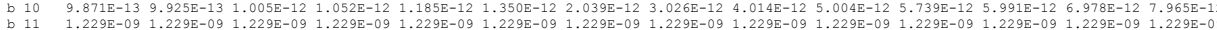

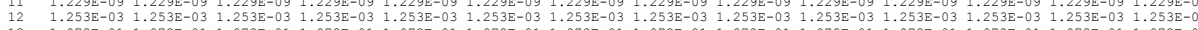

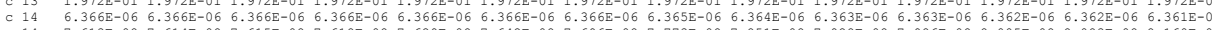

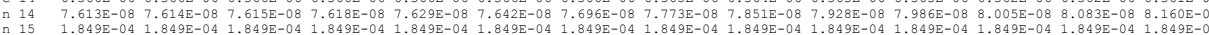

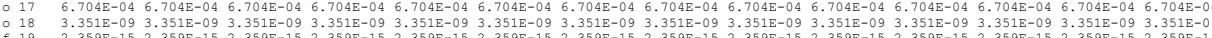

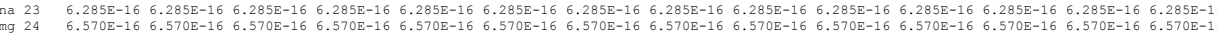

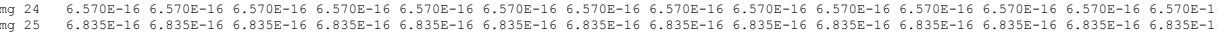

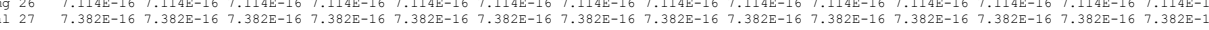

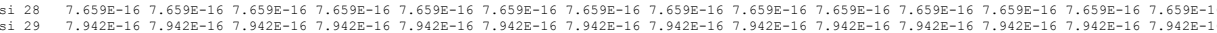

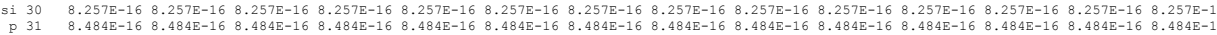

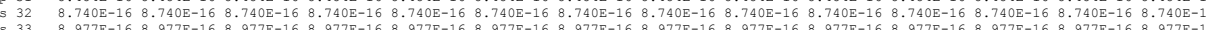

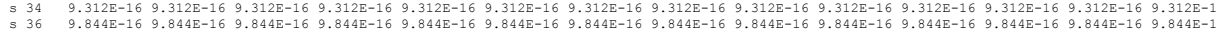

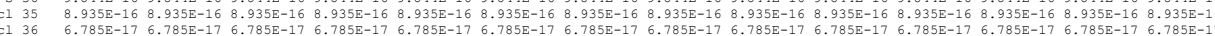

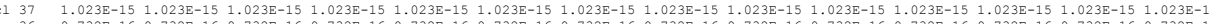

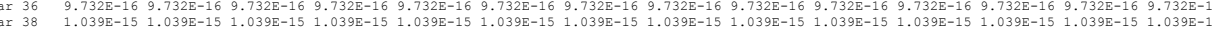

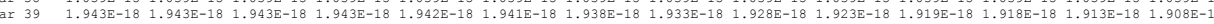

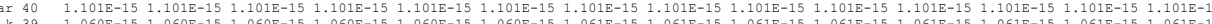

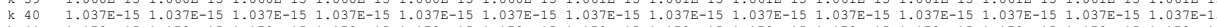

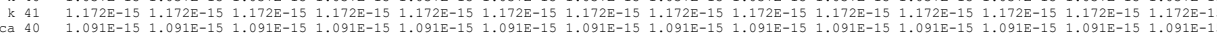

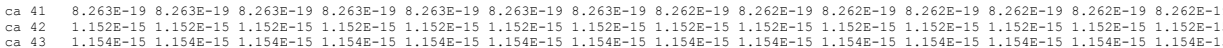

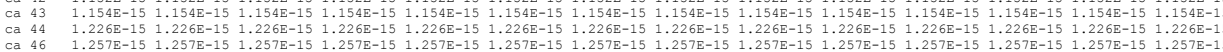

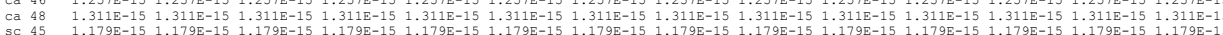

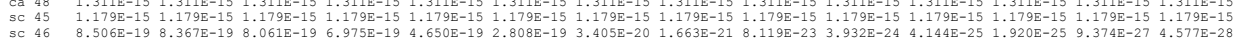
5 more years for cooling used fuel material

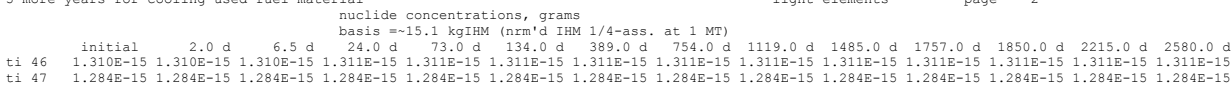

light elements page 2

B-19 


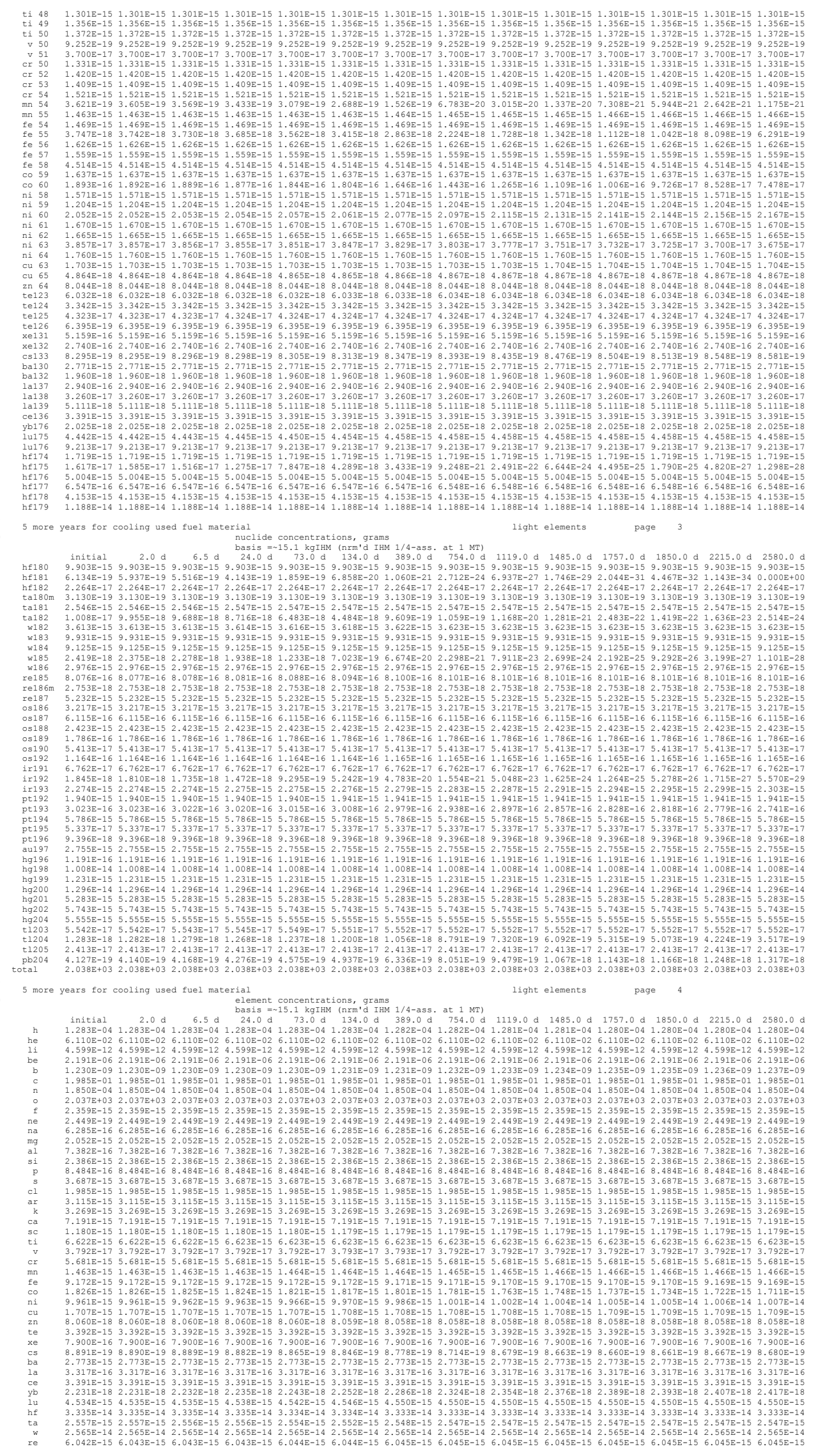

B-20 


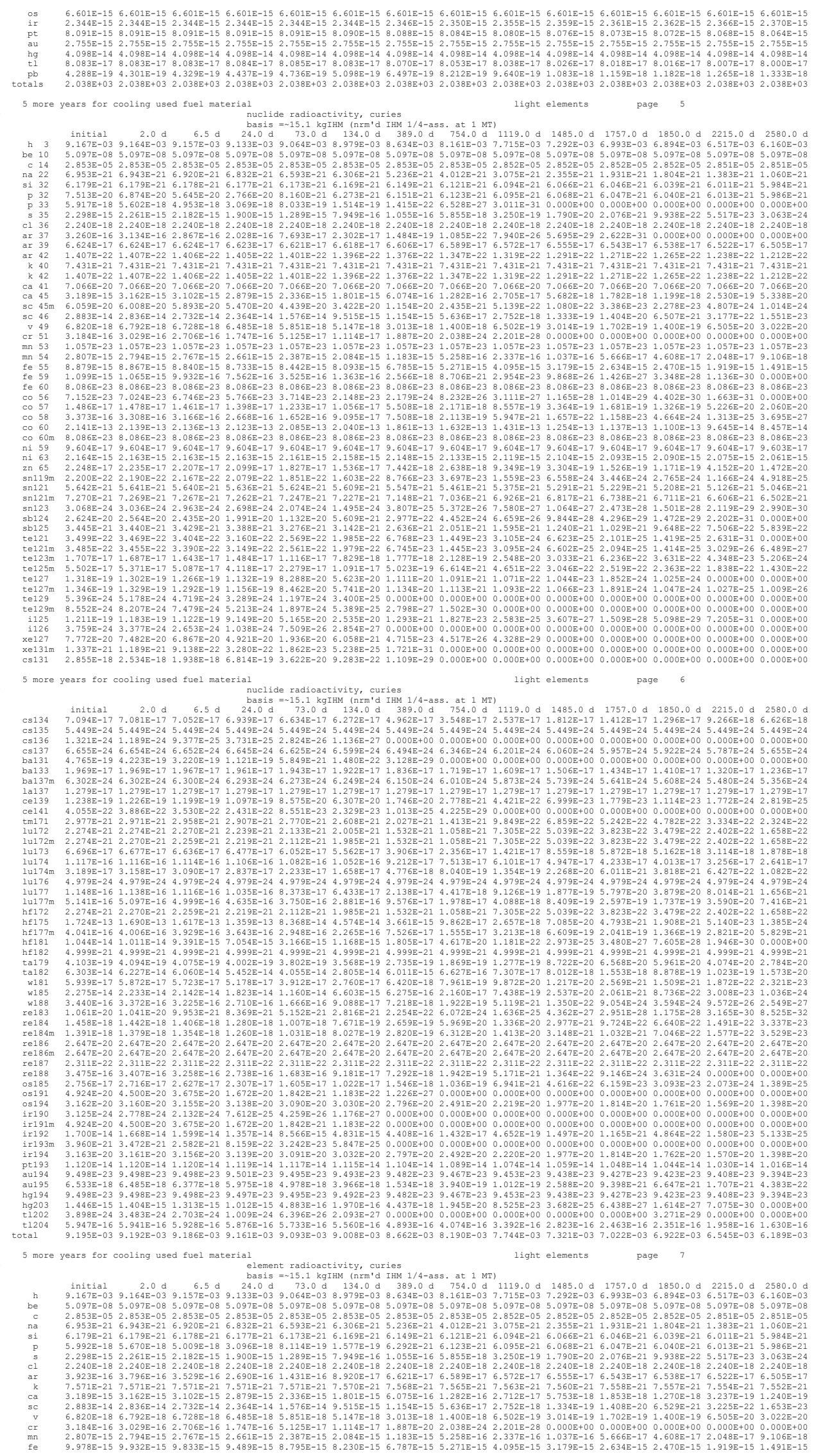

B-21 


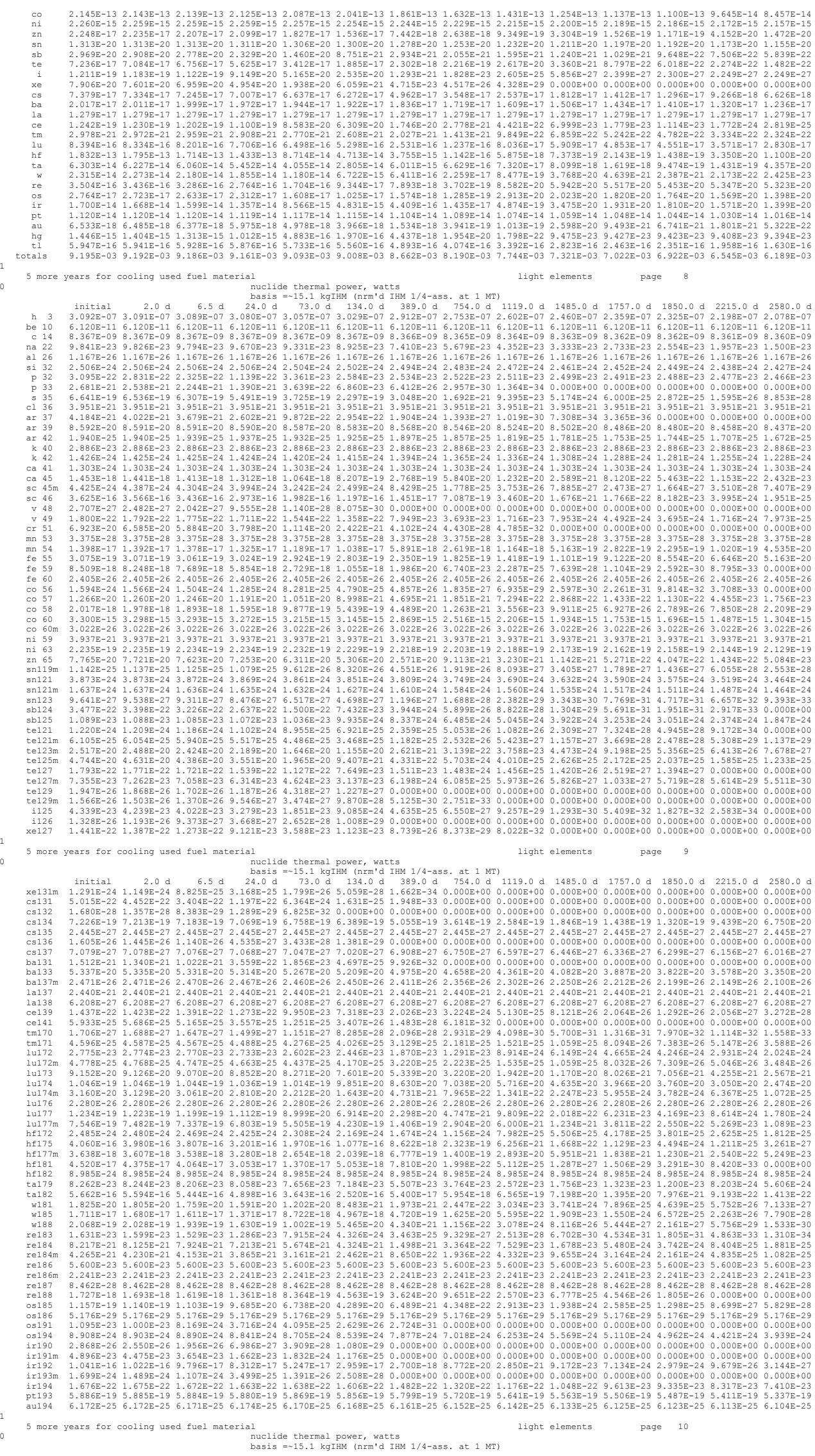

B-22 


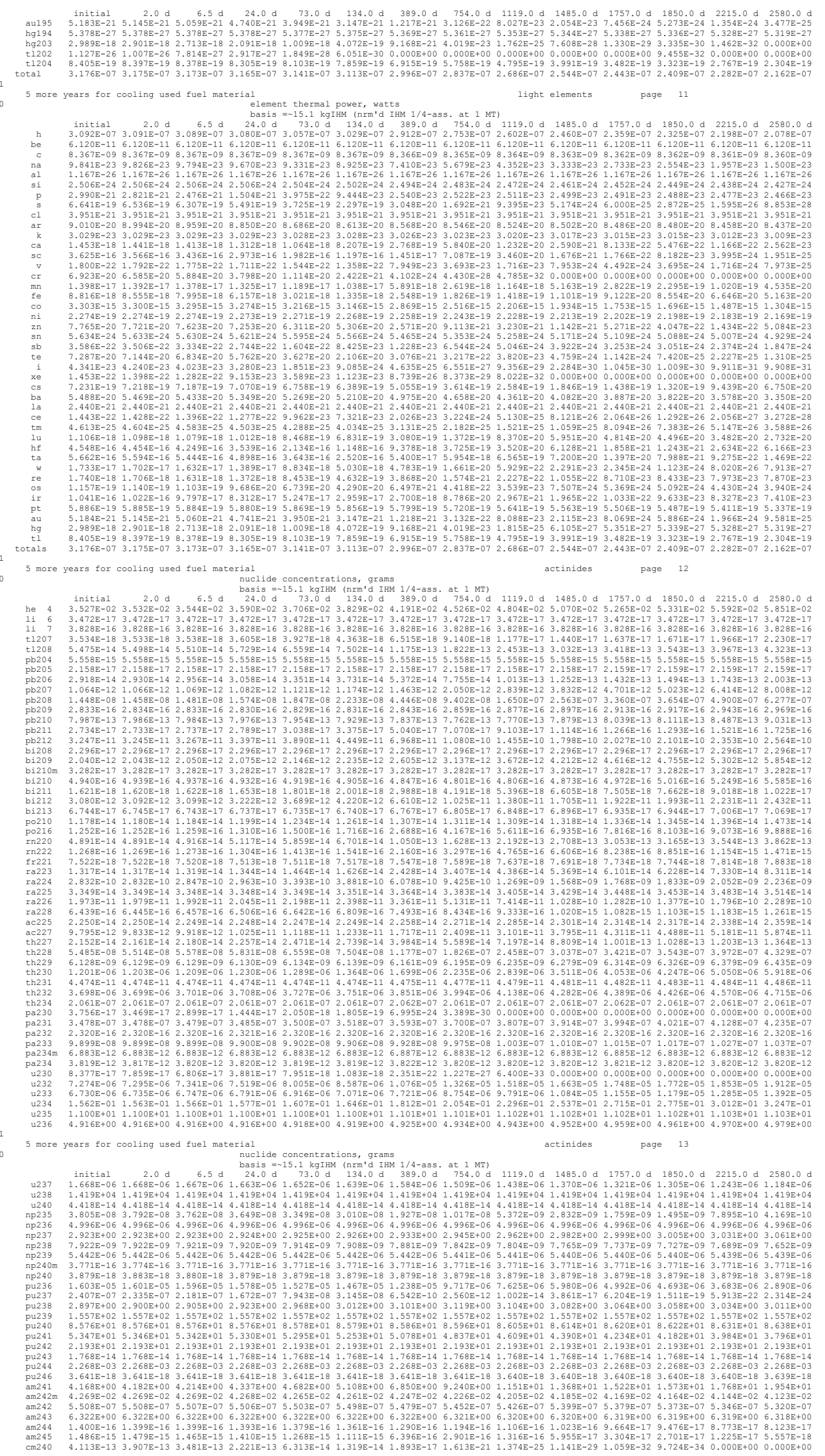

B-23 


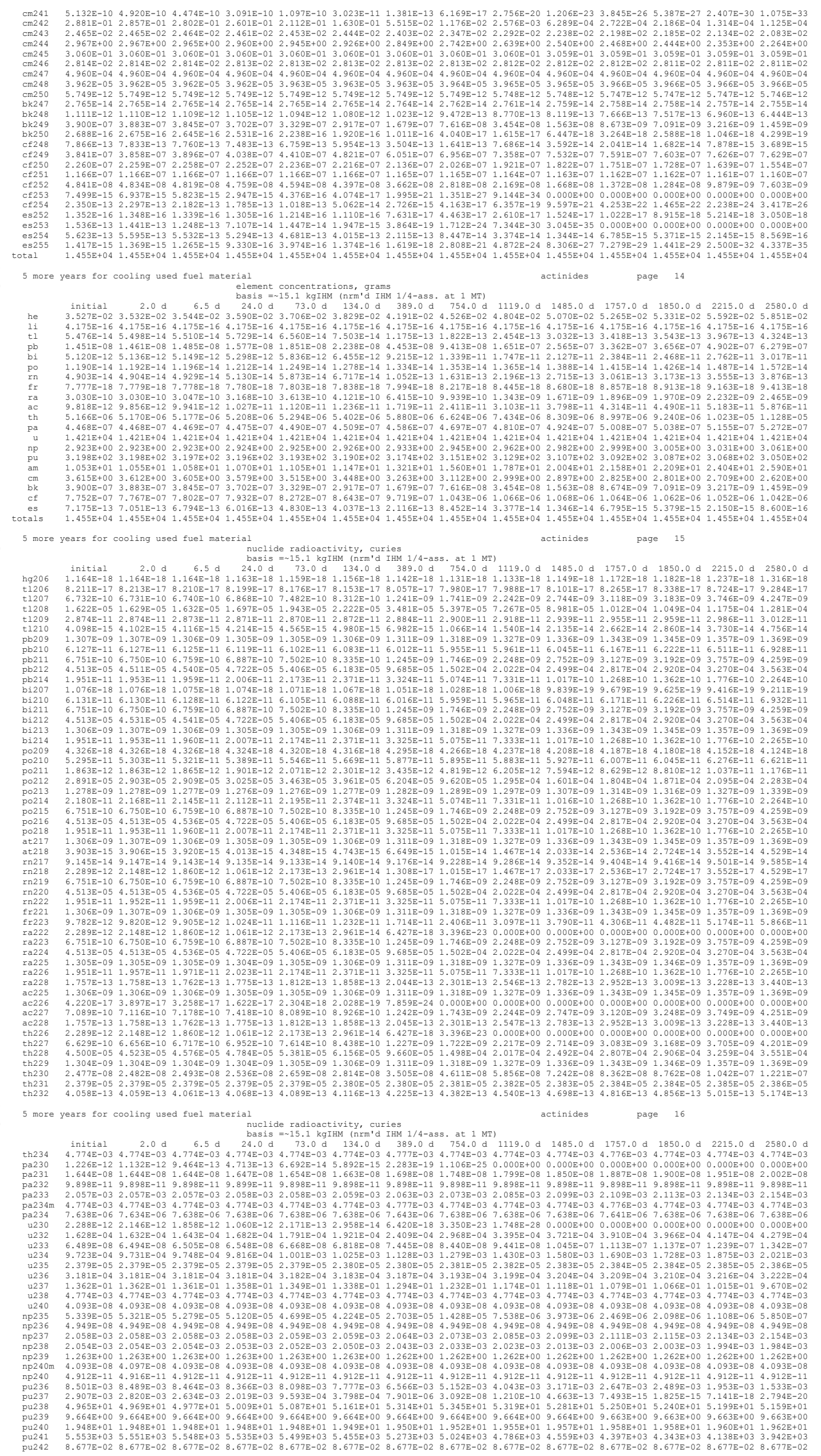

B-24 

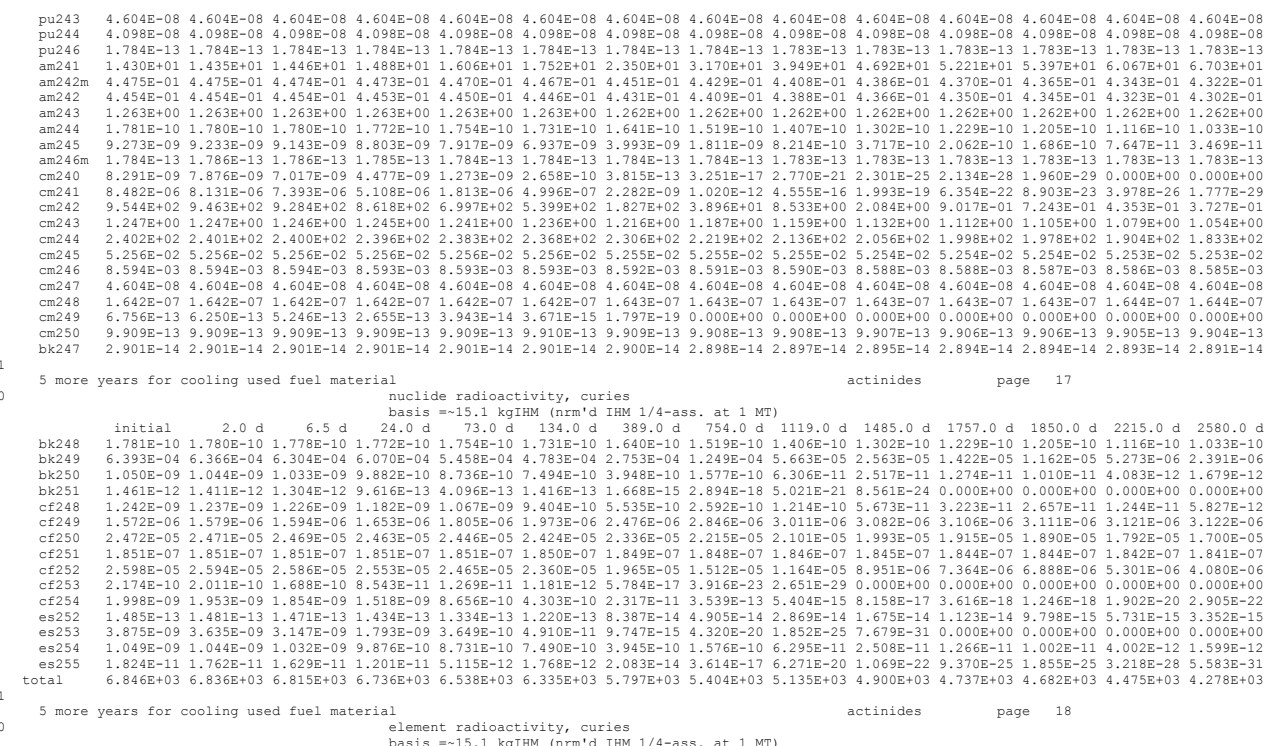

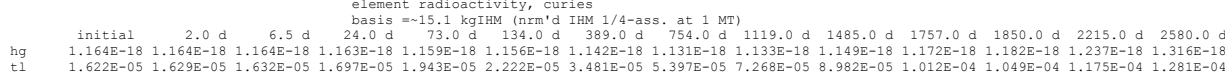

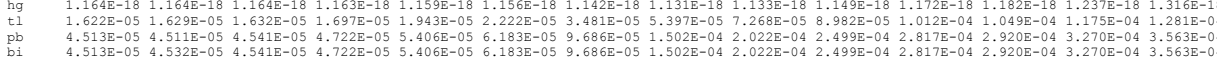

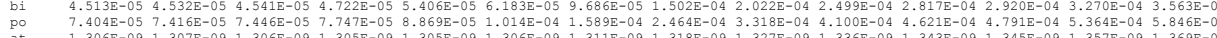

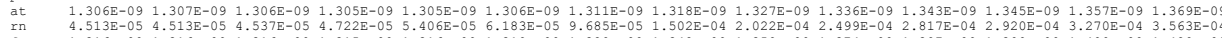
0
0

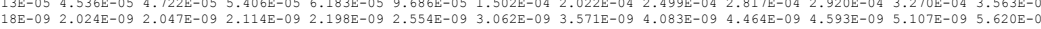

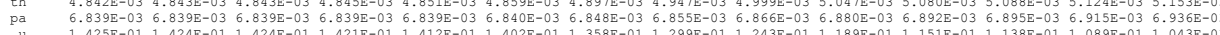

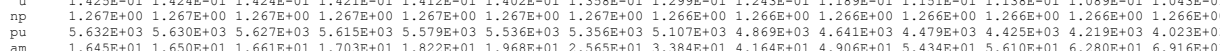

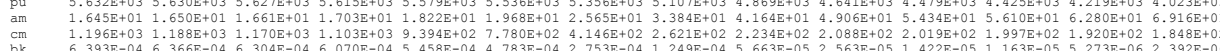

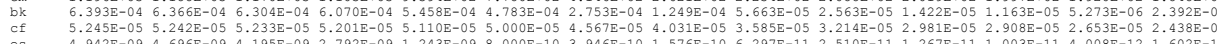

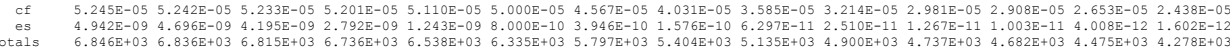
5 more years for cooling used fuel material

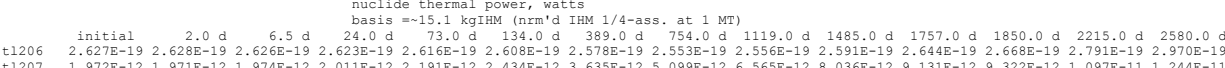

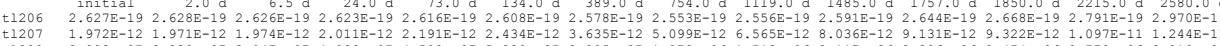

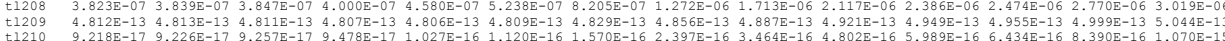

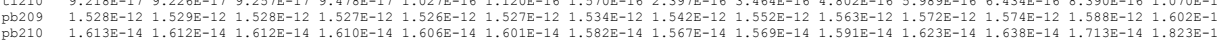

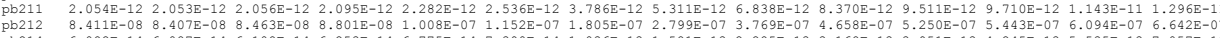

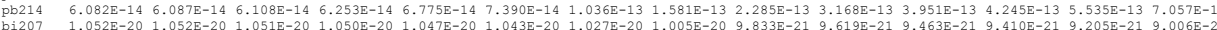

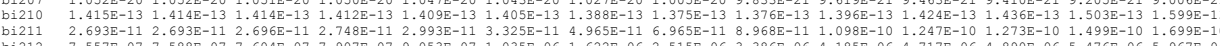

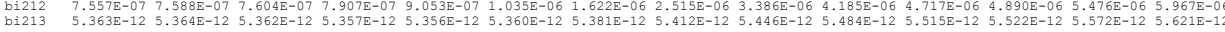
$\begin{array}{llll} & \end{array}$

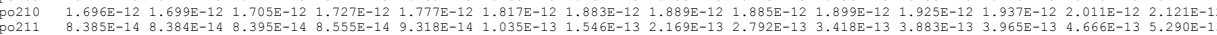

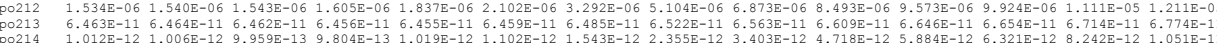

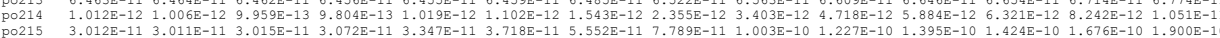

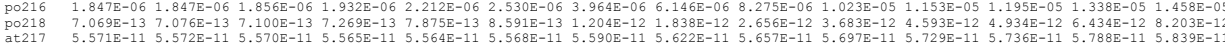

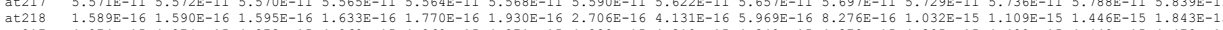

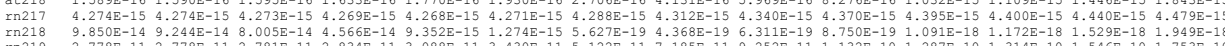

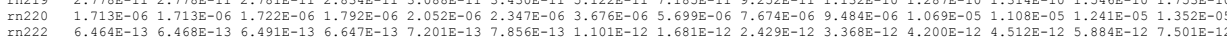

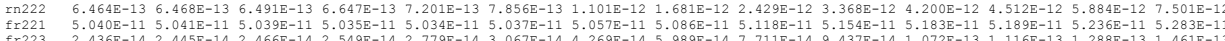

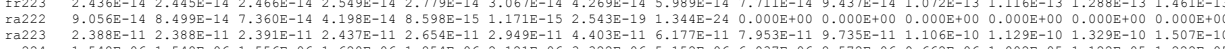

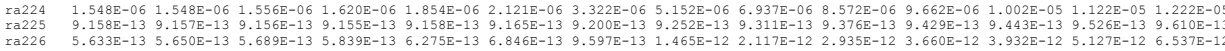

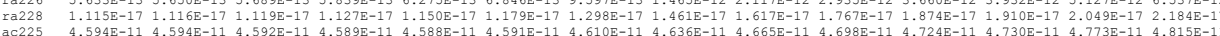

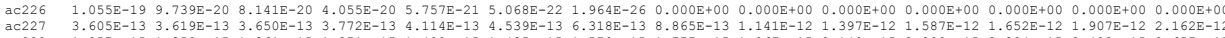

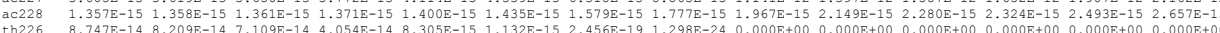

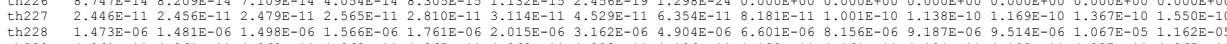

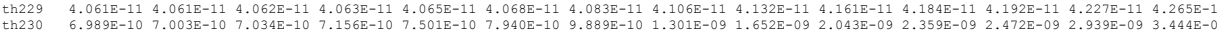

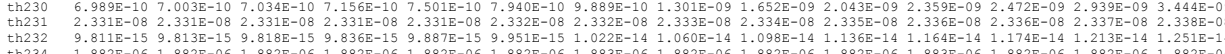

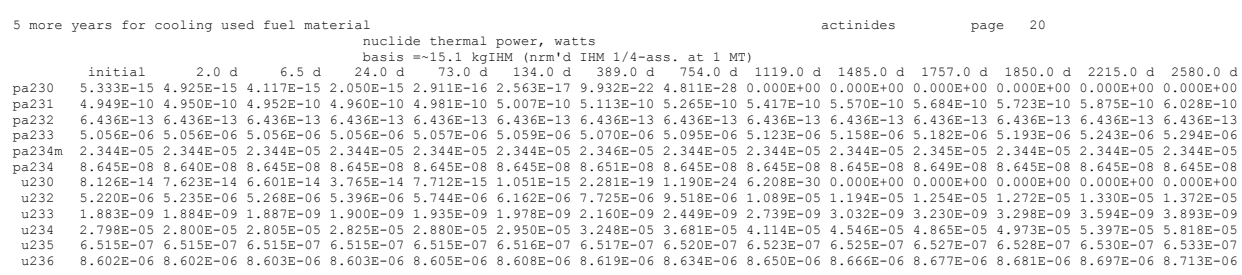




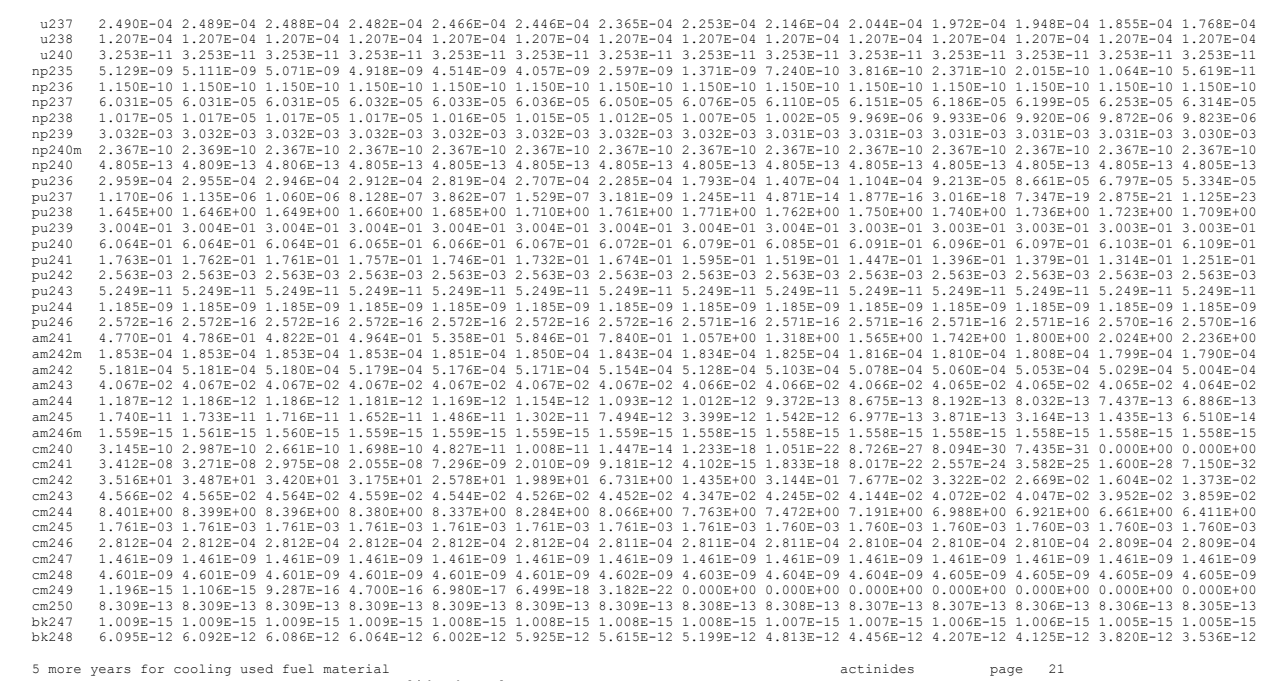

5 more years for cooling used fuel material
nuclide thermal power, watts
basis $=215.1 \mathrm{~kg}$ HM (nrm'd IHM 1/4-ass. at $1 \mathrm{MT}$ )

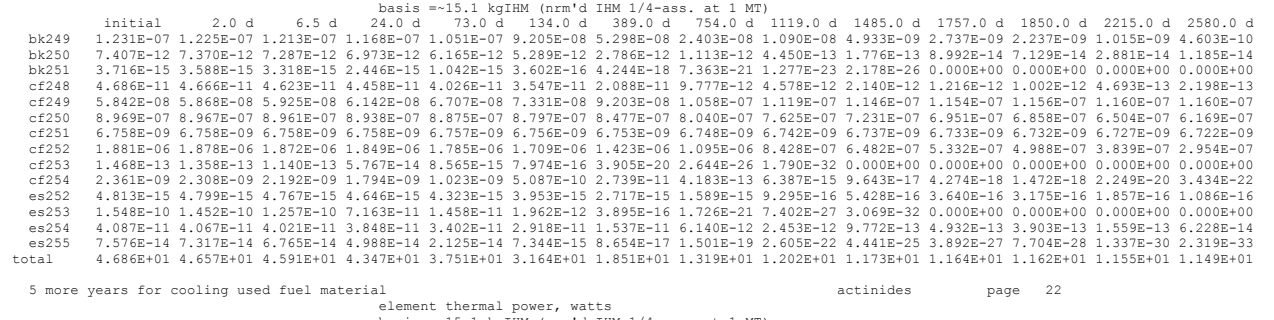

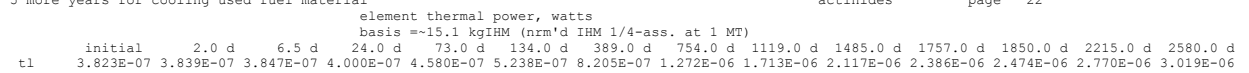

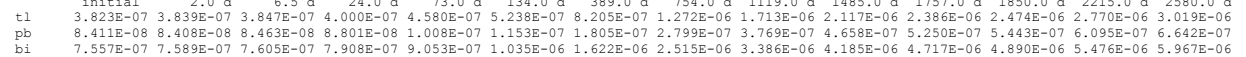

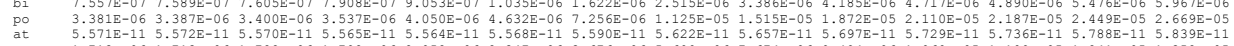

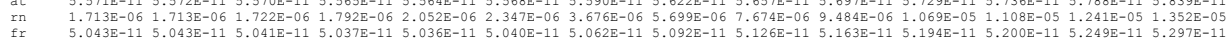
$\begin{array}{lllllllllllllllll}\mathrm{ra} & 1.548 \mathrm{E}-06 & 1.548 \mathrm{E}-06 & 1.556 \mathrm{E}-06 & 1.620 \mathrm{E}-06 & 1.854 \mathrm{E}-06 & 2.121 \mathrm{E}-06 & 3.323 \mathrm{E}-06 & 5.152 \mathrm{E}-06 & 6.937 \mathrm{E}-06 & 8.572 \mathrm{E}-06 & 9.662 \mathrm{E}-06 & 1.002 \mathrm{E}-05 & 1.122 \mathrm{E}-05 & 1.222 \mathrm{E}-05 \\ \mathrm{ac} & 4.630 \mathrm{E}-11 & 4.630 \mathrm{E}-11 & 4.629 \mathrm{E}-11 & 4.627 \mathrm{E}-11 & 4.629 \mathrm{E}-11 & 4.637 \mathrm{E}-11 & 4.673 \mathrm{E}-11 & 4.725 \mathrm{E}-11 & 4.779 \mathrm{E}-11 & 4.838 \mathrm{E}-11 & 4.883 \mathrm{E}-11 & 4.895 \mathrm{E}-11 & 4.964 \mathrm{E}-11 & 5.032 \mathrm{E}-11\end{array}$

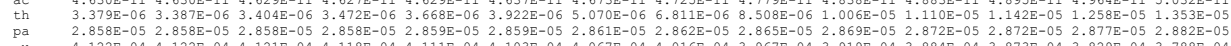

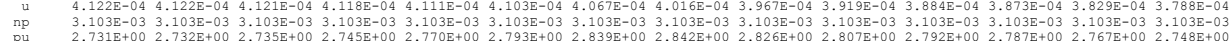

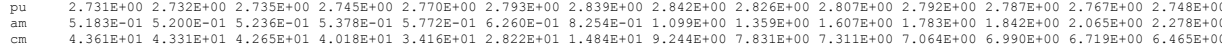

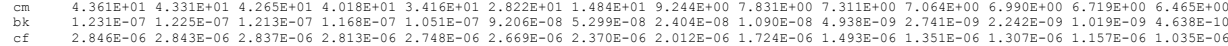

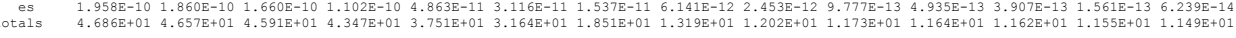
5 more years for cooling used fuel material

$$
\text { allide concentrations, grams page } 23
$$

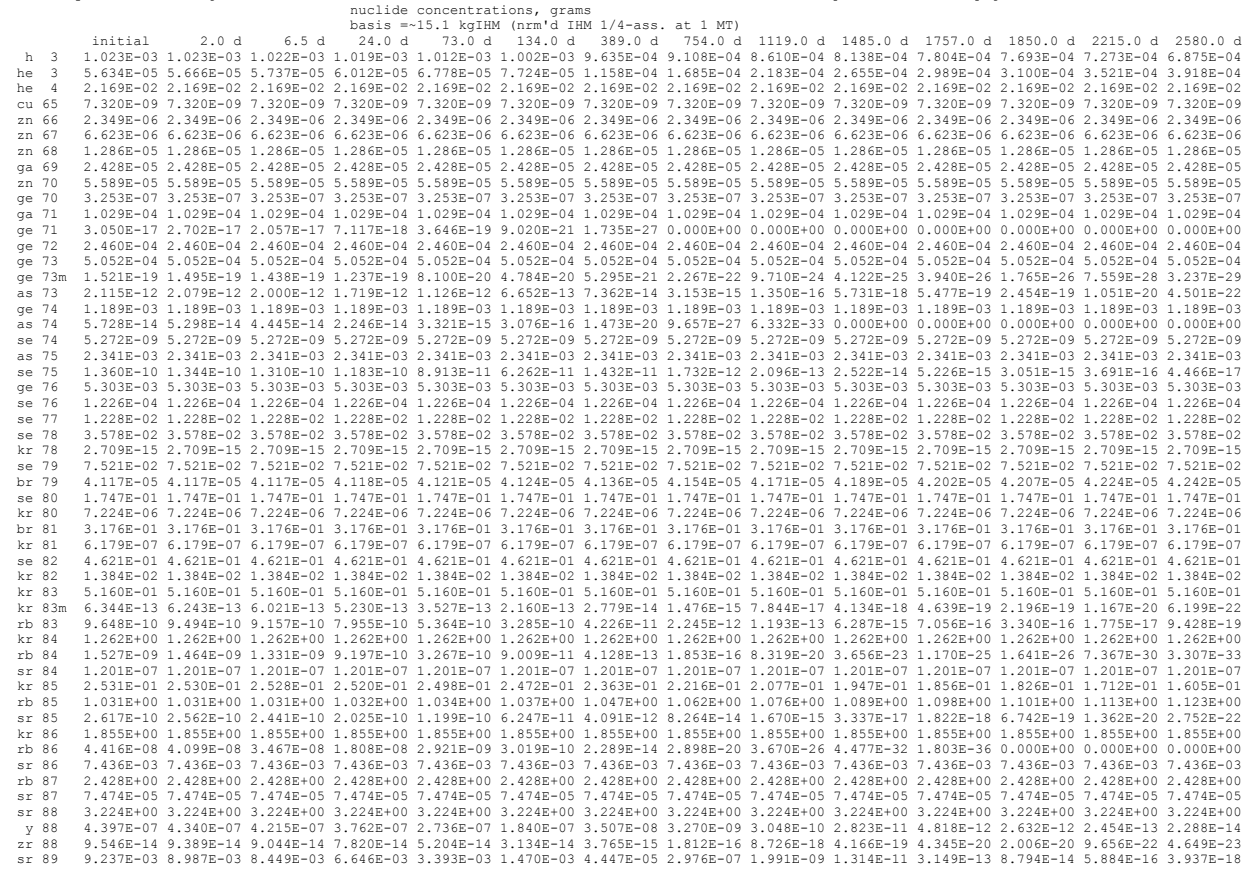



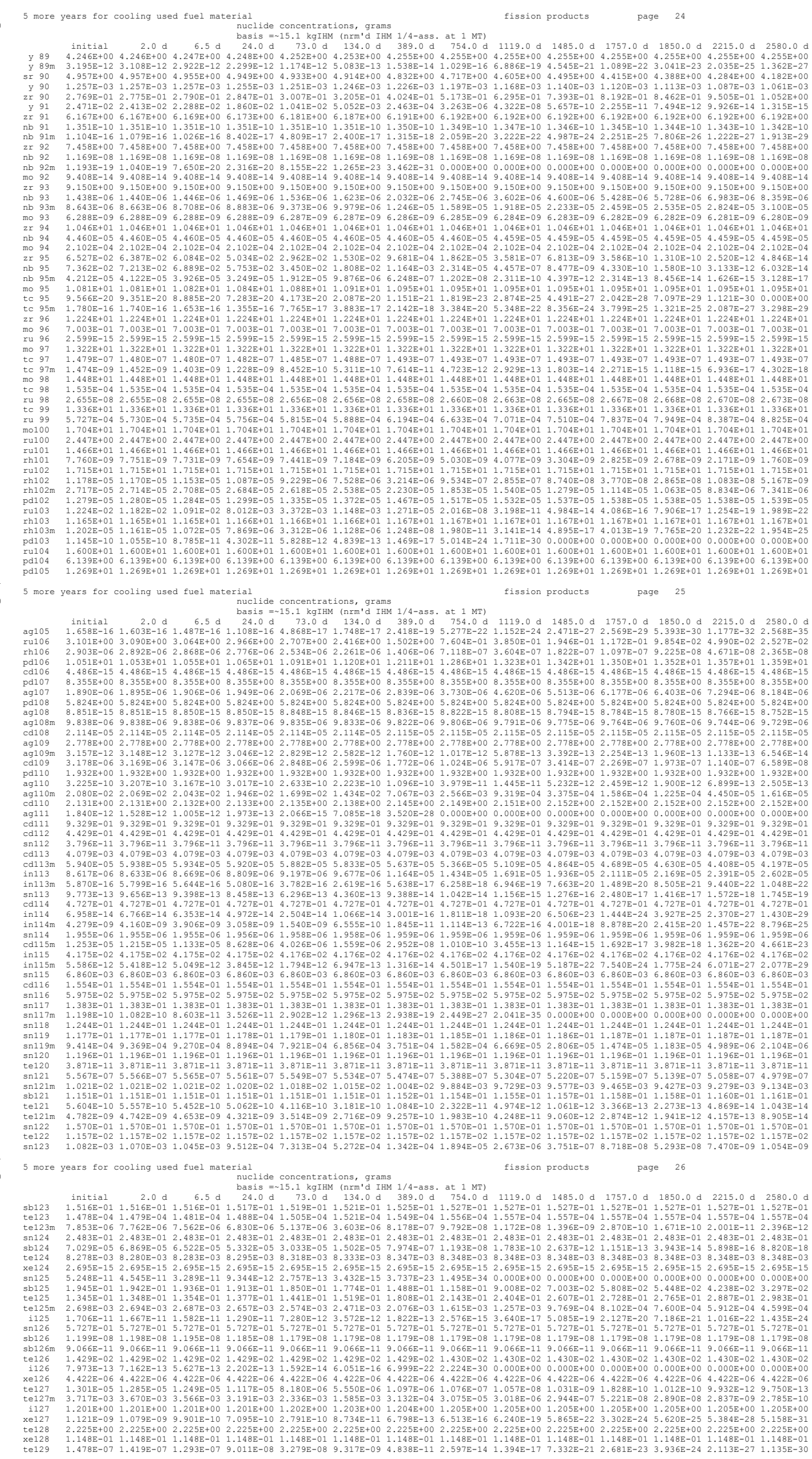

B-27 

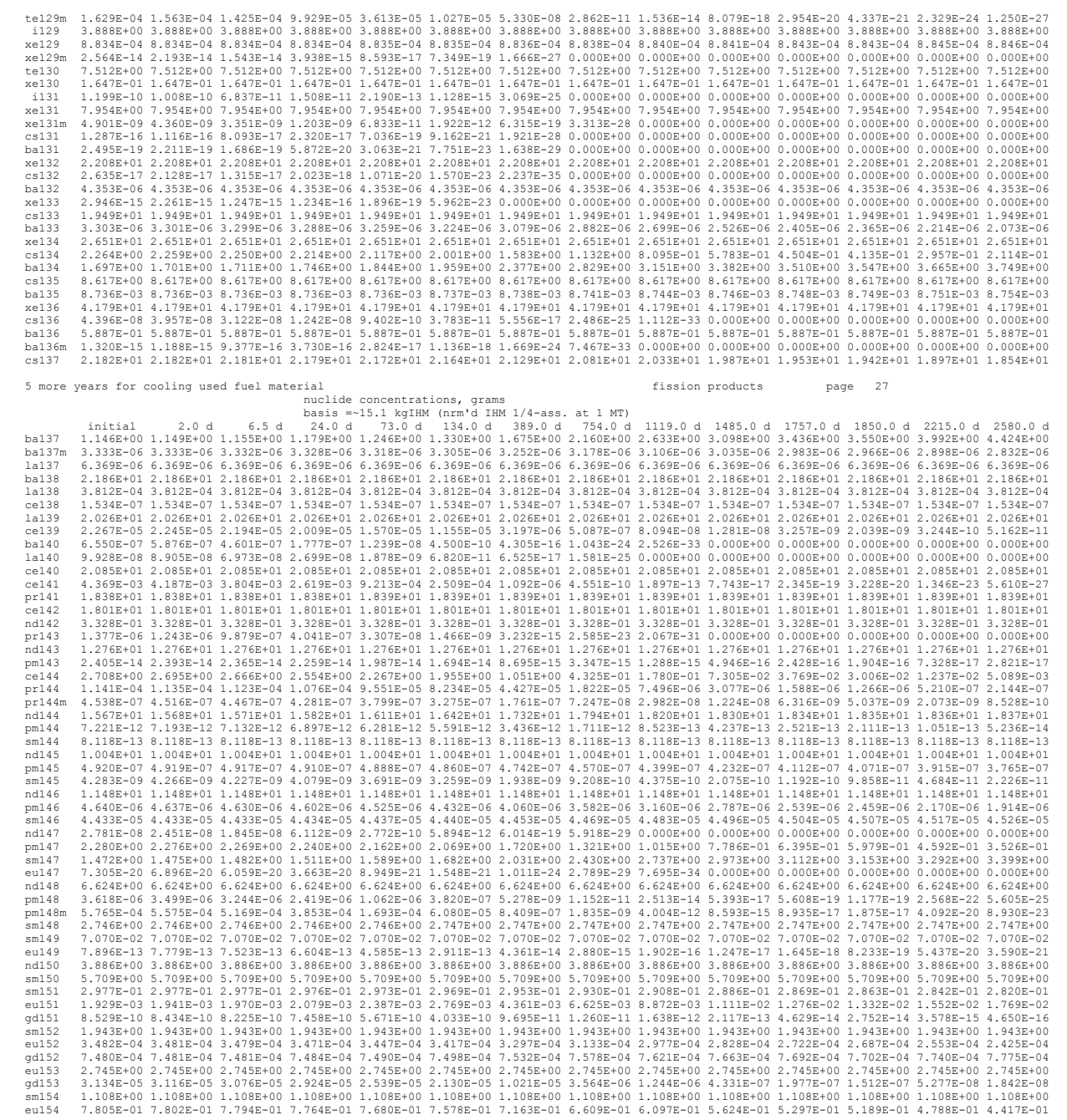

fission products page 2

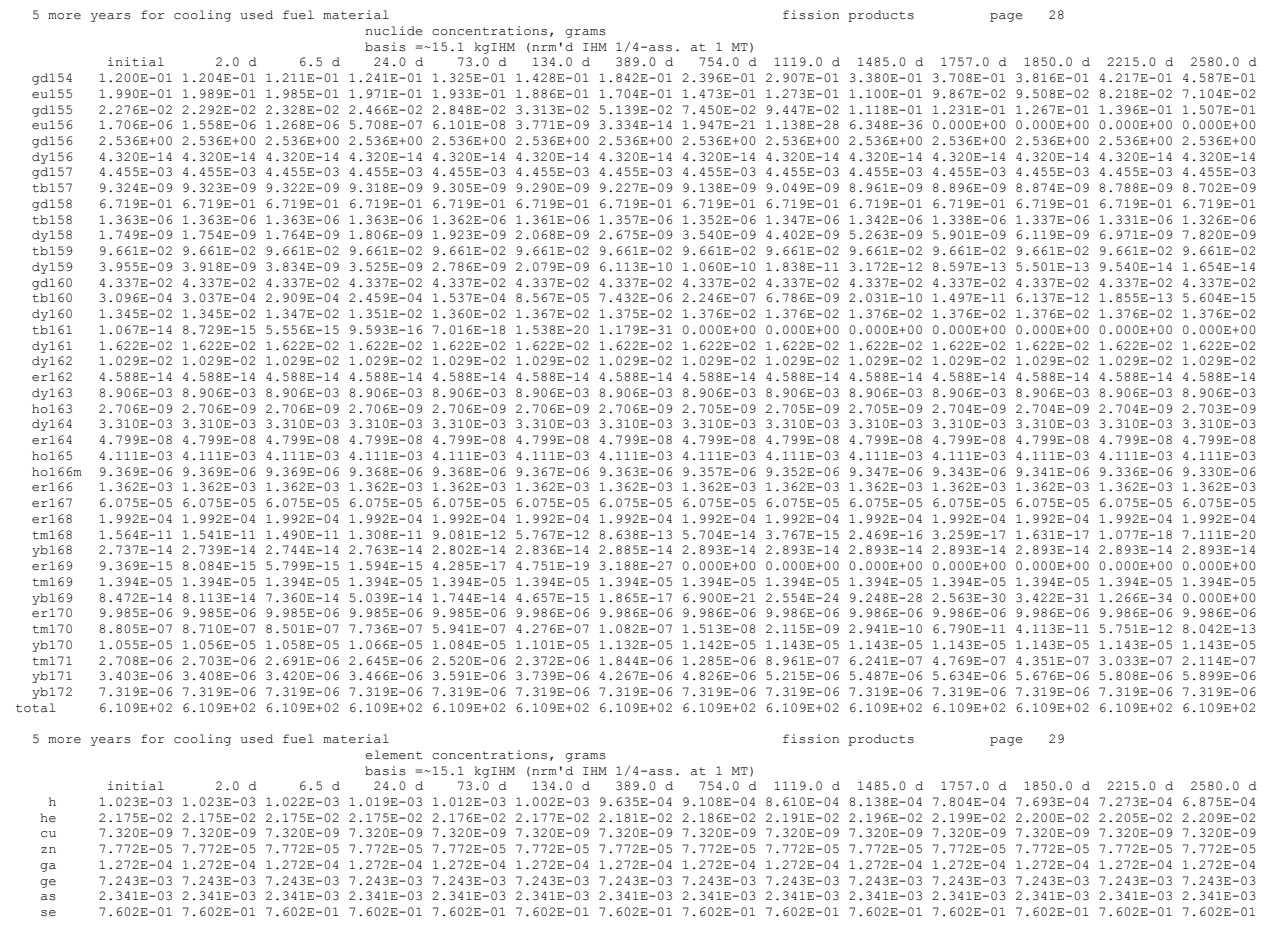

B-28 


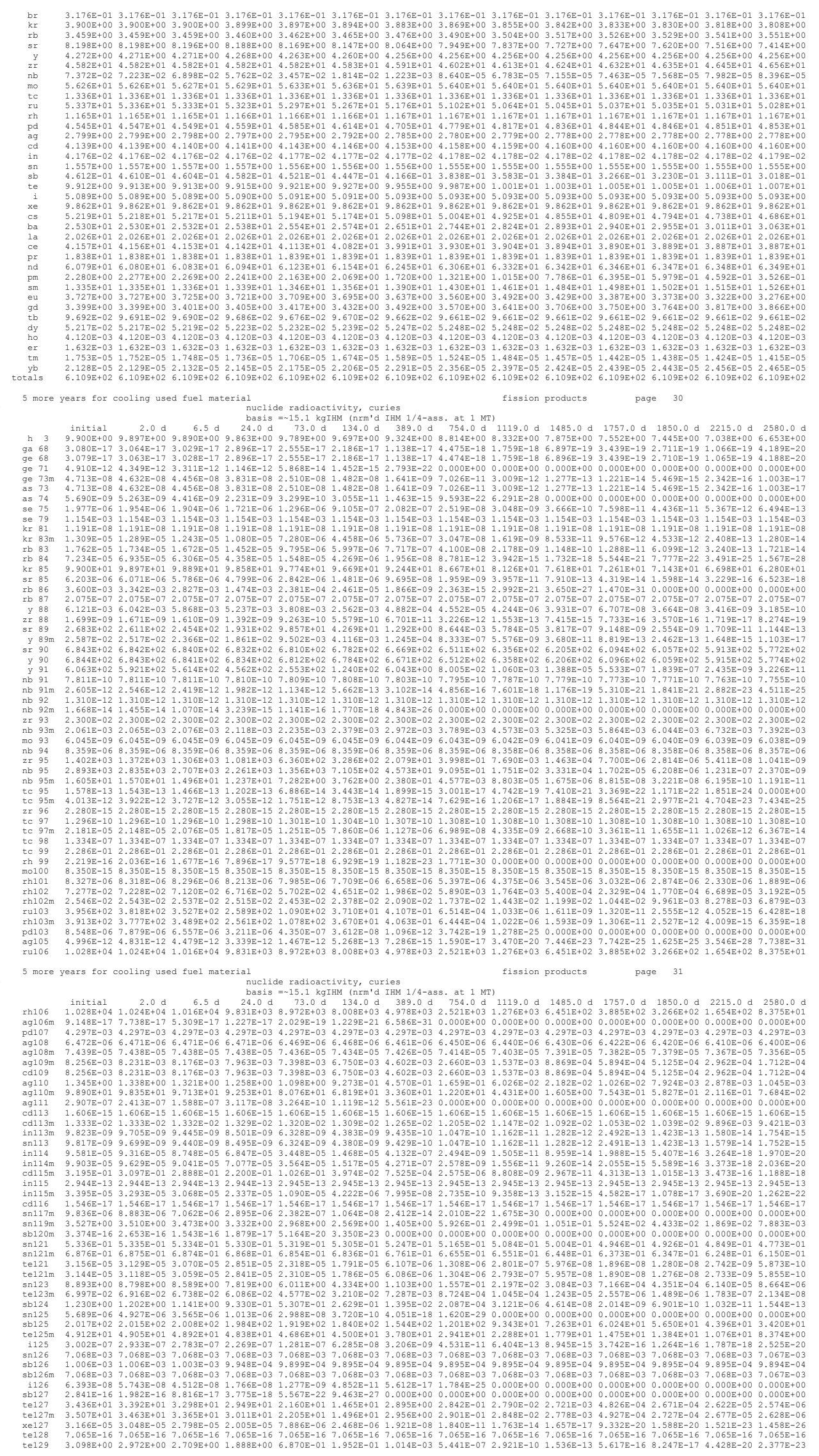

B-29 


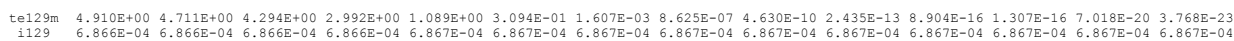

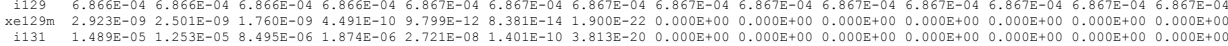

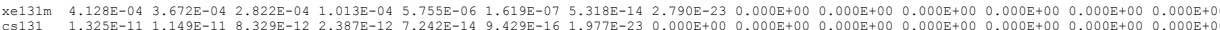
$\begin{array}{lllllllllllllllll}\text { ba131 } & 2.163 \mathrm{E}-14 & 1.918 \mathrm{E}-14 & 1.462 \mathrm{E}-14 & 5.092 \mathrm{E}-15 & 2.656 \mathrm{E}-16 & 6.721 \mathrm{E}-18 & 1.420 \mathrm{E}-24 & 0.000 \mathrm{E}+00 & 0.000 \mathrm{E}+00 & 0.000 \mathrm{E}+00 & 0.000 \mathrm{E}+00 & 0.000 \mathrm{E}+00 & 0.000 \mathrm{E}+00 & 0.000 \mathrm{E}+00 \\ \mathrm{cs} 132 & 4.025 \mathrm{E}-12 & 3.250 \mathrm{E}-12 & 2.008 \mathrm{E}-12 & 3.089 \mathrm{E}-13 & 1.635 \mathrm{E}-15 & 2.397 \mathrm{E}-18 & 3.416 \mathrm{E}-30 & 0.000 \mathrm{E}+00 & 0.000 \mathrm{E}+00 & 0.000 \mathrm{E}+00 & 0.000 \mathrm{E}+00 & 0.000 \mathrm{E}+00 & 0.000 \mathrm{E}+00 & 0.000 \mathrm{E}+00\end{array}$ 5 more years for cooling used fuel material nuclide radioactivity, curies
basis $=\sim 15.1 \mathrm{~kg}$ HMM (nrm'd IHM $1 / 4-$ ass. at 1 .

$$
\text { fission products page } 32
$$

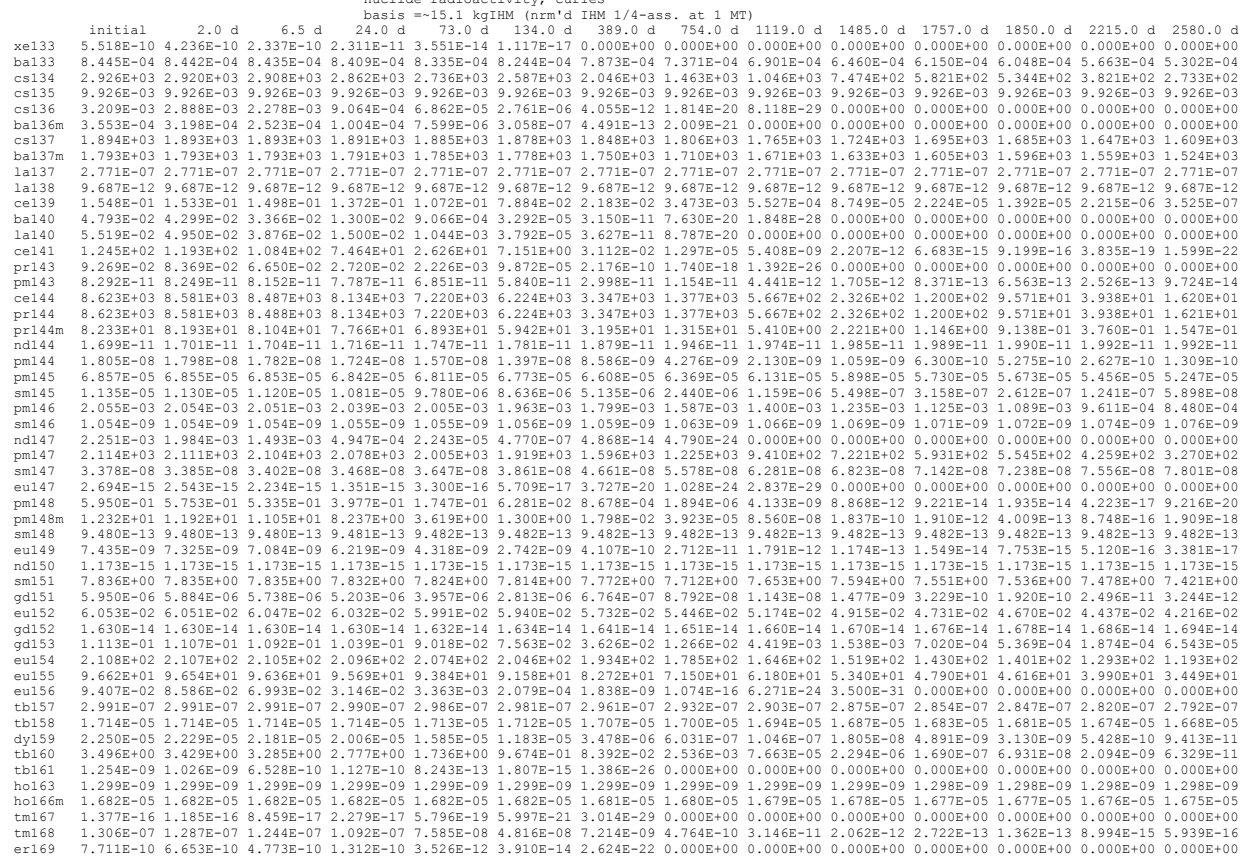

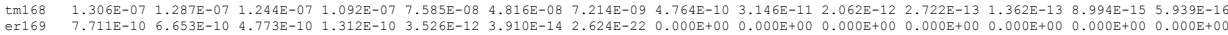

5 more years for cooling used fuel material nuclide radioactivity, curies
basis $=\sim 15.1 \mathrm{~kg}$ HMM (nrm'd IHM $1 / 4$-ass. at $1 \mathrm{MT}$ )

fission products page 33

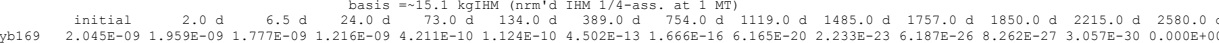

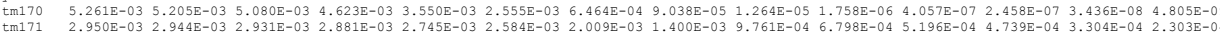

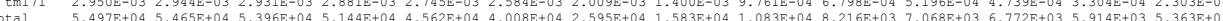

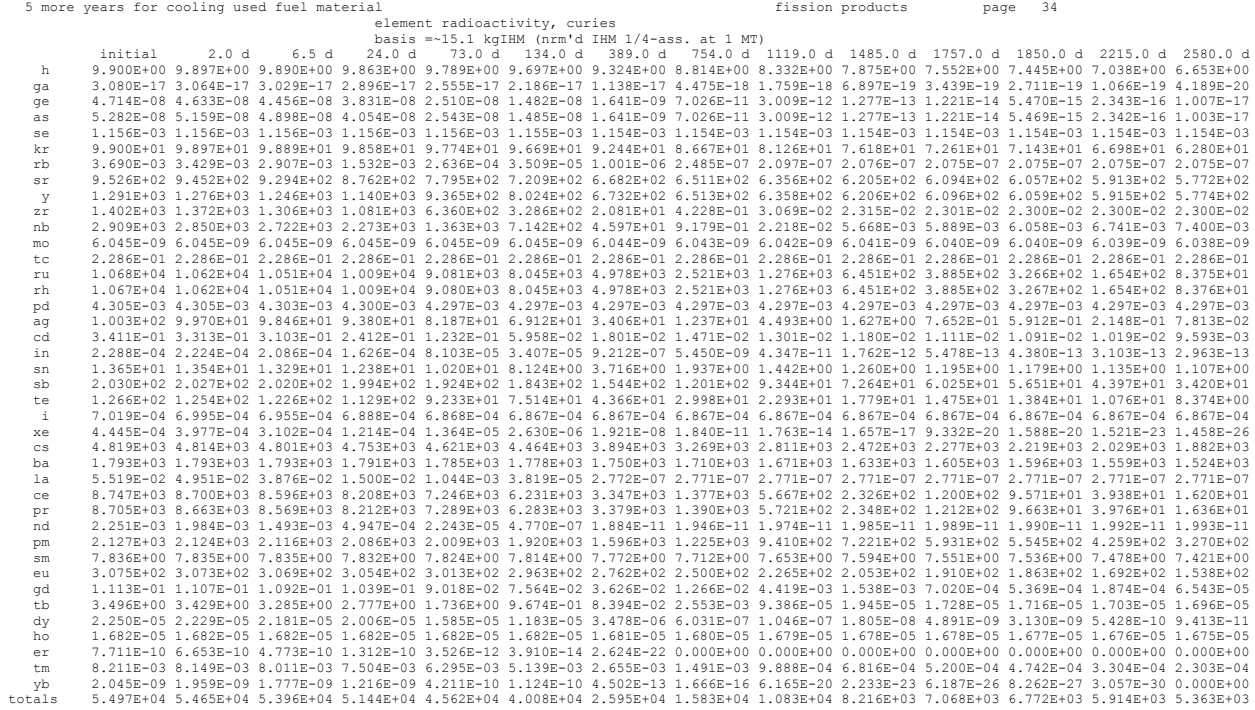

$\begin{array}{llll}-13 & 1.666-16 & 0 & 0\end{array}$

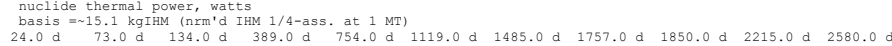

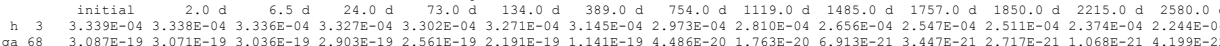

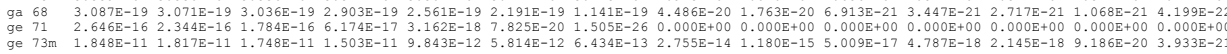

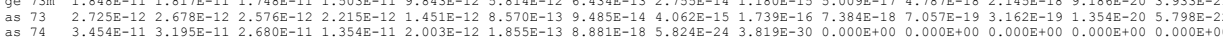

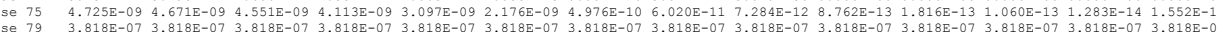

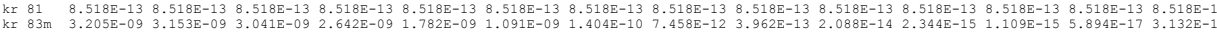

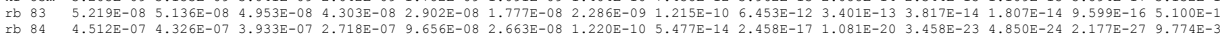

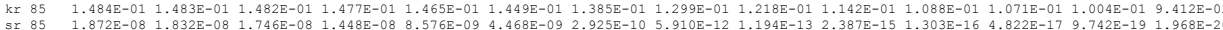

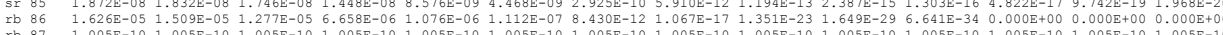

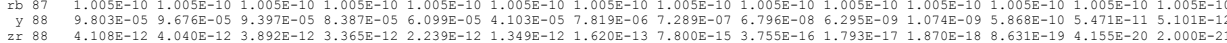

B-30 


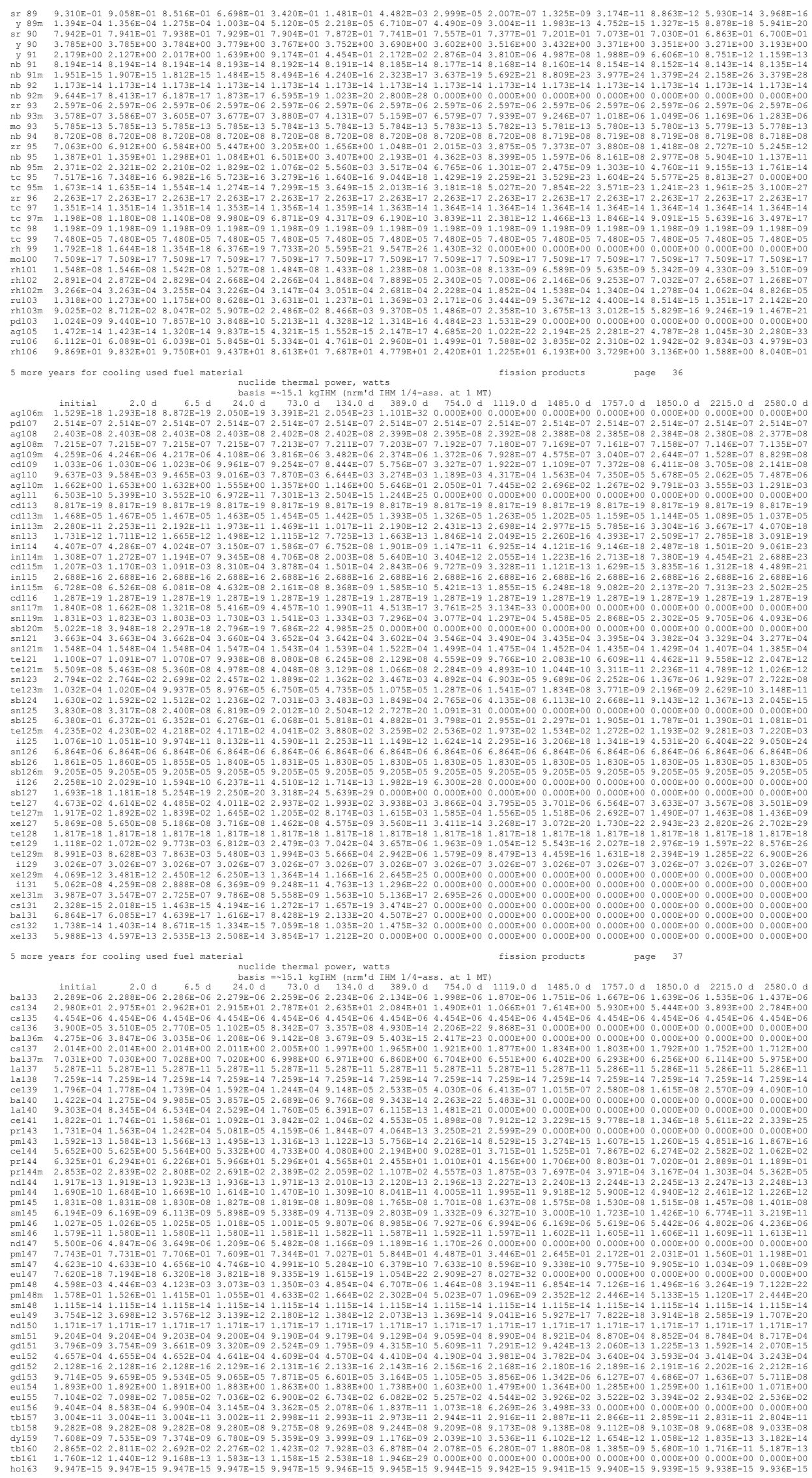

B-31 


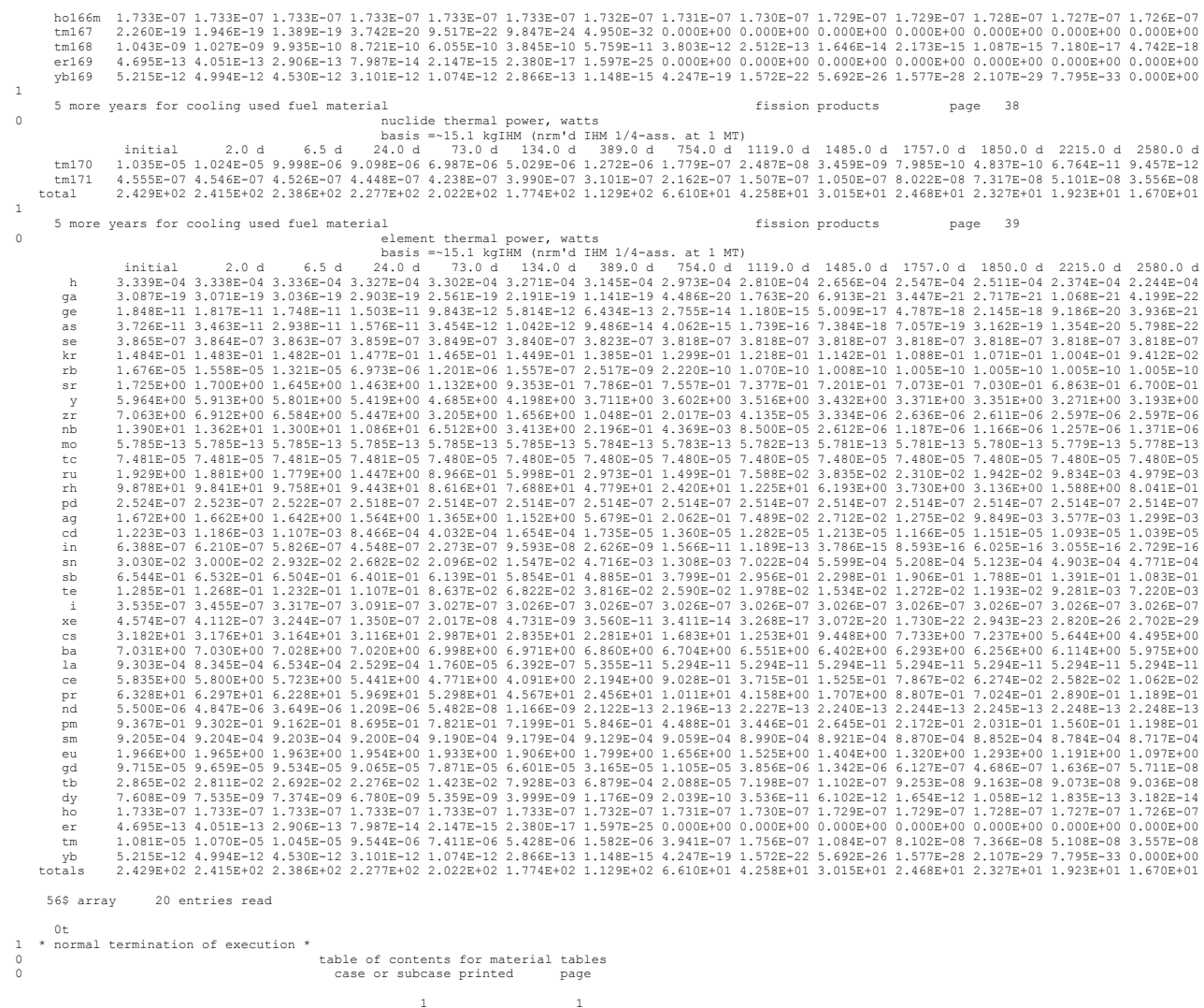

B-32 


\section{B.3 ORIGEN RESULTS FOR COOLING MOX FUEL ROD C1}
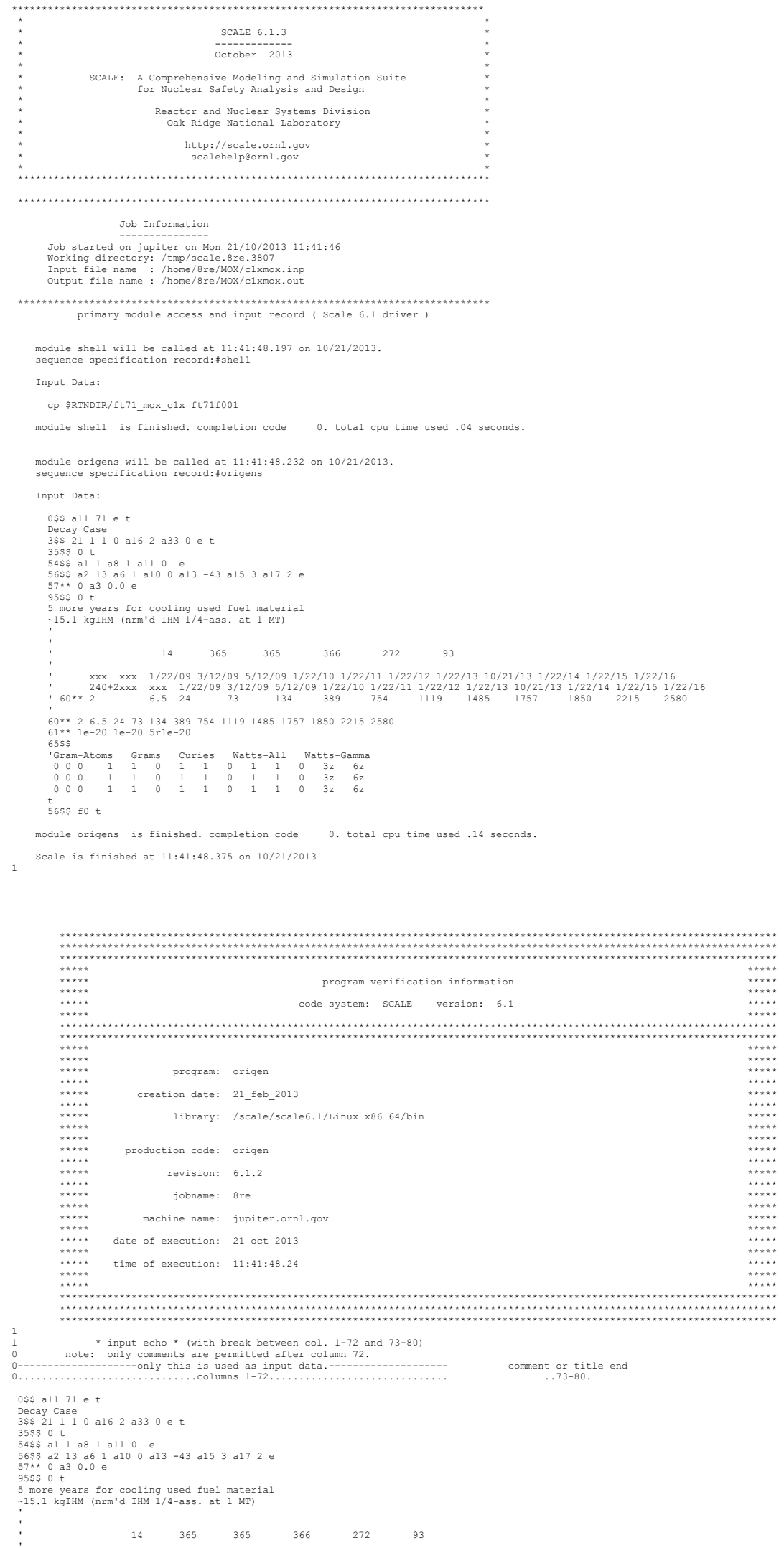


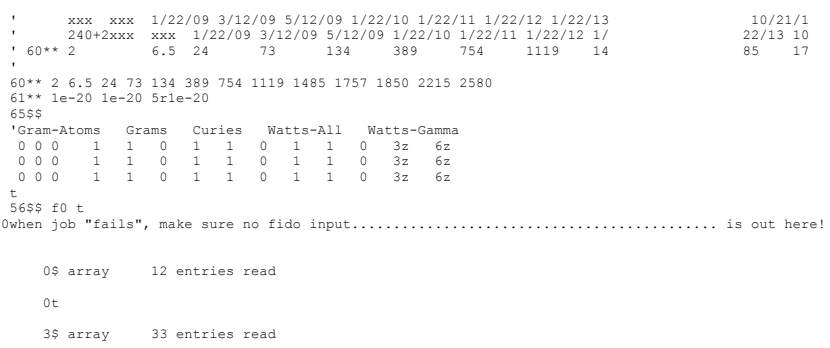




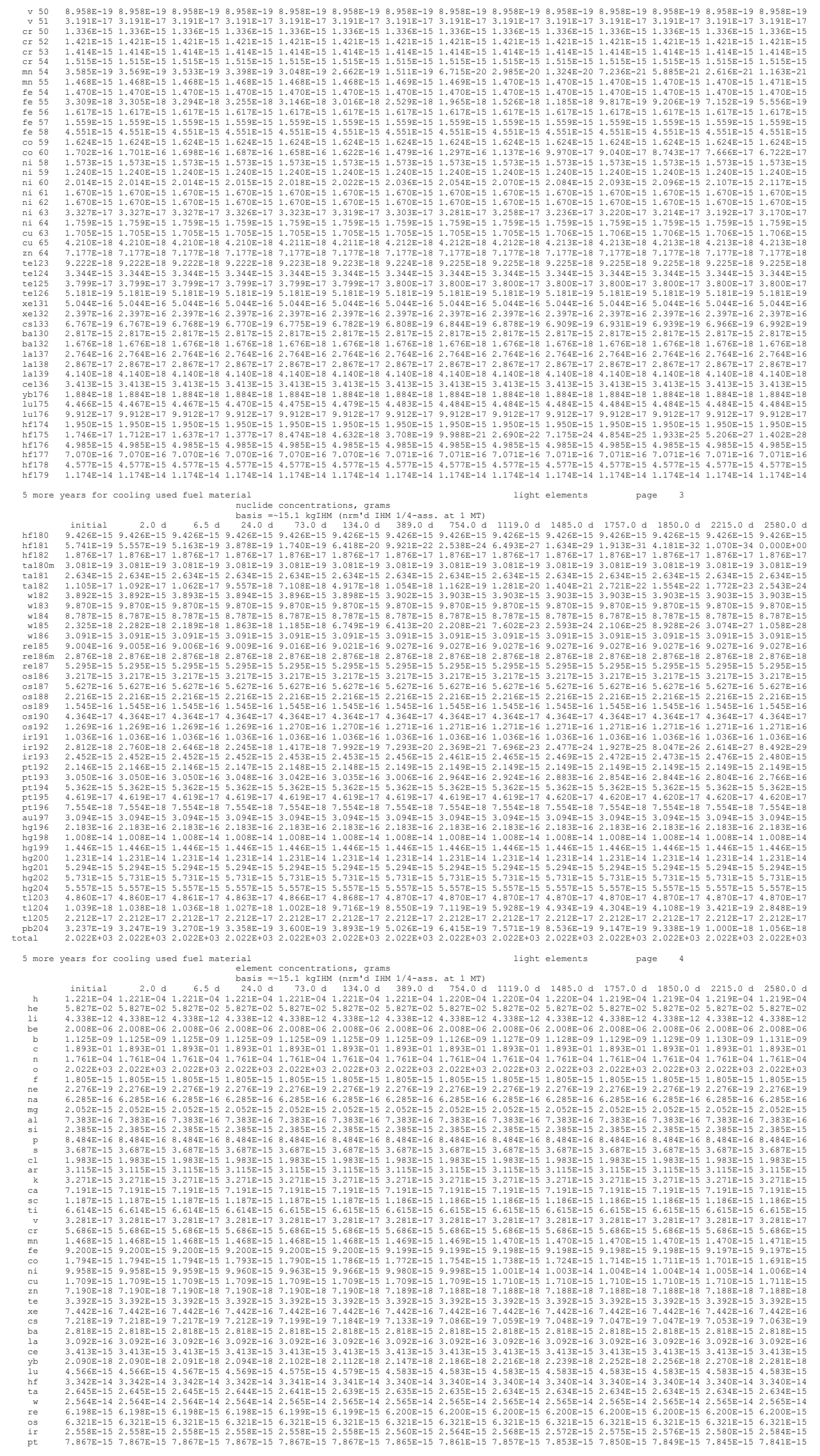

B-35 


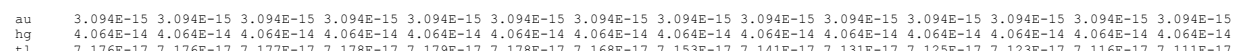

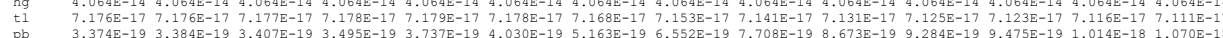

$\begin{array}{llllll} & \end{array}$ 5 more years for cooling used fuel materia

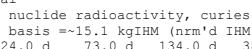
light elements page 5

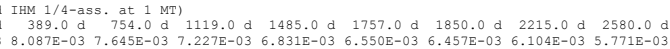

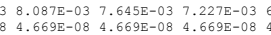

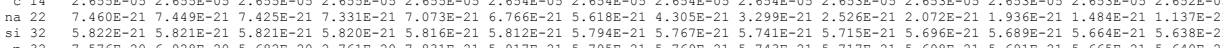

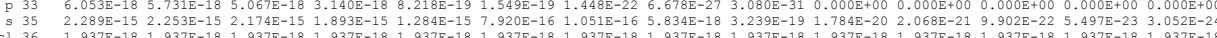

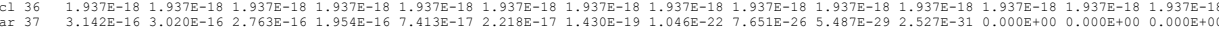

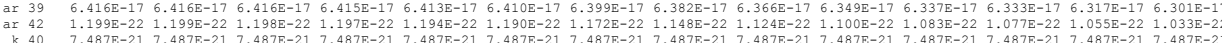

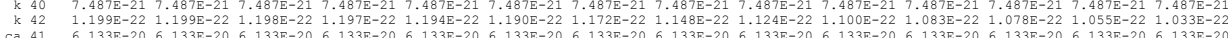

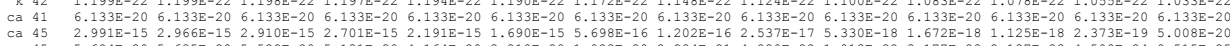

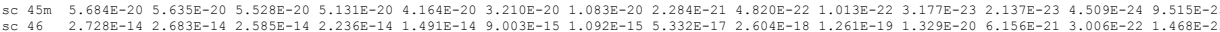

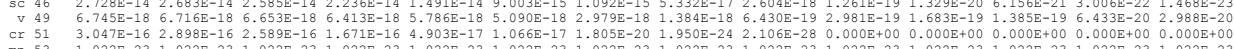

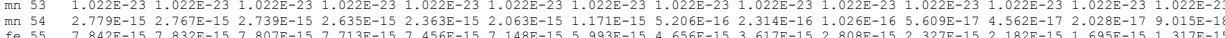

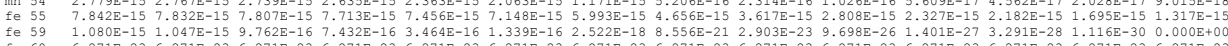

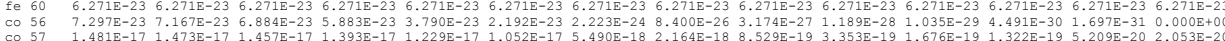

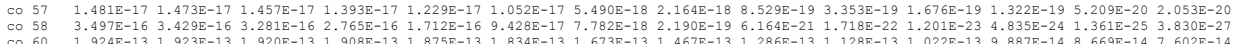

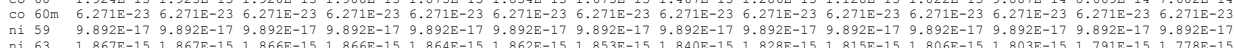

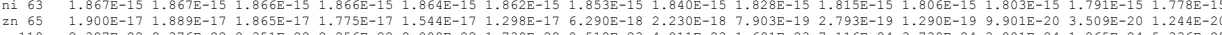

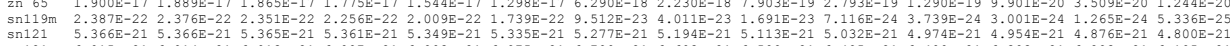

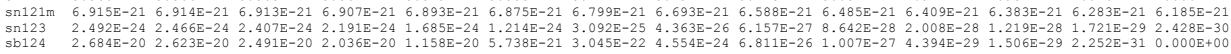

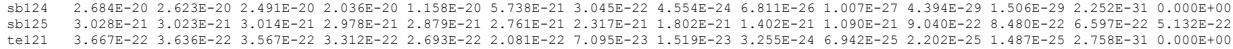

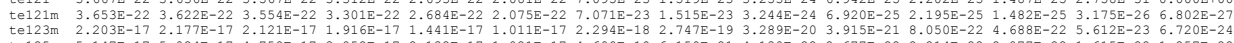

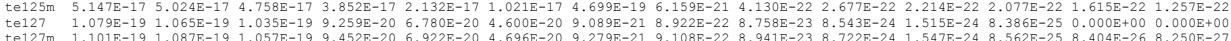

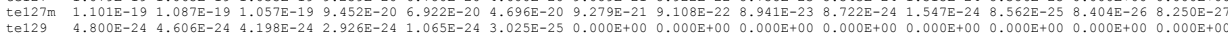

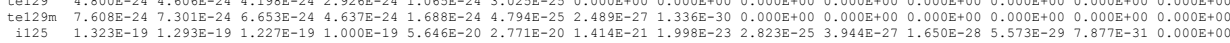

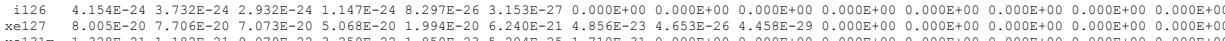

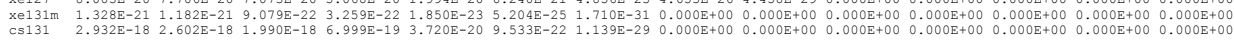

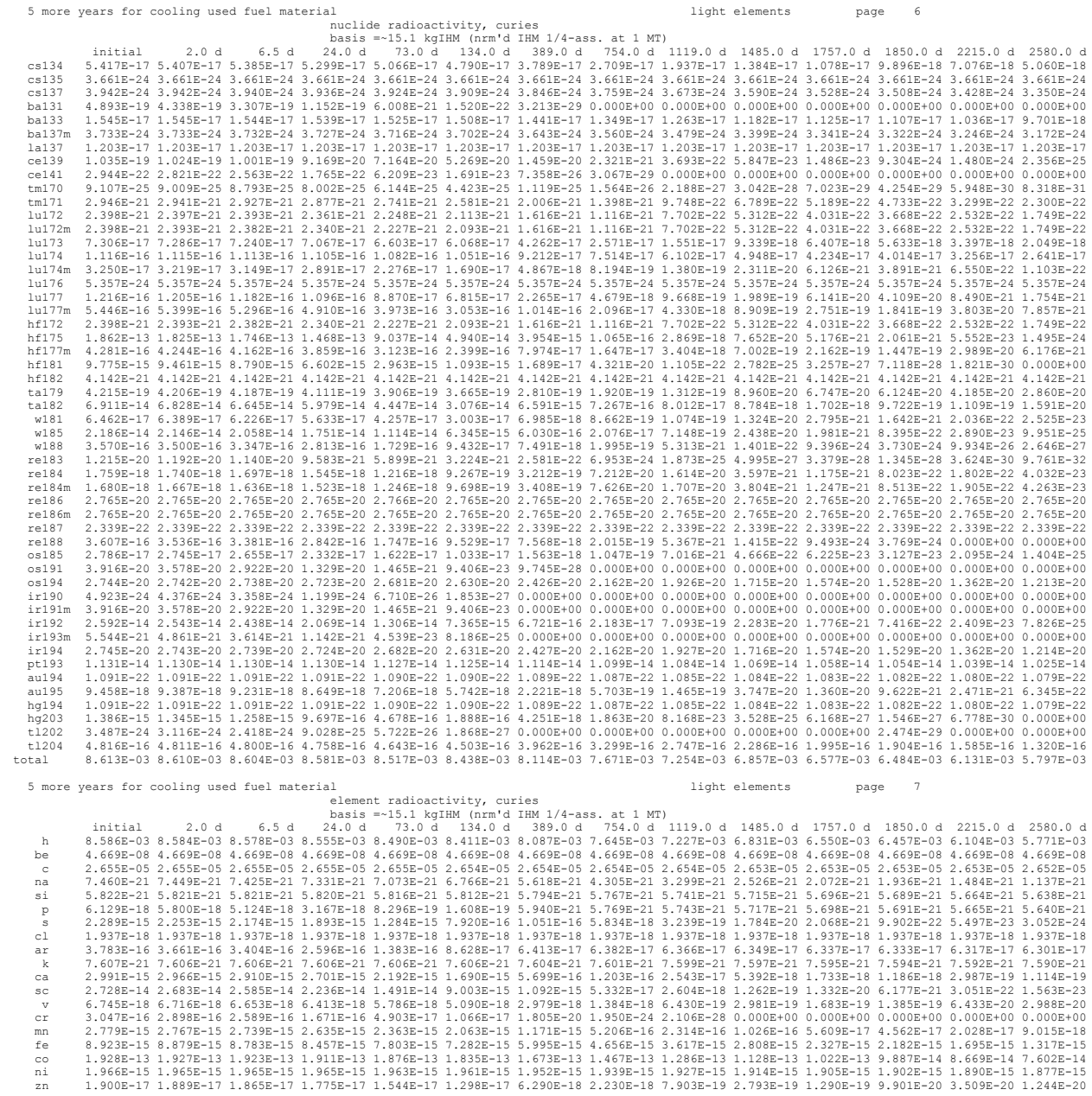

B-36 


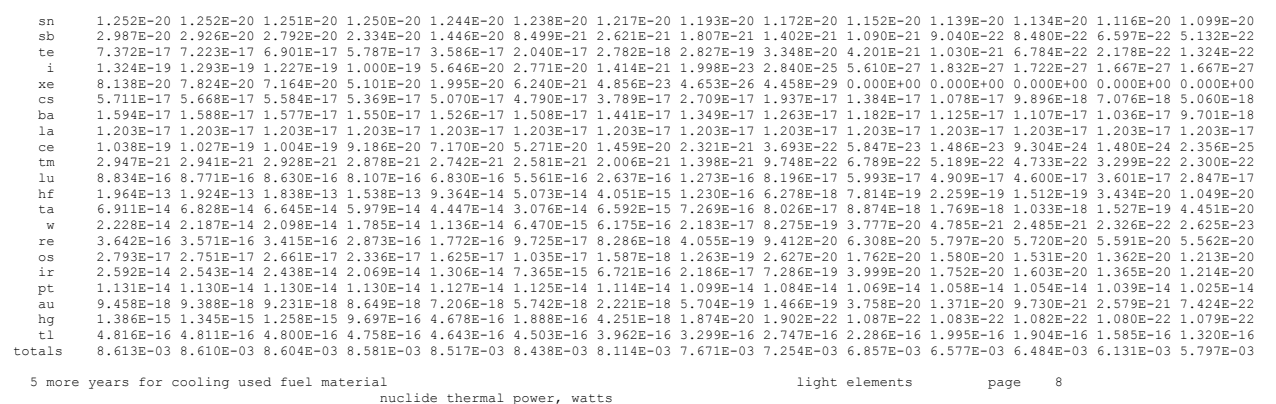

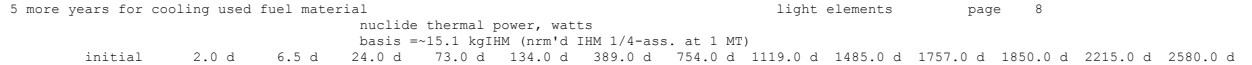

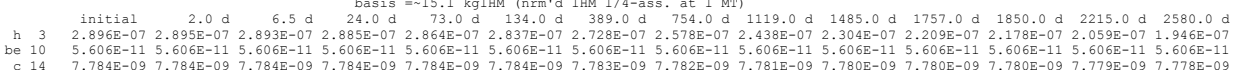

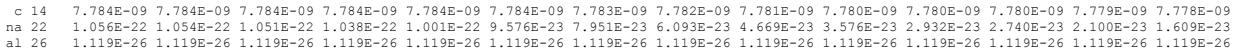

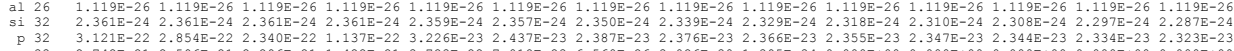

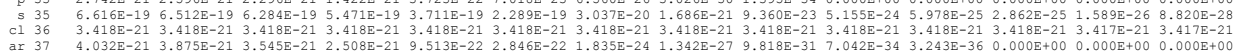

ax
ax 39
8

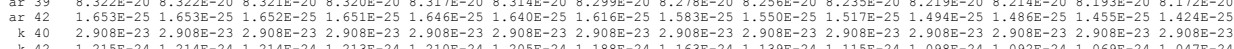

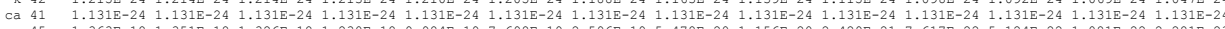

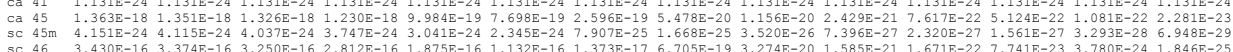

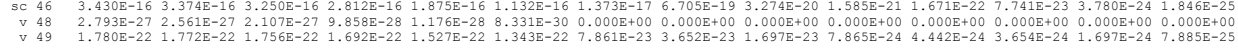

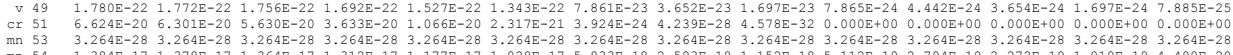

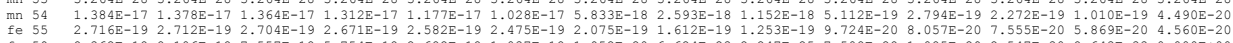

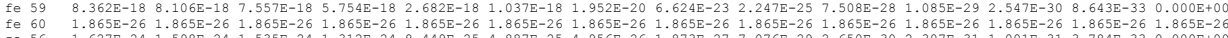

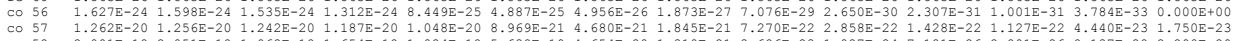

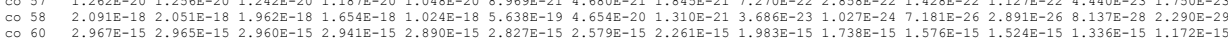

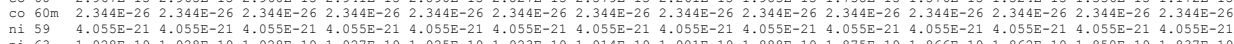

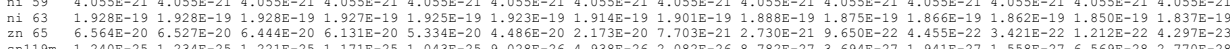

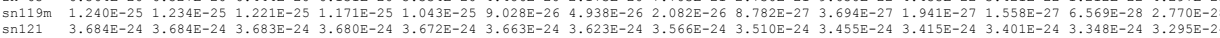

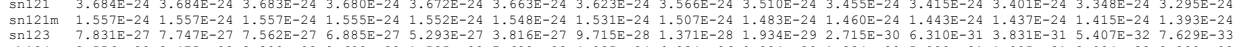

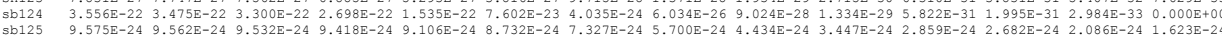

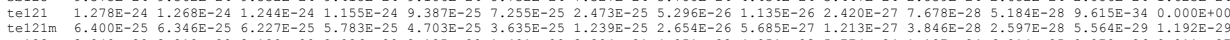

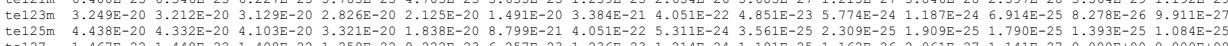

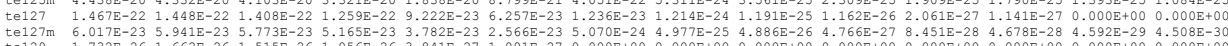

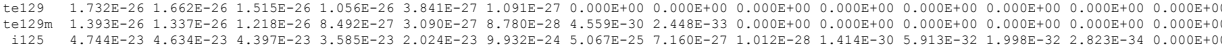

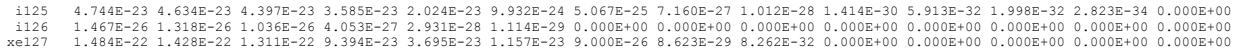

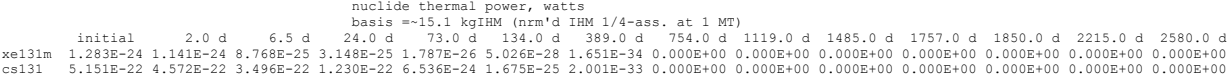

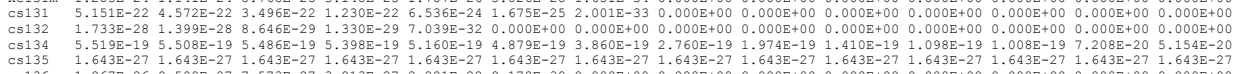

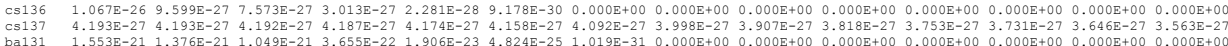

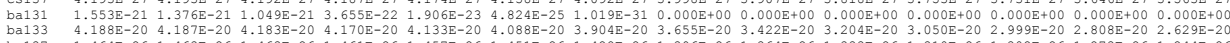

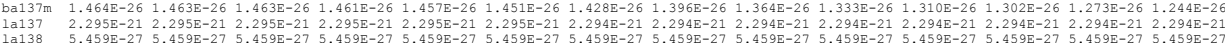

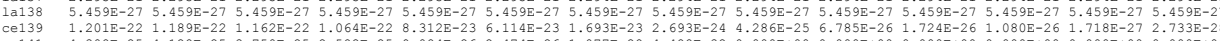

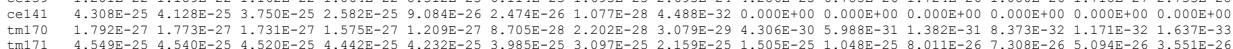

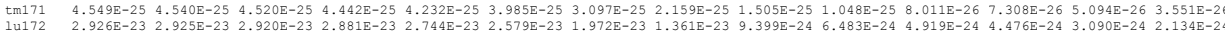

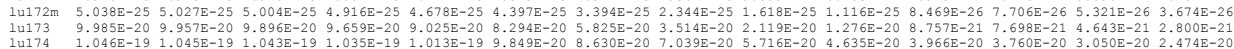

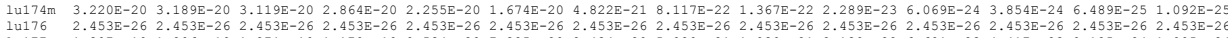

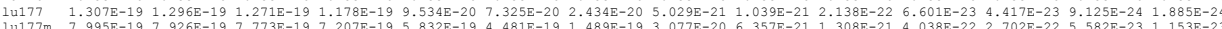

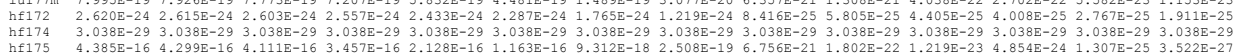

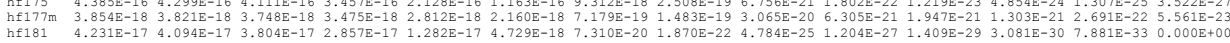

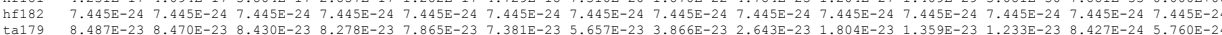

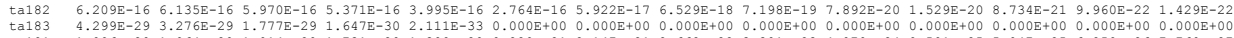

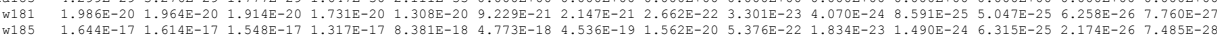

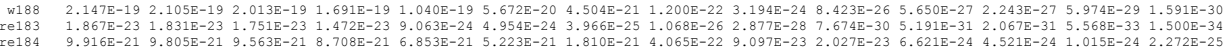

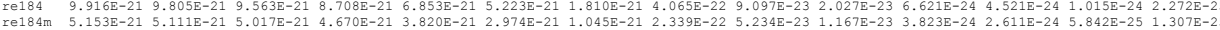

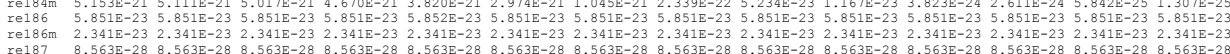

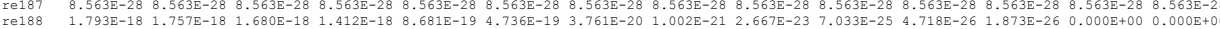

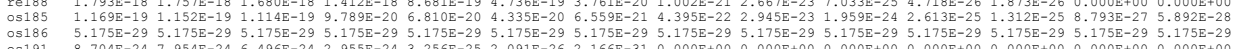

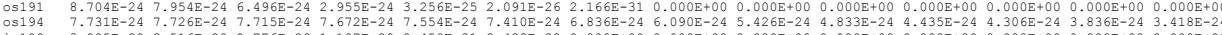

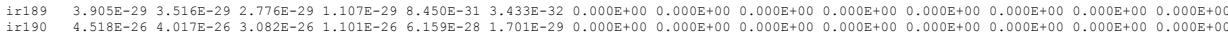

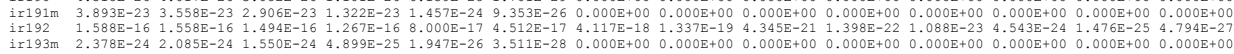
5 more years for cooling used fuel materiat nuclide thernal power, watts light elements page 10

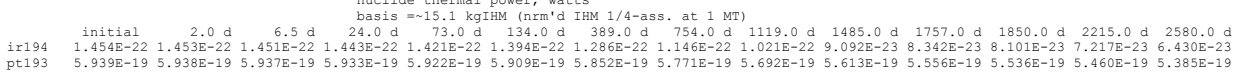




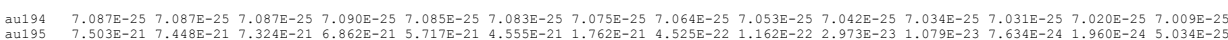

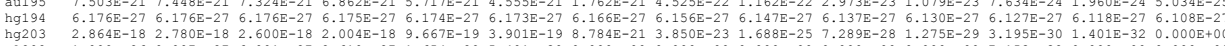
$\begin{array}{llllllllllllllllll}\text { total } & 2.974 \mathrm{E}-07 & 2.974 \mathrm{E}-07 & 2.972 \mathrm{E}-07 & 2.964 \mathrm{E}-07 & 2.942 \mathrm{E}-07 & 2.915 \mathrm{E}-07 & 2.806 \mathrm{E}-07 & 2.657 \mathrm{E}-07 & 2.516 \mathrm{E}-07 & 2.382 \mathrm{E}-07 & 2.288 \mathrm{E}-07 & 2.256 \mathrm{E}-07 & 2.137 \mathrm{E}-07 & 2.025 \mathrm{E}-07\end{array}$

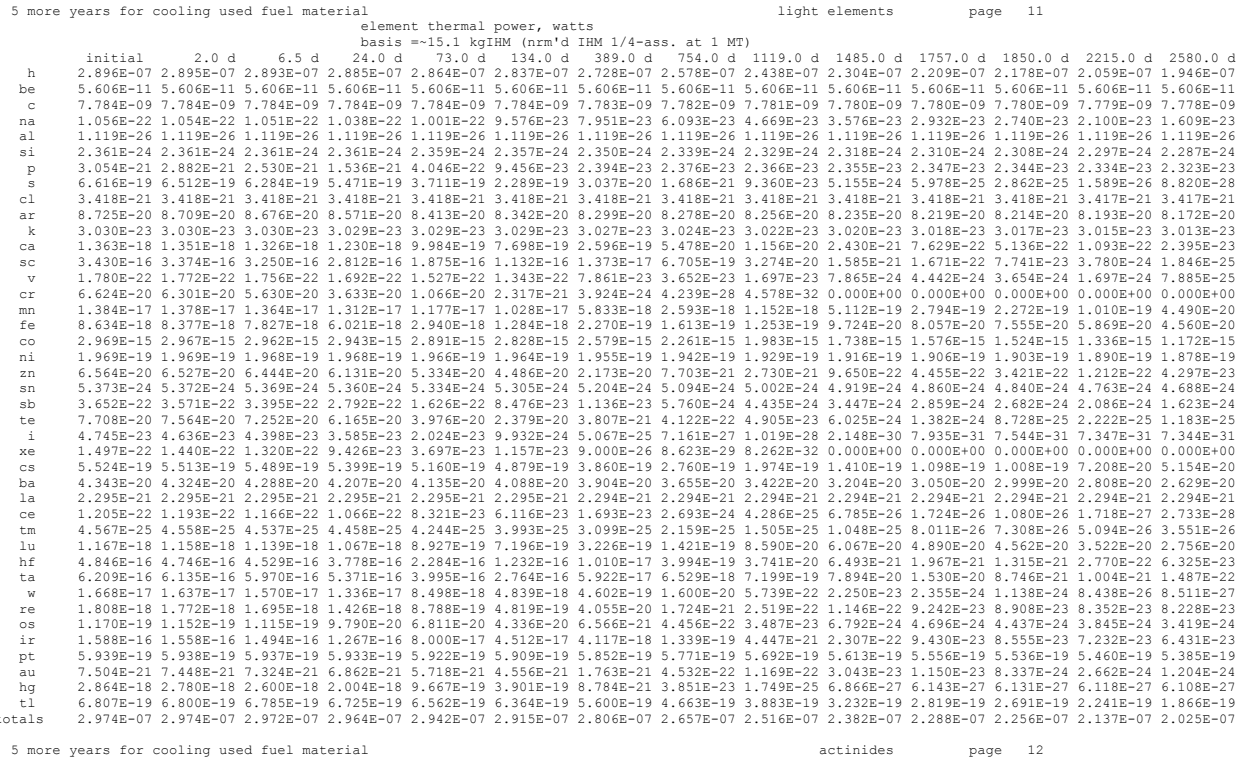
5

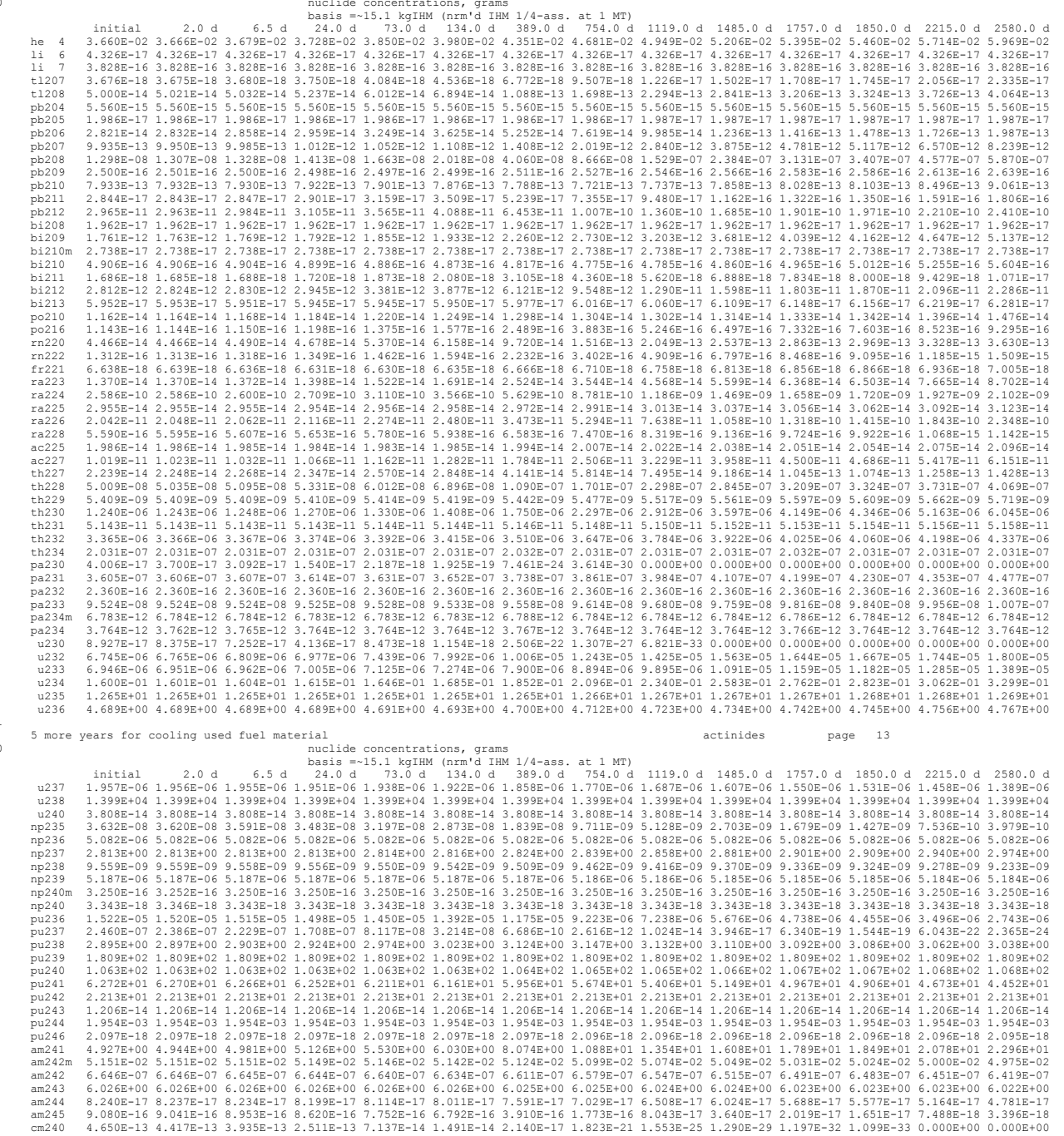

B-38 


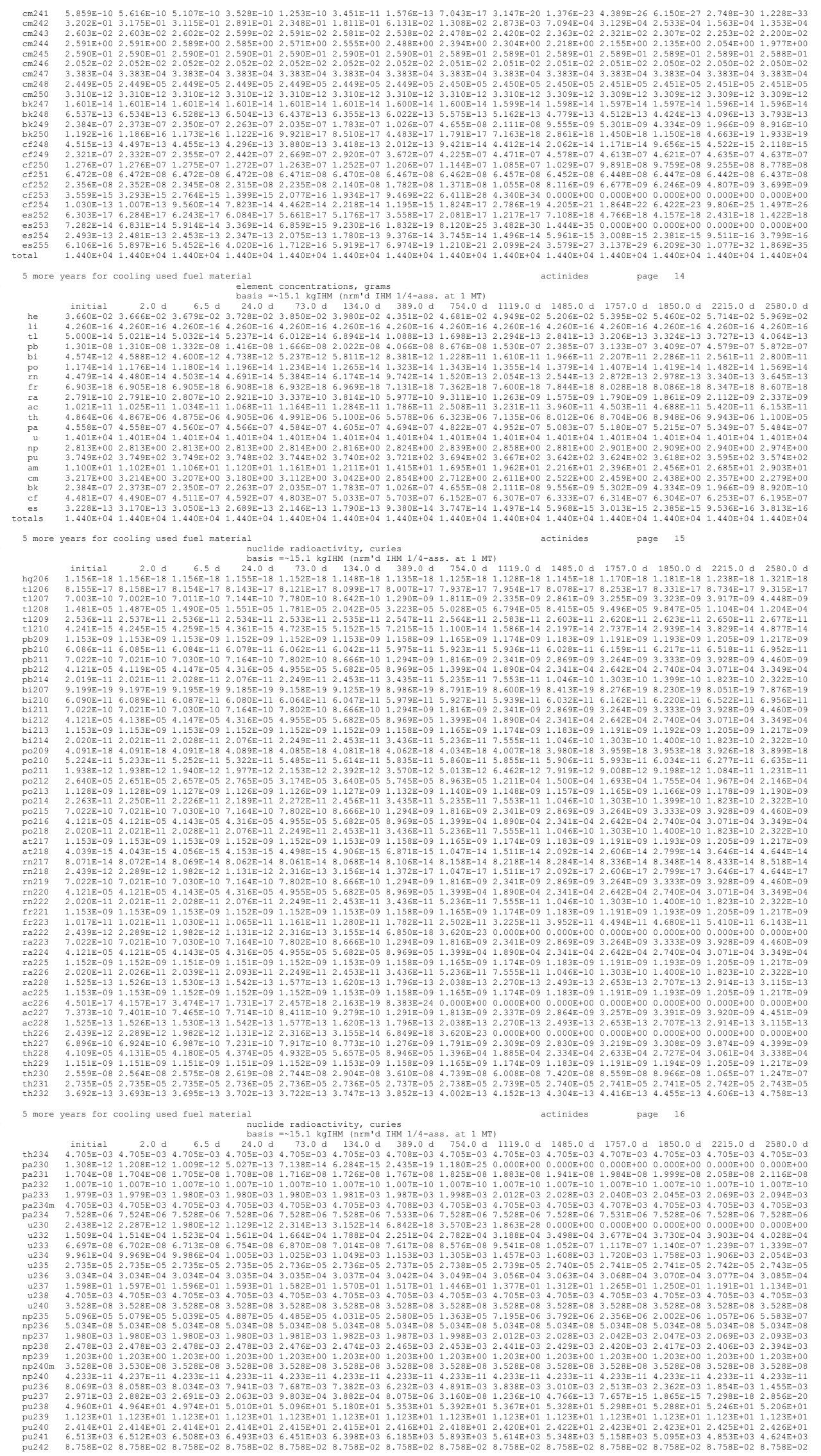

B-39 


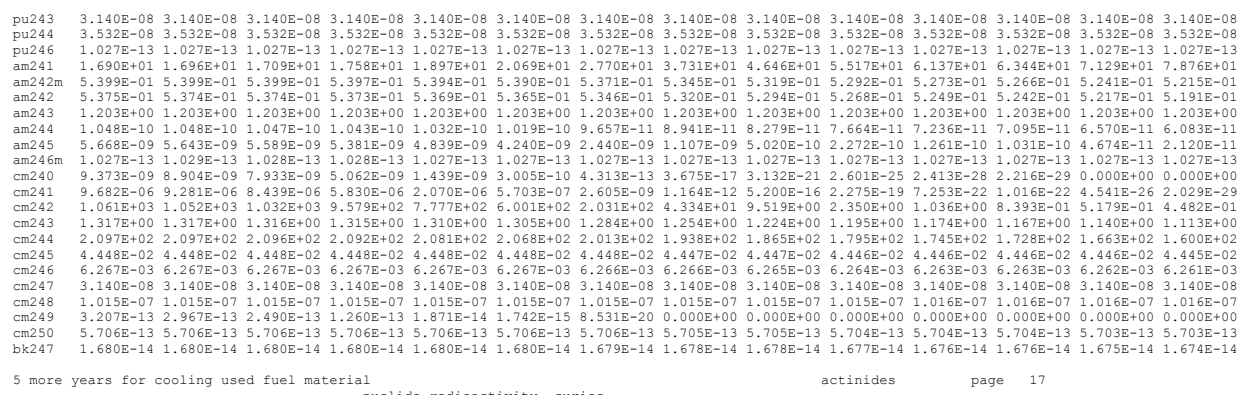

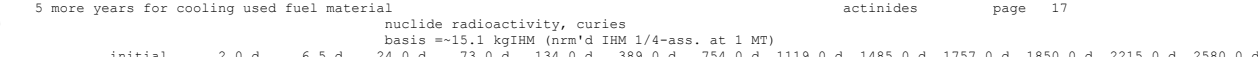

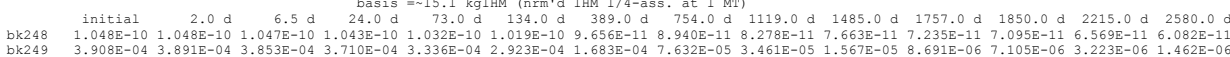

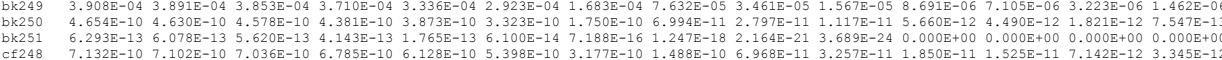

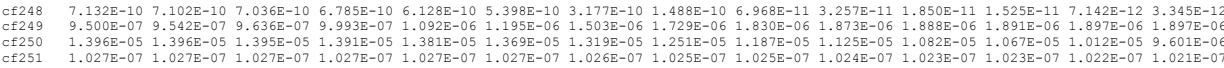

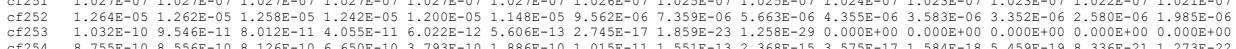

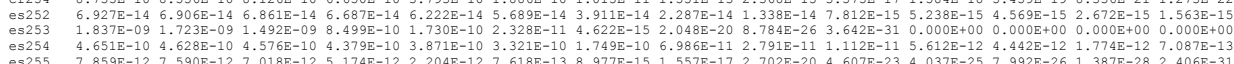

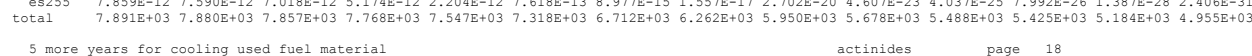

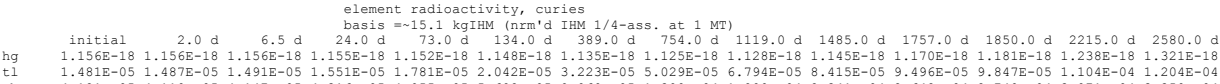

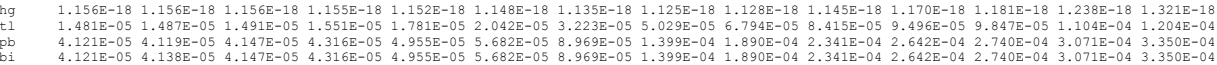

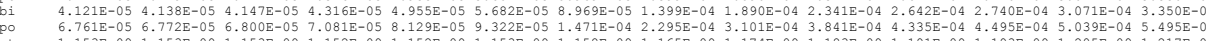

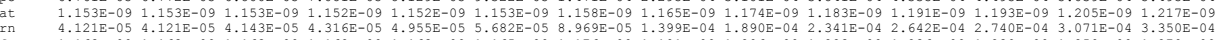

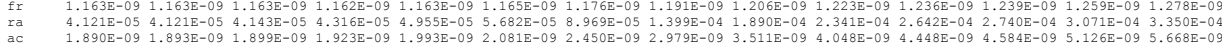

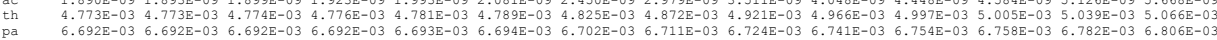

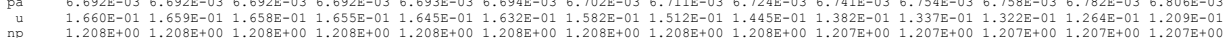

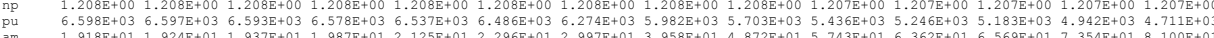

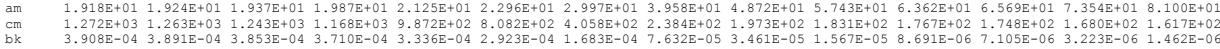

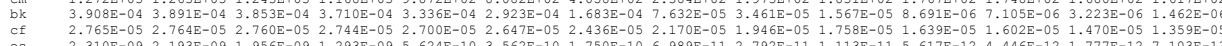

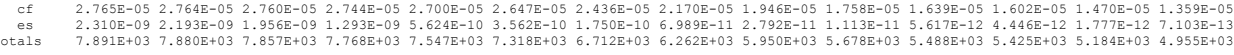

5 more years for cooling used fuel material $\quad$ actinides page 19

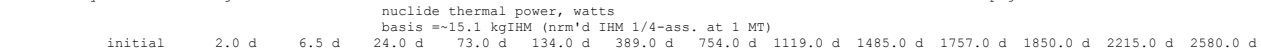

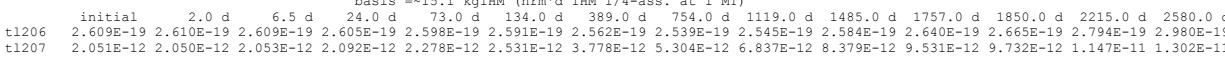

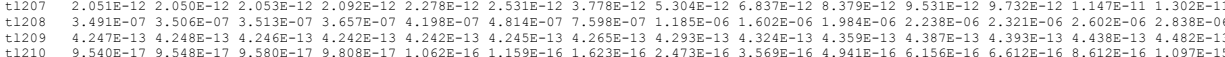

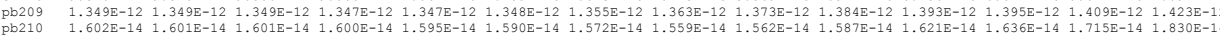

$\begin{array}{lllllllllllllll}\mathrm{pb} 211 & 2.136 \mathrm{E}-12 & 2.136 \mathrm{E}-12 & 2.139 \mathrm{E}-12 & 2.179 \mathrm{E}-12 & 2.373 \mathrm{E}-12 & 2.636 \mathrm{E}-12 & 3.935 \mathrm{E}-12 & 5.525 \mathrm{E}-12 & 7.121 \mathrm{E}-12 & 8.728 \mathrm{E}-12 & 9.928 \mathrm{E}-12 & 1.014 \mathrm{E}-11 & 1.195 \mathrm{E}-11 & 1.357 \mathrm{E}-1\end{array}$

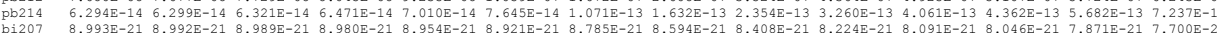

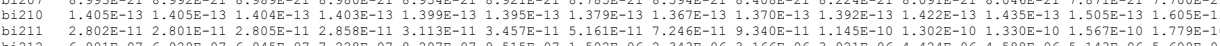

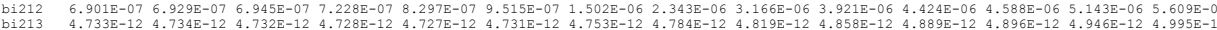

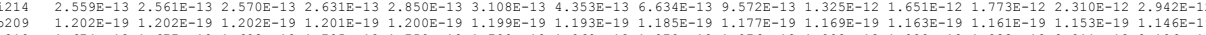

$\begin{array}{lllllllllllllllll}p 0210 & 1.674 \mathrm{E}-12 & 1.677 \mathrm{E}-12 & 1.68 \mathrm{E}-12 & 1.705 \mathrm{E}-12 & 1.758 \mathrm{E}-12 & 1.799 \mathrm{E}-12 & 1.869 \mathrm{E}-12 & 1.878 \mathrm{E}-12 & 1.876 \mathrm{E}-12 & 1.892 \mathrm{E}-12 & 1.920 \mathrm{E}-12 & 1.93 \mathrm{E}-12 & 2.011 \mathrm{E}-12 & 2.126 \mathrm{E}-12 \\ \mathrm{p} 0211 & 8.722 \mathrm{E}-14 & 8.721 \mathrm{E}-14 & 8.732 \mathrm{E}-14 & 8.898 \mathrm{E}-14 & 9.690 \mathrm{E}-14 & 1.076 \mathrm{E}-13 & 1.607 \mathrm{E}-13 & 2.256 \mathrm{E}-13 & 2.908 \mathrm{E}-13 & 3.564 \mathrm{E}-13 & 4.054 \mathrm{E}-13 & 4.139 \mathrm{E}-13 & 4.879 \mathrm{E}-13 & 5.540 \mathrm{E}-1\end{array}$

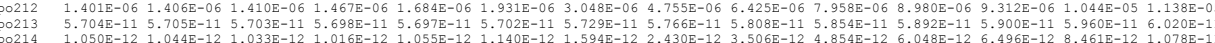

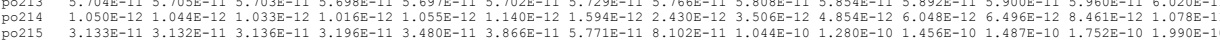

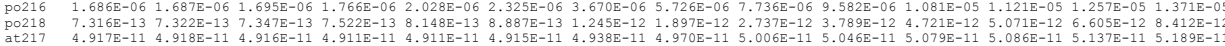

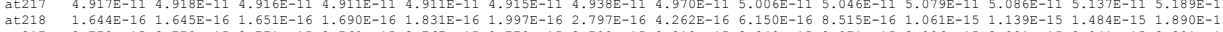

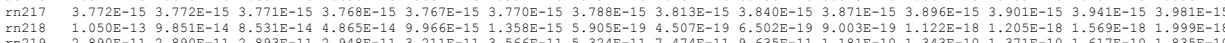

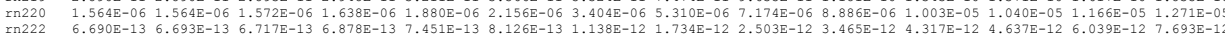

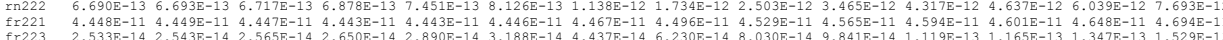

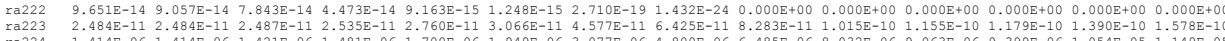

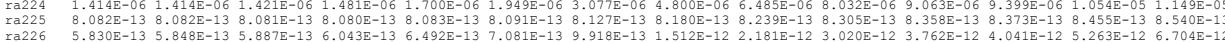

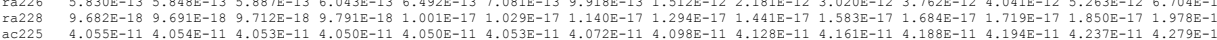

$\begin{array}{llllllllllllllllll}\text { ac226 } & 1.125 \mathrm{E}-19 & 1.039 \mathrm{E}-19 & 8.683 \mathrm{E}-20 & 4.325 \mathrm{E}-20 & 6.140 \mathrm{E}-21 & 5.405 \mathrm{E}-22 & 2.095 \mathrm{E}-26 & 0.000 \mathrm{E}+00 & 0.000 \mathrm{E}+00 & 0.000 \mathrm{E}+00 & 0.000 \mathrm{E}+00 & 0.000 \mathrm{E}+00 & 0.000 \mathrm{E}+00 & 0.000 \mathrm{E}+0 \\ \text { ac227 } & 3.749 \mathrm{E}-13 & 3.764 \mathrm{E}-13 & 3.796 \mathrm{E}-13 & 3.923 \mathrm{E}-13 & 4.277 \mathrm{E}-13 & 4.719 \mathrm{E}-13 & 6.567 \mathrm{E}-13 & 9.221 \mathrm{E}-13 & 1.188 \mathrm{E}-12 & 1.456 \mathrm{E}-12 & 1.656 \mathrm{E}-12 & 1.725 \mathrm{E}-12 & 1.994 \mathrm{E}-12 & 2.264 \mathrm{E}-1\end{array}$

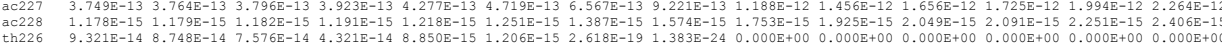

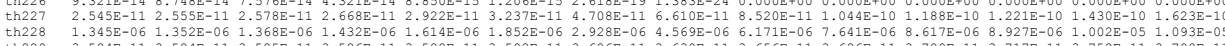

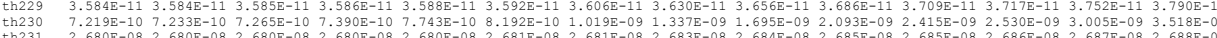

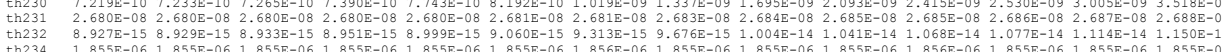
5 more years for cooling used fuel material
nuclide thermal power, watts
actinides page 20

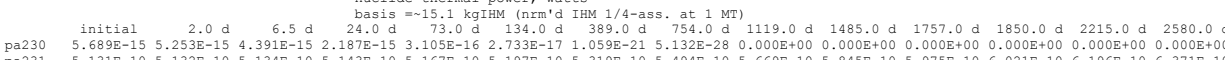

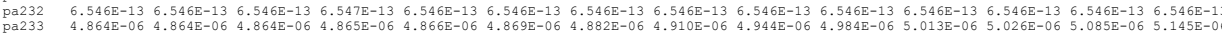

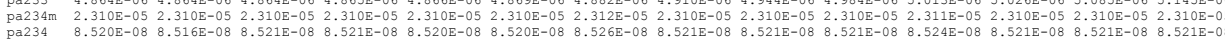

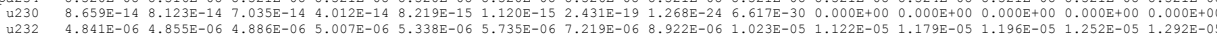

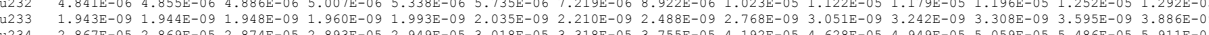

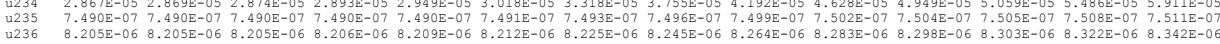




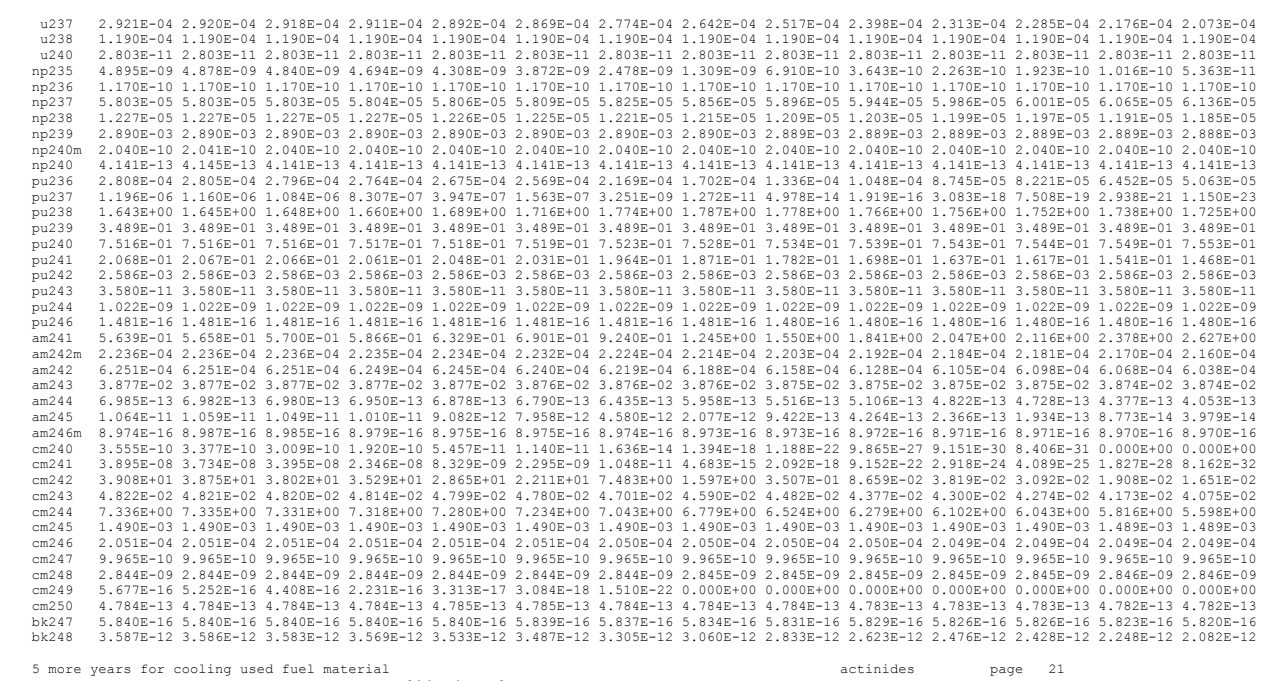

5 more years for cooling used fuel material
nuclide thermal power, watts
basis $=\sim 15.1 \mathrm{kgIHM}$ (nrm'd IHM 1/4-ass. at $1 \mathrm{MT}$ )

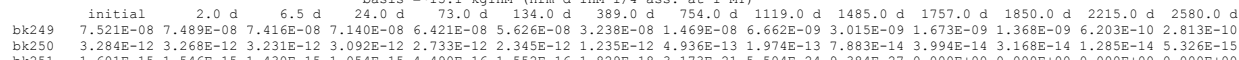

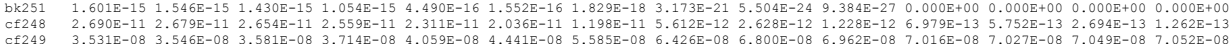

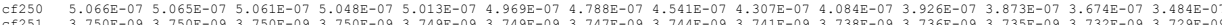

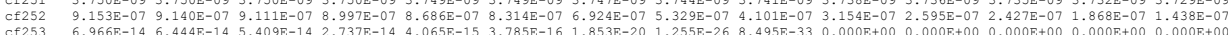

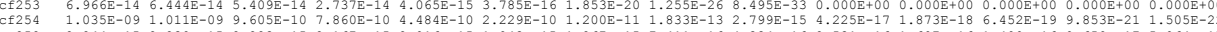

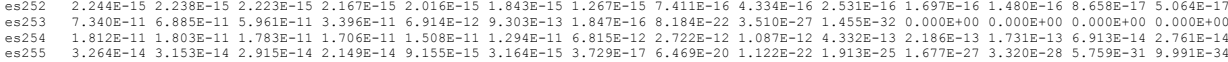

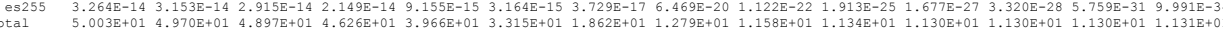
5 more years for cooling used fuel material

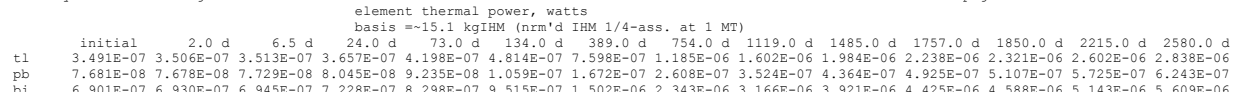

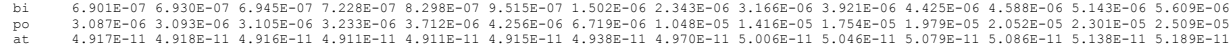

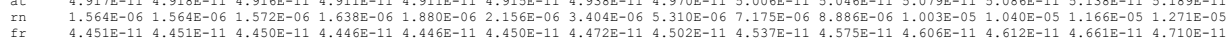

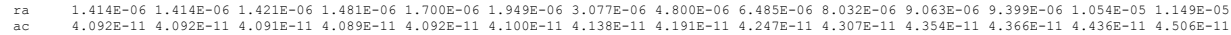

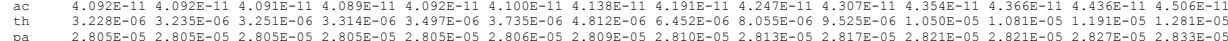

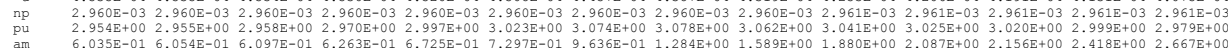

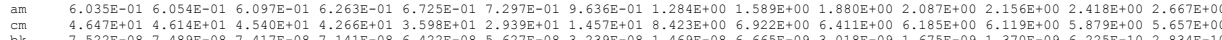

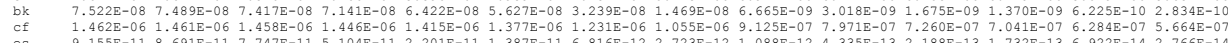

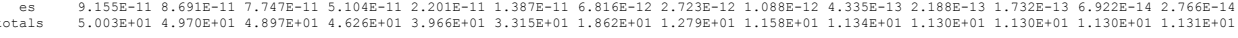
5 more years for cooling used fuel materia

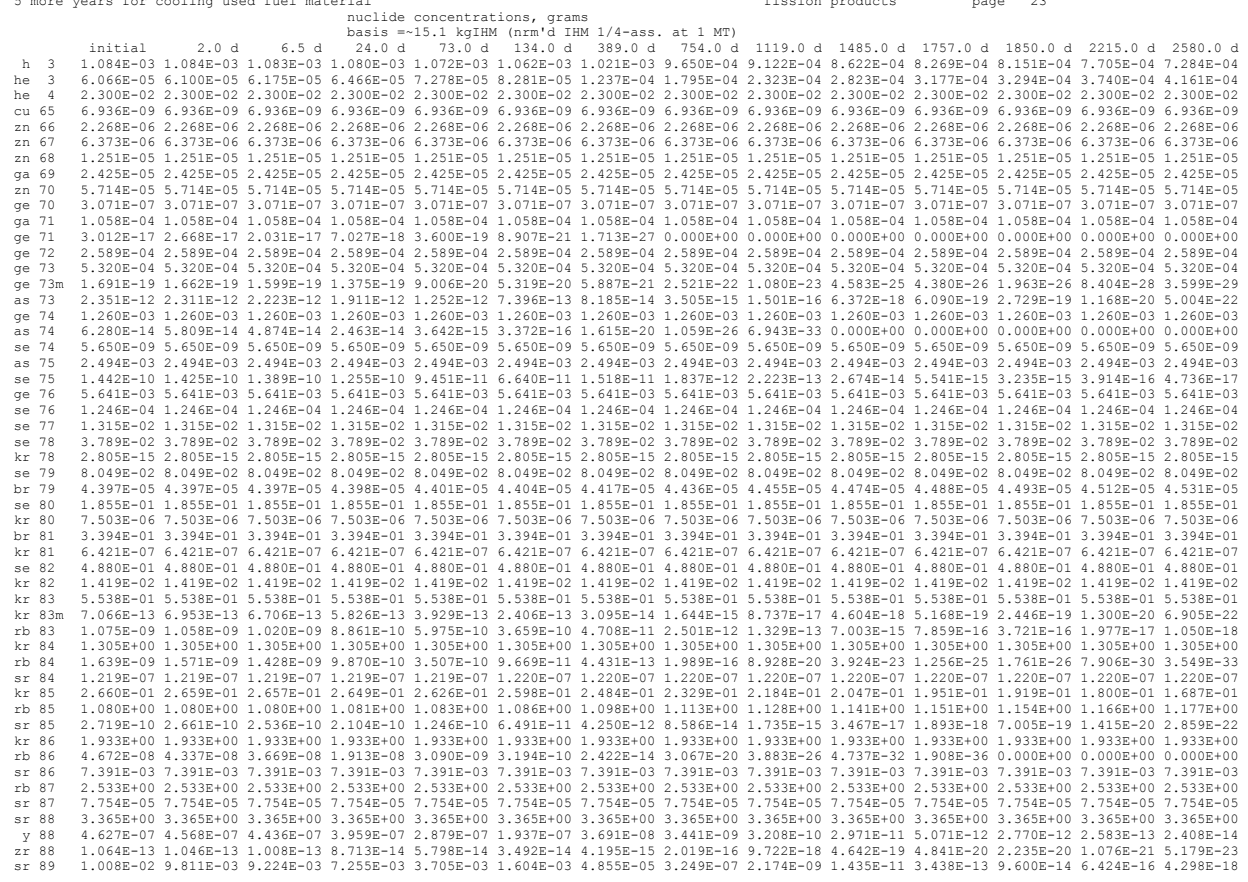

$$
\text { fission products page } 23
$$




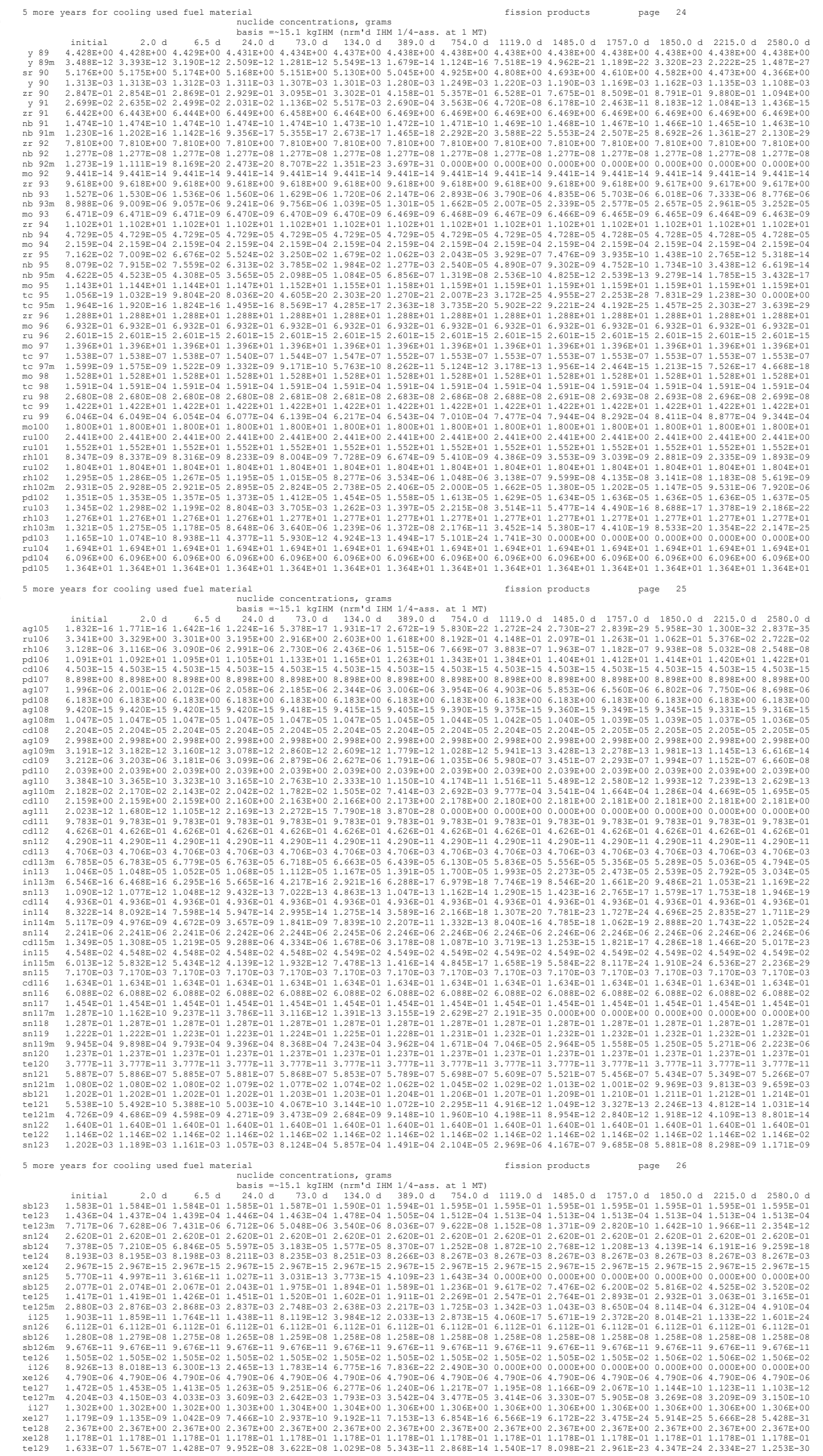

B-42 

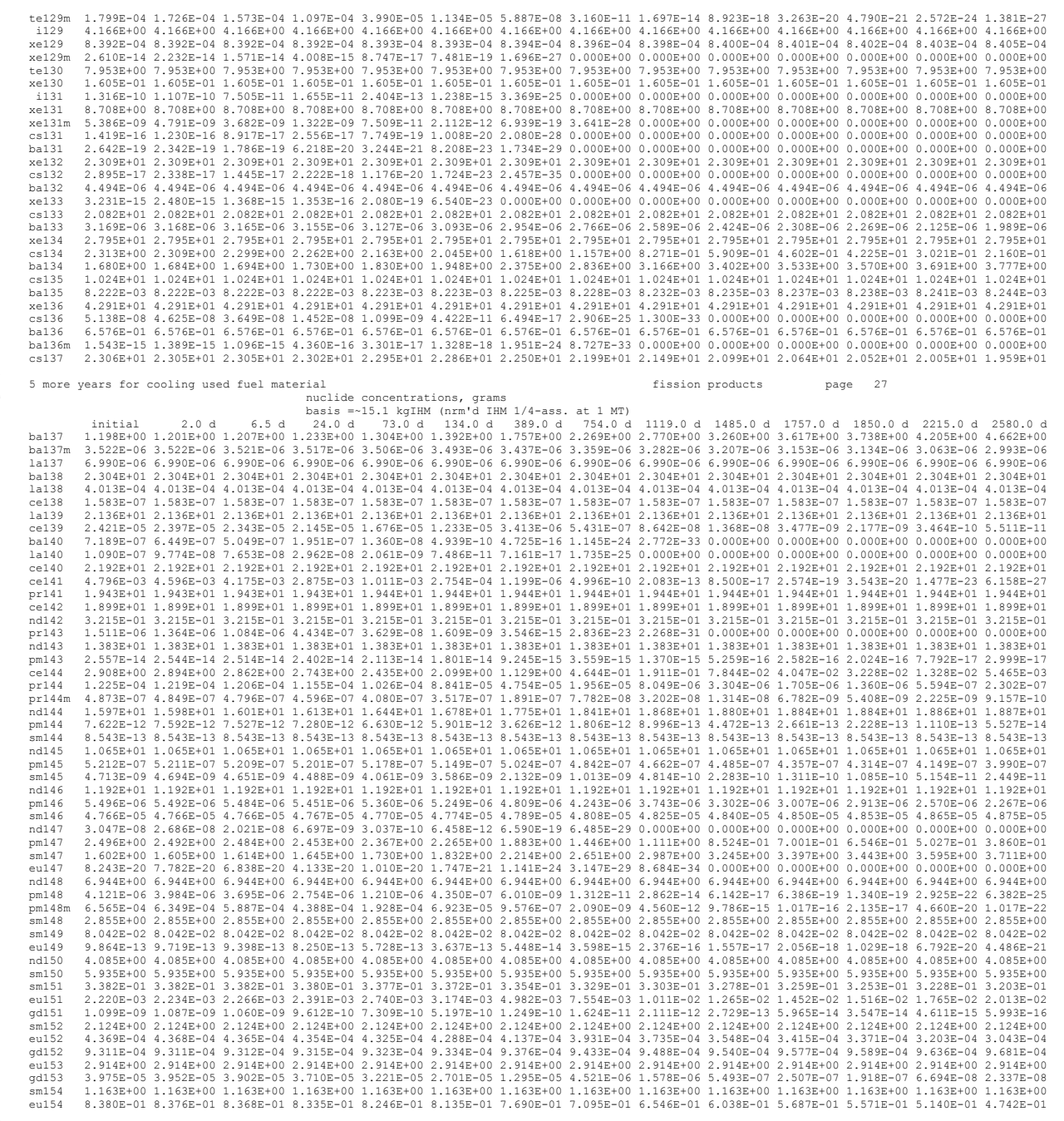

fission products page 2

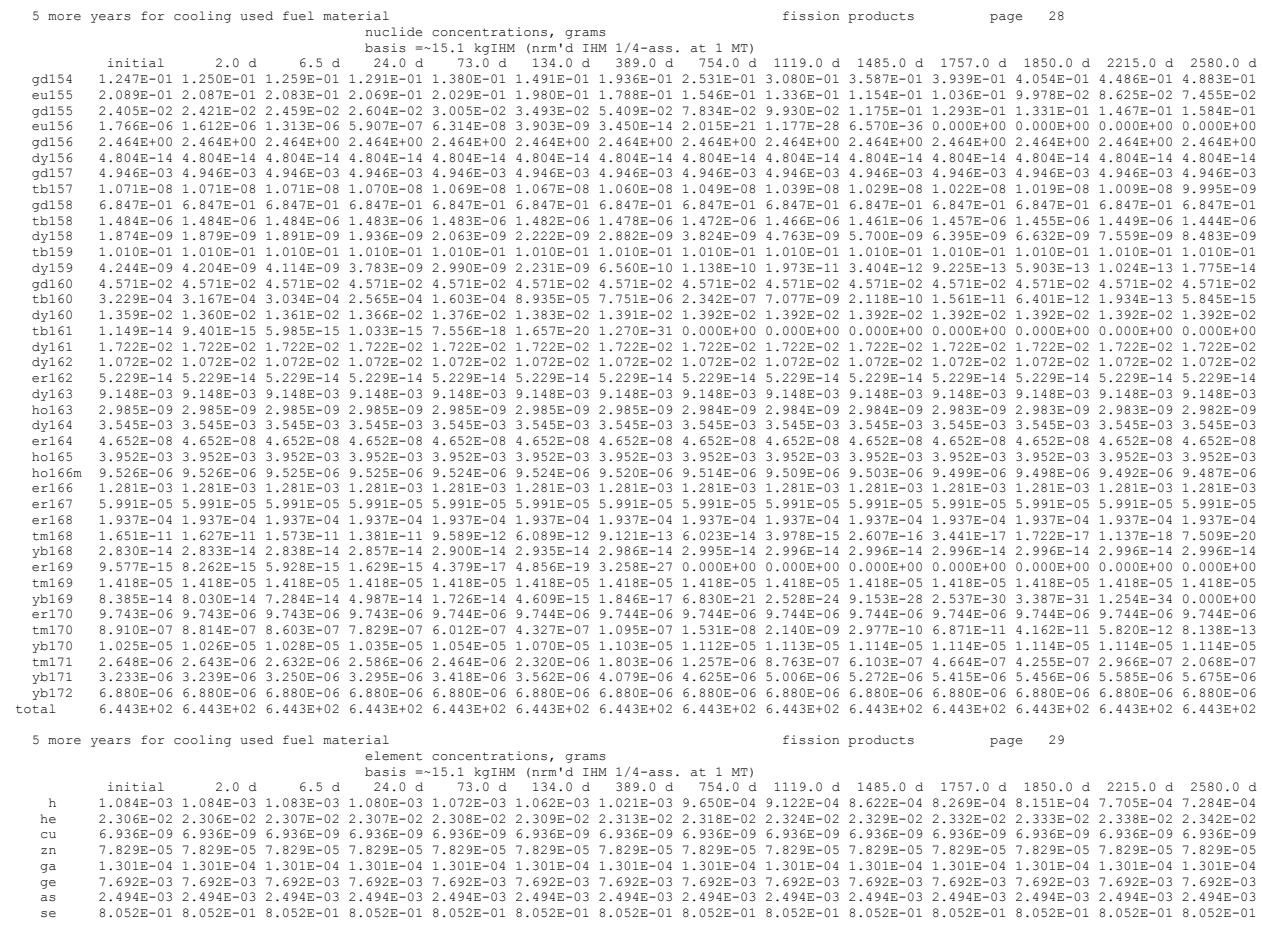

B-43 


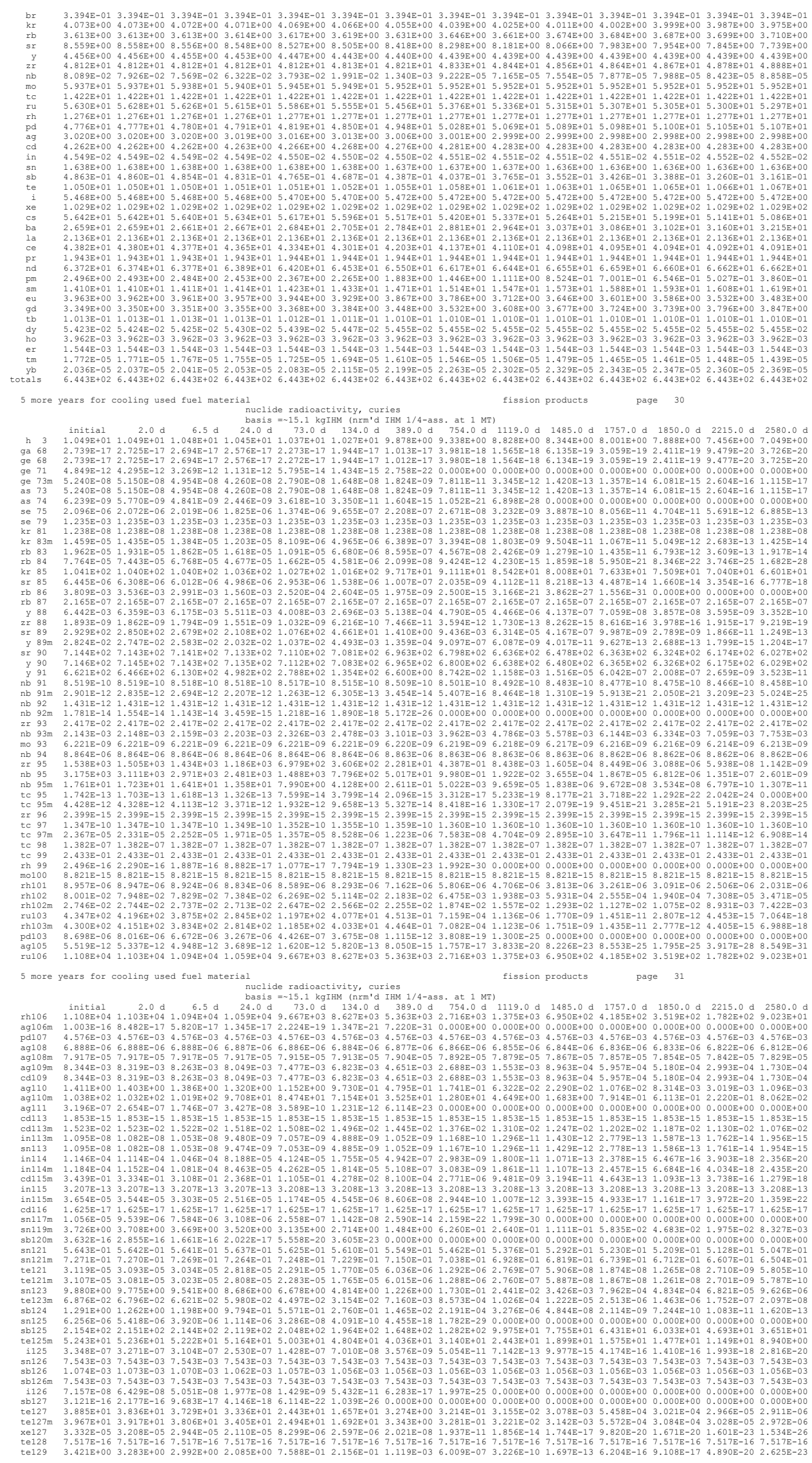

B-44 


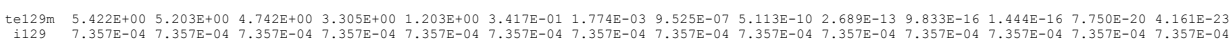

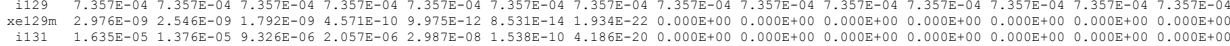

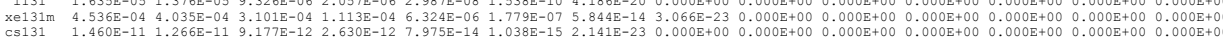

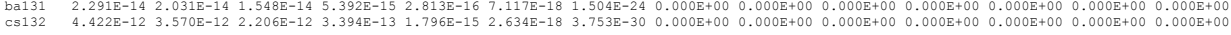
5 more years for cooling used fuel material

fission products page 32

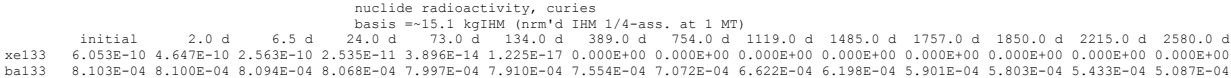

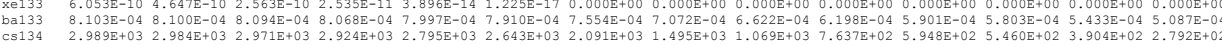

$\begin{array}{llllllllllllllllll}\text { Cs135 } & 1.180 \mathrm{E}-02 & 1.180 \mathrm{E}-02 & 1.180 \mathrm{E}-02 & 1.180 \mathrm{E}-02 & 1.180 \mathrm{E}-02 & 1.180 \mathrm{E}-02 & 1.180 \mathrm{E}-02 & 1.180 \mathrm{E}-02 & 1.180 \mathrm{E}-02 & 1.180 \mathrm{E}-02 & 1.180 \mathrm{E}-02 & 1.180 \mathrm{E}-02 & 1.180 \mathrm{E}-02 & 1.180 \mathrm{E}-02 \\ \mathrm{Cs} 136 & 3.750 \mathrm{E}-03 & 3.375 \mathrm{E}-03 & 2.663 \mathrm{E}-03 & 1.059 \mathrm{E}-03 & 8.021 \mathrm{E}-05 & 3.227 \mathrm{E}-06 & 4.740 \mathrm{E}-12 & 2.121 \mathrm{E}-20 & 9.488 \mathrm{E}-29 & 0.000 \mathrm{E}+00 & 0.000 \mathrm{E}+00 & 0.000 \mathrm{E}+00 & 0.000 \mathrm{E}+00 & 0.000 \mathrm{E}+0\end{array}$

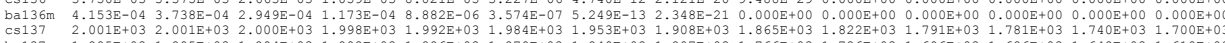

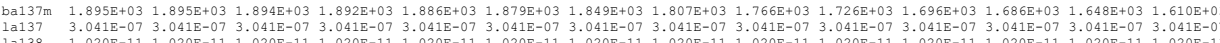

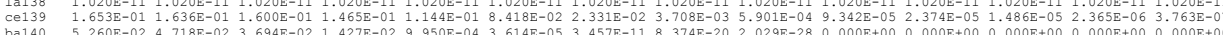

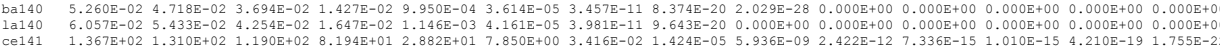

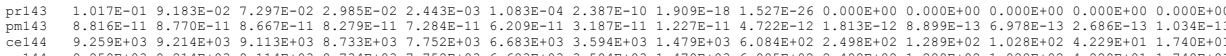

$\begin{array}{llllllllllllllll}\text { pr144m } & 8.840 \mathrm{E}+01 & 8.797 \mathrm{E}+01 & 8.701 \mathrm{E}+01 & 8.339 \mathrm{E}+01 & 7.402 \mathrm{E}+01 & 6.381 \mathrm{E}+01 & 3.431 \mathrm{E}+01 & 1.412 \mathrm{E}+01 & 5.809 \mathrm{E}+00 & 2.398 \mathrm{E}+00 & 1.230 \mathrm{E}+00 & 9.812 \mathrm{E}-01 & 4.037 \mathrm{E}-01 & 1.661 \mathrm{E}-01\end{array}$

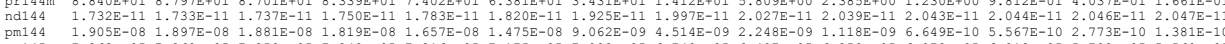

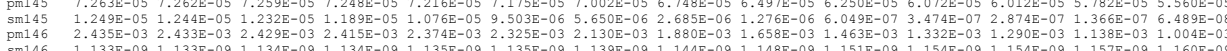

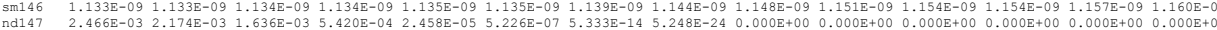

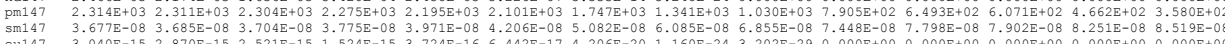

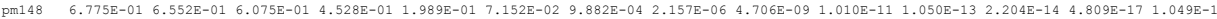

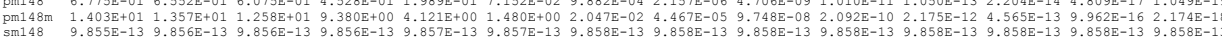

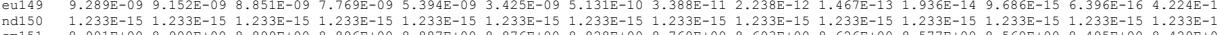

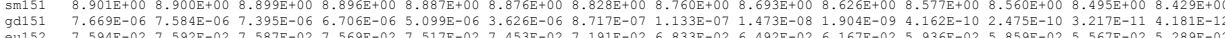

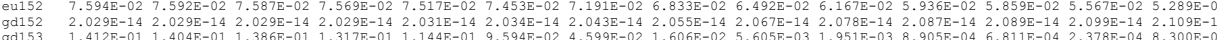

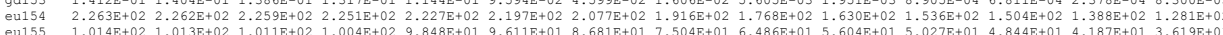

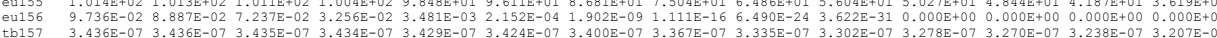

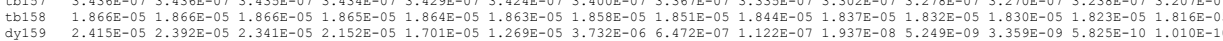

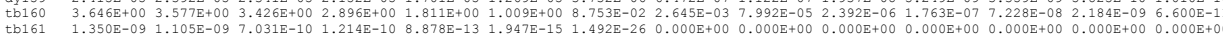

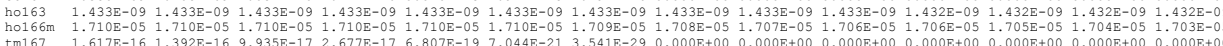

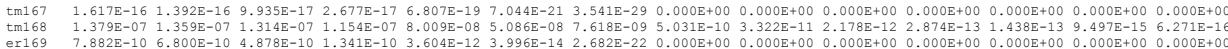

5 more years for cooling used fuel nateria

nuclide radioactivity, curies
basis $=\sim 15.1 \mathrm{kgHM}$ (nrm'd IHM $1 / 4$-ass. at $1 \mathrm{MT}$ )

fission products page 33

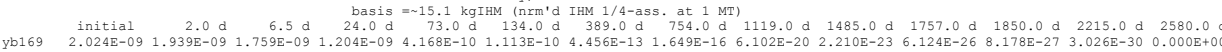

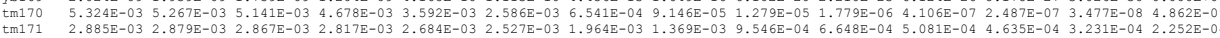

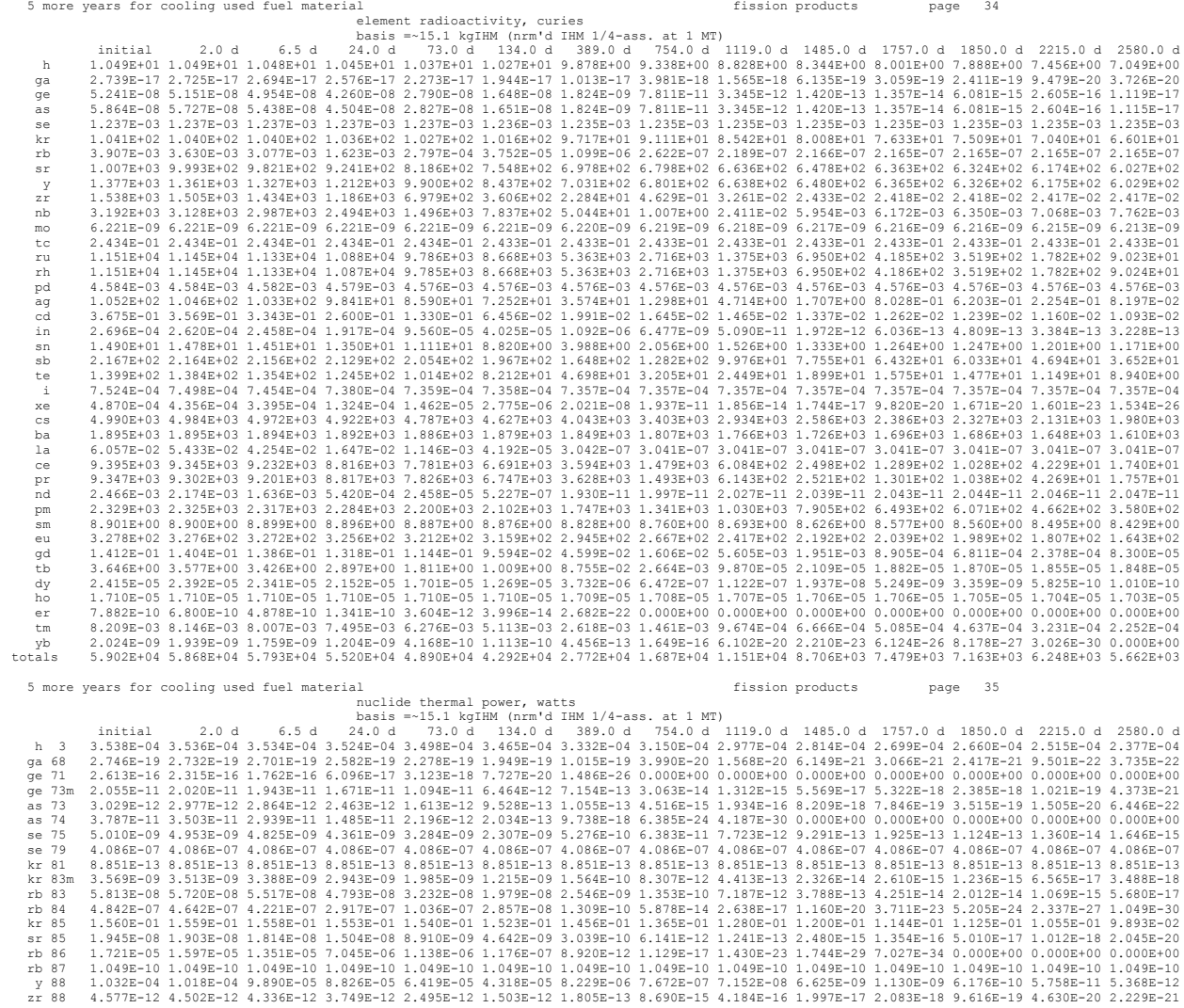

B-45 


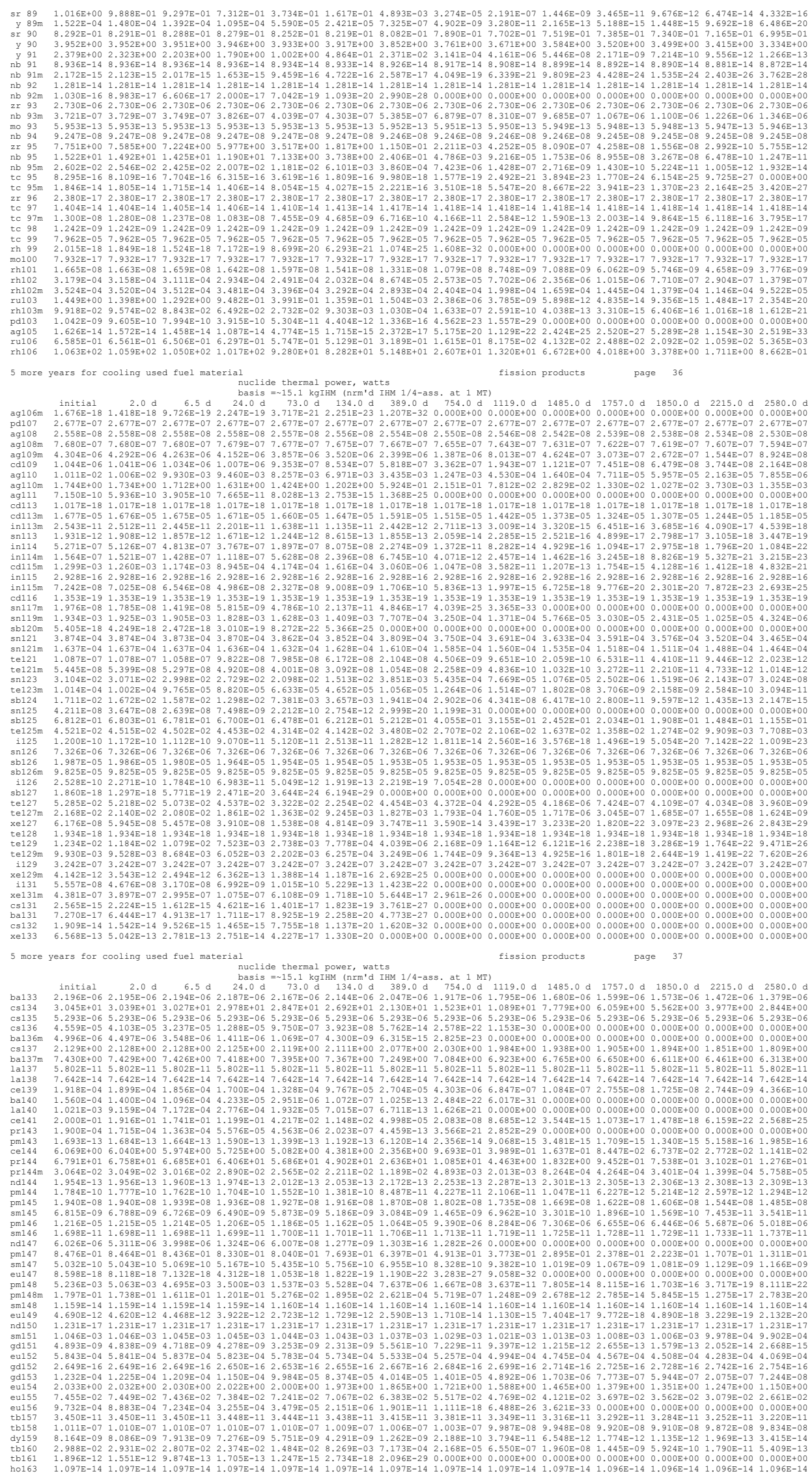




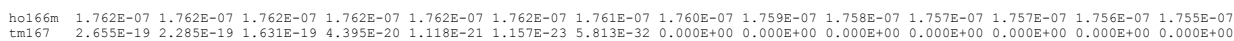

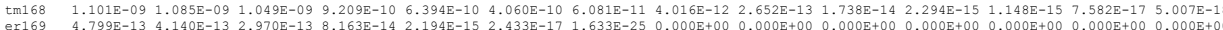
Yh169-5.96E 5 more years for cooling used fuel material huclide thermal power, watts
fission product
page 38

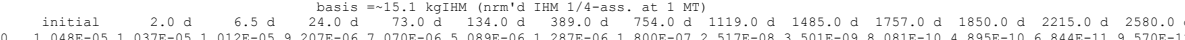

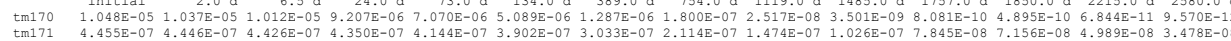

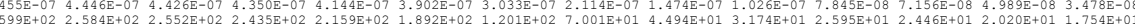

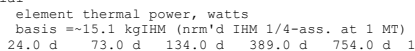
fission products page 39

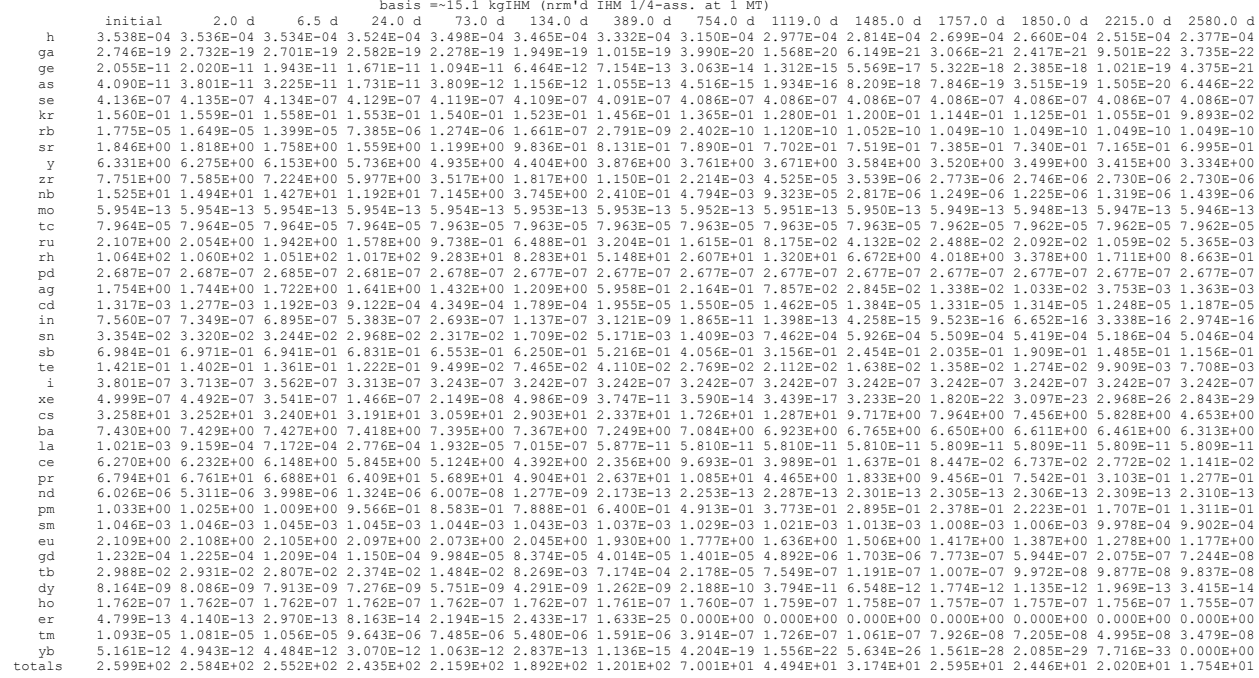

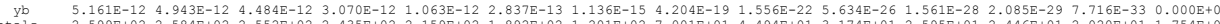
56 array 20 entries read

1 * ${ }_{0}$ normal termination of execution * table of contents for material tables
case or subcase printed page 


\section{B.4 ORIGEN RESULTS FOR COOLING MOX FUEL ROD B14}
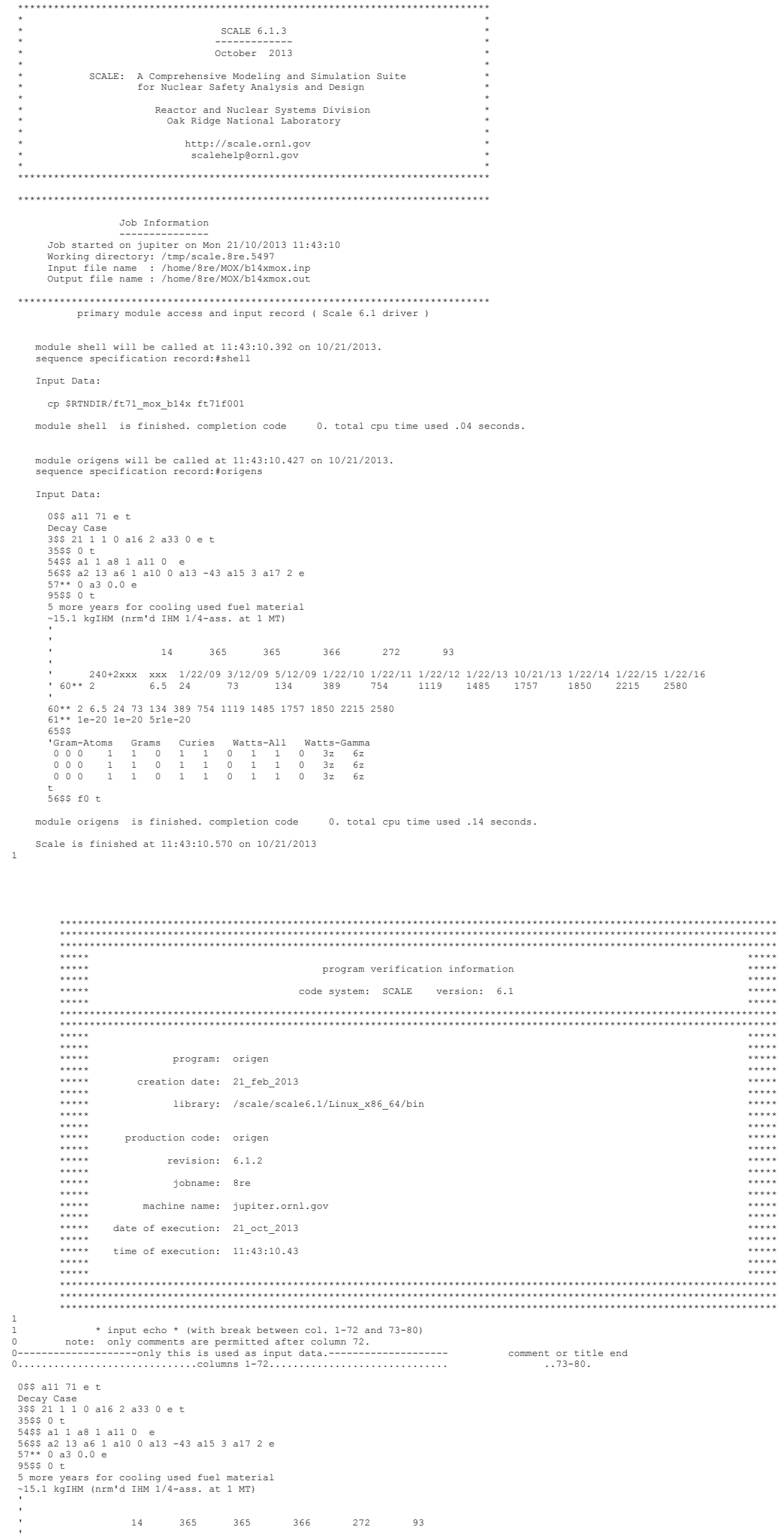


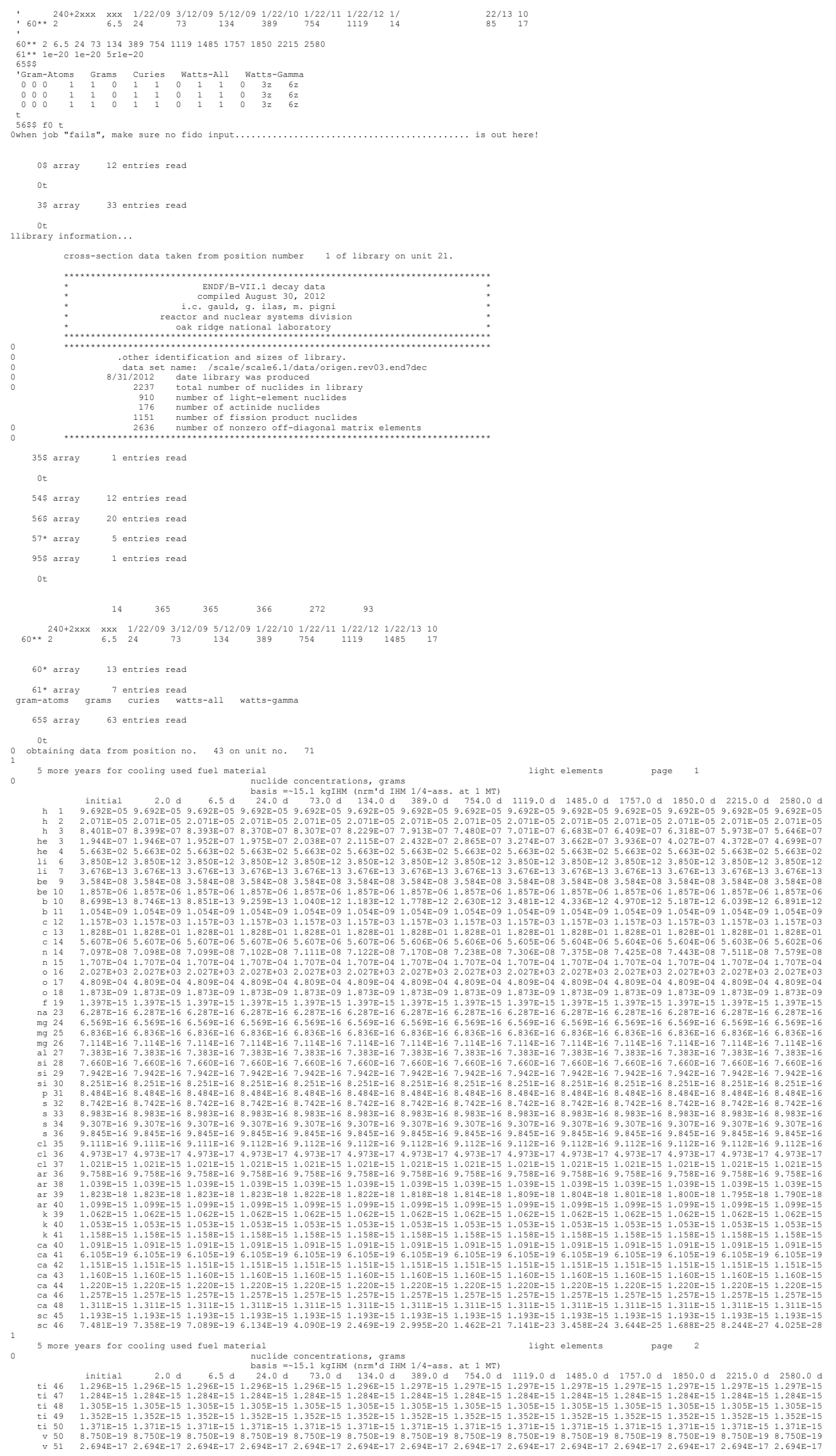

B-49 


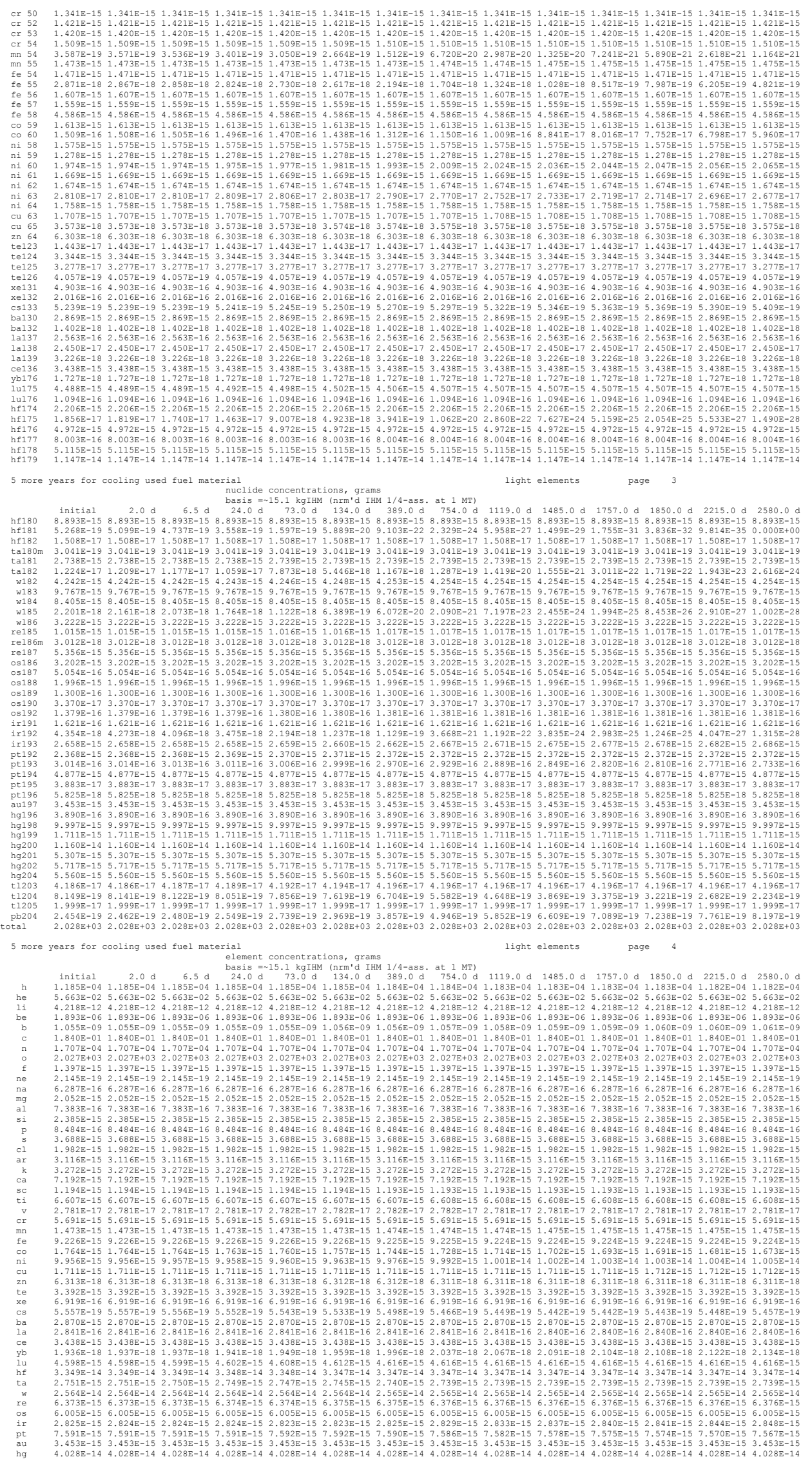

B-50 
$\begin{array}{lllllllllllllllll}\mathrm{t} 1 & 6.267 \mathrm{E}-17 & 6.267 \mathrm{E}-17 & 6.267 \mathrm{E}-17 & 6.269 \mathrm{E}-17 & 6.270 \mathrm{E}-17 & 6.270 \mathrm{E}-17 & 6.262 \mathrm{E}-17 & 6.251 \mathrm{E}-17 & 6.241 \mathrm{E}-17 & 6.233 \mathrm{E}-17 & 6.228 \mathrm{E}-17 & 6.227 \mathrm{E}-17 & 6.222 \mathrm{E}-17 & 6.217 \mathrm{E}-17 \\ \mathrm{pb} & 2.568 \mathrm{E}-19 & 2.576 \mathrm{E}-19 & 2.593 \mathrm{E}-19 & 2.662 \mathrm{E}-19 & 2.852 \mathrm{E}-19 & 3.082 \mathrm{E}-19 & 3.970 \mathrm{E}-19 & 5.059 \mathrm{E}-19 & 5.966 \mathrm{E}-19 & 6.723 \mathrm{E}-19 & 7.202 \mathrm{E}-19 & 7.351 \mathrm{E}-19 & 7.875 \mathrm{E}-19 & 8.310 \mathrm{E}-1\end{array}$

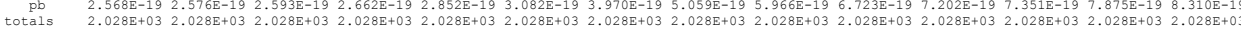
5 more years for cooling used fuel material

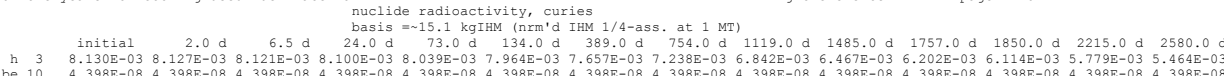

light elements page 5

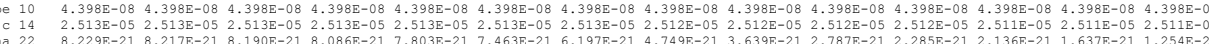

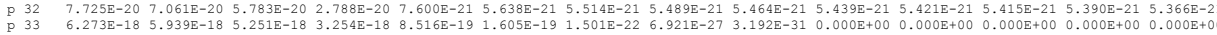

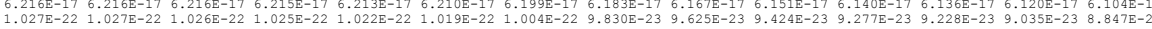

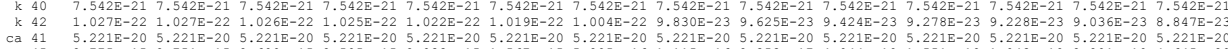

$\begin{array}{lll}\text { Ca } 45 & 2.775 \mathrm{E}-15 & 2.751 \\ \text { sc } 45 \mathrm{~m} & 5.272 \mathrm{E}-20 & 5.227\end{array}$

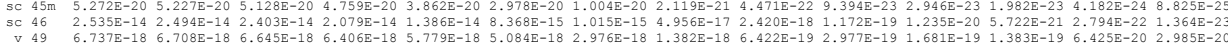

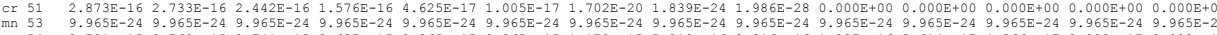

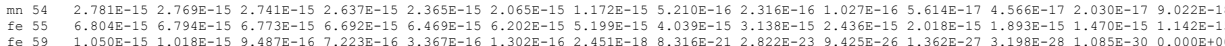

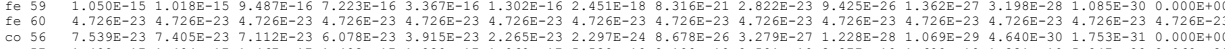

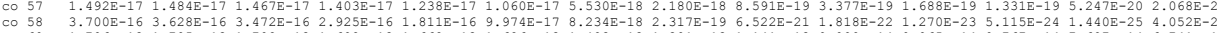

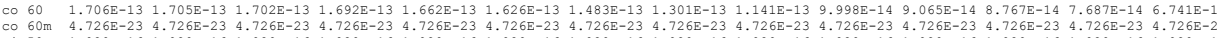

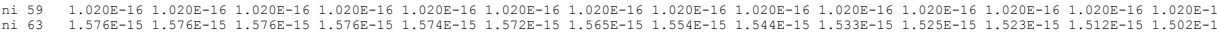

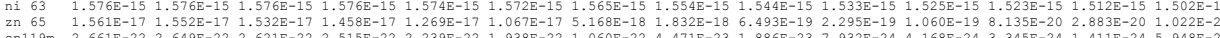

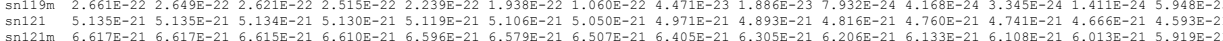

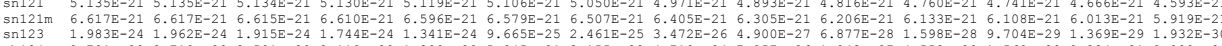

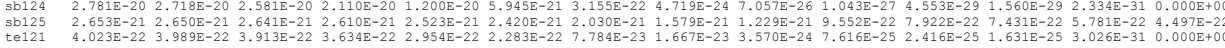

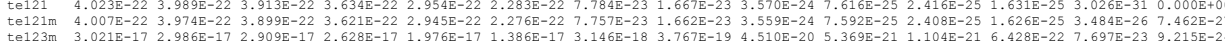

$\begin{array}{llllllllll}t & 1\end{array}$

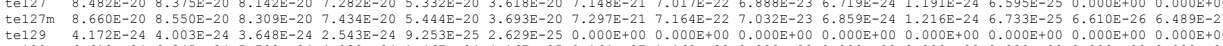

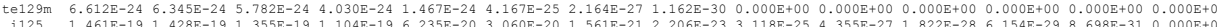

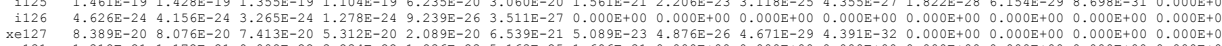

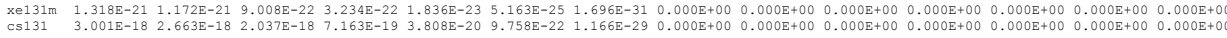

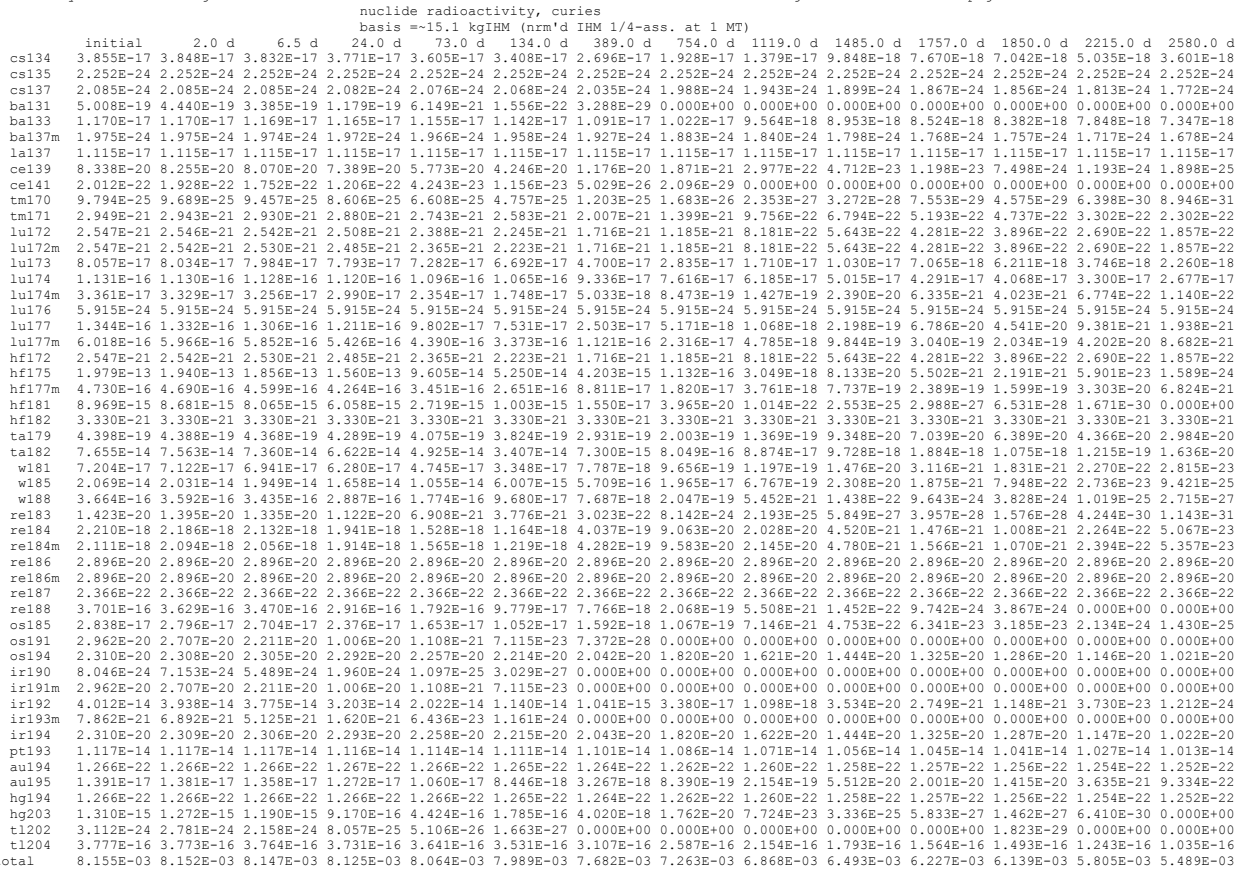

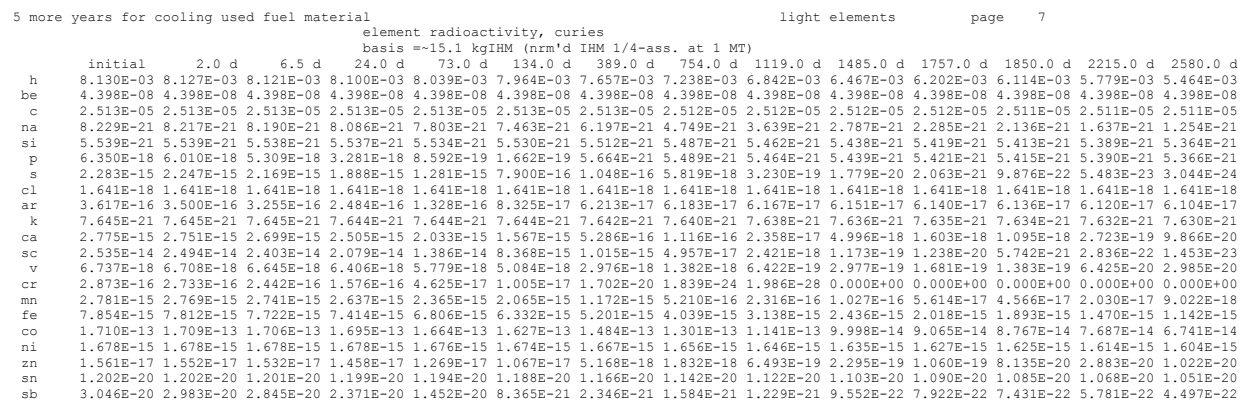

B-51 


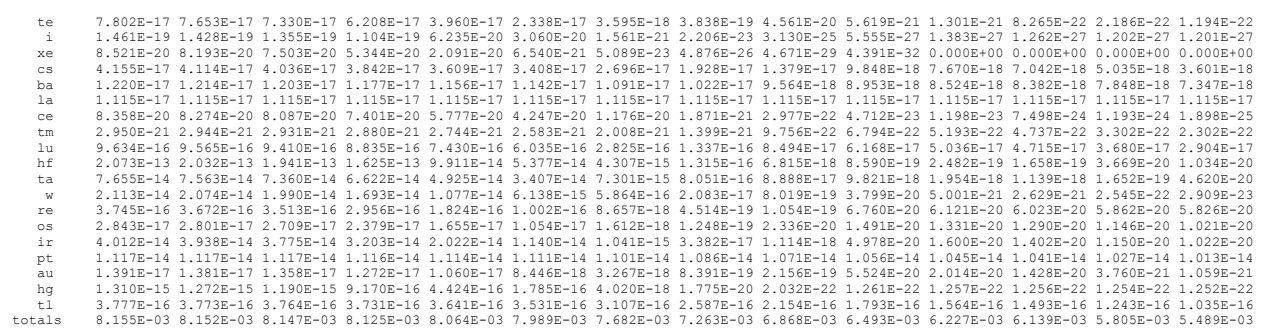
totals $\quad \begin{array}{llllllll} & \end{array}$

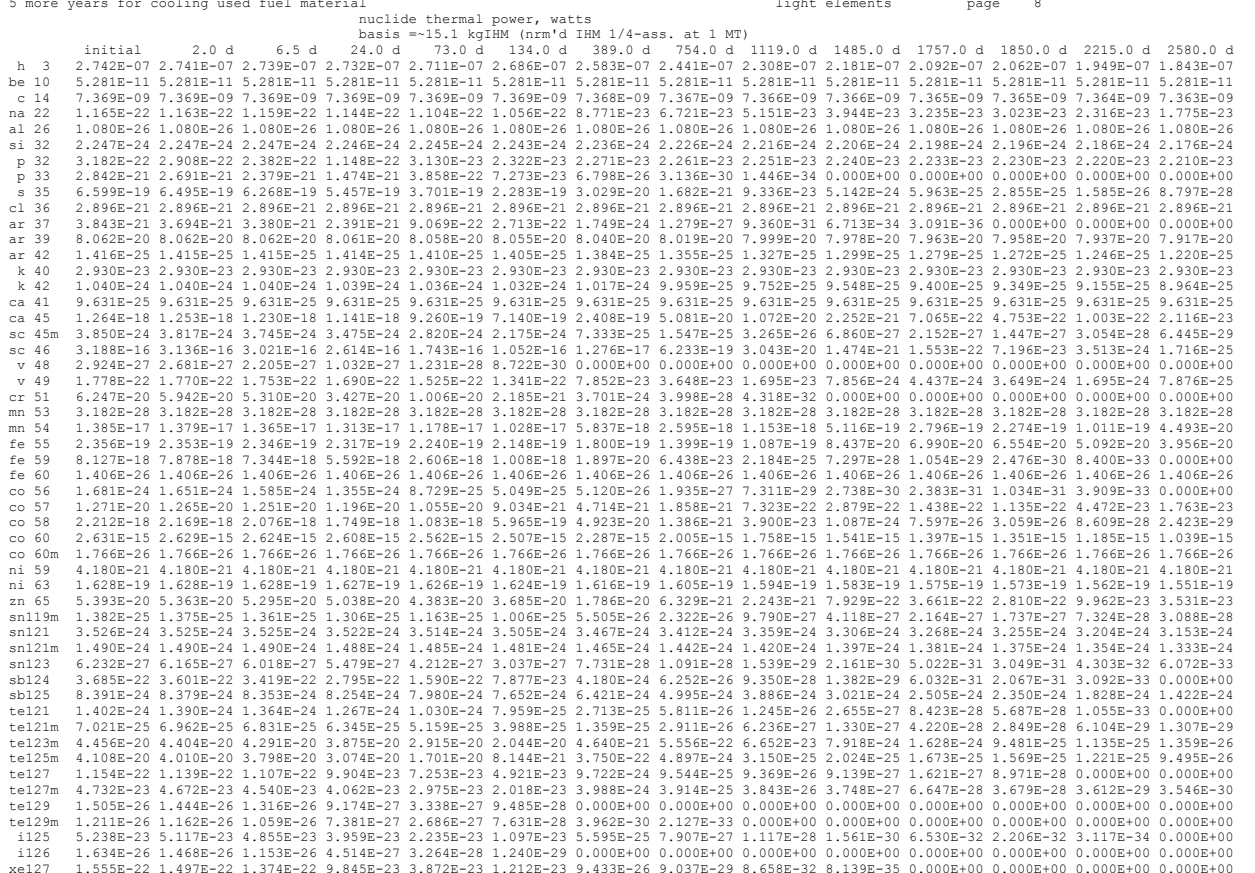

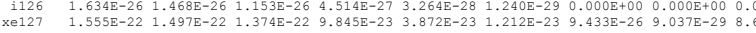

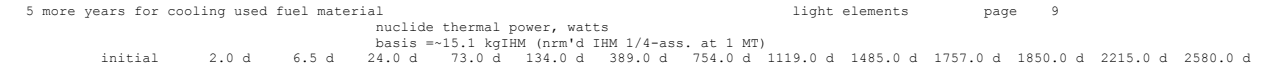

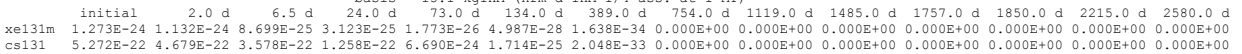

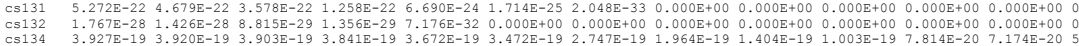

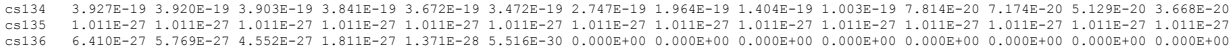

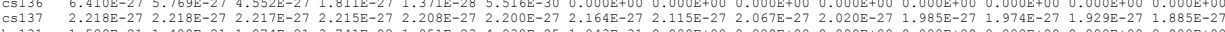

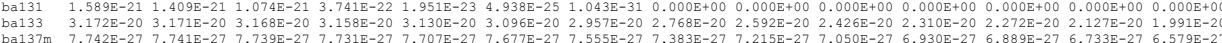

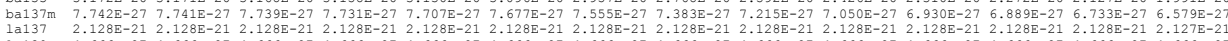

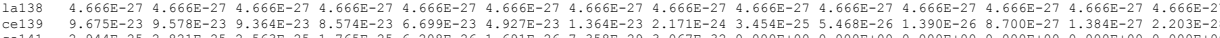

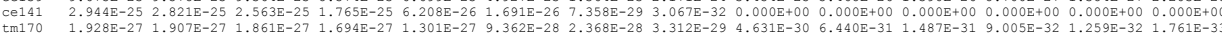

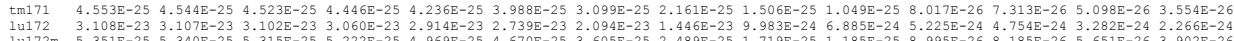

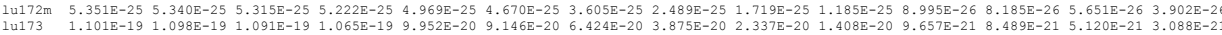

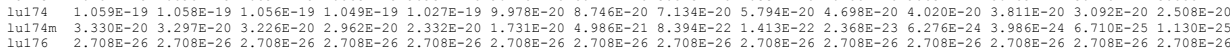

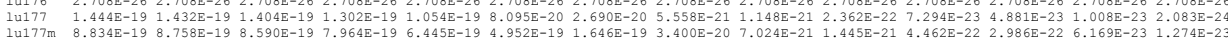

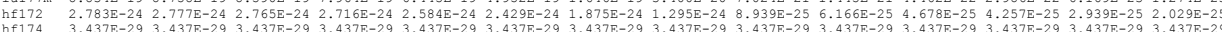

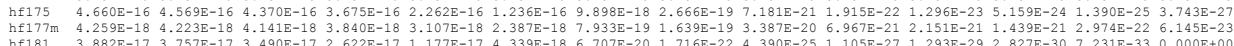

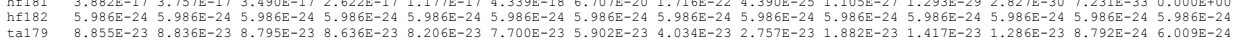

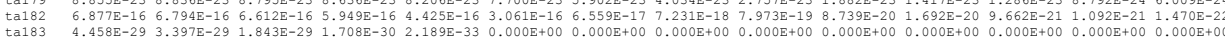

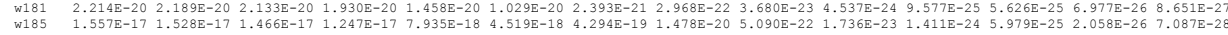

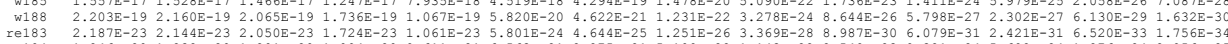

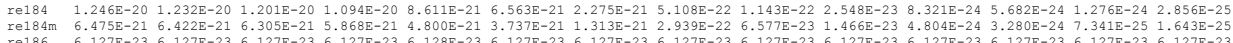

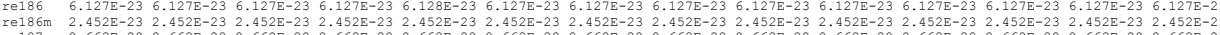

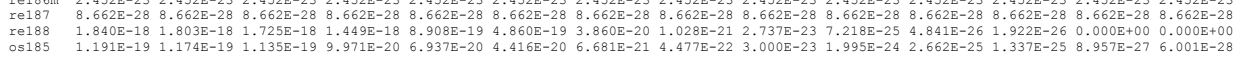

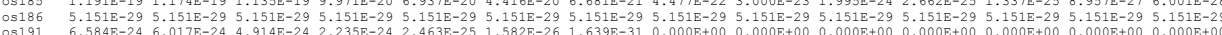

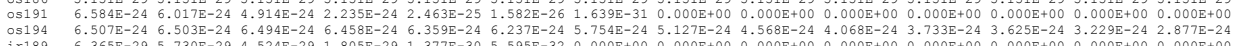

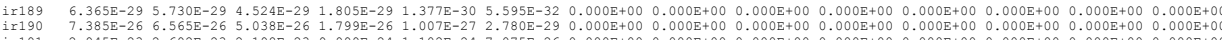

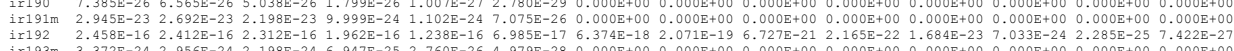
5 more years for cooling used fuel

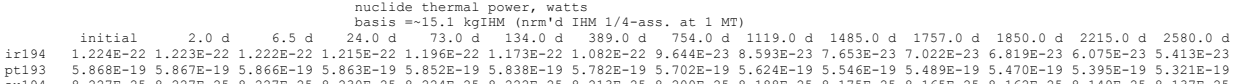

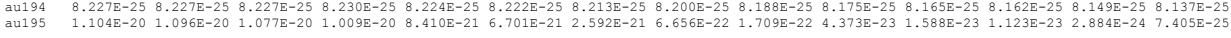

B-52 


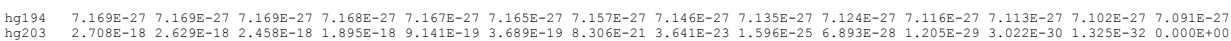

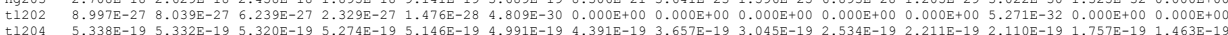
(1) 5 more years for cooling used fuel material light elements page 11 element thermal power, watts
basis $=\sim 15.1 \mathrm{~kg}$ IHM $($ nrm'd $\mathrm{THM}$

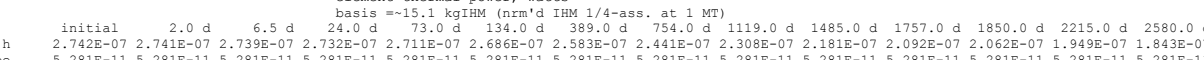

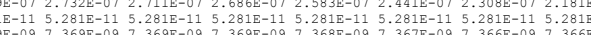

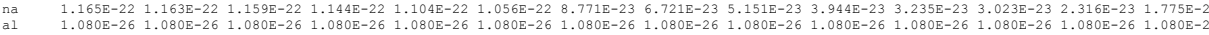

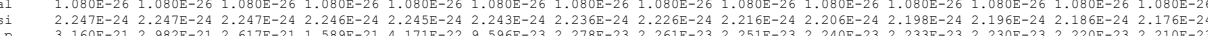

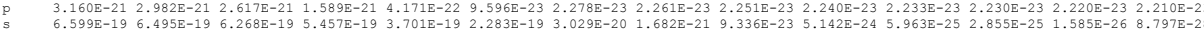

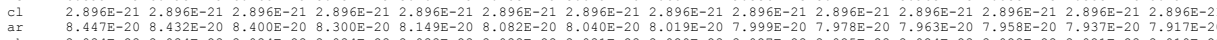

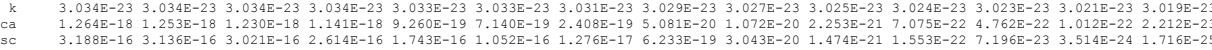

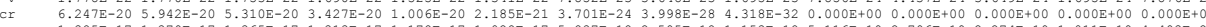

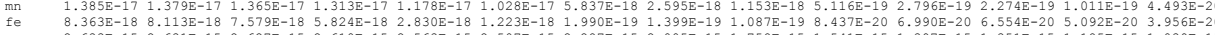

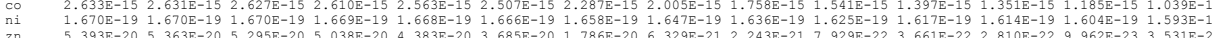

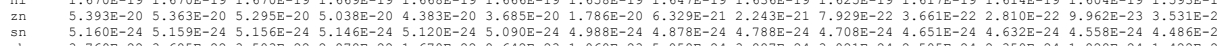

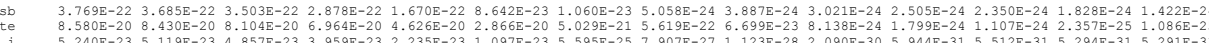
$\begin{array}{ccc}\text { i } & 5.240 \mathrm{E}-23 & 5.11 \\ \mathrm{xe} & 1.568 \mathrm{E}-22 & 1.50\end{array}$

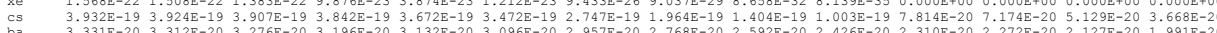

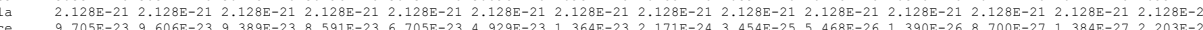

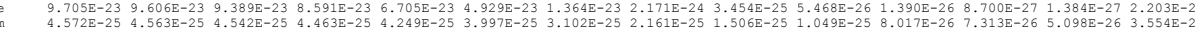

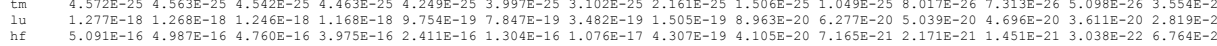

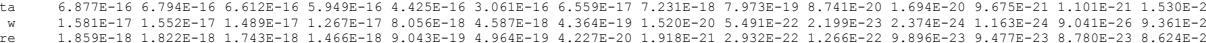

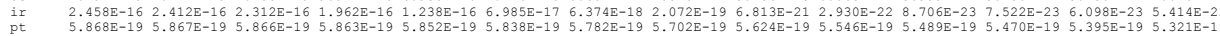

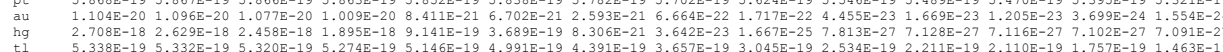

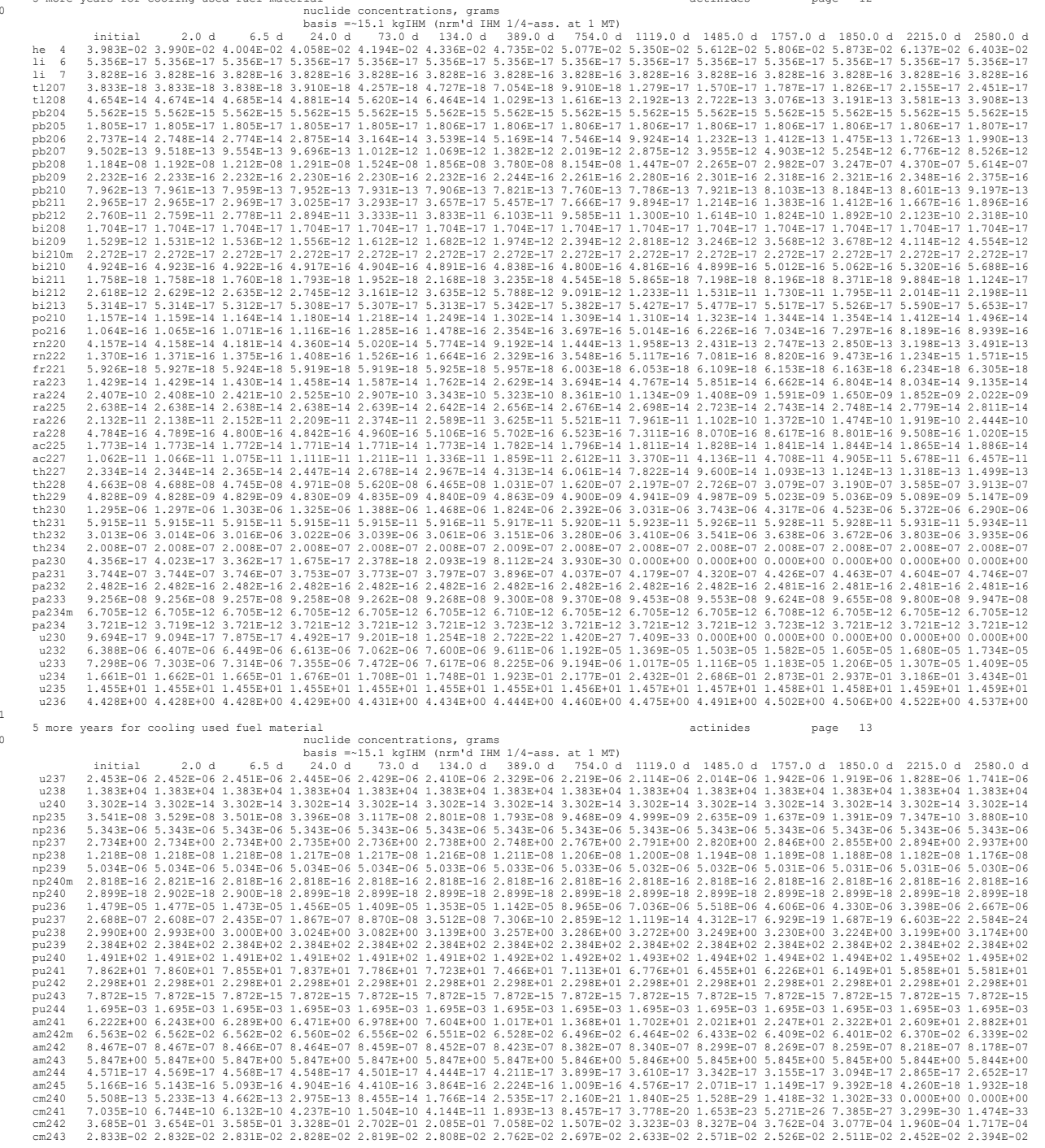

B-53 


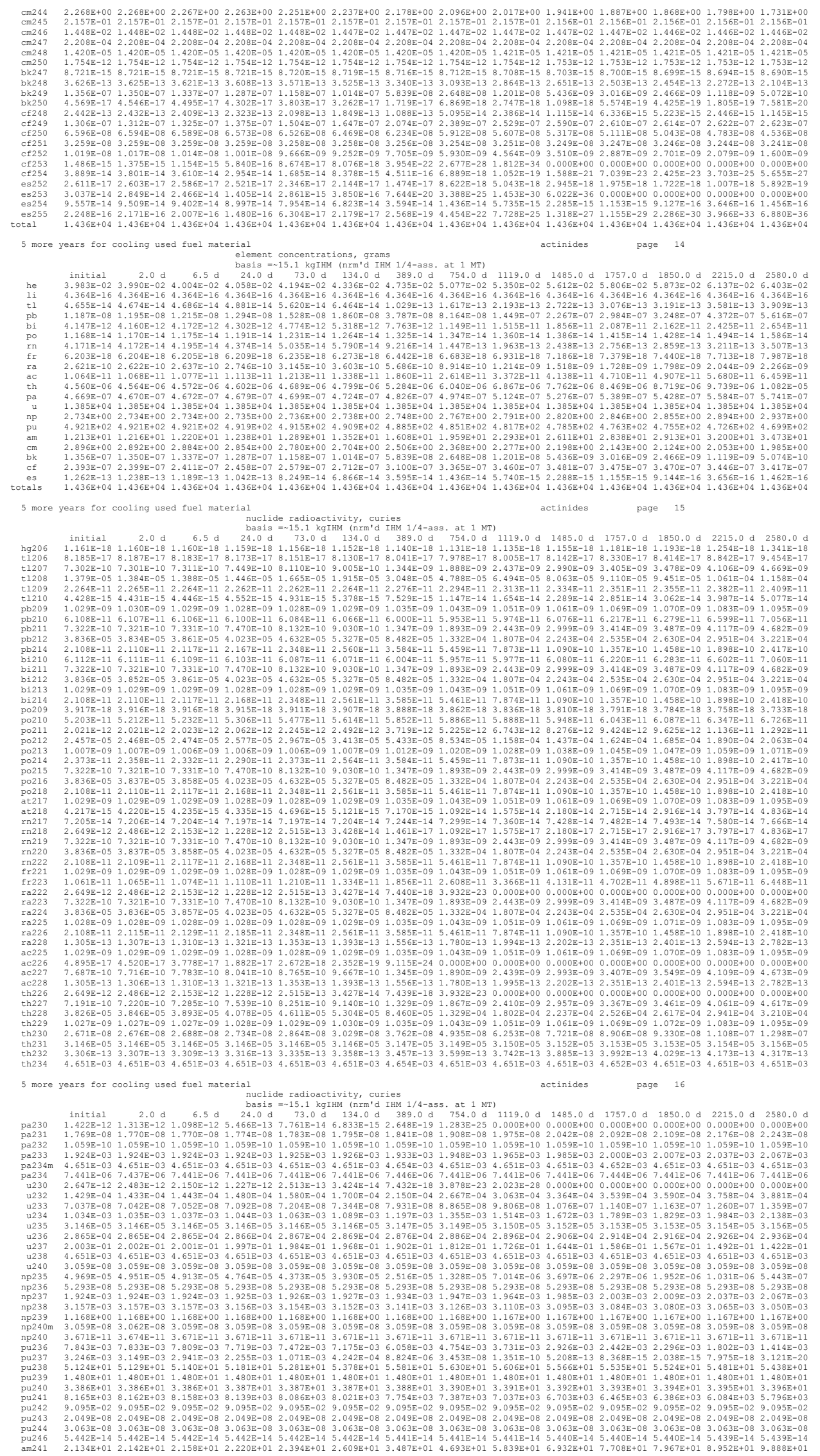

B-54 

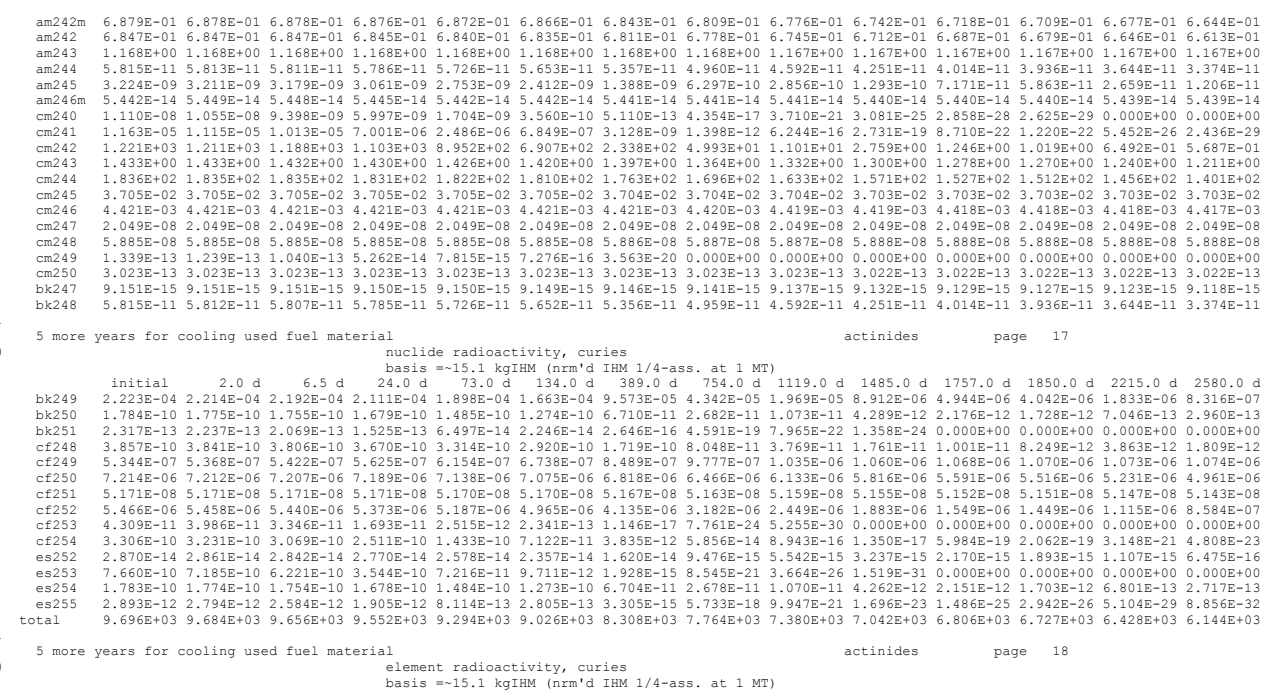

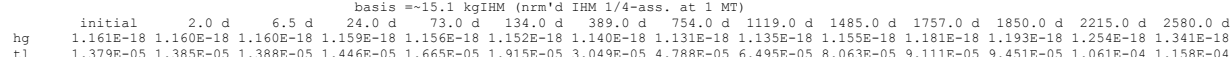

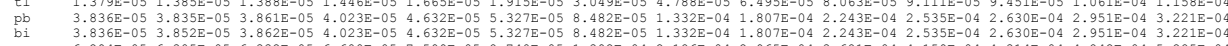

pot
pot

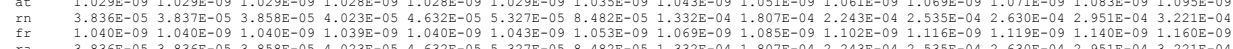

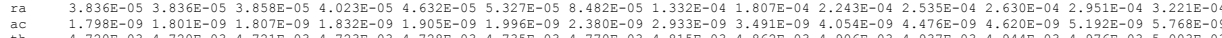

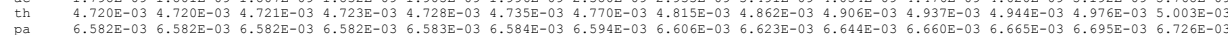

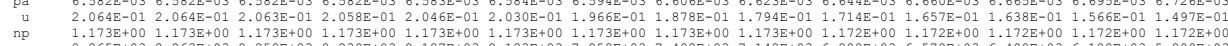

$\begin{array}{lllllllllllllll}\text { pu } & 8.265 \mathrm{E}+03 & 8.263 \mathrm{E}+03 & 8.258 \mathrm{E}+03 & 8.239 \mathrm{E}+03 & 8.187 \mathrm{E}+03 & 8.123 \mathrm{E}+03 & 7.858 \mathrm{E}+03 & 7.492 \mathrm{E}+03 & 7.142 \mathrm{E}+03 & 6.808 \mathrm{E}+03 & 6.570 \mathrm{E}+03 & 6.490 \mathrm{E}+03 & 6.188 \mathrm{E}+03 & 5.899 \mathrm{E}+03 \\ \mathrm{am} & 2.388 \mathrm{E}+01 & 2.396 \mathrm{E}+01 & 2.412 \mathrm{E}+01 & 2.474 \mathrm{E}+01 & 2.648 \mathrm{E}+01 & 2.862 \mathrm{E}+01 & 3.741 \mathrm{E}+01 & 4.945 \mathrm{E}+01 & 6.091 \mathrm{E}+01 & 7.183 \mathrm{E}+01 & 7.959 \mathrm{E}+01 & 8.218 \mathrm{E}+01 & 9.202 \mathrm{E}+01 & 1.014 \mathrm{E}+02\end{array}$

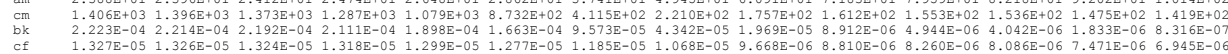

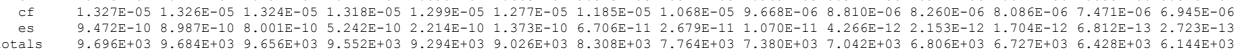

5 more years for cooling used fuel material $\quad$ actinides page 19

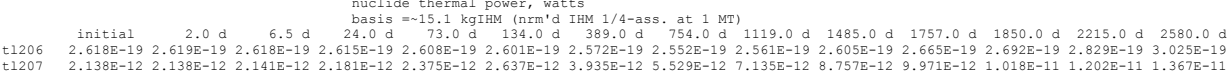

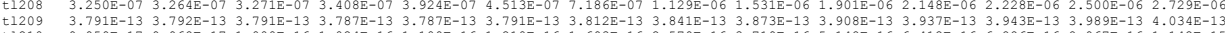

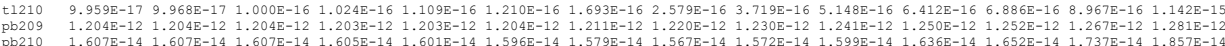

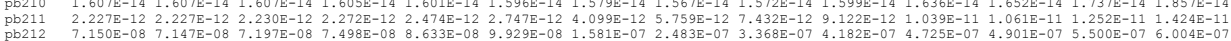

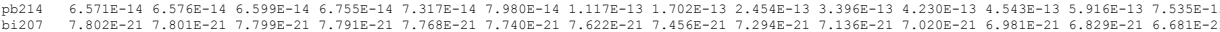

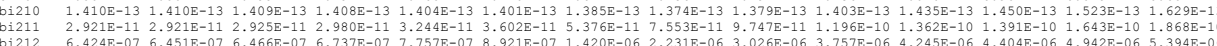

$\begin{array}{lllllllllllllllllll}0.1212 & 6.424 \mathrm{E}-07 & 6.451 \mathrm{E}-07 & 6.466 \mathrm{E}-07 & 6.737 \mathrm{E}-07 & 7.757 \mathrm{E}-07 & 8.921 \mathrm{E}-07 & 1.420 \mathrm{E}-06 & 2.231 \mathrm{E}-06 & 3.026 \mathrm{E}-06 & 3.757 \mathrm{E}-06 & 4.245 \mathrm{E}-06 & 4.404 \mathrm{E}-06 & 4.942 \mathrm{E}-06 & 5.394 \mathrm{E}-06\end{array}$

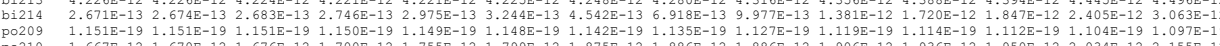

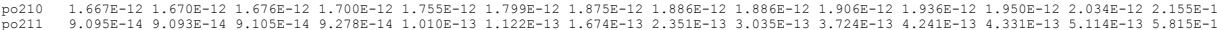

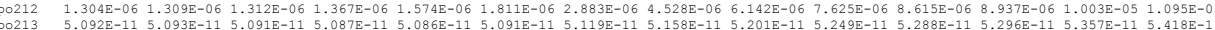

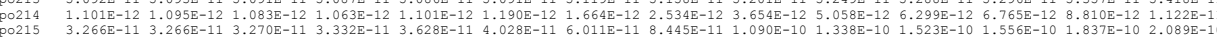

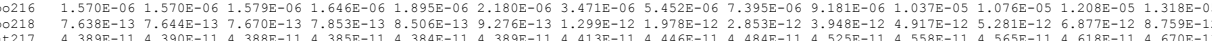

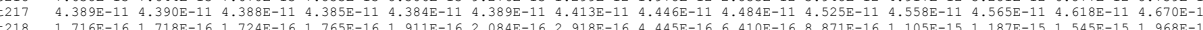

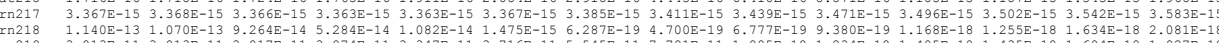

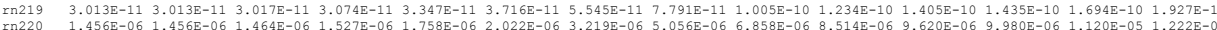

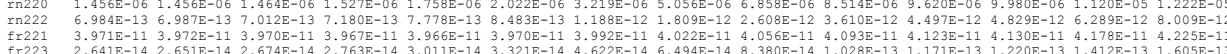

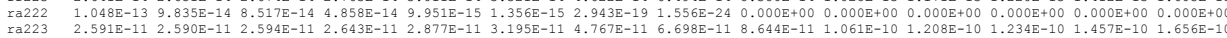

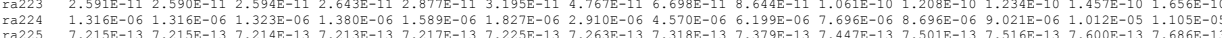

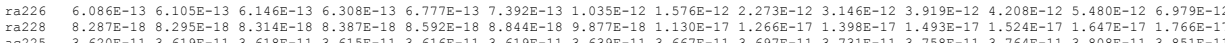

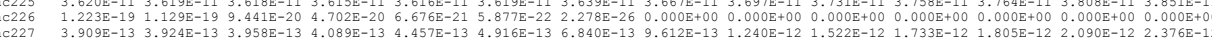

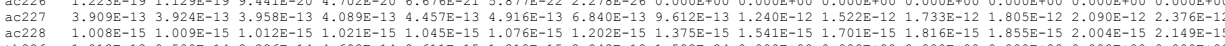

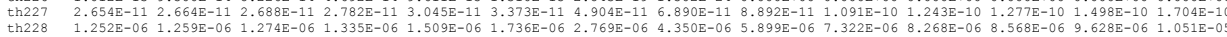

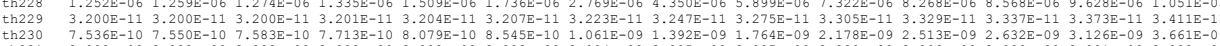

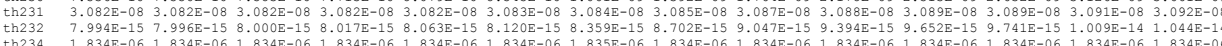

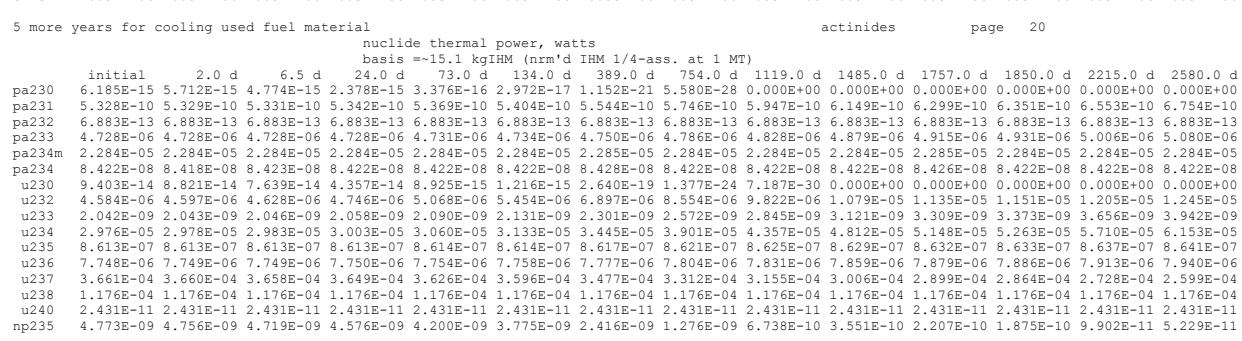

B-55 


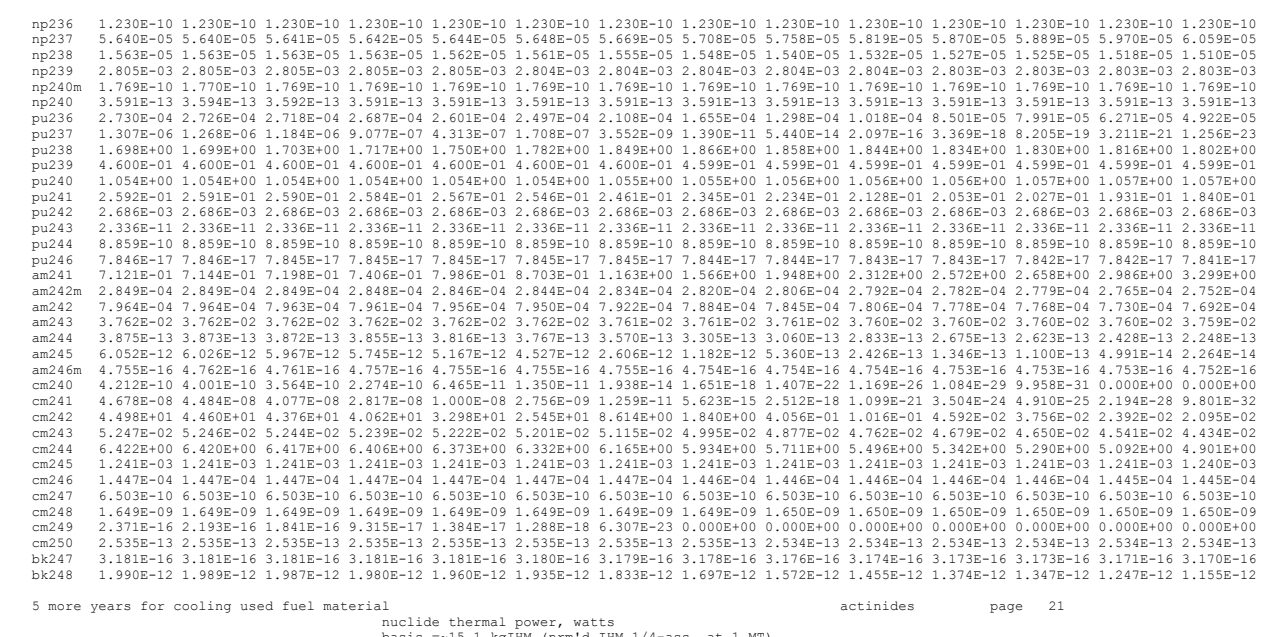

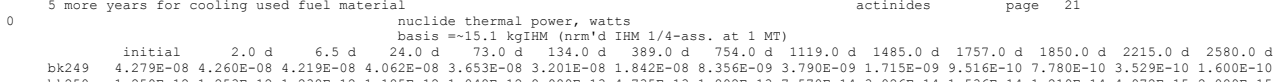

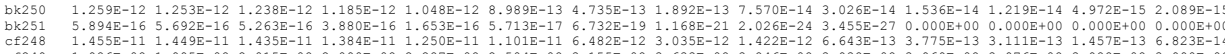

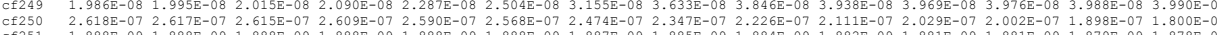

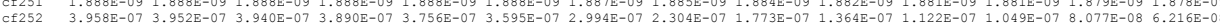

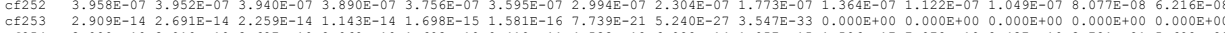

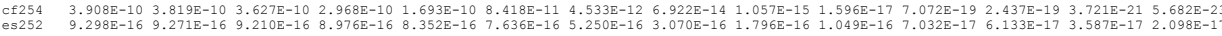

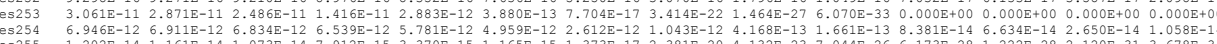
$\begin{array}{lllllllllll} & & & \end{array}$

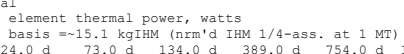
actinides page 22

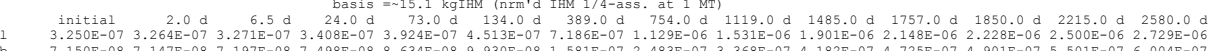

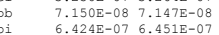

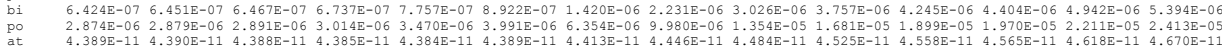

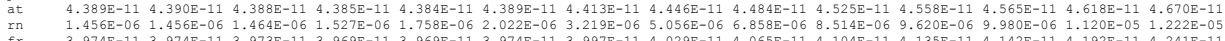

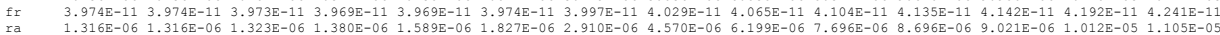

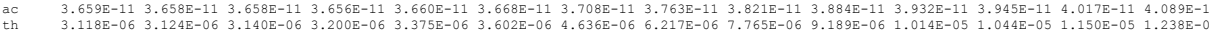

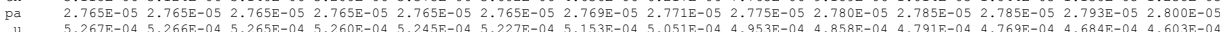

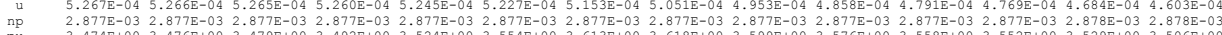

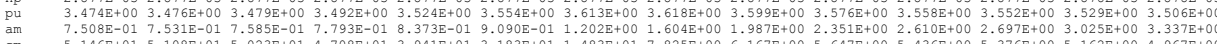

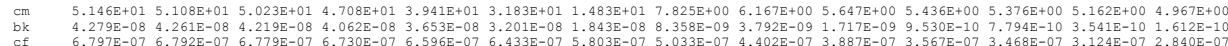

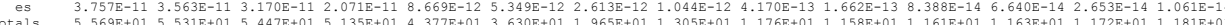
5 more years for cooling used fuel matería

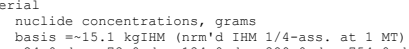

$$
\text { fission products page } 23
$$

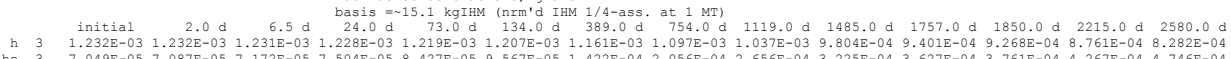

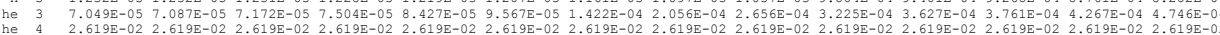

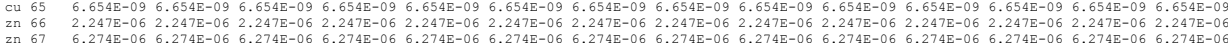

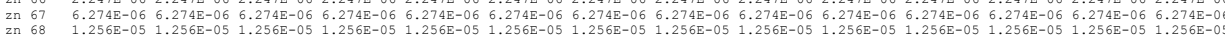

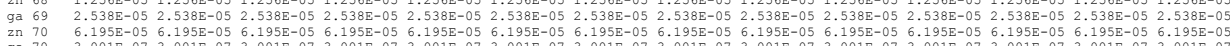

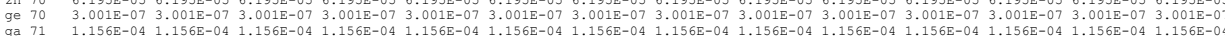

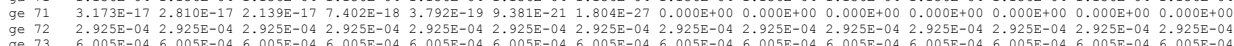

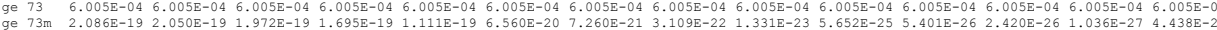

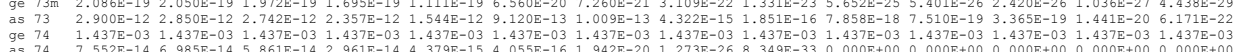

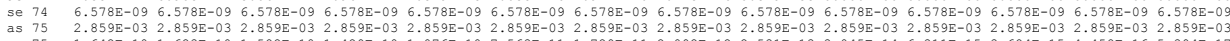

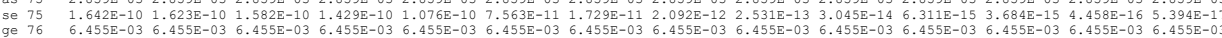

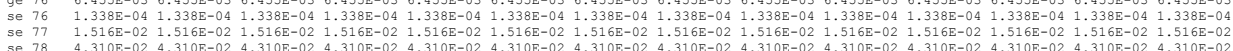

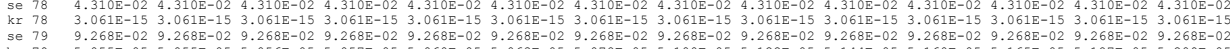

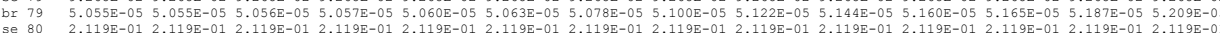

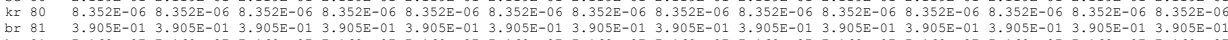

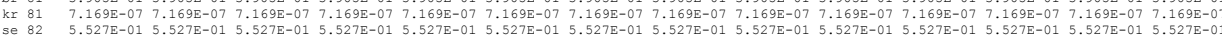

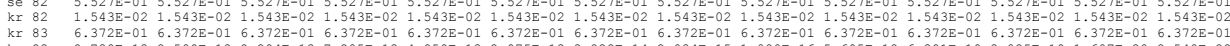

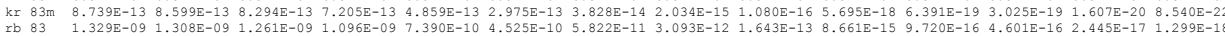

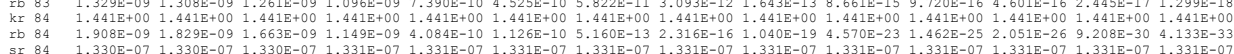

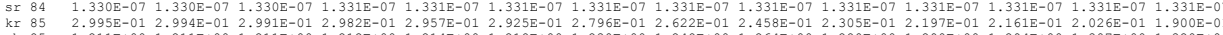

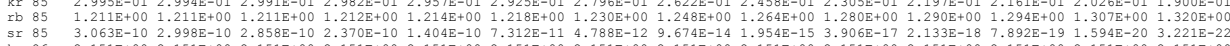

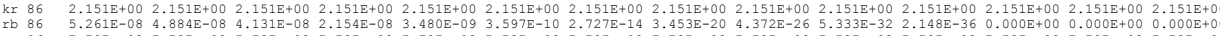

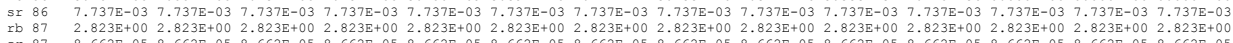

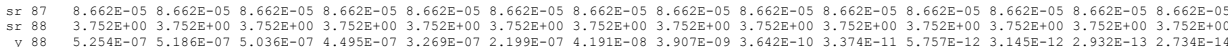

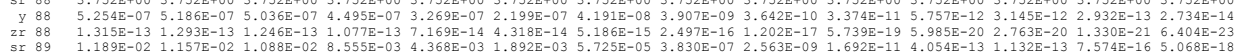

5 more years for cooling used fuel material $\underset{\text { nuclide concentrations, grams }}{\text { fission products }}$ page 24

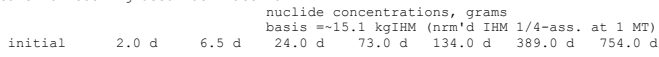




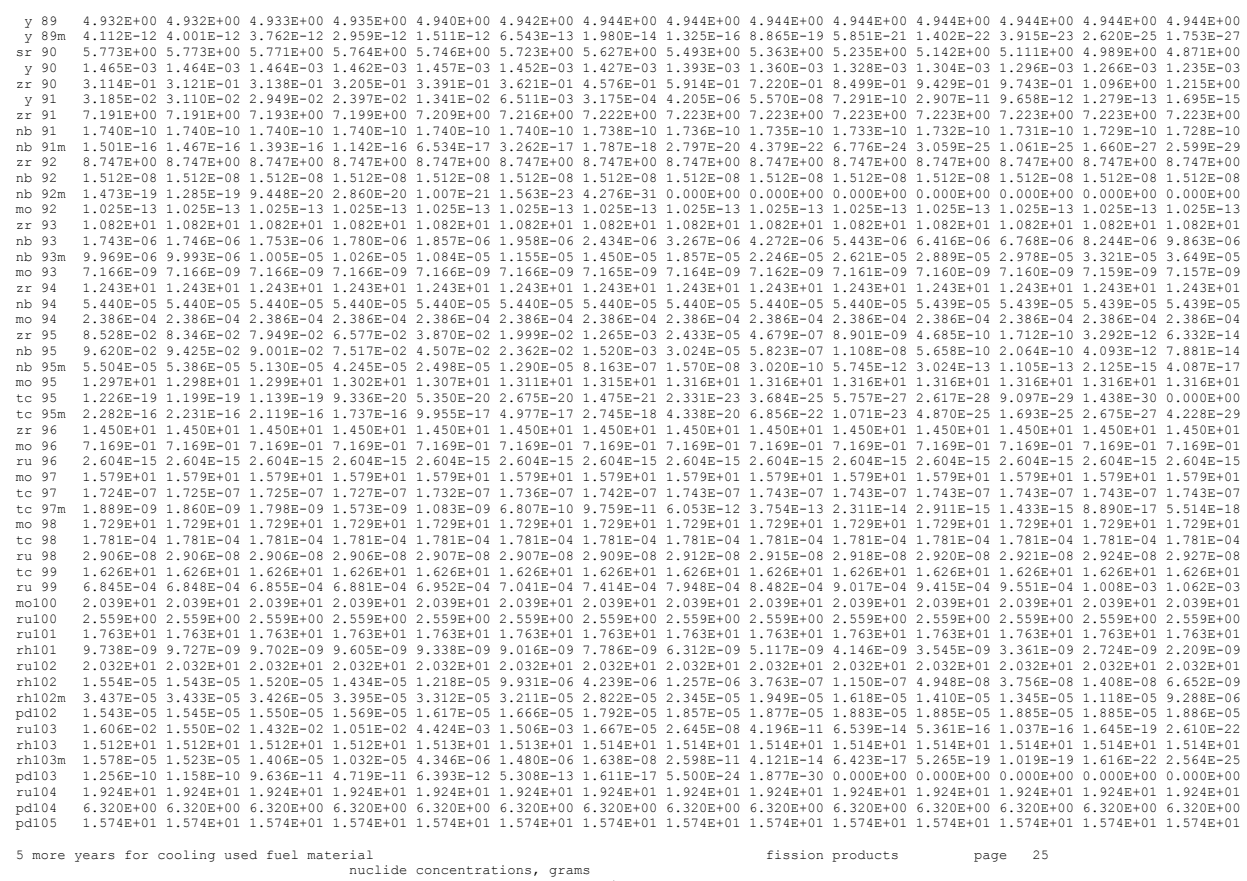

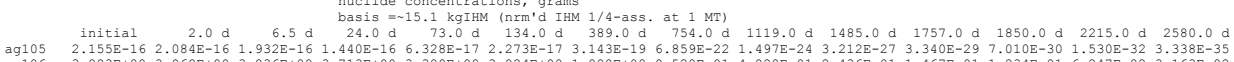

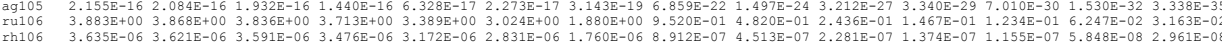

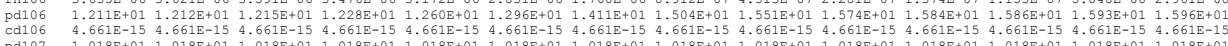

$\begin{array}{lll} & \\ & \end{array}$

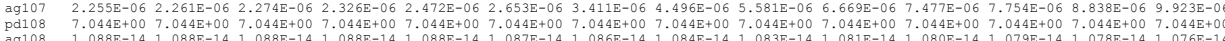

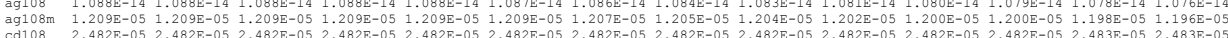

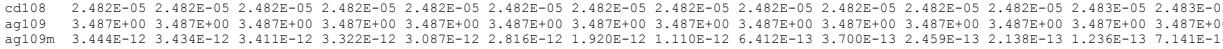

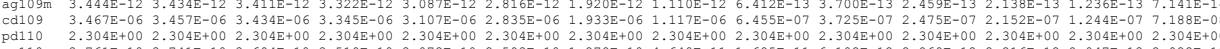

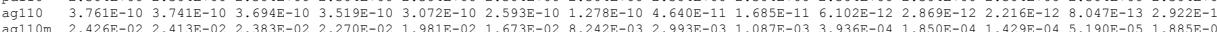

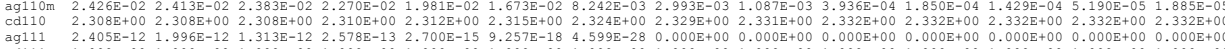

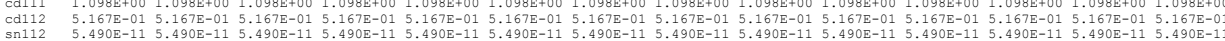

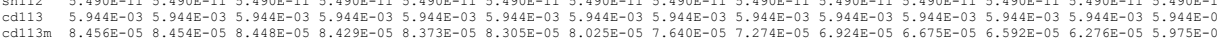

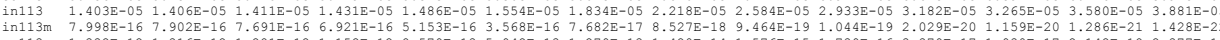

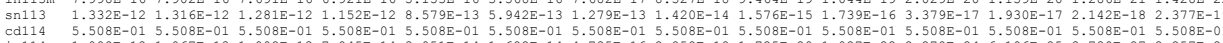

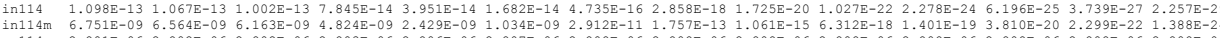

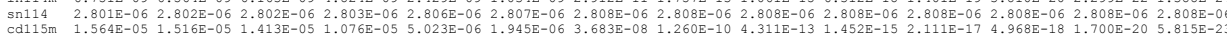

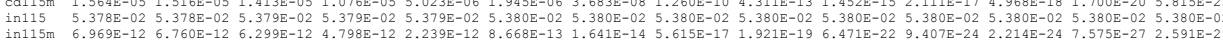

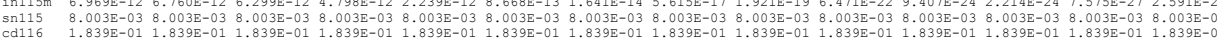

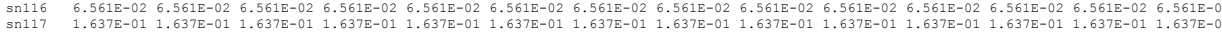

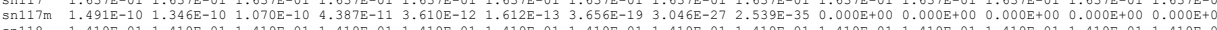

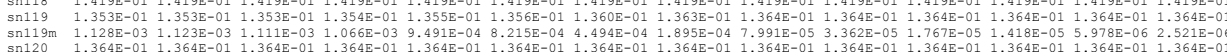

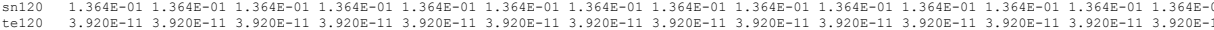

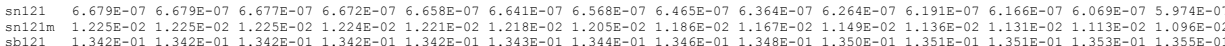

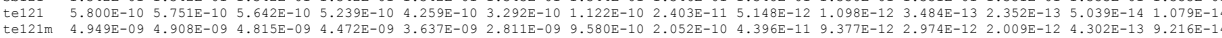

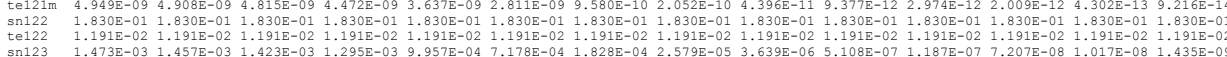

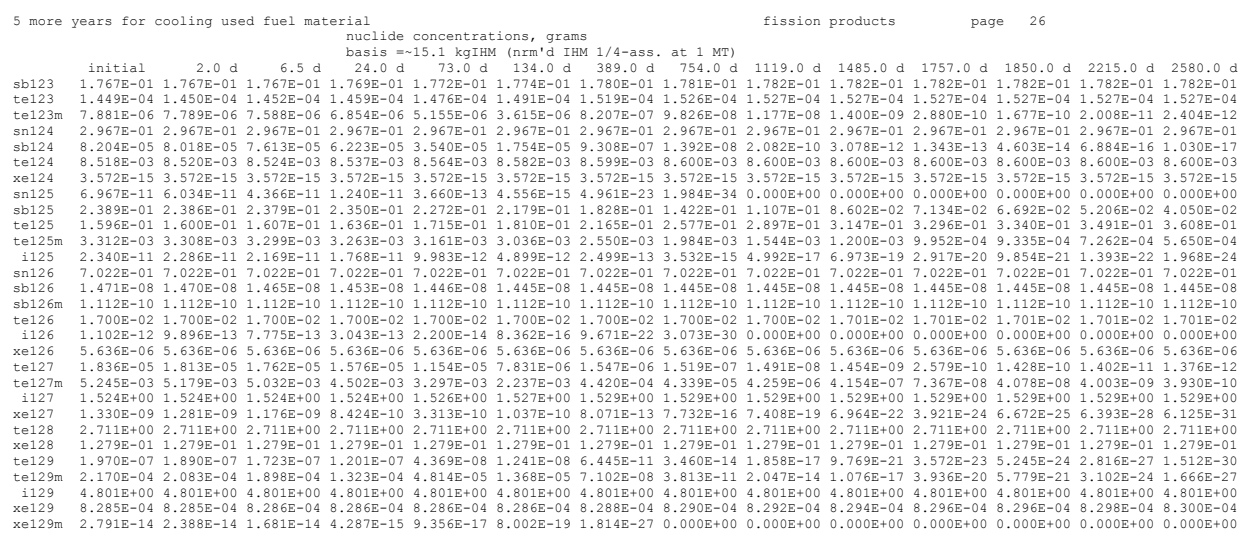

B-57 


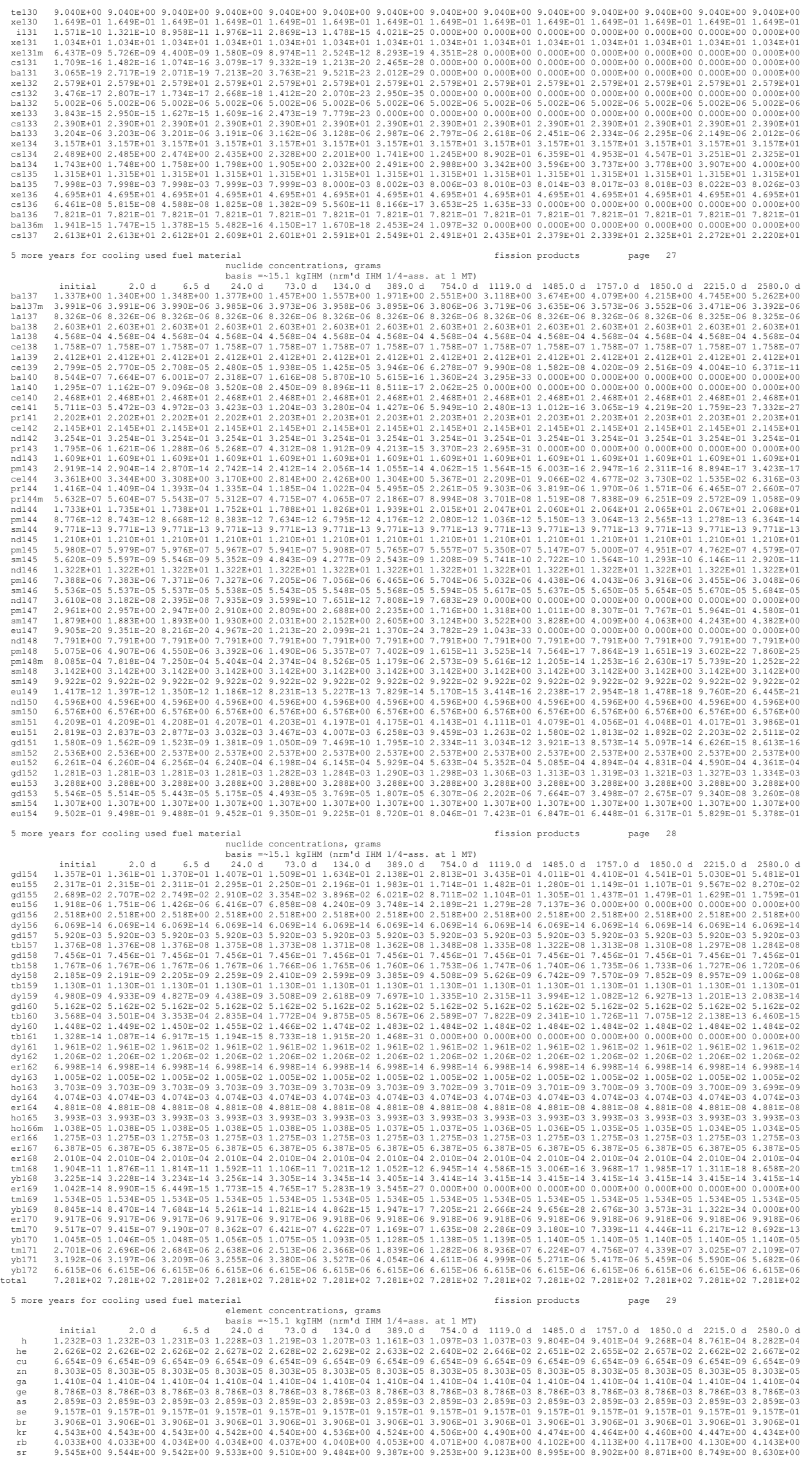

B-58 


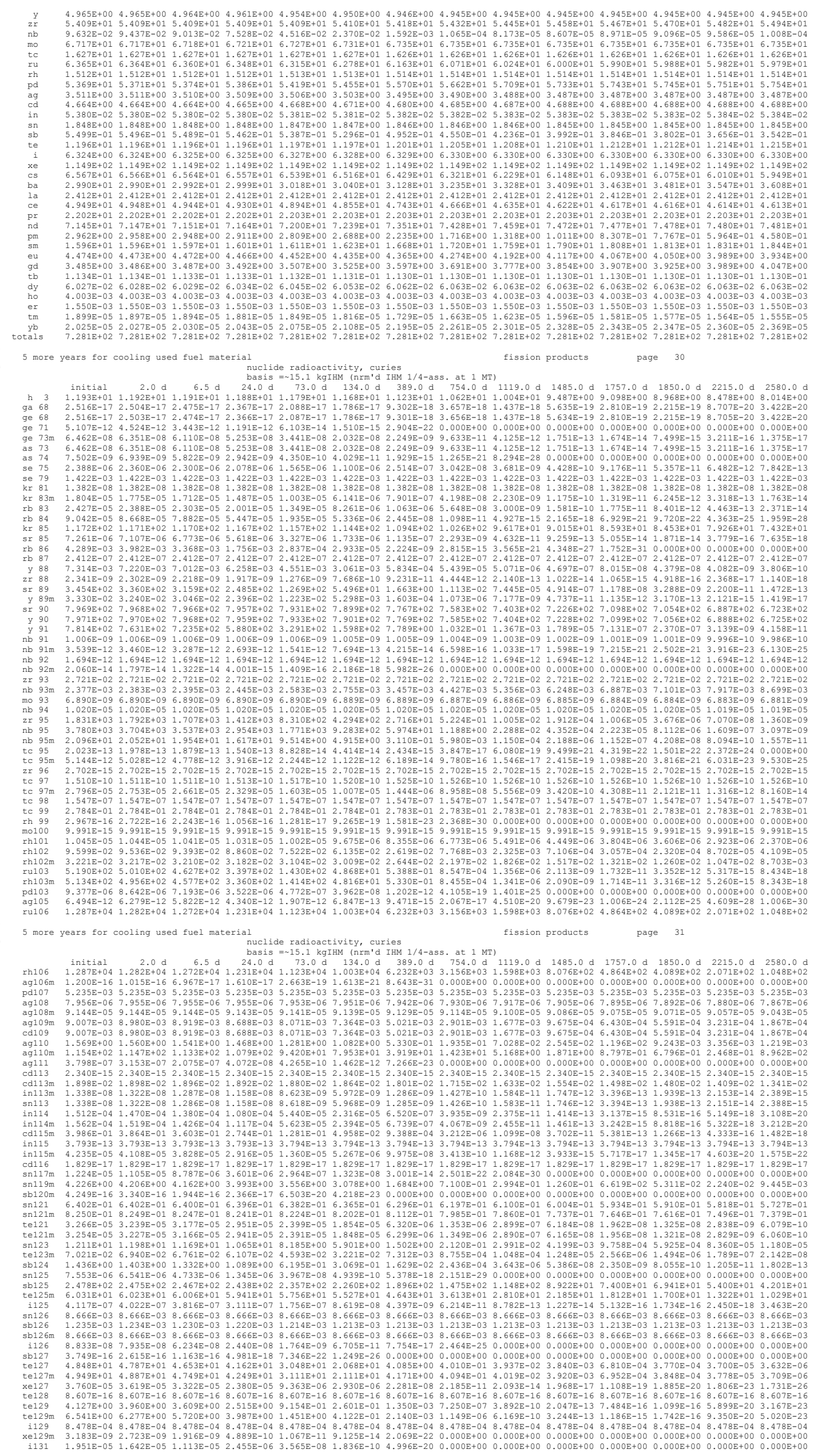

B-59 


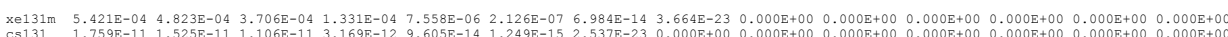

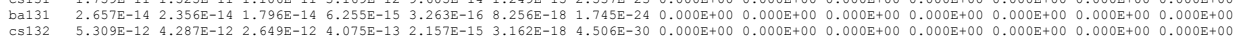

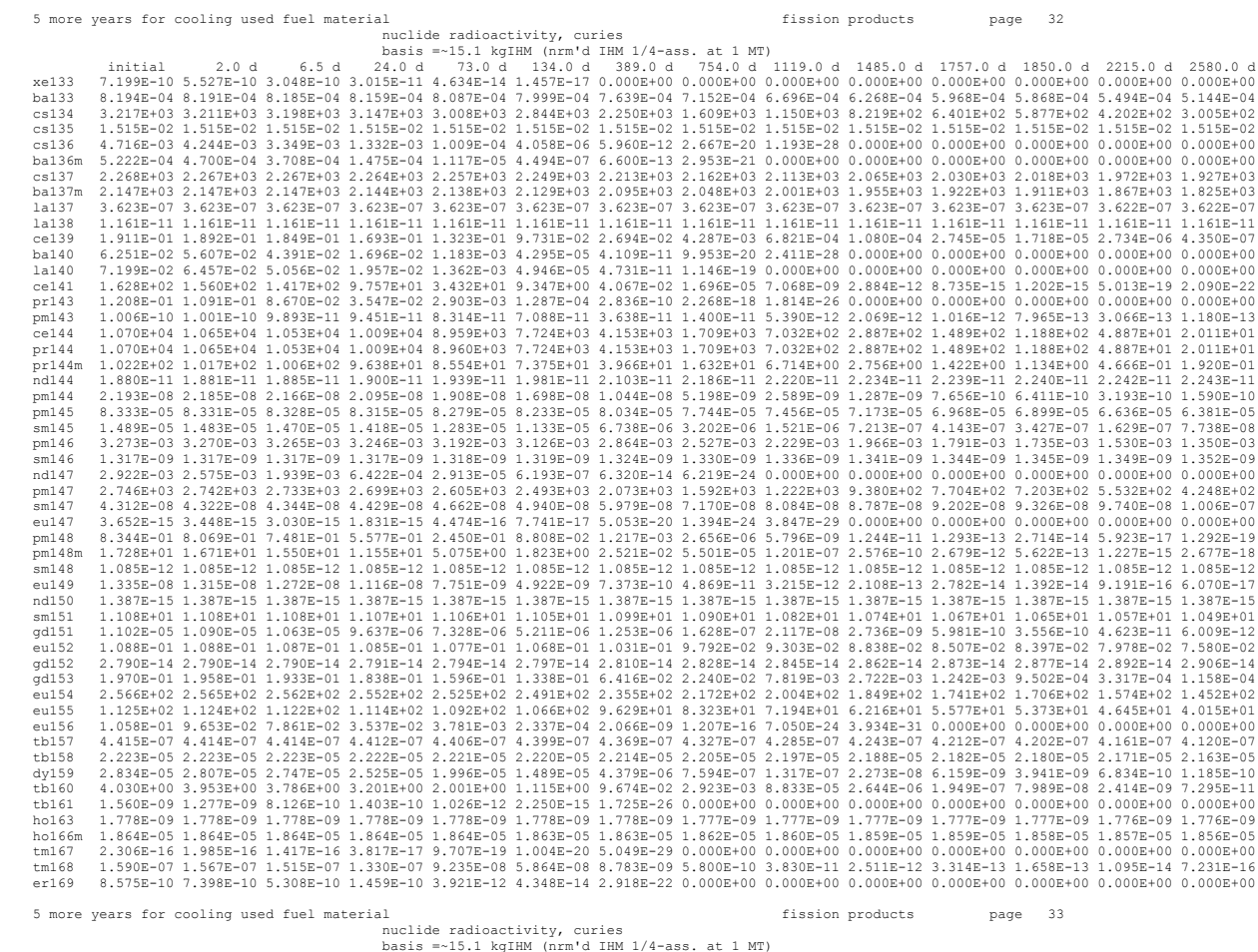

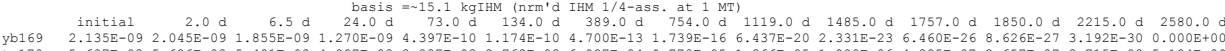

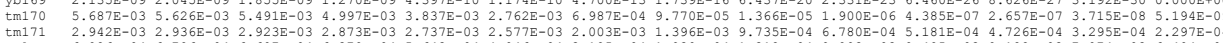

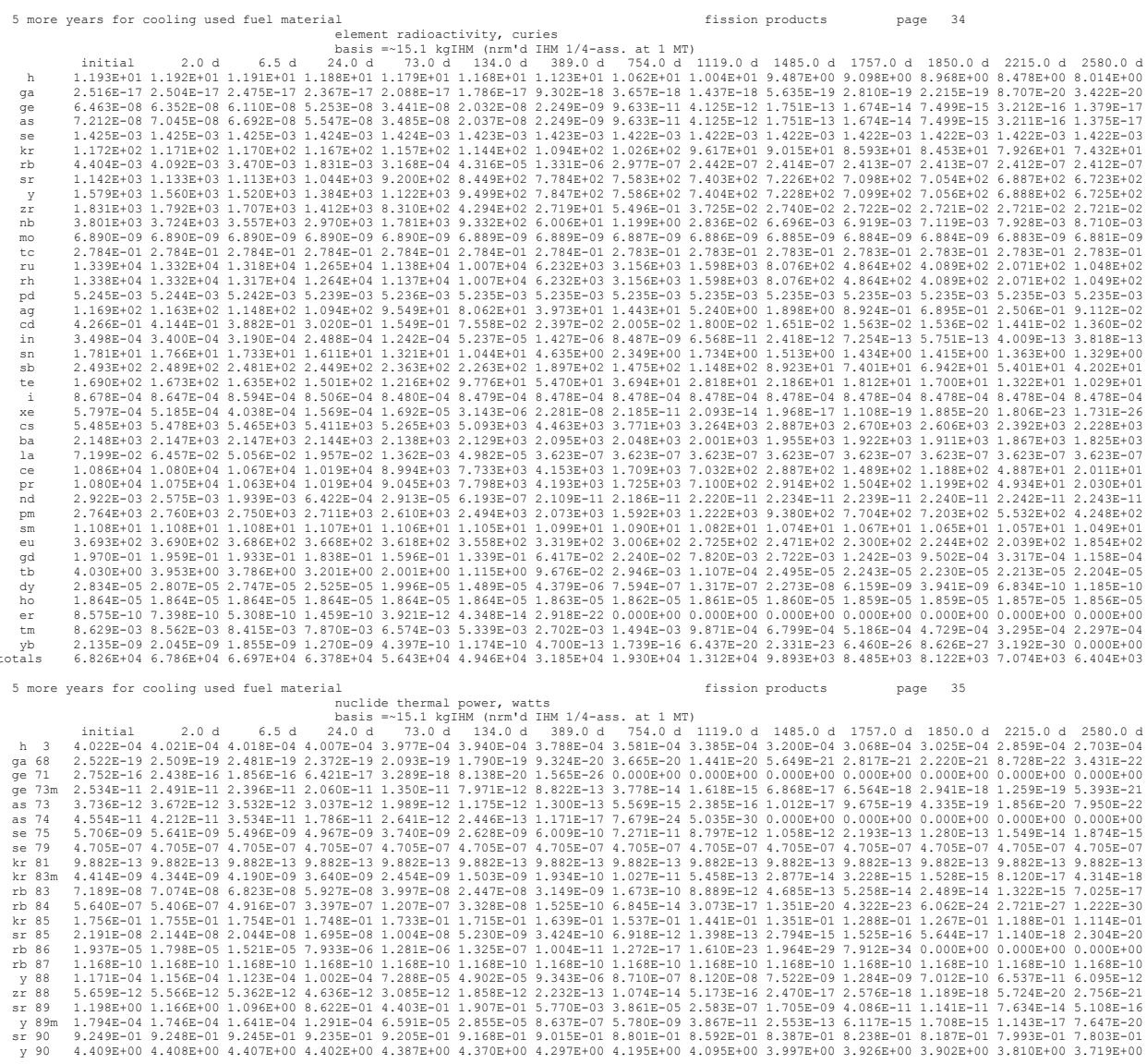

B-60 


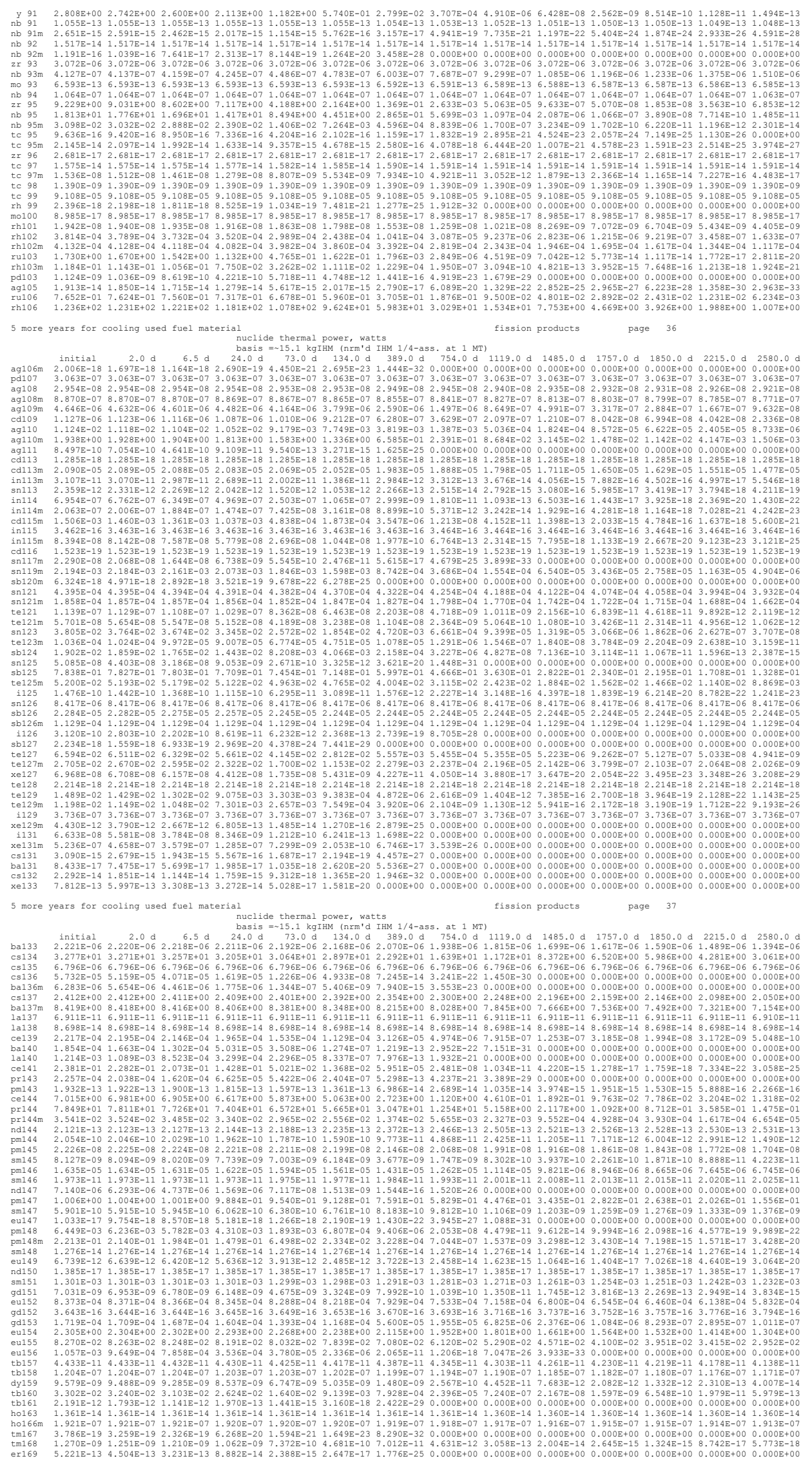

B-61 


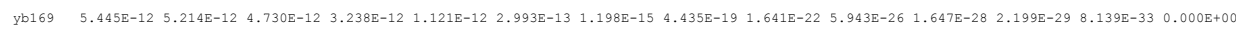
5 more years for cooling used fuel material huclide thermal power, watts

fission products page 3

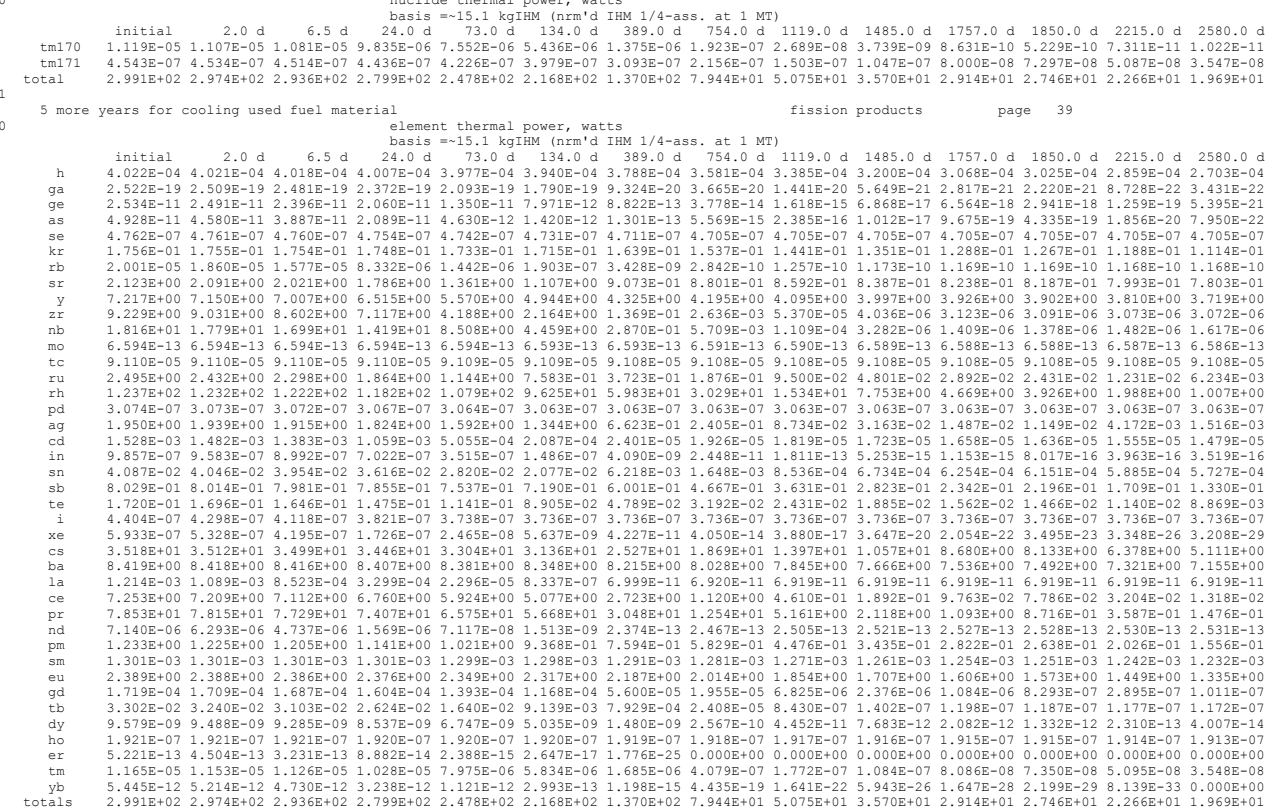

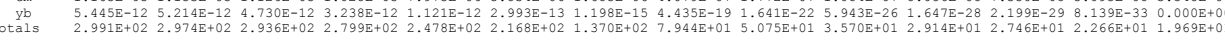

565 array 20 entries read 1 * 0 normal termination of execution * table of contents for material tables
case or subcase printed page 


\section{B.5 ORIGEN RESULTS FOR COOLING MOX FUEL ROD K5}
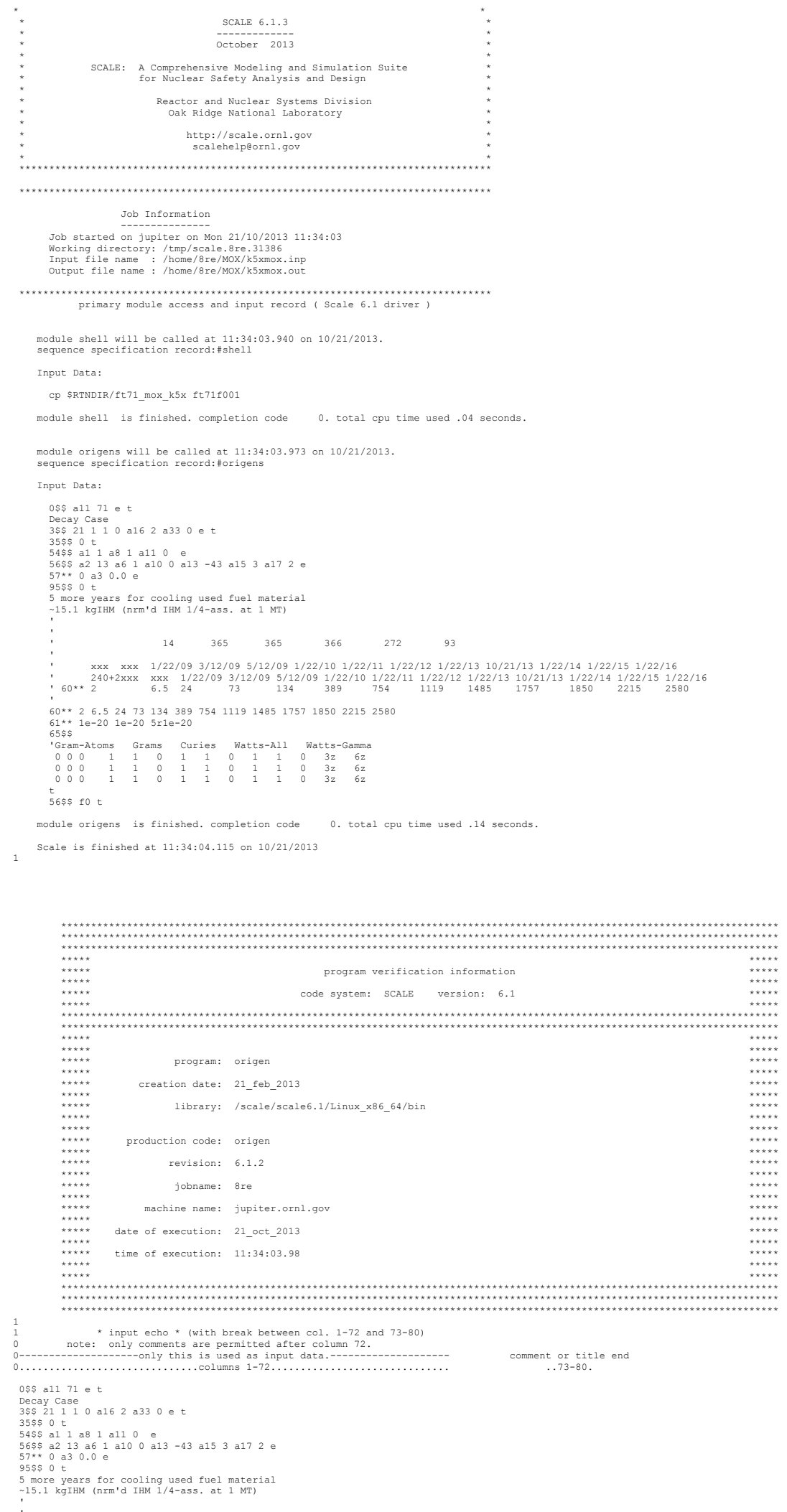


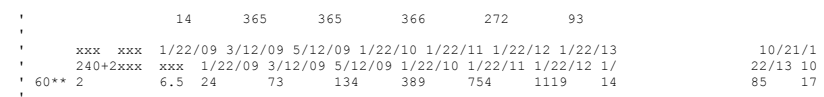

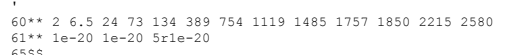

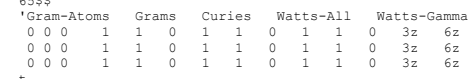

$t$
565 s fo $t$
owhen job "fails", make sure no fido input................................. is out here!

0 array $\quad 12$ entries read
ot

$3 \$$ array 33 entries read

llibrary information...
at

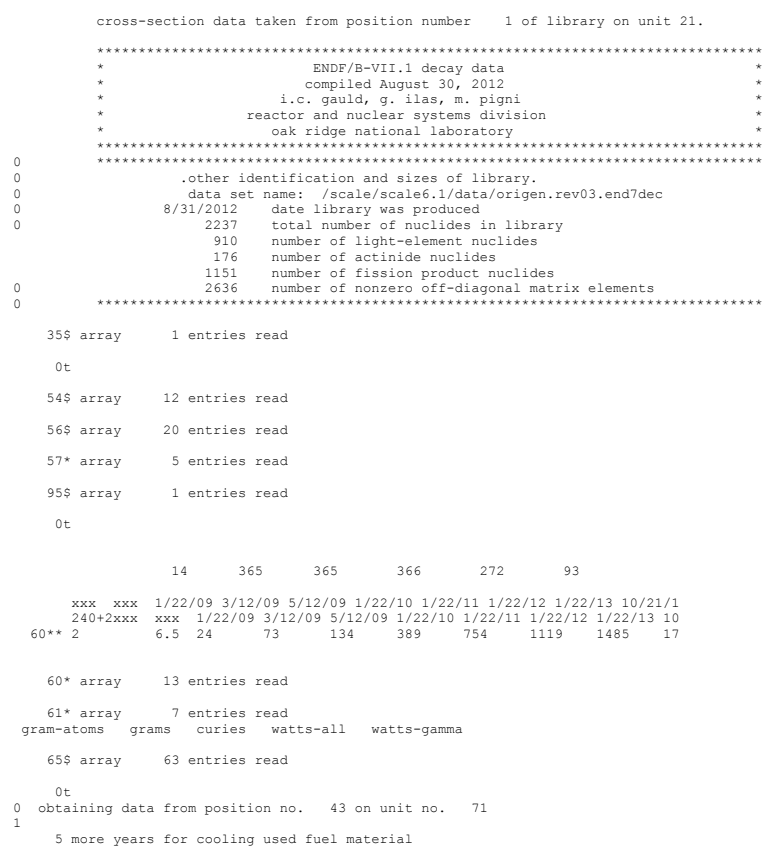

$$
\text { light elements page } 1
$$

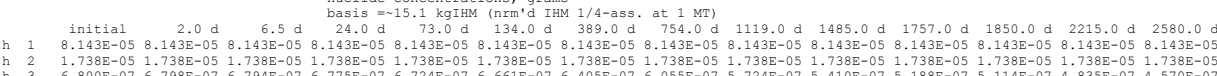

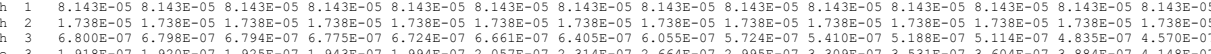

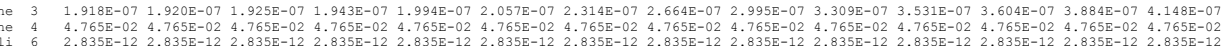

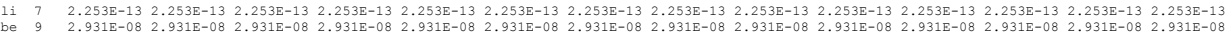

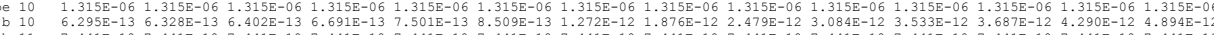

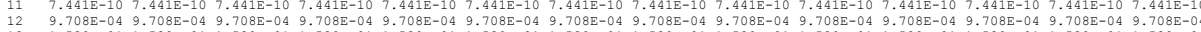

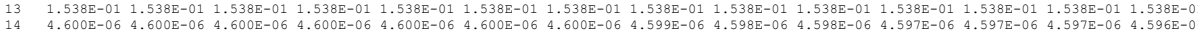

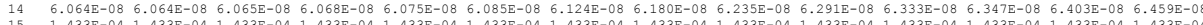

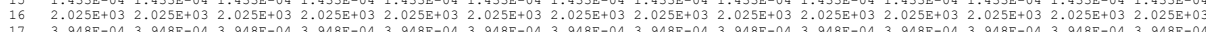

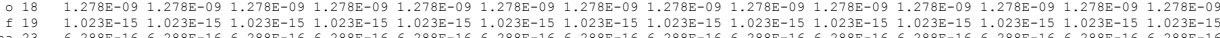

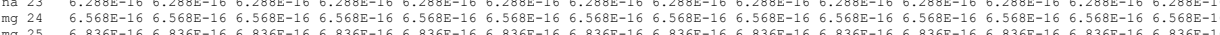

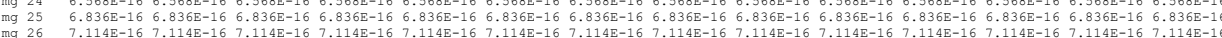

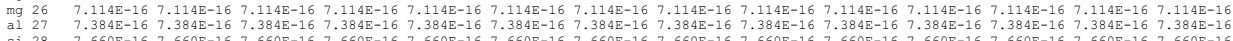

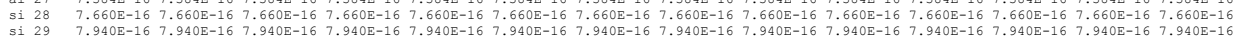

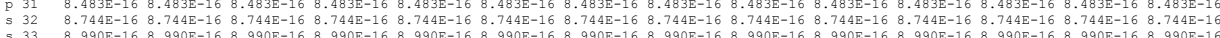

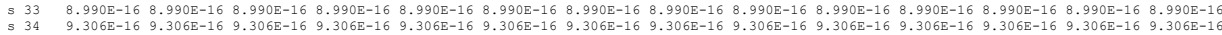

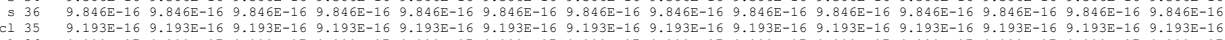

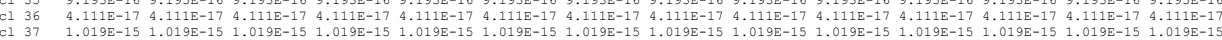

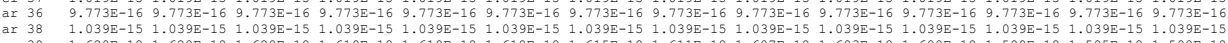

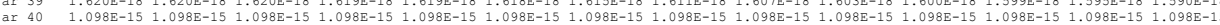

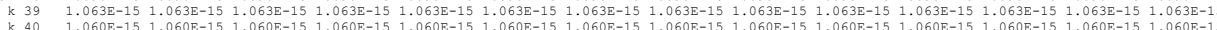

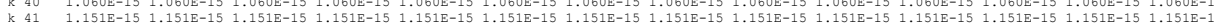

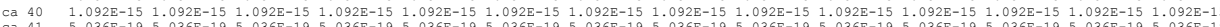

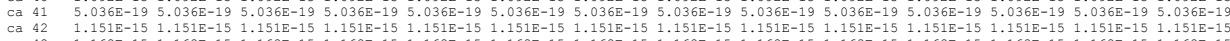

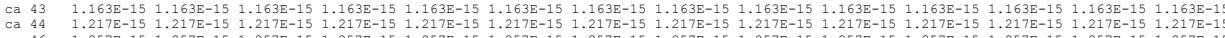

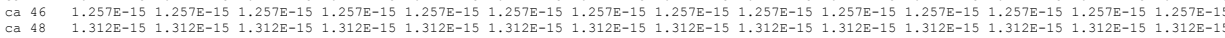

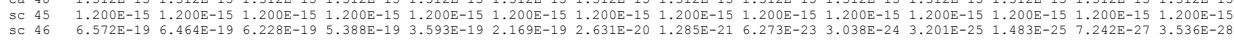

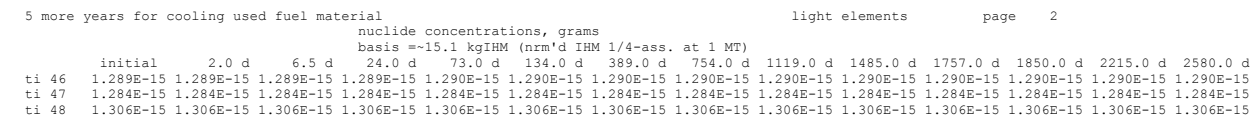




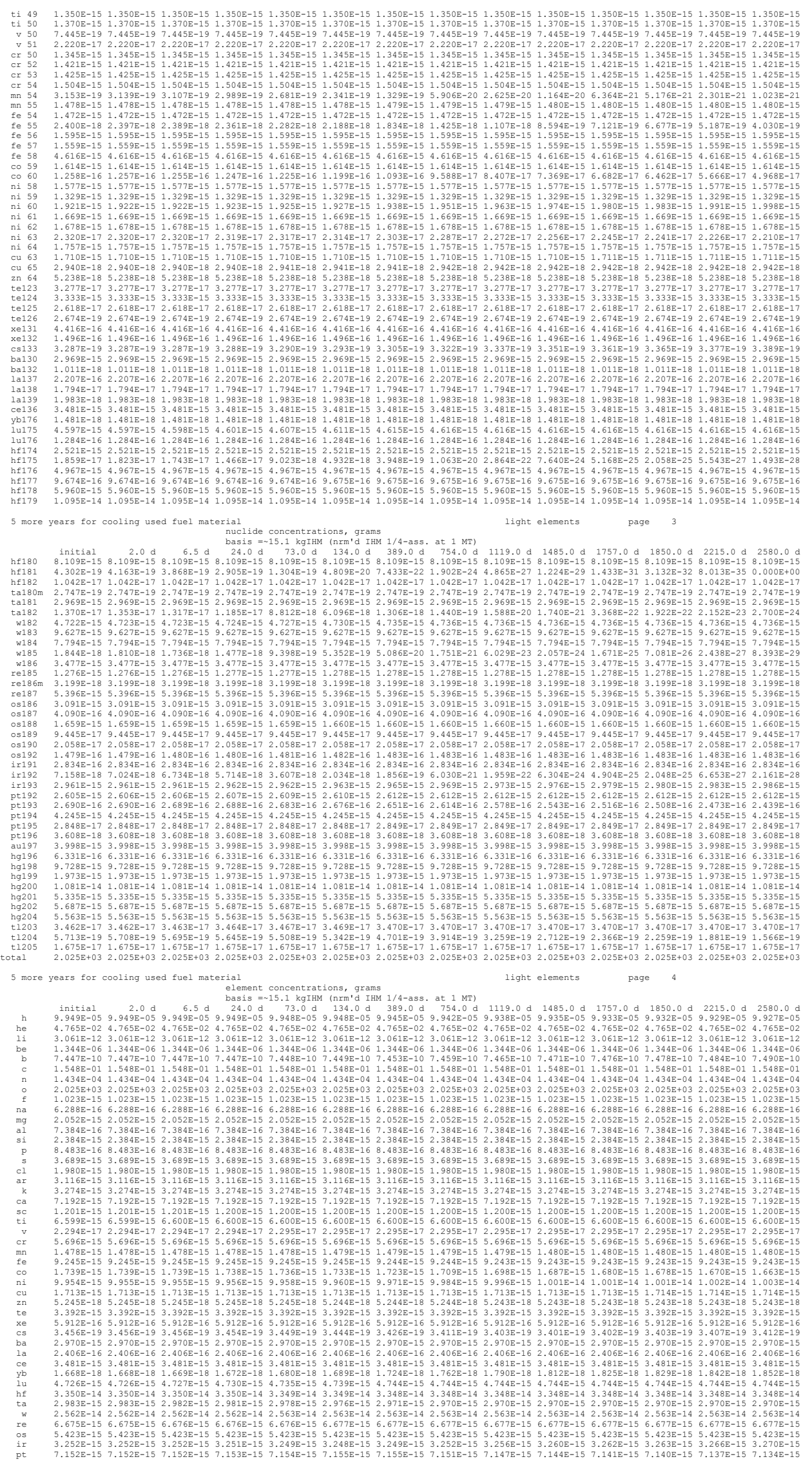

B-65 


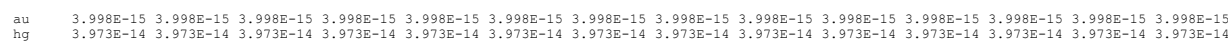

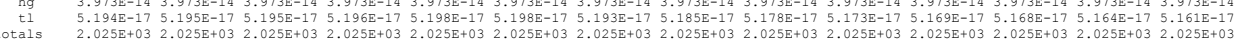

5 more years for cooling used fuel material

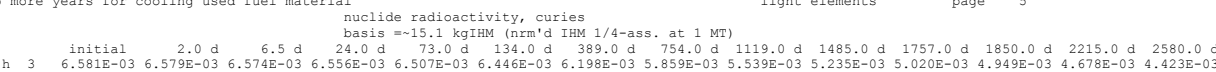

light elements page 5

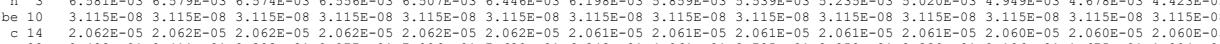

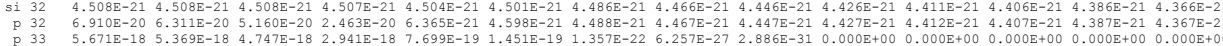

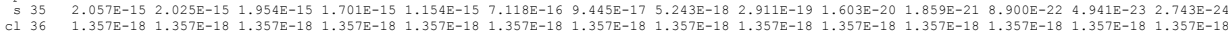

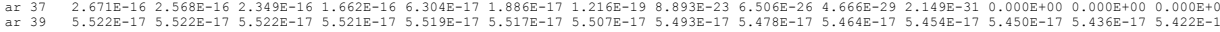

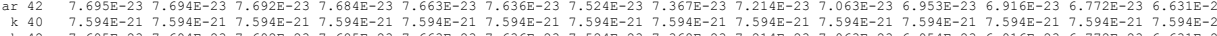

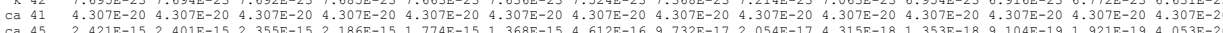

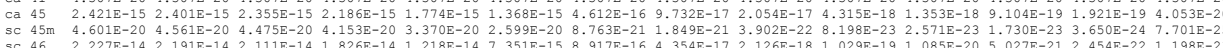

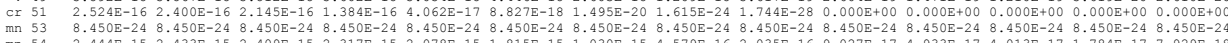

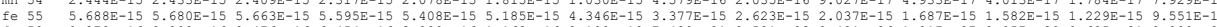

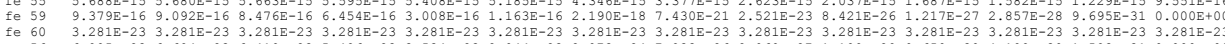

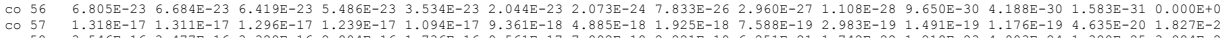

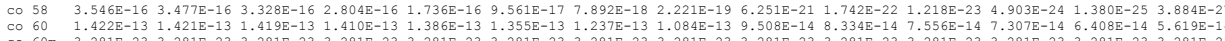

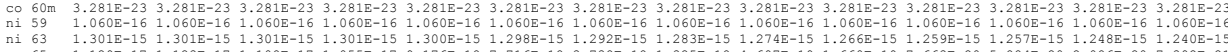

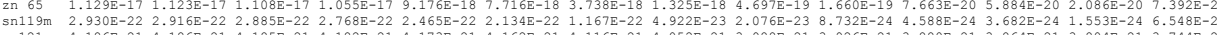

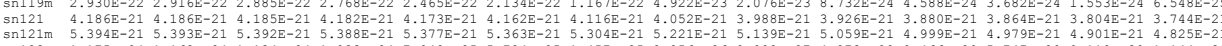

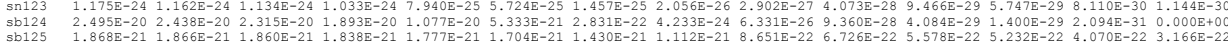

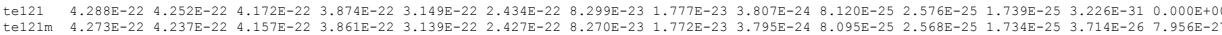

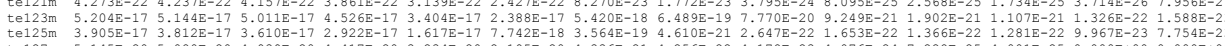

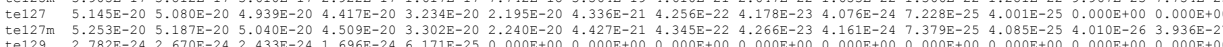

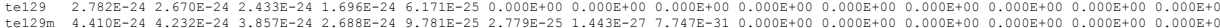

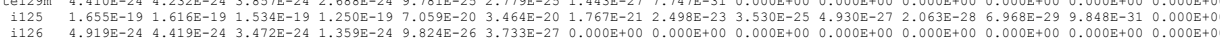

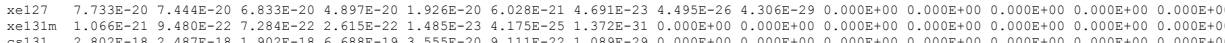

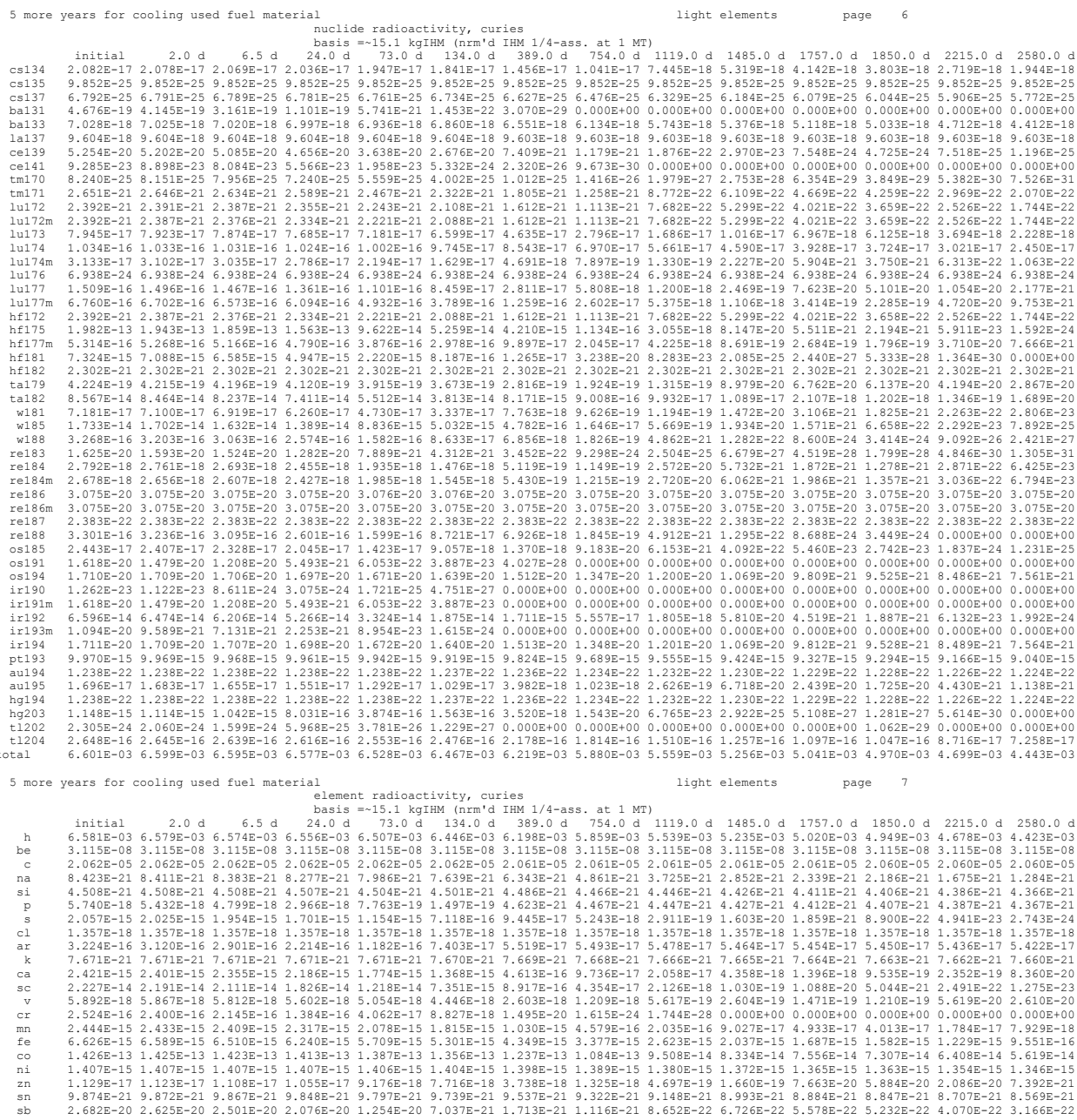

B-66 


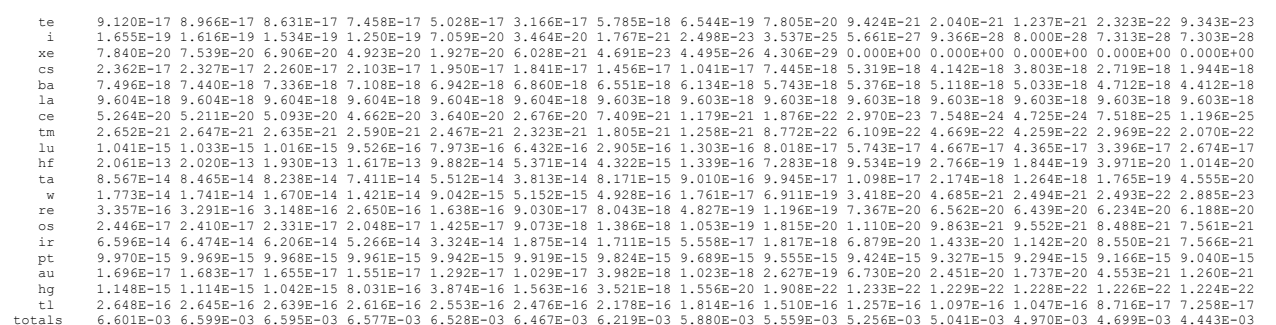

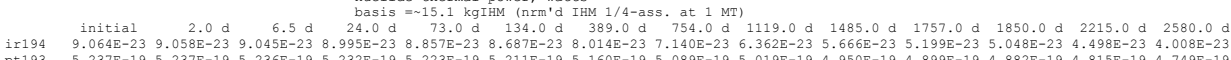

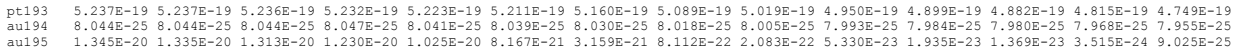


$\begin{array}{lllllllllllllllll}\mathrm{h} & 194 & 7.010 \mathrm{E}-27 & 7.010 \mathrm{E}-27 & 7.009 \mathrm{E}-27 & 7.009 \mathrm{E}-27 & 7.007 \mathrm{E}-27 & 7.006 \mathrm{E}-27 & 6.998 \mathrm{E}-27 & 6.987 \mathrm{E}-27 & 6.976 \mathrm{E}-27 & 6.965 \mathrm{E}-27 & 6.957 \mathrm{E}-27 & 6.954 \mathrm{E}-27 & 6.944 \mathrm{E}-27 & 6.933 \mathrm{E}-27 \\ \mathrm{hg} 203 & 2.372 \mathrm{E}-18 & 2.302 \mathrm{E}-18 & 2.153 \mathrm{E}-18 & 1.660 \mathrm{E}-18 & 8.006 \mathrm{E}-19 & 3.231 \mathrm{E}-19 & 7.275 \mathrm{E}-21 & 3.189 \mathrm{E}-23 & 1.398 \mathrm{E}-25 & 6.037 \mathrm{E}-28 & 1.056 \mathrm{E}-29 & 2.646 \mathrm{E}-30 & 1.160 \mathrm{E}-32 & 0.000 \mathrm{E}+00\end{array}$

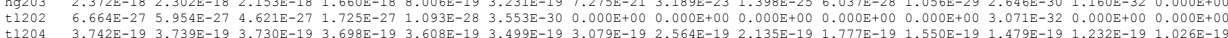

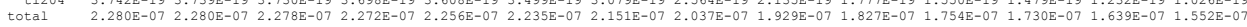
5 more years for cooling used fuel materia light elements page 11

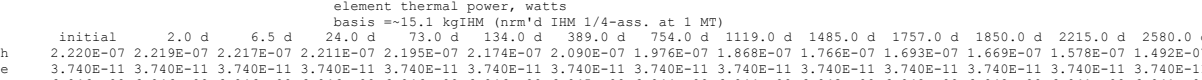

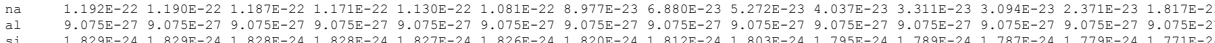

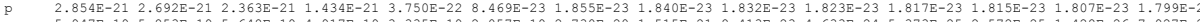

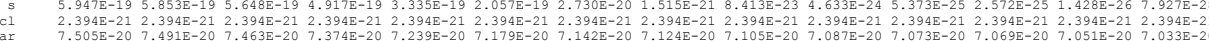

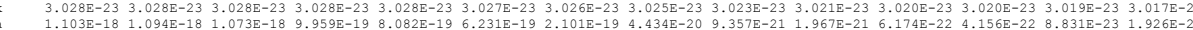

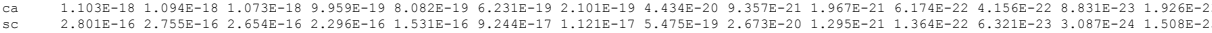

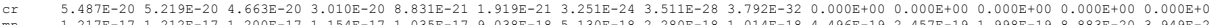

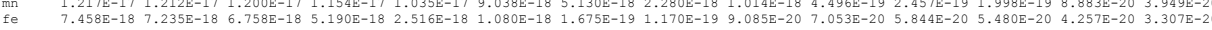

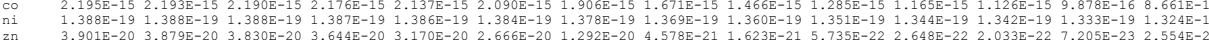

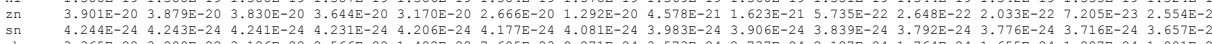

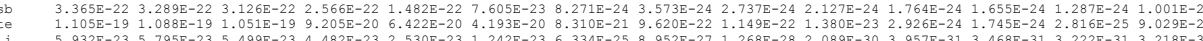
$\begin{array}{llllllll}1 & 5.932 \mathrm{E}-23 & 5 & & \end{array}$

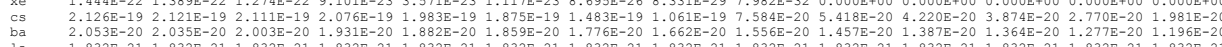

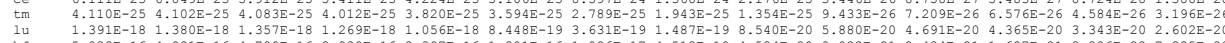

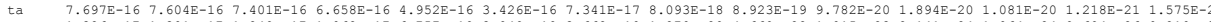

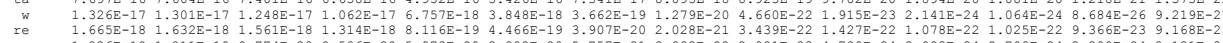

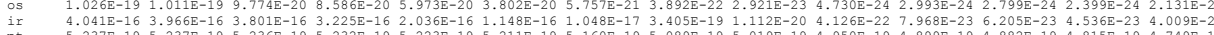

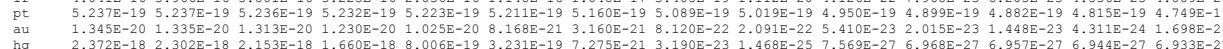

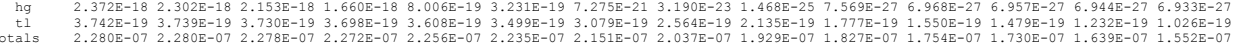
nuclide concentrations, grams actinides page 12

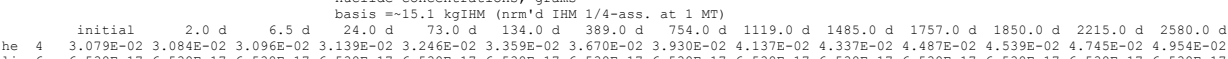

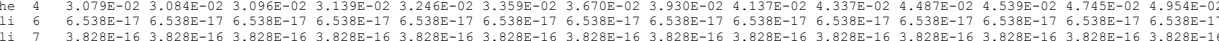

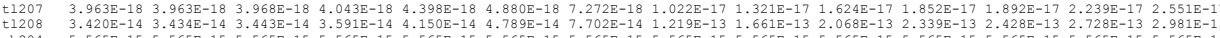

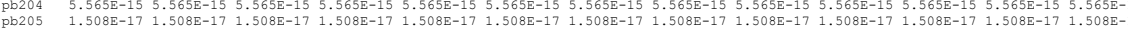

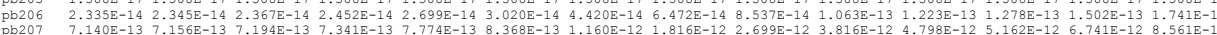

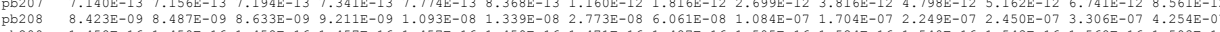

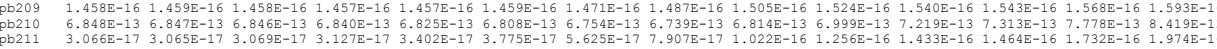

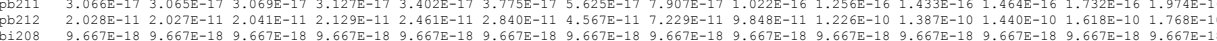

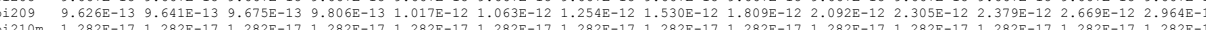

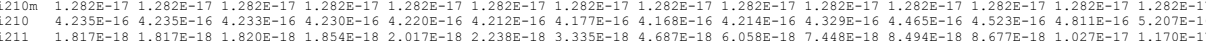

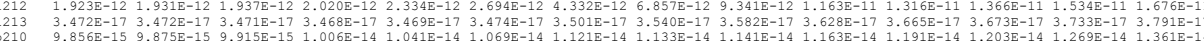

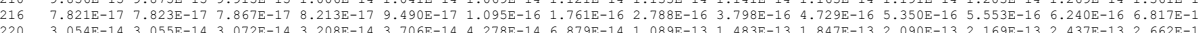
$\begin{array}{lllllllll} & \end{array}$

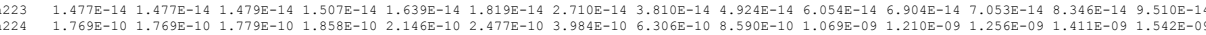

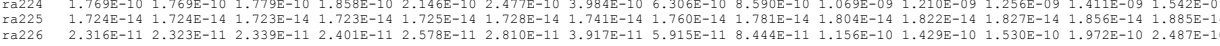

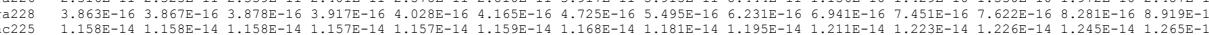

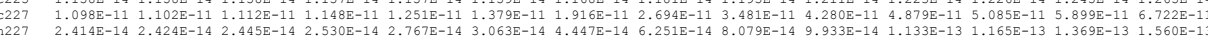

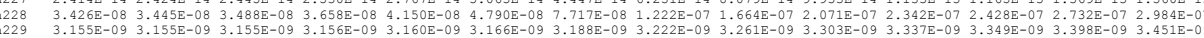

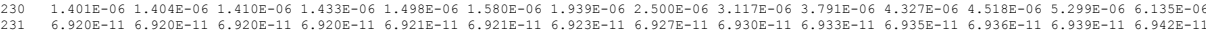

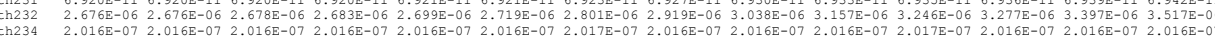

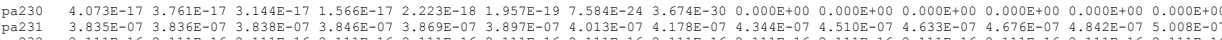

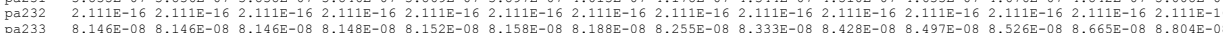

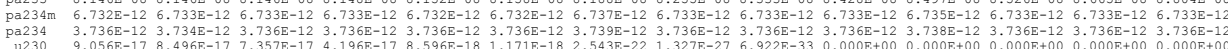

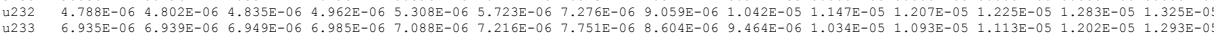

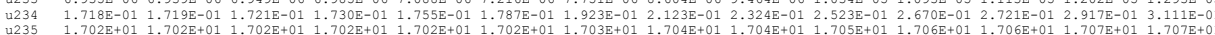

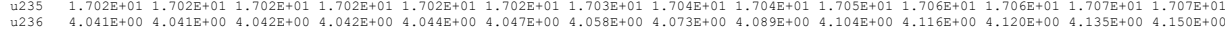
5 more years for cooling used fuel material

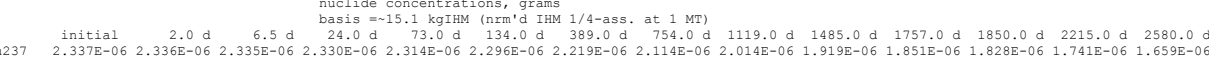

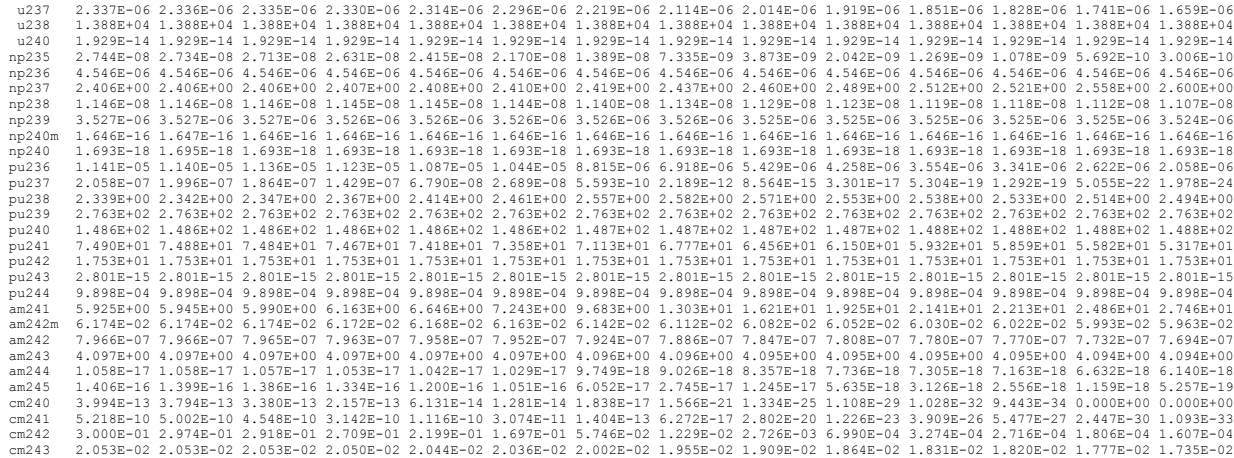




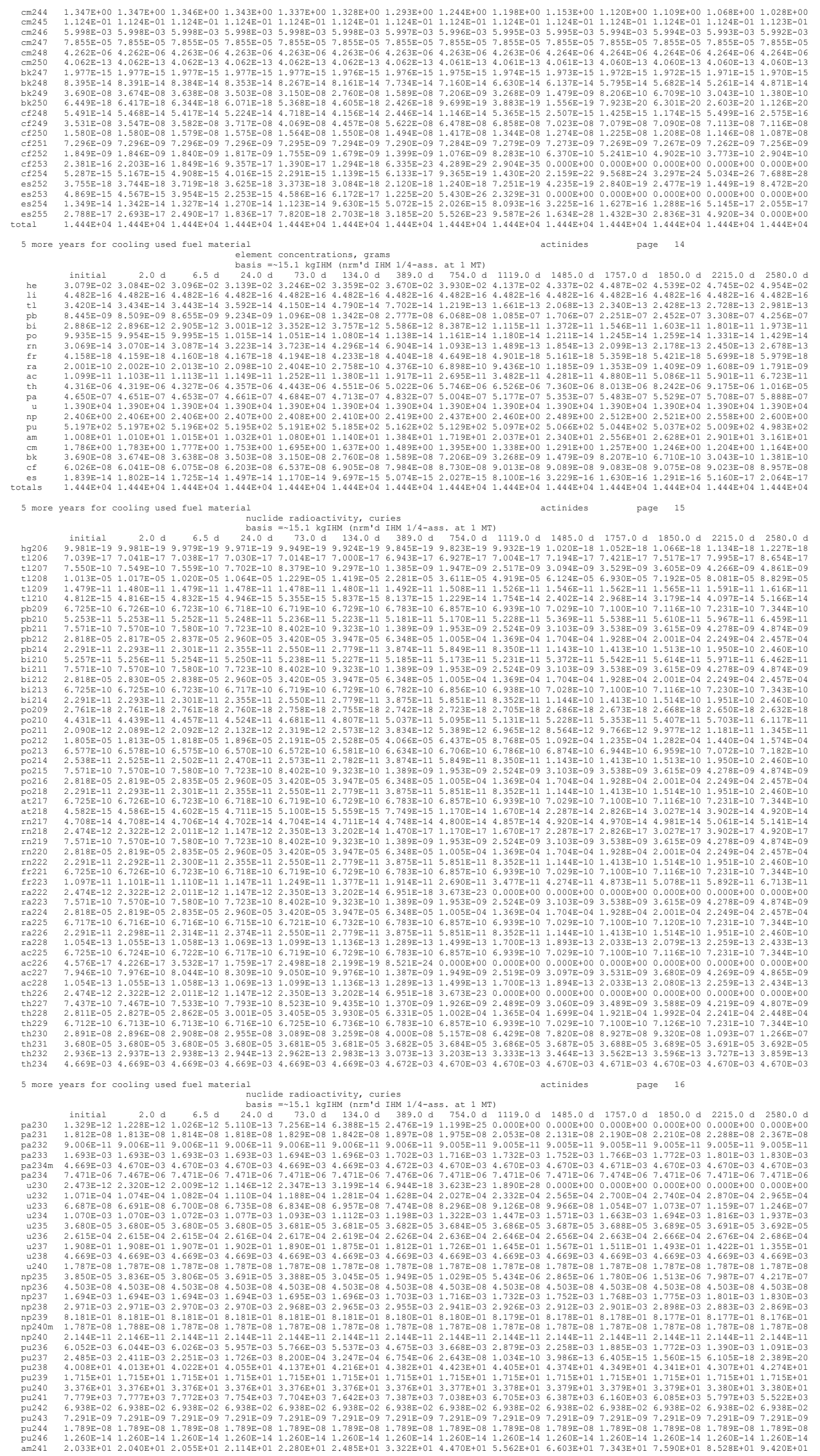

B-69 

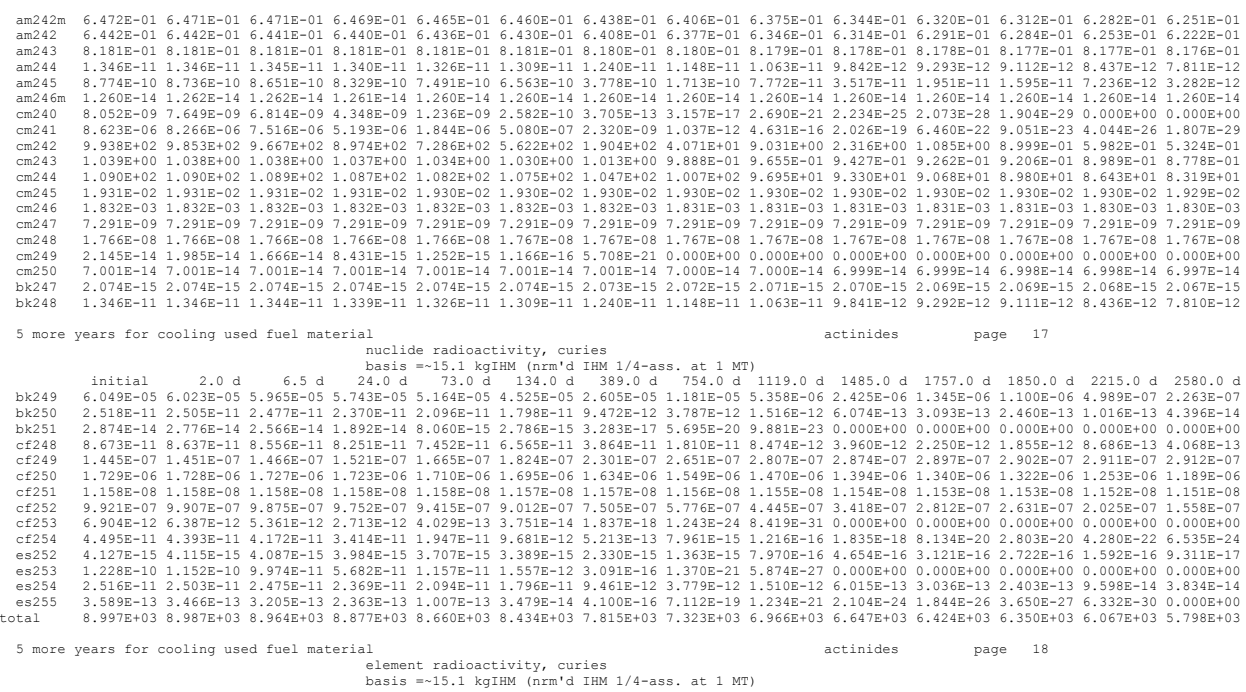

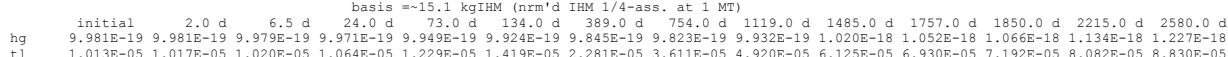

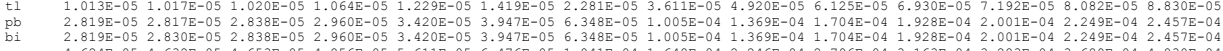

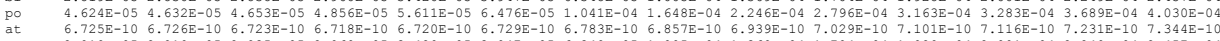

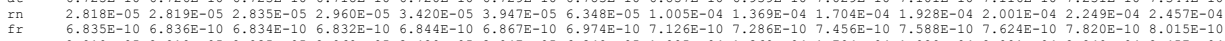

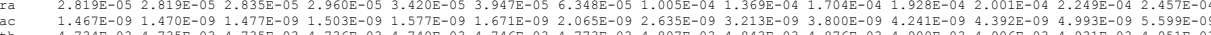

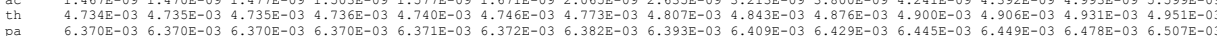

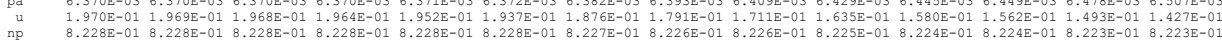

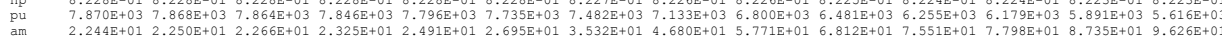

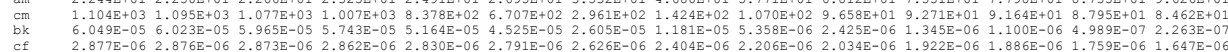

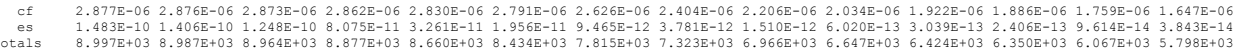

5 more years for cooling used fuel material

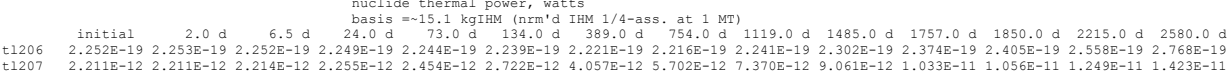

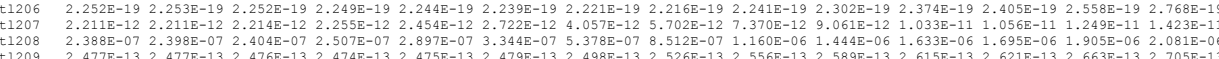

$\begin{array}{ll} & \\ & \end{array}$

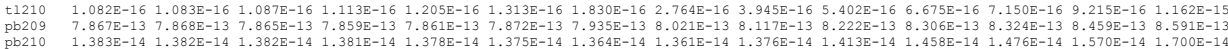

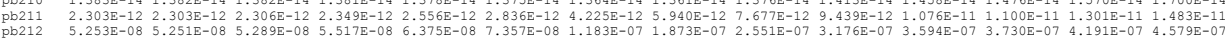

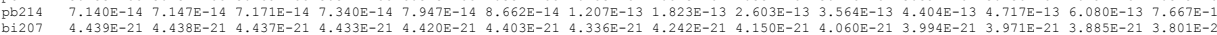

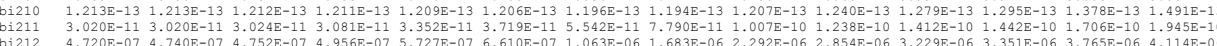

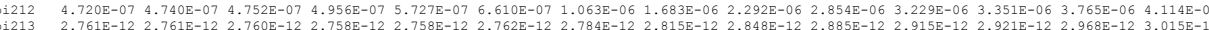

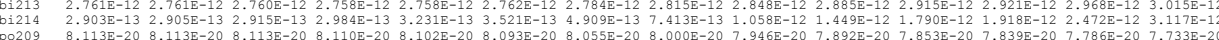

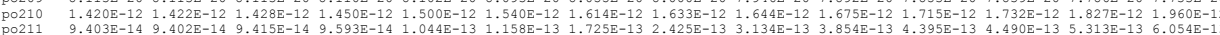

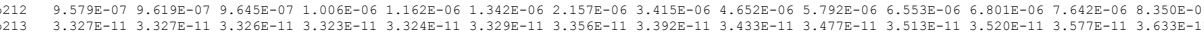

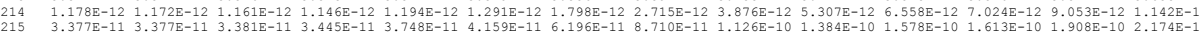

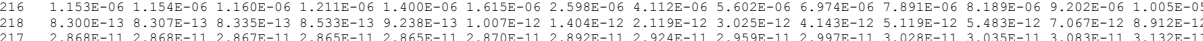

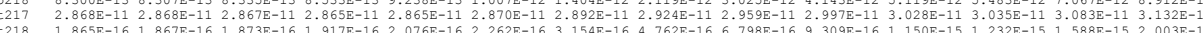

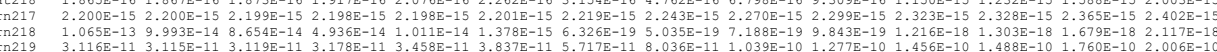

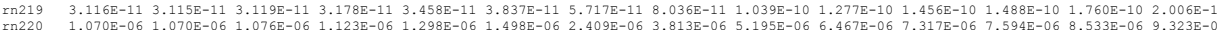

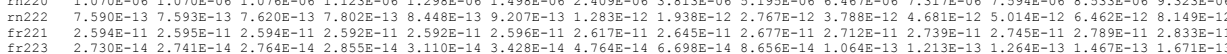

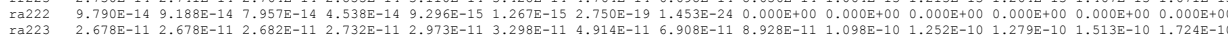

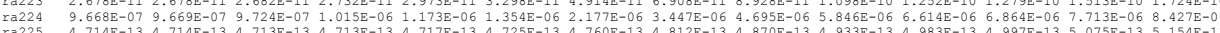

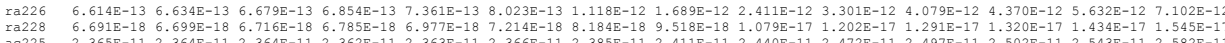

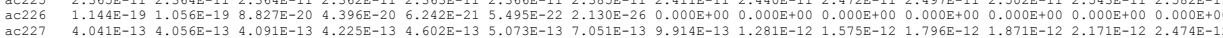

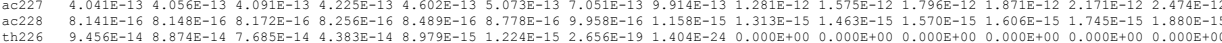

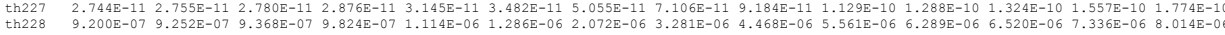

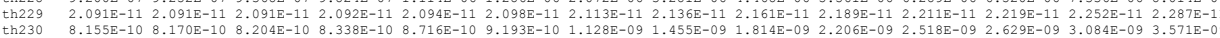

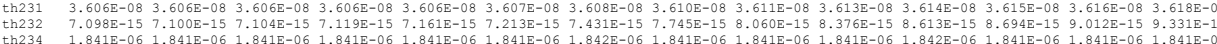

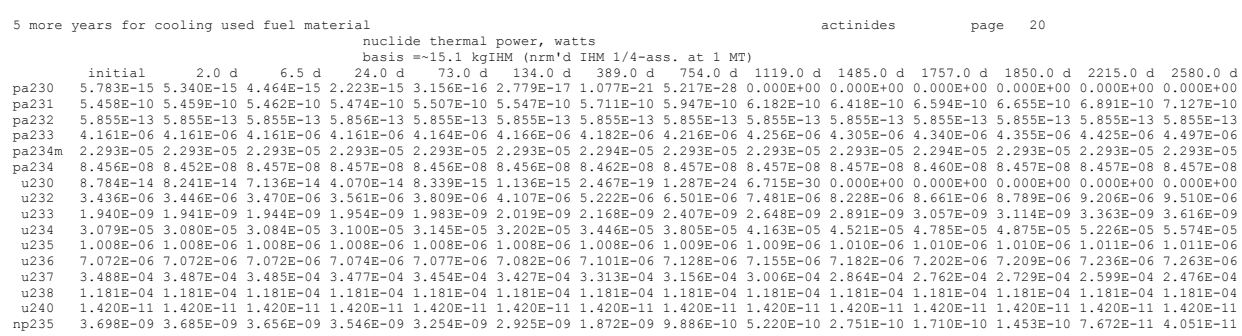

B-70 


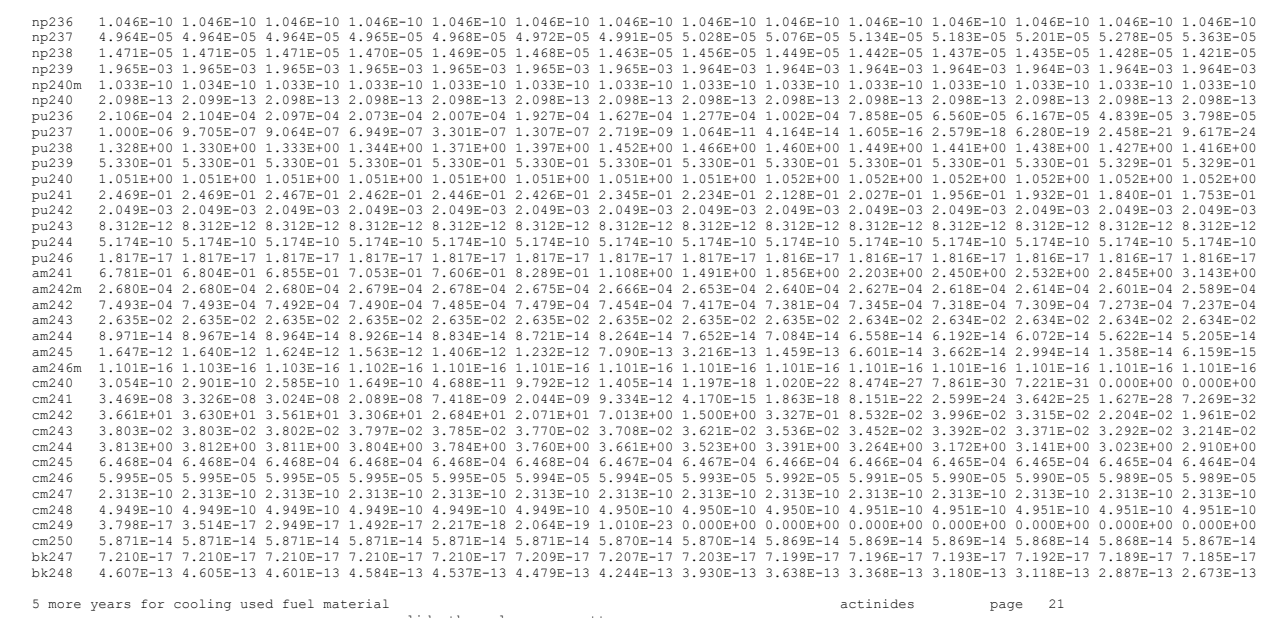

5 more years for cooling used fuel material
nuclide thermal power, watts
basis $=\sim 15.1 \mathrm{~kg}$ IMM (ntm'd IHM 1/4-ass. at $1 \mathrm{MT}$ )

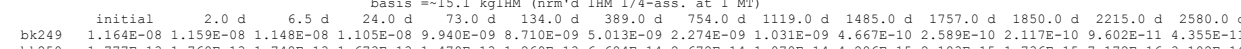

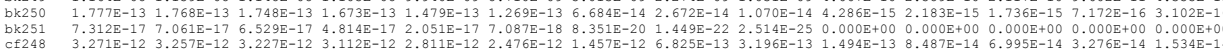

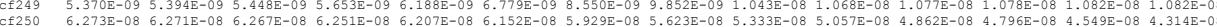

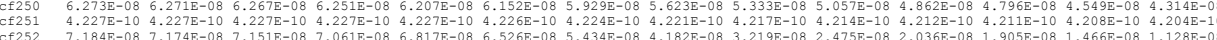

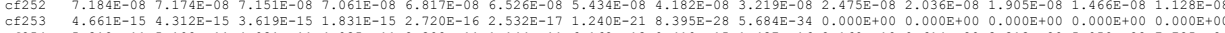

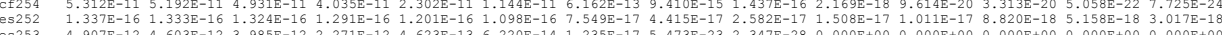

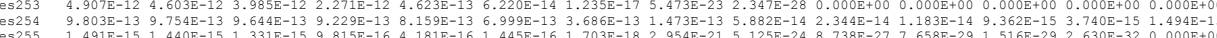

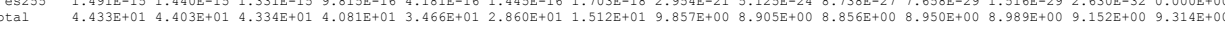
5 more years for cooling used fuel material

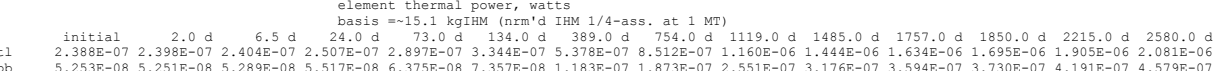

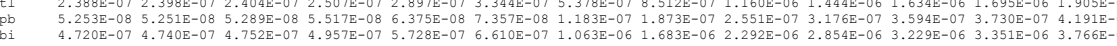

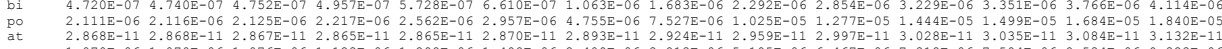

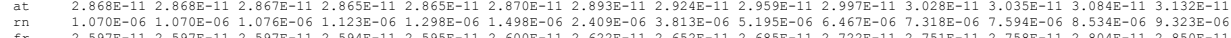

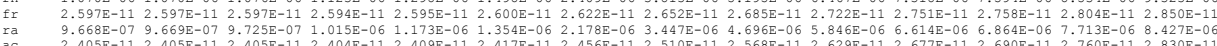

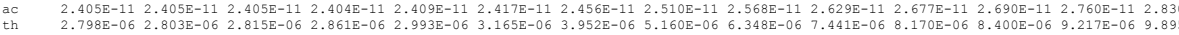

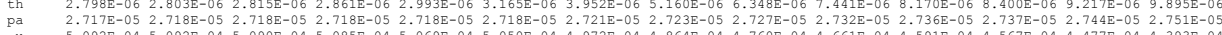

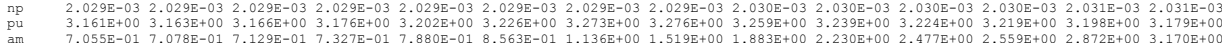

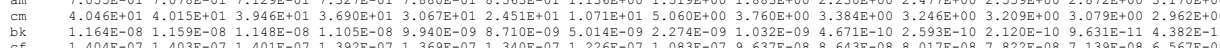

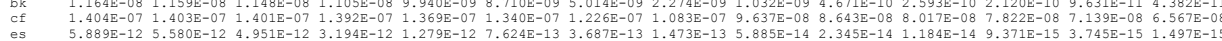

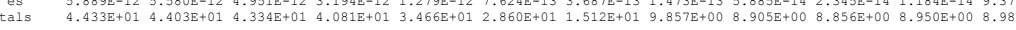
5 more years for cooling used fuel materit huclide concentrations, grams fission products page 23

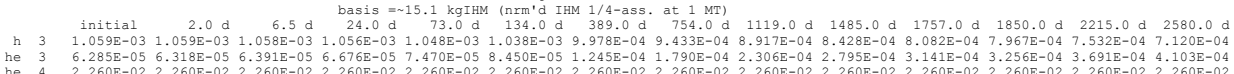

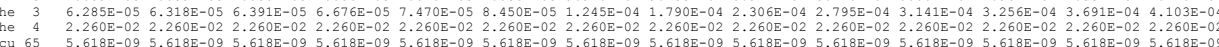

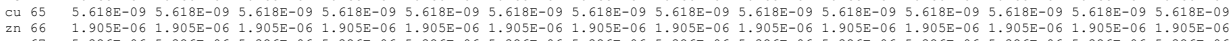

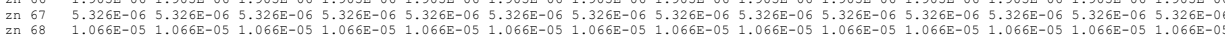

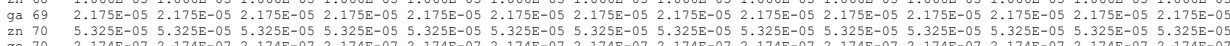

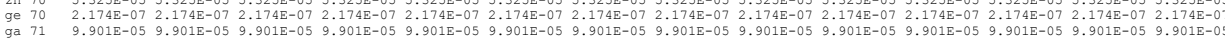

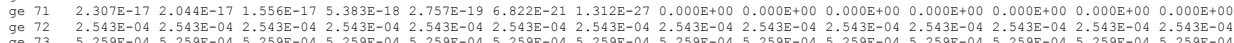

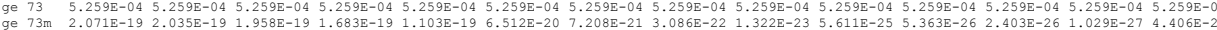

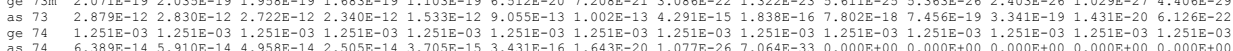

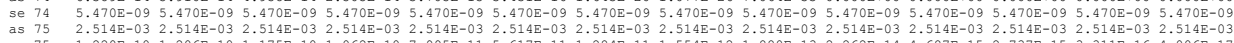

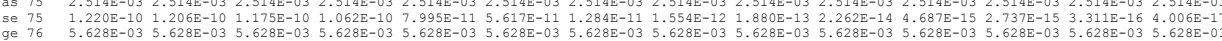

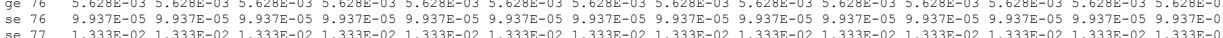

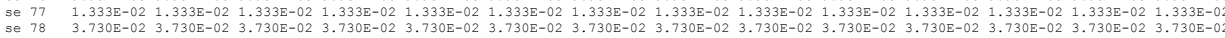

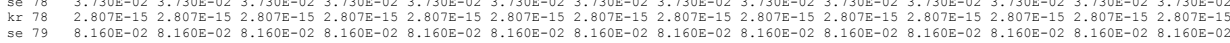

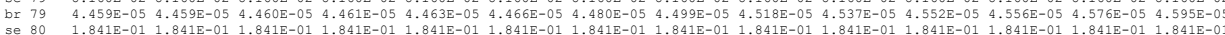

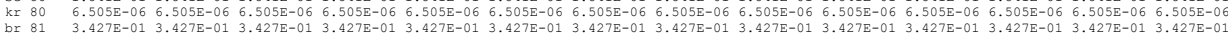

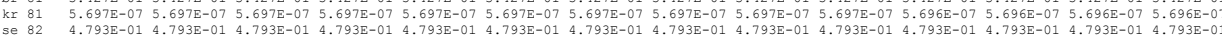

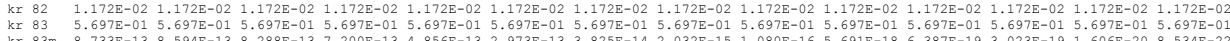

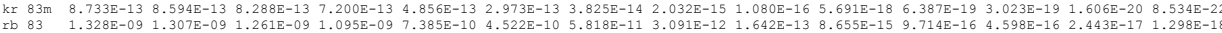

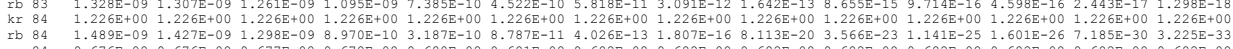

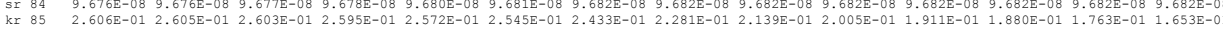

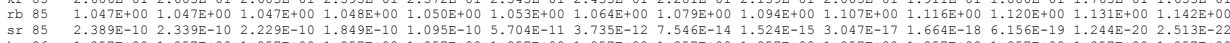

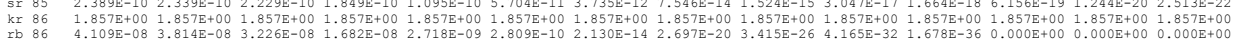

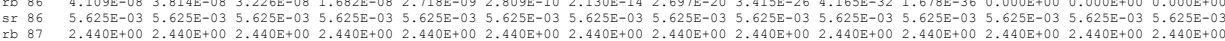

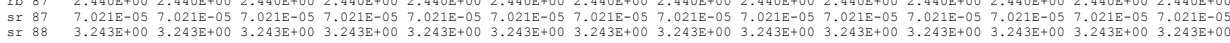

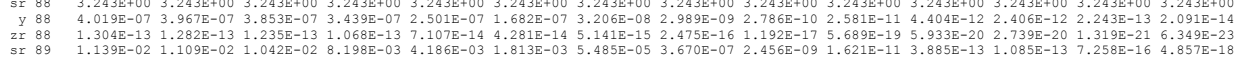
5 more years for cooling used fuel material $\quad$ fission products $\quad$ page 24

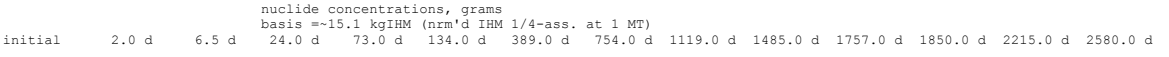

B-71 


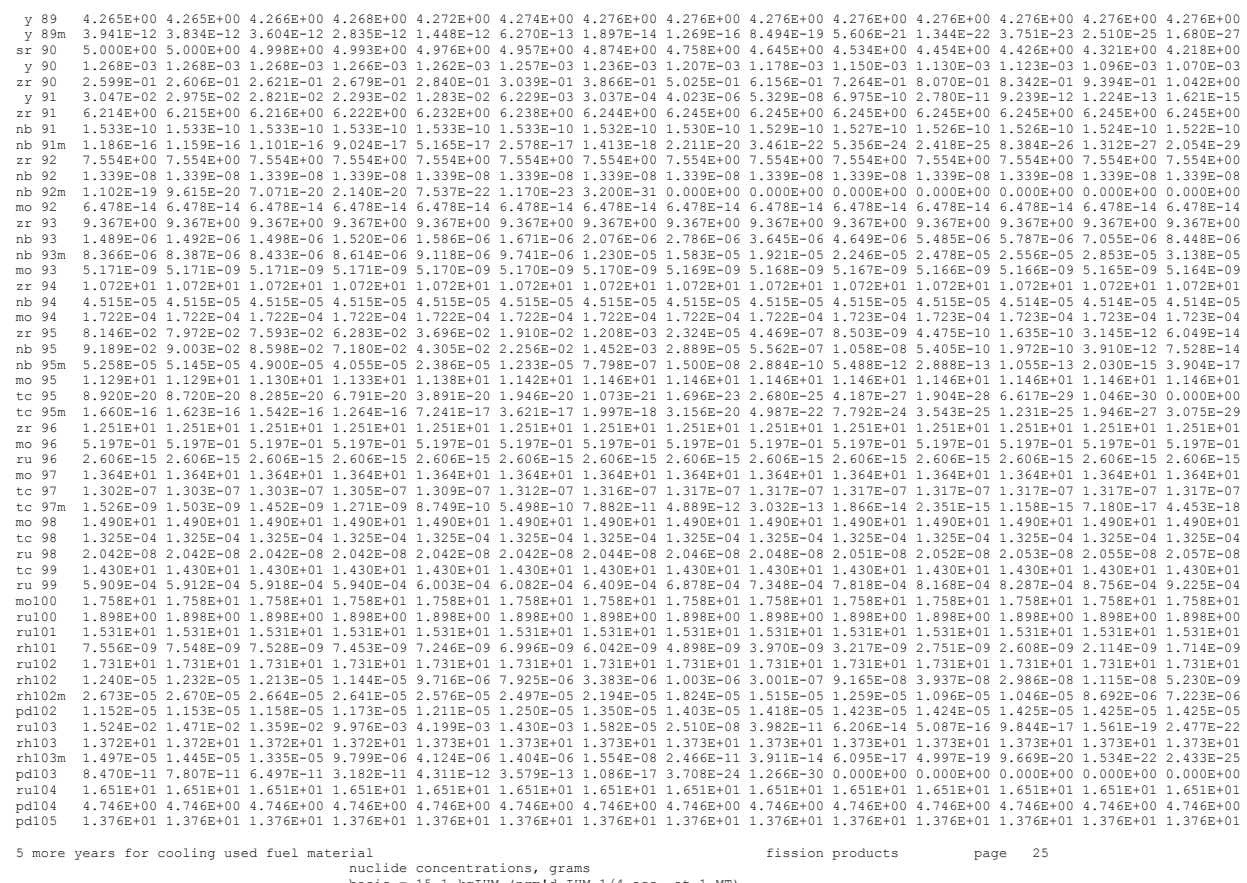

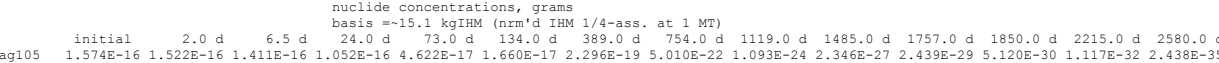

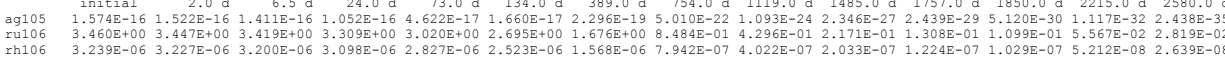

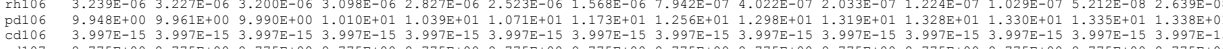

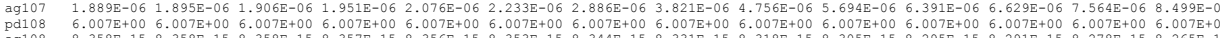

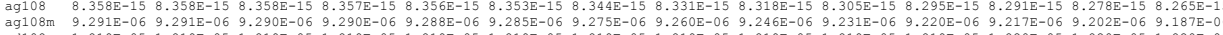

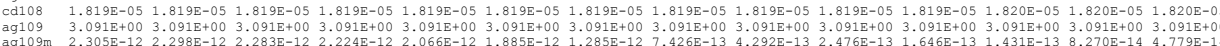

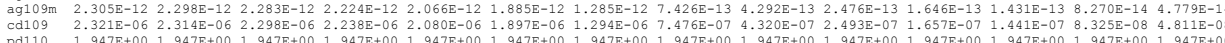

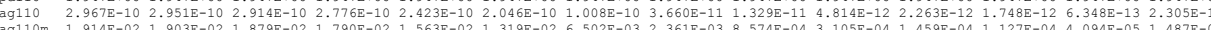

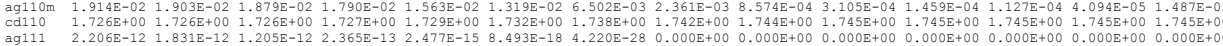

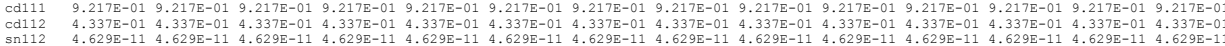

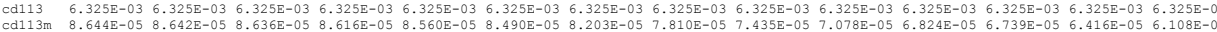

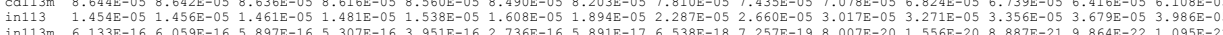

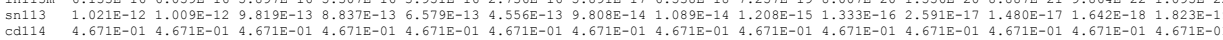

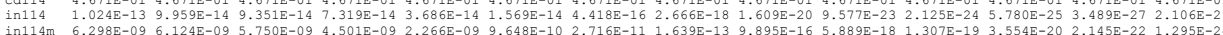

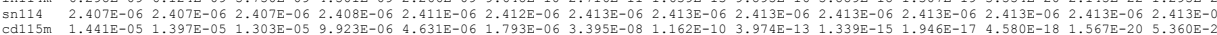

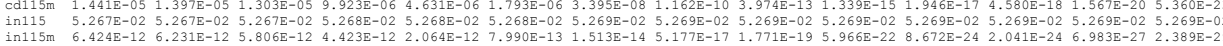

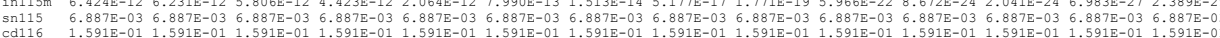

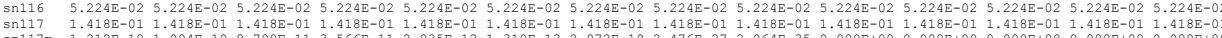

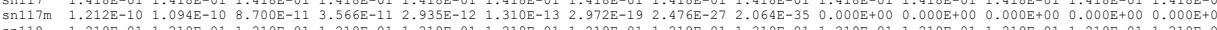

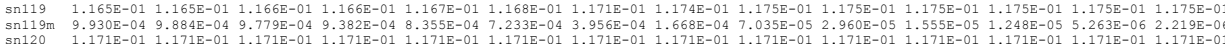

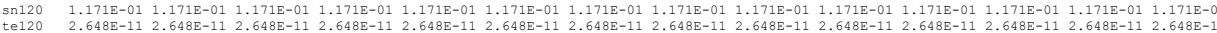

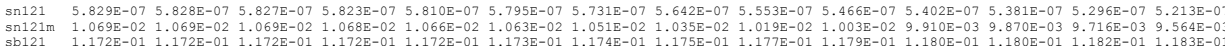

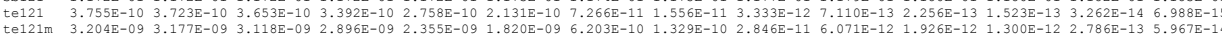

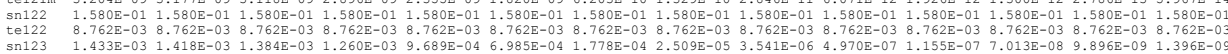

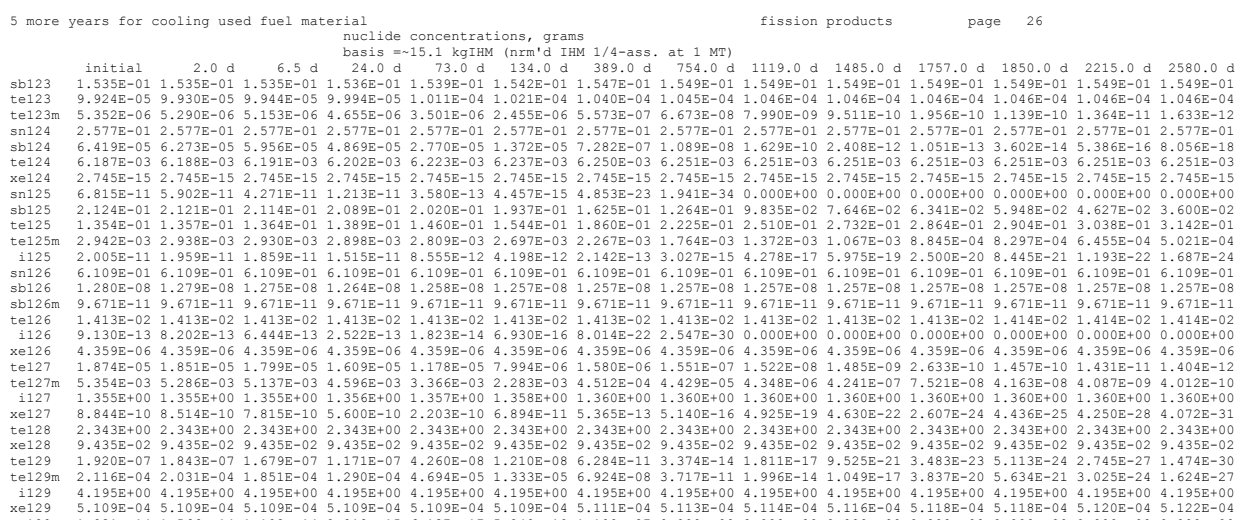

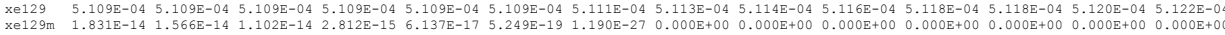

B-72 


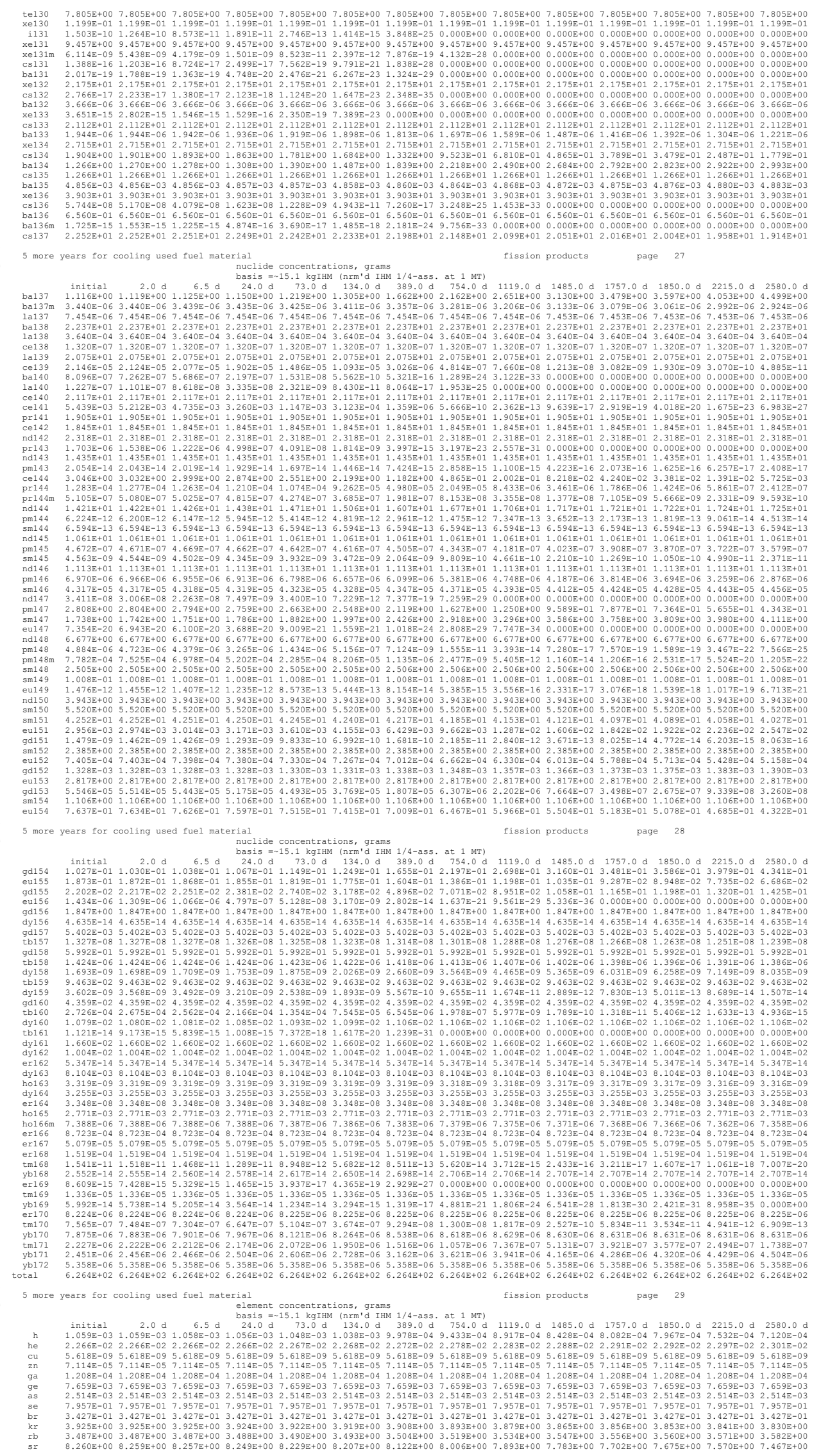

B-73 


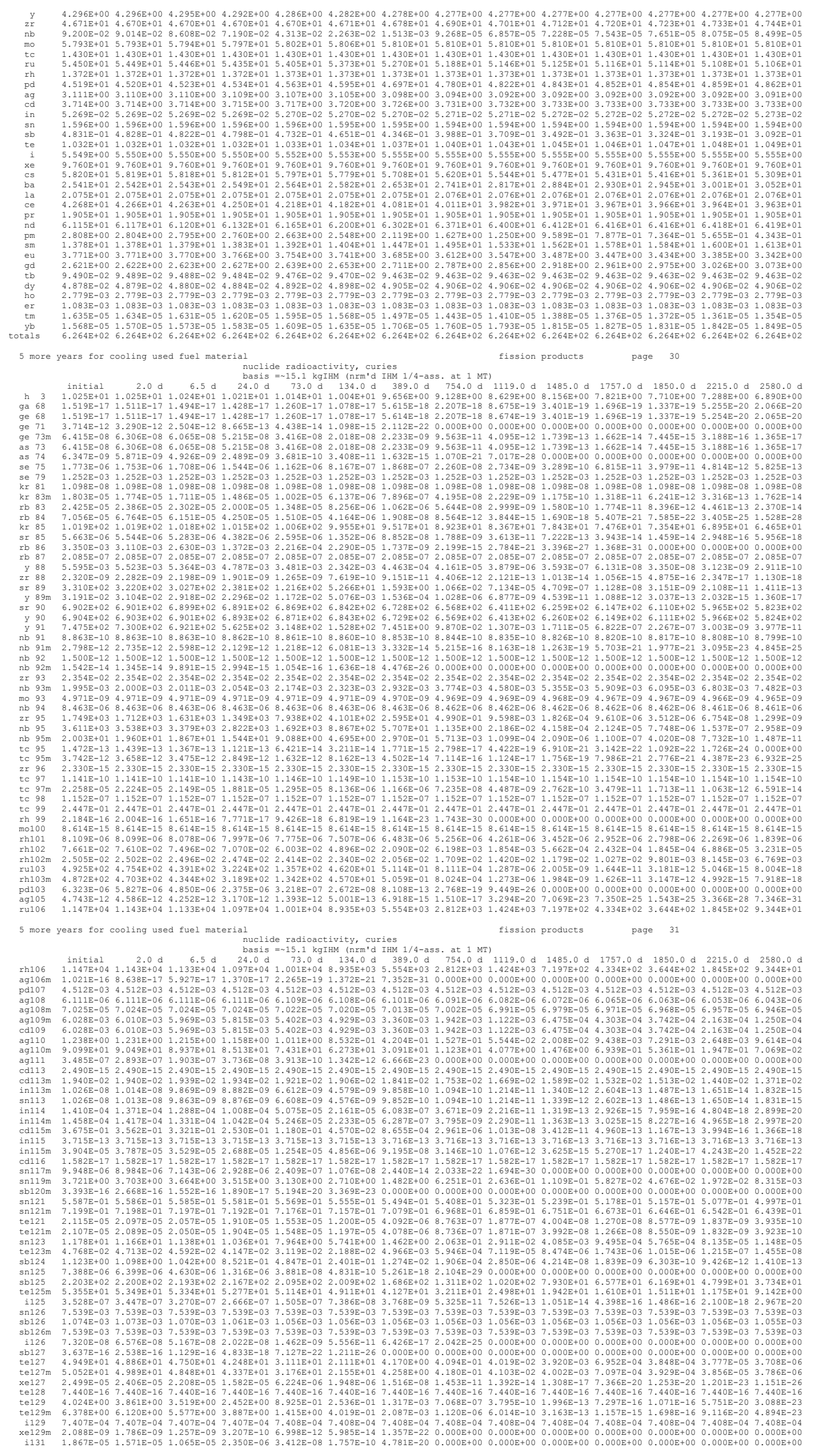

B-74 


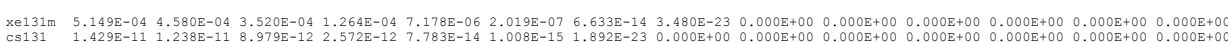

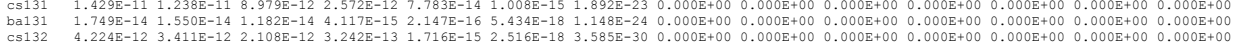
5 more years for cooling used fuel materia

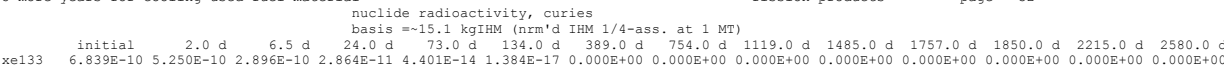
fission products page 32

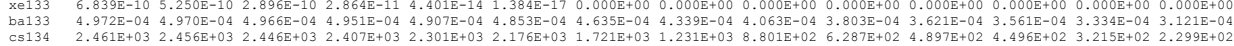
$\begin{array}{llllllllllllllll}\text { cs } 135 & 1.458 \mathrm{E}-02 & 1.458 \mathrm{E}-02 & 1.458 \mathrm{E}-02 & 1.458 \mathrm{E}-02 & 1.458 \mathrm{E}-02 & 1.458 \mathrm{E}-02 & 1.458 \mathrm{E}-02 & 1.458 \mathrm{E}-02 & 1.458 \mathrm{E}-02 & 1.458 \mathrm{E}-02 & 1.458 \mathrm{E}-02 & 1.458 \mathrm{E}-02 & 1.458 \mathrm{E}-02 & 1.458 \mathrm{E}-02 \\ \mathrm{cs} 136 & 4.193 \mathrm{E}-03 & 3.773 \mathrm{E}-03 & 2.977 \mathrm{E}-03 & 1.184 \mathrm{E}-03 & 8.967 \mathrm{E}-05 & 3.608 \mathrm{E}-06 & 5.299 \mathrm{E}-12 & 2.371 \mathrm{E}-20 & 1.061 \mathrm{E}-28 & 0.000 \mathrm{E}+00 & 0.000 \mathrm{E}+00 & 0.000 \mathrm{E}+00 & 0.000 \mathrm{E}+00 & 0.000 \mathrm{E}+0\end{array}$

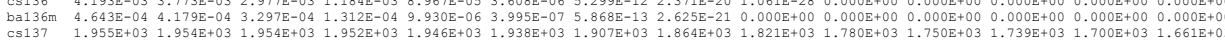

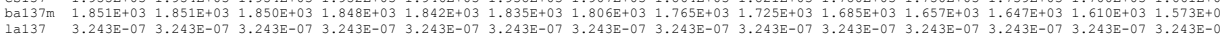

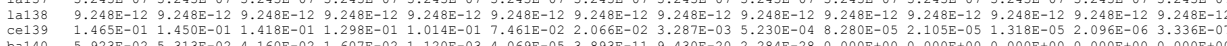

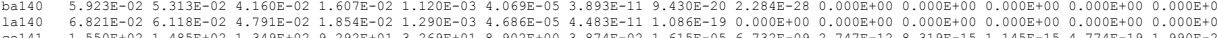

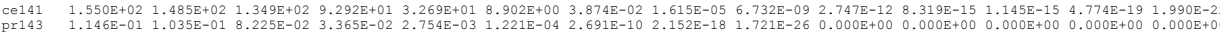

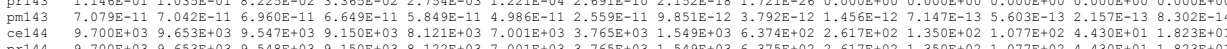

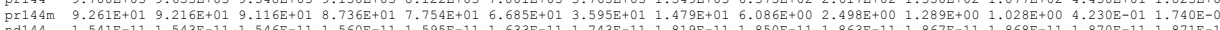

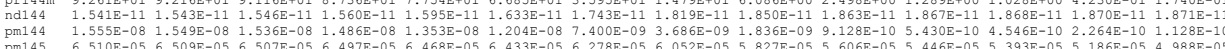

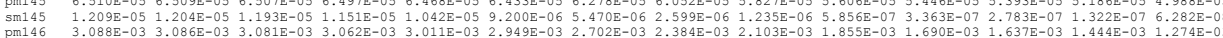

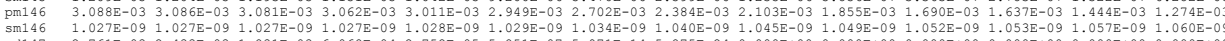

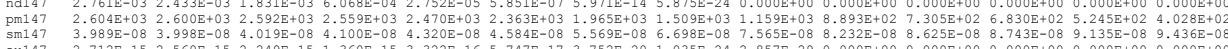

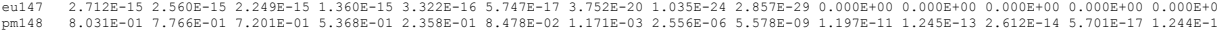

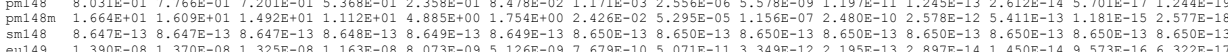

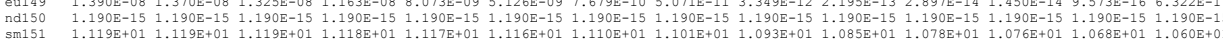
$\begin{array}{llllllllllllll}\begin{array}{l}\text { sm151 } \\ \text { gd151 }\end{array} 1.119 \mathrm{E}+01 & 1.119 \mathrm{E}+01 & 1.119 \mathrm{E}+01 & 1.118 \mathrm{E}+01 & 1.117 \mathrm{E}+01 & 1.116 \mathrm{E}+01 & 1.110 \mathrm{E}+01 & 1.101 \mathrm{E}+01 & 1.093 \mathrm{E}+01 & 1.085 \mathrm{E}+01 & 1.078 \mathrm{E}+01 & 1.076 \mathrm{E}+01 & 1.068 \mathrm{E}+01 & 1.060 \mathrm{E}+0 \\ 1.020 \mathrm{E}-05 & 9.949 \mathrm{E}-06 & 9.022 \mathrm{E}-06 & 6.860 \mathrm{E}-06 & 4.878 \mathrm{E}-06 & 1.173 \mathrm{E}-06 & 1.524 \mathrm{E}-07 & 1.981 \mathrm{E}-08 & 2.561 \mathrm{E}-09 & 5.599 \mathrm{E}-10 & 3.329 \mathrm{E}-10 & 4.327 \mathrm{E}-11 & 5.625 \mathrm{E}-1\end{array}$

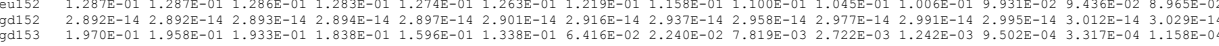

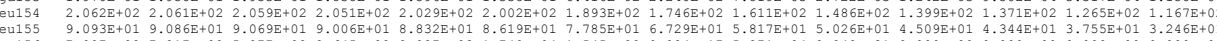

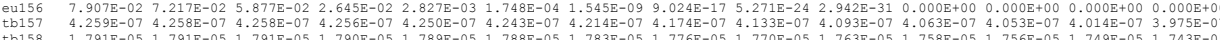

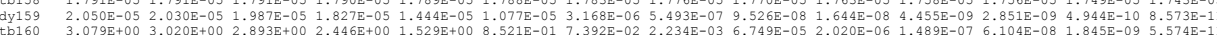

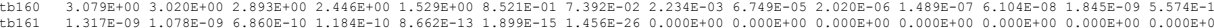

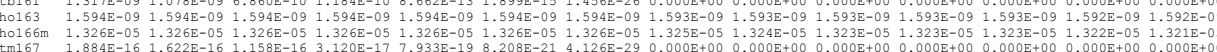

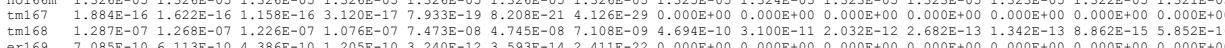
5 more years for cooling used fuet material
nuclide radioactivity, curies
basis $=\sim 15.1 \mathrm{~kg}$ HMM (nrm'd IHM $1 / 4$-ass. at 1 M 1 )
fission products page 33

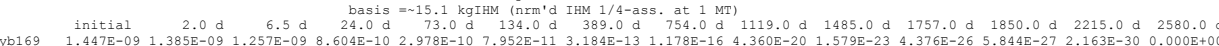

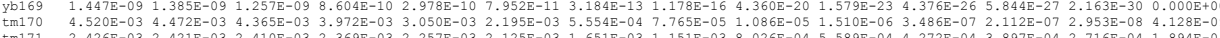

$\begin{array}{lllllllll} & \end{array}$

5 more years for cooling used fuel material element radioactivity, curies
basis $=\sim 15.1 \mathrm{kgIHM}$ (nrm'd IHM $1 / 4$-ass. at $1 \mathrm{MT}$ )

fission products page 34

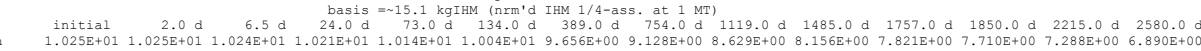

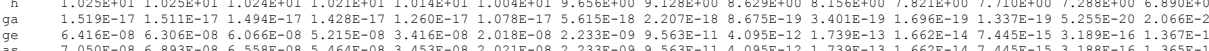

$\begin{array}{llllllllllllllll}\text { as } & 7.050 \mathrm{E}-08 & 6.893 \mathrm{E}-08 & 6.558 \mathrm{E}-08 & 5.465 \mathrm{E}-08 & 3.453 \mathrm{E}-08 & 2.021 \mathrm{E}-08 & 2.233 \mathrm{E}-09 & 9.563 \mathrm{E}-11 & 4.095 \mathrm{E}-12 & 1.739 \mathrm{E}-13 & 1.662 \mathrm{E}-14 & 7.445 \mathrm{E}-15 & 3.188 \mathrm{E}-16 & 1.365 \mathrm{E}-1\end{array}$

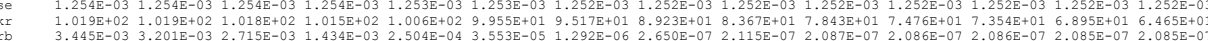

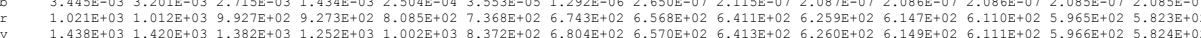

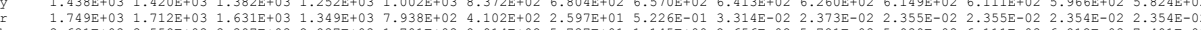

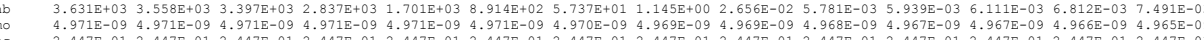

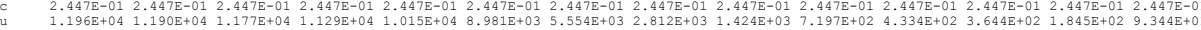

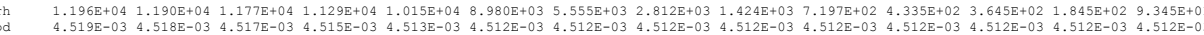

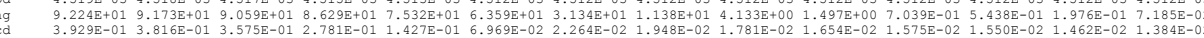

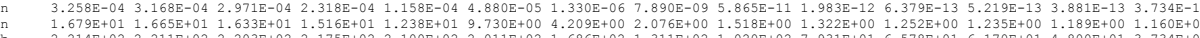

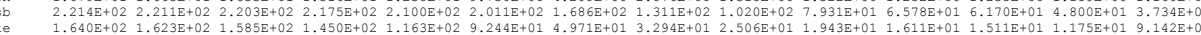

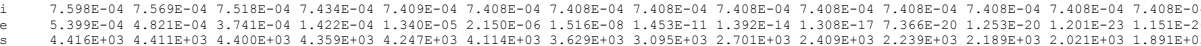

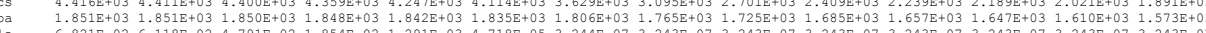

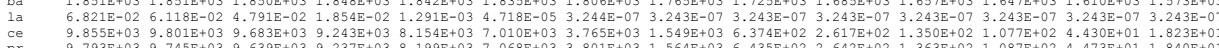

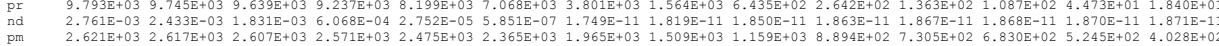

$\begin{array}{llllllllllllllll}\mathrm{pm} & 2.621 \mathrm{E}+03 & 2.617 \mathrm{E}+03 & 2.607 \mathrm{E}+03 & 2.571 \mathrm{E}+03 & 2.475 \mathrm{E}+03 & 2.365 \mathrm{E}+03 & 1.965 \mathrm{E}+03 & 1.509 \mathrm{E}+03 & 1.159 \mathrm{E}+03 & 8.894 \mathrm{E}+02 & 7.305 \mathrm{E}+02 & 6.830 \mathrm{E}+02 & 5.245 \mathrm{E}+02 & 4.028 \mathrm{E}+02 \\ \mathrm{sm} & 1.119 \mathrm{E}+01 & 1.119 \mathrm{E}+01 & 1.119 \mathrm{E}+01 & 1.118 \mathrm{E}+01 & 1.117 \mathrm{E}+01 & 1.116 \mathrm{E}+01 & 1.110 \mathrm{E}+01 & 1.101 \mathrm{E}+01 & 1.093 \mathrm{E}+01 & 1.085 \mathrm{E}+01 & 1.078 \mathrm{E}+01 & 1.076 \mathrm{E}+01 & 1.068 \mathrm{E}+01 & 1.060 \mathrm{E}+0\end{array}$

$\begin{array}{lllllllllllllll}\mathrm{sm} & 1.119 \mathrm{E}+01 & 1.972 \mathrm{E}+02 & 2.968 \mathrm{E}+02 & 2.954 \mathrm{E}+02 & 2.914 \mathrm{E}+02 & 2.865 \mathrm{E}+02 & 2.672 \mathrm{E}+02 & 2.420 \mathrm{E}+02 & 2.194 \mathrm{E}+02 & 1.990 \mathrm{E}+02 & 1.851 \mathrm{E}+02 & 1.006 \mathrm{E}+02 & 1.641 \mathrm{E}+02 & 1.493 \mathrm{E}+02\end{array}$

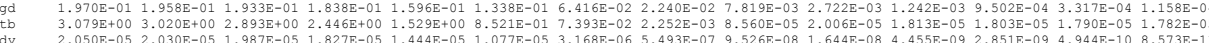

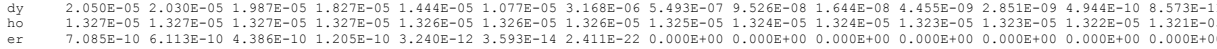

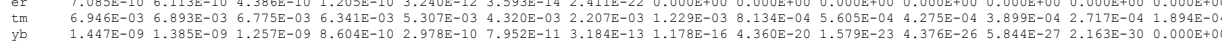

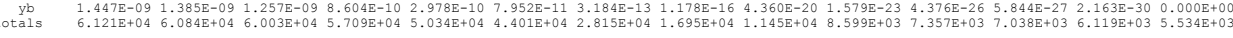

5 more years for cooling used fuel material

fission products

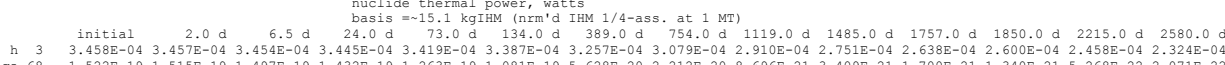

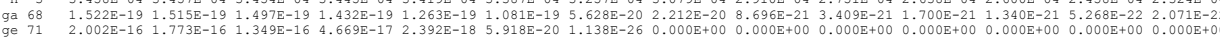

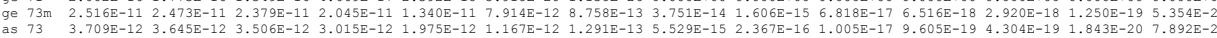

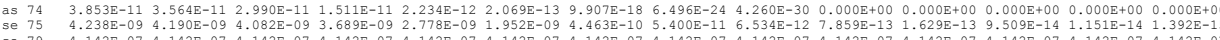

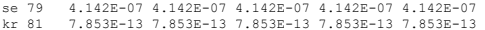

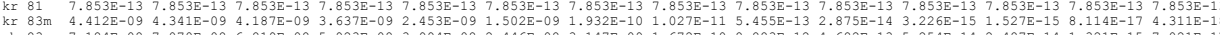

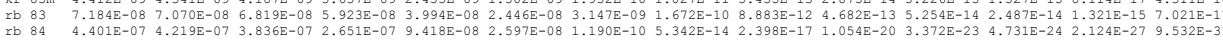

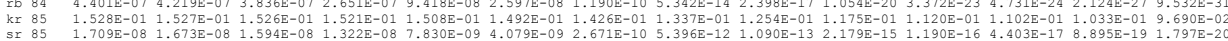

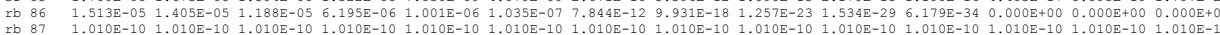

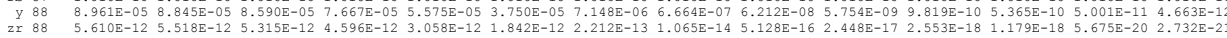

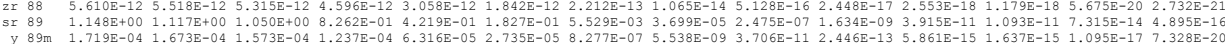

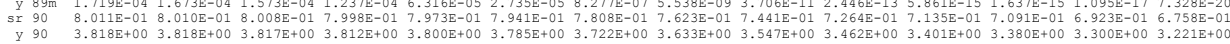

B-75 


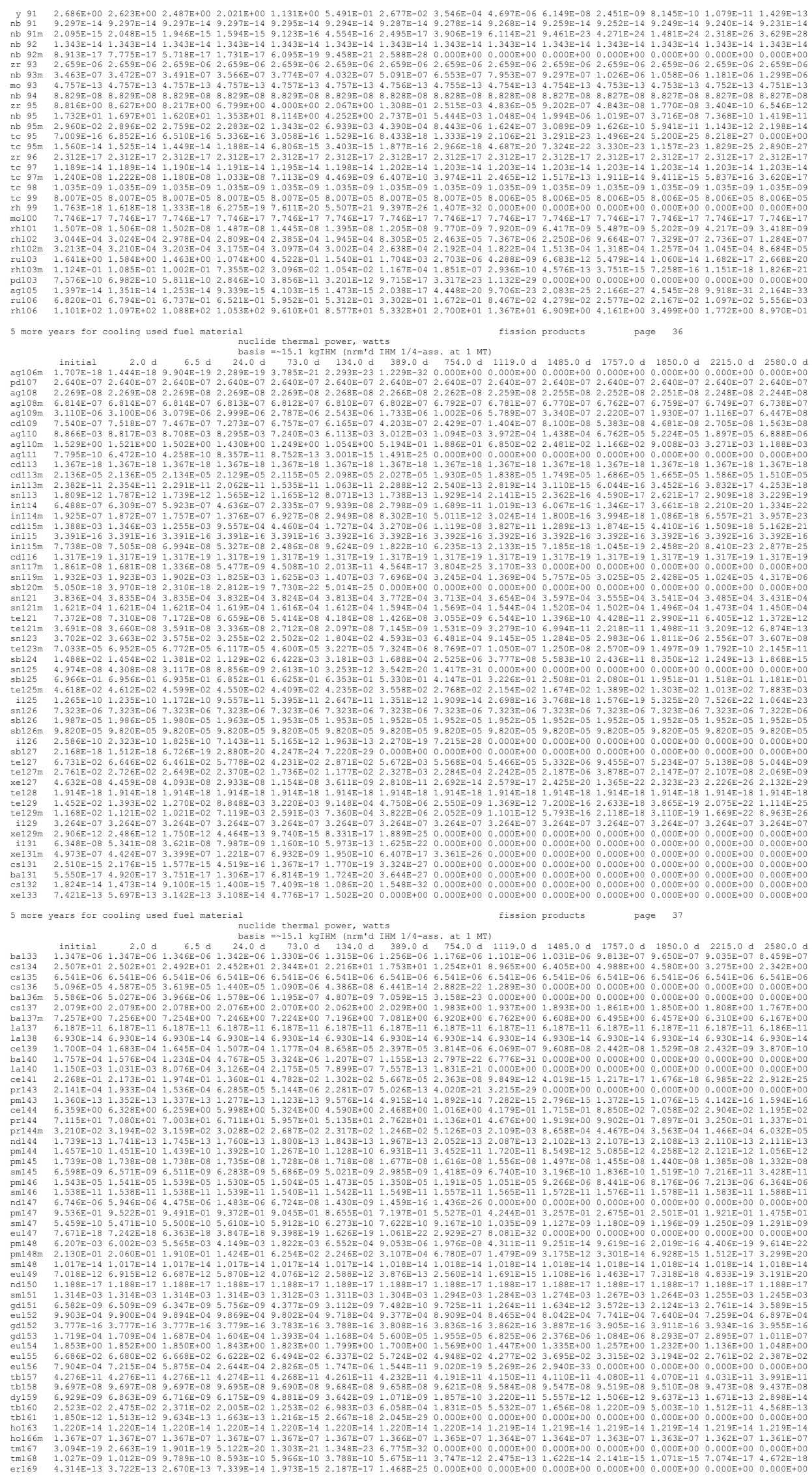

B-76 


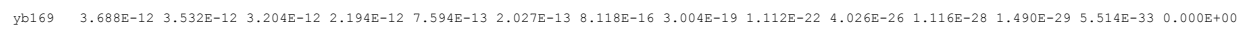
5 more years for cooling used fuel material

fission products page 38

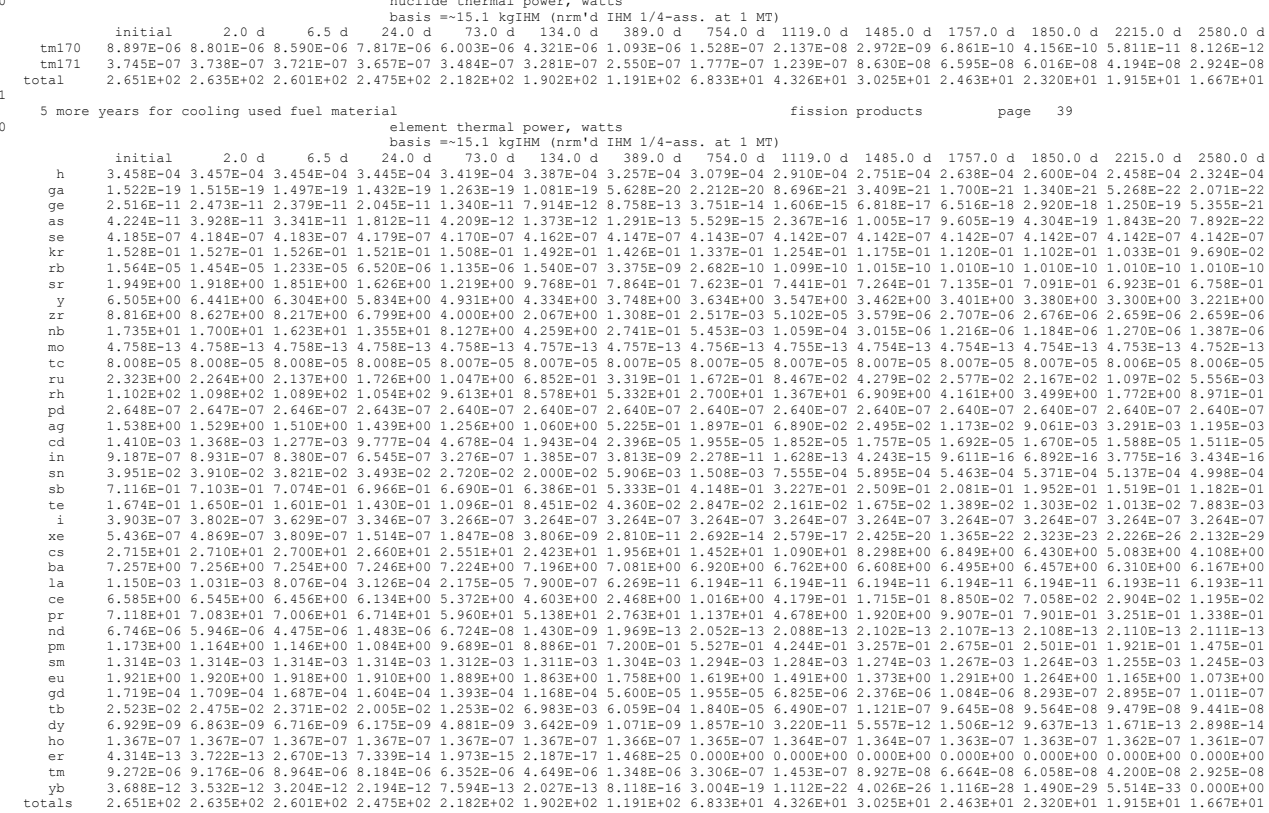

$56 \$$ array 20 entries read

1 * 0 normal termination of execution *

table of contents for material tables
case or subcase printed 



\section{APPENDIX C.}

\section{MOX FUEL ROD NUCLIDE WEIGHT FRACTIONS AND ACTIVITIES}





\section{APPENDIX C. MOX FUEL ROD NUCLIDE WEIGHT FRACTIONS AND ACTIVITIES}

Table C-1. MOX Fuel Rod B4 (high Pu): Nuclide mass in grams per MTIHM

\begin{tabular}{|c|c|c|c|c|c|}
\hline & $1 / 22 / 2009$ & $10 / 21 / 2013$ & $1 / 22 / 2014$ & $1 / 22 / 2015$ & $1 / 22 / 2016$ \\
\hline ac225 & $1.169 \mathrm{E}-12$ & $1.215 \mathrm{E}-12$ & $1.217 \mathrm{E}-12$ & $1.232 \mathrm{E}-12$ & $1.246 \mathrm{E}-12$ \\
\hline ac227 & 7.377E-10 & $3.125 \mathrm{E}-09$ & $3.255 \mathrm{E}-09$ & 3.769E-09 & 4.286E-09 \\
\hline $\operatorname{ag} 105$ & $9.491 \mathrm{E}-15$ & $2.200 \mathrm{E}-27$ & $4.618 \mathrm{E}-28$ & $1.008 \mathrm{E}-30$ & $2.199 \mathrm{E}-33$ \\
\hline ag107 & $1.543 \mathrm{E}-04$ & $4.958 \mathrm{E}-04$ & 5.142E-04 & 5.861E-04 & $6.581 \mathrm{E}-04$ \\
\hline $\operatorname{ag} 108$ & $7.206 \mathrm{E}-13$ & $7.153 \mathrm{E}-13$ & $7.153 \mathrm{E}-13$ & $7.140 \mathrm{E}-13$ & $7.126 \mathrm{E}-13$ \\
\hline ag108m & $8.011 \mathrm{E}-04$ & $7.952 \mathrm{E}-04$ & 7.952E-04 & 7.939E-04 & $7.926 \mathrm{E}-04$ \\
\hline $\operatorname{ag} 109$ & $2.314 \mathrm{E}+02$ & $2.314 \mathrm{E}+02$ & $2.314 \mathrm{E}+02$ & $2.314 \mathrm{E}+02$ & $2.314 \mathrm{E}+02$ \\
\hline $\operatorname{ag} 109 m$ & $2.195 \mathrm{E}-10$ & $1.624 \mathrm{E}-11$ & $1.412 \mathrm{E}-11$ & $8.163 \mathrm{E}-12$ & $4.716 \mathrm{E}-12$ \\
\hline $\operatorname{ag} 110$ & $2.327 \mathrm{E}-08$ & $1.897 \mathrm{E}-10$ & $1.466 \mathrm{E}-10$ & $5.321 \mathrm{E}-11$ & $1.933 \mathrm{E}-11$ \\
\hline $\operatorname{ag} 110 \mathrm{~m}$ & $1.501 \mathrm{E}+00$ & $1.224 \mathrm{E}-02$ & $9.451 \mathrm{E}-03$ & $3.432 \mathrm{E}-03$ & $1.246 \mathrm{E}-03$ \\
\hline ag111 & $1.708 \mathrm{E}-11$ & $0.000 \mathrm{E}+00$ & $0.000 \mathrm{E}+00$ & $0.000 \mathrm{E}+00$ & $0.000 \mathrm{E}+00$ \\
\hline al27 & $4.876 \mathrm{E}-14$ & $4.876 \mathrm{E}-14$ & $4.876 \mathrm{E}-14$ & $4.876 \mathrm{E}-14$ & $4.876 \mathrm{E}-14$ \\
\hline am241 & $4.308 \mathrm{E}+02$ & $1.495 \mathrm{E}+03$ & $1.545 \mathrm{E}+03$ & $1.736 \mathrm{E}+03$ & $1.917 \mathrm{E}+03$ \\
\hline am242 & 5.635E-05 & $5.505 \mathrm{E}-05$ & $5.498 \mathrm{E}-05$ & $5.471 \mathrm{E}-05$ & 5.444E-05 \\
\hline $\operatorname{am} 242 m$ & $4.368 \mathrm{E}+00$ & $4.267 \mathrm{E}+00$ & $4.261 \mathrm{E}+00$ & $4.241 \mathrm{E}+00$ & $4.220 \mathrm{E}+00$ \\
\hline $\operatorname{am} 243$ & $3.849 \mathrm{E}+02$ & $3.848 \mathrm{E}+02$ & $3.847 \mathrm{E}+02$ & $3.847 \mathrm{E}+02$ & $3.847 \mathrm{E}+02$ \\
\hline $\operatorname{am} 244$ & $2.919 \mathrm{E}-15$ & $2.024 \mathrm{E}-15$ & $1.985 \mathrm{E}-15$ & $1.838 \mathrm{E}-15$ & $1.702 \mathrm{E}-15$ \\
\hline am245 & $3.154 \mathrm{E}-14$ & $7.391 \mathrm{E}-16$ & $6.040 \mathrm{E}-16$ & $2.740 \mathrm{E}-16$ & $1.242 \mathrm{E}-16$ \\
\hline $\operatorname{ar} 36$ & $6.445 \mathrm{E}-14$ & $6.445 \mathrm{E}-14$ & $6.445 \mathrm{E}-14$ & $6.445 \mathrm{E}-14$ & $6.445 \mathrm{E}-14$ \\
\hline $\operatorname{ar} 38$ & $6.862 \mathrm{E}-14$ & $6.862 \mathrm{E}-14$ & $6.862 \mathrm{E}-14$ & $6.862 \mathrm{E}-14$ & $6.862 \mathrm{E}-14$ \\
\hline ar39 & $1.202 \mathrm{E}-16$ & $1.188 \mathrm{E}-16$ & $1.187 \mathrm{E}-16$ & $1.184 \mathrm{E}-16$ & $1.180 \mathrm{E}-16$ \\
\hline $\operatorname{ar} 40$ & $7.258 \mathrm{E}-14$ & $7.258 \mathrm{E}-14$ & $7.258 \mathrm{E}-14$ & $7.258 \mathrm{E}-14$ & $7.258 \mathrm{E}-14$ \\
\hline as73 & $1.565 \mathrm{E}-10$ & $4.988 \mathrm{E}-17$ & $2.235 \mathrm{E}-17$ & $9.570 \mathrm{E}-19$ & $4.098 \mathrm{E}-20$ \\
\hline as74 & $1.962 \mathrm{E}-12$ & $0.000 \mathrm{E}+00$ & $0.000 \mathrm{E}+00$ & $0.000 \mathrm{E}+00$ & $0.000 \mathrm{E}+00$ \\
\hline as75 & $1.898 \mathrm{E}-01$ & $1.898 \mathrm{E}-01$ & $1.898 \mathrm{E}-01$ & $1.898 \mathrm{E}-01$ & $1.898 \mathrm{E}-01$ \\
\hline au197 & $2.289 \mathrm{E}-13$ & $2.289 \mathrm{E}-13$ & $2.289 \mathrm{E}-13$ & $2.289 \mathrm{E}-13$ & $2.289 \mathrm{E}-13$ \\
\hline b10 & $6.123 \mathrm{E}-11$ & $3.283 \mathrm{E}-10$ & $3.426 \mathrm{E}-10$ & $3.989 \mathrm{E}-10$ & $4.551 \mathrm{E}-10$ \\
\hline b11 & $6.961 \mathrm{E}-08$ & $6.961 \mathrm{E}-08$ & $6.961 \mathrm{E}-08$ & $6.961 \mathrm{E}-08$ & $6.961 \mathrm{E}-08$ \\
\hline ba130 & $1.896 \mathrm{E}-13$ & $1.896 \mathrm{E}-13$ & $1.896 \mathrm{E}-13$ & $1.896 \mathrm{E}-13$ & $1.896 \mathrm{E}-13$ \\
\hline ba131 & 4.764E-18 & $0.000 \mathrm{E}+00$ & $0.000 \mathrm{E}+00$ & $0.000 \mathrm{E}+00$ & $0.000 \mathrm{E}+00$ \\
\hline ba132 & $3.311 \mathrm{E}-04$ & $3.311 \mathrm{E}-04$ & $3.311 \mathrm{E}-04$ & $3.311 \mathrm{E}-04$ & $3.311 \mathrm{E}-04$ \\
\hline ba133 & $2.104 \mathrm{E}-04$ & $1.539 \mathrm{E}-04$ & $1.513 \mathrm{E}-04$ & $1.417 \mathrm{E}-04$ & $1.326 \mathrm{E}-04$ \\
\hline ba134 & $1.188 \mathrm{E}+02$ & $2.470 \mathrm{E}+02$ & $2.497 \mathrm{E}+02$ & $2.582 \mathrm{E}+02$ & $2.644 \mathrm{E}+02$ \\
\hline ba135 & $5.269 \mathrm{E}-01$ & $5.280 \mathrm{E}-01$ & $5.281 \mathrm{E}-01$ & $5.284 \mathrm{E}-01$ & $5.286 \mathrm{E}-01$ \\
\hline ba136 & $5.189 \mathrm{E}+01$ & $5.189 \mathrm{E}+01$ & $5.189 \mathrm{E}+01$ & $5.189 \mathrm{E}+01$ & $5.189 \mathrm{E}+01$ \\
\hline ba136m & $3.638 \mathrm{E}-14$ & $0.000 \mathrm{E}+00$ & $0.000 \mathrm{E}+00$ & $0.000 \mathrm{E}+00$ & $0.000 \mathrm{E}+00$ \\
\hline ba137 & $9.128 \mathrm{E}+01$ & $2.705 \mathrm{E}+02$ & $2.795 \mathrm{E}+02$ & $3.146 \mathrm{E}+02$ & $3.489 \mathrm{E}+02$ \\
\hline
\end{tabular}




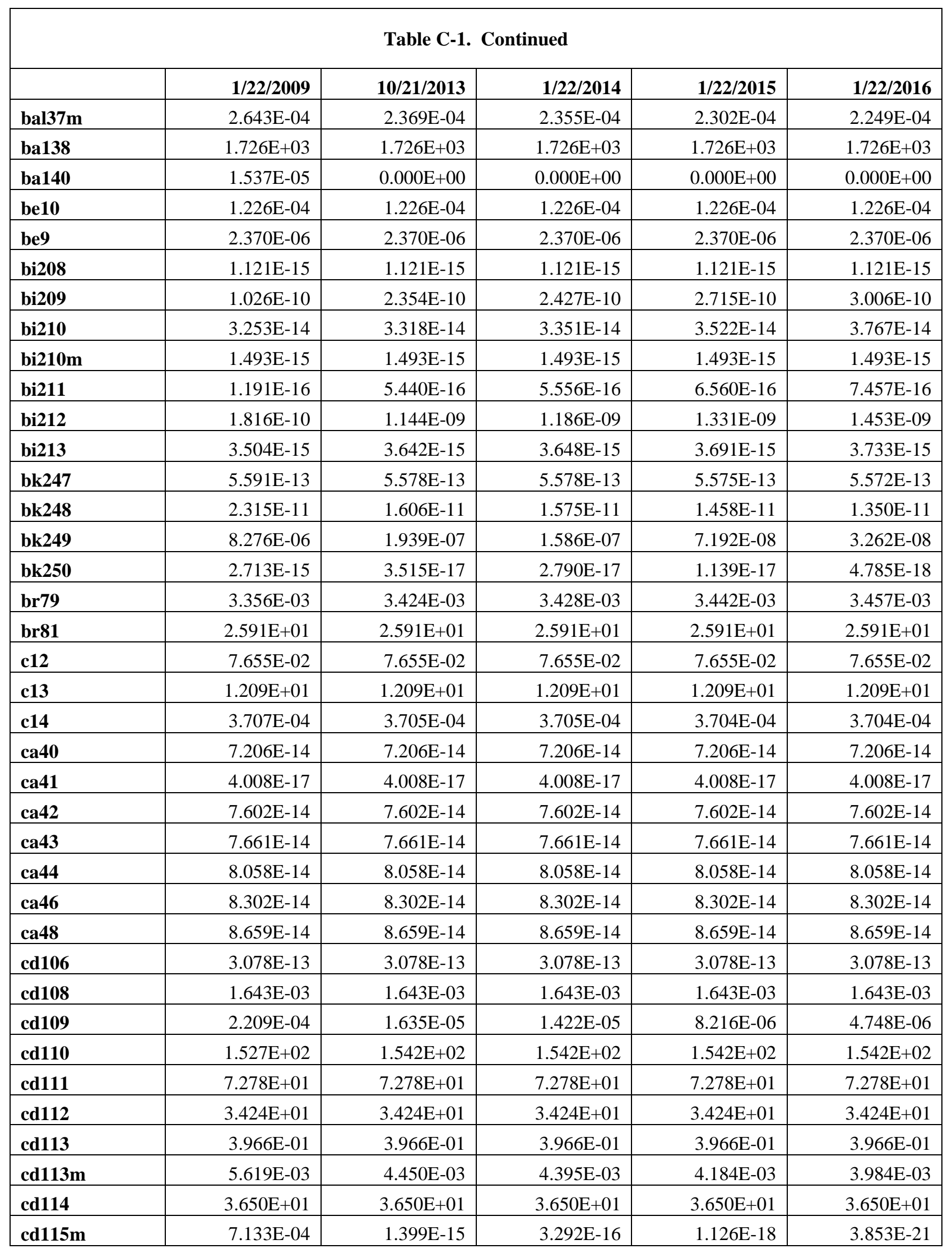




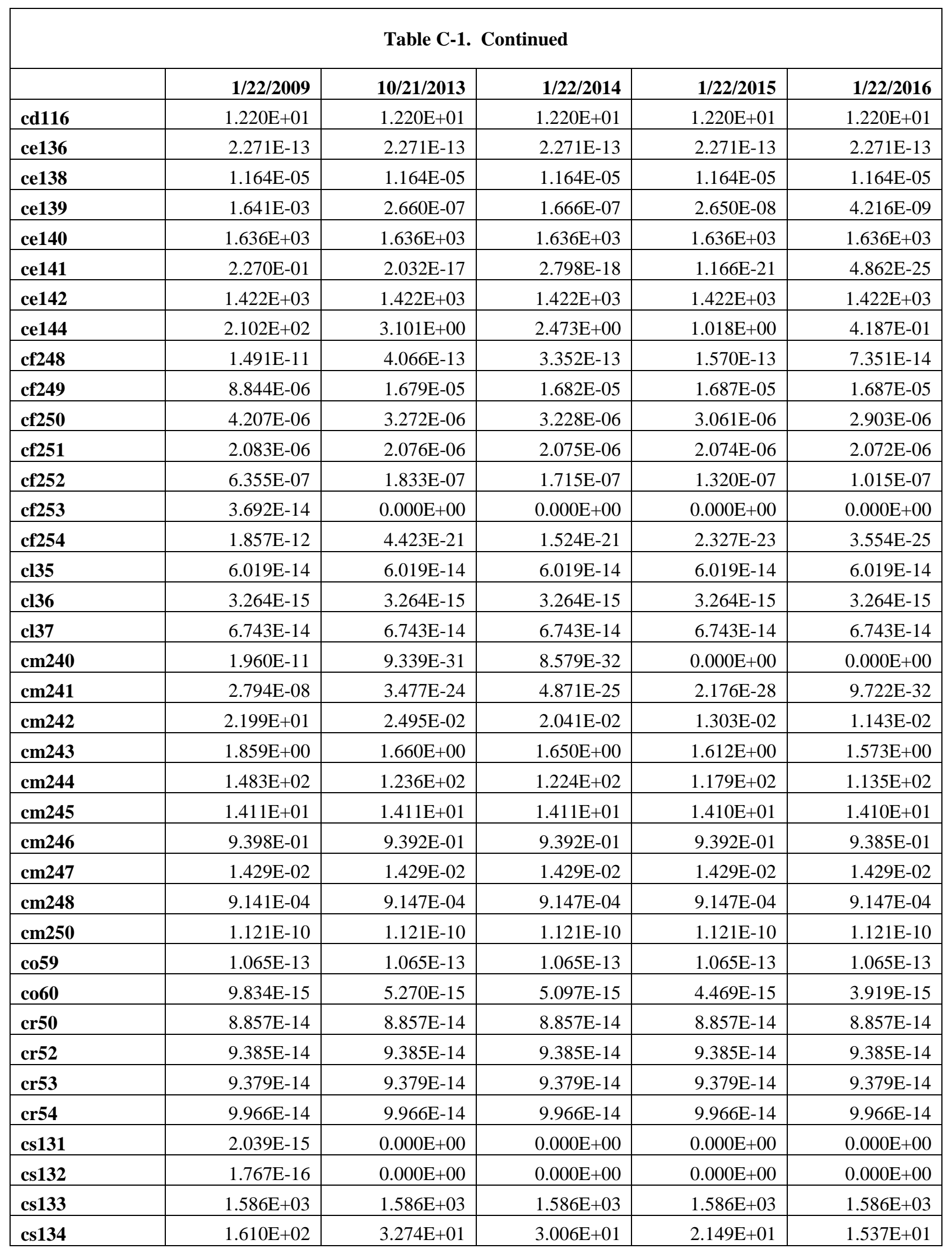




\begin{tabular}{|c|c|c|c|c|c|}
\hline \multicolumn{6}{|c|}{ Table C-1. Continued } \\
\hline & $1 / 22 / 2009$ & $10 / 21 / 2013$ & $1 / 22 / 2014$ & $1 / 22 / 2015$ & $1 / 22 / 2016$ \\
\hline $\operatorname{cs} 135$ & $8.758 \mathrm{E}+02$ & $8.758 \mathrm{E}+02$ & $8.758 \mathrm{E}+02$ & $8.758 \mathrm{E}+02$ & $8.758 \mathrm{E}+02$ \\
\hline $\operatorname{cs} 136$ & $1.211 \mathrm{E}-06$ & $0.000 \mathrm{E}+00$ & $0.000 \mathrm{E}+00$ & $0.000 \mathrm{E}+00$ & $0.000 \mathrm{E}+00$ \\
\hline $\operatorname{cs} 137$ & $1.730 \mathrm{E}+03$ & $1.551 \mathrm{E}+03$ & $1.542 \mathrm{E}+03$ & $1.507 \mathrm{E}+03$ & $1.472 \mathrm{E}+03$ \\
\hline cu63 & $1.127 \mathrm{E}-13$ & $1.128 \mathrm{E}-13$ & $1.128 \mathrm{E}-13$ & $1.128 \mathrm{E}-13$ & $1.128 \mathrm{E}-13$ \\
\hline cu65 & 4.401E-07 & 4.401E-07 & 4.401E-07 & 4.401E-07 & 4.401E-07 \\
\hline dy156 & $4.008 \mathrm{E}-12$ & $4.008 \mathrm{E}-12$ & $4.008 \mathrm{E}-12$ & $4.008 \mathrm{E}-12$ & $4.008 \mathrm{E}-12$ \\
\hline dy158 & $1.499 \mathrm{E}-07$ & $5.023 \mathrm{E}-07$ & $5.210 \mathrm{E}-07$ & $5.944 \mathrm{E}-07$ & $6.677 \mathrm{E}-07$ \\
\hline dy159 & $2.944 \mathrm{E}-07$ & $7.179 \mathrm{E}-11$ & $4.595 \mathrm{E}-11$ & 7.972E-12 & $1.382 \mathrm{E}-12$ \\
\hline dy160 & $9.623 \mathrm{E}-01$ & $9.808 \mathrm{E}-01$ & $9.808 \mathrm{E}-01$ & $9.808 \mathrm{E}-01$ & $9.808 \mathrm{E}-01$ \\
\hline dy161 & $1.300 \mathrm{E}+00$ & $1.300 \mathrm{E}+00$ & $1.300 \mathrm{E}+00$ & $1.300 \mathrm{E}+00$ & $1.300 \mathrm{E}+00$ \\
\hline dy162 & 7.992E-01 & 7.992E-01 & $7.992 \mathrm{E}-01$ & 7.992E-01 & $7.992 \mathrm{E}-01$ \\
\hline dy163 & $6.657 \mathrm{E}-01$ & $6.657 \mathrm{E}-01$ & $6.657 \mathrm{E}-01$ & $6.657 \mathrm{E}-01$ & $6.657 \mathrm{E}-01$ \\
\hline dy164 & $2.703 \mathrm{E}-01$ & 2.703E-01 & $2.703 \mathrm{E}-01$ & $2.703 \mathrm{E}-01$ & $2.703 \mathrm{E}-01$ \\
\hline er162 & $4.631 \mathrm{E}-12$ & 4.631E-12 & $4.631 \mathrm{E}-12$ & $4.631 \mathrm{E}-12$ & $4.631 \mathrm{E}-12$ \\
\hline er164 & $3.222 \mathrm{E}-06$ & $3.222 \mathrm{E}-06$ & $3.222 \mathrm{E}-06$ & $3.222 \mathrm{E}-06$ & $3.222 \mathrm{E}-06$ \\
\hline er166 & $8.414 \mathrm{E}-02$ & $8.414 \mathrm{E}-02$ & $8.414 \mathrm{E}-02$ & $8.414 \mathrm{E}-02$ & $8.414 \mathrm{E}-02$ \\
\hline er167 & $4.228 \mathrm{E}-03$ & $4.228 \mathrm{E}-03$ & $4.228 \mathrm{E}-03$ & $4.228 \mathrm{E}-03$ & $4.228 \mathrm{E}-03$ \\
\hline er168 & $1.329 \mathrm{E}-02$ & $1.329 \mathrm{E}-02$ & $1.329 \mathrm{E}-02$ & $1.329 \mathrm{E}-02$ & $1.329 \mathrm{E}-02$ \\
\hline er169 & $1.172 \mathrm{E}-13$ & $0.000 \mathrm{E}+00$ & $0.000 \mathrm{E}+00$ & $0.000 \mathrm{E}+00$ & $0.000 \mathrm{E}+00$ \\
\hline er170 & $6.564 \mathrm{E}-04$ & $6.564 \mathrm{E}-04$ & $6.564 \mathrm{E}-04$ & $6.564 \mathrm{E}-04$ & $6.564 \mathrm{E}-04$ \\
\hline es252 & $1.594 \mathrm{E}-15$ & $1.248 \mathrm{E}-16$ & $1.089 \mathrm{E}-16$ & $6.370 \mathrm{E}-17$ & $3.726 \mathrm{E}-17$ \\
\hline es 253 & $8.883 \mathrm{E}-13$ & $0.000 \mathrm{E}+00$ & $0.000 \mathrm{E}+00$ & $0.000 \mathrm{E}+00$ & $0.000 \mathrm{E}+00$ \\
\hline es254 & $5.673 \mathrm{E}-12$ & 7.272E-14 & $5.755 \mathrm{E}-14$ & $2.299 \mathrm{E}-14$ & $9.180 \mathrm{E}-15$ \\
\hline es255 & $9.266 \mathrm{E}-15$ & 7.232E-28 & $1.431 \mathrm{E}-28$ & $2.483 \mathrm{E}-31$ & $4.309 \mathrm{E}-34$ \\
\hline eu147 & $3.275 \mathrm{E}-18$ & $0.000 \mathrm{E}+00$ & $0.000 \mathrm{E}+00$ & $0.000 \mathrm{E}+00$ & $0.000 \mathrm{E}+00$ \\
\hline eu149 & $7.932 \mathrm{E}-11$ & $1.976 \mathrm{E}-16$ & $9.887 \mathrm{E}-17$ & $6.530 \mathrm{E}-18$ & $4.312 \mathrm{E}-19$ \\
\hline eu151 & $2.024 \mathrm{E}-01$ & $1.209 \mathrm{E}+00$ & $1.261 \mathrm{E}+00$ & $1.468 \mathrm{E}+00$ & $1.674 \mathrm{E}+00$ \\
\hline eu152 & 4.193E-02 & $3.288 \mathrm{E}-02$ & $3.246 \mathrm{E}-02$ & $3.084 \mathrm{E}-02$ & $2.930 \mathrm{E}-02$ \\
\hline eu153 & $2.180 \mathrm{E}+02$ & $2.180 \mathrm{E}+02$ & $2.180 \mathrm{E}+02$ & $2.180 \mathrm{E}+02$ & $2.180 \mathrm{E}+02$ \\
\hline eu154 & $6.265 \mathrm{E}+01$ & $4.275 \mathrm{E}+01$ & $4.187 \mathrm{E}+01$ & $3.864 \mathrm{E}+01$ & $3.565 \mathrm{E}+01$ \\
\hline eu155 & $1.520 \mathrm{E}+01$ & $7.609 \mathrm{E}+00$ & $7.331 \mathrm{E}+00$ & $6.335 \mathrm{E}+00$ & $5.476 \mathrm{E}+00$ \\
\hline eu156 & 4.232E-05 & $0.000 \mathrm{E}+00$ & $0.000 \mathrm{E}+00$ & $0.000 \mathrm{E}+00$ & $0.000 \mathrm{E}+00$ \\
\hline f19 & $9.167 \mathrm{E}-14$ & $9.167 \mathrm{E}-14$ & $9.167 \mathrm{E}-14$ & $9.167 \mathrm{E}-14$ & $9.167 \mathrm{E}-14$ \\
\hline fe54 & $9.715 \mathrm{E}-14$ & $9.715 \mathrm{E}-14$ & $9.715 \mathrm{E}-14$ & $9.715 \mathrm{E}-14$ & $9.715 \mathrm{E}-14$ \\
\hline fe55 & $1.854 \mathrm{E}-16$ & $5.592 \mathrm{E}-17$ & $5.244 \mathrm{E}-17$ & $4.074 \mathrm{E}-17$ & $3.165 \mathrm{E}-17$ \\
\hline fe56 & $1.061 \mathrm{E}-13$ & $1.061 \mathrm{E}-13$ & $1.061 \mathrm{E}-13$ & $1.061 \mathrm{E}-13$ & $1.061 \mathrm{E}-13$ \\
\hline fe57 & $1.030 \mathrm{E}-13$ & $1.030 \mathrm{E}-13$ & $1.030 \mathrm{E}-13$ & $1.030 \mathrm{E}-13$ & $1.030 \mathrm{E}-13$ \\
\hline fe58 & $3.029 \mathrm{E}-13$ & $3.029 \mathrm{E}-13$ & $3.029 \mathrm{E}-13$ & $3.029 \mathrm{E}-13$ & $3.029 \mathrm{E}-13$ \\
\hline
\end{tabular}




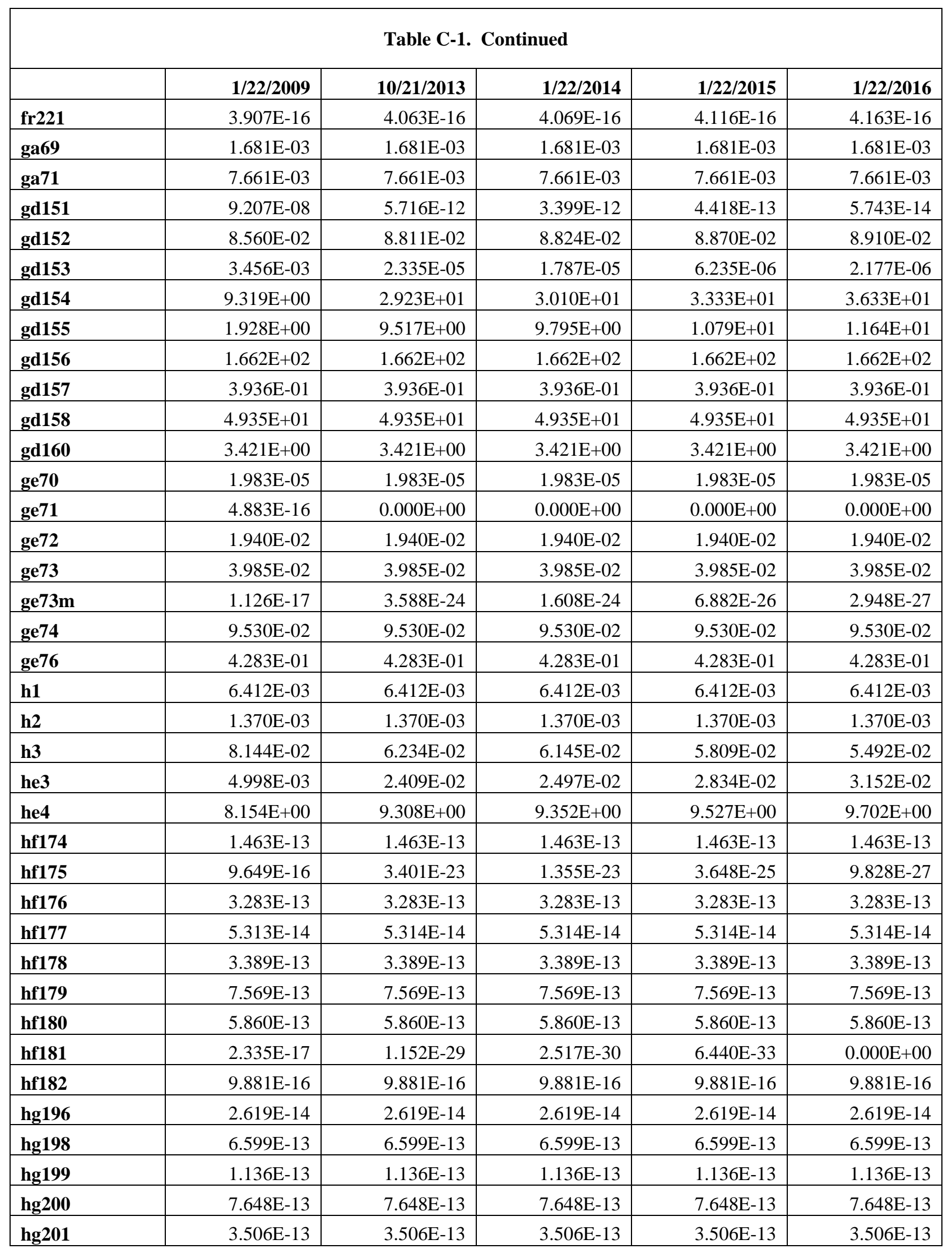




\begin{tabular}{|c|c|c|c|c|c|}
\hline \multicolumn{6}{|c|}{ Table C-1. Continued } \\
\hline & $1 / 22 / 2009$ & $10 / 21 / 2013$ & $1 / 22 / 2014$ & $1 / 22 / 2015$ & $1 / 22 / 2016$ \\
\hline hg202 & $3.775 \mathrm{E}-13$ & $3.775 \mathrm{E}-13$ & $3.775 \mathrm{E}-13$ & $3.775 \mathrm{E}-13$ & $3.775 \mathrm{E}-13$ \\
\hline hg204 & $3.672 \mathrm{E}-13$ & $3.672 \mathrm{E}-13$ & $3.672 \mathrm{E}-13$ & $3.672 \mathrm{E}-13$ & $3.672 \mathrm{E}-13$ \\
\hline ho163 & $2.459 \mathrm{E}-07$ & $2.457 \mathrm{E}-07$ & $2.457 \mathrm{E}-07$ & $2.456 \mathrm{E}-07$ & $2.456 \mathrm{E}-07$ \\
\hline ho165 & $2.633 \mathrm{E}-01$ & $2.633 \mathrm{E}-01$ & 2.633E-01 & $2.633 \mathrm{E}-01$ & $2.633 \mathrm{E}-01$ \\
\hline ho166m & $6.869 \mathrm{E}-04$ & $6.849 \mathrm{E}-04$ & $6.849 \mathrm{E}-04$ & $6.849 \mathrm{E}-04$ & $6.842 \mathrm{E}-04$ \\
\hline i125 & $1.174 \mathrm{E}-09$ & $1.936 \mathrm{E}-18$ & $6.541 \mathrm{E}-19$ & $9.246 \mathrm{E}-21$ & $1.306 \mathrm{E}-22$ \\
\hline i126 & $2.019 \mathrm{E}-11$ & $0.000 \mathrm{E}+00$ & $0.000 \mathrm{E}+00$ & $0.000 \mathrm{E}+00$ & $0.000 \mathrm{E}+00$ \\
\hline i127 & $1.012 \mathrm{E}+02$ & $1.015 \mathrm{E}+02$ & $1.015 \mathrm{E}+02$ & $1.015 \mathrm{E}+02$ & $1.015 \mathrm{E}+02$ \\
\hline i129 & $3.185 \mathrm{E}+02$ & $3.185 \mathrm{E}+02$ & $3.185 \mathrm{E}+02$ & $3.185 \mathrm{E}+02$ & $3.185 \mathrm{E}+02$ \\
\hline i131 & $1.310 \mathrm{E}-09$ & $0.000 \mathrm{E}+00$ & $0.000 \mathrm{E}+00$ & $0.000 \mathrm{E}+00$ & $0.000 \mathrm{E}+00$ \\
\hline in113 & $9.550 \mathrm{E}-04$ & $2.123 \mathrm{E}-03$ & $2.178 \mathrm{E}-03$ & $2.388 \mathrm{E}-03$ & $2.588 \mathrm{E}-03$ \\
\hline in113m & $4.585 \mathrm{E}-14$ & $1.344 \mathrm{E}-18$ & $7.675 \mathrm{E}-19$ & $8.520 \mathrm{E}-20$ & $9.458 \mathrm{E}-21$ \\
\hline in114 & $5.218 \mathrm{E}-12$ & $1.515 \mathrm{E}-22$ & $4.121 \mathrm{E}-23$ & $2.487 \mathrm{E}-25$ & $1.501 \mathrm{E}-27$ \\
\hline in114m & $3.209 \mathrm{E}-07$ & $9.313 \mathrm{E}-18$ & $2.534 \mathrm{E}-18$ & $1.529 \mathrm{E}-20$ & $9.227 \mathrm{E}-23$ \\
\hline in115 & $3.576 \mathrm{E}+00$ & $3.577 \mathrm{E}+00$ & $3.577 \mathrm{E}+00$ & $3.577 \mathrm{E}+00$ & $3.577 \mathrm{E}+00$ \\
\hline in115m & $3.179 \mathrm{E}-10$ & $6.233 \mathrm{E}-22$ & $1.467 \mathrm{E}-22$ & $5.020 \mathrm{E}-25$ & $1.717 \mathrm{E}-27$ \\
\hline ir191 & $1.088 \mathrm{E}-14$ & $1.088 \mathrm{E}-14$ & $1.088 \mathrm{E}-14$ & $1.088 \mathrm{E}-14$ & $1.088 \mathrm{E}-14$ \\
\hline ir192 & $2.325 \mathrm{E}-16$ & $1.996 \mathrm{E}-23$ & $8.335 \mathrm{E}-24$ & $2.708 \mathrm{E}-25$ & $8.797 \mathrm{E}-27$ \\
\hline ir193 & $1.761 \mathrm{E}-13$ & $1.773 \mathrm{E}-13$ & $1.774 \mathrm{E}-13$ & $1.777 \mathrm{E}-13$ & $1.779 \mathrm{E}-13$ \\
\hline k39 & 7.014E-14 & $7.014 \mathrm{E}-14$ & 7.014E-14 & $7.014 \mathrm{E}-14$ & 7.014E-14 \\
\hline $\mathbf{k 4 0}$ & $6.955 \mathrm{E}-14$ & $6.955 \mathrm{E}-14$ & $6.955 \mathrm{E}-14$ & $6.955 \mathrm{E}-14$ & $6.955 \mathrm{E}-14$ \\
\hline k41 & 7.642E-14 & 7.642E-14 & 7.642E-14 & 7.642E-14 & 7.642E-14 \\
\hline kr78 & $2.022 \mathrm{E}-13$ & $2.022 \mathrm{E}-13$ & $2.022 \mathrm{E}-13$ & $2.022 \mathrm{E}-13$ & $2.022 \mathrm{E}-13$ \\
\hline kr80 & $5.531 \mathrm{E}-04$ & $5.531 \mathrm{E}-04$ & $5.531 \mathrm{E}-04$ & $5.531 \mathrm{E}-04$ & $5.531 \mathrm{E}-04$ \\
\hline kr81 & 4.748E-05 & $4.748 \mathrm{E}-05$ & 4.748E-05 & $4.748 \mathrm{E}-05$ & 4.748E-05 \\
\hline kr82 & $1.021 \mathrm{E}+00$ & $1.021 \mathrm{E}+00$ & $1.021 \mathrm{E}+00$ & $1.021 \mathrm{E}+00$ & $1.021 \mathrm{E}+00$ \\
\hline kr83 & $4.230 \mathrm{E}+01$ & $4.230 \mathrm{E}+01$ & $4.230 \mathrm{E}+01$ & $4.230 \mathrm{E}+01$ & $4.230 \mathrm{E}+01$ \\
\hline kr83m & $4.786 \mathrm{E}-11$ & $4.245 \mathrm{E}-17$ & $2.010 \mathrm{E}-17$ & $1.068 \mathrm{E}-18$ & $5.673 \mathrm{E}-20$ \\
\hline kr84 & $9.550 \mathrm{E}+01$ & $9.550 \mathrm{E}+01$ & $9.550 \mathrm{E}+01$ & $9.550 \mathrm{E}+01$ & $9.550 \mathrm{E}+01$ \\
\hline kr85 & $1.977 \mathrm{E}+01$ & $1.456 \mathrm{E}+01$ & $1.433 \mathrm{E}+01$ & $1.343 \mathrm{E}+01$ & $1.260 \mathrm{E}+01$ \\
\hline kr86 & $1.426 \mathrm{E}+02$ & $1.426 \mathrm{E}+02$ & $1.426 \mathrm{E}+02$ & $1.426 \mathrm{E}+02$ & $1.426 \mathrm{E}+02$ \\
\hline la137 & $5.529 \mathrm{E}-04$ & $5.529 \mathrm{E}-04$ & 5.529E-04 & $5.529 \mathrm{E}-04$ & $5.529 \mathrm{E}-04$ \\
\hline la138 & $3.028 \mathrm{E}-02$ & $3.028 \mathrm{E}-02$ & $3.028 \mathrm{E}-02$ & $3.028 \mathrm{E}-02$ & $3.028 \mathrm{E}-02$ \\
\hline la139 & $1.599 \mathrm{E}+03$ & $1.599 \mathrm{E}+03$ & $1.599 \mathrm{E}+03$ & $1.599 \mathrm{E}+03$ & $1.599 \mathrm{E}+03$ \\
\hline la140 & $2.334 \mathrm{E}-06$ & $0.000 \mathrm{E}+00$ & $0.000 \mathrm{E}+00$ & $0.000 \mathrm{E}+00$ & $0.000 \mathrm{E}+00$ \\
\hline li6 & $2.547 \mathrm{E}-10$ & $2.547 \mathrm{E}-10$ & $2.547 \mathrm{E}-10$ & $2.547 \mathrm{E}-10$ & $2.547 \mathrm{E}-10$ \\
\hline li7 & $2.423 \mathrm{E}-11$ & $2.423 \mathrm{E}-11$ & $2.423 \mathrm{E}-11$ & $2.423 \mathrm{E}-11$ & $2.423 \mathrm{E}-11$ \\
\hline lu175 & $2.967 \mathrm{E}-13$ & $2.977 \mathrm{E}-13$ & $2.977 \mathrm{E}-13$ & $2.977 \mathrm{E}-13$ & $2.977 \mathrm{E}-13$ \\
\hline
\end{tabular}




\begin{tabular}{|c|c|c|c|c|c|}
\hline \multicolumn{6}{|c|}{ Table C-1. Continued } \\
\hline & $1 / 22 / 2009$ & $10 / 21 / 2013$ & $1 / 22 / 2014$ & $1 / 22 / 2015$ & $1 / 22 / 2016$ \\
\hline lu176 & $7.272 \mathrm{E}-15$ & $7.272 \mathrm{E}-15$ & $7.272 \mathrm{E}-15$ & $7.272 \mathrm{E}-15$ & $7.272 \mathrm{E}-15$ \\
\hline mg24 & $4.338 \mathrm{E}-14$ & $4.338 \mathrm{E}-14$ & $4.338 \mathrm{E}-14$ & $4.338 \mathrm{E}-14$ & $4.338 \mathrm{E}-14$ \\
\hline mg25 & $4.515 \mathrm{E}-14$ & $4.515 \mathrm{E}-14$ & $4.515 \mathrm{E}-14$ & $4.515 \mathrm{E}-14$ & $4.515 \mathrm{E}-14$ \\
\hline mg26 & 4.699E-14 & $4.699 \mathrm{E}-14$ & $4.699 \mathrm{E}-14$ & 4.699E-14 & 4.699E-14 \\
\hline mn54 & $2.242 \mathrm{E}-17$ & 4.773E-19 & $3.882 \mathrm{E}-19$ & $1.725 \mathrm{E}-19$ & $7.668 \mathrm{E}-20$ \\
\hline mn55 & $9.729 \mathrm{E}-14$ & $9.742 \mathrm{E}-14$ & $9.742 \mathrm{E}-14$ & $9.742 \mathrm{E}-14$ & $9.742 \mathrm{E}-14$ \\
\hline mo100 & $1.353 \mathrm{E}+03$ & $1.353 \mathrm{E}+03$ & $1.353 \mathrm{E}+03$ & $1.353 \mathrm{E}+03$ & $1.353 \mathrm{E}+03$ \\
\hline mo92 & $6.763 \mathrm{E}-12$ & $6.763 \mathrm{E}-12$ & $6.763 \mathrm{E}-12$ & $6.763 \mathrm{E}-12$ & $6.763 \mathrm{E}-12$ \\
\hline mo93 & 4.741E-07 & 4.737E-07 & 4.737E-07 & 4.736E-07 & $4.736 \mathrm{E}-07$ \\
\hline mo94 & $1.579 \mathrm{E}-02$ & $1.579 \mathrm{E}-02$ & $1.579 \mathrm{E}-02$ & $1.579 \mathrm{E}-02$ & $1.579 \mathrm{E}-02$ \\
\hline mo95 & $8.632 \mathrm{E}+02$ & $8.725 \mathrm{E}+02$ & $8.725 \mathrm{E}+02$ & $8.725 \mathrm{E}+02$ & $8.725 \mathrm{E}+02$ \\
\hline mo96 & $4.736 \mathrm{E}+01$ & $4.736 \mathrm{E}+01$ & $4.736 \mathrm{E}+01$ & $4.736 \mathrm{E}+01$ & $4.736 \mathrm{E}+01$ \\
\hline mo97 & $1.047 \mathrm{E}+03$ & $1.047 \mathrm{E}+03$ & $1.047 \mathrm{E}+03$ & $1.047 \mathrm{E}+03$ & $1.047 \mathrm{E}+03$ \\
\hline mo98 & $1.147 \mathrm{E}+03$ & $1.147 \mathrm{E}+03$ & $1.147 \mathrm{E}+03$ & $1.147 \mathrm{E}+03$ & $1.147 \mathrm{E}+03$ \\
\hline n14 & 4.700E-06 & 4.913E-06 & $4.925 \mathrm{E}-06$ & $4.970 \mathrm{E}-06$ & $5.015 \mathrm{E}-06$ \\
\hline n15 & $1.129 \mathrm{E}-02$ & $1.129 \mathrm{E}-02$ & $1.129 \mathrm{E}-02$ & $1.129 \mathrm{E}-02$ & $1.129 \mathrm{E}-02$ \\
\hline na23 & 4.152E-14 & $4.152 \mathrm{E}-14$ & $4.152 \mathrm{E}-14$ & 4.152E-14 & 4.152E-14 \\
\hline nb91 & $1.155 \mathrm{E}-08$ & $1.149 \mathrm{E}-08$ & $1.149 \mathrm{E}-08$ & $1.148 \mathrm{E}-08$ & $1.147 \mathrm{E}-08$ \\
\hline nb91m & $7.562 \mathrm{E}-15$ & $2.026 \mathrm{E}-23$ & $7.027 \mathrm{E}-24$ & $1.100 \mathrm{E}-25$ & $1.721 \mathrm{E}-27$ \\
\hline nb92 & $1.004 \mathrm{E}-06$ & $1.004 \mathrm{E}-06$ & $1.004 \mathrm{E}-06$ & $1.004 \mathrm{E}-06$ & $1.004 \mathrm{E}-06$ \\
\hline nb92m & $1.892 \mathrm{E}-18$ & $0.000 \mathrm{E}+00$ & $0.000 \mathrm{E}+00$ & $0.000 \mathrm{E}+00$ & $0.000 \mathrm{E}+00$ \\
\hline nb93 & $1.181 \mathrm{E}-04$ & $4.255 \mathrm{E}-04$ & 4.489E-04 & $5.467 \mathrm{E}-04$ & $6.541 \mathrm{E}-04$ \\
\hline nb93m & $6.803 \mathrm{E}-04$ & $1.915 \mathrm{E}-03$ & $1.975 \mathrm{E}-03$ & $2.202 \mathrm{E}-03$ & $2.419 \mathrm{E}-03$ \\
\hline nb94 & $3.608 \mathrm{E}-03$ & 3.607E-03 & $3.607 \mathrm{E}-03$ & $3.607 \mathrm{E}-03$ & 3.607E-03 \\
\hline nb95 & $4.985 \mathrm{E}+00$ & $3.752 \mathrm{E}-08$ & $1.369 \mathrm{E}-08$ & $2.715 \mathrm{E}-10$ & $5.227 \mathrm{E}-12$ \\
\hline nb95m & $2.815 \mathrm{E}-03$ & $2.005 \mathrm{E}-11$ & $7.325 \mathrm{E}-12$ & $1.409 \mathrm{E}-13$ & $2.711 \mathrm{E}-15$ \\
\hline nd142 & $2.148 \mathrm{E}+01$ & $2.148 \mathrm{E}+01$ & $2.148 \mathrm{E}+01$ & $2.148 \mathrm{E}+01$ & $2.148 \mathrm{E}+01$ \\
\hline nd143 & $1.068 \mathrm{E}+03$ & $1.068 \mathrm{E}+03$ & $1.068 \mathrm{E}+03$ & $1.068 \mathrm{E}+03$ & $1.068 \mathrm{E}+03$ \\
\hline nd144 & $1.160 \mathrm{E}+03$ & $1.367 \mathrm{E}+03$ & $1.368 \mathrm{E}+03$ & $1.369 \mathrm{E}+03$ & $1.370 \mathrm{E}+03$ \\
\hline nd145 & $8.031 \mathrm{E}+02$ & $8.031 \mathrm{E}+02$ & $8.031 \mathrm{E}+02$ & $8.031 \mathrm{E}+02$ & $8.031 \mathrm{E}+02$ \\
\hline nd146 & $8.758 \mathrm{E}+02$ & $8.758 \mathrm{E}+02$ & $8.758 \mathrm{E}+02$ & $8.758 \mathrm{E}+02$ & $8.758 \mathrm{E}+02$ \\
\hline nd147 & $5.261 \mathrm{E}-07$ & $0.000 \mathrm{E}+00$ & $0.000 \mathrm{E}+00$ & $0.000 \mathrm{E}+00$ & $0.000 \mathrm{E}+00$ \\
\hline nd148 & $5.165 \mathrm{E}+02$ & $5.165 \mathrm{E}+02$ & $5.165 \mathrm{E}+02$ & $5.165 \mathrm{E}+02$ & $5.165 \mathrm{E}+02$ \\
\hline nd150 & $3.047 \mathrm{E}+02$ & $3.047 \mathrm{E}+02$ & $3.047 \mathrm{E}+02$ & $3.047 \mathrm{E}+02$ & $3.047 \mathrm{E}+02$ \\
\hline ni58 & $1.040 \mathrm{E}-13$ & $1.040 \mathrm{E}-13$ & $1.040 \mathrm{E}-13$ & $1.040 \mathrm{E}-13$ & $1.040 \mathrm{E}-13$ \\
\hline ni59 & $8.454 \mathrm{E}-14$ & $8.454 \mathrm{E}-14$ & $8.454 \mathrm{E}-14$ & $8.454 \mathrm{E}-14$ & $8.454 \mathrm{E}-14$ \\
\hline ni60 & $1.303 \mathrm{E}-13$ & $1.349 \mathrm{E}-13$ & $1.351 \mathrm{E}-13$ & $1.357 \mathrm{E}-13$ & $1.363 \mathrm{E}-13$ \\
\hline ni61 & $1.102 \mathrm{E}-13$ & $1.102 \mathrm{E}-13$ & $1.102 \mathrm{E}-13$ & $1.102 \mathrm{E}-13$ & $1.102 \mathrm{E}-13$ \\
\hline
\end{tabular}




\begin{tabular}{|c|c|c|c|c|c|}
\hline \multicolumn{6}{|c|}{ Table C-1. Continued } \\
\hline & $1 / 22 / 2009$ & $10 / 21 / 2013$ & $1 / 22 / 2014$ & $1 / 22 / 2015$ & $1 / 22 / 2016$ \\
\hline ni62 & $1.106 \mathrm{E}-13$ & $1.106 \mathrm{E}-13$ & $1.106 \mathrm{E}-13$ & $1.106 \mathrm{E}-13$ & $1.106 \mathrm{E}-13$ \\
\hline ni63 & $1.843 \mathrm{E}-15$ & $1.785 \mathrm{E}-15$ & $1.781 \mathrm{E}-15$ & $1.769 \mathrm{E}-15$ & $1.757 \mathrm{E}-15$ \\
\hline ni64 & $1.161 \mathrm{E}-13$ & $1.161 \mathrm{E}-13$ & $1.161 \mathrm{E}-13$ & $1.161 \mathrm{E}-13$ & $1.161 \mathrm{E}-13$ \\
\hline np235 & $2.244 \mathrm{E}-06$ & $1.082 \mathrm{E}-07$ & 9.194E-08 & $4.855 \mathrm{E}-08$ & $2.564 \mathrm{E}-08$ \\
\hline np236 & $3.546 \mathrm{E}-04$ & $3.546 \mathrm{E}-04$ & $3.546 \mathrm{E}-04$ & $3.546 \mathrm{E}-04$ & $3.546 \mathrm{E}-04$ \\
\hline np237 & $1.810 \mathrm{E}+02$ & $1.884 \mathrm{E}+02$ & $1.890 \mathrm{E}+02$ & $1.916 \mathrm{E}+02$ & $1.945 \mathrm{E}+02$ \\
\hline np238 & 8.104E-07 & $7.919 \mathrm{E}-07$ & $7.906 \mathrm{E}-07$ & $7.866 \mathrm{E}-07$ & $7.833 \mathrm{E}-07$ \\
\hline np239 & $3.314 \mathrm{E}-04$ & $3.312 \mathrm{E}-04$ & 3.312E-04 & $3.312 \mathrm{E}-04$ & 3.312E-04 \\
\hline np240 & $1.899 \mathrm{E}-16$ & $1.899 \mathrm{E}-16$ & $1.899 \mathrm{E}-16$ & $1.899 \mathrm{E}-16$ & $1.899 \mathrm{E}-16$ \\
\hline np240m & $1.845 \mathrm{E}-14$ & $1.845 \mathrm{E}-14$ & $1.845 \mathrm{E}-14$ & $1.845 \mathrm{E}-14$ & $1.845 \mathrm{E}-14$ \\
\hline 016 & $1.344 \mathrm{E}+05$ & $1.344 \mathrm{E}+05$ & $1.344 \mathrm{E}+05$ & $1.344 \mathrm{E}+05$ & $1.344 \mathrm{E}+05$ \\
\hline o17 & $3.168 \mathrm{E}-02$ & $3.168 \mathrm{E}-02$ & $3.168 \mathrm{E}-02$ & $3.168 \mathrm{E}-02$ & $3.168 \mathrm{E}-02$ \\
\hline o18 & $1.228 \mathrm{E}-07$ & $1.228 \mathrm{E}-07$ & $1.228 \mathrm{E}-07$ & $1.228 \mathrm{E}-07$ & $1.228 \mathrm{E}-07$ \\
\hline os186 & $2.113 \mathrm{E}-13$ & $2.113 \mathrm{E}-13$ & $2.113 \mathrm{E}-13$ & $2.113 \mathrm{E}-13$ & $2.113 \mathrm{E}-13$ \\
\hline os187 & $3.323 \mathrm{E}-14$ & $3.323 \mathrm{E}-14$ & $3.323 \mathrm{E}-14$ & $3.323 \mathrm{E}-14$ & 3.323E-14 \\
\hline os188 & $1.313 \mathrm{E}-13$ & $1.313 \mathrm{E}-13$ & $1.313 \mathrm{E}-13$ & $1.313 \mathrm{E}-13$ & $1.313 \mathrm{E}-13$ \\
\hline os189 & $8.527 \mathrm{E}-15$ & $8.527 \mathrm{E}-15$ & $8.527 \mathrm{E}-15$ & $8.527 \mathrm{E}-15$ & $8.527 \mathrm{E}-15$ \\
\hline os190 & $2.204 \mathrm{E}-15$ & $2.204 \mathrm{E}-15$ & $2.204 \mathrm{E}-15$ & $2.204 \mathrm{E}-15$ & $2.204 \mathrm{E}-15$ \\
\hline os192 & $9.128 \mathrm{E}-15$ & $9.134 \mathrm{E}-15$ & $9.134 \mathrm{E}-15$ & $9.134 \mathrm{E}-15$ & $9.134 \mathrm{E}-15$ \\
\hline p31 & $5.603 \mathrm{E}-14$ & $5.603 \mathrm{E}-14$ & $5.603 \mathrm{E}-14$ & $5.603 \mathrm{E}-14$ & $5.603 \mathrm{E}-14$ \\
\hline pa230 & $1.109 \mathrm{E}-15$ & $0.000 \mathrm{E}+00$ & $0.000 \mathrm{E}+00$ & $0.000 \mathrm{E}+00$ & $0.000 \mathrm{E}+00$ \\
\hline pa231 & $2.490 \mathrm{E}-05$ & $2.938 \mathrm{E}-05$ & $2.962 \mathrm{E}-05$ & $3.057 \mathrm{E}-05$ & $3.151 \mathrm{E}-05$ \\
\hline pa232 & $1.647 \mathrm{E}-14$ & $1.647 \mathrm{E}-14$ & $1.647 \mathrm{E}-14$ & $1.647 \mathrm{E}-14$ & $1.647 \mathrm{E}-14$ \\
\hline pa233 & $6.128 \mathrm{E}-06$ & $6.372 \mathrm{E}-06$ & $6.392 \mathrm{E}-06$ & $6.489 \mathrm{E}-06$ & $6.587 \mathrm{E}-06$ \\
\hline pa234 & $2.467 \mathrm{E}-10$ & $2.467 \mathrm{E}-10$ & $2.467 \mathrm{E}-10$ & $2.467 \mathrm{E}-10$ & $2.467 \mathrm{E}-10$ \\
\hline pa234m & $4.445 \mathrm{E}-10$ & $4.446 \mathrm{E}-10$ & $4.445 \mathrm{E}-10$ & $4.445 \mathrm{E}-10$ & $4.445 \mathrm{E}-10$ \\
\hline pb204 & $3.673 \mathrm{E}-13$ & $3.673 \mathrm{E}-13$ & $3.673 \mathrm{E}-13$ & 3.673E-13 & $3.673 \mathrm{E}-13$ \\
\hline pb205 & $1.188 \mathrm{E}-15$ & $1.189 \mathrm{E}-15$ & $1.189 \mathrm{E}-15$ & $1.189 \mathrm{E}-15$ & $1.189 \mathrm{E}-15$ \\
\hline pb206 & $1.901 \mathrm{E}-12$ & $9.346 \mathrm{E}-12$ & $9.755 \mathrm{E}-12$ & $1.142 \mathrm{E}-11$ & $1.316 \mathrm{E}-11$ \\
\hline pb207 & $6.415 \mathrm{E}-11$ & $3.252 \mathrm{E}-10$ & $3.485 \mathrm{E}-10$ & 4.495E-10 & 5.657E-10 \\
\hline pb208 & $8.540 \mathrm{E}-07$ & $1.971 \mathrm{E}-05$ & $2.147 \mathrm{E}-05$ & $2.890 \mathrm{E}-05$ & $3.711 \mathrm{E}-05$ \\
\hline pb209 & $1.472 \mathrm{E}-14$ & $1.530 \mathrm{E}-14$ & $1.533 \mathrm{E}-14$ & $1.551 \mathrm{E}-14$ & $1.568 \mathrm{E}-14$ \\
\hline pb210 & $5.260 \mathrm{E}-11$ & $5.364 \mathrm{E}-11$ & $5.417 \mathrm{E}-11$ & $5.695 \mathrm{E}-11$ & $6.090 \mathrm{E}-11$ \\
\hline pb211 & $2.008 \mathrm{E}-15$ & $9.174 \mathrm{E}-15$ & 9.372E-15 & $1.107 \mathrm{E}-14$ & $1.258 \mathrm{E}-14$ \\
\hline pb212 & $1.915 \mathrm{E}-09$ & $1.206 \mathrm{E}-08$ & $1.251 \mathrm{E}-08$ & $1.403 \mathrm{E}-08$ & $1.532 \mathrm{E}-08$ \\
\hline pd102 & $1.040 \mathrm{E}-03$ & $1.249 \mathrm{E}-03$ & $1.249 \mathrm{E}-03$ & $1.250 \mathrm{E}-03$ & $1.250 \mathrm{E}-03$ \\
\hline pd103 & $3.111 \mathrm{E}-09$ & $0.000 \mathrm{E}+00$ & $0.000 \mathrm{E}+00$ & $0.000 \mathrm{E}+00$ & $0.000 \mathrm{E}+00$ \\
\hline pd104 & $4.175 \mathrm{E}+02$ & $4.175 \mathrm{E}+02$ & $4.175 \mathrm{E}+02$ & $4.175 \mathrm{E}+02$ & $4.175 \mathrm{E}+02$ \\
\hline
\end{tabular}




\begin{tabular}{|c|c|c|c|c|c|}
\hline \multicolumn{6}{|c|}{ Table C-1. Continued } \\
\hline & $1 / 22 / 2009$ & $10 / 21 / 2013$ & $1 / 22 / 2014$ & $1 / 22 / 2015$ & $1 / 22 / 2016$ \\
\hline pd105 & $1.044 \mathrm{E}+03$ & $1.044 \mathrm{E}+03$ & $1.044 \mathrm{E}+03$ & $1.044 \mathrm{E}+03$ & $1.044 \mathrm{E}+03$ \\
\hline pd106 & $8.137 \mathrm{E}+02$ & $1.049 \mathrm{E}+03$ & $1.051 \mathrm{E}+03$ & $1.055 \mathrm{E}+03$ & $1.057 \mathrm{E}+03$ \\
\hline pd107 & $6.750 \mathrm{E}+02$ & $6.750 \mathrm{E}+02$ & $6.750 \mathrm{E}+02$ & $6.750 \mathrm{E}+02$ & $6.750 \mathrm{E}+02$ \\
\hline pd108 & $4.669 \mathrm{E}+02$ & $4.669 \mathrm{E}+02$ & $4.669 \mathrm{E}+02$ & $4.669 \mathrm{E}+02$ & $4.669 \mathrm{E}+02$ \\
\hline pd110 & $1.527 \mathrm{E}+02$ & $1.527 \mathrm{E}+02$ & $1.527 \mathrm{E}+02$ & $1.527 \mathrm{E}+02$ & $1.527 \mathrm{E}+02$ \\
\hline pm143 & $1.806 \mathrm{E}-12$ & $1.941 \mathrm{E}-14$ & $1.522 \mathrm{E}-14$ & $5.858 \mathrm{E}-15$ & $2.255 \mathrm{E}-15$ \\
\hline pm144 & $5.543 \mathrm{E}-10$ & $2.026 \mathrm{E}-11$ & $1.696 \mathrm{E}-11$ & $8.447 \mathrm{E}-12$ & $4.208 \mathrm{E}-12$ \\
\hline pm145 & $3.955 \mathrm{E}-05$ & 3.314E-05 & $3.281 \mathrm{E}-05$ & $3.156 \mathrm{E}-05$ & $3.035 \mathrm{E}-05$ \\
\hline pm146 & 4.887E-04 & $2.697 \mathrm{E}-04$ & $2.612 \mathrm{E}-04$ & $2.304 \mathrm{E}-04$ & $2.033 \mathrm{E}-04$ \\
\hline pm147 & $1.933 \mathrm{E}+02$ & $5.519 \mathrm{E}+01$ & $5.160 \mathrm{E}+01$ & $3.962 \mathrm{E}+01$ & $3.043 \mathrm{E}+01$ \\
\hline pm148 & $2.255 \mathrm{E}-04$ & $5.230 \mathrm{E}-17$ & $1.098 \mathrm{E}-17$ & $2.395 \mathrm{E}-20$ & $5.228 \mathrm{E}-23$ \\
\hline pm148m & $3.594 \mathrm{E}-02$ & $8.335 \mathrm{E}-15$ & $1.749 \mathrm{E}-15$ & $3.817 \mathrm{E}-18$ & $8.328 \mathrm{E}-21$ \\
\hline po210 & $7.807 \mathrm{E}-13$ & $8.896 \mathrm{E}-13$ & $8.962 \mathrm{E}-13$ & $9.346 \mathrm{E}-13$ & $9.907 \mathrm{E}-13$ \\
\hline po216 & 7.384E-15 & $4.650 \mathrm{E}-14$ & $4.824 \mathrm{E}-14$ & $5.414 \mathrm{E}-14$ & $5.909 \mathrm{E}-14$ \\
\hline pr141 & $1.460 \mathrm{E}+03$ & $1.461 \mathrm{E}+03$ & $1.461 \mathrm{E}+03$ & $1.461 \mathrm{E}+03$ & $1.461 \mathrm{E}+03$ \\
\hline pr143 & $3.493 \mathrm{E}-05$ & $0.000 \mathrm{E}+00$ & $0.000 \mathrm{E}+00$ & $0.000 \mathrm{E}+00$ & $0.000 \mathrm{E}+00$ \\
\hline pr144 & $8.850 \mathrm{E}-03$ & $1.306 \mathrm{E}-04$ & $1.042 \mathrm{E}-04$ & $4.286 \mathrm{E}-05$ & $1.763 \mathrm{E}-05$ \\
\hline pr144m & $3.522 \mathrm{E}-05$ & $5.197 \mathrm{E}-07$ & 4.144E-07 & $1.705 \mathrm{E}-07$ & $7.014 \mathrm{E}-08$ \\
\hline pt192 & $1.569 \mathrm{E}-13$ & $1.571 \mathrm{E}-13$ & $1.571 \mathrm{E}-13$ & $1.571 \mathrm{E}-13$ & $1.571 \mathrm{E}-13$ \\
\hline pt193 & $1.985 \mathrm{E}-14$ & $1.859 \mathrm{E}-14$ & $1.852 \mathrm{E}-14$ & $1.826 \mathrm{E}-14$ & $1.801 \mathrm{E}-14$ \\
\hline pt194 & $3.211 \mathrm{E}-13$ & $3.211 \mathrm{E}-13$ & $3.211 \mathrm{E}-13$ & $3.211 \mathrm{E}-13$ & $3.211 \mathrm{E}-13$ \\
\hline pt195 & $2.549 \mathrm{E}-15$ & $2.549 \mathrm{E}-15$ & $2.549 \mathrm{E}-15$ & $2.549 \mathrm{E}-15$ & $2.549 \mathrm{E}-15$ \\
\hline pt196 & $3.811 \mathrm{E}-16$ & $3.811 \mathrm{E}-16$ & $3.811 \mathrm{E}-16$ & $3.811 \mathrm{E}-16$ & $3.811 \mathrm{E}-16$ \\
\hline pu236 & $9.623 \mathrm{E}-04$ & $3.045 \mathrm{E}-04$ & $2.862 \mathrm{E}-04$ & $2.246 \mathrm{E}-04$ & $1.763 \mathrm{E}-04$ \\
\hline pu237 & $1.234 \mathrm{E}-05$ & $4.578 \mathrm{E}-17$ & $1.115 \mathrm{E}-17$ & $4.362 \mathrm{E}-20$ & $1.707 \mathrm{E}-22$ \\
\hline pu238 & $1.996 \mathrm{E}+02$ & $2.133 \mathrm{E}+02$ & $2.129 \mathrm{E}+02$ & $2.112 \mathrm{E}+02$ & $2.096 \mathrm{E}+02$ \\
\hline pu239 & $1.595 \mathrm{E}+04$ & $1.595 \mathrm{E}+04$ & $1.595 \mathrm{E}+04$ & $1.595 \mathrm{E}+04$ & $1.595 \mathrm{E}+04$ \\
\hline pu240 & $9.960 \mathrm{E}+03$ & $9.980 \mathrm{E}+03$ & $9.980 \mathrm{E}+03$ & $9.980 \mathrm{E}+03$ & $9.986 \mathrm{E}+03$ \\
\hline pu241 & $5.212 \mathrm{E}+03$ & $4.141 \mathrm{E}+03$ & $4.090 \mathrm{E}+03$ & $3.897 \mathrm{E}+03$ & $3.712 \mathrm{E}+03$ \\
\hline pu242 & $1.516 \mathrm{E}+03$ & $1.516 \mathrm{E}+03$ & $1.516 \mathrm{E}+03$ & $1.516 \mathrm{E}+03$ & $1.516 \mathrm{E}+03$ \\
\hline pu243 & $5.093 \mathrm{E}-13$ & $5.093 \mathrm{E}-13$ & $5.093 \mathrm{E}-13$ & $5.093 \mathrm{E}-13$ & $5.093 \mathrm{E}-13$ \\
\hline pu244 & $1.110 \mathrm{E}-01$ & $1.110 \mathrm{E}-01$ & $1.110 \mathrm{E}-01$ & $1.110 \mathrm{E}-01$ & $1.110 \mathrm{E}-01$ \\
\hline ra223 & $9.676 \mathrm{E}-13$ & 4.422E-12 & $4.516 \mathrm{E}-12$ & $5.332 \mathrm{E}-12$ & $6.064 \mathrm{E}-12$ \\
\hline ra224 & $1.670 \mathrm{E}-08$ & $1.051 \mathrm{E}-07$ & $1.091 \mathrm{E}-07$ & $1.224 \mathrm{E}-07$ & $1.337 \mathrm{E}-07$ \\
\hline ra225 & $1.741 \mathrm{E}-12$ & $1.811 \mathrm{E}-12$ & $1.814 \mathrm{E}-12$ & $1.835 \mathrm{E}-12$ & $1.856 \mathrm{E}-12$ \\
\hline ra226 & $1.468 \mathrm{E}-09$ & $9.108 \mathrm{E}-09$ & $9.781 \mathrm{E}-09$ & $1.273 \mathrm{E}-08$ & $1.621 \mathrm{E}-08$ \\
\hline ra228 & $3.193 \mathrm{E}-14$ & $5.689 \mathrm{E}-14$ & $5.810 \mathrm{E}-14$ & $6.278 \mathrm{E}-14$ & $6.730 \mathrm{E}-14$ \\
\hline rb83 & $7.278 \mathrm{E}-08$ & $6.457 \mathrm{E}-14$ & $3.057 \mathrm{E}-14$ & $1.624 \mathrm{E}-15$ & $8.626 \mathrm{E}-17$ \\
\hline
\end{tabular}




\begin{tabular}{|c|c|c|c|c|c|}
\hline \multicolumn{6}{|c|}{ Table C-1. Continued } \\
\hline & $1 / 22 / 2009$ & $10 / 21 / 2013$ & $1 / 22 / 2014$ & $1 / 22 / 2015$ & $1 / 22 / 2016$ \\
\hline rb85 & $8.038 \mathrm{E}+01$ & $8.560 \mathrm{E}+01$ & $8.579 \mathrm{E}+01$ & $8.672 \mathrm{E}+01$ & $8.751 \mathrm{E}+01$ \\
\hline rb84 & $7.609 \mathrm{E}-08$ & $9.676 \mathrm{E}-24$ & $1.357 \mathrm{E}-24$ & $6.092 \mathrm{E}-28$ & $2.735 \mathrm{E}-31$ \\
\hline rb87 & $1.872 \mathrm{E}+02$ & $1.872 \mathrm{E}+02$ & $1.872 \mathrm{E}+02$ & $1.872 \mathrm{E}+02$ & $1.872 \mathrm{E}+02$ \\
\hline re185 & $6.737 \mathrm{E}-14$ & $6.750 \mathrm{E}-14$ & $6.750 \mathrm{E}-14$ & $6.750 \mathrm{E}-14$ & $6.750 \mathrm{E}-14$ \\
\hline re186m & $1.993 \mathrm{E}-16$ & $1.993 \mathrm{E}-16$ & $1.993 \mathrm{E}-16$ & $1.993 \mathrm{E}-16$ & $1.993 \mathrm{E}-16$ \\
\hline re187 & $3.538 \mathrm{E}-13$ & $3.538 \mathrm{E}-13$ & $3.538 \mathrm{E}-13$ & $3.538 \mathrm{E}-13$ & $3.538 \mathrm{E}-13$ \\
\hline rh101 & $6.366 \mathrm{E}-07$ & $2.350 \mathrm{E}-07$ & $2.227 \mathrm{E}-07$ & $1.806 \mathrm{E}-07$ & $1.464 \mathrm{E}-07$ \\
\hline rh102 & $9.504 \mathrm{E}-04$ & $3.280 \mathrm{E}-06$ & $2.489 \mathrm{E}-06$ & $9.339 \mathrm{E}-07$ & 4.409E-07 \\
\hline rh102m & $2.251 \mathrm{E}-03$ & $9.346 \mathrm{E}-04$ & 8.916E-04 & $7.410 \mathrm{E}-04$ & $6.157 \mathrm{E}-04$ \\
\hline rh103 & $1.005 \mathrm{E}+03$ & $1.005 \mathrm{E}+03$ & $1.005 \mathrm{E}+03$ & $1.005 \mathrm{E}+03$ & $1.005 \mathrm{E}+03$ \\
\hline rh103m & $6.849 \mathrm{E}-04$ & $3.492 \mathrm{E}-17$ & $6.757 \mathrm{E}-18$ & $1.072 \mathrm{E}-20$ & $1.700 \mathrm{E}-23$ \\
\hline rh106 & $2.304 \mathrm{E}-04$ & $9.108 \mathrm{E}-06$ & 7.655E-06 & $3.877 \mathrm{E}-06$ & $1.963 \mathrm{E}-06$ \\
\hline rn220 & $2.884 \mathrm{E}-12$ & $1.816 \mathrm{E}-11$ & $1.884 \mathrm{E}-11$ & $2.114 \mathrm{E}-11$ & $2.308 \mathrm{E}-11$ \\
\hline rn222 & $9.352 \mathrm{E}-15$ & $5.854 \mathrm{E}-14$ & $6.286 \mathrm{E}-14$ & $8.183 \mathrm{E}-14$ & $1.042 \mathrm{E}-13$ \\
\hline ru100 & $1.691 \mathrm{E}+02$ & $1.691 \mathrm{E}+02$ & $1.691 \mathrm{E}+02$ & $1.691 \mathrm{E}+02$ & $1.691 \mathrm{E}+02$ \\
\hline ru101 & $1.170 \mathrm{E}+03$ & $1.170 \mathrm{E}+03$ & $1.170 \mathrm{E}+03$ & $1.170 \mathrm{E}+03$ & $1.170 \mathrm{E}+03$ \\
\hline ru102 & $1.347 \mathrm{E}+03$ & $1.347 \mathrm{E}+03$ & $1.347 \mathrm{E}+03$ & $1.347 \mathrm{E}+03$ & $1.347 \mathrm{E}+03$ \\
\hline ru103 & $6.968 \mathrm{E}-01$ & $3.555 \mathrm{E}-14$ & $6.875 \mathrm{E}-15$ & $1.091 \mathrm{E}-17$ & $1.730 \mathrm{E}-20$ \\
\hline ru104 & $1.276 \mathrm{E}+03$ & $1.276 \mathrm{E}+03$ & $1.276 \mathrm{E}+03$ & $1.276 \mathrm{E}+03$ & $1.276 \mathrm{E}+03$ \\
\hline ru106 & $2.462 \mathrm{E}+02$ & $9.729 \mathrm{E}+00$ & $8.177 \mathrm{E}+00$ & $4.141 \mathrm{E}+00$ & $2.097 \mathrm{E}+00$ \\
\hline ru96 & $1.720 \mathrm{E}-13$ & $1.720 \mathrm{E}-13$ & $1.720 \mathrm{E}-13$ & $1.720 \mathrm{E}-13$ & $1.720 \mathrm{E}-13$ \\
\hline ru98 & $1.922 \mathrm{E}-06$ & $1.931 \mathrm{E}-06$ & $1.931 \mathrm{E}-06$ & $1.933 \mathrm{E}-06$ & $1.935 \mathrm{E}-06$ \\
\hline ru99 & $4.563 \mathrm{E}-02$ & $6.244 \mathrm{E}-02$ & $6.334 \mathrm{E}-02$ & $6.690 \mathrm{E}-02$ & $7.041 \mathrm{E}-02$ \\
\hline s32 & $5.774 \mathrm{E}-14$ & $5.774 \mathrm{E}-14$ & $5.774 \mathrm{E}-14$ & $5.774 \mathrm{E}-14$ & $5.774 \mathrm{E}-14$ \\
\hline s33 & $5.933 \mathrm{E}-14$ & 5.933E-14 & 5.933E-14 & $5.933 \mathrm{E}-14$ & $5.933 \mathrm{E}-14$ \\
\hline s34 & $6.147 \mathrm{E}-14$ & $6.147 \mathrm{E}-14$ & $6.147 \mathrm{E}-14$ & $6.147 \mathrm{E}-14$ & $6.147 \mathrm{E}-14$ \\
\hline s36 & $6.502 \mathrm{E}-14$ & $6.502 \mathrm{E}-14$ & $6.502 \mathrm{E}-14$ & $6.502 \mathrm{E}-14$ & $6.502 \mathrm{E}-14$ \\
\hline sb121 & $8.903 \mathrm{E}+00$ & $8.962 \mathrm{E}+00$ & $8.962 \mathrm{E}+00$ & $8.976 \mathrm{E}+00$ & $8.989 \mathrm{E}+00$ \\
\hline sb123 & $1.173 \mathrm{E}+01$ & $1.182 \mathrm{E}+01$ & $1.182 \mathrm{E}+01$ & $1.182 \mathrm{E}+01$ & $1.182 \mathrm{E}+01$ \\
\hline sb124 & 4.112E-03 & $8.877 \mathrm{E}-12$ & $3.041 \mathrm{E}-12$ & $4.549 \mathrm{E}-14$ & $6.803 \mathrm{E}-16$ \\
\hline sb125 & $1.559 \mathrm{E}+01$ & $4.733 \mathrm{E}+00$ & $4.440 \mathrm{E}+00$ & $3.454 \mathrm{E}+00$ & $2.687 \mathrm{E}+00$ \\
\hline sb126 & $9.643 \mathrm{E}-07$ & $9.590 \mathrm{E}-07$ & $9.590 \mathrm{E}-07$ & $9.590 \mathrm{E}-07$ & $9.590 \mathrm{E}-07$ \\
\hline sb126m & 7.377E-09 & 7.377E-09 & 7.377E-09 & 7.377E-09 & 7.377E-09 \\
\hline sc45 & $7.879 \mathrm{E}-14$ & $7.886 \mathrm{E}-14$ & $7.886 \mathrm{E}-14$ & $7.886 \mathrm{E}-14$ & $7.886 \mathrm{E}-14$ \\
\hline sc46 & $4.026 \mathrm{E}-17$ & $2.392 \mathrm{E}-23$ & $1.108 \mathrm{E}-23$ & $5.411 \mathrm{E}-25$ & $2.642 \mathrm{E}-26$ \\
\hline se74 & 4.363E-07 & 4.364E-07 & 4.364E-07 & 4.364E-07 & 4.364E-07 \\
\hline se75 & $9.451 \mathrm{E}-09$ & $4.173 \mathrm{E}-13$ & $2.436 \mathrm{E}-13$ & $2.948 \mathrm{E}-14$ & $3.567 \mathrm{E}-15$ \\
\hline se76 & $8.857 \mathrm{E}-03$ & $8.857 \mathrm{E}-03$ & 8.857E-03 & $8.857 \mathrm{E}-03$ & 8.857E-03 \\
\hline
\end{tabular}




\begin{tabular}{|c|c|c|c|c|c|}
\hline \multicolumn{6}{|c|}{ Table C-1. Continued } \\
\hline & $1 / 22 / 2009$ & $10 / 21 / 2013$ & $1 / 22 / 2014$ & $1 / 22 / 2015$ & $1 / 22 / 2016$ \\
\hline se77 & $1.006 \mathrm{E}+00$ & $1.006 \mathrm{E}+00$ & $1.006 \mathrm{E}+00$ & $1.006 \mathrm{E}+00$ & $1.006 \mathrm{E}+00$ \\
\hline se78 & $2.858 \mathrm{E}+00$ & $2.858 \mathrm{E}+00$ & $2.858 \mathrm{E}+00$ & $2.858 \mathrm{E}+00$ & $2.858 \mathrm{E}+00$ \\
\hline se79 & $6.151 \mathrm{E}+00$ & $6.150 \mathrm{E}+00$ & $6.150 \mathrm{E}+00$ & $6.150 \mathrm{E}+00$ & $6.150 \mathrm{E}+00$ \\
\hline se80 & $1.405 \mathrm{E}+01$ & $1.405 \mathrm{E}+01$ & $1.405 \mathrm{E}+01$ & $1.405 \mathrm{E}+01$ & $1.405 \mathrm{E}+01$ \\
\hline se82 & $3.666 \mathrm{E}+01$ & $3.666 \mathrm{E}+01$ & $3.666 \mathrm{E}+01$ & $3.666 \mathrm{E}+01$ & $3.666 \mathrm{E}+01$ \\
\hline si28 & $5.058 \mathrm{E}-14$ & $5.058 \mathrm{E}-14$ & $5.058 \mathrm{E}-14$ & $5.058 \mathrm{E}-14$ & $5.058 \mathrm{E}-14$ \\
\hline si29 & $5.245 \mathrm{E}-14$ & $5.245 \mathrm{E}-14$ & $5.245 \mathrm{E}-14$ & $5.245 \mathrm{E}-14$ & $5.245 \mathrm{E}-14$ \\
\hline si30 & $5.449 \mathrm{E}-14$ & $5.449 \mathrm{E}-14$ & 5.449E-14 & $5.449 \mathrm{E}-14$ & $5.449 \mathrm{E}-14$ \\
\hline sm144 & $6.459 \mathrm{E}-11$ & $6.459 \mathrm{E}-11$ & $6.459 \mathrm{E}-11$ & $6.459 \mathrm{E}-11$ & $6.459 \mathrm{E}-11$ \\
\hline $\operatorname{sm145}$ & $3.549 \mathrm{E}-07$ & $1.037 \mathrm{E}-08$ & 8.579E-09 & 4.076E-09 & $1.936 \mathrm{E}-09$ \\
\hline $\operatorname{sm} 146$ & $3.671 \mathrm{E}-03$ & $3.745 \mathrm{E}-03$ & $3.748 \mathrm{E}-03$ & $3.758 \mathrm{E}-03$ & 3.767E-03 \\
\hline sm147 & $1.282 \mathrm{E}+02$ & $2.664 \mathrm{E}+02$ & $2.699 \mathrm{E}+02$ & $2.820 \mathrm{E}+02$ & $2.911 \mathrm{E}+02$ \\
\hline sm148 & $2.080 \mathrm{E}+02$ & $2.081 \mathrm{E}+02$ & $2.081 \mathrm{E}+02$ & $2.081 \mathrm{E}+02$ & $2.081 \mathrm{E}+02$ \\
\hline sm149 & $6.605 \mathrm{E}+00$ & $6.605 \mathrm{E}+00$ & $6.605 \mathrm{E}+00$ & $6.605 \mathrm{E}+00$ & $6.605 \mathrm{E}+00$ \\
\hline sm150 & $4.357 \mathrm{E}+02$ & $4.357 \mathrm{E}+02$ & $4.357 \mathrm{E}+02$ & $4.357 \mathrm{E}+02$ & $4.357 \mathrm{E}+02$ \\
\hline sm151 & $2.804 \mathrm{E}+01$ & $2.703 \mathrm{E}+01$ & $2.697 \mathrm{E}+01$ & $2.677 \mathrm{E}+01$ & $2.656 \mathrm{E}+01$ \\
\hline sm152 & $1.686 \mathrm{E}+02$ & $1.686 \mathrm{E}+02$ & $1.686 \mathrm{E}+02$ & $1.686 \mathrm{E}+02$ & $1.686 \mathrm{E}+02$ \\
\hline sm154 & $8.659 \mathrm{E}+01$ & $8.659 \mathrm{E}+01$ & $8.659 \mathrm{E}+01$ & $8.659 \mathrm{E}+01$ & $8.659 \mathrm{E}+01$ \\
\hline sn112 & 3.648E-09 & $3.648 \mathrm{E}-09$ & 3.648E-09 & $3.648 \mathrm{E}-09$ & 3.648E-09 \\
\hline sn113 & $7.635 \mathrm{E}-11$ & $2.238 \mathrm{E}-15$ & $1.279 \mathrm{E}-15$ & $1.419 \mathrm{E}-16$ & $1.575 \mathrm{E}-17$ \\
\hline sn114 & $1.863 \mathrm{E}-04$ & $1.866 \mathrm{E}-04$ & $1.866 \mathrm{E}-04$ & $1.866 \mathrm{E}-04$ & $1.866 \mathrm{E}-04$ \\
\hline sn115 & $5.306 \mathrm{E}-01$ & $5.306 \mathrm{E}-01$ & $5.306 \mathrm{E}-01$ & $5.306 \mathrm{E}-01$ & $5.306 \mathrm{E}-01$ \\
\hline sn116 & $4.343 \mathrm{E}+00$ & $4.343 \mathrm{E}+00$ & $4.343 \mathrm{E}+00$ & $4.343 \mathrm{E}+00$ & $4.343 \mathrm{E}+00$ \\
\hline sn117 & $1.086 \mathrm{E}+01$ & $1.086 \mathrm{E}+01$ & $1.086 \mathrm{E}+01$ & $1.086 \mathrm{E}+01$ & $1.086 \mathrm{E}+01$ \\
\hline sn117m & $2.904 \mathrm{E}-09$ & $0.000 \mathrm{E}+00$ & $0.000 \mathrm{E}+00$ & $0.000 \mathrm{E}+00$ & $0.000 \mathrm{E}+00$ \\
\hline sn118 & $9.405 \mathrm{E}+00$ & $9.405 \mathrm{E}+00$ & $9.405 \mathrm{E}+00$ & $9.405 \mathrm{E}+00$ & $9.405 \mathrm{E}+00$ \\
\hline sn119 & $8.976 \mathrm{E}+00$ & $9.048 \mathrm{E}+00$ & $9.048 \mathrm{E}+00$ & $9.048 \mathrm{E}+00$ & $9.048 \mathrm{E}+00$ \\
\hline sn119m & $7.060 \mathrm{E}-02$ & $1.171 \mathrm{E}-03$ & $9.398 \mathrm{E}-04$ & $3.962 \mathrm{E}-04$ & $1.671 \mathrm{E}-04$ \\
\hline sn120 & $9.042 \mathrm{E}+00$ & $9.042 \mathrm{E}+00$ & $9.042 \mathrm{E}+00$ & $9.042 \mathrm{E}+00$ & $9.042 \mathrm{E}+00$ \\
\hline sn121 & 4.426E-05 & 4.107E-05 & 4.090E-05 & $4.026 \mathrm{E}-05$ & $3.963 \mathrm{E}-05$ \\
\hline sn121m & $8.117 \mathrm{E}-01$ & $7.536 \mathrm{E}-01$ & $7.503 \mathrm{E}-01$ & 7.384E-01 & 7.272E-01 \\
\hline sn122 & $1.213 \mathrm{E}+01$ & $1.213 \mathrm{E}+01$ & $1.213 \mathrm{E}+01$ & $1.213 \mathrm{E}+01$ & $1.213 \mathrm{E}+01$ \\
\hline sn123 & $8.599 \mathrm{E}-02$ & $7.879 \mathrm{E}-06$ & 4.786E-06 & $6.750 \mathrm{E}-07$ & $9.530 \mathrm{E}-08$ \\
\hline sn124 & $1.968 \mathrm{E}+01$ & $1.968 \mathrm{E}+01$ & $1.968 \mathrm{E}+01$ & $1.968 \mathrm{E}+01$ & $1.968 \mathrm{E}+01$ \\
\hline sn125 & $8.236 \mathrm{E}-10$ & $0.000 \mathrm{E}+00$ & $0.000 \mathrm{E}+00$ & $0.000 \mathrm{E}+00$ & $0.000 \mathrm{E}+00$ \\
\hline sn126 & $4.659 \mathrm{E}+01$ & $4.659 \mathrm{E}+01$ & $4.659 \mathrm{E}+01$ & $4.659 \mathrm{E}+01$ & $4.659 \mathrm{E}+01$ \\
\hline sr84 & 8.804E-06 & 8.804E-06 & 8.804E-06 & $8.804 \mathrm{E}-06$ & 8.804E-06 \\
\hline sr85 & $1.567 \mathrm{E}-08$ & $1.411 \mathrm{E}-16$ & $5.220 \mathrm{E}-17$ & $1.055 \mathrm{E}-18$ & $2.131 \mathrm{E}-20$ \\
\hline
\end{tabular}

C-13 


\begin{tabular}{|c|c|c|c|c|c|}
\hline \multicolumn{6}{|c|}{ Table C-1. Continued } \\
\hline & $1 / 22 / 2009$ & $10 / 21 / 2013$ & $1 / 22 / 2014$ & $1 / 22 / 2015$ & $1 / 22 / 2016$ \\
\hline sr86 & $5.116 \mathrm{E}-01$ & $5.116 \mathrm{E}-01$ & $5.116 \mathrm{E}-01$ & $5.116 \mathrm{E}-01$ & $5.116 \mathrm{E}-01$ \\
\hline sr87 & $5.740 \mathrm{E}-03$ & $5.740 \mathrm{E}-03$ & $5.740 \mathrm{E}-03$ & $5.740 \mathrm{E}-03$ & $5.740 \mathrm{E}-03$ \\
\hline sr88 & $2.488 \mathrm{E}+02$ & $2.488 \mathrm{E}+02$ & $2.488 \mathrm{E}+02$ & $2.488 \mathrm{E}+02$ & $2.488 \mathrm{E}+02$ \\
\hline sr89 & $5.673 \mathrm{E}-01$ & $2.688 \mathrm{E}-11$ & $7.509 \mathrm{E}-12$ & $5.023 \mathrm{E}-14$ & $3.361 \mathrm{E}-16$ \\
\hline sr90 & $3.822 \mathrm{E}+02$ & $3.409 \mathrm{E}+02$ & $3.388 \mathrm{E}+02$ & $3.308 \mathrm{E}+02$ & $3.229 \mathrm{E}+02$ \\
\hline ta180m & $2.007 \mathrm{E}-17$ & $2.007 \mathrm{E}-17$ & $2.007 \mathrm{E}-17$ & $2.007 \mathrm{E}-17$ & $2.007 \mathrm{E}-17$ \\
\hline ta181 & $1.811 \mathrm{E}-13$ & $1.812 \mathrm{E}-13$ & $1.812 \mathrm{E}-13$ & $1.812 \mathrm{E}-13$ & $1.812 \mathrm{E}-13$ \\
\hline ta182 & $7.021 \mathrm{E}-16$ & $1.997 \mathrm{E}-20$ & $1.140 \mathrm{E}-20$ & $1.288 \mathrm{E}-21$ & $1.730 \mathrm{E}-22$ \\
\hline tb157 & $9.154 \mathrm{E}-07$ & $8.745 \mathrm{E}-07$ & $8.718 \mathrm{E}-07$ & 8.632E-07 & 8.553E-07 \\
\hline tb158 & $1.172 \mathrm{E}-04$ & $1.151 \mathrm{E}-04$ & $1.151 \mathrm{E}-04$ & $1.146 \mathrm{E}-04$ & $1.141 \mathrm{E}-04$ \\
\hline tb159 & $7.490 \mathrm{E}+00$ & $7.490 \mathrm{E}+00$ & $7.490 \mathrm{E}+00$ & $7.490 \mathrm{E}+00$ & $7.490 \mathrm{E}+00$ \\
\hline tb160 & $1.873 \mathrm{E}-02$ & 1.140E-09 & 4.674E-10 & $1.413 \mathrm{E}-11$ & $4.269 \mathrm{E}-13$ \\
\hline tb161 & $7.893 \mathrm{E}-14$ & $0.000 \mathrm{E}+00$ & $0.000 \mathrm{E}+00$ & $0.000 \mathrm{E}+00$ & $0.000 \mathrm{E}+00$ \\
\hline tc95 & $6.148 \mathrm{E}-18$ & $1.724 \mathrm{E}-26$ & $5.991 \mathrm{E}-27$ & $9.464 \mathrm{E}-29$ & $0.000 \mathrm{E}+00$ \\
\hline tc95m & $1.144 \mathrm{E}-14$ & $3.207 \mathrm{E}-23$ & $1.115 \mathrm{E}-23$ & $1.761 \mathrm{E}-25$ & $2.784 \mathrm{E}-27$ \\
\hline tc97 & $1.144 \mathrm{E}-05$ & $1.154 \mathrm{E}-05$ & $1.154 \mathrm{E}-05$ & $1.154 \mathrm{E}-05$ & $1.154 \mathrm{E}-05$ \\
\hline tc97m & $1.042 \mathrm{E}-07$ & $1.927 \mathrm{E}-13$ & $9.491 \mathrm{E}-14$ & $5.887 \mathrm{E}-15$ & $3.651 \mathrm{E}-16$ \\
\hline tc98 & $1.179 \mathrm{E}-02$ & $1.179 \mathrm{E}-02$ & $1.179 \mathrm{E}-02$ & $1.179 \mathrm{E}-02$ & $1.179 \mathrm{E}-02$ \\
\hline tc99 & $1.079 \mathrm{E}+03$ & $1.079 \mathrm{E}+03$ & $1.079 \mathrm{E}+03$ & $1.079 \mathrm{E}+03$ & $1.079 \mathrm{E}+03$ \\
\hline te120 & $2.588 \mathrm{E}-09$ & $2.588 \mathrm{E}-09$ & $2.588 \mathrm{E}-09$ & $2.588 \mathrm{E}-09$ & $2.588 \mathrm{E}-09$ \\
\hline te121 & 3.455E-08 & $2.297 \mathrm{E}-11$ & $1.551 \mathrm{E}-11$ & $3.323 \mathrm{E}-12$ & $7.120 \mathrm{E}-13$ \\
\hline te121m & $2.949 \mathrm{E}-07$ & $1.961 \mathrm{E}-10$ & $1.324 \mathrm{E}-10$ & $2.837 \mathrm{E}-11$ & $6.077 \mathrm{E}-12$ \\
\hline te122 & 7.873E-01 & $7.873 \mathrm{E}-01$ & $7.873 \mathrm{E}-01$ & 7.873E-01 & 7.873E-01 \\
\hline te123 & $9.630 \mathrm{E}-03$ & $1.009 \mathrm{E}-02$ & $1.009 \mathrm{E}-02$ & $1.009 \mathrm{E}-02$ & $1.009 \mathrm{E}-02$ \\
\hline te123m & $4.520 \mathrm{E}-04$ & $1.899 \mathrm{E}-08$ & $1.106 \mathrm{E}-08$ & $1.324 \mathrm{E}-09$ & $1.586 \mathrm{E}-10$ \\
\hline te124 & $5.641 \mathrm{E}-01$ & $5.682 \mathrm{E}-01$ & $5.682 \mathrm{E}-01$ & 5.682E-01 & $5.682 \mathrm{E}-01$ \\
\hline te125 & $1.085 \mathrm{E}+01$ & $2.186 \mathrm{E}+01$ & $2.216 \mathrm{E}+01$ & $2.316 \mathrm{E}+01$ & $2.394 \mathrm{E}+01$ \\
\hline te125m & $2.165 \mathrm{E}-01$ & $6.603 \mathrm{E}-02$ & $6.194 \mathrm{E}-02$ & $4.818 \mathrm{E}-02$ & $3.748 \mathrm{E}-02$ \\
\hline te126 & $1.127 \mathrm{E}+00$ & $1.127 \mathrm{E}+00$ & $1.127 \mathrm{E}+00$ & $1.127 \mathrm{E}+00$ & $1.128 \mathrm{E}+00$ \\
\hline te127 & $1.049 \mathrm{E}-03$ & $1.716 \mathrm{E}-08$ & $9.497 \mathrm{E}-09$ & $9.326 \mathrm{E}-10$ & 9.154E-11 \\
\hline te $127 \mathrm{~m}$ & $2.996 \mathrm{E}-01$ & $4.902 \mathrm{E}-06$ & $2.713 \mathrm{E}-06$ & $2.664 \mathrm{E}-07$ & $2.615 \mathrm{E}-08$ \\
\hline te128 & $1.798 \mathrm{E}+02$ & $1.798 \mathrm{E}+02$ & $1.798 \mathrm{E}+02$ & $1.798 \mathrm{E}+02$ & $1.798 \mathrm{E}+02$ \\
\hline te129 & 7.972E-06 & $2.371 \mathrm{E}-21$ & $3.481 \mathrm{E}-22$ & $1.869 \mathrm{E}-25$ & $1.003 \mathrm{E}-28$ \\
\hline te129m & $8.784 \mathrm{E}-03$ & $2.613 \mathrm{E}-18$ & $3.836 \mathrm{E}-19$ & $2.059 \mathrm{E}-22$ & $1.106 \mathrm{E}-25$ \\
\hline te130 & $5.996 \mathrm{E}+02$ & $5.996 \mathrm{E}+02$ & $5.996 \mathrm{E}+02$ & $5.996 \mathrm{E}+02$ & $5.996 \mathrm{E}+02$ \\
\hline th227 & $1.625 \mathrm{E}-12$ & $7.252 \mathrm{E}-12$ & 7.457E-12 & $8.751 \mathrm{E}-12$ & $9.947 \mathrm{E}-12$ \\
\hline th228 & $3.288 \mathrm{E}-06$ & $2.036 \mathrm{E}-05$ & $2.110 \mathrm{E}-05$ & $2.370 \mathrm{E}-05$ & $2.587 \mathrm{E}-05$ \\
\hline th229 & $3.189 \mathrm{E}-07$ & $3.316 \mathrm{E}-07$ & $3.325 \mathrm{E}-07$ & $3.360 \mathrm{E}-07$ & $3.399 \mathrm{E}-07$ \\
\hline
\end{tabular}




\begin{tabular}{|c|c|c|c|c|c|}
\hline & $1 / 22 / 2009$ & $10 / 21 / 2013$ & $1 / 22 / 2014$ & $1 / 22 / 2015$ & $1 / 22 / 2016$ \\
\hline th230 & 8.804E-05 & $2.863 \mathrm{E}-04$ & $2.999 \mathrm{E}-04$ & $3.561 \mathrm{E}-04$ & 4.169E-04 \\
\hline th231 & $3.941 \mathrm{E}-09$ & $3.950 \mathrm{E}-09$ & $3.950 \mathrm{E}-09$ & $3.952 \mathrm{E}-09$ & $3.954 \mathrm{E}-09$ \\
\hline th232 & $1.995 \mathrm{E}-04$ & $2.403 \mathrm{E}-04$ & $2.425 \mathrm{E}-04$ & $2.512 \mathrm{E}-04$ & $2.600 \mathrm{E}-04$ \\
\hline th234 & $1.331 \mathrm{E}-05$ & $1.331 \mathrm{E}-05$ & $1.331 \mathrm{E}-05$ & $1.331 \mathrm{E}-05$ & $1.331 \mathrm{E}-05$ \\
\hline ti46 & $8.560 \mathrm{E}-14$ & $8.560 \mathrm{E}-14$ & $8.560 \mathrm{E}-14$ & $8.560 \mathrm{E}-14$ & $8.560 \mathrm{E}-14$ \\
\hline ti47 & $8.480 \mathrm{E}-14$ & $8.480 \mathrm{E}-14$ & $8.480 \mathrm{E}-14$ & $8.480 \mathrm{E}-14$ & $8.480 \mathrm{E}-14$ \\
\hline ti48 & $8.619 \mathrm{E}-14$ & 8.619E-14 & 8.619E-14 & $8.619 \mathrm{E}-14$ & $8.619 \mathrm{E}-14$ \\
\hline ti49 & $8.923 \mathrm{E}-14$ & $8.923 \mathrm{E}-14$ & 8.923E-14 & 8.923E-14 & $8.923 \mathrm{E}-14$ \\
\hline ti50 & $9.048 \mathrm{E}-14$ & $9.048 \mathrm{E}-14$ & $9.048 \mathrm{E}-14$ & $9.048 \mathrm{E}-14$ & $9.048 \mathrm{E}-14$ \\
\hline tl203 & $2.751 \mathrm{E}-15$ & $2.756 \mathrm{E}-15$ & $2.756 \mathrm{E}-15$ & $2.756 \mathrm{E}-15$ & $2.756 \mathrm{E}-15$ \\
\hline tl204 & $5.270 \mathrm{E}-17$ & $2.209 \mathrm{E}-17$ & $2.109 \mathrm{E}-17$ & $1.756 \mathrm{E}-17$ & $1.462 \mathrm{E}-17$ \\
\hline tl205 & $1.315 \mathrm{E}-15$ & $1.315 \mathrm{E}-15$ & $1.315 \mathrm{E}-15$ & $1.315 \mathrm{E}-15$ & $1.315 \mathrm{E}-15$ \\
\hline tl207 & $2.596 \mathrm{E}-16$ & $1.186 \mathrm{E}-15$ & $1.211 \mathrm{E}-15$ & $1.431 \mathrm{E}-15$ & $1.627 \mathrm{E}-15$ \\
\hline tl208 & $3.228 \mathrm{E}-12$ & $2.034 \mathrm{E}-11$ & $2.110 \mathrm{E}-11$ & $2.367 \mathrm{E}-11$ & $2.584 \mathrm{E}-11$ \\
\hline $\operatorname{tm} 168$ & $1.055 \mathrm{E}-09$ & $2.629 \mathrm{E}-15$ & $1.316 \mathrm{E}-15$ & $8.685 \mathrm{E}-17$ & $5.737 \mathrm{E}-18$ \\
\hline $\operatorname{tm} 169$ & $1.016 \mathrm{E}-03$ & $1.016 \mathrm{E}-03$ & $1.016 \mathrm{E}-03$ & $1.016 \mathrm{E}-03$ & $1.016 \mathrm{E}-03$ \\
\hline $\operatorname{tm} 170$ & $5.528 \mathrm{E}-05$ & $4.852 \mathrm{E}-09$ & $2.939 \mathrm{E}-09$ & $4.109 \mathrm{E}-10$ & $5.746 \mathrm{E}-11$ \\
\hline $\operatorname{tm} 171$ & $1.744 \mathrm{E}-04$ & $3.146 \mathrm{E}-05$ & $2.870 \mathrm{E}-05$ & $2.001 \mathrm{E}-05$ & $1.395 \mathrm{E}-05$ \\
\hline u230 & $2.975 \mathrm{E}-15$ & $0.000 \mathrm{E}+00$ & $0.000 \mathrm{E}+00$ & $0.000 \mathrm{E}+00$ & $0.000 \mathrm{E}+00$ \\
\hline $\mathbf{u} 232$ & 4.373E-04 & $1.046 \mathrm{E}-03$ & $1.061 \mathrm{E}-03$ & $1.110 \mathrm{E}-03$ & $1.147 \mathrm{E}-03$ \\
\hline $\mathbf{u} 233$ & $4.883 \mathrm{E}-04$ & $7.846 \mathrm{E}-04$ & $7.998 \mathrm{E}-04$ & 8.665E-04 & $9.339 \mathrm{E}-04$ \\
\hline u234 & $1.112 \mathrm{E}+01$ & $1.903 \mathrm{E}+01$ & $1.945 \mathrm{E}+01$ & $2.110 \mathrm{E}+01$ & $2.273 \mathrm{E}+01$ \\
\hline $\mathbf{u} 235$ & $9.689 \mathrm{E}+02$ & $9.715 \mathrm{E}+02$ & $9.715 \mathrm{E}+02$ & $9.722 \mathrm{E}+02$ & $9.722 \mathrm{E}+02$ \\
\hline u236 & $2.930 \mathrm{E}+02$ & $2.979 \mathrm{E}+02$ & $2.981 \mathrm{E}+02$ & $2.992 \mathrm{E}+02$ & $3.002 \mathrm{E}+02$ \\
\hline $\mathbf{u} 237$ & $1.626 \mathrm{E}-04$ & $1.292 \mathrm{E}-04$ & $1.276 \mathrm{E}-04$ & $1.216 \mathrm{E}-04$ & $1.158 \mathrm{E}-04$ \\
\hline u238 & $9.167 \mathrm{E}+05$ & $9.167 \mathrm{E}+05$ & $9.167 \mathrm{E}+05$ & $9.167 \mathrm{E}+05$ & $9.167 \mathrm{E}+05$ \\
\hline u240 & $2.162 \mathrm{E}-12$ & $2.162 \mathrm{E}-12$ & $2.162 \mathrm{E}-12$ & $2.162 \mathrm{E}-12$ & $2.162 \mathrm{E}-12$ \\
\hline v50 & $5.768 \mathrm{E}-17$ & $5.768 \mathrm{E}-17$ & $5.768 \mathrm{E}-17$ & $5.768 \mathrm{E}-17$ & $5.768 \mathrm{E}-17$ \\
\hline v51 & $1.768 \mathrm{E}-15$ & $1.768 \mathrm{E}-15$ & $1.768 \mathrm{E}-15$ & $1.768 \mathrm{E}-15$ & $1.768 \mathrm{E}-15$ \\
\hline w182 & $2.810 \mathrm{E}-13$ & $2.817 \mathrm{E}-13$ & $2.817 \mathrm{E}-13$ & $2.817 \mathrm{E}-13$ & $2.817 \mathrm{E}-13$ \\
\hline w183 & $6.447 \mathrm{E}-13$ & $6.447 \mathrm{E}-13$ & $6.447 \mathrm{E}-13$ & $6.447 \mathrm{E}-13$ & $6.447 \mathrm{E}-13$ \\
\hline w184 & $5.542 \mathrm{E}-13$ & $5.542 \mathrm{E}-13$ & $5.542 \mathrm{E}-13$ & $5.542 \mathrm{E}-13$ & $5.542 \mathrm{E}-13$ \\
\hline w185 & $1.159 \mathrm{E}-16$ & $1.311 \mathrm{E}-23$ & $5.556 \mathrm{E}-24$ & $1.913 \mathrm{E}-25$ & $6.586 \mathrm{E}-27$ \\
\hline w186 & $2.131 \mathrm{E}-13$ & $2.131 \mathrm{E}-13$ & $2.131 \mathrm{E}-13$ & $2.131 \mathrm{E}-13$ & $2.131 \mathrm{E}-13$ \\
\hline xe124 & $2.368 \mathrm{E}-13$ & $2.368 \mathrm{E}-13$ & $2.368 \mathrm{E}-13$ & $2.368 \mathrm{E}-13$ & $2.368 \mathrm{E}-13$ \\
\hline xe126 & $3.738 \mathrm{E}-04$ & $3.738 \mathrm{E}-04$ & $3.738 \mathrm{E}-04$ & $3.738 \mathrm{E}-04$ & $3.738 \mathrm{E}-04$ \\
\hline xe127 & $5.560 \mathrm{E}-08$ & $2.588 \mathrm{E}-22$ & $4.404 \mathrm{E}-23$ & $4.220 \mathrm{E}-26$ & $4.043 \mathrm{E}-29$ \\
\hline xe128 & $8.461 \mathrm{E}+00$ & $8.461 \mathrm{E}+00$ & $8.461 \mathrm{E}+00$ & $8.461 \mathrm{E}+00$ & $8.461 \mathrm{E}+00$ \\
\hline
\end{tabular}




\begin{tabular}{|c|c|c|c|c|c|}
\hline \multicolumn{6}{|c|}{ Table C-1. Continued } \\
\hline & $1 / 22 / 2009$ & $10 / 21 / 2013$ & $1 / 22 / 2014$ & $1 / 22 / 2015$ & $1 / 22 / 2016$ \\
\hline xe129 & $5.459 \mathrm{E}-02$ & $5.465 \mathrm{E}-02$ & $5.466 \mathrm{E}-02$ & $5.467 \mathrm{E}-02$ & $5.469 \mathrm{E}-02$ \\
\hline xe129m & $2.824 \mathrm{E}-13$ & $0.000 \mathrm{E}+00$ & $0.000 \mathrm{E}+00$ & $0.000 \mathrm{E}+00$ & $0.000 \mathrm{E}+00$ \\
\hline xe130 & $1.089 \mathrm{E}+01$ & $1.089 \mathrm{E}+01$ & $1.089 \mathrm{E}+01$ & $1.089 \mathrm{E}+01$ & $1.089 \mathrm{E}+01$ \\
\hline xe131 & $3.234 \mathrm{E}-14$ & $3.234 \mathrm{E}-14$ & $3.234 \mathrm{E}-14$ & $3.234 \mathrm{E}-14$ & 3.234E-14 \\
\hline xe131 & $6.869 \mathrm{E}+02$ & $6.869 \mathrm{E}+02$ & $6.869 \mathrm{E}+02$ & $6.869 \mathrm{E}+02$ & $6.869 \mathrm{E}+02$ \\
\hline xe131m & $1.048 \mathrm{E}-07$ & $0.000 \mathrm{E}+00$ & $0.000 \mathrm{E}+00$ & $0.000 \mathrm{E}+00$ & $0.000 \mathrm{E}+00$ \\
\hline xe132 & $1.323 \mathrm{E}-14$ & $1.323 \mathrm{E}-14$ & $1.323 \mathrm{E}-14$ & $1.323 \mathrm{E}-14$ & $1.323 \mathrm{E}-14$ \\
\hline xe132 & $1.709 \mathrm{E}+03$ & $1.709 \mathrm{E}+03$ & $1.709 \mathrm{E}+03$ & $1.709 \mathrm{E}+03$ & $1.709 \mathrm{E}+03$ \\
\hline xe133 & $1.067 \mathrm{E}-14$ & $0.000 \mathrm{E}+00$ & $0.000 \mathrm{E}+00$ & $0.000 \mathrm{E}+00$ & $0.000 \mathrm{E}+00$ \\
\hline xe134 & $2.093 \mathrm{E}+03$ & $2.093 \mathrm{E}+03$ & $2.093 \mathrm{E}+03$ & $2.093 \mathrm{E}+03$ & $2.093 \mathrm{E}+03$ \\
\hline xe136 & $3.109 \mathrm{E}+03$ & $3.109 \mathrm{E}+03$ & $3.109 \mathrm{E}+03$ & $3.109 \mathrm{E}+03$ & $3.109 \mathrm{E}+03$ \\
\hline y88 & $2.973 \mathrm{E}-05$ & $3.808 \mathrm{E}-10$ & $2.080 \mathrm{E}-10$ & $1.940 \mathrm{E}-11$ & $1.808 \mathrm{E}-12$ \\
\hline y89 & $3.273 \mathrm{E}+02$ & $3.278 \mathrm{E}+02$ & $3.278 \mathrm{E}+02$ & $3.278 \mathrm{E}+02$ & $3.278 \mathrm{E}+02$ \\
\hline y89m & $1.962 \mathrm{E}-10$ & $9.299 \mathrm{E}-21$ & $2.596 \mathrm{E}-21$ & $1.737 \mathrm{E}-23$ & $1.162 \mathrm{E}-25$ \\
\hline y90 & $9.696 \mathrm{E}-02$ & $8.645 \mathrm{E}-02$ & $8.593 \mathrm{E}-02$ & $8.388 \mathrm{E}-02$ & $8.190 \mathrm{E}-02$ \\
\hline y91 & $1.589 \mathrm{E}+00$ & $1.927 \mathrm{E}-09$ & $6.405 \mathrm{E}-10$ & $8.480 \mathrm{E}-12$ & $1.123 \mathrm{E}-13$ \\
\hline yb168 & $2.160 \mathrm{E}-12$ & $2.266 \mathrm{E}-12$ & $2.266 \mathrm{E}-12$ & $2.266 \mathrm{E}-12$ & $2.266 \mathrm{E}-12$ \\
\hline yb169 & $3.475 \mathrm{E}-12$ & $1.767 \mathrm{E}-28$ & $2.360 \mathrm{E}-29$ & $8.738 \mathrm{E}-33$ & $0.000 \mathrm{E}+00$ \\
\hline yb170 & $6.981 \mathrm{E}-04$ & $7.536 \mathrm{E}-04$ & $7.536 \mathrm{E}-04$ & $7.536 \mathrm{E}-04$ & $7.536 \mathrm{E}-04$ \\
\hline yb171 & $2.152 \mathrm{E}-04$ & $3.583 \mathrm{E}-04$ & $3.610 \mathrm{E}-04$ & $3.697 \mathrm{E}-04$ & $3.758 \mathrm{E}-04$ \\
\hline yb172 & 4.374E-04 & 4.374E-04 & 4.374E-04 & 4.374E-04 & 4.374E-04 \\
\hline yb176 & $1.137 \mathrm{E}-16$ & $1.137 \mathrm{E}-16$ & $1.137 \mathrm{E}-16$ & $1.137 \mathrm{E}-16$ & $1.137 \mathrm{E}-16$ \\
\hline zn64 & $4.143 \mathrm{E}-16$ & $4.143 \mathrm{E}-16$ & $4.143 \mathrm{E}-16$ & 4.143E-16 & 4.143E-16 \\
\hline zn66 & $1.487 \mathrm{E}-04$ & $1.487 \mathrm{E}-04$ & $1.487 \mathrm{E}-04$ & $1.487 \mathrm{E}-04$ & $1.487 \mathrm{E}-04$ \\
\hline zn67 & $4.152 \mathrm{E}-04$ & $4.152 \mathrm{E}-04$ & $4.152 \mathrm{E}-04$ & $4.152 \mathrm{E}-04$ & 4.152E-04 \\
\hline zn68 & $8.315 \mathrm{E}-04$ & $8.315 \mathrm{E}-04$ & $8.315 \mathrm{E}-04$ & $8.315 \mathrm{E}-04$ & $8.315 \mathrm{E}-04$ \\
\hline zn70 & 4.107E-03 & 4.107E-03 & $4.107 \mathrm{E}-03$ & 4.107E-03 & 4.107E-03 \\
\hline zr88 & $7.153 \mathrm{E}-12$ & $3.975 \mathrm{E}-18$ & $1.835 \mathrm{E}-18$ & $8.837 \mathrm{E}-20$ & 4.253E-21 \\
\hline zr90 & $2.125 \mathrm{E}+01$ & $6.251 \mathrm{E}+01$ & $6.460 \mathrm{E}+01$ & $7.265 \mathrm{E}+01$ & $8.051 \mathrm{E}+01$ \\
\hline zr91 & $4.773 \mathrm{E}+02$ & $4.789 \mathrm{E}+02$ & $4.789 \mathrm{E}+02$ & $4.789 \mathrm{E}+02$ & $4.789 \mathrm{E}+02$ \\
\hline zr92 & $5.800 \mathrm{E}+02$ & $5.800 \mathrm{E}+02$ & $5.800 \mathrm{E}+02$ & $5.800 \mathrm{E}+02$ & $5.800 \mathrm{E}+02$ \\
\hline zr93 & $7.179 \mathrm{E}+02$ & $7.179 \mathrm{E}+02$ & $7.179 \mathrm{E}+02$ & $7.179 \mathrm{E}+02$ & $7.179 \mathrm{E}+02$ \\
\hline zr94 & $8.243 \mathrm{E}+02$ & $8.243 \mathrm{E}+02$ & $8.243 \mathrm{E}+02$ & $8.243 \mathrm{E}+02$ & $8.243 \mathrm{E}+02$ \\
\hline zr95 & $4.362 \mathrm{E}+00$ & $3.107 \mathrm{E}-08$ & $1.135 \mathrm{E}-08$ & $2.183 \mathrm{E}-10$ & 4.199E-12 \\
\hline zr96 & $9.616 \mathrm{E}+02$ & $9.616 \mathrm{E}+02$ & $9.616 \mathrm{E}+02$ & $9.616 \mathrm{E}+02$ & $9.616 \mathrm{E}+02$ \\
\hline Total & $1.1347 \mathrm{E}+06$ & $1.1347 E+06$ & $1.1347 E+06$ & $1.1347 \mathrm{E}+06$ & $1.1347 E+06$ \\
\hline
\end{tabular}


Table C-2. MOX Fuel Rod Al (low Pu): Nuclide mass in grams per MTIHM

\begin{tabular}{|c|c|c|c|c|c|}
\hline & $1 / 22 / 2009$ & $10 / 21 / 2013$ & $1 / 22 / 2014$ & $1 / 22 / 2015$ & $1 / 22 / 2016$ \\
\hline ac225 & $1.483 \mathrm{E}-12$ & $1.526 \mathrm{E}-12$ & $1.528 \mathrm{E}-12$ & $1.542 \mathrm{E}-12$ & $1.556 \mathrm{E}-12$ \\
\hline ac227 & $6.761 \mathrm{E}-10$ & 2.843E-09 & $2.960 \mathrm{E}-09$ & 3.417E-09 & $3.874 \mathrm{E}-09$ \\
\hline $\operatorname{ag} 105$ & $7.308 \mathrm{E}-15$ & $1.694 \mathrm{E}-27$ & $3.557 \mathrm{E}-28$ & 7.763E-31 & $1.694 \mathrm{E}-33$ \\
\hline $\operatorname{ag} 107$ & $1.286 \mathrm{E}-04$ & 4.074E-04 & 4.223E-04 & $4.811 \mathrm{E}-04$ & $5.398 \mathrm{E}-04$ \\
\hline $\operatorname{ag} 108$ & $5.837 \mathrm{E}-13$ & $5.794 \mathrm{E}-13$ & $5.791 \mathrm{E}-13$ & $5.782 \mathrm{E}-13$ & $5.773 \mathrm{E}-13$ \\
\hline ag108m & $6.488 \mathrm{E}-04$ & $6.440 \mathrm{E}-04$ & $6.438 \mathrm{E}-04$ & $6.427 \mathrm{E}-04$ & $6.417 \mathrm{E}-04$ \\
\hline ag109 & $1.832 \mathrm{E}+02$ & $1.832 \mathrm{E}+02$ & $1.832 \mathrm{E}+02$ & $1.832 \mathrm{E}+02$ & $1.832 \mathrm{E}+02$ \\
\hline $\operatorname{ag} 109 m$ & $2.009 \mathrm{E}-10$ & $1.487 \mathrm{E}-11$ & $1.293 \mathrm{E}-11$ & $7.473 \mathrm{E}-12$ & $4.318 \mathrm{E}-12$ \\
\hline $\operatorname{ag} 110$ & $1.990 \mathrm{E}-08$ & $1.622 \mathrm{E}-10$ & $1.253 \mathrm{E}-10$ & $4.551 \mathrm{E}-11$ & $1.652 \mathrm{E}-11$ \\
\hline ag110m & $1.284 \mathrm{E}+00$ & $1.046 \mathrm{E}-02$ & $8.080 \mathrm{E}-03$ & $2.935 \mathrm{E}-03$ & $1.066 \mathrm{E}-03$ \\
\hline $\operatorname{ag} 111$ & $1.301 \mathrm{E}-11$ & $0.000 \mathrm{E}+00$ & $0.000 \mathrm{E}+00$ & $0.000 \mathrm{E}+00$ & $0.000 \mathrm{E}+00$ \\
\hline al 27 & $4.869 \mathrm{E}-14$ & $4.869 \mathrm{E}-14$ & $4.869 \mathrm{E}-14$ & $4.869 \mathrm{E}-14$ & $4.869 \mathrm{E}-14$ \\
\hline am241 & $2.861 \mathrm{E}+02$ & $1.004 \mathrm{E}+03$ & $1.038 \mathrm{E}+03$ & $1.166 \mathrm{E}+03$ & $1.289 \mathrm{E}+03$ \\
\hline am242 & $3.632 \mathrm{E}-05$ & $3.548 \mathrm{E}-05$ & $3.544 \mathrm{E}-05$ & $3.526 \mathrm{E}-05$ & $3.509 \mathrm{E}-05$ \\
\hline am242m & $2.815 \mathrm{E}+00$ & $2.750 \mathrm{E}+00$ & $2.747 \mathrm{E}+00$ & $2.733 \mathrm{E}+00$ & $2.719 \mathrm{E}+00$ \\
\hline $\operatorname{am} 243$ & $4.170 \mathrm{E}+02$ & $4.168 \mathrm{E}+02$ & $4.168 \mathrm{E}+02$ & $4.168 \mathrm{E}+02$ & $4.167 \mathrm{E}+02$ \\
\hline am244 & $9.188 \mathrm{E}-15$ & $6.374 \mathrm{E}-15$ & $6.250 \mathrm{E}-15$ & $5.787 \mathrm{E}-15$ & $5.358 \mathrm{E}-15$ \\
\hline am245 & $9.300 \mathrm{E}-14$ & $2.179 \mathrm{E}-15$ & $1.782 \mathrm{E}-15$ & $8.080 \mathrm{E}-16$ & $3.665 \mathrm{E}-16$ \\
\hline ar 36 & $6.419 \mathrm{E}-14$ & $6.419 \mathrm{E}-14$ & $6.419 \mathrm{E}-14$ & $6.419 \mathrm{E}-14$ & $6.419 \mathrm{E}-14$ \\
\hline ar 38 & $6.853 \mathrm{E}-14$ & $6.853 \mathrm{E}-14$ & $6.853 \mathrm{E}-14$ & $6.853 \mathrm{E}-14$ & $6.853 \mathrm{E}-14$ \\
\hline ar 39 & $1.282 \mathrm{E}-16$ & $1.266 \mathrm{E}-16$ & $1.265 \mathrm{E}-16$ & $1.262 \mathrm{E}-16$ & $1.259 \mathrm{E}-16$ \\
\hline ar 40 & $7.262 \mathrm{E}-14$ & $7.262 \mathrm{E}-14$ & $7.262 \mathrm{E}-14$ & $7.262 \mathrm{E}-14$ & $7.262 \mathrm{E}-14$ \\
\hline as 73 & $1.134 \mathrm{E}-10$ & $3.613 \mathrm{E}-17$ & $1.619 \mathrm{E}-17$ & $6.932 \mathrm{E}-19$ & $2.969 \mathrm{E}-20$ \\
\hline as 74 & $1.481 \mathrm{E}-12$ & $0.000 \mathrm{E}+00$ & $0.000 \mathrm{E}+00$ & $0.000 \mathrm{E}+00$ & $0.000 \mathrm{E}+00$ \\
\hline as 75 & $1.544 \mathrm{E}-01$ & $1.544 \mathrm{E}-01$ & $1.544 \mathrm{E}-01$ & $1.544 \mathrm{E}-01$ & $1.544 \mathrm{E}-01$ \\
\hline au197 & $1.817 \mathrm{E}-13$ & $1.817 \mathrm{E}-13$ & $1.817 \mathrm{E}-13$ & $1.817 \mathrm{E}-13$ & $1.817 \mathrm{E}-13$ \\
\hline b 10 & $6.939 \mathrm{E}-11$ & $3.785 \mathrm{E}-10$ & $3.952 \mathrm{E}-10$ & 4.603E-10 & $5.254 \mathrm{E}-10$ \\
\hline b 11 & $8.106 \mathrm{E}-08$ & $8.106 \mathrm{E}-08$ & $8.106 \mathrm{E}-08$ & $8.106 \mathrm{E}-08$ & $8.106 \mathrm{E}-08$ \\
\hline ba130 & $1.828 \mathrm{E}-13$ & $1.828 \mathrm{E}-13$ & $1.828 \mathrm{E}-13$ & $1.828 \mathrm{E}-13$ & $1.828 \mathrm{E}-13$ \\
\hline ba131 & 3.873E-18 & $0.000 \mathrm{E}+00$ & $0.000 \mathrm{E}+00$ & $0.000 \mathrm{E}+00$ & $0.000 \mathrm{E}+00$ \\
\hline ba132 & $2.871 \mathrm{E}-04$ & $2.871 \mathrm{E}-04$ & $2.871 \mathrm{E}-04$ & $2.871 \mathrm{E}-04$ & $2.871 \mathrm{E}-04$ \\
\hline ba133 & $2.169 \mathrm{E}-04$ & $1.586 \mathrm{E}-04$ & $1.560 \mathrm{E}-04$ & $1.460 \mathrm{E}-04$ & $1.367 \mathrm{E}-04$ \\
\hline ba134 & $1.152 \mathrm{E}+02$ & $2.315 \mathrm{E}+02$ & $2.340 \mathrm{E}+02$ & $2.417 \mathrm{E}+02$ & $2.473 \mathrm{E}+02$ \\
\hline ba135 & $5.762 \mathrm{E}-01$ & $5.770 \mathrm{E}-01$ & $5.771 \mathrm{E}-01$ & 5.772E-01 & $5.774 \mathrm{E}-01$ \\
\hline ba136 & $3.883 \mathrm{E}+01$ & $3.883 \mathrm{E}+01$ & $3.883 \mathrm{E}+01$ & $3.883 \mathrm{E}+01$ & $3.883 \mathrm{E}+01$ \\
\hline ba136m & $2.460 \mathrm{E}-14$ & $0.000 \mathrm{E}+00$ & $0.000 \mathrm{E}+00$ & $0.000 \mathrm{E}+00$ & $0.000 \mathrm{E}+00$ \\
\hline ba137 & $7.777 \mathrm{E}+01$ & $2.266 \mathrm{E}+02$ & $2.342 \mathrm{E}+02$ & $2.633 \mathrm{E}+02$ & $2.918 \mathrm{E}+02$ \\
\hline
\end{tabular}




\begin{tabular}{|c|c|c|c|c|c|}
\hline \multicolumn{6}{|c|}{ Table C-2. Continued } \\
\hline & $1 / 22 / 2009$ & $10 / 21 / 2013$ & $1 / 22 / 2014$ & $1 / 22 / 2015$ & $1 / 22 / 2016$ \\
\hline ba137m & $2.195 \mathrm{E}-04$ & $1.968 \mathrm{E}-04$ & $1.956 \mathrm{E}-04$ & $1.911 \mathrm{E}-04$ & $1.868 \mathrm{E}-04$ \\
\hline ba138 & $1.442 \mathrm{E}+03$ & $1.442 \mathrm{E}+03$ & $1.442 \mathrm{E}+03$ & $1.442 \mathrm{E}+03$ & $1.442 \mathrm{E}+03$ \\
\hline ba140 & $1.172 \mathrm{E}-05$ & $0.000 \mathrm{E}+00$ & $0.000 \mathrm{E}+00$ & $0.000 \mathrm{E}+00$ & $0.000 \mathrm{E}+00$ \\
\hline be 9 & $2.587 \mathrm{E}-06$ & $2.587 \mathrm{E}-06$ & $2.587 \mathrm{E}-06$ & $2.587 \mathrm{E}-06$ & $2.587 \mathrm{E}-06$ \\
\hline be 10 & $1.419 \mathrm{E}-04$ & $1.419 \mathrm{E}-04$ & $1.419 \mathrm{E}-04$ & $1.419 \mathrm{E}-04$ & $1.419 \mathrm{E}-04$ \\
\hline bi208 & $1.514 \mathrm{E}-15$ & $1.514 \mathrm{E}-15$ & $1.514 \mathrm{E}-15$ & $1.514 \mathrm{E}-15$ & $1.514 \mathrm{E}-15$ \\
\hline bi209 & $1.369 \mathrm{E}-10$ & $3.045 \mathrm{E}-10$ & $3.136 \mathrm{E}-10$ & $3.497 \mathrm{E}-10$ & $3.861 \mathrm{E}-10$ \\
\hline bi210 & $3.253 \mathrm{E}-14$ & $3.279 \mathrm{E}-14$ & $3.309 \mathrm{E}-14$ & $3.462 \mathrm{E}-14$ & $3.684 \mathrm{E}-14$ \\
\hline bi210m & $2.165 \mathrm{E}-15$ & $2.165 \mathrm{E}-15$ & $2.165 \mathrm{E}-15$ & $2.165 \mathrm{E}-15$ & $2.165 \mathrm{E}-15$ \\
\hline bi211 & $1.090 \mathrm{E}-16$ & $4.950 \mathrm{E}-16$ & $5.054 \mathrm{E}-16$ & $5.948 \mathrm{E}-16$ & $6.741 \mathrm{E}-16$ \\
\hline bi212 & $2.125 \mathrm{E}-10$ & $1.268 \mathrm{E}-09$ & $1.315 \mathrm{E}-09$ & $1.472 \mathrm{E}-09$ & $1.604 \mathrm{E}-09$ \\
\hline bi213 & 4.444E-15 & $4.574 \mathrm{E}-15$ & $4.580 \mathrm{E}-15$ & $4.621 \mathrm{E}-15$ & 4.663E-15 \\
\hline bk247 & $1.824 \mathrm{E}-12$ & $1.819 \mathrm{E}-12$ & $1.819 \mathrm{E}-12$ & $1.818 \mathrm{E}-12$ & $1.817 \mathrm{E}-12$ \\
\hline bk248 & $7.288 \mathrm{E}-11$ & $5.056 \mathrm{E}-11$ & $4.958 \mathrm{E}-11$ & $4.591 \mathrm{E}-11$ & $4.250 \mathrm{E}-11$ \\
\hline bk249 & $2.442 \mathrm{E}-05$ & $5.721 \mathrm{E}-07$ & 4.677E-07 & $2.121 \mathrm{E}-07$ & $9.623 \mathrm{E}-08$ \\
\hline bk250 & $1.669 \mathrm{E}-14$ & $2.153 \mathrm{E}-16$ & $1.707 \mathrm{E}-16$ & $6.899 \mathrm{E}-17$ & $2.836 \mathrm{E}-17$ \\
\hline br 79 & $2.716 \mathrm{E}-03$ & $2.772 \mathrm{E}-03$ & $2.775 \mathrm{E}-03$ & $2.786 \mathrm{E}-03$ & $2.798 \mathrm{E}-03$ \\
\hline br 81 & $2.095 \mathrm{E}+01$ & $2.095 \mathrm{E}+01$ & $2.095 \mathrm{E}+01$ & $2.095 \mathrm{E}+01$ & $2.095 \mathrm{E}+01$ \\
\hline c 12 & $8.265 \mathrm{E}-02$ & $8.265 \mathrm{E}-02$ & $8.265 \mathrm{E}-02$ & $8.265 \mathrm{E}-02$ & $8.265 \mathrm{E}-02$ \\
\hline c 13 & $1.301 \mathrm{E}+01$ & $1.301 \mathrm{E}+01$ & $1.301 \mathrm{E}+01$ & $1.301 \mathrm{E}+01$ & $1.301 \mathrm{E}+01$ \\
\hline c 14 & 4.199E-04 & 4.197E-04 & $4.196 \mathrm{E}-04$ & 4.196E-04 & $4.196 \mathrm{E}-04$ \\
\hline ca 40 & $7.196 \mathrm{E}-14$ & $7.196 \mathrm{E}-14$ & $7.196 \mathrm{E}-14$ & $7.196 \mathrm{E}-14$ & $7.196 \mathrm{E}-14$ \\
\hline ca 41 & $5.450 \mathrm{E}-17$ & $5.450 \mathrm{E}-17$ & $5.450 \mathrm{E}-17$ & $5.450 \mathrm{E}-17$ & $5.450 \mathrm{E}-17$ \\
\hline ca 42 & $7.598 \mathrm{E}-14$ & $7.598 \mathrm{E}-14$ & $7.598 \mathrm{E}-14$ & $7.598 \mathrm{E}-14$ & $7.598 \mathrm{E}-14$ \\
\hline ca 43 & 7.612E-14 & 7.612E-14 & 7.612E-14 & 7.612E-14 & 7.612E-14 \\
\hline ca 44 & $8.087 \mathrm{E}-14$ & $8.087 \mathrm{E}-14$ & $8.087 \mathrm{E}-14$ & $8.087 \mathrm{E}-14$ & $8.087 \mathrm{E}-14$ \\
\hline ca 46 & $8.291 \mathrm{E}-14$ & $8.291 \mathrm{E}-14$ & $8.291 \mathrm{E}-14$ & $8.291 \mathrm{E}-14$ & $8.291 \mathrm{E}-14$ \\
\hline ca 48 & 8.647E-14 & $8.647 \mathrm{E}-14$ & $8.647 \mathrm{E}-14$ & 8.647E-14 & 8.647E-14 \\
\hline cd106 & $2.959 \mathrm{E}-13$ & $2.959 \mathrm{E}-13$ & $2.959 \mathrm{E}-13$ & $2.959 \mathrm{E}-13$ & $2.959 \mathrm{E}-13$ \\
\hline cd108 & $1.394 \mathrm{E}-03$ & $1.395 \mathrm{E}-03$ & $1.395 \mathrm{E}-03$ & $1.395 \mathrm{E}-03$ & $1.395 \mathrm{E}-03$ \\
\hline cd109 & $2.022 \mathrm{E}-04$ & $1.497 \mathrm{E}-05$ & $1.301 \mathrm{E}-05$ & 7.519E-06 & $4.346 \mathrm{E}-06$ \\
\hline cd110 & $1.407 \mathrm{E}+02$ & $1.419 \mathrm{E}+02$ & $1.419 \mathrm{E}+02$ & $1.419 \mathrm{E}+02$ & $1.419 \mathrm{E}+02$ \\
\hline cd111 & $6.153 \mathrm{E}+01$ & $6.153 \mathrm{E}+01$ & $6.153 \mathrm{E}+01$ & $6.153 \mathrm{E}+01$ & $6.153 \mathrm{E}+01$ \\
\hline cd112 & $2.921 \mathrm{E}+01$ & $2.921 \mathrm{E}+01$ & $2.921 \mathrm{E}+01$ & $2.921 \mathrm{E}+01$ & $2.921 \mathrm{E}+01$ \\
\hline cd113 & $2.690 \mathrm{E}-01$ & $2.690 \mathrm{E}-01$ & $2.690 \mathrm{E}-01$ & $2.690 \mathrm{E}-01$ & $2.690 \mathrm{E}-01$ \\
\hline cd113m & $3.905 \mathrm{E}-03$ & $3.093 \mathrm{E}-03$ & $3.054 \mathrm{E}-03$ & $2.907 \mathrm{E}-03$ & $2.768 \mathrm{E}-03$ \\
\hline cd114 & $3.118 \mathrm{E}+01$ & $3.118 \mathrm{E}+01$ & $3.118 \mathrm{E}+01$ & $3.118 \mathrm{E}+01$ & $3.118 \mathrm{E}+01$ \\
\hline cd115m & $5.691 \mathrm{E}-04$ & $1.116 \mathrm{E}-15$ & $2.626 \mathrm{E}-16$ & 8.984E-19 & $3.074 \mathrm{E}-21$ \\
\hline
\end{tabular}




\begin{tabular}{|c|c|c|c|c|c|}
\hline \multicolumn{6}{|c|}{ Table C-2. Continued } \\
\hline & $1 / 22 / 2009$ & $10 / 21 / 2013$ & $1 / 22 / 2014$ & $1 / 22 / 2015$ & $1 / 22 / 2016$ \\
\hline cd116 & $1.025 \mathrm{E}+01$ & $1.025 \mathrm{E}+01$ & $1.025 \mathrm{E}+01$ & $1.025 \mathrm{E}+01$ & $1.025 \mathrm{E}+01$ \\
\hline ce136 & $2.237 \mathrm{E}-13$ & $2.237 \mathrm{E}-13$ & $2.237 \mathrm{E}-13$ & $2.237 \mathrm{E}-13$ & $2.237 \mathrm{E}-13$ \\
\hline ce138 & $1.012 \mathrm{E}-05$ & $1.012 \mathrm{E}-05$ & $1.012 \mathrm{E}-05$ & $1.012 \mathrm{E}-05$ & $1.012 \mathrm{E}-05$ \\
\hline ce139 & $1.325 \mathrm{E}-03$ & $2.148 \mathrm{E}-07$ & $1.345 \mathrm{E}-07$ & $2.140 \mathrm{E}-08$ & 3.405E-09 \\
\hline ce140 & $1.375 \mathrm{E}+03$ & $1.375 \mathrm{E}+03$ & $1.375 \mathrm{E}+03$ & $1.375 \mathrm{E}+03$ & $1.375 \mathrm{E}+03$ \\
\hline ce141 & $1.727 \mathrm{E}-01$ & $1.547 \mathrm{E}-17$ & $2.129 \mathrm{E}-18$ & $8.878 \mathrm{E}-22$ & $3.700 \mathrm{E}-25$ \\
\hline ce142 & $1.188 \mathrm{E}+03$ & $1.188 \mathrm{E}+03$ & $1.188 \mathrm{E}+03$ & $1.188 \mathrm{E}+03$ & $1.188 \mathrm{E}+03$ \\
\hline ce144 & $1.685 \mathrm{E}+02$ & $2.486 \mathrm{E}+00$ & $1.983 \mathrm{E}+00$ & $8.159 \mathrm{E}-01$ & 3.357E-01 \\
\hline cf248 & $4.936 \mathrm{E}-11$ & $1.346 \mathrm{E}-12$ & $1.109 \mathrm{E}-12$ & $5.196 \mathrm{E}-13$ & $2.433 \mathrm{E}-13$ \\
\hline cf249 & $2.663 \mathrm{E}-05$ & $5.007 \mathrm{E}-05$ & $5.015 \mathrm{E}-05$ & $5.030 \mathrm{E}-05$ & $5.032 \mathrm{E}-05$ \\
\hline cf250 & $1.485 \mathrm{E}-05$ & $1.155 \mathrm{E}-05$ & $1.140 \mathrm{E}-05$ & $1.081 \mathrm{E}-05$ & $1.025 \mathrm{E}-05$ \\
\hline cf251 & 7.691E-06 & 7.664E-06 & 7.664E-06 & $7.658 \mathrm{E}-06$ & 7.651E-06 \\
\hline cf252 & $3.139 \mathrm{E}-06$ & $9.050 \mathrm{E}-07$ & $8.469 \mathrm{E}-07$ & $6.516 \mathrm{E}-07$ & $5.015 \mathrm{E}-07$ \\
\hline cf253 & $1.944 \mathrm{E}-13$ & $0.000 \mathrm{E}+00$ & $0.000 \mathrm{E}+00$ & $0.000 \mathrm{E}+00$ & $0.000 \mathrm{E}+00$ \\
\hline cf254 & $1.177 \mathrm{E}-11$ & $2.805 \mathrm{E}-20$ & $9.663 \mathrm{E}-21$ & $1.476 \mathrm{E}-22$ & $2.254 \mathrm{E}-24$ \\
\hline cl 35 & $5.893 \mathrm{E}-14$ & $5.893 \mathrm{E}-14$ & 5.893E-14 & $5.893 \mathrm{E}-14$ & $5.893 \mathrm{E}-14$ \\
\hline cl 36 & $4.475 \mathrm{E}-15$ & $4.475 \mathrm{E}-15$ & $4.475 \mathrm{E}-15$ & $4.475 \mathrm{E}-15$ & $4.475 \mathrm{E}-15$ \\
\hline cl 37 & $6.748 \mathrm{E}-14$ & $6.748 \mathrm{E}-14$ & $6.748 \mathrm{E}-14$ & $6.748 \mathrm{E}-14$ & $6.748 \mathrm{E}-14$ \\
\hline cm240 & $1.465 \mathrm{E}-11$ & $6.985 \mathrm{E}-31$ & $6.414 \mathrm{E}-32$ & $0.000 \mathrm{E}+00$ & $0.000 \mathrm{E}+00$ \\
\hline $\mathrm{cm} 241$ & $2.039 \mathrm{E}-08$ & $2.536 \mathrm{E}-24$ & $3.553 \mathrm{E}-25$ & $1.588 \mathrm{E}-28$ & $7.091 \mathrm{E}-32$ \\
\hline cm242 & $1.716 \mathrm{E}+01$ & $1.795 \mathrm{E}-02$ & $1.442 \mathrm{E}-02$ & 8.667E-03 & $7.420 \mathrm{E}-03$ \\
\hline cm243 & $1.623 \mathrm{E}+00$ & $1.450 \mathrm{E}+00$ & $1.441 \mathrm{E}+00$ & $1.408 \mathrm{E}+00$ & $1.374 \mathrm{E}+00$ \\
\hline cm244 & $1.952 \mathrm{E}+02$ & $1.628 \mathrm{E}+02$ & $1.612 \mathrm{E}+02$ & $1.552 \mathrm{E}+02$ & $1.493 \mathrm{E}+02$ \\
\hline cm245 & $2.018 \mathrm{E}+01$ & $2.018 \mathrm{E}+01$ & $2.018 \mathrm{E}+01$ & $2.018 \mathrm{E}+01$ & $2.018 \mathrm{E}+01$ \\
\hline cm246 & $1.855 \mathrm{E}+00$ & $1.855 \mathrm{E}+00$ & $1.854 \mathrm{E}+00$ & $1.854 \mathrm{E}+00$ & $1.854 \mathrm{E}+00$ \\
\hline cm247 & $3.272 \mathrm{E}-02$ & $3.272 \mathrm{E}-02$ & $3.272 \mathrm{E}-02$ & $3.272 \mathrm{E}-02$ & $3.272 \mathrm{E}-02$ \\
\hline $\mathrm{cm} 248$ & $2.613 \mathrm{E}-03$ & $2.616 \mathrm{E}-03$ & $2.616 \mathrm{E}-03$ & $2.616 \mathrm{E}-03$ & $2.616 \mathrm{E}-03$ \\
\hline cm250 & $3.792 \mathrm{E}-10$ & $3.791 \mathrm{E}-10$ & $3.791 \mathrm{E}-10$ & $3.791 \mathrm{E}-10$ & $3.790 \mathrm{E}-10$ \\
\hline co 59 & $1.080 \mathrm{E}-13$ & $1.080 \mathrm{E}-13$ & $1.080 \mathrm{E}-13$ & $1.080 \mathrm{E}-13$ & $1.080 \mathrm{E}-13$ \\
\hline co 60 & $1.238 \mathrm{E}-14$ & $6.635 \mathrm{E}-15$ & $6.415 \mathrm{E}-15$ & $5.625 \mathrm{E}-15$ & $4.932 \mathrm{E}-15$ \\
\hline cr 50 & $8.779 \mathrm{E}-14$ & $8.779 \mathrm{E}-14$ & 8.779E-14 & $8.779 \mathrm{E}-14$ & $8.779 \mathrm{E}-14$ \\
\hline cr 52 & $9.366 \mathrm{E}-14$ & $9.366 \mathrm{E}-14$ & $9.366 \mathrm{E}-14$ & $9.366 \mathrm{E}-14$ & $9.366 \mathrm{E}-14$ \\
\hline cr 53 & $9.294 \mathrm{E}-14$ & $9.294 \mathrm{E}-14$ & $9.294 \mathrm{E}-14$ & $9.294 \mathrm{E}-14$ & $9.294 \mathrm{E}-14$ \\
\hline cr 54 & $1.003 \mathrm{E}-13$ & $1.003 \mathrm{E}-13$ & $1.003 \mathrm{E}-13$ & $1.003 \mathrm{E}-13$ & $1.003 \mathrm{E}-13$ \\
\hline $\operatorname{cs} 131$ & $1.530 \mathrm{E}-15$ & $0.000 \mathrm{E}+00$ & $0.000 \mathrm{E}+00$ & $0.000 \mathrm{E}+00$ & $0.000 \mathrm{E}+00$ \\
\hline $\operatorname{cs} 132$ & $1.334 \mathrm{E}-16$ & $0.000 \mathrm{E}+00$ & $0.000 \mathrm{E}+00$ & $0.000 \mathrm{E}+00$ & $0.000 \mathrm{E}+00$ \\
\hline $\operatorname{cs} 133$ & $1.286 \mathrm{E}+03$ & $1.286 \mathrm{E}+03$ & $1.286 \mathrm{E}+03$ & $1.286 \mathrm{E}+03$ & $1.286 \mathrm{E}+03$ \\
\hline $\operatorname{cs} 134$ & $1.460 \mathrm{E}+02$ & $2.971 \mathrm{E}+01$ & $2.727 \mathrm{E}+01$ & $1.950 \mathrm{E}+01$ & $1.394 \mathrm{E}+01$ \\
\hline
\end{tabular}




\begin{tabular}{|c|c|c|c|c|c|}
\hline \multicolumn{6}{|c|}{ Table C-2. Continued } \\
\hline & $1 / 22 / 2009$ & $10 / 21 / 2013$ & $1 / 22 / 2014$ & $1 / 22 / 2015$ & $1 / 22 / 2016$ \\
\hline $\operatorname{cs} 135$ & $5.684 \mathrm{E}+02$ & $5.684 \mathrm{E}+02$ & $5.684 \mathrm{E}+02$ & $5.684 \mathrm{E}+02$ & $5.684 \mathrm{E}+02$ \\
\hline $\operatorname{cs} 136$ & 8.192E-07 & $0.000 \mathrm{E}+00$ & $0.000 \mathrm{E}+00$ & $0.000 \mathrm{E}+00$ & $0.000 \mathrm{E}+00$ \\
\hline $\operatorname{cs} 137$ & $1.437 \mathrm{E}+03$ & $1.288 \mathrm{E}+03$ & $1.281 \mathrm{E}+03$ & $1.251 \mathrm{E}+03$ & $1.223 \mathrm{E}+03$ \\
\hline cu 63 & $1.123 \mathrm{E}-13$ & $1.124 \mathrm{E}-13$ & $1.124 \mathrm{E}-13$ & $1.124 \mathrm{E}-13$ & $1.124 \mathrm{E}-13$ \\
\hline cu 65 & $4.828 \mathrm{E}-07$ & $4.828 \mathrm{E}-07$ & $4.828 \mathrm{E}-07$ & $4.828 \mathrm{E}-07$ & $4.828 \mathrm{E}-07$ \\
\hline dy156 & $2.849 \mathrm{E}-12$ & $2.849 \mathrm{E}-12$ & $2.849 \mathrm{E}-12$ & $2.849 \mathrm{E}-12$ & $2.849 \mathrm{E}-12$ \\
\hline dy158 & $1.191 \mathrm{E}-07$ & $3.892 \mathrm{E}-07$ & 4.036E-07 & $4.598 \mathrm{E}-07$ & $5.158 \mathrm{E}-07$ \\
\hline dy159 & $2.325 \mathrm{E}-07$ & $5.671 \mathrm{E}-11$ & $3.628 \mathrm{E}-11$ & $6.293 \mathrm{E}-12$ & $1.091 \mathrm{E}-12$ \\
\hline dy160 & 8.911E-01 & $9.076 \mathrm{E}-01$ & $9.076 \mathrm{E}-01$ & $9.076 \mathrm{E}-01$ & $9.076 \mathrm{E}-01$ \\
\hline dy161 & $1.070 \mathrm{E}+00$ & $1.070 \mathrm{E}+00$ & $1.070 \mathrm{E}+00$ & $1.070 \mathrm{E}+00$ & $1.070 \mathrm{E}+00$ \\
\hline dy162 & $6.787 \mathrm{E}-01$ & $6.787 \mathrm{E}-01$ & $6.787 \mathrm{E}-01$ & $6.787 \mathrm{E}-01$ & $6.787 \mathrm{E}-01$ \\
\hline dy163 & $5.874 \mathrm{E}-01$ & $5.874 \mathrm{E}-01$ & $5.874 \mathrm{E}-01$ & $5.874 \mathrm{E}-01$ & $5.874 \mathrm{E}-01$ \\
\hline dy164 & $2.183 \mathrm{E}-01$ & $2.183 \mathrm{E}-01$ & $2.183 \mathrm{E}-01$ & $2.183 \mathrm{E}-01$ & $2.183 \mathrm{E}-01$ \\
\hline er162 & $3.026 \mathrm{E}-12$ & $3.026 \mathrm{E}-12$ & $3.026 \mathrm{E}-12$ & $3.026 \mathrm{E}-12$ & $3.026 \mathrm{E}-12$ \\
\hline er164 & $3.165 \mathrm{E}-06$ & $3.165 \mathrm{E}-06$ & $3.165 \mathrm{E}-06$ & $3.165 \mathrm{E}-06$ & $3.165 \mathrm{E}-06$ \\
\hline er166 & $8.984 \mathrm{E}-02$ & 8.984E-02 & 8.984E-02 & $8.984 \mathrm{E}-02$ & 8.984E-02 \\
\hline er167 & 4.007E-03 & $4.007 \mathrm{E}-03$ & 4.007E-03 & 4.007E-03 & 4.007E-03 \\
\hline er168 & $1.314 \mathrm{E}-02$ & $1.314 \mathrm{E}-02$ & $1.314 \mathrm{E}-02$ & $1.314 \mathrm{E}-02$ & $1.314 \mathrm{E}-02$ \\
\hline er169 & $1.051 \mathrm{E}-13$ & $0.000 \mathrm{E}+00$ & $0.000 \mathrm{E}+00$ & $0.000 \mathrm{E}+00$ & $0.000 \mathrm{E}+00$ \\
\hline er170 & $6.586 \mathrm{E}-04$ & $6.587 \mathrm{E}-04$ & $6.587 \mathrm{E}-04$ & $6.587 \mathrm{E}-04$ & $6.587 \mathrm{E}-04$ \\
\hline es252 & $8.608 \mathrm{E}-15$ & $6.741 \mathrm{E}-16$ & $5.880 \mathrm{E}-16$ & $3.439 \mathrm{E}-16$ & $2.012 \mathrm{E}-16$ \\
\hline es 253 & $4.688 \mathrm{E}-12$ & $0.000 \mathrm{E}+00$ & $0.000 \mathrm{E}+00$ & $0.000 \mathrm{E}+00$ & $0.000 \mathrm{E}+00$ \\
\hline es 254 & $3.492 \mathrm{E}-11$ & $4.475 \mathrm{E}-13$ & $3.543 \mathrm{E}-13$ & $1.415 \mathrm{E}-13$ & $5.652 \mathrm{E}-14$ \\
\hline es 255 & $6.154 \mathrm{E}-14$ & $4.801 \mathrm{E}-27$ & $9.505 \mathrm{E}-28$ & $1.649 \mathrm{E}-30$ & $2.861 \mathrm{E}-33$ \\
\hline eu147 & $2.416 \mathrm{E}-18$ & $0.000 \mathrm{E}+00$ & $0.000 \mathrm{E}+00$ & $0.000 \mathrm{E}+00$ & $0.000 \mathrm{E}+00$ \\
\hline eu149 & $4.356 \mathrm{E}-11$ & $1.085 \mathrm{E}-16$ & $5.430 \mathrm{E}-17$ & $3.586 \mathrm{E}-18$ & $2.368 \mathrm{E}-19$ \\
\hline eu151 & $1.371 \mathrm{E}-01$ & $8.416 \mathrm{E}-01$ & $8.786 \mathrm{E}-01$ & $1.024 \mathrm{E}+00$ & $1.167 \mathrm{E}+00$ \\
\hline eu152 & $2.289 \mathrm{E}-02$ & $1.795 \mathrm{E}-02$ & $1.772 \mathrm{E}-02$ & $1.684 \mathrm{E}-02$ & $1.600 \mathrm{E}-02$ \\
\hline eu153 & $1.811 \mathrm{E}+02$ & $1.811 \mathrm{E}+02$ & $1.811 \mathrm{E}+02$ & $1.811 \mathrm{E}+02$ & $1.811 \mathrm{E}+02$ \\
\hline eu154 & $5.121 \mathrm{E}+01$ & $3.494 \mathrm{E}+01$ & $3.423 \mathrm{E}+01$ & $3.158 \mathrm{E}+01$ & $2.913 \mathrm{E}+01$ \\
\hline eu155 & $1.300 \mathrm{E}+01$ & $6.508 \mathrm{E}+00$ & $6.271 \mathrm{E}+00$ & $5.421 \mathrm{E}+00$ & $4.686 \mathrm{E}+00$ \\
\hline eu156 & $3.765 \mathrm{E}-05$ & $0.000 \mathrm{E}+00$ & $0.000 \mathrm{E}+00$ & $0.000 \mathrm{E}+00$ & $0.000 \mathrm{E}+00$ \\
\hline f 19 & $1.556 \mathrm{E}-13$ & $1.556 \mathrm{E}-13$ & $1.556 \mathrm{E}-13$ & $1.556 \mathrm{E}-13$ & $1.556 \mathrm{E}-13$ \\
\hline fe 54 & $9.689 \mathrm{E}-14$ & $9.689 \mathrm{E}-14$ & $9.689 \mathrm{E}-14$ & $9.689 \mathrm{E}-14$ & $9.689 \mathrm{E}-14$ \\
\hline fe 55 & $2.431 \mathrm{E}-16$ & 7.335E-17 & $6.873 \mathrm{E}-17$ & $5.341 \mathrm{E}-17$ & 4.149E-17 \\
\hline fe 56 & $1.072 \mathrm{E}-13$ & $1.072 \mathrm{E}-13$ & $1.072 \mathrm{E}-13$ & $1.072 \mathrm{E}-13$ & $1.072 \mathrm{E}-13$ \\
\hline fe 57 & $1.028 \mathrm{E}-13$ & $1.028 \mathrm{E}-13$ & $1.028 \mathrm{E}-13$ & $1.028 \mathrm{E}-13$ & $1.028 \mathrm{E}-13$ \\
\hline fe 58 & $2.977 \mathrm{E}-13$ & $2.977 \mathrm{E}-13$ & $2.977 \mathrm{E}-13$ & $2.977 \mathrm{E}-13$ & $2.977 \mathrm{E}-13$ \\
\hline
\end{tabular}




\begin{tabular}{|c|c|c|c|c|c|}
\hline \multicolumn{6}{|c|}{ Table C-2. Continued } \\
\hline & $1 / 22 / 2009$ & $10 / 21 / 2013$ & $1 / 22 / 2014$ & $1 / 22 / 2015$ & $1 / 22 / 2016$ \\
\hline fr221 & $4.956 \mathrm{E}-16$ & 5.101E-16 & $5.108 \mathrm{E}-16$ & $5.154 \mathrm{E}-16$ & $5.200 \mathrm{E}-16$ \\
\hline ga 69 & $1.601 \mathrm{E}-03$ & $1.601 \mathrm{E}-03$ & $1.601 \mathrm{E}-03$ & $1.601 \mathrm{E}-03$ & $1.601 \mathrm{E}-03$ \\
\hline ga 71 & $6.787 \mathrm{E}-03$ & $6.787 \mathrm{E}-03$ & $6.787 \mathrm{E}-03$ & $6.787 \mathrm{E}-03$ & $6.787 \mathrm{E}-03$ \\
\hline $\operatorname{gd151}$ & 4.919E-08 & $3.053 \mathrm{E}-12$ & $1.815 \mathrm{E}-12$ & $2.360 \mathrm{E}-13$ & 3.067E-14 \\
\hline gd152 & $4.936 \mathrm{E}-02$ & $5.074 \mathrm{E}-02$ & $5.080 \mathrm{E}-02$ & $5.105 \mathrm{E}-02$ & $5.128 \mathrm{E}-02$ \\
\hline $\operatorname{gd153}$ & $1.929 \mathrm{E}-03$ & $1.304 \mathrm{E}-05$ & 9.973E-06 & $3.481 \mathrm{E}-06$ & $1.215 \mathrm{E}-06$ \\
\hline $\operatorname{gd154}$ & $8.186 \mathrm{E}+00$ & $2.446 \mathrm{E}+01$ & $2.517 \mathrm{E}+01$ & $2.781 \mathrm{E}+01$ & $3.026 \mathrm{E}+01$ \\
\hline $\operatorname{gd155}$ & $1.627 \mathrm{E}+00$ & $8.120 \mathrm{E}+00$ & $8.357 \mathrm{E}+00$ & $9.208 \mathrm{E}+00$ & $9.940 \mathrm{E}+00$ \\
\hline $\operatorname{gd156}$ & $1.673 \mathrm{E}+02$ & $1.673 \mathrm{E}+02$ & $1.673 \mathrm{E}+02$ & $1.673 \mathrm{E}+02$ & $1.673 \mathrm{E}+02$ \\
\hline gd157 & $2.938 \mathrm{E}-01$ & $2.938 \mathrm{E}-01$ & $2.938 \mathrm{E}-01$ & $2.938 \mathrm{E}-01$ & $2.938 \mathrm{E}-01$ \\
\hline $\operatorname{gd158}$ & $4.432 \mathrm{E}+01$ & $4.432 \mathrm{E}+01$ & $4.432 \mathrm{E}+01$ & $4.432 \mathrm{E}+01$ & $4.432 \mathrm{E}+01$ \\
\hline gd160 & $2.861 \mathrm{E}+00$ & $2.861 \mathrm{E}+00$ & $2.861 \mathrm{E}+00$ & $2.861 \mathrm{E}+00$ & $2.861 \mathrm{E}+00$ \\
\hline ge 70 & $2.146 \mathrm{E}-05$ & $2.146 \mathrm{E}-05$ & $2.146 \mathrm{E}-05$ & $2.146 \mathrm{E}-05$ & $2.146 \mathrm{E}-05$ \\
\hline ge 71 & $4.694 \mathrm{E}-16$ & $0.000 \mathrm{E}+00$ & $0.000 \mathrm{E}+00$ & $0.000 \mathrm{E}+00$ & $0.000 \mathrm{E}+00$ \\
\hline ge 72 & $1.623 \mathrm{E}-02$ & $1.623 \mathrm{E}-02$ & $1.623 \mathrm{E}-02$ & $1.623 \mathrm{E}-02$ & $1.623 \mathrm{E}-02$ \\
\hline ge 73 & $3.332 \mathrm{E}-02$ & 3.332E-02 & $3.332 \mathrm{E}-02$ & $3.332 \mathrm{E}-02$ & 3.332E-02 \\
\hline ge 73m & $8.159 \mathrm{E}-18$ & $2.599 \mathrm{E}-24$ & $1.164 \mathrm{E}-24$ & $4.986 \mathrm{E}-26$ & $2.135 \mathrm{E}-27$ \\
\hline ge 74 & $7.843 \mathrm{E}-02$ & $7.843 \mathrm{E}-02$ & $7.843 \mathrm{E}-02$ & $7.843 \mathrm{E}-02$ & $7.843 \mathrm{E}-02$ \\
\hline ge 76 & $3.498 \mathrm{E}-01$ & $3.498 \mathrm{E}-01$ & $3.498 \mathrm{E}-01$ & $3.498 \mathrm{E}-01$ & 3.498E-01 \\
\hline h 1 & $6.919 \mathrm{E}-03$ & $6.919 \mathrm{E}-03$ & $6.919 \mathrm{E}-03$ & $6.919 \mathrm{E}-03$ & $6.919 \mathrm{E}-03$ \\
\hline h 2 & $1.481 \mathrm{E}-03$ & $1.481 \mathrm{E}-03$ & $1.481 \mathrm{E}-03$ & $1.481 \mathrm{E}-03$ & $1.481 \mathrm{E}-03$ \\
\hline h3 & $6.727 \mathrm{E}-02$ & 5.152E-02 & $5.079 \mathrm{E}-02$ & 4.802E-02 & $4.539 \mathrm{E}-02$ \\
\hline he3 & $3.977 \mathrm{E}-03$ & $1.974 \mathrm{E}-02$ & $2.047 \mathrm{E}-02$ & $2.325 \mathrm{E}-02$ & $2.587 \mathrm{E}-02$ \\
\hline he4 & $7.829 \mathrm{E}+00$ & $8.934 \mathrm{E}+00$ & $8.977 \mathrm{E}+00$ & $9.149 \mathrm{E}+00$ & $9.320 \mathrm{E}+00$ \\
\hline hf174 & $1.134 \mathrm{E}-13$ & $1.134 \mathrm{E}-13$ & $1.134 \mathrm{E}-13$ & $1.134 \mathrm{E}-13$ & $1.134 \mathrm{E}-13$ \\
\hline hf175 & $8.410 \mathrm{E}-16$ & $2.965 \mathrm{E}-23$ & $1.181 \mathrm{E}-23$ & $3.179 \mathrm{E}-25$ & $8.561 \mathrm{E}-27$ \\
\hline hf176 & $3.301 \mathrm{E}-13$ & $3.301 \mathrm{E}-13$ & $3.301 \mathrm{E}-13$ & $3.301 \mathrm{E}-13$ & $3.301 \mathrm{E}-13$ \\
\hline hf177 & $4.318 \mathrm{E}-14$ & $4.319 \mathrm{E}-14$ & $4.319 \mathrm{E}-14$ & $4.319 \mathrm{E}-14$ & $4.319 \mathrm{E}-14$ \\
\hline hf178 & $2.739 \mathrm{E}-13$ & $2.739 \mathrm{E}-13$ & $2.739 \mathrm{E}-13$ & $2.739 \mathrm{E}-13$ & $2.739 \mathrm{E}-13$ \\
\hline hf179 & $7.836 \mathrm{E}-13$ & $7.836 \mathrm{E}-13$ & $7.836 \mathrm{E}-13$ & $7.836 \mathrm{E}-13$ & $7.836 \mathrm{E}-13$ \\
\hline hf180 & $6.532 \mathrm{E}-13$ & $6.532 \mathrm{E}-13$ & $6.532 \mathrm{E}-13$ & $6.532 \mathrm{E}-13$ & $6.532 \mathrm{E}-13$ \\
\hline hf181 & $2.733 \mathrm{E}-17$ & $1.348 \mathrm{E}-29$ & $2.946 \mathrm{E}-30$ & $7.539 \mathrm{E}-33$ & $0.000 \mathrm{E}+00$ \\
\hline hf182 & $1.493 \mathrm{E}-15$ & $1.493 \mathrm{E}-15$ & $1.493 \mathrm{E}-15$ & $1.493 \mathrm{E}-15$ & $1.493 \mathrm{E}-15$ \\
\hline hg196 & $7.856 \mathrm{E}-15$ & $7.856 \mathrm{E}-15$ & $7.856 \mathrm{E}-15$ & $7.856 \mathrm{E}-15$ & $7.856 \mathrm{E}-15$ \\
\hline hg198 & $6.649 \mathrm{E}-13$ & $6.649 \mathrm{E}-13$ & $6.649 \mathrm{E}-13$ & $6.649 \mathrm{E}-13$ & $6.649 \mathrm{E}-13$ \\
\hline hg199 & $8.120 \mathrm{E}-14$ & $8.120 \mathrm{E}-14$ & $8.120 \mathrm{E}-14$ & $8.120 \mathrm{E}-14$ & $8.120 \mathrm{E}-14$ \\
\hline hg200 & $8.548 \mathrm{E}-13$ & $8.548 \mathrm{E}-13$ & $8.548 \mathrm{E}-13$ & $8.548 \mathrm{E}-13$ & $8.548 \mathrm{E}-13$ \\
\hline hg201 & $3.485 \mathrm{E}-13$ & $3.485 \mathrm{E}-13$ & $3.485 \mathrm{E}-13$ & $3.485 \mathrm{E}-13$ & $3.485 \mathrm{E}-13$ \\
\hline
\end{tabular}




\begin{tabular}{|c|c|c|c|c|c|}
\hline & $1 / 22 / 2009$ & $10 / 21 / 2013$ & $1 / 22 / 2014$ & $1 / 22 / 2015$ & $1 / 22 / 2016$ \\
\hline hg202 & $3.788 \mathrm{E}-13$ & $3.788 \mathrm{E}-13$ & $3.788 \mathrm{E}-13$ & $3.788 \mathrm{E}-13$ & $3.788 \mathrm{E}-13$ \\
\hline hg204 & $3.664 \mathrm{E}-13$ & 3.664E-13 & $3.664 \mathrm{E}-13$ & $3.664 \mathrm{E}-13$ & $3.664 \mathrm{E}-13$ \\
\hline ho163 & $1.785 \mathrm{E}-07$ & $1.784 \mathrm{E}-07$ & 1.784E-07 & $1.784 \mathrm{E}-07$ & $1.783 \mathrm{E}-07$ \\
\hline ho165 & $2.712 \mathrm{E}-01$ & $2.712 \mathrm{E}-01$ & $2.712 \mathrm{E}-01$ & $2.712 \mathrm{E}-01$ & $2.712 \mathrm{E}-01$ \\
\hline ho166m & $6.179 \mathrm{E}-04$ & $6.163 \mathrm{E}-04$ & $6.161 \mathrm{E}-04$ & $6.158 \mathrm{E}-04$ & $6.154 \mathrm{E}-04$ \\
\hline i125 & $8.509 \mathrm{E}-10$ & $1.403 \mathrm{E}-18$ & $4.740 \mathrm{E}-19$ & $6.701 \mathrm{E}-21$ & $9.465 \mathrm{E}-23$ \\
\hline i126 & $1.452 \mathrm{E}-11$ & $0.000 \mathrm{E}+00$ & $0.000 \mathrm{E}+00$ & $0.000 \mathrm{E}+00$ & $0.000 \mathrm{E}+00$ \\
\hline i127 & $7.922 \mathrm{E}+01$ & $7.948 \mathrm{E}+01$ & $7.948 \mathrm{E}+01$ & $7.948 \mathrm{E}+01$ & $7.948 \mathrm{E}+01$ \\
\hline i129 & $2.564 \mathrm{E}+02$ & $2.564 \mathrm{E}+02$ & $2.564 \mathrm{E}+02$ & $2.564 \mathrm{E}+02$ & $2.564 \mathrm{E}+02$ \\
\hline i131 & $9.947 \mathrm{E}-10$ & $0.000 \mathrm{E}+00$ & $0.000 \mathrm{E}+00$ & $0.000 \mathrm{E}+00$ & $0.000 \mathrm{E}+00$ \\
\hline in113 & $5.810 \mathrm{E}-04$ & $1.392 \mathrm{E}-03$ & $1.431 \mathrm{E}-03$ & $1.577 \mathrm{E}-03$ & $1.716 \mathrm{E}-03$ \\
\hline in113m & $3.351 \mathrm{E}-14$ & $9.821 \mathrm{E}-19$ & $5.610 \mathrm{E}-19$ & $6.227 \mathrm{E}-20$ & $6.913 \mathrm{E}-21$ \\
\hline in114 & $3.279 \mathrm{E}-12$ & $9.525 \mathrm{E}-23$ & $2.590 \mathrm{E}-23$ & $1.563 \mathrm{E}-25$ & $9.432 \mathrm{E}-28$ \\
\hline in $114 m$ & $2.017 \mathrm{E}-07$ & $5.856 \mathrm{E}-18$ & $1.593 \mathrm{E}-18$ & $9.610 \mathrm{E}-21$ & $5.802 \mathrm{E}-23$ \\
\hline in115 & $2.754 \mathrm{E}+00$ & $2.754 \mathrm{E}+00$ & $2.754 \mathrm{E}+00$ & $2.754 \mathrm{E}+00$ & $2.754 \mathrm{E}+00$ \\
\hline in $115 m$ & $2.536 \mathrm{E}-10$ & 4.973E-22 & $1.171 \mathrm{E}-22$ & $4.004 \mathrm{E}-25$ & $1.370 \mathrm{E}-27$ \\
\hline ir191 & $4.460 \mathrm{E}-15$ & $4.460 \mathrm{E}-15$ & $4.460 \mathrm{E}-15$ & $4.460 \mathrm{E}-15$ & $4.460 \mathrm{E}-15$ \\
\hline ir192 & $9.709 \mathrm{E}-17$ & 8.337E-24 & $3.481 \mathrm{E}-24$ & $1.131 \mathrm{E}-25$ & $3.674 \mathrm{E}-27$ \\
\hline ir193 & $1.501 \mathrm{E}-13$ & $1.513 \mathrm{E}-13$ & $1.514 \mathrm{E}-13$ & $1.516 \mathrm{E}-13$ & $1.519 \mathrm{E}-13$ \\
\hline k 39 & $6.992 \mathrm{E}-14$ & $6.998 \mathrm{E}-14$ & $6.998 \mathrm{E}-14$ & $6.998 \mathrm{E}-14$ & $6.998 \mathrm{E}-14$ \\
\hline k 40 & $6.840 \mathrm{E}-14$ & $6.840 \mathrm{E}-14$ & $6.840 \mathrm{E}-14$ & $6.840 \mathrm{E}-14$ & $6.840 \mathrm{E}-14$ \\
\hline k 41 & $7.730 \mathrm{E}-14$ & $7.730 \mathrm{E}-14$ & $7.730 \mathrm{E}-14$ & $7.730 \mathrm{E}-14$ & $7.730 \mathrm{E}-14$ \\
\hline kr 78 & $1.787 \mathrm{E}-13$ & $1.787 \mathrm{E}-13$ & $1.787 \mathrm{E}-13$ & $1.787 \mathrm{E}-13$ & $1.787 \mathrm{E}-13$ \\
\hline kr 80 & $4.765 \mathrm{E}-04$ & $4.765 \mathrm{E}-04$ & $4.765 \mathrm{E}-04$ & $4.765 \mathrm{E}-04$ & $4.765 \mathrm{E}-04$ \\
\hline kr 81 & $4.076 \mathrm{E}-05$ & $4.076 \mathrm{E}-05$ & $4.076 \mathrm{E}-05$ & $4.076 \mathrm{E}-05$ & $4.076 \mathrm{E}-05$ \\
\hline kr 82 & $9.129 \mathrm{E}-01$ & $9.129 \mathrm{E}-01$ & $9.129 \mathrm{E}-01$ & $9.129 \mathrm{E}-01$ & $9.129 \mathrm{E}-01$ \\
\hline kr 83 & $3.403 \mathrm{E}+01$ & $3.403 \mathrm{E}+01$ & $3.403 \mathrm{E}+01$ & $3.403 \mathrm{E}+01$ & $3.403 \mathrm{E}+01$ \\
\hline kr 83m & $3.450 \mathrm{E}-11$ & $3.060 \mathrm{E}-17$ & $1.448 \mathrm{E}-17$ & $7.697 \mathrm{E}-19$ & $4.089 \mathrm{E}-20$ \\
\hline kr 84 & $8.324 \mathrm{E}+01$ & $8.324 \mathrm{E}+01$ & $8.324 \mathrm{E}+01$ & $8.324 \mathrm{E}+01$ & $8.324 \mathrm{E}+01$ \\
\hline kr 85 & $1.662 \mathrm{E}+01$ & $1.224 \mathrm{E}+01$ & $1.204 \mathrm{E}+01$ & $1.129 \mathrm{E}+01$ & $1.059 \mathrm{E}+01$ \\
\hline kr 86 & $1.224 \mathrm{E}+02$ & $1.224 \mathrm{E}+02$ & $1.224 \mathrm{E}+02$ & $1.224 \mathrm{E}+02$ & $1.224 \mathrm{E}+02$ \\
\hline la137 & $1.939 \mathrm{E}-14$ & $1.939 \mathrm{E}-14$ & $1.939 \mathrm{E}-14$ & $1.939 \mathrm{E}-14$ & $1.939 \mathrm{E}-14$ \\
\hline la137 & $4.201 \mathrm{E}-04$ & 4.201E-04 & 4.201E-04 & $4.201 \mathrm{E}-04$ & 4.201E-04 \\
\hline la138 & $2.514 \mathrm{E}-02$ & $2.514 \mathrm{E}-02$ & $2.514 \mathrm{E}-02$ & $2.514 \mathrm{E}-02$ & $2.514 \mathrm{E}-02$ \\
\hline la139 & $1.336 \mathrm{E}+03$ & $1.336 \mathrm{E}+03$ & $1.336 \mathrm{E}+03$ & $1.336 \mathrm{E}+03$ & $1.336 \mathrm{E}+03$ \\
\hline la140 & $1.780 \mathrm{E}-06$ & $0.000 \mathrm{E}+00$ & $0.000 \mathrm{E}+00$ & $0.000 \mathrm{E}+00$ & $0.000 \mathrm{E}+00$ \\
\hline li6 & $2.700 \mathrm{E}-10$ & $2.700 \mathrm{E}-10$ & $2.700 \mathrm{E}-10$ & $2.700 \mathrm{E}-10$ & $2.700 \mathrm{E}-10$ \\
\hline li7 & $3.333 \mathrm{E}-11$ & 3.333E-11 & 3.333E-11 & 3.333E-11 & 3.333E-11 \\
\hline
\end{tabular}




\begin{tabular}{|c|c|c|c|c|c|}
\hline & $1 / 22 / 2009$ & $10 / 21 / 2013$ & $1 / 22 / 2014$ & $1 / 22 / 2015$ & $1 / 22 / 2016$ \\
\hline lu175 & $2.932 \mathrm{E}-13$ & $2.940 \mathrm{E}-13$ & $2.940 \mathrm{E}-13$ & $2.940 \mathrm{E}-13$ & $2.940 \mathrm{E}-13$ \\
\hline lu176 & $6.077 \mathrm{E}-15$ & $6.077 \mathrm{E}-15$ & $6.077 \mathrm{E}-15$ & $6.077 \mathrm{E}-15$ & $6.077 \mathrm{E}-15$ \\
\hline mg 24 & 4.334E-14 & 4.334E-14 & 4.334E-14 & $4.334 \mathrm{E}-14$ & $4.334 \mathrm{E}-14$ \\
\hline mg 25 & $4.508 \mathrm{E}-14$ & $4.508 \mathrm{E}-14$ & $4.508 \mathrm{E}-14$ & $4.508 \mathrm{E}-14$ & $4.508 \mathrm{E}-14$ \\
\hline mg 26 & 4.692E-14 & 4.692E-14 & 4.692E-14 & 4.692E-14 & 4.692E-14 \\
\hline mn 54 & $2.264 \mathrm{E}-17$ & $4.820 \mathrm{E}-19$ & $3.921 \mathrm{E}-19$ & $1.743 \mathrm{E}-19$ & $7.750 \mathrm{E}-20$ \\
\hline mn 55 & $9.650 \mathrm{E}-14$ & $9.670 \mathrm{E}-14$ & $9.670 \mathrm{E}-14$ & $9.670 \mathrm{E}-14$ & $9.670 \mathrm{E}-14$ \\
\hline mo 92 & $6.205 \mathrm{E}-12$ & $6.205 \mathrm{E}-12$ & $6.205 \mathrm{E}-12$ & $6.205 \mathrm{E}-12$ & $6.205 \mathrm{E}-12$ \\
\hline mo 93 & $4.148 \mathrm{E}-07$ & 4.144E-07 & 4.144E-07 & 4.143E-07 & 4.142E-07 \\
\hline mo 94 & $1.386 \mathrm{E}-02$ & $1.386 \mathrm{E}-02$ & $1.386 \mathrm{E}-02$ & $1.386 \mathrm{E}-02$ & $1.386 \mathrm{E}-02$ \\
\hline mo 95 & $7.150 \mathrm{E}+02$ & $7.223 \mathrm{E}+02$ & $7.223 \mathrm{E}+02$ & $7.223 \mathrm{E}+02$ & $7.223 \mathrm{E}+02$ \\
\hline mo 96 & $4.619 \mathrm{E}+01$ & $4.619 \mathrm{E}+01$ & $4.619 \mathrm{E}+01$ & $4.619 \mathrm{E}+01$ & $4.619 \mathrm{E}+01$ \\
\hline mo 97 & $8.720 \mathrm{E}+02$ & $8.720 \mathrm{E}+02$ & $8.720 \mathrm{E}+02$ & $8.720 \mathrm{E}+02$ & $8.720 \mathrm{E}+02$ \\
\hline mo 98 & $9.551 \mathrm{E}+02$ & $9.551 \mathrm{E}+02$ & $9.551 \mathrm{E}+02$ & $9.551 \mathrm{E}+02$ & $9.551 \mathrm{E}+02$ \\
\hline mo100 & $1.124 \mathrm{E}+03$ & $1.124 \mathrm{E}+03$ & $1.124 \mathrm{E}+03$ & $1.124 \mathrm{E}+03$ & $1.124 \mathrm{E}+03$ \\
\hline n 14 & $5.025 \mathrm{E}-06$ & 5.267E-06 & $5.280 \mathrm{E}-06$ & 5.331E-06 & $5.382 \mathrm{E}-06$ \\
\hline n 15 & $1.220 \mathrm{E}-02$ & $1.220 \mathrm{E}-02$ & $1.220 \mathrm{E}-02$ & $1.220 \mathrm{E}-02$ & $1.220 \mathrm{E}-02$ \\
\hline na 23 & $4.146 \mathrm{E}-14$ & 4.146E-14 & $4.146 \mathrm{E}-14$ & 4.146E-14 & $4.146 \mathrm{E}-14$ \\
\hline nb 91 & $8.911 \mathrm{E}-09$ & 8.872E-09 & $8.865 \mathrm{E}-09$ & 8.858E-09 & 8.852E-09 \\
\hline nb 91m & $5.542 \mathrm{E}-15$ & $1.485 \mathrm{E}-23$ & $5.149 \mathrm{E}-24$ & $8.060 \mathrm{E}-26$ & $1.262 \mathrm{E}-27$ \\
\hline nb 92 & $7.711 \mathrm{E}-07$ & $7.711 \mathrm{E}-07$ & $7.711 \mathrm{E}-07$ & $7.711 \mathrm{E}-07$ & $7.711 \mathrm{E}-07$ \\
\hline nb 92m & $1.528 \mathrm{E}-18$ & $0.000 \mathrm{E}+00$ & $0.000 \mathrm{E}+00$ & $0.000 \mathrm{E}+00$ & $0.000 \mathrm{E}+00$ \\
\hline nb 93 & $9.689 \mathrm{E}-05$ & $3.580 \mathrm{E}-04$ & $3.778 \mathrm{E}-04$ & 4.606E-04 & $5.514 \mathrm{E}-04$ \\
\hline nb 93m & $5.859 \mathrm{E}-04$ & $1.622 \mathrm{E}-03$ & $1.672 \mathrm{E}-03$ & $1.863 \mathrm{E}-03$ & $2.045 \mathrm{E}-03$ \\
\hline nb 94 & $2.942 \mathrm{E}-03$ & $2.941 \mathrm{E}-03$ & $2.941 \mathrm{E}-03$ & $2.941 \mathrm{E}-03$ & $2.941 \mathrm{E}-03$ \\
\hline nb 95 & $3.795 \mathrm{E}+00$ & $2.856 \mathrm{E}-08$ & $1.042 \mathrm{E}-08$ & $2.067 \mathrm{E}-10$ & $3.979 \mathrm{E}-12$ \\
\hline nb 95m & $2.143 \mathrm{E}-03$ & $1.526 \mathrm{E}-11$ & $5.578 \mathrm{E}-12$ & $1.072 \mathrm{E}-13$ & $2.063 \mathrm{E}-15$ \\
\hline nd142 & $2.195 \mathrm{E}+01$ & $2.195 \mathrm{E}+01$ & $2.195 \mathrm{E}+01$ & $2.195 \mathrm{E}+01$ & $2.195 \mathrm{E}+01$ \\
\hline nd143 & $8.416 \mathrm{E}+02$ & $8.416 \mathrm{E}+02$ & $8.416 \mathrm{E}+02$ & $8.416 \mathrm{E}+02$ & $8.416 \mathrm{E}+02$ \\
\hline nd144 & $1.043 \mathrm{E}+03$ & $1.210 \mathrm{E}+03$ & $1.210 \mathrm{E}+03$ & $1.211 \mathrm{E}+03$ & $1.212 \mathrm{E}+03$ \\
\hline nd145 & $6.622 \mathrm{E}+02$ & $6.622 \mathrm{E}+02$ & $6.622 \mathrm{E}+02$ & $6.622 \mathrm{E}+02$ & $6.622 \mathrm{E}+02$ \\
\hline nd146 & $7.572 \mathrm{E}+02$ & $7.572 \mathrm{E}+02$ & $7.572 \mathrm{E}+02$ & $7.572 \mathrm{E}+02$ & $7.572 \mathrm{E}+02$ \\
\hline nd147 & $4.031 \mathrm{E}-07$ & $0.000 \mathrm{E}+00$ & $0.000 \mathrm{E}+00$ & $0.000 \mathrm{E}+00$ & $0.000 \mathrm{E}+00$ \\
\hline nd148 & $4.369 \mathrm{E}+02$ & $4.369 \mathrm{E}+02$ & $4.369 \mathrm{E}+02$ & $4.369 \mathrm{E}+02$ & $4.369 \mathrm{E}+02$ \\
\hline nd150 & $2.563 \mathrm{E}+02$ & $2.563 \mathrm{E}+02$ & $2.563 \mathrm{E}+02$ & $2.563 \mathrm{E}+02$ & $2.563 \mathrm{E}+02$ \\
\hline ni 58 & $1.036 \mathrm{E}-13$ & $1.036 \mathrm{E}-13$ & $1.036 \mathrm{E}-13$ & $1.036 \mathrm{E}-13$ & $1.036 \mathrm{E}-13$ \\
\hline ni 59 & $7.941 \mathrm{E}-14$ & $7.941 \mathrm{E}-14$ & $7.941 \mathrm{E}-14$ & $7.941 \mathrm{E}-14$ & $7.941 \mathrm{E}-14$ \\
\hline ni 60 & $1.355 \mathrm{E}-13$ & $1.412 \mathrm{E}-13$ & $1.414 \mathrm{E}-13$ & $1.422 \mathrm{E}-13$ & $1.429 \mathrm{E}-13$ \\
\hline
\end{tabular}




\begin{tabular}{|c|c|c|c|c|c|}
\hline \multicolumn{6}{|c|}{ Table C-2. Continued } \\
\hline & $1 / 22 / 2009$ & $10 / 21 / 2013$ & $1 / 22 / 2014$ & $1 / 22 / 2015$ & $1 / 22 / 2016$ \\
\hline ni 61 & $1.102 \mathrm{E}-13$ & $1.102 \mathrm{E}-13$ & $1.102 \mathrm{E}-13$ & $1.102 \mathrm{E}-13$ & $1.102 \mathrm{E}-13$ \\
\hline ni 62 & $1.098 \mathrm{E}-13$ & $1.098 \mathrm{E}-13$ & $1.098 \mathrm{E}-13$ & $1.098 \mathrm{E}-13$ & $1.098 \mathrm{E}-13$ \\
\hline ni 63 & $2.543 \mathrm{E}-15$ & $2.462 \mathrm{E}-15$ & $2.457 \mathrm{E}-15$ & $2.440 \mathrm{E}-15$ & $2.424 \mathrm{E}-15$ \\
\hline ni 64 & $1.161 \mathrm{E}-13$ & $1.161 \mathrm{E}-13$ & $1.161 \mathrm{E}-13$ & $1.161 \mathrm{E}-13$ & $1.161 \mathrm{E}-13$ \\
\hline np235 & $2.407 \mathrm{E}-06$ & $1.160 \mathrm{E}-07$ & $9.861 \mathrm{E}-08$ & 5.207E-08 & $2.750 \mathrm{E}-08$ \\
\hline np236 & $3.295 \mathrm{E}-04$ & $3.295 \mathrm{E}-04$ & $3.295 \mathrm{E}-04$ & $3.295 \mathrm{E}-04$ & $3.295 \mathrm{E}-04$ \\
\hline np237 & $1.929 \mathrm{E}+02$ & $1.978 \mathrm{E}+02$ & $1.982 \mathrm{E}+02$ & $1.999 \mathrm{E}+02$ & $2.019 \mathrm{E}+02$ \\
\hline np238 & $5.224 \mathrm{E}-07$ & $5.103 \mathrm{E}-07$ & 5.097E-07 & $5.072 \mathrm{E}-07$ & $5.047 \mathrm{E}-07$ \\
\hline np239 & $3.589 \mathrm{E}-04$ & $3.588 \mathrm{E}-04$ & $3.588 \mathrm{E}-04$ & $3.588 \mathrm{E}-04$ & $3.588 \mathrm{E}-04$ \\
\hline np240 & $2.559 \mathrm{E}-16$ & $2.559 \mathrm{E}-16$ & $2.559 \mathrm{E}-16$ & $2.559 \mathrm{E}-16$ & $2.559 \mathrm{E}-16$ \\
\hline np240m & $2.487 \mathrm{E}-14$ & $2.487 \mathrm{E}-14$ & $2.487 \mathrm{E}-14$ & $2.487 \mathrm{E}-14$ & $2.487 \mathrm{E}-14$ \\
\hline о 16 & $1.344 \mathrm{E}+05$ & $1.344 \mathrm{E}+05$ & $1.344 \mathrm{E}+05$ & $1.344 \mathrm{E}+05$ & $1.344 \mathrm{E}+05$ \\
\hline o 17 & 4.422E-02 & 4.422E-02 & 4.422E-02 & $4.422 \mathrm{E}-02$ & 4.422E-02 \\
\hline о 18 & $2.210 \mathrm{E}-07$ & $2.210 \mathrm{E}-07$ & $2.210 \mathrm{E}-07$ & $2.210 \mathrm{E}-07$ & $2.210 \mathrm{E}-07$ \\
\hline os186 & $2.122 \mathrm{E}-13$ & $2.122 \mathrm{E}-13$ & $2.122 \mathrm{E}-13$ & $2.122 \mathrm{E}-13$ & $2.122 \mathrm{E}-13$ \\
\hline os187 & $4.033 \mathrm{E}-14$ & $4.033 \mathrm{E}-14$ & $4.033 \mathrm{E}-14$ & $4.033 \mathrm{E}-14$ & $4.033 \mathrm{E}-14$ \\
\hline os188 & $1.598 \mathrm{E}-13$ & $1.598 \mathrm{E}-13$ & $1.598 \mathrm{E}-13$ & $1.598 \mathrm{E}-13$ & $1.598 \mathrm{E}-13$ \\
\hline os189 & $1.178 \mathrm{E}-14$ & $1.178 \mathrm{E}-14$ & $1.178 \mathrm{E}-14$ & $1.178 \mathrm{E}-14$ & $1.178 \mathrm{E}-14$ \\
\hline os190 & $3.570 \mathrm{E}-15$ & $3.570 \mathrm{E}-15$ & $3.570 \mathrm{E}-15$ & $3.570 \mathrm{E}-15$ & $3.570 \mathrm{E}-15$ \\
\hline os192 & $7.678 \mathrm{E}-15$ & $7.684 \mathrm{E}-15$ & 7.684E-15 & $7.684 \mathrm{E}-15$ & $7.684 \mathrm{E}-15$ \\
\hline p 31 & $5.596 \mathrm{E}-14$ & $5.596 \mathrm{E}-14$ & $5.596 \mathrm{E}-14$ & $5.596 \mathrm{E}-14$ & $5.596 \mathrm{E}-14$ \\
\hline pa230 & $9.525 \mathrm{E}-16$ & $0.000 \mathrm{E}+00$ & $0.000 \mathrm{E}+00$ & $0.000 \mathrm{E}+00$ & $0.000 \mathrm{E}+00$ \\
\hline pa231 & $2.299 \mathrm{E}-05$ & 2.634E-05 & 2.652E-05 & $2.723 \mathrm{E}-05$ & $2.793 \mathrm{E}-05$ \\
\hline pa232 & $1.531 \mathrm{E}-14$ & $1.530 \mathrm{E}-14$ & $1.530 \mathrm{E}-14$ & $1.530 \mathrm{E}-14$ & $1.530 \mathrm{E}-14$ \\
\hline pa233 & $6.530 \mathrm{E}-06$ & $6.695 \mathrm{E}-06$ & $6.708 \mathrm{E}-06$ & $6.774 \mathrm{E}-06$ & $6.840 \mathrm{E}-06$ \\
\hline pa234 & $2.520 \mathrm{E}-10$ & $2.520 \mathrm{E}-10$ & $2.520 \mathrm{E}-10$ & $2.520 \mathrm{E}-10$ & $2.520 \mathrm{E}-10$ \\
\hline pa234m & $4.540 \mathrm{E}-10$ & $4.541 \mathrm{E}-10$ & $4.540 \mathrm{E}-10$ & $4.540 \mathrm{E}-10$ & $4.540 \mathrm{E}-10$ \\
\hline pb204 & $3.666 \mathrm{E}-13$ & $3.666 \mathrm{E}-13$ & $3.666 \mathrm{E}-13$ & $3.666 \mathrm{E}-13$ & $3.666 \mathrm{E}-13$ \\
\hline pb205 & $1.423 \mathrm{E}-15$ & $1.424 \mathrm{E}-15$ & $1.424 \mathrm{E}-15$ & $1.424 \mathrm{E}-15$ & $1.424 \mathrm{E}-15$ \\
\hline pb206 & $2.017 \mathrm{E}-12$ & $9.445 \mathrm{E}-12$ & $9.854 \mathrm{E}-12$ & $1.150 \mathrm{E}-11$ & $1.321 \mathrm{E}-11$ \\
\hline pb207 & $7.137 \mathrm{E}-11$ & $3.101 \mathrm{E}-10$ & $3.313 \mathrm{E}-10$ & $4.231 \mathrm{E}-10$ & $5.282 \mathrm{E}-10$ \\
\hline pb208 & $1.038 \mathrm{E}-06$ & $2.216 \mathrm{E}-05$ & $2.410 \mathrm{E}-05$ & $3.232 \mathrm{E}-05$ & $4.140 \mathrm{E}-05$ \\
\hline pb209 & $1.867 \mathrm{E}-14$ & $1.921 \mathrm{E}-14$ & $1.924 \mathrm{E}-14$ & $1.941 \mathrm{E}-14$ & $1.958 \mathrm{E}-14$ \\
\hline pb210 & 5.261E-11 & $5.302 \mathrm{E}-11$ & $5.350 \mathrm{E}-11$ & $5.598 \mathrm{E}-11$ & $5.957 \mathrm{E}-11$ \\
\hline pb211 & $1.840 \mathrm{E}-15$ & $8.350 \mathrm{E}-15$ & $8.529 \mathrm{E}-15$ & $1.003 \mathrm{E}-14$ & $1.138 \mathrm{E}-14$ \\
\hline pb212 & $2.241 \mathrm{E}-09$ & $1.337 \mathrm{E}-08$ & $1.386 \mathrm{E}-08$ & $1.552 \mathrm{E}-08$ & $1.691 \mathrm{E}-08$ \\
\hline pd102 & $8.568 \mathrm{E}-04$ & $1.014 \mathrm{E}-03$ & $1.014 \mathrm{E}-03$ & $1.014 \mathrm{E}-03$ & $1.015 \mathrm{E}-03$ \\
\hline pd103 & $2.838 \mathrm{E}-09$ & $0.000 \mathrm{E}+00$ & $0.000 \mathrm{E}+00$ & $0.000 \mathrm{E}+00$ & $0.000 \mathrm{E}+00$ \\
\hline
\end{tabular}




\begin{tabular}{|c|c|c|c|c|c|}
\hline & $1 / 22 / 2009$ & $10 / 21 / 2013$ & $1 / 22 / 2014$ & $1 / 22 / 2015$ & $1 / 22 / 2016$ \\
\hline pd104 & $4.049 \mathrm{E}+02$ & $4.049 \mathrm{E}+02$ & $4.049 \mathrm{E}+02$ & $4.049 \mathrm{E}+02$ & $4.049 \mathrm{E}+02$ \\
\hline pd105 & $8.370 \mathrm{E}+02$ & $8.370 \mathrm{E}+02$ & $8.370 \mathrm{E}+02$ & $8.370 \mathrm{E}+02$ & $8.370 \mathrm{E}+02$ \\
\hline pd106 & $7.025 \mathrm{E}+02$ & $8.904 \mathrm{E}+02$ & $8.918 \mathrm{E}+02$ & $8.951 \mathrm{E}+02$ & $8.964 \mathrm{E}+02$ \\
\hline pd107 & $5.511 \mathrm{E}+02$ & $5.511 \mathrm{E}+02$ & $5.511 \mathrm{E}+02$ & $5.511 \mathrm{E}+02$ & $5.511 \mathrm{E}+02$ \\
\hline pd108 & $3.841 \mathrm{E}+02$ & $3.841 \mathrm{E}+02$ & $3.841 \mathrm{E}+02$ & $3.841 \mathrm{E}+02$ & $3.841 \mathrm{E}+02$ \\
\hline pd110 & $1.274 \mathrm{E}+02$ & $1.274 \mathrm{E}+02$ & $1.274 \mathrm{E}+02$ & $1.274 \mathrm{E}+02$ & $1.274 \mathrm{E}+02$ \\
\hline pm143 & $1.490 \mathrm{E}-12$ & $1.601 \mathrm{E}-14$ & $1.256 \mathrm{E}-14$ & $4.833 \mathrm{E}-15$ & $1.861 \mathrm{E}-15$ \\
\hline pm144 & $4.549 \mathrm{E}-10$ & $1.663 \mathrm{E}-11$ & $1.392 \mathrm{E}-11$ & $6.932 \mathrm{E}-12$ & $3.454 \mathrm{E}-12$ \\
\hline pm145 & $3.239 \mathrm{E}-05$ & $2.712 \mathrm{E}-05$ & $2.685 \mathrm{E}-05$ & $2.582 \mathrm{E}-05$ & $2.483 \mathrm{E}-05$ \\
\hline pm146 & $3.035 \mathrm{E}-04$ & $1.675 \mathrm{E}-04$ & $1.622 \mathrm{E}-04$ & $1.431 \mathrm{E}-04$ & $1.262 \mathrm{E}-04$ \\
\hline pm147 & $1.477 \mathrm{E}+02$ & $4.218 \mathrm{E}+01$ & $3.944 \mathrm{E}+01$ & $3.029 \mathrm{E}+01$ & $2.326 \mathrm{E}+01$ \\
\hline pm148 & $1.596 \mathrm{E}-04$ & $3.699 \mathrm{E}-17$ & 7.763E-18 & $1.694 \mathrm{E}-20$ & $3.697 \mathrm{E}-23$ \\
\hline pm148m & $2.541 \mathrm{E}-02$ & $5.893 \mathrm{E}-15$ & $1.237 \mathrm{E}-15$ & $2.699 \mathrm{E}-18$ & $5.890 \mathrm{E}-21$ \\
\hline po210 & $7.909 \mathrm{E}-13$ & $8.812 \mathrm{E}-13$ & 8.872E-13 & $9.208 \mathrm{E}-13$ & $9.716 \mathrm{E}-13$ \\
\hline po216 & $8.641 \mathrm{E}-15$ & $5.155 \mathrm{E}-14$ & $5.345 \mathrm{E}-14$ & $5.984 \mathrm{E}-14$ & $6.522 \mathrm{E}-14$ \\
\hline pr141 & $1.212 \mathrm{E}+03$ & $1.213 \mathrm{E}+03$ & $1.213 \mathrm{E}+03$ & $1.213 \mathrm{E}+03$ & $1.213 \mathrm{E}+03$ \\
\hline pr143 & $2.665 \mathrm{E}-05$ & $0.000 \mathrm{E}+00$ & $0.000 \mathrm{E}+00$ & $0.000 \mathrm{E}+00$ & $0.000 \mathrm{E}+00$ \\
\hline pr144 & 7.097E-03 & $1.047 \mathrm{E}-04$ & $8.350 \mathrm{E}-05$ & $3.436 \mathrm{E}-05$ & $1.414 \mathrm{E}-05$ \\
\hline pr144m & $2.824 \mathrm{E}-05$ & $4.166 \mathrm{E}-07$ & 3.322E-07 & $1.367 \mathrm{E}-07$ & $5.625 \mathrm{E}-08$ \\
\hline pt192 & $1.280 \mathrm{E}-13$ & $1.280 \mathrm{E}-13$ & $1.280 \mathrm{E}-13$ & $1.280 \mathrm{E}-13$ & $1.280 \mathrm{E}-13$ \\
\hline pt193 & $1.992 \mathrm{E}-14$ & $1.865 \mathrm{E}-14$ & $1.859 \mathrm{E}-14$ & $1.833 \mathrm{E}-14$ & $1.808 \mathrm{E}-14$ \\
\hline pt194 & $3.816 \mathrm{E}-13$ & $3.816 \mathrm{E}-13$ & $3.816 \mathrm{E}-13$ & $3.816 \mathrm{E}-13$ & $3.816 \mathrm{E}-13$ \\
\hline pt195 & $3.520 \mathrm{E}-15$ & $3.520 \mathrm{E}-15$ & $3.520 \mathrm{E}-15$ & $3.520 \mathrm{E}-15$ & $3.520 \mathrm{E}-15$ \\
\hline pt196 & $6.198 \mathrm{E}-16$ & $6.198 \mathrm{E}-16$ & $6.198 \mathrm{E}-16$ & $6.198 \mathrm{E}-16$ & $6.198 \mathrm{E}-16$ \\
\hline pu236 & $1.041 \mathrm{E}-03$ & 3.293E-04 & $3.095 \mathrm{E}-04$ & $2.429 \mathrm{E}-04$ & $1.906 \mathrm{E}-04$ \\
\hline pu237 & $1.103 \mathrm{E}-05$ & $4.092 \mathrm{E}-17$ & $9.966 \mathrm{E}-18$ & $3.900 \mathrm{E}-20$ & $1.526 \mathrm{E}-22$ \\
\hline pu238 & $1.928 \mathrm{E}+02$ & $2.021 \mathrm{E}+02$ & $2.017 \mathrm{E}+02$ & $2.001 \mathrm{E}+02$ & $1.986 \mathrm{E}+02$ \\
\hline pu239 & $1.027 \mathrm{E}+04$ & $1.027 \mathrm{E}+04$ & $1.027 \mathrm{E}+04$ & $1.027 \mathrm{E}+04$ & $1.027 \mathrm{E}+04$ \\
\hline pu240 & $5.657 \mathrm{E}+03$ & $5.686 \mathrm{E}+03$ & $5.687 \mathrm{E}+03$ & $5.693 \mathrm{E}+03$ & $5.698 \mathrm{E}+03$ \\
\hline pu241 & $3.516 \mathrm{E}+03$ & $2.793 \mathrm{E}+03$ & $2.758 \mathrm{E}+03$ & $2.628 \mathrm{E}+03$ & $2.504 \mathrm{E}+03$ \\
\hline pu242 & $1.446 \mathrm{E}+03$ & $1.446 \mathrm{E}+03$ & $1.446 \mathrm{E}+03$ & $1.446 \mathrm{E}+03$ & $1.446 \mathrm{E}+03$ \\
\hline pu243 & $1.166 \mathrm{E}-12$ & $1.166 \mathrm{E}-12$ & $1.166 \mathrm{E}-12$ & $1.166 \mathrm{E}-12$ & $1.166 \mathrm{E}-12$ \\
\hline pu244 & $1.496 \mathrm{E}-01$ & $1.496 \mathrm{E}-01$ & $1.496 \mathrm{E}-01$ & $1.496 \mathrm{E}-01$ & $1.496 \mathrm{E}-01$ \\
\hline pu246 & $2.402 \mathrm{E}-16$ & $2.401 \mathrm{E}-16$ & $2.401 \mathrm{E}-16$ & $2.401 \mathrm{E}-16$ & $2.400 \mathrm{E}-16$ \\
\hline ra223 & $8.865 \mathrm{E}-13$ & $4.024 \mathrm{E}-12$ & $4.108 \mathrm{E}-12$ & $4.835 \mathrm{E}-12$ & $5.482 \mathrm{E}-12$ \\
\hline ra224 & $1.954 \mathrm{E}-08$ & $1.166 \mathrm{E}-07$ & $1.209 \mathrm{E}-07$ & $1.353 \mathrm{E}-07$ & $1.475 \mathrm{E}-07$ \\
\hline ra225 & $2.208 \mathrm{E}-12$ & $2.274 \mathrm{E}-12$ & $2.278 \mathrm{E}-12$ & $2.297 \mathrm{E}-12$ & $2.318 \mathrm{E}-12$ \\
\hline ra226 & $1.349 \mathrm{E}-09$ & $8.456 \mathrm{E}-09$ & 9.083E-09 & $1.185 \mathrm{E}-08$ & $1.510 \mathrm{E}-08$ \\
\hline
\end{tabular}




\begin{tabular}{|c|c|c|c|c|c|}
\hline \multicolumn{6}{|c|}{ Table C-2. Continued } \\
\hline & $1 / 22 / 2009$ & $10 / 21 / 2013$ & $1 / 22 / 2014$ & $1 / 22 / 2015$ & $1 / 22 / 2016$ \\
\hline ra228 & 4.291E-14 & 7.137E-14 & $7.275 \mathrm{E}-14$ & $7.803 \mathrm{E}-14$ & $8.317 \mathrm{E}-14$ \\
\hline rb 83 & $5.247 \mathrm{E}-08$ & $4.654 \mathrm{E}-14$ & $2.203 \mathrm{E}-14$ & $1.171 \mathrm{E}-15$ & $6.219 \mathrm{E}-17$ \\
\hline rb 84 & $6.066 \mathrm{E}-08$ & 7.717E-24 & $1.082 \mathrm{E}-24$ & $4.859 \mathrm{E}-28$ & $2.181 \mathrm{E}-31$ \\
\hline rb 85 & $6.807 \mathrm{E}+01$ & $7.242 \mathrm{E}+01$ & $7.262 \mathrm{E}+01$ & $7.341 \mathrm{E}+01$ & $7.407 \mathrm{E}+01$ \\
\hline rb 86 & $1.193 \mathrm{E}-06$ & $1.189 \mathrm{E}-34$ & $0.000 \mathrm{E}+00$ & $0.000 \mathrm{E}+00$ & $0.000 \mathrm{E}+00$ \\
\hline rb 87 & $1.601 \mathrm{E}+02$ & $1.601 \mathrm{E}+02$ & $1.601 \mathrm{E}+02$ & $1.601 \mathrm{E}+02$ & $1.601 \mathrm{E}+02$ \\
\hline re185 & $5.330 \mathrm{E}-14$ & $5.343 \mathrm{E}-14$ & $5.343 \mathrm{E}-14$ & $5.343 \mathrm{E}-14$ & $5.343 \mathrm{E}-14$ \\
\hline re186m & $1.816 \mathrm{E}-16$ & $1.816 \mathrm{E}-16$ & $1.816 \mathrm{E}-16$ & $1.816 \mathrm{E}-16$ & $1.816 \mathrm{E}-16$ \\
\hline re187 & $3.451 \mathrm{E}-13$ & $3.451 \mathrm{E}-13$ & $3.451 \mathrm{E}-13$ & $3.451 \mathrm{E}-13$ & $3.451 \mathrm{E}-13$ \\
\hline rh101 & $5.049 \mathrm{E}-07$ & $1.863 \mathrm{E}-07$ & $1.766 \mathrm{E}-07$ & $1.432 \mathrm{E}-07$ & $1.161 \mathrm{E}-07$ \\
\hline rh102 & $7.170 \mathrm{E}-04$ & $2.487 \mathrm{E}-06$ & $1.890 \mathrm{E}-06$ & $7.143 \mathrm{E}-07$ & $3.408 \mathrm{E}-07$ \\
\hline rh102m & $1.770 \mathrm{E}-03$ & $7.348 \mathrm{E}-04$ & 7.011E-04 & $5.827 \mathrm{E}-04$ & 4.842E-04 \\
\hline rh103 & $7.691 \mathrm{E}+02$ & $7.697 \mathrm{E}+02$ & $7.697 \mathrm{E}+02$ & $7.697 \mathrm{E}+02$ & $7.697 \mathrm{E}+02$ \\
\hline rh103m & $5.190 \mathrm{E}-04$ & $2.647 \mathrm{E}-17$ & 5.122E-18 & $8.126 \mathrm{E}-21$ & $1.289 \mathrm{E}-23$ \\
\hline rh106 & $1.831 \mathrm{E}-04$ & $7.236 \mathrm{E}-06$ & $6.085 \mathrm{E}-06$ & $3.081 \mathrm{E}-06$ & $1.560 \mathrm{E}-06$ \\
\hline rn220 & $3.375 \mathrm{E}-12$ & $2.014 \mathrm{E}-11$ & $2.088 \mathrm{E}-11$ & $2.338 \mathrm{E}-11$ & $2.547 \mathrm{E}-11$ \\
\hline rn222 & $8.601 \mathrm{E}-15$ & $5.434 \mathrm{E}-14$ & $5.838 \mathrm{E}-14$ & $7.612 \mathrm{E}-14$ & $9.703 \mathrm{E}-14$ \\
\hline ru 96 & $1.714 \mathrm{E}-13$ & $1.714 \mathrm{E}-13$ & $1.714 \mathrm{E}-13$ & $1.714 \mathrm{E}-13$ & $1.714 \mathrm{E}-13$ \\
\hline ru 98 & $1.751 \mathrm{E}-06$ & $1.759 \mathrm{E}-06$ & $1.760 \mathrm{E}-06$ & $1.761 \mathrm{E}-06$ & $1.763 \mathrm{E}-06$ \\
\hline ru 99 & $3.797 \mathrm{E}-02$ & $5.169 \mathrm{E}-02$ & 5.243E-02 & $5.532 \mathrm{E}-02$ & $5.821 \mathrm{E}-02$ \\
\hline ru100 & $1.614 \mathrm{E}+02$ & $1.614 \mathrm{E}+02$ & $1.614 \mathrm{E}+02$ & $1.614 \mathrm{E}+02$ & $1.614 \mathrm{E}+02$ \\
\hline ru101 & $9.670 \mathrm{E}+02$ & $9.670 \mathrm{E}+02$ & $9.670 \mathrm{E}+02$ & $9.670 \mathrm{E}+02$ & $9.670 \mathrm{E}+02$ \\
\hline ru102 & $1.131 \mathrm{E}+03$ & $1.131 \mathrm{E}+03$ & $1.131 \mathrm{E}+03$ & $1.131 \mathrm{E}+03$ & $1.131 \mathrm{E}+03$ \\
\hline ru103 & $5.285 \mathrm{E}-01$ & $2.695 \mathrm{E}-14$ & $5.215 \mathrm{E}-15$ & $8.271 \mathrm{E}-18$ & $1.312 \mathrm{E}-20$ \\
\hline ru104 & $1.055 \mathrm{E}+03$ & $1.055 \mathrm{E}+03$ & $1.055 \mathrm{E}+03$ & $1.055 \mathrm{E}+03$ & $1.055 \mathrm{E}+03$ \\
\hline ru106 & $1.956 \mathrm{E}+02$ & $7.730 \mathrm{E}+00$ & $6.500 \mathrm{E}+00$ & $3.291 \mathrm{E}+00$ & $1.667 \mathrm{E}+00$ \\
\hline s 32 & $5.765 \mathrm{E}-14$ & $5.765 \mathrm{E}-14$ & $5.765 \mathrm{E}-14$ & $5.765 \mathrm{E}-14$ & $5.765 \mathrm{E}-14$ \\
\hline s 33 & $5.921 \mathrm{E}-14$ & $5.921 \mathrm{E}-14$ & $5.921 \mathrm{E}-14$ & $5.921 \mathrm{E}-14$ & $5.921 \mathrm{E}-14$ \\
\hline s 34 & $6.142 \mathrm{E}-14$ & $6.142 \mathrm{E}-14$ & 6.142E-14 & $6.142 \mathrm{E}-14$ & $6.142 \mathrm{E}-14$ \\
\hline s 36 & $6.493 \mathrm{E}-14$ & $6.493 \mathrm{E}-14$ & $6.493 \mathrm{E}-14$ & $6.493 \mathrm{E}-14$ & $6.493 \mathrm{E}-14$ \\
\hline sb121 & $7.592 \mathrm{E}+00$ & $7.638 \mathrm{E}+00$ & $7.638 \mathrm{E}+00$ & $7.651 \mathrm{E}+00$ & $7.658 \mathrm{E}+00$ \\
\hline sb123 & $1.001 \mathrm{E}+01$ & $1.007 \mathrm{E}+01$ & $1.007 \mathrm{E}+01$ & $1.007 \mathrm{E}+01$ & $1.007 \mathrm{E}+01$ \\
\hline sb124 & $3.517 \mathrm{E}-03$ & 7.592E-12 & $2.601 \mathrm{E}-12$ & $3.890 \mathrm{E}-14$ & $5.818 \mathrm{E}-16$ \\
\hline sb125 & $1.262 \mathrm{E}+01$ & $3.831 \mathrm{E}+00$ & $3.593 \mathrm{E}+00$ & $2.795 \mathrm{E}+00$ & $2.175 \mathrm{E}+00$ \\
\hline sb126 & $7.816 \mathrm{E}-07$ & 7.777E-07 & 7.777E-07 & 7.777E-07 & 7.777E-07 \\
\hline sb126m & $5.980 \mathrm{E}-09$ & $5.980 \mathrm{E}-09$ & $5.980 \mathrm{E}-09$ & $5.980 \mathrm{E}-09$ & $5.980 \mathrm{E}-09$ \\
\hline sc 45 & 7.777E-14 & 7.777E-14 & 7.777E-14 & 7.777E-14 & 7.777E-14 \\
\hline sc 46 & $4.601 \mathrm{E}-17$ & $2.733 \mathrm{E}-23$ & $1.266 \mathrm{E}-23$ & $6.183 \mathrm{E}-25$ & $3.019 \mathrm{E}-26$ \\
\hline
\end{tabular}




\begin{tabular}{|c|c|c|c|c|c|}
\hline \multicolumn{6}{|c|}{ Table C-2. Continued } \\
\hline & $1 / 22 / 2009$ & $10 / 21 / 2013$ & $1 / 22 / 2014$ & $1 / 22 / 2015$ & $1 / 22 / 2016$ \\
\hline se 74 & $3.477 \mathrm{E}-07$ & $3.477 \mathrm{E}-07$ & $3.477 \mathrm{E}-07$ & $3.477 \mathrm{E}-07$ & 3.477E-07 \\
\hline se 75 & 7.803E-09 & $3.447 \mathrm{E}-13$ & $2.012 \mathrm{E}-13$ & $2.435 \mathrm{E}-14$ & $2.946 \mathrm{E}-15$ \\
\hline se 76 & $8.087 \mathrm{E}-03$ & $8.087 \mathrm{E}-03$ & 8.087E-03 & $8.087 \mathrm{E}-03$ & $8.087 \mathrm{E}-03$ \\
\hline se 77 & $8.100 \mathrm{E}-01$ & $8.100 \mathrm{E}-01$ & $8.100 \mathrm{E}-01$ & $8.100 \mathrm{E}-01$ & $8.100 \mathrm{E}-01$ \\
\hline se 78 & $2.360 \mathrm{E}+00$ & $2.360 \mathrm{E}+00$ & $2.360 \mathrm{E}+00$ & $2.360 \mathrm{E}+00$ & $2.360 \mathrm{E}+00$ \\
\hline se 79 & $4.961 \mathrm{E}+00$ & $4.961 \mathrm{E}+00$ & $4.961 \mathrm{E}+00$ & $4.961 \mathrm{E}+00$ & $4.961 \mathrm{E}+00$ \\
\hline se 80 & $1.152 \mathrm{E}+01$ & $1.152 \mathrm{E}+01$ & $1.152 \mathrm{E}+01$ & $1.152 \mathrm{E}+01$ & $1.152 \mathrm{E}+01$ \\
\hline se 82 & $3.048 \mathrm{E}+01$ & $3.048 \mathrm{E}+01$ & $3.048 \mathrm{E}+01$ & $3.048 \mathrm{E}+01$ & $3.048 \mathrm{E}+01$ \\
\hline si 28 & $5.052 \mathrm{E}-14$ & $5.052 \mathrm{E}-14$ & 5.052E-14 & $5.052 \mathrm{E}-14$ & $5.052 \mathrm{E}-14$ \\
\hline si 29 & $5.238 \mathrm{E}-14$ & $5.238 \mathrm{E}-14$ & $5.238 \mathrm{E}-14$ & $5.238 \mathrm{E}-14$ & $5.238 \mathrm{E}-14$ \\
\hline si 30 & $5.446 \mathrm{E}-14$ & $5.446 \mathrm{E}-14$ & $5.446 \mathrm{E}-14$ & $5.446 \mathrm{E}-14$ & $5.446 \mathrm{E}-14$ \\
\hline sm144 & $5.355 \mathrm{E}-11$ & $5.355 \mathrm{E}-11$ & $5.355 \mathrm{E}-11$ & $5.355 \mathrm{E}-11$ & $5.355 \mathrm{E}-11$ \\
\hline sm145 & $2.690 \mathrm{E}-07$ & $7.862 \mathrm{E}-09$ & $6.502 \mathrm{E}-09$ & $3.090 \mathrm{E}-09$ & $1.468 \mathrm{E}-09$ \\
\hline $\operatorname{sm} 146$ & $2.925 \mathrm{E}-03$ & $2.971 \mathrm{E}-03$ & $2.973 \mathrm{E}-03$ & $2.979 \mathrm{E}-03$ & $2.985 \mathrm{E}-03$ \\
\hline sm147 & $9.966 \mathrm{E}+01$ & $2.053 \mathrm{E}+02$ & $2.080 \mathrm{E}+02$ & $2.171 \mathrm{E}+02$ & $2.242 \mathrm{E}+02$ \\
\hline sm148 & $1.811 \mathrm{E}+02$ & $1.812 \mathrm{E}+02$ & $1.812 \mathrm{E}+02$ & $1.812 \mathrm{E}+02$ & $1.812 \mathrm{E}+02$ \\
\hline sm149 & $4.663 \mathrm{E}+00$ & $4.663 \mathrm{E}+00$ & $4.663 \mathrm{E}+00$ & $4.663 \mathrm{E}+00$ & $4.663 \mathrm{E}+00$ \\
\hline sm150 & $3.766 \mathrm{E}+02$ & $3.766 \mathrm{E}+02$ & $3.766 \mathrm{E}+02$ & $3.766 \mathrm{E}+02$ & $3.766 \mathrm{E}+02$ \\
\hline sm151 & $1.963 \mathrm{E}+01$ & $1.892 \mathrm{E}+01$ & $1.888 \mathrm{E}+01$ & $1.875 \mathrm{E}+01$ & $1.860 \mathrm{E}+01$ \\
\hline sm152 & $1.282 \mathrm{E}+02$ & $1.282 \mathrm{E}+02$ & $1.282 \mathrm{E}+02$ & $1.282 \mathrm{E}+02$ & $1.282 \mathrm{E}+02$ \\
\hline sm154 & $7.308 \mathrm{E}+01$ & $7.308 \mathrm{E}+01$ & $7.308 \mathrm{E}+01$ & $7.308 \mathrm{E}+01$ & $7.308 \mathrm{E}+01$ \\
\hline sn112 & $2.504 \mathrm{E}-09$ & $2.504 \mathrm{E}-09$ & $2.504 \mathrm{E}-09$ & $2.504 \mathrm{E}-09$ & $2.504 \mathrm{E}-09$ \\
\hline sn113 & $5.579 \mathrm{E}-11$ & $1.636 \mathrm{E}-15$ & $9.340 \mathrm{E}-16$ & $1.037 \mathrm{E}-16$ & $1.151 \mathrm{E}-17$ \\
\hline sn114 & $1.290 \mathrm{E}-04$ & $1.292 \mathrm{E}-04$ & $1.292 \mathrm{E}-04$ & $1.292 \mathrm{E}-04$ & $1.292 \mathrm{E}-04$ \\
\hline sn115 & $4.525 \mathrm{E}-01$ & $4.525 \mathrm{E}-01$ & $4.525 \mathrm{E}-01$ & $4.525 \mathrm{E}-01$ & $4.525 \mathrm{E}-01$ \\
\hline sn116 & $3.941 \mathrm{E}+00$ & $3.941 \mathrm{E}+00$ & $3.941 \mathrm{E}+00$ & $3.941 \mathrm{E}+00$ & $3.941 \mathrm{E}+00$ \\
\hline sn117 & $9.122 \mathrm{E}+00$ & $9.122 \mathrm{E}+00$ & $9.122 \mathrm{E}+00$ & $9.122 \mathrm{E}+00$ & $9.122 \mathrm{E}+00$ \\
\hline sn117m & $2.326 \mathrm{E}-09$ & $0.000 \mathrm{E}+00$ & $0.000 \mathrm{E}+00$ & $0.000 \mathrm{E}+00$ & $0.000 \mathrm{E}+00$ \\
\hline sn118 & $8.205 \mathrm{E}+00$ & $8.205 \mathrm{E}+00$ & $8.205 \mathrm{E}+00$ & $8.205 \mathrm{E}+00$ & $8.205 \mathrm{E}+00$ \\
\hline sn119 & $7.770 \mathrm{E}+00$ & $7.829 \mathrm{E}+00$ & $7.829 \mathrm{E}+00$ & $7.829 \mathrm{E}+00$ & $7.829 \mathrm{E}+00$ \\
\hline sn119m & $5.866 \mathrm{E}-02$ & $9.722 \mathrm{E}-04$ & 7.803E-04 & $3.291 \mathrm{E}-04$ & $1.388 \mathrm{E}-04$ \\
\hline sn120 & $7.889 \mathrm{E}+00$ & $7.889 \mathrm{E}+00$ & $7.889 \mathrm{E}+00$ & $7.889 \mathrm{E}+00$ & $7.889 \mathrm{E}+00$ \\
\hline sn121 & $3.668 \mathrm{E}-05$ & $3.403 \mathrm{E}-05$ & $3.390 \mathrm{E}-05$ & $3.336 \mathrm{E}-05$ & $3.284 \mathrm{E}-05$ \\
\hline sn121m & $6.728 \mathrm{E}-01$ & $6.243 \mathrm{E}-01$ & $6.218 \mathrm{E}-01$ & $6.120 \mathrm{E}-01$ & $6.025 \mathrm{E}-01$ \\
\hline sn122 & $1.036 \mathrm{E}+01$ & $1.036 \mathrm{E}+01$ & $1.036 \mathrm{E}+01$ & $1.036 \mathrm{E}+01$ & $1.036 \mathrm{E}+01$ \\
\hline sn123 & $6.274 \mathrm{E}-02$ & $5.750 \mathrm{E}-06$ & $3.491 \mathrm{E}-06$ & $4.927 \mathrm{E}-07$ & $6.952 \mathrm{E}-08$ \\
\hline sn124 & $1.638 \mathrm{E}+01$ & $1.638 \mathrm{E}+01$ & $1.638 \mathrm{E}+01$ & $1.638 \mathrm{E}+01$ & $1.638 \mathrm{E}+01$ \\
\hline sn125 & $6.163 \mathrm{E}-10$ & $0.000 \mathrm{E}+00$ & $0.000 \mathrm{E}+00$ & $0.000 \mathrm{E}+00$ & $0.000 \mathrm{E}+00$ \\
\hline
\end{tabular}




\begin{tabular}{|c|c|c|c|c|c|}
\hline \multicolumn{6}{|c|}{ Table C-2. Continued } \\
\hline & $1 / 22 / 2009$ & $10 / 21 / 2013$ & $1 / 22 / 2014$ & $1 / 22 / 2015$ & $1 / 22 / 2016$ \\
\hline sn126 & $3.777 \mathrm{E}+01$ & $3.777 \mathrm{E}+01$ & $3.777 \mathrm{E}+01$ & $3.777 \mathrm{E}+01$ & $3.777 \mathrm{E}+01$ \\
\hline sr 84 & $7.922 \mathrm{E}-06$ & $7.922 \mathrm{E}-06$ & $7.922 \mathrm{E}-06$ & $7.922 \mathrm{E}-06$ & $7.922 \mathrm{E}-06$ \\
\hline sr 85 & $1.336 \mathrm{E}-08$ & $1.202 \mathrm{E}-16$ & 4.447E-17 & 8.984E-19 & $1.815 \mathrm{E}-20$ \\
\hline sr 86 & 4.905E-01 & 4.905E-01 & 4.905E-01 & 4.905E-01 & 4.905E-01 \\
\hline sr 87 & $4.930 \mathrm{E}-03$ & 4.930E-03 & $4.930 \mathrm{E}-03$ & $4.930 \mathrm{E}-03$ & $4.930 \mathrm{E}-03$ \\
\hline sr 88 & $2.127 \mathrm{E}+02$ & $2.127 \mathrm{E}+02$ & $2.127 \mathrm{E}+02$ & $2.127 \mathrm{E}+02$ & $2.127 \mathrm{E}+02$ \\
\hline sr 89 & 4.384E-01 & $2.077 \mathrm{E}-11$ & $5.800 \mathrm{E}-12$ & $3.881 \mathrm{E}-14$ & $2.597 \mathrm{E}-16$ \\
\hline sr 90 & $3.264 \mathrm{E}+02$ & $2.912 \mathrm{E}+02$ & $2.894 \mathrm{E}+02$ & $2.826 \mathrm{E}+02$ & $2.758 \mathrm{E}+02$ \\
\hline ta180m & $2.065 \mathrm{E}-17$ & $2.065 \mathrm{E}-17$ & $2.065 \mathrm{E}-17$ & $2.065 \mathrm{E}-17$ & $2.065 \mathrm{E}-17$ \\
\hline ta181 & $1.680 \mathrm{E}-13$ & $1.680 \mathrm{E}-13$ & $1.680 \mathrm{E}-13$ & $1.680 \mathrm{E}-13$ & $1.680 \mathrm{E}-13$ \\
\hline ta182 & $5.749 \mathrm{E}-16$ & $1.638 \mathrm{E}-20$ & $9.360 \mathrm{E}-21$ & $1.079 \mathrm{E}-21$ & $1.658 \mathrm{E}-22$ \\
\hline tb157 & $6.146 \mathrm{E}-07$ & $5.868 \mathrm{E}-07$ & $5.853 \mathrm{E}-07$ & $5.796 \mathrm{E}-07$ & $5.740 \mathrm{E}-07$ \\
\hline tb158 & $8.990 \mathrm{E}-05$ & $8.825 \mathrm{E}-05$ & 8.819E-05 & 8.779E-05 & $8.746 \mathrm{E}-05$ \\
\hline tb159 & $6.372 \mathrm{E}+00$ & $6.372 \mathrm{E}+00$ & $6.372 \mathrm{E}+00$ & $6.372 \mathrm{E}+00$ & $6.372 \mathrm{E}+00$ \\
\hline tb160 & $1.622 \mathrm{E}-02$ & $9.874 \mathrm{E}-10$ & $4.048 \mathrm{E}-10$ & $1.224 \mathrm{E}-11$ & $3.696 \mathrm{E}-13$ \\
\hline tb161 & $6.327 \mathrm{E}-14$ & $0.000 \mathrm{E}+00$ & $0.000 \mathrm{E}+00$ & $0.000 \mathrm{E}+00$ & $0.000 \mathrm{E}+00$ \\
\hline tc 95 & 4.804E-18 & $1.347 \mathrm{E}-26$ & $4.681 \mathrm{E}-27$ & 7.394E-29 & $0.000 \mathrm{E}+00$ \\
\hline tc $95 \mathrm{~m}$ & $8.937 \mathrm{E}-15$ & $2.506 \mathrm{E}-23$ & $8.713 \mathrm{E}-24$ & $1.377 \mathrm{E}-25$ & $2.175 \mathrm{E}-27$ \\
\hline tc 97 & $9.775 \mathrm{E}-06$ & $9.848 \mathrm{E}-06$ & $9.848 \mathrm{E}-06$ & $9.848 \mathrm{E}-06$ & $9.848 \mathrm{E}-06$ \\
\hline tc $97 \mathrm{~m}$ & $8.100 \mathrm{E}-08$ & $1.498 \mathrm{E}-13$ & 7.374E-14 & $4.575 \mathrm{E}-15$ & $2.838 \mathrm{E}-16$ \\
\hline tc 98 & $1.012 \mathrm{E}-02$ & $1.012 \mathrm{E}-02$ & $1.012 \mathrm{E}-02$ & $1.012 \mathrm{E}-02$ & $1.012 \mathrm{E}-02$ \\
\hline tc 99 & $8.812 \mathrm{E}+02$ & $8.812 \mathrm{E}+02$ & $8.812 \mathrm{E}+02$ & $8.812 \mathrm{E}+02$ & $8.812 \mathrm{E}+02$ \\
\hline te120 & $2.553 \mathrm{E}-09$ & 2.553E-09 & $2.553 \mathrm{E}-09$ & $2.553 \mathrm{E}-09$ & $2.553 \mathrm{E}-09$ \\
\hline te121 & 3.339E-08 & $2.220 \mathrm{E}-11$ & $1.499 \mathrm{E}-11$ & $3.212 \mathrm{E}-12$ & $6.880 \mathrm{E}-13$ \\
\hline te121m & $2.850 \mathrm{E}-07$ & $1.896 \mathrm{E}-10$ & $1.280 \mathrm{E}-10$ & $2.742 \mathrm{E}-11$ & $5.874 \mathrm{E}-12$ \\
\hline te122 & $7.631 \mathrm{E}-01$ & 7.631E-01 & 7.631E-01 & 7.631E-01 & 7.631E-01 \\
\hline te123 & $9.815 \mathrm{E}-03$ & $1.027 \mathrm{E}-02$ & $1.027 \mathrm{E}-02$ & $1.027 \mathrm{E}-02$ & $1.027 \mathrm{E}-02$ \\
\hline te123m & $4.505 \mathrm{E}-04$ & $1.893 \mathrm{E}-08$ & $1.102 \mathrm{E}-08$ & $1.320 \mathrm{E}-09$ & $1.580 \mathrm{E}-10$ \\
\hline te124 & $5.471 \mathrm{E}-01$ & $5.506 \mathrm{E}-01$ & $5.506 \mathrm{E}-01$ & $5.506 \mathrm{E}-01$ & $5.506 \mathrm{E}-01$ \\
\hline te125 & $9.083 \mathrm{E}+00$ & $1.799 \mathrm{E}+01$ & $1.824 \mathrm{E}+01$ & $1.904 \mathrm{E}+01$ & $1.968 \mathrm{E}+01$ \\
\hline te $125 \mathrm{~m}$ & $1.753 \mathrm{E}-01$ & 5.344E-02 & 5.013E-02 & $3.900 \mathrm{E}-02$ & $3.033 \mathrm{E}-02$ \\
\hline te126 & $9.426 \mathrm{E}-01$ & $9.432 \mathrm{E}-01$ & 9.432E-01 & $9.432 \mathrm{E}-01$ & 9.432E-01 \\
\hline te127 & $7.368 \mathrm{E}-04$ & $1.206 \mathrm{E}-08$ & $6.675 \mathrm{E}-09$ & $6.551 \mathrm{E}-10$ & $6.431 \mathrm{E}-11$ \\
\hline te127m & $2.105 \mathrm{E}-01$ & 3.444E-06 & $1.906 \mathrm{E}-06$ & $1.871 \mathrm{E}-07$ & $1.837 \mathrm{E}-08$ \\
\hline te128 & $1.468 \mathrm{E}+02$ & $1.468 \mathrm{E}+02$ & $1.468 \mathrm{E}+02$ & $1.468 \mathrm{E}+02$ & $1.468 \mathrm{E}+02$ \\
\hline te129 & $5.944 \mathrm{E}-06$ & $1.768 \mathrm{E}-21$ & $2.596 \mathrm{E}-22$ & $1.394 \mathrm{E}-25$ & $7.486 \mathrm{E}-29$ \\
\hline te129m & $6.549 \mathrm{E}-03$ & $1.948 \mathrm{E}-18$ & $2.861 \mathrm{E}-19$ & $1.536 \mathrm{E}-22$ & $8.245 \mathrm{E}-26$ \\
\hline te130 & $4.955 \mathrm{E}+02$ & $4.955 \mathrm{E}+02$ & $4.955 \mathrm{E}+02$ & $4.955 \mathrm{E}+02$ & $4.955 \mathrm{E}+02$ \\
\hline
\end{tabular}




\begin{tabular}{|c|c|c|c|c|c|}
\hline \multicolumn{6}{|c|}{ Table C-2. Continued } \\
\hline & $1 / 22 / 2009$ & $10 / 21 / 2013$ & $1 / 22 / 2014$ & $1 / 22 / 2 / 15$ & $1 / 22 / 2016$ \\
\hline th227 & $1.489 \mathrm{E}-12$ & $6.603 \mathrm{E}-12$ & $6.781 \mathrm{E}-12$ & $7.935 \mathrm{E}-12$ & 8.997E-12 \\
\hline th228 & $3.846 \mathrm{E}-06$ & $2.256 \mathrm{E}-05$ & $2.337 \mathrm{E}-05$ & $2.620 \mathrm{E}-05$ & $2.855 \mathrm{E}-05$ \\
\hline th229 & 4.043E-07 & $4.165 \mathrm{E}-07$ & 4.173E-07 & 4.208E-07 & 4.244E-07 \\
\hline th230 & 8.113E-05 & $2.673 \mathrm{E}-04$ & $2.801 \mathrm{E}-04$ & $3.331 \mathrm{E}-04$ & 3.903E-04 \\
\hline th231 & $2.951 \mathrm{E}-09$ & $2.956 \mathrm{E}-09$ & $2.957 \mathrm{E}-09$ & $2.958 \mathrm{E}-09$ & $2.959 \mathrm{E}-09$ \\
\hline th232 & $2.446 \mathrm{E}-04$ & $2.895 \mathrm{E}-04$ & $2.919 \mathrm{E}-04$ & $3.014 \mathrm{E}-04$ & $3.110 \mathrm{E}-04$ \\
\hline th234 & $1.359 \mathrm{E}-05$ & $1.360 \mathrm{E}-05$ & $1.359 \mathrm{E}-05$ & $1.359 \mathrm{E}-05$ & $1.359 \mathrm{E}-05$ \\
\hline ti 46 & 8.647E-14 & 8.647E-14 & $8.647 \mathrm{E}-14$ & 8.647E-14 & 8.647E-14 \\
\hline ti 47 & $8.469 \mathrm{E}-14$ & $8.469 \mathrm{E}-14$ & $8.469 \mathrm{E}-14$ & $8.469 \mathrm{E}-14$ & $8.469 \mathrm{E}-14$ \\
\hline ti 48 & $8.581 \mathrm{E}-14$ & $8.581 \mathrm{E}-14$ & $8.581 \mathrm{E}-14$ & $8.581 \mathrm{E}-14$ & $8.581 \mathrm{E}-14$ \\
\hline ti 49 & $8.944 \mathrm{E}-14$ & $8.944 \mathrm{E}-14$ & $8.944 \mathrm{E}-14$ & 8.944E-14 & $8.944 \mathrm{E}-14$ \\
\hline ti 50 & $9.050 \mathrm{E}-14$ & $9.050 \mathrm{E}-14$ & $9.050 \mathrm{E}-14$ & $9.050 \mathrm{E}-14$ & $9.050 \mathrm{E}-14$ \\
\hline tl203 & 3.657E-15 & $3.662 \mathrm{E}-15$ & $3.662 \mathrm{E}-15$ & 3.662E-15 & $3.662 \mathrm{E}-15$ \\
\hline tl204 & $8.364 \mathrm{E}-17$ & $3.506 \mathrm{E}-17$ & $3.346 \mathrm{E}-17$ & $2.786 \mathrm{E}-17$ & $2.320 \mathrm{E}-17$ \\
\hline tl205 & $1.592 \mathrm{E}-15$ & $1.592 \mathrm{E}-15$ & $1.592 \mathrm{E}-15$ & $1.592 \mathrm{E}-15$ & $1.592 \mathrm{E}-15$ \\
\hline tl207 & $2.378 \mathrm{E}-16$ & $1.080 \mathrm{E}-15$ & $1.102 \mathrm{E}-15$ & $1.297 \mathrm{E}-15$ & $1.471 \mathrm{E}-15$ \\
\hline tl208 & $3.779 \mathrm{E}-12$ & $2.254 \mathrm{E}-11$ & $2.337 \mathrm{E}-11$ & $2.617 \mathrm{E}-11$ & $2.851 \mathrm{E}-11$ \\
\hline $\operatorname{tm} 168$ & $8.627 \mathrm{E}-10$ & $2.150 \mathrm{E}-15$ & $1.076 \mathrm{E}-15$ & 7.104E-17 & $4.690 \mathrm{E}-18$ \\
\hline $\operatorname{tm} 169$ & $9.195 \mathrm{E}-04$ & $9.195 \mathrm{E}-04$ & $9.195 \mathrm{E}-04$ & $9.195 \mathrm{E}-04$ & $9.195 \mathrm{E}-04$ \\
\hline $\operatorname{tm} 170$ & $5.103 \mathrm{E}-05$ & 4.479E-09 & $2.713 \mathrm{E}-09$ & $3.793 \mathrm{E}-10$ & 5.304E-11 \\
\hline $\operatorname{tm} 171$ & $1.745 \mathrm{E}-04$ & $3.146 \mathrm{E}-05$ & $2.870 \mathrm{E}-05$ & $2.001 \mathrm{E}-05$ & $1.394 \mathrm{E}-05$ \\
\hline $\mathbf{u} 230$ & $2.560 \mathrm{E}-15$ & $0.000 \mathrm{E}+00$ & $0.000 \mathrm{E}+00$ & $0.000 \mathrm{E}+00$ & $0.000 \mathrm{E}+00$ \\
\hline $\mathrm{u} 232$ & 4.959E-04 & $1.153 \mathrm{E}-03$ & $1.169 \mathrm{E}-03$ & $1.222 \mathrm{E}-03$ & $1.261 \mathrm{E}-03$ \\
\hline u233 & 4.479E-04 & $7.618 \mathrm{E}-04$ & 7.777E-04 & $8.476 \mathrm{E}-04$ & $9.182 \mathrm{E}-04$ \\
\hline u234 & $1.040 \mathrm{E}+01$ & $1.791 \mathrm{E}+01$ & $1.830 \mathrm{E}+01$ & $1.987 \mathrm{E}+01$ & $2.142 \mathrm{E}+01$ \\
\hline $\mathbf{u} 235$ & $7.256 \mathrm{E}+02$ & $7.269 \mathrm{E}+02$ & $7.269 \mathrm{E}+02$ & $7.275 \mathrm{E}+02$ & $7.275 \mathrm{E}+02$ \\
\hline u236 & $3.243 \mathrm{E}+02$ & $3.271 \mathrm{E}+02$ & $3.272 \mathrm{E}+02$ & $3.278 \mathrm{E}+02$ & $3.284 \mathrm{E}+02$ \\
\hline $\mathbf{u} 237$ & $1.097 \mathrm{E}-04$ & $8.713 \mathrm{E}-05$ & $8.608 \mathrm{E}-05$ & 8.199E-05 & $7.810 \mathrm{E}-05$ \\
\hline u238 & $9.360 \mathrm{E}+05$ & $9.360 \mathrm{E}+05$ & $9.360 \mathrm{E}+05$ & $9.360 \mathrm{E}+05$ & $9.360 \mathrm{E}+05$ \\
\hline $\mathrm{u} 240$ & $2.914 \mathrm{E}-12$ & $2.914 \mathrm{E}-12$ & $2.914 \mathrm{E}-12$ & $2.914 \mathrm{E}-12$ & $2.914 \mathrm{E}-12$ \\
\hline v 50 & $6.103 \mathrm{E}-17$ & $6.103 \mathrm{E}-17$ & $6.103 \mathrm{E}-17$ & $6.103 \mathrm{E}-17$ & $6.103 \mathrm{E}-17$ \\
\hline v 51 & $2.440 \mathrm{E}-15$ & $2.440 \mathrm{E}-15$ & $2.440 \mathrm{E}-15$ & $2.440 \mathrm{E}-15$ & $2.440 \mathrm{E}-15$ \\
\hline w182 & $2.384 \mathrm{E}-13$ & $2.390 \mathrm{E}-13$ & $2.390 \mathrm{E}-13$ & $2.390 \mathrm{E}-13$ & $2.390 \mathrm{E}-13$ \\
\hline w183 & $6.550 \mathrm{E}-13$ & $6.550 \mathrm{E}-13$ & $6.550 \mathrm{E}-13$ & $6.550 \mathrm{E}-13$ & $6.550 \mathrm{E}-13$ \\
\hline w184 & $6.019 \mathrm{E}-13$ & $6.019 \mathrm{E}-13$ & $6.019 \mathrm{E}-13$ & $6.019 \mathrm{E}-13$ & $6.019 \mathrm{E}-13$ \\
\hline w185 & $1.278 \mathrm{E}-16$ & $1.446 \mathrm{E}-23$ & $6.129 \mathrm{E}-24$ & $2.110 \mathrm{E}-25$ & $7.262 \mathrm{E}-27$ \\
\hline w186 & $1.963 \mathrm{E}-13$ & $1.963 \mathrm{E}-13$ & $1.963 \mathrm{E}-13$ & $1.963 \mathrm{E}-13$ & $1.963 \mathrm{E}-13$ \\
\hline xe124 & $1.778 \mathrm{E}-13$ & $1.778 \mathrm{E}-13$ & $1.778 \mathrm{E}-13$ & $1.778 \mathrm{E}-13$ & $1.778 \mathrm{E}-13$ \\
\hline
\end{tabular}




\begin{tabular}{|c|c|c|c|c|c|}
\hline \multicolumn{6}{|c|}{ Table C-2. Continued } \\
\hline & $1 / 22 / 2009$ & $10 / 21 / 2013$ & $1 / 22 / 2014$ & $1 / 22 / 2015$ & $1 / 22 / 2016$ \\
\hline xe126 & $2.917 \mathrm{E}-04$ & 2.917E-04 & $2.917 \mathrm{E}-04$ & $2.917 \mathrm{E}-04$ & 2.917E-04 \\
\hline xe127 & $4.680 \mathrm{E}-08$ & $2.178 \mathrm{E}-22$ & $3.707 \mathrm{E}-23$ & $3.551 \mathrm{E}-26$ & $3.402 \mathrm{E}-29$ \\
\hline xe128 & $7.572 \mathrm{E}+00$ & $7.572 \mathrm{E}+00$ & $7.572 \mathrm{E}+00$ & $7.572 \mathrm{E}+00$ & $7.572 \mathrm{E}+00$ \\
\hline xe129 & $5.827 \mathrm{E}-02$ & $5.833 \mathrm{E}-02$ & $5.833 \mathrm{E}-02$ & $5.834 \mathrm{E}-02$ & $5.835 \mathrm{E}-02$ \\
\hline xe129m & $2.597 \mathrm{E}-13$ & $0.000 \mathrm{E}+00$ & $0.000 \mathrm{E}+00$ & $0.000 \mathrm{E}+00$ & $0.000 \mathrm{E}+00$ \\
\hline xe130 & $1.086 \mathrm{E}+01$ & $1.086 \mathrm{E}+01$ & $1.086 \mathrm{E}+01$ & $1.086 \mathrm{E}+01$ & $1.086 \mathrm{E}+01$ \\
\hline xe131 & $5.246 \mathrm{E}+02$ & $5.246 \mathrm{E}+02$ & $5.246 \mathrm{E}+02$ & $5.246 \mathrm{E}+02$ & $5.246 \mathrm{E}+02$ \\
\hline xe131m & 7.935E-08 & $0.000 \mathrm{E}+00$ & $0.000 \mathrm{E}+00$ & $0.000 \mathrm{E}+00$ & $0.000 \mathrm{E}+00$ \\
\hline xe132 & $1.456 \mathrm{E}+03$ & $1.456 \mathrm{E}+03$ & $1.456 \mathrm{E}+03$ & $1.456 \mathrm{E}+03$ & $1.456 \mathrm{E}+03$ \\
\hline xe133 & $8.139 \mathrm{E}-15$ & $0.000 \mathrm{E}+00$ & $0.000 \mathrm{E}+00$ & $0.000 \mathrm{E}+00$ & $0.000 \mathrm{E}+00$ \\
\hline xe134 & $1.749 \mathrm{E}+03$ & $1.749 \mathrm{E}+03$ & $1.749 \mathrm{E}+03$ & $1.749 \mathrm{E}+03$ & $1.749 \mathrm{E}+03$ \\
\hline xe136 & $2.756 \mathrm{E}+03$ & $2.756 \mathrm{E}+03$ & $2.756 \mathrm{E}+03$ & $2.756 \mathrm{E}+03$ & $2.756 \mathrm{E}+03$ \\
\hline y 88 & $2.481 \mathrm{E}-05$ & $3.178 \mathrm{E}-10$ & $1.736 \mathrm{E}-10$ & $1.619 \mathrm{E}-11$ & $1.509 \mathrm{E}-12$ \\
\hline y 89 & $2.802 \mathrm{E}+02$ & $2.807 \mathrm{E}+02$ & $2.807 \mathrm{E}+02$ & $2.807 \mathrm{E}+02$ & $2.807 \mathrm{E}+02$ \\
\hline y 89m & $1.516 \mathrm{E}-10$ & $7.183 \mathrm{E}-21$ & $2.006 \mathrm{E}-21$ & $1.342 \mathrm{E}-23$ & $8.984 \mathrm{E}-26$ \\
\hline y 90 & $8.278 \mathrm{E}-02$ & 7.387E-02 & $7.341 \mathrm{E}-02$ & $7.170 \mathrm{E}-02$ & $6.998 \mathrm{E}-02$ \\
\hline y 91 & $1.227 \mathrm{E}+00$ & $1.487 \mathrm{E}-09$ & $4.943 \mathrm{E}-10$ & $6.547 \mathrm{E}-12$ & $8.674 \mathrm{E}-14$ \\
\hline yb168 & $1.822 \mathrm{E}-12$ & $1.908 \mathrm{E}-12$ & $1.908 \mathrm{E}-12$ & $1.908 \mathrm{E}-12$ & $1.908 \mathrm{E}-12$ \\
\hline yb169 & $3.324 \mathrm{E}-12$ & $1.691 \mathrm{E}-28$ & 2.257E-29 & $8.350 \mathrm{E}-33$ & $0.000 \mathrm{E}+00$ \\
\hline yb170 & $7.031 \mathrm{E}-04$ & $7.539 \mathrm{E}-04$ & $7.539 \mathrm{E}-04$ & $7.539 \mathrm{E}-04$ & $7.539 \mathrm{E}-04$ \\
\hline yb171 & $2.286 \mathrm{E}-04$ & $3.716 \mathrm{E}-04$ & $3.744 \mathrm{E}-04$ & $3.831 \mathrm{E}-04$ & 3.891E-04 \\
\hline yb172 & $4.828 \mathrm{E}-04$ & $4.828 \mathrm{E}-04$ & $4.828 \mathrm{E}-04$ & $4.828 \mathrm{E}-04$ & $4.828 \mathrm{E}-04$ \\
\hline yb176 & $1.336 \mathrm{E}-16$ & $1.336 \mathrm{E}-16$ & $1.336 \mathrm{E}-16$ & $1.336 \mathrm{E}-16$ & $1.336 \mathrm{E}-16$ \\
\hline zn 64 & $5.306 \mathrm{E}-16$ & $5.306 \mathrm{E}-16$ & $5.306 \mathrm{E}-16$ & $5.306 \mathrm{E}-16$ & $5.306 \mathrm{E}-16$ \\
\hline zn 66 & $1.549 \mathrm{E}-04$ & $1.549 \mathrm{E}-04$ & $1.549 \mathrm{E}-04$ & $1.549 \mathrm{E}-04$ & $1.549 \mathrm{E}-04$ \\
\hline zn 67 & $4.368 \mathrm{E}-04$ & $4.368 \mathrm{E}-04$ & $4.368 \mathrm{E}-04$ & $4.368 \mathrm{E}-04$ & $4.368 \mathrm{E}-04$ \\
\hline zn 68 & $8.482 \mathrm{E}-04$ & $8.482 \mathrm{E}-04$ & $8.482 \mathrm{E}-04$ & $8.482 \mathrm{E}-04$ & 8.482E-04 \\
\hline zn 70 & $3.686 \mathrm{E}-03$ & $3.686 \mathrm{E}-03$ & $3.686 \mathrm{E}-03$ & $3.686 \mathrm{E}-03$ & $3.686 \mathrm{E}-03$ \\
\hline zr 88 & $5.158 \mathrm{E}-12$ & $2.866 \mathrm{E}-18$ & $1.323 \mathrm{E}-18$ & $6.369 \mathrm{E}-20$ & $3.066 \mathrm{E}-21$ \\
\hline $\operatorname{zr} 90$ & $1.878 \mathrm{E}+01$ & $5.403 \mathrm{E}+01$ & $5.581 \mathrm{E}+01$ & $6.269 \mathrm{E}+01$ & $6.939 \mathrm{E}+01$ \\
\hline $\operatorname{zr} 91$ & $4.072 \mathrm{E}+02$ & $4.084 \mathrm{E}+02$ & $4.084 \mathrm{E}+02$ & $4.084 \mathrm{E}+02$ & $4.084 \mathrm{E}+02$ \\
\hline $\operatorname{zr} 92$ & $4.919 \mathrm{E}+02$ & $4.919 \mathrm{E}+02$ & $4.919 \mathrm{E}+02$ & $4.919 \mathrm{E}+02$ & $4.919 \mathrm{E}+02$ \\
\hline $\operatorname{zr} 93$ & $6.035 \mathrm{E}+02$ & $6.035 \mathrm{E}+02$ & $6.035 \mathrm{E}+02$ & $6.035 \mathrm{E}+02$ & $6.035 \mathrm{E}+02$ \\
\hline zr 94 & $6.899 \mathrm{E}+02$ & $6.899 \mathrm{E}+02$ & $6.899 \mathrm{E}+02$ & $6.899 \mathrm{E}+02$ & $6.899 \mathrm{E}+02$ \\
\hline $\operatorname{zr} 95$ & $3.320 \mathrm{E}+00$ & $2.365 \mathrm{E}-08$ & $8.641 \mathrm{E}-09$ & $1.662 \mathrm{E}-10$ & $3.196 \mathrm{E}-12$ \\
\hline zr 96 & $8.073 \mathrm{E}+02$ & $8.073 E+02$ & $8.073 \mathrm{E}+02$ & $8.073 \mathrm{E}+02$ & $8.073 \mathrm{E}+02$ \\
\hline Total & $1.1339 \mathrm{E}+06$ & $1.1339 \mathrm{E}+06$ & $1.1339 \mathrm{E}+06$ & $1.1339 \mathrm{E}+06$ & $1.1339 \mathrm{E}+06$ \\
\hline
\end{tabular}


Table C-3. MOX Fuel Rod Cl (medium Pu): Nuclide mass in grams per MTIHM

\begin{tabular}{|c|c|c|c|c|c|}
\hline & $1 / 22 / 2009$ & $10 / 21 / 2013$ & $1 / 22 / 2014$ & $1 / 22 / 2015$ & $1 / 22 / 2016$ \\
\hline ac225 & $1.319 \mathrm{E}-12$ & $1.363 \mathrm{E}-12$ & $1.365 \mathrm{E}-12$ & $1.379 \mathrm{E}-12$ & $1.393 \mathrm{E}-12$ \\
\hline ac227 & $7.086 \mathrm{E}-10$ & 2.991E-09 & $3.115 \mathrm{E}-09$ & $3.601 \mathrm{E}-09$ & $4.089 \mathrm{E}-09$ \\
\hline $\operatorname{ag} 105$ & $8.136 \mathrm{E}-15$ & $1.887 \mathrm{E}-27$ & $3.960 \mathrm{E}-28$ & $8.641 \mathrm{E}-31$ & $1.886 \mathrm{E}-33$ \\
\hline $\operatorname{ag} 107$ & $1.368 \mathrm{E}-04$ & $4.360 \mathrm{E}-04$ & $4.521 \mathrm{E}-04$ & 5.151E-04 & 5.782E-04 \\
\hline $\operatorname{ag} 108$ & $6.262 \mathrm{E}-13$ & $6.214 \mathrm{E}-13$ & $6.212 \mathrm{E}-13$ & $6.202 \mathrm{E}-13$ & $6.192 \mathrm{E}-13$ \\
\hline ag108m & $6.959 \mathrm{E}-04$ & $6.906 \mathrm{E}-04$ & $6.906 \mathrm{E}-04$ & $6.893 \mathrm{E}-04$ & $6.886 \mathrm{E}-04$ \\
\hline ag109 & $1.993 \mathrm{E}+02$ & $1.993 \mathrm{E}+02$ & $1.993 \mathrm{E}+02$ & $1.993 \mathrm{E}+02$ & $1.993 \mathrm{E}+02$ \\
\hline $\operatorname{ag} 109 m$ & $2.046 \mathrm{E}-10$ & $1.514 \mathrm{E}-11$ & $1.317 \mathrm{E}-11$ & $7.611 \mathrm{E}-12$ & $4.398 \mathrm{E}-12$ \\
\hline $\operatorname{ag} 110$ & $2.104 \mathrm{E}-08$ & $1.715 \mathrm{E}-10$ & $1.325 \mathrm{E}-10$ & $4.812 \mathrm{E}-11$ & $1.748 \mathrm{E}-11$ \\
\hline $\operatorname{ag} 110 \mathrm{~m}$ & $1.357 \mathrm{E}+00$ & $1.106 \mathrm{E}-02$ & $8.548 \mathrm{E}-03$ & $3.104 \mathrm{E}-03$ & $1.127 \mathrm{E}-03$ \\
\hline ag111 & $1.442 \mathrm{E}-11$ & $0.000 \mathrm{E}+00$ & $0.000 \mathrm{E}+00$ & $0.000 \mathrm{E}+00$ & $0.000 \mathrm{E}+00$ \\
\hline al 27 & $4.908 \mathrm{E}-14$ & $4.908 \mathrm{E}-14$ & $4.908 \mathrm{E}-14$ & $4.908 \mathrm{E}-14$ & $4.908 \mathrm{E}-14$ \\
\hline $\operatorname{am} 241$ & $3.407 \mathrm{E}+02$ & $1.189 \mathrm{E}+03$ & $1.229 \mathrm{E}+03$ & $1.381 \mathrm{E}+03$ & $1.526 \mathrm{E}+03$ \\
\hline $\operatorname{am} 242$ & $4.416 \mathrm{E}-05$ & $4.315 \mathrm{E}-05$ & 4.309E-05 & $4.288 \mathrm{E}-05$ & 4.267E-05 \\
\hline $\operatorname{am} 242 m$ & $3.423 \mathrm{E}+00$ & $3.344 \mathrm{E}+00$ & $3.339 \mathrm{E}+00$ & $3.324 \mathrm{E}+00$ & $3.307 \mathrm{E}+00$ \\
\hline am243 & $4.006 \mathrm{E}+02$ & $4.004 \mathrm{E}+02$ & $4.004 \mathrm{E}+02$ & $4.004 \mathrm{E}+02$ & $4.003 \mathrm{E}+02$ \\
\hline $\operatorname{am} 244$ & $5.450 \mathrm{E}-15$ & $3.781 \mathrm{E}-15$ & $3.707 \mathrm{E}-15$ & $3.433 \mathrm{E}-15$ & $3.178 \mathrm{E}-15$ \\
\hline am245 & $5.730 \mathrm{E}-14$ & $1.342 \mathrm{E}-15$ & $1.097 \mathrm{E}-15$ & $4.977 \mathrm{E}-16$ & $2.257 \mathrm{E}-16$ \\
\hline $\operatorname{ar} 36$ & $6.478 \mathrm{E}-14$ & $6.478 \mathrm{E}-14$ & $6.478 \mathrm{E}-14$ & $6.478 \mathrm{E}-14$ & $6.478 \mathrm{E}-14$ \\
\hline ar 38 & $6.906 \mathrm{E}-14$ & $6.906 \mathrm{E}-14$ & $6.906 \mathrm{E}-14$ & $6.906 \mathrm{E}-14$ & $6.906 \mathrm{E}-14$ \\
\hline ar 39 & $1.251 \mathrm{E}-16$ & $1.236 \mathrm{E}-16$ & $1.235 \mathrm{E}-16$ & $1.232 \mathrm{E}-16$ & $1.228 \mathrm{E}-16$ \\
\hline ar 40 & $7.312 \mathrm{E}-14$ & $7.312 \mathrm{E}-14$ & $7.312 \mathrm{E}-14$ & $7.312 \mathrm{E}-14$ & $7.312 \mathrm{E}-14$ \\
\hline as 73 & $1.270 \mathrm{E}-10$ & $4.048 \mathrm{E}-17$ & $1.814 \mathrm{E}-17$ & 7.764E-19 & $3.326 \mathrm{E}-20$ \\
\hline as 74 & $1.637 \mathrm{E}-12$ & $0.000 \mathrm{E}+00$ & $0.000 \mathrm{E}+00$ & $0.000 \mathrm{E}+00$ & $0.000 \mathrm{E}+00$ \\
\hline as 75 & $1.658 \mathrm{E}-01$ & $1.658 \mathrm{E}-01$ & $1.658 \mathrm{E}-01$ & $1.658 \mathrm{E}-01$ & $1.658 \mathrm{E}-01$ \\
\hline au197 & $2.057 \mathrm{E}-13$ & $2.057 \mathrm{E}-13$ & $2.057 \mathrm{E}-13$ & $2.057 \mathrm{E}-13$ & $2.057 \mathrm{E}-13$ \\
\hline b 10 & $6.460 \mathrm{E}-11$ & $3.500 \mathrm{E}-10$ & $3.653 \mathrm{E}-10$ & 4.254E-10 & $4.856 \mathrm{E}-10$ \\
\hline b 11 & 7.471E-08 & $7.471 \mathrm{E}-08$ & $7.471 \mathrm{E}-08$ & $7.471 \mathrm{E}-08$ & $7.471 \mathrm{E}-08$ \\
\hline ba130 & $1.872 \mathrm{E}-13$ & $1.872 \mathrm{E}-13$ & $1.872 \mathrm{E}-13$ & $1.872 \mathrm{E}-13$ & $1.872 \mathrm{E}-13$ \\
\hline ba131 & 4.133E-18 & $0.000 \mathrm{E}+00$ & $0.000 \mathrm{E}+00$ & $0.000 \mathrm{E}+00$ & $0.000 \mathrm{E}+00$ \\
\hline ba132 & $2.987 \mathrm{E}-04$ & $2.987 \mathrm{E}-04$ & $2.987 \mathrm{E}-04$ & $2.987 \mathrm{E}-04$ & $2.987 \mathrm{E}-04$ \\
\hline ba133 & $2.097 \mathrm{E}-04$ & $1.534 \mathrm{E}-04$ & $1.508 \mathrm{E}-04$ & $1.412 \mathrm{E}-04$ & $1.322 \mathrm{E}-04$ \\
\hline ba134 & $1.150 \mathrm{E}+02$ & $2.348 \mathrm{E}+02$ & $2.373 \mathrm{E}+02$ & $2.453 \mathrm{E}+02$ & $2.511 \mathrm{E}+02$ \\
\hline ba135 & $5.465 \mathrm{E}-01$ & $5.475 \mathrm{E}-01$ & $5.476 \mathrm{E}-01$ & $5.478 \mathrm{E}-01$ & $5.480 \mathrm{E}-01$ \\
\hline ba136 & $4.371 \mathrm{E}+01$ & $4.371 \mathrm{E}+01$ & $4.371 \mathrm{E}+01$ & $4.371 \mathrm{E}+01$ & $4.371 \mathrm{E}+01$ \\
\hline ba136m & $2.898 \mathrm{E}-14$ & $0.000 \mathrm{E}+00$ & $0.000 \mathrm{E}+00$ & $0.000 \mathrm{E}+00$ & $0.000 \mathrm{E}+00$ \\
\hline ba137 & $8.196 \mathrm{E}+01$ & $2.404 \mathrm{E}+02$ & $2.485 \mathrm{E}+02$ & $2.795 \mathrm{E}+02$ & $3.099 \mathrm{E}+02$ \\
\hline
\end{tabular}




\begin{tabular}{|c|c|c|c|c|c|}
\hline \multicolumn{6}{|c|}{ Table C-3. Continued } \\
\hline & $1 / 22 / 2009$ & $10 / 21 / 2013$ & $1 / 22 / 2014$ & $1 / 22 / 2015$ & $1 / 22 / 2016$ \\
\hline ba137m & $2.338 \mathrm{E}-04$ & 2.096E-04 & $2.083 \mathrm{E}-04$ & $2.036 \mathrm{E}-04$ & $1.989 \mathrm{E}-04$ \\
\hline ba138 & $1.531 \mathrm{E}+03$ & $1.531 \mathrm{E}+03$ & $1.531 \mathrm{E}+03$ & $1.531 \mathrm{E}+03$ & $1.531 \mathrm{E}+03$ \\
\hline ba140 & $1.297 \mathrm{E}-05$ & $0.000 \mathrm{E}+00$ & $0.000 \mathrm{E}+00$ & $0.000 \mathrm{E}+00$ & $0.000 \mathrm{E}+00$ \\
\hline be 9 & $2.467 \mathrm{E}-06$ & 2.467E-06 & $2.467 \mathrm{E}-06$ & 2.467E-06 & $2.467 \mathrm{E}-06$ \\
\hline be 10 & $1.310 \mathrm{E}-04$ & $1.310 \mathrm{E}-04$ & $1.310 \mathrm{E}-04$ & $1.310 \mathrm{E}-04$ & $1.310 \mathrm{E}-04$ \\
\hline bi208 & $1.304 \mathrm{E}-15$ & $1.304 \mathrm{E}-15$ & $1.304 \mathrm{E}-15$ & $1.304 \mathrm{E}-15$ & $1.304 \mathrm{E}-15$ \\
\hline bi209 & $1.191 \mathrm{E}-10$ & $2.685 \mathrm{E}-10$ & $2.766 \mathrm{E}-10$ & $3.089 \mathrm{E}-10$ & $3.415 \mathrm{E}-10$ \\
\hline bi210 & $3.256 \mathrm{E}-14$ & $3.300 \mathrm{E}-14$ & $3.331 \mathrm{E}-14$ & $3.493 \mathrm{E}-14$ & $3.725 \mathrm{E}-14$ \\
\hline bi210m & $1.820 \mathrm{E}-15$ & $1.820 \mathrm{E}-15$ & $1.820 \mathrm{E}-15$ & $1.820 \mathrm{E}-15$ & $1.820 \mathrm{E}-15$ \\
\hline bi211 & $1.143 \mathrm{E}-16$ & $5.207 \mathrm{E}-16$ & $5.318 \mathrm{E}-16$ & $6.267 \mathrm{E}-16$ & $7.119 \mathrm{E}-16$ \\
\hline bi212 & $1.958 \mathrm{E}-10$ & 1.198E-09 & $1.243 \mathrm{E}-09$ & 1.393E-09 & $1.520 \mathrm{E}-09$ \\
\hline bi213 & $3.952 \mathrm{E}-15$ & $4.087 \mathrm{E}-15$ & $4.092 \mathrm{E}-15$ & $4.134 \mathrm{E}-15$ & $4.175 \mathrm{E}-15$ \\
\hline bk247 & $1.064 \mathrm{E}-12$ & $1.062 \mathrm{E}-12$ & $1.062 \mathrm{E}-12$ & $1.061 \mathrm{E}-12$ & $1.061 \mathrm{E}-12$ \\
\hline bk248 & $4.323 \mathrm{E}-11$ & $2.999 \mathrm{E}-11$ & $2.941 \mathrm{E}-11$ & $2.723 \mathrm{E}-11$ & $2.521 \mathrm{E}-11$ \\
\hline bk249 & $1.504 \mathrm{E}-05$ & $3.524 \mathrm{E}-07$ & $2.881 \mathrm{E}-07$ & $1.307 \mathrm{E}-07$ & $5.926 \mathrm{E}-08$ \\
\hline bk250 & $7.458 \mathrm{E}-15$ & $9.638 \mathrm{E}-17$ & $7.644 \mathrm{E}-17$ & $3.100 \mathrm{E}-17$ & $1.285 \mathrm{E}-17$ \\
\hline br 79 & $2.923 \mathrm{E}-03$ & $2.983 \mathrm{E}-03$ & $2.987 \mathrm{E}-03$ & $2.999 \mathrm{E}-03$ & $3.012 \mathrm{E}-03$ \\
\hline br 81 & $2.256 \mathrm{E}+01$ & $2.256 \mathrm{E}+01$ & $2.256 \mathrm{E}+01$ & $2.256 \mathrm{E}+01$ & $2.256 \mathrm{E}+01$ \\
\hline c 12 & 7.930E-02 & $7.930 \mathrm{E}-02$ & $7.930 \mathrm{E}-02$ & $7.930 \mathrm{E}-02$ & $7.930 \mathrm{E}-02$ \\
\hline c 13 & $1.250 \mathrm{E}+01$ & $1.250 \mathrm{E}+01$ & $1.250 \mathrm{E}+01$ & $1.250 \mathrm{E}+01$ & $1.250 \mathrm{E}+01$ \\
\hline c 14 & $3.937 \mathrm{E}-04$ & $3.934 \mathrm{E}-04$ & $3.934 \mathrm{E}-04$ & $3.934 \mathrm{E}-04$ & $3.934 \mathrm{E}-04$ \\
\hline ca 40 & $7.252 \mathrm{E}-14$ & 7.252E-14 & $7.252 \mathrm{E}-14$ & 7.252E-14 & $7.252 \mathrm{E}-14$ \\
\hline ca 41 & $4.767 \mathrm{E}-17$ & 4.767E-17 & 4.767E-17 & 4.767E-17 & $4.767 \mathrm{E}-17$ \\
\hline ca 42 & $7.657 \mathrm{E}-14$ & 7.657E-14 & 7.657E-14 & 7.657E-14 & $7.657 \mathrm{E}-14$ \\
\hline ca 43 & $7.691 \mathrm{E}-14$ & $7.691 \mathrm{E}-14$ & $7.691 \mathrm{E}-14$ & $7.691 \mathrm{E}-14$ & $7.691 \mathrm{E}-14$ \\
\hline ca 44 & $8.129 \mathrm{E}-14$ & $8.129 \mathrm{E}-14$ & $8.129 \mathrm{E}-14$ & $8.129 \mathrm{E}-14$ & $8.129 \mathrm{E}-14$ \\
\hline ca 46 & $8.355 \mathrm{E}-14$ & $8.355 \mathrm{E}-14$ & $8.355 \mathrm{E}-14$ & $8.355 \mathrm{E}-14$ & $8.355 \mathrm{E}-14$ \\
\hline ca 48 & $8.714 \mathrm{E}-14$ & $8.714 \mathrm{E}-14$ & $8.714 \mathrm{E}-14$ & $8.714 \mathrm{E}-14$ & $8.714 \mathrm{E}-14$ \\
\hline cd106 & $2.993 \mathrm{E}-13$ & $2.993 \mathrm{E}-13$ & $2.993 \mathrm{E}-13$ & $2.993 \mathrm{E}-13$ & $2.993 \mathrm{E}-13$ \\
\hline cd108 & $1.465 \mathrm{E}-03$ & $1.466 \mathrm{E}-03$ & $1.466 \mathrm{E}-03$ & $1.466 \mathrm{E}-03$ & $1.466 \mathrm{E}-03$ \\
\hline cd109 & $2.060 \mathrm{E}-04$ & $1.524 \mathrm{E}-05$ & $1.325 \mathrm{E}-05$ & 7.657E-06 & 4.427E-06 \\
\hline cd110 & $1.436 \mathrm{E}+02$ & $1.450 \mathrm{E}+02$ & $1.450 \mathrm{E}+02$ & $1.450 \mathrm{E}+02$ & $1.450 \mathrm{E}+02$ \\
\hline cd111 & $6.503 \mathrm{E}+01$ & $6.503 \mathrm{E}+01$ & $6.503 \mathrm{E}+01$ & $6.503 \mathrm{E}+01$ & $6.503 \mathrm{E}+01$ \\
\hline cd112 & $3.075 \mathrm{E}+01$ & $3.075 \mathrm{E}+01$ & $3.075 \mathrm{E}+01$ & $3.075 \mathrm{E}+01$ & $3.075 \mathrm{E}+01$ \\
\hline cd113 & $3.128 \mathrm{E}-01$ & $3.128 \mathrm{E}-01$ & $3.128 \mathrm{E}-01$ & $3.128 \mathrm{E}-01$ & $3.128 \mathrm{E}-01$ \\
\hline cd113m & 4.495E-03 & $3.560 \mathrm{E}-03$ & $3.516 \mathrm{E}-03$ & 3.347E-03 & $3.187 \mathrm{E}-03$ \\
\hline cd114 & $3.281 \mathrm{E}+01$ & $3.281 \mathrm{E}+01$ & $3.281 \mathrm{E}+01$ & $3.281 \mathrm{E}+01$ & $3.281 \mathrm{E}+01$ \\
\hline cd115m & $6.174 \mathrm{E}-04$ & $1.210 \mathrm{E}-15$ & $2.849 \mathrm{E}-16$ & $9.745 \mathrm{E}-19$ & $3.335 \mathrm{E}-21$ \\
\hline
\end{tabular}




\begin{tabular}{|c|c|c|c|c|c|}
\hline \multicolumn{6}{|c|}{ Table C-3. Continued } \\
\hline & $1 / 22 / 2009$ & $10 / 21 / 2013$ & $1 / 22 / 2014$ & $1 / 22 / 2015$ & $1 / 22 / 2016$ \\
\hline cd116 & $1.086 \mathrm{E}+01$ & $1.086 \mathrm{E}+01$ & $1.086 \mathrm{E}+01$ & $1.086 \mathrm{E}+01$ & $1.086 \mathrm{E}+01$ \\
\hline ce136 & $2.269 \mathrm{E}-13$ & $2.269 \mathrm{E}-13$ & $2.269 \mathrm{E}-13$ & $2.269 \mathrm{E}-13$ & $2.269 \mathrm{E}-13$ \\
\hline ce138 & $1.052 \mathrm{E}-05$ & $1.052 \mathrm{E}-05$ & $1.052 \mathrm{E}-05$ & $1.052 \mathrm{E}-05$ & $1.052 \mathrm{E}-05$ \\
\hline ce139 & $1.426 \mathrm{E}-03$ & $2.311 \mathrm{E}-07$ & $1.447 \mathrm{E}-07$ & 2.303E-08 & 3.663E-09 \\
\hline ce140 & $1.457 \mathrm{E}+03$ & $1.457 \mathrm{E}+03$ & $1.457 \mathrm{E}+03$ & $1.457 \mathrm{E}+03$ & $1.457 \mathrm{E}+03$ \\
\hline ce141 & $1.911 \mathrm{E}-01$ & $1.711 \mathrm{E}-17$ & $2.355 \mathrm{E}-18$ & $9.818 \mathrm{E}-22$ & $4.093 \mathrm{E}-25$ \\
\hline ce142 & $1.262 \mathrm{E}+03$ & $1.262 \mathrm{E}+03$ & $1.262 \mathrm{E}+03$ & $1.262 \mathrm{E}+03$ & $1.262 \mathrm{E}+03$ \\
\hline ce144 & $1.823 \mathrm{E}+02$ & $2.690 \mathrm{E}+00$ & $2.146 \mathrm{E}+00$ & 8.827E-01 & 3.633E-01 \\
\hline cf 248 & $2.856 \mathrm{E}-11$ & $7.784 \mathrm{E}-13$ & $6.418 \mathrm{E}-13$ & $3.006 \mathrm{E}-13$ & $1.408 \mathrm{E}-13$ \\
\hline cf249 & $1.623 \mathrm{E}-05$ & $3.066 \mathrm{E}-05$ & 3.072E-05 & $3.081 \mathrm{E}-05$ & $3.082 \mathrm{E}-05$ \\
\hline cf250 & $8.455 \mathrm{E}-06$ & $6.575 \mathrm{E}-06$ & $6.487 \mathrm{E}-06$ & $6.152 \mathrm{E}-06$ & $5.835 \mathrm{E}-06$ \\
\hline cf251 & 4.302E-06 & $4.286 \mathrm{E}-06$ & 4.285E-06 & 4.282E-06 & 4.279E-06 \\
\hline cf 252 & $1.539 \mathrm{E}-06$ & $4.438 \mathrm{E}-07$ & 4.152E-07 & $3.195 \mathrm{E}-07$ & $2.459 \mathrm{E}-07$ \\
\hline cf 253 & $9.299 \mathrm{E}-14$ & $0.000 \mathrm{E}+00$ & $0.000 \mathrm{E}+00$ & $0.000 \mathrm{E}+00$ & $0.000 \mathrm{E}+00$ \\
\hline cf254 & $5.200 \mathrm{E}-12$ & $1.239 \mathrm{E}-20$ & 4.269E-21 & $6.518 \mathrm{E}-23$ & $9.951 \mathrm{E}-25$ \\
\hline cl 35 & $5.998 \mathrm{E}-14$ & $5.998 \mathrm{E}-14$ & $5.998 \mathrm{E}-14$ & $5.998 \mathrm{E}-14$ & $5.998 \mathrm{E}-14$ \\
\hline cl 36 & $3.901 \mathrm{E}-15$ & $3.901 \mathrm{E}-15$ & $3.901 \mathrm{E}-15$ & $3.901 \mathrm{E}-15$ & $3.901 \mathrm{E}-15$ \\
\hline cl 37 & $6.793 \mathrm{E}-14$ & $6.793 \mathrm{E}-14$ & $6.793 \mathrm{E}-14$ & $6.793 \mathrm{E}-14$ & $6.793 \mathrm{E}-14$ \\
\hline cm240 & $1.669 \mathrm{E}-11$ & 7.957E-31 & $7.305 \mathrm{E}-32$ & $0.000 \mathrm{E}+00$ & $0.000 \mathrm{E}+00$ \\
\hline cm241 & $2.345 \mathrm{E}-08$ & $2.917 \mathrm{E}-24$ & $4.088 \mathrm{E}-25$ & $1.827 \mathrm{E}-28$ & $8.163 \mathrm{E}-32$ \\
\hline cm242 & $1.922 \mathrm{E}+01$ & $2.080 \mathrm{E}-02$ & $1.684 \mathrm{E}-02$ & $1.039 \mathrm{E}-02$ & 8.993E-03 \\
\hline cm243 & $1.728 \mathrm{E}+00$ & $1.543 \mathrm{E}+00$ & $1.533 \mathrm{E}+00$ & $1.498 \mathrm{E}+00$ & $1.462 \mathrm{E}+00$ \\
\hline $\mathrm{cm} 244$ & $1.718 \mathrm{E}+02$ & $1.432 \mathrm{E}+02$ & $1.419 \mathrm{E}+02$ & $1.365 \mathrm{E}+02$ & $1.314 \mathrm{E}+02$ \\
\hline cm245 & $1.722 \mathrm{E}+01$ & $1.721 \mathrm{E}+01$ & $1.721 \mathrm{E}+01$ & $1.721 \mathrm{E}+01$ & $1.720 \mathrm{E}+01$ \\
\hline cm246 & $1.364 \mathrm{E}+00$ & $1.363 \mathrm{E}+00$ & $1.363 \mathrm{E}+00$ & $1.363 \mathrm{E}+00$ & $1.363 \mathrm{E}+00$ \\
\hline cm247 & $2.249 \mathrm{E}-02$ & $2.249 \mathrm{E}-02$ & $2.249 \mathrm{E}-02$ & $2.249 \mathrm{E}-02$ & $2.249 \mathrm{E}-02$ \\
\hline $\mathrm{cm} 248$ & $1.628 \mathrm{E}-03$ & $1.629 \mathrm{E}-03$ & $1.629 \mathrm{E}-03$ & $1.629 \mathrm{E}-03$ & $1.629 \mathrm{E}-03$ \\
\hline cm250 & $2.200 \mathrm{E}-10$ & $2.200 \mathrm{E}-10$ & $2.200 \mathrm{E}-10$ & $2.200 \mathrm{E}-10$ & $2.200 \mathrm{E}-10$ \\
\hline co 59 & $1.079 \mathrm{E}-13$ & $1.079 \mathrm{E}-13$ & $1.079 \mathrm{E}-13$ & $1.079 \mathrm{E}-13$ & $1.079 \mathrm{E}-13$ \\
\hline co 60 & $1.121 \mathrm{E}-14$ & $6.009 \mathrm{E}-15$ & 5.812E-15 & $5.096 \mathrm{E}-15$ & $4.468 \mathrm{E}-15$ \\
\hline cr 50 & $8.880 \mathrm{E}-14$ & $8.880 \mathrm{E}-14$ & $8.880 \mathrm{E}-14$ & $8.880 \mathrm{E}-14$ & $8.880 \mathrm{E}-14$ \\
\hline cr 52 & $9.445 \mathrm{E}-14$ & $9.445 \mathrm{E}-14$ & $9.445 \mathrm{E}-14$ & $9.445 \mathrm{E}-14$ & $9.445 \mathrm{E}-14$ \\
\hline cr 53 & $9.399 \mathrm{E}-14$ & $9.399 \mathrm{E}-14$ & $9.399 \mathrm{E}-14$ & $9.399 \mathrm{E}-14$ & $9.399 \mathrm{E}-14$ \\
\hline cr 54 & $1.007 \mathrm{E}-13$ & $1.007 \mathrm{E}-13$ & $1.007 \mathrm{E}-13$ & $1.007 \mathrm{E}-13$ & $1.007 \mathrm{E}-13$ \\
\hline $\operatorname{cs} 131$ & $1.699 \mathrm{E}-15$ & $0.000 \mathrm{E}+00$ & $0.000 \mathrm{E}+00$ & $0.000 \mathrm{E}+00$ & $0.000 \mathrm{E}+00$ \\
\hline $\operatorname{cs} 132$ & $1.477 \mathrm{E}-16$ & $0.000 \mathrm{E}+00$ & $0.000 \mathrm{E}+00$ & $0.000 \mathrm{E}+00$ & $0.000 \mathrm{E}+00$ \\
\hline cs133 & $1.384 \mathrm{E}+03$ & $1.384 \mathrm{E}+03$ & $1.384 \mathrm{E}+03$ & $1.384 \mathrm{E}+03$ & $1.384 \mathrm{E}+03$ \\
\hline $\operatorname{cs} 134$ & $1.504 \mathrm{E}+02$ & $3.059 \mathrm{E}+01$ & $2.808 \mathrm{E}+01$ & $2.008 \mathrm{E}+01$ & $1.436 \mathrm{E}+01$ \\
\hline
\end{tabular}




\begin{tabular}{|c|c|c|c|c|c|}
\hline \multicolumn{6}{|c|}{ Table C-3. Continued } \\
\hline & $1 / 22 / 2009$ & $10 / 21 / 2013$ & $1 / 22 / 2014$ & $1 / 22 / 2015$ & $1 / 22 / 2016$ \\
\hline $\operatorname{cs135}$ & $6.807 \mathrm{E}+02$ & $6.807 \mathrm{E}+02$ & $6.807 \mathrm{E}+02$ & $6.807 \mathrm{E}+02$ & $6.807 \mathrm{E}+02$ \\
\hline $\operatorname{cs136}$ & $9.651 \mathrm{E}-07$ & $0.000 \mathrm{E}+00$ & $0.000 \mathrm{E}+00$ & $0.000 \mathrm{E}+00$ & $0.000 \mathrm{E}+00$ \\
\hline $\operatorname{cs137}$ & $1.530 \mathrm{E}+03$ & $1.372 \mathrm{E}+03$ & $1.364 \mathrm{E}+03$ & $1.333 \mathrm{E}+03$ & $1.302 \mathrm{E}+03$ \\
\hline cu 63 & $1.133 \mathrm{E}-13$ & $1.134 \mathrm{E}-13$ & $1.134 \mathrm{E}-13$ & $1.134 \mathrm{E}-13$ & $1.134 \mathrm{E}-13$ \\
\hline cu 65 & $4.610 \mathrm{E}-07$ & $4.610 \mathrm{E}-07$ & 4.610E-07 & $4.610 \mathrm{E}-07$ & 4.610E-07 \\
\hline dy156 & $3.193 \mathrm{E}-12$ & $3.193 \mathrm{E}-12$ & $3.193 \mathrm{E}-12$ & $3.193 \mathrm{E}-12$ & $3.193 \mathrm{E}-12$ \\
\hline dy158 & $1.287 \mathrm{E}-07$ & $4.251 \mathrm{E}-07$ & 4.408E-07 & $5.024 \mathrm{E}-07$ & $5.639 \mathrm{E}-07$ \\
\hline dy159 & $2.515 \mathrm{E}-07$ & $6.132 \mathrm{E}-11$ & $3.924 \mathrm{E}-11$ & $6.807 \mathrm{E}-12$ & $1.180 \mathrm{E}-12$ \\
\hline dy160 & $9.080 \mathrm{E}-01$ & $9.253 \mathrm{E}-01$ & $9.253 \mathrm{E}-01$ & $9.253 \mathrm{E}-01$ & 9.253E-01 \\
\hline dy161 & $1.145 \mathrm{E}+00$ & $1.145 \mathrm{E}+00$ & $1.145 \mathrm{E}+00$ & $1.145 \mathrm{E}+00$ & $1.145 \mathrm{E}+00$ \\
\hline dy162 & $7.126 \mathrm{E}-01$ & $7.126 \mathrm{E}-01$ & $7.126 \mathrm{E}-01$ & $7.126 \mathrm{E}-01$ & $7.126 \mathrm{E}-01$ \\
\hline dy163 & $6.081 \mathrm{E}-01$ & $6.081 \mathrm{E}-01$ & $6.081 \mathrm{E}-01$ & $6.081 \mathrm{E}-01$ & $6.081 \mathrm{E}-01$ \\
\hline dy164 & $2.356 \mathrm{E}-01$ & $2.356 \mathrm{E}-01$ & $2.356 \mathrm{E}-01$ & $2.356 \mathrm{E}-01$ & $2.356 \mathrm{E}-01$ \\
\hline er162 & $3.476 \mathrm{E}-12$ & $3.476 \mathrm{E}-12$ & $3.476 \mathrm{E}-12$ & $3.476 \mathrm{E}-12$ & $3.476 \mathrm{E}-12$ \\
\hline er164 & $3.092 \mathrm{E}-06$ & $3.092 \mathrm{E}-06$ & $3.092 \mathrm{E}-06$ & $3.092 \mathrm{E}-06$ & $3.092 \mathrm{E}-06$ \\
\hline er166 & $8.515 \mathrm{E}-02$ & $8.515 \mathrm{E}-02$ & $8.515 \mathrm{E}-02$ & $8.515 \mathrm{E}-02$ & $8.515 \mathrm{E}-02$ \\
\hline er167 & $3.982 \mathrm{E}-03$ & $3.982 \mathrm{E}-03$ & $3.982 \mathrm{E}-03$ & $3.982 \mathrm{E}-03$ & $3.982 \mathrm{E}-03$ \\
\hline er168 & $1.288 \mathrm{E}-02$ & $1.288 \mathrm{E}-02$ & $1.288 \mathrm{E}-02$ & $1.288 \mathrm{E}-02$ & $1.288 \mathrm{E}-02$ \\
\hline er169 & $1.083 \mathrm{E}-13$ & $0.000 \mathrm{E}+00$ & $0.000 \mathrm{E}+00$ & $0.000 \mathrm{E}+00$ & $0.000 \mathrm{E}+00$ \\
\hline er170 & $6.476 \mathrm{E}-04$ & $6.477 \mathrm{E}-04$ & $6.477 \mathrm{E}-04$ & $6.477 \mathrm{E}-04$ & $6.477 \mathrm{E}-04$ \\
\hline es252 & $4.044 \mathrm{E}-15$ & $3.168 \mathrm{E}-16$ & $2.763 \mathrm{E}-16$ & $1.616 \mathrm{E}-16$ & $9.452 \mathrm{E}-17$ \\
\hline es253 & $2.239 \mathrm{E}-12$ & $0.000 \mathrm{E}+00$ & $0.000 \mathrm{E}+00$ & $0.000 \mathrm{E}+00$ & $0.000 \mathrm{E}+00$ \\
\hline es254 & $1.560 \mathrm{E}-11$ & $1.999 \mathrm{E}-13$ & $1.583 \mathrm{E}-13$ & $6.322 \mathrm{E}-14$ & $2.525 \mathrm{E}-14$ \\
\hline es255 & $2.672 \mathrm{E}-14$ & $2.085 \mathrm{E}-27$ & 4.127E-28 & $7.159 \mathrm{E}-31$ & $1.242 \mathrm{E}-33$ \\
\hline eu147 & $2.747 \mathrm{E}-18$ & $0.000 \mathrm{E}+00$ & $0.000 \mathrm{E}+00$ & $0.000 \mathrm{E}+00$ & $0.000 \mathrm{E}+00$ \\
\hline eu149 & $5.484 \mathrm{E}-11$ & $1.367 \mathrm{E}-16$ & $6.840 \mathrm{E}-17$ & $4.515 \mathrm{E}-18$ & $2.982 \mathrm{E}-19$ \\
\hline eu151 & $1.589 \mathrm{E}-01$ & $9.651 \mathrm{E}-01$ & $1.008 \mathrm{E}+00$ & $1.173 \mathrm{E}+00$ & $1.338 \mathrm{E}+00$ \\
\hline eu152 & $2.894 \mathrm{E}-02$ & $2.270 \mathrm{E}-02$ & $2.241 \mathrm{E}-02$ & $2.129 \mathrm{E}-02$ & $2.023 \mathrm{E}-02$ \\
\hline eu153 & $1.937 \mathrm{E}+02$ & $1.937 \mathrm{E}+02$ & $1.937 \mathrm{E}+02$ & $1.937 \mathrm{E}+02$ & $1.937 \mathrm{E}+02$ \\
\hline eu154 & $5.540 \mathrm{E}+01$ & $3.780 \mathrm{E}+01$ & $3.703 \mathrm{E}+01$ & $3.417 \mathrm{E}+01$ & $3.152 \mathrm{E}+01$ \\
\hline eu155 & $1.375 \mathrm{E}+01$ & $6.886 \mathrm{E}+00$ & $6.632 \mathrm{E}+00$ & $5.733 \mathrm{E}+00$ & $4.955 \mathrm{E}+00$ \\
\hline eu156 & $3.926 \mathrm{E}-05$ & $0.000 \mathrm{E}+00$ & $0.000 \mathrm{E}+00$ & $0.000 \mathrm{E}+00$ & $0.000 \mathrm{E}+00$ \\
\hline f 19 & $1.200 \mathrm{E}-13$ & $1.200 \mathrm{E}-13$ & $1.200 \mathrm{E}-13$ & $1.200 \mathrm{E}-13$ & $1.200 \mathrm{E}-13$ \\
\hline fe 54 & $9.771 \mathrm{E}-14$ & $9.771 \mathrm{E}-14$ & $9.771 \mathrm{E}-14$ & $9.771 \mathrm{E}-14$ & $9.771 \mathrm{E}-14$ \\
\hline fe 55 & $2.164 \mathrm{E}-16$ & $6.525 \mathrm{E}-17$ & $6.119 \mathrm{E}-17$ & $4.754 \mathrm{E}-17$ & $3.693 \mathrm{E}-17$ \\
\hline fe 56 & $1.075 \mathrm{E}-13$ & $1.075 \mathrm{E}-13$ & $1.075 \mathrm{E}-13$ & $1.075 \mathrm{E}-13$ & $1.075 \mathrm{E}-13$ \\
\hline fe 57 & $1.036 \mathrm{E}-13$ & $1.036 \mathrm{E}-13$ & $1.036 \mathrm{E}-13$ & $1.036 \mathrm{E}-13$ & $1.036 \mathrm{E}-13$ \\
\hline fe 58 & $3.025 \mathrm{E}-13$ & $3.025 \mathrm{E}-13$ & $3.025 \mathrm{E}-13$ & $3.025 \mathrm{E}-13$ & $3.025 \mathrm{E}-13$ \\
\hline
\end{tabular}




\begin{tabular}{|c|c|c|c|c|c|}
\hline \multicolumn{6}{|c|}{ Table C-3. Continued } \\
\hline & $1 / 22 / 2009$ & $10 / 21 / 2013$ & $1 / 22 / 2014$ & $1 / 22 / 2015$ & $1 / 22 / 2016$ \\
\hline fr221 & $4.408 \mathrm{E}-16$ & $4.557 \mathrm{E}-16$ & $4.564 \mathrm{E}-16$ & $4.610 \mathrm{E}-16$ & $4.656 \mathrm{E}-16$ \\
\hline ga 69 & $1.612 \mathrm{E}-03$ & $1.612 \mathrm{E}-03$ & $1.612 \mathrm{E}-03$ & $1.612 \mathrm{E}-03$ & $1.612 \mathrm{E}-03$ \\
\hline ga 71 & $7.033 \mathrm{E}-03$ & $7.033 \mathrm{E}-03$ & 7.033E-03 & $7.033 \mathrm{E}-03$ & $7.033 \mathrm{E}-03$ \\
\hline gd151 & $6.389 \mathrm{E}-08$ & $3.965 \mathrm{E}-12$ & $2.358 \mathrm{E}-12$ & $3.065 \mathrm{E}-13$ & $3.984 \mathrm{E}-14$ \\
\hline gd152 & $6.192 \mathrm{E}-02$ & $6.366 \mathrm{E}-02$ & $6.374 \mathrm{E}-02$ & $6.405 \mathrm{E}-02$ & $6.435 \mathrm{E}-02$ \\
\hline $\operatorname{gd153}$ & $2.466 \mathrm{E}-03$ & $1.666 \mathrm{E}-05$ & $1.275 \mathrm{E}-05$ & 4.450E-06 & $1.553 \mathrm{E}-06$ \\
\hline gd154 & $8.581 \mathrm{E}+00$ & $2.618 \mathrm{E}+01$ & $2.695 \mathrm{E}+01$ & $2.982 \mathrm{E}+01$ & $3.246 \mathrm{E}+01$ \\
\hline gd155 & $1.731 \mathrm{E}+00$ & $8.595 \mathrm{E}+00$ & $8.847 \mathrm{E}+00$ & $9.751 \mathrm{E}+00$ & $1.053 \mathrm{E}+01$ \\
\hline gd156 & $1.638 \mathrm{E}+02$ & $1.638 \mathrm{E}+02$ & $1.638 \mathrm{E}+02$ & $1.638 \mathrm{E}+02$ & $1.638 \mathrm{E}+02$ \\
\hline gd157 & $3.288 \mathrm{E}-01$ & $3.288 \mathrm{E}-01$ & $3.288 \mathrm{E}-01$ & $3.288 \mathrm{E}-01$ & $3.288 \mathrm{E}-01$ \\
\hline gd158 & $4.551 \mathrm{E}+01$ & $4.551 \mathrm{E}+01$ & $4.551 \mathrm{E}+01$ & $4.551 \mathrm{E}+01$ & $4.551 \mathrm{E}+01$ \\
\hline gd160 & $3.038 \mathrm{E}+00$ & $3.038 \mathrm{E}+00$ & $3.038 \mathrm{E}+00$ & $3.038 \mathrm{E}+00$ & $3.038 \mathrm{E}+00$ \\
\hline ge 70 & $2.041 \mathrm{E}-05$ & $2.041 \mathrm{E}-05$ & $2.041 \mathrm{E}-05$ & $2.041 \mathrm{E}-05$ & $2.041 \mathrm{E}-05$ \\
\hline ge 71 & $4.671 \mathrm{E}-16$ & $0.000 \mathrm{E}+00$ & $0.000 \mathrm{E}+00$ & $0.000 \mathrm{E}+00$ & $0.000 \mathrm{E}+00$ \\
\hline ge 72 & $1.721 \mathrm{E}-02$ & $1.721 \mathrm{E}-02$ & $1.721 \mathrm{E}-02$ & $1.721 \mathrm{E}-02$ & $1.721 \mathrm{E}-02$ \\
\hline ge 73 & $3.536 \mathrm{E}-02$ & $3.536 \mathrm{E}-02$ & $3.536 \mathrm{E}-02$ & $3.536 \mathrm{E}-02$ & $3.536 \mathrm{E}-02$ \\
\hline ge 73m & $9.140 \mathrm{E}-18$ & $2.911 \mathrm{E}-24$ & $1.305 \mathrm{E}-24$ & $5.586 \mathrm{E}-26$ & $2.392 \mathrm{E}-27$ \\
\hline ge 74 & $8.375 \mathrm{E}-02$ & $8.375 \mathrm{E}-02$ & $8.375 \mathrm{E}-02$ & $8.375 \mathrm{E}-02$ & $8.375 \mathrm{E}-02$ \\
\hline ge 76 & $3.750 \mathrm{E}-01$ & $3.750 \mathrm{E}-01$ & $3.750 \mathrm{E}-01$ & $3.750 \mathrm{E}-01$ & $3.750 \mathrm{E}-01$ \\
\hline h 1 & $6.640 \mathrm{E}-03$ & $6.640 \mathrm{E}-03$ & $6.640 \mathrm{E}-03$ & $6.640 \mathrm{E}-03$ & $6.640 \mathrm{E}-03$ \\
\hline h 2 & $1.420 \mathrm{E}-03$ & $1.420 \mathrm{E}-03$ & $1.420 \mathrm{E}-03$ & $1.420 \mathrm{E}-03$ & $1.420 \mathrm{E}-03$ \\
\hline h3 & $7.185 \mathrm{E}-02$ & $5.501 \mathrm{E}-02$ & $5.422 \mathrm{E}-02$ & $5.126 \mathrm{E}-02$ & $4.846 \mathrm{E}-02$ \\
\hline he3 & $4.310 \mathrm{E}-03$ & $2.114 \mathrm{E}-02$ & 2.192E-02 & $2.489 \mathrm{E}-02$ & $2.769 \mathrm{E}-02$ \\
\hline he4 & $7.879 \mathrm{E}+00$ & $8.987 \mathrm{E}+00$ & $9.031 \mathrm{E}+00$ & $9.199 \mathrm{E}+00$ & $9.369 \mathrm{E}+00$ \\
\hline hf174 & $1.296 \mathrm{E}-13$ & $1.296 \mathrm{E}-13$ & $1.296 \mathrm{E}-13$ & $1.296 \mathrm{E}-13$ & $1.296 \mathrm{E}-13$ \\
\hline hf175 & $9.153 \mathrm{E}-16$ & $3.226 \mathrm{E}-23$ & $1.285 \mathrm{E}-23$ & $3.460 \mathrm{E}-25$ & $9.319 \mathrm{E}-27$ \\
\hline hf176 & $3.314 \mathrm{E}-13$ & $3.314 \mathrm{E}-13$ & $3.314 \mathrm{E}-13$ & $3.314 \mathrm{E}-13$ & $3.314 \mathrm{E}-13$ \\
\hline hf177 & $4.699 \mathrm{E}-14$ & $4.700 \mathrm{E}-14$ & $4.700 \mathrm{E}-14$ & $4.700 \mathrm{E}-14$ & $4.700 \mathrm{E}-14$ \\
\hline hf178 & $3.042 \mathrm{E}-13$ & $3.042 \mathrm{E}-13$ & $3.042 \mathrm{E}-13$ & $3.042 \mathrm{E}-13$ & $3.042 \mathrm{E}-13$ \\
\hline hf179 & 7.804E-13 & $7.804 \mathrm{E}-13$ & 7.804E-13 & $7.804 \mathrm{E}-13$ & $7.804 \mathrm{E}-13$ \\
\hline hf180 & $6.265 \mathrm{E}-13$ & $6.265 \mathrm{E}-13$ & $6.265 \mathrm{E}-13$ & $6.265 \mathrm{E}-13$ & $6.265 \mathrm{E}-13$ \\
\hline hf181 & $2.578 \mathrm{E}-17$ & $1.272 \mathrm{E}-29$ & $2.779 \mathrm{E}-30$ & $7.112 \mathrm{E}-33$ & $0.000 \mathrm{E}+00$ \\
\hline hf182 & $1.247 \mathrm{E}-15$ & $1.247 \mathrm{E}-15$ & $1.247 \mathrm{E}-15$ & $1.247 \mathrm{E}-15$ & $1.247 \mathrm{E}-15$ \\
\hline hg196 & $1.451 \mathrm{E}-14$ & $1.451 \mathrm{E}-14$ & $1.451 \mathrm{E}-14$ & $1.451 \mathrm{E}-14$ & $1.451 \mathrm{E}-14$ \\
\hline hg198 & $6.700 \mathrm{E}-13$ & $6.700 \mathrm{E}-13$ & $6.700 \mathrm{E}-13$ & $6.700 \mathrm{E}-13$ & $6.700 \mathrm{E}-13$ \\
\hline hg199 & $9.612 \mathrm{E}-14$ & $9.612 \mathrm{E}-14$ & $9.612 \mathrm{E}-14$ & $9.612 \mathrm{E}-14$ & $9.612 \mathrm{E}-14$ \\
\hline hg200 & $8.183 \mathrm{E}-13$ & $8.183 \mathrm{E}-13$ & $8.183 \mathrm{E}-13$ & $8.183 \mathrm{E}-13$ & $8.183 \mathrm{E}-13$ \\
\hline hg201 & $3.519 \mathrm{E}-13$ & $3.519 \mathrm{E}-13$ & $3.519 \mathrm{E}-13$ & $3.519 \mathrm{E}-13$ & $3.519 \mathrm{E}-13$ \\
\hline
\end{tabular}




\begin{tabular}{|c|c|c|c|c|c|}
\hline \multicolumn{6}{|c|}{ Table C-3. Continued } \\
\hline & $1 / 22 / 2009$ & $10 / 21 / 2013$ & $1 / 22 / 2014$ & $1 / 22 / 2015$ & $1 / 22 / 2016$ \\
\hline hg202 & $3.809 \mathrm{E}-13$ & $3.809 \mathrm{E}-13$ & $3.809 \mathrm{E}-13$ & $3.809 \mathrm{E}-13$ & $3.809 \mathrm{E}-13$ \\
\hline hg204 & $3.694 \mathrm{E}-13$ & $3.694 \mathrm{E}-13$ & $3.694 \mathrm{E}-13$ & $3.694 \mathrm{E}-13$ & $3.694 \mathrm{E}-13$ \\
\hline ho163 & $1.984 \mathrm{E}-07$ & $1.983 \mathrm{E}-07$ & $1.983 \mathrm{E}-07$ & $1.983 \mathrm{E}-07$ & $1.982 \mathrm{E}-07$ \\
\hline ho165 & $2.627 \mathrm{E}-01$ & $2.627 \mathrm{E}-01$ & 2.627E-01 & $2.627 \mathrm{E}-01$ & $2.627 \mathrm{E}-01$ \\
\hline ho166m & $6.331 \mathrm{E}-04$ & $6.314 \mathrm{E}-04$ & 6.313E-04 & $6.309 \mathrm{E}-04$ & $6.306 \mathrm{E}-04$ \\
\hline i125 & $9.558 \mathrm{E}-10$ & $1.577 \mathrm{E}-18$ & $5.327 \mathrm{E}-19$ & $7.531 \mathrm{E}-21$ & $1.064 \mathrm{E}-22$ \\
\hline i126 & $1.638 \mathrm{E}-11$ & $0.000 \mathrm{E}+00$ & $0.000 \mathrm{E}+00$ & $0.000 \mathrm{E}+00$ & $0.000 \mathrm{E}+00$ \\
\hline i127 & $8.661 \mathrm{E}+01$ & $8.681 \mathrm{E}+01$ & $8.681 \mathrm{E}+01$ & $8.681 \mathrm{E}+01$ & $8.681 \mathrm{E}+01$ \\
\hline i129 & $2.769 \mathrm{E}+02$ & $2.769 \mathrm{E}+02$ & $2.769 \mathrm{E}+02$ & $2.769 \mathrm{E}+02$ & $2.769 \mathrm{E}+02$ \\
\hline i131 & $1.100 \mathrm{E}-09$ & $0.000 \mathrm{E}+00$ & $0.000 \mathrm{E}+00$ & $0.000 \mathrm{E}+00$ & $0.000 \mathrm{E}+00$ \\
\hline in113 & 7.099E-04 & $1.644 \mathrm{E}-03$ & $1.688 \mathrm{E}-03$ & $1.856 \mathrm{E}-03$ & $2.017 \mathrm{E}-03$ \\
\hline in113m & $3.766 \mathrm{E}-14$ & $1.104 \mathrm{E}-18$ & $6.305 \mathrm{E}-19$ & $6.999 \mathrm{E}-20$ & 7.770E-21 \\
\hline in114 & $3.953 \mathrm{E}-12$ & $1.148 \mathrm{E}-22$ & $3.121 \mathrm{E}-23$ & $1.884 \mathrm{E}-25$ & $1.137 \mathrm{E}-27$ \\
\hline in114m & $2.431 \mathrm{E}-07$ & $7.059 \mathrm{E}-18$ & $1.920 \mathrm{E}-18$ & $1.159 \mathrm{E}-20$ & $6.993 \mathrm{E}-23$ \\
\hline in115 & $3.023 \mathrm{E}+00$ & $3.024 \mathrm{E}+00$ & $3.024 \mathrm{E}+00$ & $3.024 \mathrm{E}+00$ & $3.024 \mathrm{E}+00$ \\
\hline in115m & $2.751 \mathrm{E}-10$ & $5.395 \mathrm{E}-22$ & $1.270 \mathrm{E}-22$ & $4.345 \mathrm{E}-25$ & $1.486 \mathrm{E}-27$ \\
\hline ir191 & $6.886 \mathrm{E}-15$ & $6.886 \mathrm{E}-15$ & $6.886 \mathrm{E}-15$ & $6.886 \mathrm{E}-15$ & $6.886 \mathrm{E}-15$ \\
\hline ir192 & $1.492 \mathrm{E}-16$ & $1.281 \mathrm{E}-23$ & $5.349 \mathrm{E}-24$ & $1.738 \mathrm{E}-25$ & $5.645 \mathrm{E}-27$ \\
\hline ir193 & $1.630 \mathrm{E}-13$ & $1.643 \mathrm{E}-13$ & $1.644 \mathrm{E}-13$ & $1.646 \mathrm{E}-13$ & $1.648 \mathrm{E}-13$ \\
\hline k 39 & $7.053 \mathrm{E}-14$ & $7.053 \mathrm{E}-14$ & 7.053E-14 & $7.053 \mathrm{E}-14$ & $7.053 \mathrm{E}-14$ \\
\hline k 40 & $6.946 \mathrm{E}-14$ & $6.946 \mathrm{E}-14$ & $6.946 \mathrm{E}-14$ & $6.946 \mathrm{E}-14$ & $6.946 \mathrm{E}-14$ \\
\hline k 41 & 7.737E-14 & 7.737E-14 & 7.737E-14 & 7.737E-14 & 7.737E-14 \\
\hline kr 78 & $1.864 \mathrm{E}-13$ & $1.864 \mathrm{E}-13$ & $1.864 \mathrm{E}-13$ & $1.864 \mathrm{E}-13$ & $1.864 \mathrm{E}-13$ \\
\hline kr 80 & 4.987E-04 & 4.987E-04 & 4.987E-04 & 4.987E-04 & 4.987E-04 \\
\hline kr 81 & $4.268 \mathrm{E}-05$ & $4.268 \mathrm{E}-05$ & $4.268 \mathrm{E}-05$ & $4.268 \mathrm{E}-05$ & $4.268 \mathrm{E}-05$ \\
\hline kr 82 & $9.432 \mathrm{E}-01$ & $9.432 \mathrm{E}-01$ & $9.432 \mathrm{E}-01$ & $9.432 \mathrm{E}-01$ & $9.432 \mathrm{E}-01$ \\
\hline kr 83 & $3.681 \mathrm{E}+01$ & $3.681 \mathrm{E}+01$ & $3.681 \mathrm{E}+01$ & $3.681 \mathrm{E}+01$ & $3.681 \mathrm{E}+01$ \\
\hline kr 83m & $3.873 \mathrm{E}-11$ & $3.435 \mathrm{E}-17$ & $1.626 \mathrm{E}-17$ & $8.641 \mathrm{E}-19$ & $4.590 \mathrm{E}-20$ \\
\hline kr 84 & $8.674 \mathrm{E}+01$ & $8.674 \mathrm{E}+01$ & $8.674 \mathrm{E}+01$ & $8.674 \mathrm{E}+01$ & $8.674 \mathrm{E}+01$ \\
\hline kr 85 & $1.761 \mathrm{E}+01$ & $1.297 \mathrm{E}+01$ & $1.276 \mathrm{E}+01$ & $1.196 \mathrm{E}+01$ & $1.121 \mathrm{E}+01$ \\
\hline kr 86 & $1.285 \mathrm{E}+02$ & $1.285 \mathrm{E}+02$ & $1.285 \mathrm{E}+02$ & $1.285 \mathrm{E}+02$ & $1.285 \mathrm{E}+02$ \\
\hline la137 & 4.646E-04 & 4.646E-04 & 4.646E-04 & 4.646E-04 & 4.646E-04 \\
\hline la138 & $2.667 \mathrm{E}-02$ & $2.667 \mathrm{E}-02$ & $2.667 \mathrm{E}-02$ & $2.667 \mathrm{E}-02$ & $2.667 \mathrm{E}-02$ \\
\hline la139 & $1.420 \mathrm{E}+03$ & $1.420 \mathrm{E}+03$ & $1.420 \mathrm{E}+03$ & $1.420 \mathrm{E}+03$ & $1.420 \mathrm{E}+03$ \\
\hline la140 & $1.969 \mathrm{E}-06$ & $0.000 \mathrm{E}+00$ & $0.000 \mathrm{E}+00$ & $0.000 \mathrm{E}+00$ & $0.000 \mathrm{E}+00$ \\
\hline li6 & $2.598 \mathrm{E}-10$ & $2.598 \mathrm{E}-10$ & $2.598 \mathrm{E}-10$ & $2.598 \mathrm{E}-10$ & $2.598 \mathrm{E}-10$ \\
\hline li7 & $2.849 \mathrm{E}-11$ & $2.849 \mathrm{E}-11$ & $2.849 \mathrm{E}-11$ & $2.849 \mathrm{E}-11$ & $2.849 \mathrm{E}-11$ \\
\hline lu175 & $2.971 \mathrm{E}-13$ & $2.981 \mathrm{E}-13$ & $2.981 \mathrm{E}-13$ & $2.981 \mathrm{E}-13$ & $2.981 \mathrm{E}-13$ \\
\hline
\end{tabular}




\begin{tabular}{|c|c|c|c|c|c|}
\hline \multicolumn{6}{|c|}{ Table C-3. Continued } \\
\hline & $1 / 22 / 2009$ & $10 / 21 / 2013$ & $1 / 22 / 2014$ & $1 / 22 / 2015$ & $1 / 22 / 2016$ \\
\hline lu176 & $6.589 \mathrm{E}-15$ & $6.589 \mathrm{E}-15$ & $6.589 \mathrm{E}-15$ & $6.589 \mathrm{E}-15$ & $6.589 \mathrm{E}-15$ \\
\hline mg 24 & $4.366 \mathrm{E}-14$ & $4.366 \mathrm{E}-14$ & $4.366 \mathrm{E}-14$ & $4.366 \mathrm{E}-14$ & $4.366 \mathrm{E}-14$ \\
\hline mg 25 & $4.543 \mathrm{E}-14$ & $4.543 \mathrm{E}-14$ & $4.543 \mathrm{E}-14$ & $4.543 \mathrm{E}-14$ & $4.543 \mathrm{E}-14$ \\
\hline mg 26 & 4.729E-14 & $4.729 \mathrm{E}-14$ & 4.729E-14 & $4.729 \mathrm{E}-14$ & 4.729E-14 \\
\hline mn 54 & $2.259 \mathrm{E}-17$ & $4.810 \mathrm{E}-19$ & $3.912 \mathrm{E}-19$ & $1.739 \mathrm{E}-19$ & $7.731 \mathrm{E}-20$ \\
\hline mn 55 & $9.758 \mathrm{E}-14$ & $9.771 \mathrm{E}-14$ & $9.771 \mathrm{E}-14$ & $9.771 \mathrm{E}-14$ & $9.778 \mathrm{E}-14$ \\
\hline mo 92 & $6.275 \mathrm{E}-12$ & $6.275 \mathrm{E}-12$ & $6.275 \mathrm{E}-12$ & $6.275 \mathrm{E}-12$ & $6.275 \mathrm{E}-12$ \\
\hline mo 93 & 4.301E-07 & 4.297E-07 & 4.297E-07 & 4.297E-07 & $4.296 \mathrm{E}-07$ \\
\hline mo 94 & $1.435 \mathrm{E}-02$ & $1.435 \mathrm{E}-02$ & $1.435 \mathrm{E}-02$ & $1.435 \mathrm{E}-02$ & $1.435 \mathrm{E}-02$ \\
\hline mo 95 & $7.624 \mathrm{E}+02$ & $7.704 \mathrm{E}+02$ & $7.704 \mathrm{E}+02$ & $7.704 \mathrm{E}+02$ & $7.704 \mathrm{E}+02$ \\
\hline mo 96 & $4.608 \mathrm{E}+01$ & $4.608 \mathrm{E}+01$ & $4.608 \mathrm{E}+01$ & $4.608 \mathrm{E}+01$ & $4.608 \mathrm{E}+01$ \\
\hline mo 97 & $9.279 \mathrm{E}+02$ & $9.279 \mathrm{E}+02$ & $9.279 \mathrm{E}+02$ & $9.279 \mathrm{E}+02$ & $9.279 \mathrm{E}+02$ \\
\hline mo 98 & $1.016 \mathrm{E}+03$ & $1.016 \mathrm{E}+03$ & $1.016 \mathrm{E}+03$ & $1.016 \mathrm{E}+03$ & $1.016 \mathrm{E}+03$ \\
\hline mo100 & $1.196 \mathrm{E}+03$ & $1.196 \mathrm{E}+03$ & $1.196 \mathrm{E}+03$ & $1.196 \mathrm{E}+03$ & $1.196 \mathrm{E}+03$ \\
\hline n 14 & 4.846E-06 & $5.073 \mathrm{E}-06$ & $5.085 \mathrm{E}-06$ & 5.133E-06 & $5.181 \mathrm{E}-06$ \\
\hline n 15 & $1.170 \mathrm{E}-02$ & $1.170 \mathrm{E}-02$ & $1.170 \mathrm{E}-02$ & $1.170 \mathrm{E}-02$ & $1.170 \mathrm{E}-02$ \\
\hline na 23 & $4.178 \mathrm{E}-14$ & $4.178 \mathrm{E}-14$ & $4.178 \mathrm{E}-14$ & $4.178 \mathrm{E}-14$ & 4.178E-14 \\
\hline nb 91 & $9.798 \mathrm{E}-09$ & $9.751 \mathrm{E}-09$ & $9.745 \mathrm{E}-09$ & $9.738 \mathrm{E}-09$ & $9.725 \mathrm{E}-09$ \\
\hline nb 91m & $6.219 \mathrm{E}-15$ & $1.666 \mathrm{E}-23$ & $5.778 \mathrm{E}-24$ & $9.047 \mathrm{E}-26$ & $1.416 \mathrm{E}-27$ \\
\hline nb 92 & $8.488 \mathrm{E}-07$ & $8.488 \mathrm{E}-07$ & $8.488 \mathrm{E}-07$ & $8.488 \mathrm{E}-07$ & $8.488 \mathrm{E}-07$ \\
\hline nb 92m & $1.644 \mathrm{E}-18$ & $0.000 \mathrm{E}+00$ & $0.000 \mathrm{E}+00$ & $0.000 \mathrm{E}+00$ & $0.000 \mathrm{E}+00$ \\
\hline nb 93 & $1.037 \mathrm{E}-04$ & $3.791 \mathrm{E}-04$ & $4.000 \mathrm{E}-04$ & 4.874E-04 & $5.833 \mathrm{E}-04$ \\
\hline nb 93m & $6.143 \mathrm{E}-04$ & $1.713 \mathrm{E}-03$ & $1.766 \mathrm{E}-03$ & $1.968 \mathrm{E}-03$ & $2.162 \mathrm{E}-03$ \\
\hline nb 94 & $3.143 \mathrm{E}-03$ & $3.143 \mathrm{E}-03$ & $3.143 \mathrm{E}-03$ & $3.143 \mathrm{E}-03$ & $3.143 \mathrm{E}-03$ \\
\hline nb 95 & $4.196 \mathrm{E}+00$ & $3.159 \mathrm{E}-08$ & $1.153 \mathrm{E}-08$ & $2.285 \mathrm{E}-10$ & $4.400 \mathrm{E}-12$ \\
\hline nb 95m & $2.370 \mathrm{E}-03$ & $1.688 \mathrm{E}-11$ & $6.168 \mathrm{E}-12$ & $1.186 \mathrm{E}-13$ & $2.281 \mathrm{E}-15$ \\
\hline nd142 & $2.137 \mathrm{E}+01$ & $2.137 \mathrm{E}+01$ & $2.137 \mathrm{E}+01$ & $2.137 \mathrm{E}+01$ & $2.137 \mathrm{E}+01$ \\
\hline nd143 & $9.193 \mathrm{E}+02$ & $9.193 \mathrm{E}+02$ & $9.193 \mathrm{E}+02$ & $9.193 \mathrm{E}+02$ & $9.193 \mathrm{E}+02$ \\
\hline nd144 & $1.072 \mathrm{E}+03$ & $1.252 \mathrm{E}+03$ & $1.252 \mathrm{E}+03$ & $1.254 \mathrm{E}+03$ & $1.254 \mathrm{E}+03$ \\
\hline nd145 & $7.079 \mathrm{E}+02$ & $7.079 \mathrm{E}+02$ & $7.079 \mathrm{E}+02$ & $7.079 \mathrm{E}+02$ & $7.079 \mathrm{E}+02$ \\
\hline nd146 & $7.923 \mathrm{E}+02$ & $7.923 \mathrm{E}+02$ & $7.923 \mathrm{E}+02$ & $7.923 \mathrm{E}+02$ & $7.923 \mathrm{E}+02$ \\
\hline nd147 & 4.452E-07 & $0.000 \mathrm{E}+00$ & $0.000 \mathrm{E}+00$ & $0.000 \mathrm{E}+00$ & $0.000 \mathrm{E}+00$ \\
\hline nd148 & $4.616 \mathrm{E}+02$ & $4.616 \mathrm{E}+02$ & $4.616 \mathrm{E}+02$ & $4.616 \mathrm{E}+02$ & $4.616 \mathrm{E}+02$ \\
\hline nd150 & $2.715 \mathrm{E}+02$ & $2.715 \mathrm{E}+02$ & $2.715 \mathrm{E}+02$ & $2.715 \mathrm{E}+02$ & $2.715 \mathrm{E}+02$ \\
\hline ni 58 & $1.046 \mathrm{E}-13$ & $1.046 \mathrm{E}-13$ & $1.046 \mathrm{E}-13$ & $1.046 \mathrm{E}-13$ & $1.046 \mathrm{E}-13$ \\
\hline ni 59 & $8.242 \mathrm{E}-14$ & $8.242 \mathrm{E}-14$ & $8.242 \mathrm{E}-14$ & $8.242 \mathrm{E}-14$ & $8.242 \mathrm{E}-14$ \\
\hline ni 60 & $1.339 \mathrm{E}-13$ & $1.391 \mathrm{E}-13$ & $1.393 \mathrm{E}-13$ & $1.401 \mathrm{E}-13$ & $1.407 \mathrm{E}-13$ \\
\hline ni 61 & $1.110 \mathrm{E}-13$ & $1.110 \mathrm{E}-13$ & $1.110 \mathrm{E}-13$ & $1.110 \mathrm{E}-13$ & $1.110 \mathrm{E}-13$ \\
\hline
\end{tabular}




\begin{tabular}{|c|c|c|c|c|c|}
\hline \multicolumn{6}{|c|}{ Table C-3. Continued } \\
\hline & $1 / 22 / 2009$ & $10 / 21 / 2013$ & $1 / 22 / 2014$ & $1 / 22 / 2015$ & $1 / 22 / 2016$ \\
\hline ni 62 & $1.110 \mathrm{E}-13$ & $1.110 \mathrm{E}-13$ & $1.110 \mathrm{E}-13$ & $1.110 \mathrm{E}-13$ & $1.110 \mathrm{E}-13$ \\
\hline ni 63 & $2.211 \mathrm{E}-15$ & $2.140 \mathrm{E}-15$ & $2.136 \mathrm{E}-15$ & $2.122 \mathrm{E}-15$ & $2.107 \mathrm{E}-15$ \\
\hline ni 64 & $1.169 \mathrm{E}-13$ & $1.169 \mathrm{E}-13$ & $1.169 \mathrm{E}-13$ & $1.169 \mathrm{E}-13$ & $1.169 \mathrm{E}-13$ \\
\hline np235 & $2.315 \mathrm{E}-06$ & $1.116 \mathrm{E}-07$ & $9.485 \mathrm{E}-08$ & $5.009 \mathrm{E}-08$ & $2.645 \mathrm{E}-08$ \\
\hline np236 & $3.378 \mathrm{E}-04$ & $3.378 \mathrm{E}-04$ & $3.378 \mathrm{E}-04$ & $3.378 \mathrm{E}-04$ & $3.378 \mathrm{E}-04$ \\
\hline np237 & $1.870 \mathrm{E}+02$ & $1.928 \mathrm{E}+02$ & $1.934 \mathrm{E}+02$ & $1.954 \mathrm{E}+02$ & $1.977 \mathrm{E}+02$ \\
\hline np238 & $6.352 \mathrm{E}-07$ & $6.206 \mathrm{E}-07$ & $6.198 \mathrm{E}-07$ & $6.167 \mathrm{E}-07$ & $6.137 \mathrm{E}-07$ \\
\hline np239 & $3.448 \mathrm{E}-04$ & $3.446 \mathrm{E}-04$ & $3.446 \mathrm{E}-04$ & $3.446 \mathrm{E}-04$ & $3.446 \mathrm{E}-04$ \\
\hline np240 & $2.222 \mathrm{E}-16$ & $2.222 \mathrm{E}-16$ & $2.222 \mathrm{E}-16$ & $2.222 \mathrm{E}-16$ & $2.222 \mathrm{E}-16$ \\
\hline np240m & $2.160 \mathrm{E}-14$ & $2.160 \mathrm{E}-14$ & $2.160 \mathrm{E}-14$ & $2.160 \mathrm{E}-14$ & $2.160 \mathrm{E}-14$ \\
\hline o 16 & $1.344 \mathrm{E}+05$ & $1.344 \mathrm{E}+05$ & $1.344 \mathrm{E}+05$ & $1.344 \mathrm{E}+05$ & $1.344 \mathrm{E}+05$ \\
\hline o 17 & $3.796 \mathrm{E}-02$ & $3.796 \mathrm{E}-02$ & $3.796 \mathrm{E}-02$ & $3.796 \mathrm{E}-02$ & $3.796 \mathrm{E}-02$ \\
\hline o 18 & $1.683 \mathrm{E}-07$ & $1.683 \mathrm{E}-07$ & $1.683 \mathrm{E}-07$ & $1.683 \mathrm{E}-07$ & $1.683 \mathrm{E}-07$ \\
\hline os186 & $2.138 \mathrm{E}-13$ & $2.138 \mathrm{E}-13$ & $2.138 \mathrm{E}-13$ & $2.138 \mathrm{E}-13$ & $2.138 \mathrm{E}-13$ \\
\hline os187 & $3.740 \mathrm{E}-14$ & $3.740 \mathrm{E}-14$ & $3.740 \mathrm{E}-14$ & $3.740 \mathrm{E}-14$ & $3.740 \mathrm{E}-14$ \\
\hline os188 & $1.473 \mathrm{E}-13$ & $1.473 \mathrm{E}-13$ & $1.473 \mathrm{E}-13$ & $1.473 \mathrm{E}-13$ & $1.473 \mathrm{E}-13$ \\
\hline os189 & $1.027 \mathrm{E}-14$ & $1.027 \mathrm{E}-14$ & $1.027 \mathrm{E}-14$ & $1.027 \mathrm{E}-14$ & $1.027 \mathrm{E}-14$ \\
\hline os190 & $2.901 \mathrm{E}-15$ & $2.901 \mathrm{E}-15$ & $2.901 \mathrm{E}-15$ & $2.901 \mathrm{E}-15$ & $2.901 \mathrm{E}-15$ \\
\hline os192 & $8.435 \mathrm{E}-15$ & $8.448 \mathrm{E}-15$ & $8.448 \mathrm{E}-15$ & $8.448 \mathrm{E}-15$ & $8.448 \mathrm{E}-15$ \\
\hline p 31 & $5.639 \mathrm{E}-14$ & $5.639 \mathrm{E}-14$ & $5.639 \mathrm{E}-14$ & $5.639 \mathrm{E}-14$ & $5.639 \mathrm{E}-14$ \\
\hline pa230 & $1.024 \mathrm{E}-15$ & $0.000 \mathrm{E}+00$ & $0.000 \mathrm{E}+00$ & $0.000 \mathrm{E}+00$ & $0.000 \mathrm{E}+00$ \\
\hline pa231 & 2.402E-05 & $2.791 \mathrm{E}-05$ & 2.812E-05 & $2.893 \mathrm{E}-05$ & $2.976 \mathrm{E}-05$ \\
\hline pa232 & $1.569 \mathrm{E}-14$ & $1.569 \mathrm{E}-14$ & $1.569 \mathrm{E}-14$ & $1.569 \mathrm{E}-14$ & $1.569 \mathrm{E}-14$ \\
\hline pa233 & $6.331 \mathrm{E}-06$ & $6.525 \mathrm{E}-06$ & $6.541 \mathrm{E}-06$ & $6.618 \mathrm{E}-06$ & $6.694 \mathrm{E}-06$ \\
\hline pa234 & $2.502 \mathrm{E}-10$ & $2.503 \mathrm{E}-10$ & $2.502 \mathrm{E}-10$ & $2.502 \mathrm{E}-10$ & $2.502 \mathrm{E}-10$ \\
\hline pa234m & $4.509 \mathrm{E}-10$ & $4.511 \mathrm{E}-10$ & $4.509 \mathrm{E}-10$ & $4.509 \mathrm{E}-10$ & $4.509 \mathrm{E}-10$ \\
\hline pb204 & $3.696 \mathrm{E}-13$ & $3.696 \mathrm{E}-13$ & $3.696 \mathrm{E}-13$ & $3.696 \mathrm{E}-13$ & $3.696 \mathrm{E}-13$ \\
\hline pb205 & $1.320 \mathrm{E}-15$ & $1.321 \mathrm{E}-15$ & $1.321 \mathrm{E}-15$ & $1.321 \mathrm{E}-15$ & $1.321 \mathrm{E}-15$ \\
\hline pb206 & $1.967 \mathrm{E}-12$ & $9.412 \mathrm{E}-12$ & $9.824 \mathrm{E}-12$ & $1.147 \mathrm{E}-11$ & $1.321 \mathrm{E}-11$ \\
\hline pb207 & $6.727 \mathrm{E}-11$ & $3.178 \mathrm{E}-10$ & $3.401 \mathrm{E}-10$ & 4.367E-10 & $5.476 \mathrm{E}-10$ \\
\hline pb208 & $9.392 \mathrm{E}-07$ & $2.081 \mathrm{E}-05$ & $2.265 \mathrm{E}-05$ & $3.042 \mathrm{E}-05$ & $3.902 \mathrm{E}-05$ \\
\hline pb209 & $1.660 \mathrm{E}-14$ & $1.717 \mathrm{E}-14$ & $1.719 \mathrm{E}-14$ & $1.737 \mathrm{E}-14$ & $1.754 \mathrm{E}-14$ \\
\hline pb210 & $5.266 \mathrm{E}-11$ & $5.336 \mathrm{E}-11$ & $5.386 \mathrm{E}-11$ & $5.647 \mathrm{E}-11$ & $6.023 \mathrm{E}-11$ \\
\hline pb211 & $1.928 \mathrm{E}-15$ & $8.787 \mathrm{E}-15$ & $8.973 \mathrm{E}-15$ & $1.058 \mathrm{E}-14$ & $1.200 \mathrm{E}-14$ \\
\hline pb212 & $2.064 \mathrm{E}-09$ & $1.264 \mathrm{E}-08$ & $1.310 \mathrm{E}-08$ & $1.469 \mathrm{E}-08$ & $1.602 \mathrm{E}-08$ \\
\hline pd102 & $9.126 \mathrm{E}-04$ & $1.087 \mathrm{E}-03$ & $1.087 \mathrm{E}-03$ & $1.087 \mathrm{E}-03$ & $1.088 \mathrm{E}-03$ \\
\hline pd103 & $2.909 \mathrm{E}-09$ & $0.000 \mathrm{E}+00$ & $0.000 \mathrm{E}+00$ & $0.000 \mathrm{E}+00$ & $0.000 \mathrm{E}+00$ \\
\hline pd104 & $4.052 \mathrm{E}+02$ & $4.052 \mathrm{E}+02$ & $4.052 \mathrm{E}+02$ & $4.052 \mathrm{E}+02$ & $4.052 \mathrm{E}+02$ \\
\hline
\end{tabular}




\begin{tabular}{|c|c|c|c|c|c|}
\hline \multicolumn{6}{|c|}{ Table C-3. Continued } \\
\hline & $1 / 22 / 2009$ & $10 / 21 / 2013$ & $1 / 22 / 2014$ & $1 / 22 / 2015$ & $1 / 22 / 2016$ \\
\hline pd105 & $9.067 \mathrm{E}+02$ & $9.067 \mathrm{E}+02$ & $9.067 \mathrm{E}+02$ & $9.067 \mathrm{E}+02$ & $9.067 \mathrm{E}+02$ \\
\hline pd106 & $7.345 \mathrm{E}+02$ & $9.386 \mathrm{E}+02$ & $9.399 \mathrm{E}+02$ & $9.439 \mathrm{E}+02$ & $9.452 \mathrm{E}+02$ \\
\hline pd107 & $5.915 \mathrm{E}+02$ & $5.915 \mathrm{E}+02$ & $5.915 \mathrm{E}+02$ & $5.915 \mathrm{E}+02$ & $5.915 \mathrm{E}+02$ \\
\hline pd108 & $4.110 \mathrm{E}+02$ & $4.110 \mathrm{E}+02$ & $4.110 \mathrm{E}+02$ & $4.110 \mathrm{E}+02$ & $4.110 \mathrm{E}+02$ \\
\hline pd110 & $1.355 \mathrm{E}+02$ & $1.355 \mathrm{E}+02$ & $1.355 \mathrm{E}+02$ & $1.355 \mathrm{E}+02$ & $1.355 \mathrm{E}+02$ \\
\hline pm143 & $1.597 \mathrm{E}-12$ & $1.716 \mathrm{E}-14$ & $1.345 \mathrm{E}-14$ & $5.179 \mathrm{E}-15$ & $1.993 \mathrm{E}-15$ \\
\hline pm144 & $4.839 \mathrm{E}-10$ & $1.769 \mathrm{E}-11$ & $1.481 \mathrm{E}-11$ & $7.378 \mathrm{E}-12$ & $3.674 \mathrm{E}-12$ \\
\hline pm145 & 3.457E-05 & $2.896 \mathrm{E}-05$ & $2.868 \mathrm{E}-05$ & $2.758 \mathrm{E}-05$ & $2.652 \mathrm{E}-05$ \\
\hline pm146 & $3.623 \mathrm{E}-04$ & $1.999 \mathrm{E}-04$ & $1.936 \mathrm{E}-04$ & $1.708 \mathrm{E}-04$ & $1.507 \mathrm{E}-04$ \\
\hline pm147 & $1.631 \mathrm{E}+02$ & $4.654 \mathrm{E}+01$ & $4.351 \mathrm{E}+01$ & $3.341 \mathrm{E}+01$ & $2.566 \mathrm{E}+01$ \\
\hline pm148 & $1.831 \mathrm{E}-04$ & $4.245 \mathrm{E}-17$ & $8.907 \mathrm{E}-18$ & $1.944 \mathrm{E}-20$ & $4.242 \mathrm{E}-23$ \\
\hline pm148m & $2.917 \mathrm{E}-02$ & $6.760 \mathrm{E}-15$ & $1.419 \mathrm{E}-15$ & $3.098 \mathrm{E}-18$ & $6.760 \mathrm{E}-21$ \\
\hline po210 & $7.870 \mathrm{E}-13$ & $8.860 \mathrm{E}-13$ & $8.920 \mathrm{E}-13$ & $9.279 \mathrm{E}-13$ & $9.811 \mathrm{E}-13$ \\
\hline po216 & $7.963 \mathrm{E}-15$ & 4.874E-14 & $5.054 \mathrm{E}-14$ & $5.665 \mathrm{E}-14$ & $6.178 \mathrm{E}-14$ \\
\hline pr141 & $1.292 \mathrm{E}+03$ & $1.292 \mathrm{E}+03$ & $1.292 \mathrm{E}+03$ & $1.292 \mathrm{E}+03$ & $1.292 \mathrm{E}+03$ \\
\hline pr143 & $2.947 \mathrm{E}-05$ & $0.000 \mathrm{E}+00$ & $0.000 \mathrm{E}+00$ & $0.000 \mathrm{E}+00$ & $0.000 \mathrm{E}+00$ \\
\hline pr144 & $7.677 \mathrm{E}-03$ & $1.133 \mathrm{E}-04$ & $9.040 \mathrm{E}-05$ & $3.718 \mathrm{E}-05$ & $1.530 \mathrm{E}-05$ \\
\hline pr144m & $3.055 \mathrm{E}-05$ & $4.508 \mathrm{E}-07$ & $3.595 \mathrm{E}-07$ & $1.479 \mathrm{E}-07$ & $6.087 \mathrm{E}-08$ \\
\hline pt192 & $1.427 \mathrm{E}-13$ & $1.428 \mathrm{E}-13$ & $1.428 \mathrm{E}-13$ & $1.428 \mathrm{E}-13$ & $1.428 \mathrm{E}-13$ \\
\hline pt193 & $2.026 \mathrm{E}-14$ & $1.897 \mathrm{E}-14$ & $1.890 \mathrm{E}-14$ & $1.864 \mathrm{E}-14$ & $1.839 \mathrm{E}-14$ \\
\hline pt194 & $3.564 \mathrm{E}-13$ & $3.564 \mathrm{E}-13$ & $3.564 \mathrm{E}-13$ & $3.564 \mathrm{E}-13$ & $3.564 \mathrm{E}-13$ \\
\hline pt195 & $3.070 \mathrm{E}-15$ & $3.071 \mathrm{E}-15$ & $3.071 \mathrm{E}-15$ & $3.071 \mathrm{E}-15$ & $3.071 \mathrm{E}-15$ \\
\hline pt196 & $5.021 \mathrm{E}-16$ & $5.021 \mathrm{E}-16$ & $5.021 \mathrm{E}-16$ & $5.021 \mathrm{E}-16$ & $5.021 \mathrm{E}-16$ \\
\hline pu236 & $9.957 \mathrm{E}-04$ & $3.149 \mathrm{E}-04$ & $2.961 \mathrm{E}-04$ & $2.324 \mathrm{E}-04$ & $1.823 \mathrm{E}-04$ \\
\hline pu237 & $1.135 \mathrm{E}-05$ & $4.214 \mathrm{E}-17$ & $1.026 \mathrm{E}-17$ & $4.017 \mathrm{E}-20$ & $1.572 \mathrm{E}-22$ \\
\hline pu238 & $1.944 \mathrm{E}+02$ & $2.055 \mathrm{E}+02$ & $2.051 \mathrm{E}+02$ & $2.035 \mathrm{E}+02$ & $2.019 \mathrm{E}+02$ \\
\hline pu239 & $1.202 \mathrm{E}+04$ & $1.202 \mathrm{E}+04$ & $1.202 \mathrm{E}+04$ & $1.202 \mathrm{E}+04$ & $1.202 \mathrm{E}+04$ \\
\hline pu240 & $7.066 \mathrm{E}+03$ & $7.092 \mathrm{E}+03$ & $7.092 \mathrm{E}+03$ & $7.099 \mathrm{E}+03$ & $7.099 \mathrm{E}+03$ \\
\hline pu241 & $4.156 \mathrm{E}+03$ & $3.302 \mathrm{E}+03$ & $3.261 \mathrm{E}+03$ & $3.106 \mathrm{E}+03$ & $2.959 \mathrm{E}+03$ \\
\hline pu242 & $1.471 \mathrm{E}+03$ & $1.471 \mathrm{E}+03$ & $1.471 \mathrm{E}+03$ & $1.471 \mathrm{E}+03$ & $1.471 \mathrm{E}+03$ \\
\hline pu243 & $8.016 \mathrm{E}-13$ & $8.016 \mathrm{E}-13$ & 8.016E-13 & $8.016 \mathrm{E}-13$ & $8.016 \mathrm{E}-13$ \\
\hline pu244 & $1.299 \mathrm{E}-01$ & $1.299 \mathrm{E}-01$ & $1.299 \mathrm{E}-01$ & $1.299 \mathrm{E}-01$ & $1.299 \mathrm{E}-01$ \\
\hline pu246 & $1.394 \mathrm{E}-16$ & $1.393 \mathrm{E}-16$ & $1.393 \mathrm{E}-16$ & $1.393 \mathrm{E}-16$ & $1.393 \mathrm{E}-16$ \\
\hline ra223 & $9.293 \mathrm{E}-13$ & 4.233E-12 & $4.323 \mathrm{E}-12$ & $5.095 \mathrm{E}-12$ & $5.784 \mathrm{E}-12$ \\
\hline ra224 & $1.801 \mathrm{E}-08$ & $1.102 \mathrm{E}-07$ & $1.143 \mathrm{E}-07$ & $1.281 \mathrm{E}-07$ & $1.397 \mathrm{E}-07$ \\
\hline ra225 & $1.964 \mathrm{E}-12$ & $2.031 \mathrm{E}-12$ & $2.035 \mathrm{E}-12$ & $2.055 \mathrm{E}-12$ & $2.076 \mathrm{E}-12$ \\
\hline ra226 & $1.407 \mathrm{E}-09$ & 8.761E-09 & $9.406 \mathrm{E}-09$ & $1.225 \mathrm{E}-08$ & $1.561 \mathrm{E}-08$ \\
\hline ra228 & $3.758 \mathrm{E}-14$ & $6.464 \mathrm{E}-14$ & $6.595 \mathrm{E}-14$ & $7.099 \mathrm{E}-14$ & 7.591E-14 \\
\hline
\end{tabular}




\begin{tabular}{|c|c|c|c|c|c|}
\hline \multicolumn{6}{|c|}{ Table C-3. Continued } \\
\hline & $1 / 22 / 2009$ & $10 / 21 / 2013$ & $1 / 22 / 2014$ & $1 / 22 / 2015$ & $1 / 22 / 2016$ \\
\hline rb 83 & $5.890 \mathrm{E}-08$ & $5.224 \mathrm{E}-14$ & $2.473 \mathrm{E}-14$ & $1.314 \mathrm{E}-15$ & $6.979 \mathrm{E}-17$ \\
\hline rb 84 & $6.561 \mathrm{E}-08$ & $8.349 \mathrm{E}-24$ & $1.171 \mathrm{E}-24$ & $5.255 \mathrm{E}-28$ & $2.359 \mathrm{E}-31$ \\
\hline rb 85 & $7.185 \mathrm{E}+01$ & $7.651 \mathrm{E}+01$ & $7.671 \mathrm{E}+01$ & $7.750 \mathrm{E}+01$ & $7.824 \mathrm{E}+01$ \\
\hline rb 86 & $1.272 \mathrm{E}-06$ & $1.268 \mathrm{E}-34$ & $0.000 \mathrm{E}+00$ & $0.000 \mathrm{E}+00$ & $0.000 \mathrm{E}+00$ \\
\hline rb 87 & $1.684 \mathrm{E}+02$ & $1.684 \mathrm{E}+02$ & $1.684 \mathrm{E}+02$ & $1.684 \mathrm{E}+02$ & $1.684 \mathrm{E}+02$ \\
\hline re185 & $5.988 \mathrm{E}-14$ & $6.000 \mathrm{E}-14$ & $6.000 \mathrm{E}-14$ & $6.000 \mathrm{E}-14$ & $6.000 \mathrm{E}-14$ \\
\hline re186m & $1.912 \mathrm{E}-16$ & $1.912 \mathrm{E}-16$ & $1.912 \mathrm{E}-16$ & $1.912 \mathrm{E}-16$ & $1.912 \mathrm{E}-16$ \\
\hline re187 & $3.520 \mathrm{E}-13$ & $3.520 \mathrm{E}-13$ & $3.520 \mathrm{E}-13$ & $3.520 \mathrm{E}-13$ & $3.520 \mathrm{E}-13$ \\
\hline rh101 & $5.473 \mathrm{E}-07$ & $2.020 \mathrm{E}-07$ & $1.915 \mathrm{E}-07$ & $1.552 \mathrm{E}-07$ & $1.258 \mathrm{E}-07$ \\
\hline rh102 & $7.943 \mathrm{E}-04$ & $2.749 \mathrm{E}-06$ & $2.088 \mathrm{E}-06$ & $7.863 \mathrm{E}-07$ & $3.735 \mathrm{E}-07$ \\
\hline rh102m & $1.924 \mathrm{E}-03$ & $7.990 \mathrm{E}-04$ & 7.624E-04 & $6.335 \mathrm{E}-04$ & $5.264 \mathrm{E}-04$ \\
\hline rh103 & $8.482 \mathrm{E}+02$ & $8.488 \mathrm{E}+02$ & $8.488 \mathrm{E}+02$ & $8.488 \mathrm{E}+02$ & $8.488 \mathrm{E}+02$ \\
\hline rh103m & $5.748 \mathrm{E}-04$ & $2.931 \mathrm{E}-17$ & 5.672E-18 & $9.000 \mathrm{E}-21$ & $1.427 \mathrm{E}-23$ \\
\hline rh106 & $1.988 \mathrm{E}-04$ & 7.857E-06 & $6.606 \mathrm{E}-06$ & $3.345 \mathrm{E}-06$ & $1.694 \mathrm{E}-06$ \\
\hline rn220 & $3.109 \mathrm{E}-12$ & $1.903 \mathrm{E}-11$ & $1.974 \mathrm{E}-11$ & $2.212 \mathrm{E}-11$ & $2.413 \mathrm{E}-11$ \\
\hline rn222 & $8.967 \mathrm{E}-15$ & $5.629 \mathrm{E}-14$ & $6.045 \mathrm{E}-14$ & $7.877 \mathrm{E}-14$ & $1.003 \mathrm{E}-13$ \\
\hline ru 96 & $1.729 \mathrm{E}-13$ & $1.729 \mathrm{E}-13$ & $1.729 \mathrm{E}-13$ & $1.729 \mathrm{E}-13$ & $1.729 \mathrm{E}-13$ \\
\hline ru 98 & $1.781 \mathrm{E}-06$ & $1.790 \mathrm{E}-06$ & $1.790 \mathrm{E}-06$ & $1.792 \mathrm{E}-06$ & $1.794 \mathrm{E}-06$ \\
\hline ru 99 & $4.039 \mathrm{E}-02$ & $5.512 \mathrm{E}-02$ & $5.591 \mathrm{E}-02$ & $5.901 \mathrm{E}-02$ & $6.211 \mathrm{E}-02$ \\
\hline ru100 & $1.623 \mathrm{E}+02$ & $1.623 \mathrm{E}+02$ & $1.623 \mathrm{E}+02$ & $1.623 \mathrm{E}+02$ & $1.623 \mathrm{E}+02$ \\
\hline ru101 & $1.032 \mathrm{E}+03$ & $1.032 \mathrm{E}+03$ & $1.032 \mathrm{E}+03$ & $1.032 \mathrm{E}+03$ & $1.032 \mathrm{E}+03$ \\
\hline ru102 & $1.199 \mathrm{E}+03$ & $1.199 \mathrm{E}+03$ & $1.199 \mathrm{E}+03$ & $1.199 \mathrm{E}+03$ & $1.199 \mathrm{E}+03$ \\
\hline ru103 & $5.852 \mathrm{E}-01$ & $2.985 \mathrm{E}-14$ & $5.775 \mathrm{E}-15$ & $9.160 \mathrm{E}-18$ & $1.453 \mathrm{E}-20$ \\
\hline ru104 & $1.126 \mathrm{E}+03$ & $1.126 \mathrm{E}+03$ & $1.126 \mathrm{E}+03$ & $1.126 \mathrm{E}+03$ & $1.126 \mathrm{E}+03$ \\
\hline ru106 & $2.124 \mathrm{E}+02$ & $8.395 \mathrm{E}+00$ & $7.059 \mathrm{E}+00$ & $3.573 \mathrm{E}+00$ & $1.809 \mathrm{E}+00$ \\
\hline s 32 & $5.810 \mathrm{E}-14$ & $5.810 \mathrm{E}-14$ & $5.810 \mathrm{E}-14$ & $5.810 \mathrm{E}-14$ & $5.810 \mathrm{E}-14$ \\
\hline s 33 & $5.969 \mathrm{E}-14$ & $5.969 \mathrm{E}-14$ & $5.969 \mathrm{E}-14$ & $5.969 \mathrm{E}-14$ & $5.969 \mathrm{E}-14$ \\
\hline s 34 & $6.188 \mathrm{E}-14$ & $6.188 \mathrm{E}-14$ & $6.188 \mathrm{E}-14$ & $6.188 \mathrm{E}-14$ & $6.188 \mathrm{E}-14$ \\
\hline s 36 & $6.543 \mathrm{E}-14$ & $6.543 \mathrm{E}-14$ & $6.543 \mathrm{E}-14$ & $6.543 \mathrm{E}-14$ & $6.543 \mathrm{E}-14$ \\
\hline sb121 & $7.990 \mathrm{E}+00$ & $8.043 \mathrm{E}+00$ & $8.050 \mathrm{E}+00$ & $8.056 \mathrm{E}+00$ & $8.070 \mathrm{E}+00$ \\
\hline sb123 & $1.054 \mathrm{E}+01$ & $1.060 \mathrm{E}+01$ & $1.060 \mathrm{E}+01$ & $1.060 \mathrm{E}+01$ & $1.060 \mathrm{E}+01$ \\
\hline sb124 & $3.720 \mathrm{E}-03$ & $8.030 \mathrm{E}-12$ & $2.751 \mathrm{E}-12$ & $4.115 \mathrm{E}-14$ & $6.154 \mathrm{E}-16$ \\
\hline sb125 & $1.358 \mathrm{E}+01$ & $4.121 \mathrm{E}+00$ & $3.866 \mathrm{E}+00$ & $3.008 \mathrm{E}+00$ & $2.340 \mathrm{E}+00$ \\
\hline sb126 & $8.409 \mathrm{E}-07$ & $8.362 \mathrm{E}-07$ & 8.362E-07 & $8.362 \mathrm{E}-07$ & $8.362 \mathrm{E}-07$ \\
\hline sb126m & $6.432 \mathrm{E}-09$ & $6.432 \mathrm{E}-09$ & $6.432 \mathrm{E}-09$ & $6.432 \mathrm{E}-09$ & $6.432 \mathrm{E}-09$ \\
\hline sc 45 & $7.883 \mathrm{E}-14$ & $7.883 \mathrm{E}-14$ & $7.883 \mathrm{E}-14$ & $7.883 \mathrm{E}-14$ & $7.883 \mathrm{E}-14$ \\
\hline sc 46 & $4.386 \mathrm{E}-17$ & $2.606 \mathrm{E}-23$ & $1.207 \mathrm{E}-23$ & $5.895 \mathrm{E}-25$ & $2.878 \mathrm{E}-26$ \\
\hline se 74 & $3.756 \mathrm{E}-07$ & $3.756 \mathrm{E}-07$ & $3.756 \mathrm{E}-07$ & $3.756 \mathrm{E}-07$ & $3.756 \mathrm{E}-07$ \\
\hline
\end{tabular}




\begin{tabular}{|c|c|c|c|c|c|}
\hline \multicolumn{6}{|c|}{ Table C-3. Continued } \\
\hline & $1 / 22 / 2009$ & $10 / 21 / 2013$ & $1 / 22 / 2014$ & $1 / 22 / 2015$ & $1 / 22 / 2016$ \\
\hline se 75 & $8.342 \mathrm{E}-09$ & $3.683 \mathrm{E}-13$ & $2.150 \mathrm{E}-13$ & $2.602 \mathrm{E}-14$ & $3.148 \mathrm{E}-15$ \\
\hline se 76 & $8.282 \mathrm{E}-03$ & $8.282 \mathrm{E}-03$ & $8.282 \mathrm{E}-03$ & $8.282 \mathrm{E}-03$ & $8.282 \mathrm{E}-03$ \\
\hline se 77 & $8.741 \mathrm{E}-01$ & $8.741 \mathrm{E}-01$ & $8.741 \mathrm{E}-01$ & $8.741 \mathrm{E}-01$ & $8.741 \mathrm{E}-01$ \\
\hline se 78 & $2.519 \mathrm{E}+00$ & $2.519 \mathrm{E}+00$ & $2.519 \mathrm{E}+00$ & $2.519 \mathrm{E}+00$ & $2.519 \mathrm{E}+00$ \\
\hline se 79 & $5.350 \mathrm{E}+00$ & $5.350 \mathrm{E}+00$ & $5.350 \mathrm{E}+00$ & $5.350 \mathrm{E}+00$ & $5.350 \mathrm{E}+00$ \\
\hline se 80 & $1.233 \mathrm{E}+01$ & $1.233 \mathrm{E}+01$ & $1.233 \mathrm{E}+01$ & $1.233 \mathrm{E}+01$ & $1.233 \mathrm{E}+01$ \\
\hline se 82 & $3.244 \mathrm{E}+01$ & $3.244 \mathrm{E}+01$ & $3.244 \mathrm{E}+01$ & $3.244 \mathrm{E}+01$ & $3.244 \mathrm{E}+01$ \\
\hline si 28 & $5.091 \mathrm{E}-14$ & $5.091 \mathrm{E}-14$ & $5.091 \mathrm{E}-14$ & $5.091 \mathrm{E}-14$ & $5.091 \mathrm{E}-14$ \\
\hline si 29 & $5.278 \mathrm{E}-14$ & $5.278 \mathrm{E}-14$ & $5.278 \mathrm{E}-14$ & $5.278 \mathrm{E}-14$ & $5.278 \mathrm{E}-14$ \\
\hline si 30 & $5.486 \mathrm{E}-14$ & $5.486 \mathrm{E}-14$ & $5.486 \mathrm{E}-14$ & $5.486 \mathrm{E}-14$ & $5.486 \mathrm{E}-14$ \\
\hline $\operatorname{sm144}$ & $5.679 \mathrm{E}-11$ & $5.679 \mathrm{E}-11$ & $5.679 \mathrm{E}-11$ & $5.679 \mathrm{E}-11$ & $5.679 \mathrm{E}-11$ \\
\hline $\operatorname{sm} 145$ & $2.983 \mathrm{E}-07$ & 8.714E-09 & 7.212E-09 & $3.426 \mathrm{E}-09$ & $1.628 \mathrm{E}-09$ \\
\hline sm146 & $3.169 \mathrm{E}-03$ & $3.224 \mathrm{E}-03$ & $3.226 \mathrm{E}-03$ & $3.234 \mathrm{E}-03$ & $3.240 \mathrm{E}-03$ \\
\hline $\operatorname{sm} 147$ & $1.093 \mathrm{E}+02$ & $2.258 \mathrm{E}+02$ & $2.289 \mathrm{E}+02$ & $2.390 \mathrm{E}+02$ & $2.467 \mathrm{E}+02$ \\
\hline sm148 & $1.898 \mathrm{E}+02$ & $1.898 \mathrm{E}+02$ & $1.898 \mathrm{E}+02$ & $1.898 \mathrm{E}+02$ & $1.898 \mathrm{E}+02$ \\
\hline sm149 & $5.346 \mathrm{E}+00$ & $5.346 \mathrm{E}+00$ & $5.346 \mathrm{E}+00$ & $5.346 \mathrm{E}+00$ & $5.346 \mathrm{E}+00$ \\
\hline sm150 & $3.945 \mathrm{E}+02$ & $3.945 \mathrm{E}+02$ & $3.945 \mathrm{E}+02$ & $3.945 \mathrm{E}+02$ & $3.945 \mathrm{E}+02$ \\
\hline sm151 & $2.247 \mathrm{E}+01$ & $2.166 \mathrm{E}+01$ & $2.162 \mathrm{E}+01$ & $2.146 \mathrm{E}+01$ & $2.129 \mathrm{E}+01$ \\
\hline sm152 & $1.412 \mathrm{E}+02$ & $1.412 \mathrm{E}+02$ & $1.412 \mathrm{E}+02$ & $1.412 \mathrm{E}+02$ & $1.412 \mathrm{E}+02$ \\
\hline sm154 & $7.731 \mathrm{E}+01$ & $7.731 \mathrm{E}+01$ & $7.731 \mathrm{E}+01$ & $7.731 \mathrm{E}+01$ & $7.731 \mathrm{E}+01$ \\
\hline sn112 & 2.852E-09 & 2.852E-09 & $2.852 \mathrm{E}-09$ & $2.852 \mathrm{E}-09$ & $2.852 \mathrm{E}-09$ \\
\hline sn113 & $6.269 \mathrm{E}-11$ & $1.838 \mathrm{E}-15$ & $1.050 \mathrm{E}-15$ & $1.165 \mathrm{E}-16$ & $1.294 \mathrm{E}-17$ \\
\hline sn114 & 1.490E-04 & $1.493 \mathrm{E}-04$ & 1.493E-04 & 1.493E-04 & $1.493 \mathrm{E}-04$ \\
\hline sn115 & $4.766 \mathrm{E}-01$ & $4.766 \mathrm{E}-01$ & $4.766 \mathrm{E}-01$ & $4.766 \mathrm{E}-01$ & 4.766E-01 \\
\hline sn116 & $4.047 \mathrm{E}+00$ & $4.047 \mathrm{E}+00$ & $4.047 \mathrm{E}+00$ & $4.047 \mathrm{E}+00$ & $4.047 \mathrm{E}+00$ \\
\hline sn117 & $9.665 \mathrm{E}+00$ & $9.665 \mathrm{E}+00$ & $9.665 \mathrm{E}+00$ & $9.665 \mathrm{E}+00$ & $9.665 \mathrm{E}+00$ \\
\hline sn117m & $2.517 \mathrm{E}-09$ & $0.000 \mathrm{E}+00$ & $0.000 \mathrm{E}+00$ & $0.000 \mathrm{E}+00$ & $0.000 \mathrm{E}+00$ \\
\hline sn118 & $8.555 \mathrm{E}+00$ & $8.555 \mathrm{E}+00$ & $8.555 \mathrm{E}+00$ & $8.555 \mathrm{E}+00$ & $8.555 \mathrm{E}+00$ \\
\hline sn119 & $8.129 \mathrm{E}+00$ & $8.189 \mathrm{E}+00$ & $8.189 \mathrm{E}+00$ & $8.189 \mathrm{E}+00$ & $8.189 \mathrm{E}+00$ \\
\hline sn119m & $6.246 \mathrm{E}-02$ & $1.036 \mathrm{E}-03$ & $8.309 \mathrm{E}-04$ & $3.504 \mathrm{E}-04$ & $1.478 \mathrm{E}-04$ \\
\hline sn120 & $8.222 \mathrm{E}+00$ & $8.222 \mathrm{E}+00$ & $8.222 \mathrm{E}+00$ & $8.222 \mathrm{E}+00$ & $8.222 \mathrm{E}+00$ \\
\hline sn121 & $3.909 \mathrm{E}-05$ & $3.627 \mathrm{E}-05$ & $3.612 \mathrm{E}-05$ & $3.555 \mathrm{E}-05$ & $3.500 \mathrm{E}-05$ \\
\hline sn121m & $7.172 \mathrm{E}-01$ & 6.654E-01 & $6.626 \mathrm{E}-01$ & $6.523 \mathrm{E}-01$ & $6.420 \mathrm{E}-01$ \\
\hline sn122 & $1.090 \mathrm{E}+01$ & $1.090 \mathrm{E}+01$ & $1.090 \mathrm{E}+01$ & $1.090 \mathrm{E}+01$ & $1.090 \mathrm{E}+01$ \\
\hline sn123 & $7.026 \mathrm{E}-02$ & $6.438 \mathrm{E}-06$ & $3.909 \mathrm{E}-06$ & $5.516 \mathrm{E}-07$ & $7.784 \mathrm{E}-08$ \\
\hline sn124 & $1.742 \mathrm{E}+01$ & $1.742 \mathrm{E}+01$ & $1.742 \mathrm{E}+01$ & $1.742 \mathrm{E}+01$ & $1.742 \mathrm{E}+01$ \\
\hline sn125 & $6.827 \mathrm{E}-10$ & $0.000 \mathrm{E}+00$ & $0.000 \mathrm{E}+00$ & $0.000 \mathrm{E}+00$ & $0.000 \mathrm{E}+00$ \\
\hline sn126 & $4.063 \mathrm{E}+01$ & $4.063 \mathrm{E}+01$ & $4.063 \mathrm{E}+01$ & $4.063 \mathrm{E}+01$ & $4.063 \mathrm{E}+01$ \\
\hline
\end{tabular}




\begin{tabular}{|c|c|c|c|c|c|}
\hline \multicolumn{6}{|c|}{ Table C-3. Continued } \\
\hline & $1 / 22 / 2009$ & $10 / 21 / 2013$ & $1 / 22 / 2014$ & $1 / 22 / 2015$ & $1 / 22 / 2016$ \\
\hline sr 84 & $8.103 \mathrm{E}-06$ & $8.109 \mathrm{E}-06$ & 8.109E-06 & 8.109E-06 & $8.109 \mathrm{E}-06$ \\
\hline sr 85 & $1.399 \mathrm{E}-08$ & $1.258 \mathrm{E}-16$ & $4.656 \mathrm{E}-17$ & $9.406 \mathrm{E}-19$ & $1.900 \mathrm{E}-20$ \\
\hline sr 86 & 4.913E-01 & 4.913E-01 & 4.913E-01 & 4.913E-01 & 4.913E-01 \\
\hline sr 87 & $5.154 \mathrm{E}-03$ & $5.154 \mathrm{E}-03$ & 5.154E-03 & $5.154 \mathrm{E}-03$ & $5.154 \mathrm{E}-03$ \\
\hline sr 88 & $2.237 \mathrm{E}+02$ & $2.237 \mathrm{E}+02$ & $2.237 \mathrm{E}+02$ & $2.237 \mathrm{E}+02$ & $2.237 \mathrm{E}+02$ \\
\hline sr 89 & 4.822E-01 & $2.285 \mathrm{E}-11$ & $6.381 \mathrm{E}-12$ & $4.270 \mathrm{E}-14$ & $2.857 \mathrm{E}-16$ \\
\hline sr 90 & $3.435 \mathrm{E}+02$ & $3.064 \mathrm{E}+02$ & $3.046 \mathrm{E}+02$ & $2.973 \mathrm{E}+02$ & $2.902 \mathrm{E}+02$ \\
\hline ta180m & $2.048 \mathrm{E}-17$ & $2.048 \mathrm{E}-17$ & $2.048 \mathrm{E}-17$ & $2.048 \mathrm{E}-17$ & $2.048 \mathrm{E}-17$ \\
\hline ta181 & $1.751 \mathrm{E}-13$ & $1.751 \mathrm{E}-13$ & $1.751 \mathrm{E}-13$ & $1.751 \mathrm{E}-13$ & $1.751 \mathrm{E}-13$ \\
\hline ta182 & $6.353 \mathrm{E}-16$ & $1.809 \mathrm{E}-20$ & $1.033 \mathrm{E}-20$ & $1.178 \mathrm{E}-21$ & $1.690 \mathrm{E}-22$ \\
\hline tb157 & $7.112 \mathrm{E}-07$ & $6.793 \mathrm{E}-07$ & $6.773 \mathrm{E}-07$ & $6.707 \mathrm{E}-07$ & $6.644 \mathrm{E}-07$ \\
\hline tb158 & $9.858 \mathrm{E}-05$ & $9.685 \mathrm{E}-05$ & $9.671 \mathrm{E}-05$ & 9.632E-05 & $9.598 \mathrm{E}-05$ \\
\hline tb159 & $6.714 \mathrm{E}+00$ & $6.714 \mathrm{E}+00$ & $6.714 \mathrm{E}+00$ & $6.714 \mathrm{E}+00$ & $6.714 \mathrm{E}+00$ \\
\hline tb160 & $1.705 \mathrm{E}-02$ & $1.038 \mathrm{E}-09$ & $4.255 \mathrm{E}-10$ & $1.286 \mathrm{E}-11$ & $3.885 \mathrm{E}-13$ \\
\hline tb161 & $6.866 \mathrm{E}-14$ & $0.000 \mathrm{E}+00$ & $0.000 \mathrm{E}+00$ & $0.000 \mathrm{E}+00$ & $0.000 \mathrm{E}+00$ \\
\hline tc 95 & $5.342 \mathrm{E}-18$ & $1.498 \mathrm{E}-26$ & $5.205 \mathrm{E}-27$ & $8.229 \mathrm{E}-29$ & $0.000 \mathrm{E}+00$ \\
\hline tc $95 \mathrm{~m}$ & $9.937 \mathrm{E}-15$ & $2.786 \mathrm{E}-23$ & $9.685 \mathrm{E}-24$ & $1.531 \mathrm{E}-25$ & $2.419 \mathrm{E}-27$ \\
\hline tc 97 & $1.024 \mathrm{E}-05$ & $1.032 \mathrm{E}-05$ & $1.032 \mathrm{E}-05$ & $1.032 \mathrm{E}-05$ & $1.032 \mathrm{E}-05$ \\
\hline tc $97 \mathrm{~m}$ & $8.854 \mathrm{E}-08$ & $1.638 \mathrm{E}-13$ & $8.063 \mathrm{E}-14$ & $5.003 \mathrm{E}-15$ & $3.103 \mathrm{E}-16$ \\
\hline tc 98 & $1.058 \mathrm{E}-02$ & $1.058 \mathrm{E}-02$ & $1.058 \mathrm{E}-02$ & $1.058 \mathrm{E}-02$ & $1.058 \mathrm{E}-02$ \\
\hline tc 99 & $9.452 \mathrm{E}+02$ & $9.452 \mathrm{E}+02$ & $9.452 \mathrm{E}+02$ & $9.452 \mathrm{E}+02$ & $9.452 \mathrm{E}+02$ \\
\hline te120 & $2.511 \mathrm{E}-09$ & $2.511 \mathrm{E}-09$ & $2.511 \mathrm{E}-09$ & $2.511 \mathrm{E}-09$ & $2.511 \mathrm{E}-09$ \\
\hline te121 & $3.326 \mathrm{E}-08$ & $2.211 \mathrm{E}-11$ & $1.493 \mathrm{E}-11$ & $3.199 \mathrm{E}-12$ & $6.853 \mathrm{E}-13$ \\
\hline te121m & $2.839 \mathrm{E}-07$ & $1.888 \mathrm{E}-10$ & $1.275 \mathrm{E}-10$ & $2.731 \mathrm{E}-11$ & $5.850 \mathrm{E}-12$ \\
\hline te122 & $7.618 \mathrm{E}-01$ & $7.618 \mathrm{E}-01$ & $7.618 \mathrm{E}-01$ & $7.618 \mathrm{E}-01$ & $7.618 \mathrm{E}-01$ \\
\hline te123 & $9.612 \mathrm{E}-03$ & $1.006 \mathrm{E}-02$ & $1.006 \mathrm{E}-02$ & $1.006 \mathrm{E}-02$ & $1.006 \mathrm{E}-02$ \\
\hline te $123 \mathrm{~m}$ & 4.461E-04 & $1.874 \mathrm{E}-08$ & $1.091 \mathrm{E}-08$ & $1.307 \mathrm{E}-09$ & $1.565 \mathrm{E}-10$ \\
\hline te124 & $5.458 \mathrm{E}-01$ & $5.495 \mathrm{E}-01$ & $5.495 \mathrm{E}-01$ & $5.495 \mathrm{E}-01$ & $5.495 \mathrm{E}-01$ \\
\hline te125 & $9.645 \mathrm{E}+00$ & $1.923 \mathrm{E}+01$ & $1.949 \mathrm{E}+01$ & $2.036 \mathrm{E}+01$ & $2.104 \mathrm{E}+01$ \\
\hline te125m & $1.886 \mathrm{E}-01$ & $5.750 \mathrm{E}-02$ & 5.393E-02 & 4.196E-02 & $3.264 \mathrm{E}-02$ \\
\hline te126 & $1.000 \mathrm{E}+00$ & $1.000 \mathrm{E}+00$ & $1.001 \mathrm{E}+00$ & $1.001 \mathrm{E}+00$ & $1.001 \mathrm{E}+00$ \\
\hline te127 & 8.395E-04 & $1.374 \mathrm{E}-08$ & 7.604E-09 & $7.465 \mathrm{E}-10$ & 7.332E-11 \\
\hline te127m & $2.399 \mathrm{E}-01$ & $3.925 \mathrm{E}-06$ & $2.173 \mathrm{E}-06$ & $2.133 \mathrm{E}-07$ & $2.094 \mathrm{E}-08$ \\
\hline te128 & $1.573 \mathrm{E}+02$ & $1.573 \mathrm{E}+02$ & $1.573 \mathrm{E}+02$ & $1.573 \mathrm{E}+02$ & $1.573 \mathrm{E}+02$ \\
\hline te129 & $6.615 \mathrm{E}-06$ & $1.968 \mathrm{E}-21$ & $2.889 \mathrm{E}-22$ & $1.551 \mathrm{E}-25$ & $8.329 \mathrm{E}-29$ \\
\hline te129m & $7.292 \mathrm{E}-03$ & $2.169 \mathrm{E}-18$ & $3.184 \mathrm{E}-19$ & $1.710 \mathrm{E}-22$ & $9.180 \mathrm{E}-26$ \\
\hline te130 & $5.286 \mathrm{E}+02$ & $5.286 \mathrm{E}+02$ & $5.286 \mathrm{E}+02$ & $5.286 \mathrm{E}+02$ & $5.286 \mathrm{E}+02$ \\
\hline th227 & $1.560 \mathrm{E}-12$ & $6.946 \mathrm{E}-12$ & 7.139E-12 & 8.362E-12 & $9.492 \mathrm{E}-12$ \\
\hline
\end{tabular}




\begin{tabular}{|c|c|c|c|c|c|}
\hline \multicolumn{6}{|c|}{ Table C-3. Continued } \\
\hline & $1 / 22 / 2009$ & $10 / 21 / 2013$ & $1 / 22 / 2014$ & $1 / 22 / 2015$ & $1 / 22 / 2016$ \\
\hline th228 & $3.544 \mathrm{E}-06$ & $2.133 \mathrm{E}-05$ & $2.209 \mathrm{E}-05$ & $2.480 \mathrm{E}-05$ & $2.705 \mathrm{E}-05$ \\
\hline th229 & $3.596 \mathrm{E}-07$ & $3.720 \mathrm{E}-07$ & $3.728 \mathrm{E}-07$ & $3.764 \mathrm{E}-07$ & $3.801 \mathrm{E}-07$ \\
\hline th230 & $8.442 \mathrm{E}-05$ & $2.758 \mathrm{E}-04$ & $2.889 \mathrm{E}-04$ & $3.432 \mathrm{E}-04$ & $4.018 \mathrm{E}-04$ \\
\hline th231 & 3.419E-09 & $3.425 \mathrm{E}-09$ & $3.426 \mathrm{E}-09$ & 3.427E-09 & 3.429E-09 \\
\hline th232 & $2.243 \mathrm{E}-04$ & $2.675 \mathrm{E}-04$ & $2.699 \mathrm{E}-04$ & $2.790 \mathrm{E}-04$ & $2.883 \mathrm{E}-04$ \\
\hline th234 & $1.350 \mathrm{E}-05$ & $1.351 \mathrm{E}-05$ & $1.350 \mathrm{E}-05$ & $1.350 \mathrm{E}-05$ & $1.350 \mathrm{E}-05$ \\
\hline ti 46 & $8.661 \mathrm{E}-14$ & $8.668 \mathrm{E}-14$ & $8.668 \mathrm{E}-14$ & $8.668 \mathrm{E}-14$ & $8.668 \mathrm{E}-14$ \\
\hline ti 47 & $8.535 \mathrm{E}-14$ & $8.535 \mathrm{E}-14$ & $8.535 \mathrm{E}-14$ & $8.535 \mathrm{E}-14$ & $8.535 \mathrm{E}-14$ \\
\hline ti 48 & $8.661 \mathrm{E}-14$ & $8.661 \mathrm{E}-14$ & $8.661 \mathrm{E}-14$ & $8.661 \mathrm{E}-14$ & $8.661 \mathrm{E}-14$ \\
\hline ti 49 & $9.000 \mathrm{E}-14$ & $9.000 \mathrm{E}-14$ & $9.000 \mathrm{E}-14$ & $9.000 \mathrm{E}-14$ & $9.000 \mathrm{E}-14$ \\
\hline ti 50 & $9.113 \mathrm{E}-14$ & $9.113 \mathrm{E}-14$ & $9.113 \mathrm{E}-14$ & $9.113 \mathrm{E}-14$ & $9.113 \mathrm{E}-14$ \\
\hline tl203 & $3.232 \mathrm{E}-15$ & $3.237 \mathrm{E}-15$ & $3.237 \mathrm{E}-15$ & $3.237 \mathrm{E}-15$ & $3.237 \mathrm{E}-15$ \\
\hline tl204 & $6.827 \mathrm{E}-17$ & $2.861 \mathrm{E}-17$ & $2.731 \mathrm{E}-17$ & $2.274 \mathrm{E}-17$ & $1.893 \mathrm{E}-17$ \\
\hline tl205 & $1.470 \mathrm{E}-15$ & $1.470 \mathrm{E}-15$ & $1.470 \mathrm{E}-15$ & $1.470 \mathrm{E}-15$ & $1.470 \mathrm{E}-15$ \\
\hline tl207 & $2.493 \mathrm{E}-16$ & $1.135 \mathrm{E}-15$ & $1.160 \mathrm{E}-15$ & $1.367 \mathrm{E}-15$ & $1.552 \mathrm{E}-15$ \\
\hline tl208 & $3.481 \mathrm{E}-12$ & $2.131 \mathrm{E}-11$ & $2.209 \mathrm{E}-11$ & $2.477 \mathrm{E}-11$ & $2.701 \mathrm{E}-11$ \\
\hline $\operatorname{tm} 168$ & $9.180 \mathrm{E}-10$ & $2.287 \mathrm{E}-15$ & $1.145 \mathrm{E}-15$ & $7.558 \mathrm{E}-17$ & $4.991 \mathrm{E}-18$ \\
\hline $\operatorname{tm} 169$ & $9.425 \mathrm{E}-04$ & $9.425 \mathrm{E}-04$ & $9.425 \mathrm{E}-04$ & $9.425 \mathrm{E}-04$ & $9.425 \mathrm{E}-04$ \\
\hline $\operatorname{tm} 170$ & 5.204E-05 & 4.567E-09 & $2.766 \mathrm{E}-09$ & $3.869 \mathrm{E}-10$ & $5.409 \mathrm{E}-11$ \\
\hline $\operatorname{tm} 171$ & $1.719 \mathrm{E}-04$ & $3.100 \mathrm{E}-05$ & $2.828 \mathrm{E}-05$ & $1.972 \mathrm{E}-05$ & $1.375 \mathrm{E}-05$ \\
\hline u230 & $2.749 \mathrm{E}-15$ & $0.000 \mathrm{E}+00$ & $0.000 \mathrm{E}+00$ & $0.000 \mathrm{E}+00$ & $0.000 \mathrm{E}+00$ \\
\hline u232 & $4.638 \mathrm{E}-04$ & $1.093 \mathrm{E}-03$ & $1.108 \mathrm{E}-03$ & $1.159 \mathrm{E}-03$ & $1.196 \mathrm{E}-03$ \\
\hline $\mathbf{u} 233$ & 4.656E-04 & 7.704E-04 & 7.857E-04 & $8.541 \mathrm{E}-04$ & $9.233 \mathrm{E}-04$ \\
\hline u234 & $1.073 \mathrm{E}+01$ & $1.836 \mathrm{E}+01$ & $1.876 \mathrm{E}+01$ & $2.035 \mathrm{E}+01$ & $2.193 \mathrm{E}+01$ \\
\hline $\mathbf{u} 235$ & $8.409 \mathrm{E}+02$ & $8.422 \mathrm{E}+02$ & $8.428 \mathrm{E}+02$ & $8.428 \mathrm{E}+02$ & $8.435 \mathrm{E}+02$ \\
\hline $\mathrm{u} 236$ & $3.117 \mathrm{E}+02$ & $3.152 \mathrm{E}+02$ & $3.154 \mathrm{E}+02$ & $3.161 \mathrm{E}+02$ & $3.169 \mathrm{E}+02$ \\
\hline $\mathbf{u} 237$ & $1.297 \mathrm{E}-04$ & $1.030 \mathrm{E}-04$ & $1.018 \mathrm{E}-04$ & $9.691 \mathrm{E}-05$ & $9.233 \mathrm{E}-05$ \\
\hline u238 & $9.299 \mathrm{E}+05$ & $9.299 \mathrm{E}+05$ & $9.299 \mathrm{E}+05$ & $9.299 \mathrm{E}+05$ & $9.299 \mathrm{E}+05$ \\
\hline u240 & $2.531 \mathrm{E}-12$ & $2.531 \mathrm{E}-12$ & $2.531 \mathrm{E}-12$ & $2.531 \mathrm{E}-12$ & $2.531 \mathrm{E}-12$ \\
\hline v 50 & $5.954 \mathrm{E}-17$ & $5.954 \mathrm{E}-17$ & $5.954 \mathrm{E}-17$ & 5.954E-17 & $5.954 \mathrm{E}-17$ \\
\hline v 51 & $2.121 \mathrm{E}-15$ & $2.121 \mathrm{E}-15$ & $2.121 \mathrm{E}-15$ & $2.121 \mathrm{E}-15$ & $2.121 \mathrm{E}-15$ \\
\hline w182 & $2.588 \mathrm{E}-13$ & $2.594 \mathrm{E}-13$ & $2.594 \mathrm{E}-13$ & $2.594 \mathrm{E}-13$ & $2.594 \mathrm{E}-13$ \\
\hline w183 & $6.561 \mathrm{E}-13$ & $6.561 \mathrm{E}-13$ & $6.561 \mathrm{E}-13$ & $6.561 \mathrm{E}-13$ & $6.561 \mathrm{E}-13$ \\
\hline w184 & $5.841 \mathrm{E}-13$ & $5.841 \mathrm{E}-13$ & $5.841 \mathrm{E}-13$ & $5.841 \mathrm{E}-13$ & $5.841 \mathrm{E}-13$ \\
\hline w185 & $1.238 \mathrm{E}-16$ & $1.400 \mathrm{E}-23$ & $5.934 \mathrm{E}-24$ & $2.043 \mathrm{E}-25$ & $7.033 \mathrm{E}-27$ \\
\hline w186 & $2.055 \mathrm{E}-13$ & $2.055 \mathrm{E}-13$ & $2.055 \mathrm{E}-13$ & $2.055 \mathrm{E}-13$ & $2.055 \mathrm{E}-13$ \\
\hline xe124 & $1.972 \mathrm{E}-13$ & $1.972 \mathrm{E}-13$ & $1.972 \mathrm{E}-13$ & $1.972 \mathrm{E}-13$ & $1.972 \mathrm{E}-13$ \\
\hline xe126 & $3.184 \mathrm{E}-04$ & $3.184 \mathrm{E}-04$ & 3.184E-04 & $3.184 \mathrm{E}-04$ & 3.184E-04 \\
\hline
\end{tabular}




\begin{tabular}{|c|c|c|c|c|c|}
\hline \multicolumn{6}{|c|}{ Table C-3. Continued } \\
\hline & $1 / 22 / 2009$ & $10 / 21 / 2013$ & $1 / 22 / 2014$ & $1 / 22 / 2015$ & $1 / 22 / 2016$ \\
\hline xe127 & 4.963E-08 & $2.310 \mathrm{E}-22$ & $3.931 \mathrm{E}-23$ & $3.766 \mathrm{E}-26$ & $3.608 \mathrm{E}-29$ \\
\hline xe128 & $7.830 \mathrm{E}+00$ & $7.830 \mathrm{E}+00$ & $7.830 \mathrm{E}+00$ & $7.830 \mathrm{E}+00$ & $7.830 \mathrm{E}+00$ \\
\hline xe129 & $5.578 \mathrm{E}-02$ & $5.584 \mathrm{E}-02$ & $5.585 \mathrm{E}-02$ & $5.586 \mathrm{E}-02$ & $5.587 \mathrm{E}-02$ \\
\hline xe129m & $2.664 \mathrm{E}-13$ & $0.000 \mathrm{E}+00$ & $0.000 \mathrm{E}+00$ & $0.000 \mathrm{E}+00$ & $0.000 \mathrm{E}+00$ \\
\hline xe130 & $1.067 \mathrm{E}+01$ & $1.067 \mathrm{E}+01$ & $1.067 \mathrm{E}+01$ & $1.067 \mathrm{E}+01$ & $1.067 \mathrm{E}+01$ \\
\hline xe131 & $5.788 \mathrm{E}+02$ & $5.788 \mathrm{E}+02$ & $5.788 \mathrm{E}+02$ & $5.788 \mathrm{E}+02$ & $5.788 \mathrm{E}+02$ \\
\hline xe131m & 8.787E-08 & $0.000 \mathrm{E}+00$ & $0.000 \mathrm{E}+00$ & $0.000 \mathrm{E}+00$ & $0.000 \mathrm{E}+00$ \\
\hline xe132 & $1.593 \mathrm{E}-14$ & $1.593 \mathrm{E}-14$ & $1.593 \mathrm{E}-14$ & $1.593 \mathrm{E}-14$ & $1.593 \mathrm{E}-14$ \\
\hline xe132 & $1.535 \mathrm{E}+03$ & $1.535 \mathrm{E}+03$ & $1.535 \mathrm{E}+03$ & $1.535 \mathrm{E}+03$ & $1.535 \mathrm{E}+03$ \\
\hline xe133 & $8.993 \mathrm{E}-15$ & $0.000 \mathrm{E}+00$ & $0.000 \mathrm{E}+00$ & $0.000 \mathrm{E}+00$ & $0.000 \mathrm{E}+00$ \\
\hline xe134 & $1.858 \mathrm{E}+03$ & $1.858 \mathrm{E}+03$ & $1.858 \mathrm{E}+03$ & $1.858 \mathrm{E}+03$ & $1.858 \mathrm{E}+03$ \\
\hline xe136 & $2.852 \mathrm{E}+03$ & $2.852 \mathrm{E}+03$ & $2.852 \mathrm{E}+03$ & $2.852 \mathrm{E}+03$ & $2.852 \mathrm{E}+03$ \\
\hline y 88 & $2.632 \mathrm{E}-05$ & $3.371 \mathrm{E}-10$ & $1.841 \mathrm{E}-10$ & $1.717 \mathrm{E}-11$ & $1.601 \mathrm{E}-12$ \\
\hline y 89 & $2.945 \mathrm{E}+02$ & $2.950 \mathrm{E}+02$ & $2.950 \mathrm{E}+02$ & $2.950 \mathrm{E}+02$ & $2.950 \mathrm{E}+02$ \\
\hline y 89m & $1.668 \mathrm{E}-10$ & $7.903 \mathrm{E}-21$ & $2.207 \mathrm{E}-21$ & $1.477 \mathrm{E}-23$ & $9.884 \mathrm{E}-26$ \\
\hline y 90 & 8.714E-02 & $7.770 \mathrm{E}-02$ & $7.724 \mathrm{E}-02$ & $7.544 \mathrm{E}-02$ & $7.365 \mathrm{E}-02$ \\
\hline y 91 & $1.350 \mathrm{E}+00$ & $1.637 \mathrm{E}-09$ & $5.439 \mathrm{E}-10$ & $7.205 \mathrm{E}-12$ & $9.545 \mathrm{E}-14$ \\
\hline yb168 & $1.899 \mathrm{E}-12$ & $1.991 \mathrm{E}-12$ & $1.991 \mathrm{E}-12$ & $1.991 \mathrm{E}-12$ & $1.991 \mathrm{E}-12$ \\
\hline yb169 & $3.315 \mathrm{E}-12$ & $1.686 \mathrm{E}-28$ & $2.251 \mathrm{E}-29$ & $8.335 \mathrm{E}-33$ & $0.000 \mathrm{E}+00$ \\
\hline yb170 & $6.880 \mathrm{E}-04$ & $7.405 \mathrm{E}-04$ & $7.405 \mathrm{E}-04$ & $7.405 \mathrm{E}-04$ & $7.405 \mathrm{E}-04$ \\
\hline yb171 & $2.190 \mathrm{E}-04$ & 3.599E-04 & 3.627E-04 & $3.712 \mathrm{E}-04$ & 3.772E-04 \\
\hline yb172 & $4.573 \mathrm{E}-04$ & $4.573 \mathrm{E}-04$ & $4.573 \mathrm{E}-04$ & $4.573 \mathrm{E}-04$ & 4.573E-04 \\
\hline yb176 & $1.252 \mathrm{E}-16$ & $1.252 \mathrm{E}-16$ & $1.252 \mathrm{E}-16$ & $1.252 \mathrm{E}-16$ & $1.252 \mathrm{E}-16$ \\
\hline zn 64 & $4.771 \mathrm{E}-16$ & $4.771 \mathrm{E}-16$ & $4.771 \mathrm{E}-16$ & $4.771 \mathrm{E}-16$ & $4.771 \mathrm{E}-16$ \\
\hline zn 66 & $1.508 \mathrm{E}-04$ & $1.508 \mathrm{E}-04$ & $1.508 \mathrm{E}-04$ & $1.508 \mathrm{E}-04$ & $1.508 \mathrm{E}-04$ \\
\hline zn 67 & 4.236E-04 & $4.236 \mathrm{E}-04$ & $4.236 \mathrm{E}-04$ & $4.236 \mathrm{E}-04$ & 4.236E-04 \\
\hline zn 68 & 8.315E-04 & $8.315 \mathrm{E}-04$ & $8.315 \mathrm{E}-04$ & $8.315 \mathrm{E}-04$ & 8.315E-04 \\
\hline zn 70 & $3.798 \mathrm{E}-03$ & $3.798 \mathrm{E}-03$ & $3.798 \mathrm{E}-03$ & $3.798 \mathrm{E}-03$ & 3.798E-03 \\
\hline zr 88 & $5.792 \mathrm{E}-12$ & $3.218 \mathrm{E}-18$ & $1.486 \mathrm{E}-18$ & $7.152 \mathrm{E}-20$ & $3.442 \mathrm{E}-21$ \\
\hline $\operatorname{zr} 90$ & $1.947 \mathrm{E}+01$ & $5.656 \mathrm{E}+01$ & $5.843 \mathrm{E}+01$ & $6.567 \mathrm{E}+01$ & $7.272 \mathrm{E}+01$ \\
\hline $\operatorname{zr} 91$ & $4.287 \mathrm{E}+02$ & $4.300 \mathrm{E}+02$ & $4.300 \mathrm{E}+02$ & $4.300 \mathrm{E}+02$ & $4.300 \mathrm{E}+02$ \\
\hline $\operatorname{zr} 92$ & $5.191 \mathrm{E}+02$ & $5.191 \mathrm{E}+02$ & $5.191 \mathrm{E}+02$ & $5.191 \mathrm{E}+02$ & $5.191 \mathrm{E}+02$ \\
\hline $\operatorname{zr} 93$ & $6.393 E+02$ & $6.393 \mathrm{E}+02$ & $6.392 \mathrm{E}+02$ & $6.392 \mathrm{E}+02$ & $6.392 \mathrm{E}+02$ \\
\hline zr 94 & $7.325 \mathrm{E}+02$ & $7.325 \mathrm{E}+02$ & $7.325 \mathrm{E}+02$ & $7.325 \mathrm{E}+02$ & $7.325 \mathrm{E}+02$ \\
\hline zr 95 & $3.672 \mathrm{E}+00$ & $2.616 \mathrm{E}-08$ & $9.558 \mathrm{E}-09$ & $1.838 \mathrm{E}-10$ & $3.535 \mathrm{E}-12$ \\
\hline zr 96 & $8.561 \mathrm{E}+02$ & $8.561 \mathrm{E}+02$ & $8.561 \mathrm{E}+02$ & $8.561 \mathrm{E}+02$ & $8.561 \mathrm{E}+02$ \\
\hline Total & $1.1344 \mathrm{E}+06$ & $1.1344 \mathrm{E}+06$ & $1.1344 \mathrm{E}+06$ & $1.1344 \mathrm{E}+06$ & $1.1344 \mathrm{E}+06$ \\
\hline
\end{tabular}


Table C-4. MOX Fuel Rod B14 (high Pu): Nuclide mass in grams per MTIHM

\begin{tabular}{|c|c|c|c|c|c|}
\hline & $1 / 22 / 2009$ & $10 / 21 / 2013$ & $1 / 22 / 2014$ & $1 / 22 / 2015$ & $1 / 22 / 2016$ \\
\hline ac225 & $1.174 \mathrm{E}-12$ & $1.220 \mathrm{E}-12$ & $1.222 \mathrm{E}-12$ & $1.236 \mathrm{E}-12$ & $1.250 \mathrm{E}-12$ \\
\hline ac227 & 7.363E-10 & $3.120 \mathrm{E}-09$ & $3.251 \mathrm{E}-09$ & 3.763E-09 & $4.280 \mathrm{E}-09$ \\
\hline $\operatorname{ag} 105$ & $9.544 \mathrm{E}-15$ & $2.214 \mathrm{E}-27$ & $4.646 \mathrm{E}-28$ & $1.014 \mathrm{E}-30$ & $2.212 \mathrm{E}-33$ \\
\hline $\operatorname{ag} 107$ & $1.542 \mathrm{E}-04$ & $4.956 \mathrm{E}-04$ & 5.139E-04 & $5.858 \mathrm{E}-04$ & 6.577E-04 \\
\hline ag108 & $7.211 \mathrm{E}-13$ & $7.158 \mathrm{E}-13$ & $7.151 \mathrm{E}-13$ & $7.145 \mathrm{E}-13$ & $7.131 \mathrm{E}-13$ \\
\hline ag108m & $8.013 \mathrm{E}-04$ & $7.953 \mathrm{E}-04$ & 7.953E-04 & $7.940 \mathrm{E}-04$ & 7.927E-04 \\
\hline $\operatorname{ag} 109$ & $2.311 \mathrm{E}+02$ & $2.311 \mathrm{E}+02$ & $2.311 \mathrm{E}+02$ & $2.311 \mathrm{E}+02$ & $2.311 \mathrm{E}+02$ \\
\hline $\operatorname{ag} 109 m$ & $2.202 \mathrm{E}-10$ & $1.630 \mathrm{E}-11$ & $1.417 \mathrm{E}-11$ & $8.192 \mathrm{E}-12$ & $4.733 \mathrm{E}-12$ \\
\hline $\operatorname{ag} 110$ & $2.332 \mathrm{E}-08$ & $1.901 \mathrm{E}-10$ & $1.469 \mathrm{E}-10$ & 5.333E-11 & $1.937 \mathrm{E}-11$ \\
\hline $\operatorname{ag} 110 \mathrm{~m}$ & $1.504 \mathrm{E}+00$ & $1.226 \mathrm{E}-02$ & $9.471 \mathrm{E}-03$ & $3.440 \mathrm{E}-03$ & $1.249 \mathrm{E}-03$ \\
\hline ag111 & $1.709 \mathrm{E}-11$ & $0.000 \mathrm{E}+00$ & $0.000 \mathrm{E}+00$ & $0.000 \mathrm{E}+00$ & $0.000 \mathrm{E}+00$ \\
\hline al 27 & $4.893 \mathrm{E}-14$ & 4.893E-14 & 4.893E-14 & 4.893E-14 & 4.893E-14 \\
\hline $\operatorname{am} 241$ & $4.289 \mathrm{E}+02$ & $1.489 \mathrm{E}+03$ & $1.539 \mathrm{E}+03$ & $1.729 \mathrm{E}+03$ & $1.910 \mathrm{E}+03$ \\
\hline am242 & $5.610 \mathrm{E}-05$ & $5.480 \mathrm{E}-05$ & $5.474 \mathrm{E}-05$ & $5.447 \mathrm{E}-05$ & $5.420 \mathrm{E}-05$ \\
\hline $\operatorname{am} 242 m$ & $4.348 \mathrm{E}+00$ & $4.248 \mathrm{E}+00$ & $4.242 \mathrm{E}+00$ & $4.222 \mathrm{E}+00$ & $4.201 \mathrm{E}+00$ \\
\hline $\operatorname{am} 243$ & $3.875 \mathrm{E}+02$ & $3.874 \mathrm{E}+02$ & $3.874 \mathrm{E}+02$ & $3.873 \mathrm{E}+02$ & $3.873 \mathrm{E}+02$ \\
\hline $\operatorname{am} 244$ & $3.014 \mathrm{E}-15$ & $2.091 \mathrm{E}-15$ & $2.051 \mathrm{E}-15$ & $1.899 \mathrm{E}-15$ & $1.758 \mathrm{E}-15$ \\
\hline am245 & $3.250 \mathrm{E}-14$ & $7.615 \mathrm{E}-16$ & $6.225 \mathrm{E}-16$ & $2.823 \mathrm{E}-16$ & $1.280 \mathrm{E}-16$ \\
\hline ar 36 & $6.467 \mathrm{E}-14$ & $6.467 \mathrm{E}-14$ & $6.467 \mathrm{E}-14$ & $6.467 \mathrm{E}-14$ & $6.467 \mathrm{E}-14$ \\
\hline ar 38 & $6.886 \mathrm{E}-14$ & $6.886 \mathrm{E}-14$ & $6.886 \mathrm{E}-14$ & $6.886 \mathrm{E}-14$ & $6.886 \mathrm{E}-14$ \\
\hline ar 39 & $1.208 \mathrm{E}-16$ & $1.194 \mathrm{E}-16$ & $1.193 \mathrm{E}-16$ & $1.190 \mathrm{E}-16$ & $1.186 \mathrm{E}-16$ \\
\hline ar 40 & $7.284 \mathrm{E}-14$ & $7.284 \mathrm{E}-14$ & $7.284 \mathrm{E}-14$ & $7.284 \mathrm{E}-14$ & $7.284 \mathrm{E}-14$ \\
\hline as 73 & $1.562 \mathrm{E}-10$ & 4.977E-17 & $2.230 \mathrm{E}-17$ & $9.551 \mathrm{E}-19$ & $4.090 \mathrm{E}-20$ \\
\hline as 74 & $1.962 \mathrm{E}-12$ & $0.000 \mathrm{E}+00$ & $0.000 \mathrm{E}+00$ & $0.000 \mathrm{E}+00$ & $0.000 \mathrm{E}+00$ \\
\hline as 75 & $1.895 \mathrm{E}-01$ & $1.895 \mathrm{E}-01$ & $1.895 \mathrm{E}-01$ & $1.895 \mathrm{E}-01$ & $1.895 \mathrm{E}-01$ \\
\hline au197 & $2.289 \mathrm{E}-13$ & $2.289 \mathrm{E}-13$ & $2.289 \mathrm{E}-13$ & $2.289 \mathrm{E}-13$ & $2.289 \mathrm{E}-13$ \\
\hline b 10 & $6.137 \mathrm{E}-11$ & $3.294 \mathrm{E}-10$ & $3.438 \mathrm{E}-10$ & $4.002 \mathrm{E}-10$ & $4.567 \mathrm{E}-10$ \\
\hline b 11 & $6.986 \mathrm{E}-08$ & $6.986 \mathrm{E}-08$ & $6.986 \mathrm{E}-08$ & $6.986 \mathrm{E}-08$ & $6.986 \mathrm{E}-08$ \\
\hline ba130 & $1.901 \mathrm{E}-13$ & $1.901 \mathrm{E}-13$ & $1.901 \mathrm{E}-13$ & $1.901 \mathrm{E}-13$ & $1.901 \mathrm{E}-13$ \\
\hline ba131 & $4.781 \mathrm{E}-18$ & $0.000 \mathrm{E}+00$ & $0.000 \mathrm{E}+00$ & $0.000 \mathrm{E}+00$ & $0.000 \mathrm{E}+00$ \\
\hline ba132 & $9.292 \mathrm{E}-17$ & $9.292 \mathrm{E}-17$ & $9.292 \mathrm{E}-17$ & $9.292 \mathrm{E}-17$ & $9.292 \mathrm{E}-17$ \\
\hline ba132 & $3.315 \mathrm{E}-04$ & $3.315 \mathrm{E}-04$ & $3.315 \mathrm{E}-04$ & $3.315 \mathrm{E}-04$ & $3.315 \mathrm{E}-04$ \\
\hline ba133 & $2.115 \mathrm{E}-04$ & $1.547 \mathrm{E}-04$ & $1.521 \mathrm{E}-04$ & $1.424 \mathrm{E}-04$ & $1.334 \mathrm{E}-04$ \\
\hline ba134 & $1.192 \mathrm{E}+02$ & $2.477 \mathrm{E}+02$ & $2.504 \mathrm{E}+02$ & $2.589 \mathrm{E}+02$ & $2.651 \mathrm{E}+02$ \\
\hline ba135 & 5.302E-01 & $5.313 \mathrm{E}-01$ & $5.314 \mathrm{E}-01$ & 5.317E-01 & 5.319E-01 \\
\hline ba136 & $5.184 \mathrm{E}+01$ & $5.184 \mathrm{E}+01$ & $5.184 \mathrm{E}+01$ & $5.184 \mathrm{E}+01$ & $5.184 \mathrm{E}+01$ \\
\hline ba136m & $3.633 \mathrm{E}-14$ & $0.000 \mathrm{E}+00$ & $0.000 \mathrm{E}+00$ & $0.000 \mathrm{E}+00$ & $0.000 \mathrm{E}+00$ \\
\hline ba137 & $9.126 \mathrm{E}+01$ & $2.703 E+02$ & $2.794 \mathrm{E}+02$ & $3.145 \mathrm{E}+02$ & $3.488 \mathrm{E}+02$ \\
\hline
\end{tabular}




\begin{tabular}{|c|c|c|c|c|c|}
\hline \multicolumn{6}{|c|}{ Table C-4. Continued } \\
\hline & $1 / 22 / 2009$ & $10 / 21 / 2013$ & $1 / 22 / 2014$ & $1 / 22 / 2015$ & $1 / 22 / 2016$ \\
\hline ba137m & $2.641 \mathrm{E}-04$ & $2.368 \mathrm{E}-04$ & $2.354 \mathrm{E}-04$ & $2.300 \mathrm{E}-04$ & $2.248 \mathrm{E}-04$ \\
\hline ba138 & $1.725 \mathrm{E}+03$ & $1.725 \mathrm{E}+03$ & $1.725 \mathrm{E}+03$ & $1.725 \mathrm{E}+03$ & $1.725 \mathrm{E}+03$ \\
\hline ba140 & $1.536 \mathrm{E}-05$ & $0.000 \mathrm{E}+00$ & $0.000 \mathrm{E}+00$ & $0.000 \mathrm{E}+00$ & $0.000 \mathrm{E}+00$ \\
\hline be 9 & $2.375 \mathrm{E}-06$ & $2.375 \mathrm{E}-06$ & $2.375 \mathrm{E}-06$ & $2.375 \mathrm{E}-06$ & $2.375 \mathrm{E}-06$ \\
\hline be 10 & $1.231 \mathrm{E}-04$ & $1.231 \mathrm{E}-04$ & $1.231 \mathrm{E}-04$ & $1.231 \mathrm{E}-04$ & $1.231 \mathrm{E}-04$ \\
\hline bi208 & $1.129 \mathrm{E}-15$ & $1.129 \mathrm{E}-15$ & $1.129 \mathrm{E}-15$ & $1.129 \mathrm{E}-15$ & $1.129 \mathrm{E}-15$ \\
\hline bi209 & $1.031 \mathrm{E}-10$ & $2.365 \mathrm{E}-10$ & $2.438 \mathrm{E}-10$ & $2.727 \mathrm{E}-10$ & $3.018 \mathrm{E}-10$ \\
\hline bi210 & $3.259 \mathrm{E}-14$ & 3.322E-14 & $3.355 \mathrm{E}-14$ & $3.526 \mathrm{E}-14$ & $3.770 \mathrm{E}-14$ \\
\hline bi210m & $1.506 \mathrm{E}-15$ & $1.506 \mathrm{E}-15$ & $1.506 \mathrm{E}-15$ & $1.506 \mathrm{E}-15$ & $1.506 \mathrm{E}-15$ \\
\hline bi211 & $1.188 \mathrm{E}-16$ & $5.432 \mathrm{E}-16$ & $5.548 \mathrm{E}-16$ & $6.551 \mathrm{E}-16$ & $7.450 \mathrm{E}-16$ \\
\hline bi212 & $1.819 \mathrm{E}-10$ & $1.147 \mathrm{E}-09$ & $1.190 \mathrm{E}-09$ & $1.335 \mathrm{E}-09$ & $1.457 \mathrm{E}-09$ \\
\hline bi213 & $3.518 \mathrm{E}-15$ & $3.657 \mathrm{E}-15$ & $3.662 \mathrm{E}-15$ & $3.705 \mathrm{E}-15$ & $3.747 \mathrm{E}-15$ \\
\hline bk247 & $5.780 \mathrm{E}-13$ & $5.766 \mathrm{E}-13$ & $5.765 \mathrm{E}-13$ & $5.762 \mathrm{E}-13$ & $5.760 \mathrm{E}-13$ \\
\hline bk248 & $2.391 \mathrm{E}-11$ & $1.659 \mathrm{E}-11$ & $1.626 \mathrm{E}-11$ & $1.506 \mathrm{E}-11$ & $1.394 \mathrm{E}-11$ \\
\hline bk249 & $8.530 \mathrm{E}-06$ & $1.999 \mathrm{E}-07$ & $1.634 \mathrm{E}-07$ & $7.410 \mathrm{E}-08$ & 3.362E-08 \\
\hline bk250 & $2.851 \mathrm{E}-15$ & 3.694E-17 & $2.933 \mathrm{E}-17$ & $1.196 \mathrm{E}-17$ & $5.024 \mathrm{E}-18$ \\
\hline br 79 & $3.352 \mathrm{E}-03$ & $3.420 \mathrm{E}-03$ & $3.423 \mathrm{E}-03$ & $3.438 \mathrm{E}-03$ & $3.452 \mathrm{E}-03$ \\
\hline br 81 & $2.588 \mathrm{E}+01$ & $2.588 \mathrm{E}+01$ & $2.588 \mathrm{E}+01$ & $2.588 \mathrm{E}+01$ & $2.588 \mathrm{E}+01$ \\
\hline c 12 & $7.668 \mathrm{E}-02$ & $7.668 \mathrm{E}-02$ & $7.668 \mathrm{E}-02$ & $7.668 \mathrm{E}-02$ & 7.668E-02 \\
\hline c 13 & $1.212 \mathrm{E}+01$ & $1.212 \mathrm{E}+01$ & $1.212 \mathrm{E}+01$ & $1.212 \mathrm{E}+01$ & $1.212 \mathrm{E}+01$ \\
\hline c 14 & $3.716 \mathrm{E}-04$ & 3.714E-04 & $3.714 \mathrm{E}-04$ & $3.714 \mathrm{E}-04$ & $3.713 \mathrm{E}-04$ \\
\hline ca 40 & $7.231 \mathrm{E}-14$ & $7.231 \mathrm{E}-14$ & $7.231 \mathrm{E}-14$ & $7.231 \mathrm{E}-14$ & $7.231 \mathrm{E}-14$ \\
\hline ca 41 & $4.046 \mathrm{E}-17$ & $4.046 \mathrm{E}-17$ & $4.046 \mathrm{E}-17$ & $4.046 \mathrm{E}-17$ & $4.046 \mathrm{E}-17$ \\
\hline ca 42 & $7.629 \mathrm{E}-14$ & $7.629 \mathrm{E}-14$ & $7.629 \mathrm{E}-14$ & $7.629 \mathrm{E}-14$ & $7.629 \mathrm{E}-14$ \\
\hline ca 43 & $7.688 \mathrm{E}-14$ & $7.688 \mathrm{E}-14$ & $7.688 \mathrm{E}-14$ & $7.688 \mathrm{E}-14$ & $7.688 \mathrm{E}-14$ \\
\hline ca 44 & $8.086 \mathrm{E}-14$ & $8.086 \mathrm{E}-14$ & $8.086 \mathrm{E}-14$ & $8.086 \mathrm{E}-14$ & $8.086 \mathrm{E}-14$ \\
\hline ca 46 & $8.331 \mathrm{E}-14$ & $8.331 \mathrm{E}-14$ & 8.331E-14 & $8.331 \mathrm{E}-14$ & $8.331 \mathrm{E}-14$ \\
\hline ca 48 & $8.689 \mathrm{E}-14$ & $8.689 \mathrm{E}-14$ & $8.689 \mathrm{E}-14$ & $8.689 \mathrm{E}-14$ & $8.689 \mathrm{E}-14$ \\
\hline cd106 & $3.089 \mathrm{E}-13$ & $3.089 \mathrm{E}-13$ & $3.089 \mathrm{E}-13$ & $3.089 \mathrm{E}-13$ & $3.089 \mathrm{E}-13$ \\
\hline cd108 & $1.645 \mathrm{E}-03$ & $1.645 \mathrm{E}-03$ & $1.645 \mathrm{E}-03$ & $1.646 \mathrm{E}-03$ & $1.646 \mathrm{E}-03$ \\
\hline cd109 & $2.217 \mathrm{E}-04$ & $1.640 \mathrm{E}-05$ & $1.426 \mathrm{E}-05$ & $8.245 \mathrm{E}-06$ & 4.764E-06 \\
\hline cd110 & $1.531 \mathrm{E}+02$ & $1.546 \mathrm{E}+02$ & $1.546 \mathrm{E}+02$ & $1.546 \mathrm{E}+02$ & $1.546 \mathrm{E}+02$ \\
\hline cd111 & $7.277 \mathrm{E}+01$ & $7.277 \mathrm{E}+01$ & $7.277 \mathrm{E}+01$ & $7.277 \mathrm{E}+01$ & $7.277 \mathrm{E}+01$ \\
\hline cd112 & $3.425 \mathrm{E}+01$ & $3.425 \mathrm{E}+01$ & $3.425 \mathrm{E}+01$ & $3.425 \mathrm{E}+01$ & $3.425 \mathrm{E}+01$ \\
\hline cd113 & $3.940 \mathrm{E}-01$ & $3.940 \mathrm{E}-01$ & $3.940 \mathrm{E}-01$ & $3.940 \mathrm{E}-01$ & $3.940 \mathrm{E}-01$ \\
\hline cd113m & $5.587 \mathrm{E}-03$ & $4.424 \mathrm{E}-03$ & $4.369 \mathrm{E}-03$ & $4.160 \mathrm{E}-03$ & $3.960 \mathrm{E}-03$ \\
\hline cd114 & $3.651 \mathrm{E}+01$ & $3.651 \mathrm{E}+01$ & $3.651 \mathrm{E}+01$ & $3.651 \mathrm{E}+01$ & $3.651 \mathrm{E}+01$ \\
\hline cd115m & $7.131 \mathrm{E}-04$ & $1.399 \mathrm{E}-15$ & $3.293 \mathrm{E}-16$ & $1.127 \mathrm{E}-18$ & $3.854 \mathrm{E}-21$ \\
\hline
\end{tabular}




\begin{tabular}{|c|c|c|c|c|c|}
\hline \multicolumn{6}{|c|}{ Table C-4. Continued } \\
\hline & $1 / 22 / 2009$ & $10 / 21 / 2013$ & $1 / 22 / 2014$ & $1 / 22 / 2015$ & $1 / 22 / 2016$ \\
\hline cd116 & $1.219 \mathrm{E}+01$ & $1.219 \mathrm{E}+01$ & $1.219 \mathrm{E}+01$ & $1.219 \mathrm{E}+01$ & $1.219 \mathrm{E}+01$ \\
\hline ce136 & $2.279 \mathrm{E}-13$ & $2.279 \mathrm{E}-13$ & $2.279 \mathrm{E}-13$ & $2.279 \mathrm{E}-13$ & $2.279 \mathrm{E}-13$ \\
\hline ce138 & $1.165 \mathrm{E}-05$ & $1.165 \mathrm{E}-05$ & $1.165 \mathrm{E}-05$ & $1.165 \mathrm{E}-05$ & $1.165 \mathrm{E}-05$ \\
\hline ce139 & $1.644 \mathrm{E}-03$ & $2.664 \mathrm{E}-07$ & $1.668 \mathrm{E}-07$ & $2.654 \mathrm{E}-08$ & 4.223E-09 \\
\hline ce140 & $1.636 \mathrm{E}+03$ & $1.636 \mathrm{E}+03$ & $1.636 \mathrm{E}+03$ & $1.636 \mathrm{E}+03$ & $1.636 \mathrm{E}+03$ \\
\hline ce141 & $2.269 \mathrm{E}-01$ & $2.031 \mathrm{E}-17$ & $2.796 \mathrm{E}-18$ & $1.166 \mathrm{E}-21$ & $4.859 \mathrm{E}-25$ \\
\hline ce142 & $1.422 \mathrm{E}+03$ & $1.422 \mathrm{E}+03$ & $1.422 \mathrm{E}+03$ & $1.422 \mathrm{E}+03$ & $1.422 \mathrm{E}+03$ \\
\hline ce144 & $2.101 \mathrm{E}+02$ & $3.100 \mathrm{E}+00$ & $2.472 \mathrm{E}+00$ & $1.017 \mathrm{E}+00$ & 4.186E-01 \\
\hline cf248 & $1.540 \mathrm{E}-11$ & $4.199 \mathrm{E}-13$ & $3.462 \mathrm{E}-13$ & $1.621 \mathrm{E}-13$ & $7.589 \mathrm{E}-14$ \\
\hline cf249 & $9.113 \mathrm{E}-06$ & $1.730 \mathrm{E}-05$ & $1.732 \mathrm{E}-05$ & $1.738 \mathrm{E}-05$ & $1.738 \mathrm{E}-05$ \\
\hline cf250 & $4.356 \mathrm{E}-06$ & $3.387 \mathrm{E}-06$ & 3.342E-06 & $3.170 \mathrm{E}-06$ & $3.006 \mathrm{E}-06$ \\
\hline cf251 & $2.160 \mathrm{E}-06$ & $2.152 \mathrm{E}-06$ & $2.151 \mathrm{E}-06$ & $2.150 \mathrm{E}-06$ & $2.148 \mathrm{E}-06$ \\
\hline cf252 & $6.634 \mathrm{E}-07$ & $1.913 \mathrm{E}-07$ & $1.790 \mathrm{E}-07$ & $1.378 \mathrm{E}-07$ & $1.060 \mathrm{E}-07$ \\
\hline cf253 & $3.871 \mathrm{E}-14$ & $0.000 \mathrm{E}+00$ & $0.000 \mathrm{E}+00$ & $0.000 \mathrm{E}+00$ & $0.000 \mathrm{E}+00$ \\
\hline cf254 & $1.958 \mathrm{E}-12$ & $4.665 \mathrm{E}-21$ & $1.607 \mathrm{E}-21$ & $2.454 \mathrm{E}-23$ & $3.748 \mathrm{E}-25$ \\
\hline cl 35 & $6.039 \mathrm{E}-14$ & $6.039 \mathrm{E}-14$ & $6.039 \mathrm{E}-14$ & $6.039 \mathrm{E}-14$ & $6.039 \mathrm{E}-14$ \\
\hline cl 36 & $3.296 \mathrm{E}-15$ & $3.296 \mathrm{E}-15$ & $3.296 \mathrm{E}-15$ & $3.296 \mathrm{E}-15$ & $3.296 \mathrm{E}-15$ \\
\hline cl 37 & $6.767 \mathrm{E}-14$ & $6.767 \mathrm{E}-14$ & $6.767 \mathrm{E}-14$ & $6.767 \mathrm{E}-14$ & $6.767 \mathrm{E}-14$ \\
\hline cm240 & $1.972 \mathrm{E}-11$ & $9.398 \mathrm{E}-31$ & $8.629 \mathrm{E}-32$ & $0.000 \mathrm{E}+00$ & $0.000 \mathrm{E}+00$ \\
\hline $\mathrm{cm} 241$ & $2.808 \mathrm{E}-08$ & $3.493 \mathrm{E}-24$ & $4.895 \mathrm{E}-25$ & $2.186 \mathrm{E}-28$ & $9.769 \mathrm{E}-32$ \\
\hline cm242 & $2.206 \mathrm{E}+01$ & $2.493 \mathrm{E}-02$ & $2.039 \mathrm{E}-02$ & $1.299 \mathrm{E}-02$ & $1.138 \mathrm{E}-02$ \\
\hline cm243 & $1.874 \mathrm{E}+00$ & $1.674 \mathrm{E}+00$ & $1.664 \mathrm{E}+00$ & $1.625 \mathrm{E}+00$ & $1.587 \mathrm{E}+00$ \\
\hline cm244 & $1.500 \mathrm{E}+02$ & $1.251 \mathrm{E}+02$ & $1.238 \mathrm{E}+02$ & $1.192 \mathrm{E}+02$ & $1.147 \mathrm{E}+02$ \\
\hline cm245 & $1.430 \mathrm{E}+01$ & $1.429 \mathrm{E}+01$ & $1.429 \mathrm{E}+01$ & $1.429 \mathrm{E}+01$ & $1.429 \mathrm{E}+01$ \\
\hline cm246 & $9.597 \mathrm{E}-01$ & $9.590 \mathrm{E}-01$ & $9.584 \mathrm{E}-01$ & $9.584 \mathrm{E}-01$ & $9.584 \mathrm{E}-01$ \\
\hline cm247 & $1.463 \mathrm{E}-02$ & $1.463 \mathrm{E}-02$ & $1.463 \mathrm{E}-02$ & $1.463 \mathrm{E}-02$ & $1.463 \mathrm{E}-02$ \\
\hline $\mathrm{cm} 248$ & $9.411 \mathrm{E}-04$ & $9.418 \mathrm{E}-04$ & $9.418 \mathrm{E}-04$ & $9.418 \mathrm{E}-04$ & $9.418 \mathrm{E}-04$ \\
\hline cm250 & $1.163 \mathrm{E}-10$ & $1.162 \mathrm{E}-10$ & $1.162 \mathrm{E}-10$ & $1.162 \mathrm{E}-10$ & $1.162 \mathrm{E}-10$ \\
\hline co 59 & $1.069 \mathrm{E}-13$ & $1.069 \mathrm{E}-13$ & $1.069 \mathrm{E}-13$ & $1.069 \mathrm{E}-13$ & $1.069 \mathrm{E}-13$ \\
\hline co 60 & $9.915 \mathrm{E}-15$ & $5.313 \mathrm{E}-15$ & $5.138 \mathrm{E}-15$ & $4.506 \mathrm{E}-15$ & $3.950 \mathrm{E}-15$ \\
\hline cr 50 & $8.888 \mathrm{E}-14$ & $8.888 \mathrm{E}-14$ & $8.888 \mathrm{E}-14$ & $8.888 \mathrm{E}-14$ & $8.888 \mathrm{E}-14$ \\
\hline cr 52 & $9.418 \mathrm{E}-14$ & $9.418 \mathrm{E}-14$ & $9.418 \mathrm{E}-14$ & $9.418 \mathrm{E}-14$ & $9.418 \mathrm{E}-14$ \\
\hline cr 53 & $9.411 \mathrm{E}-14$ & $9.411 \mathrm{E}-14$ & $9.411 \mathrm{E}-14$ & $9.411 \mathrm{E}-14$ & $9.411 \mathrm{E}-14$ \\
\hline cr 54 & $1.000 \mathrm{E}-13$ & $1.001 \mathrm{E}-13$ & $1.001 \mathrm{E}-13$ & $1.001 \mathrm{E}-13$ & $1.001 \mathrm{E}-13$ \\
\hline $\operatorname{cs} 131$ & $2.041 \mathrm{E}-15$ & $0.000 \mathrm{E}+00$ & $0.000 \mathrm{E}+00$ & $0.000 \mathrm{E}+00$ & $0.000 \mathrm{E}+00$ \\
\hline $\operatorname{cs} 132$ & $1.768 \mathrm{E}-16$ & $0.000 \mathrm{E}+00$ & $0.000 \mathrm{E}+00$ & $0.000 \mathrm{E}+00$ & $0.000 \mathrm{E}+00$ \\
\hline cs133 & $3.474 \mathrm{E}-17$ & $3.554 \mathrm{E}-17$ & $3.558 \mathrm{E}-17$ & $3.572 \mathrm{E}-17$ & $3.585 \mathrm{E}-17$ \\
\hline $\operatorname{cs} 133$ & $1.584 \mathrm{E}+03$ & $1.584 \mathrm{E}+03$ & $1.584 \mathrm{E}+03$ & $1.584 \mathrm{E}+03$ & $1.584 \mathrm{E}+03$ \\
\hline
\end{tabular}




\begin{tabular}{|c|c|c|c|c|c|}
\hline \multicolumn{6}{|c|}{ Table C-4. Continued } \\
\hline & $1 / 22 / 2009$ & $10 / 21 / 2013$ & $1 / 22 / 2014$ & $1 / 22 / 2015$ & $1 / 22 / 2016$ \\
\hline cs134 & $1.614 \mathrm{E}+02$ & $3.283 \mathrm{E}+01$ & $3.014 \mathrm{E}+01$ & $2.155 \mathrm{E}+01$ & $1.541 \mathrm{E}+01$ \\
\hline $\operatorname{cs} 135$ & $8.715 \mathrm{E}+02$ & $8.715 \mathrm{E}+02$ & $8.715 \mathrm{E}+02$ & $8.715 \mathrm{E}+02$ & $8.715 \mathrm{E}+02$ \\
\hline $\operatorname{cs} 136$ & $1.210 \mathrm{E}-06$ & $0.000 \mathrm{E}+00$ & $0.000 \mathrm{E}+00$ & $0.000 \mathrm{E}+00$ & $0.000 \mathrm{E}+00$ \\
\hline $\operatorname{cs137}$ & $1.729 \mathrm{E}+03$ & $1.550 \mathrm{E}+03$ & $1.541 \mathrm{E}+03$ & $1.506 \mathrm{E}+03$ & $1.471 \mathrm{E}+03$ \\
\hline cu 63 & $1.131 \mathrm{E}-13$ & $1.132 \mathrm{E}-13$ & $1.132 \mathrm{E}-13$ & $1.132 \mathrm{E}-13$ & $1.132 \mathrm{E}-13$ \\
\hline cu 65 & $2.368 \mathrm{E}-16$ & $2.369 \mathrm{E}-16$ & $2.369 \mathrm{E}-16$ & $2.369 \mathrm{E}-16$ & $2.369 \mathrm{E}-16$ \\
\hline cu 65 & $4.410 \mathrm{E}-07$ & 4.410E-07 & 4.410E-07 & $4.410 \mathrm{E}-07$ & $4.410 \mathrm{E}-07$ \\
\hline dy156 & 4.022E-12 & $4.022 \mathrm{E}-12$ & $4.022 \mathrm{E}-12$ & $4.022 \mathrm{E}-12$ & $4.022 \mathrm{E}-12$ \\
\hline dy158 & $1.497 \mathrm{E}-07$ & $5.017 \mathrm{E}-07$ & 5.204E-07 & $5.936 \mathrm{E}-07$ & $6.668 \mathrm{E}-07$ \\
\hline dy159 & $2.941 \mathrm{E}-07$ & $7.171 \mathrm{E}-11$ & $4.591 \mathrm{E}-11$ & $7.960 \mathrm{E}-12$ & $1.381 \mathrm{E}-12$ \\
\hline dy160 & $9.643 \mathrm{E}-01$ & $9.836 \mathrm{E}-01$ & $9.836 \mathrm{E}-01$ & $9.836 \mathrm{E}-01$ & $9.836 \mathrm{E}-01$ \\
\hline dy161 & $1.300 \mathrm{E}+00$ & $1.300 \mathrm{E}+00$ & $1.300 \mathrm{E}+00$ & $1.300 \mathrm{E}+00$ & $1.300 \mathrm{E}+00$ \\
\hline dy162 & 7.993E-01 & 7.993E-01 & 7.993E-01 & 7.993E-01 & 7.993E-01 \\
\hline dy163 & $6.661 \mathrm{E}-01$ & $6.661 \mathrm{E}-01$ & $6.661 \mathrm{E}-01$ & $6.661 \mathrm{E}-01$ & $6.661 \mathrm{E}-01$ \\
\hline dy164 & $2.700 \mathrm{E}-01$ & $2.700 \mathrm{E}-01$ & $2.700 \mathrm{E}-01$ & $2.700 \mathrm{E}-01$ & $2.700 \mathrm{E}-01$ \\
\hline er162 & $4.638 \mathrm{E}-12$ & $4.638 \mathrm{E}-12$ & $4.638 \mathrm{E}-12$ & $4.638 \mathrm{E}-12$ & $4.638 \mathrm{E}-12$ \\
\hline er164 & $3.235 \mathrm{E}-06$ & $3.235 \mathrm{E}-06$ & $3.235 \mathrm{E}-06$ & $3.235 \mathrm{E}-06$ & $3.235 \mathrm{E}-06$ \\
\hline er166 & $8.450 \mathrm{E}-02$ & $8.450 \mathrm{E}-02$ & $8.450 \mathrm{E}-02$ & $8.450 \mathrm{E}-02$ & $8.450 \mathrm{E}-02$ \\
\hline er167 & 4.233E-03 & $4.233 \mathrm{E}-03$ & $4.233 \mathrm{E}-03$ & 4.233E-03 & 4.233E-03 \\
\hline er168 & $1.332 \mathrm{E}-02$ & $1.332 \mathrm{E}-02$ & $1.332 \mathrm{E}-02$ & $1.332 \mathrm{E}-02$ & $1.332 \mathrm{E}-02$ \\
\hline er169 & $1.175 \mathrm{E}-13$ & $0.000 \mathrm{E}+00$ & $0.000 \mathrm{E}+00$ & $0.000 \mathrm{E}+00$ & $0.000 \mathrm{E}+00$ \\
\hline er170 & $6.573 \mathrm{E}-04$ & $6.573 \mathrm{E}-04$ & $6.573 \mathrm{E}-04$ & $6.573 \mathrm{E}-04$ & $6.573 \mathrm{E}-04$ \\
\hline es252 & $1.671 \mathrm{E}-15$ & $1.309 \mathrm{E}-16$ & $1.141 \mathrm{E}-16$ & $6.674 \mathrm{E}-17$ & $3.905 \mathrm{E}-17$ \\
\hline es 253 & $9.312 \mathrm{E}-13$ & $0.000 \mathrm{E}+00$ & $0.000 \mathrm{E}+00$ & $0.000 \mathrm{E}+00$ & $0.000 \mathrm{E}+00$ \\
\hline es254 & $5.963 \mathrm{E}-12$ & 7.642E-14 & $6.049 \mathrm{E}-14$ & $2.416 \mathrm{E}-14$ & $9.650 \mathrm{E}-15$ \\
\hline es 255 & $9.809 \mathrm{E}-15$ & $7.655 \mathrm{E}-28$ & $1.515 \mathrm{E}-28$ & $2.629 \mathrm{E}-31$ & $4.560 \mathrm{E}-34$ \\
\hline eu147 & $3.292 \mathrm{E}-18$ & $0.000 \mathrm{E}+00$ & $0.000 \mathrm{E}+00$ & $0.000 \mathrm{E}+00$ & $0.000 \mathrm{E}+00$ \\
\hline eu149 & $7.860 \mathrm{E}-11$ & $1.958 \mathrm{E}-16$ & $9.796 \mathrm{E}-17$ & $6.469 \mathrm{E}-18$ & $4.272 \mathrm{E}-19$ \\
\hline eu151 & $2.010 \mathrm{E}-01$ & $1.202 \mathrm{E}+00$ & $1.254 \mathrm{E}+00$ & $1.460 \mathrm{E}+00$ & $1.664 \mathrm{E}+00$ \\
\hline eu152 & 4.136E-02 & $3.244 \mathrm{E}-02$ & $3.202 \mathrm{E}-02$ & $3.042 \mathrm{E}-02$ & $2.890 \mathrm{E}-02$ \\
\hline eu153 & $2.179 \mathrm{E}+02$ & $2.179 \mathrm{E}+02$ & $2.179 \mathrm{E}+02$ & $2.179 \mathrm{E}+02$ & $2.179 \mathrm{E}+02$ \\
\hline eu154 & $6.265 \mathrm{E}+01$ & $4.274 \mathrm{E}+01$ & $4.187 \mathrm{E}+01$ & $3.863 \mathrm{E}+01$ & $3.564 \mathrm{E}+01$ \\
\hline eu155 & $1.521 \mathrm{E}+01$ & $7.615 \mathrm{E}+00$ & $7.337 \mathrm{E}+00$ & $6.341 \mathrm{E}+00$ & $5.481 \mathrm{E}+00$ \\
\hline eu156 & 4.252E-05 & $0.000 \mathrm{E}+00$ & $0.000 \mathrm{E}+00$ & $0.000 \mathrm{E}+00$ & $0.000 \mathrm{E}+00$ \\
\hline f 19 & $9.259 \mathrm{E}-14$ & $9.259 \mathrm{E}-14$ & $9.259 \mathrm{E}-14$ & $9.259 \mathrm{E}-14$ & $9.259 \mathrm{E}-14$ \\
\hline fe 54 & $9.749 \mathrm{E}-14$ & $9.749 \mathrm{E}-14$ & $9.749 \mathrm{E}-14$ & $9.749 \mathrm{E}-14$ & $9.749 \mathrm{E}-14$ \\
\hline fe 55 & $1.872 \mathrm{E}-16$ & $5.645 \mathrm{E}-17$ & $5.294 \mathrm{E}-17$ & 4.113E-17 & $3.195 \mathrm{E}-17$ \\
\hline fe 56 & $1.065 \mathrm{E}-13$ & $1.065 \mathrm{E}-13$ & $1.065 \mathrm{E}-13$ & $1.065 \mathrm{E}-13$ & $1.065 \mathrm{E}-13$ \\
\hline
\end{tabular}




\begin{tabular}{|c|c|c|c|c|c|}
\hline \multicolumn{6}{|c|}{ Table C-4. Continued } \\
\hline & $1 / 22 / 2009$ & $10 / 21 / 2013$ & $1 / 22 / 2014$ & $1 / 22 / 2015$ & $1 / 22 / 2016$ \\
\hline fe 57 & $1.033 \mathrm{E}-13$ & $1.033 \mathrm{E}-13$ & $1.033 \mathrm{E}-13$ & $1.033 \mathrm{E}-13$ & $1.033 \mathrm{E}-13$ \\
\hline fe 58 & $3.039 \mathrm{E}-13$ & $3.039 \mathrm{E}-13$ & $3.039 \mathrm{E}-13$ & $3.039 \mathrm{E}-13$ & $3.039 \mathrm{E}-13$ \\
\hline fr221 & $3.923 \mathrm{E}-16$ & $4.078 \mathrm{E}-16$ & $4.085 \mathrm{E}-16$ & $4.132 \mathrm{E}-16$ & $4.179 \mathrm{E}-16$ \\
\hline ga 69 & $1.682 \mathrm{E}-03$ & $1.682 \mathrm{E}-03$ & $1.682 \mathrm{E}-03$ & $1.682 \mathrm{E}-03$ & $1.682 \mathrm{E}-03$ \\
\hline ga 71 & $7.662 \mathrm{E}-03$ & $7.662 \mathrm{E}-03$ & 7.662E-03 & $7.662 \mathrm{E}-03$ & $7.662 \mathrm{E}-03$ \\
\hline gd151 & $9.153 \mathrm{E}-08$ & $5.682 \mathrm{E}-12$ & $3.378 \mathrm{E}-12$ & $4.392 \mathrm{E}-13$ & $5.708 \mathrm{E}-14$ \\
\hline $\operatorname{gd152}$ & $8.490 \mathrm{E}-02$ & $8.742 \mathrm{E}-02$ & $8.755 \mathrm{E}-02$ & $8.795 \mathrm{E}-02$ & $8.841 \mathrm{E}-02$ \\
\hline gd153 & $3.430 \mathrm{E}-03$ & $2.318 \mathrm{E}-05$ & $1.773 \mathrm{E}-05$ & $6.190 \mathrm{E}-06$ & $2.161 \mathrm{E}-06$ \\
\hline gd154 & $9.325 \mathrm{E}+00$ & $2.923 \mathrm{E}+01$ & $3.010 \mathrm{E}+01$ & $3.334 \mathrm{E}+01$ & $3.633 \mathrm{E}+01$ \\
\hline gd155 & $1.929 \mathrm{E}+00$ & $9.524 \mathrm{E}+00$ & $9.802 \mathrm{E}+00$ & $1.080 \mathrm{E}+01$ & $1.166 \mathrm{E}+01$ \\
\hline gd156 & $1.669 \mathrm{E}+02$ & $1.669 \mathrm{E}+02$ & $1.669 \mathrm{E}+02$ & $1.669 \mathrm{E}+02$ & $1.669 \mathrm{E}+02$ \\
\hline gd157 & $3.924 \mathrm{E}-01$ & $3.924 \mathrm{E}-01$ & $3.924 \mathrm{E}-01$ & $3.924 \mathrm{E}-01$ & $3.924 \mathrm{E}-01$ \\
\hline $\operatorname{gd158}$ & $4.942 \mathrm{E}+01$ & $4.942 \mathrm{E}+01$ & $4.942 \mathrm{E}+01$ & $4.942 \mathrm{E}+01$ & $4.942 \mathrm{E}+01$ \\
\hline gd160 & $3.421 \mathrm{E}+00$ & $3.421 \mathrm{E}+00$ & $3.421 \mathrm{E}+00$ & $3.421 \mathrm{E}+00$ & $3.421 \mathrm{E}+00$ \\
\hline ge 70 & $1.989 \mathrm{E}-05$ & $1.989 \mathrm{E}-05$ & $1.989 \mathrm{E}-05$ & $1.989 \mathrm{E}-05$ & $1.989 \mathrm{E}-05$ \\
\hline ge 71 & $4.906 \mathrm{E}-16$ & $0.000 \mathrm{E}+00$ & $0.000 \mathrm{E}+00$ & $0.000 \mathrm{E}+00$ & $0.000 \mathrm{E}+00$ \\
\hline ge 72 & $1.939 \mathrm{E}-02$ & $1.939 \mathrm{E}-02$ & $1.939 \mathrm{E}-02$ & $1.939 \mathrm{E}-02$ & $1.939 \mathrm{E}-02$ \\
\hline ge 73 & $3.980 \mathrm{E}-02$ & $3.980 \mathrm{E}-02$ & $3.980 \mathrm{E}-02$ & $3.980 \mathrm{E}-02$ & $3.980 \mathrm{E}-02$ \\
\hline ge $73 m$ & $1.123 \mathrm{E}-17$ & $3.580 \mathrm{E}-24$ & $1.604 \mathrm{E}-24$ & $6.866 \mathrm{E}-26$ & $2.941 \mathrm{E}-27$ \\
\hline ge 74 & $9.524 \mathrm{E}-02$ & $9.524 \mathrm{E}-02$ & $9.524 \mathrm{E}-02$ & $9.524 \mathrm{E}-02$ & $9.524 \mathrm{E}-02$ \\
\hline ge 76 & $4.278 \mathrm{E}-01$ & $4.278 \mathrm{E}-01$ & $4.278 \mathrm{E}-01$ & $4.278 \mathrm{E}-01$ & $4.278 \mathrm{E}-01$ \\
\hline h1 & $6.424 \mathrm{E}-03$ & $6.424 \mathrm{E}-03$ & $6.424 \mathrm{E}-03$ & $6.424 \mathrm{E}-03$ & $6.424 \mathrm{E}-03$ \\
\hline h2 & $1.373 \mathrm{E}-03$ & $1.373 \mathrm{E}-03$ & $1.373 \mathrm{E}-03$ & $1.373 \mathrm{E}-03$ & $1.373 \mathrm{E}-03$ \\
\hline h3 & $8.144 \mathrm{E}-02$ & $6.235 \mathrm{E}-02$ & $6.147 \mathrm{E}-02$ & $5.811 \mathrm{E}-02$ & $5.493 \mathrm{E}-02$ \\
\hline he3 & 4.987E-03 & $2.406 \mathrm{E}-02$ & $2.495 \mathrm{E}-02$ & $2.831 \mathrm{E}-02$ & $3.149 \mathrm{E}-02$ \\
\hline he4 & $8.179 \mathrm{E}+00$ & $9.337 \mathrm{E}+00$ & $9.382 \mathrm{E}+00$ & $9.557 \mathrm{E}+00$ & $9.733 \mathrm{E}+00$ \\
\hline hf174 & $1.462 \mathrm{E}-13$ & $1.462 \mathrm{E}-13$ & $1.462 \mathrm{E}-13$ & $1.462 \mathrm{E}-13$ & $1.462 \mathrm{E}-13$ \\
\hline hf175 & $9.696 \mathrm{E}-16$ & $3.419 \mathrm{E}-23$ & $1.361 \mathrm{E}-23$ & $3.667 \mathrm{E}-25$ & $9.875 \mathrm{E}-27$ \\
\hline hf176 & $3.295 \mathrm{E}-13$ & $3.295 \mathrm{E}-13$ & $3.295 \mathrm{E}-13$ & $3.295 \mathrm{E}-13$ & $3.295 \mathrm{E}-13$ \\
\hline hf177 & $5.304 \mathrm{E}-14$ & $5.305 \mathrm{E}-14$ & $5.305 \mathrm{E}-14$ & $5.305 \mathrm{E}-14$ & $5.305 \mathrm{E}-14$ \\
\hline hf178 & $3.390 \mathrm{E}-13$ & $3.390 \mathrm{E}-13$ & $3.390 \mathrm{E}-13$ & $3.390 \mathrm{E}-13$ & $3.390 \mathrm{E}-13$ \\
\hline hf179 & $7.602 \mathrm{E}-13$ & 7.602E-13 & 7.602E-13 & $7.602 \mathrm{E}-13$ & 7.602E-13 \\
\hline hf180 & $5.894 \mathrm{E}-13$ & $5.894 \mathrm{E}-13$ & $5.894 \mathrm{E}-13$ & $5.894 \mathrm{E}-13$ & $5.894 \mathrm{E}-13$ \\
\hline hf181 & $2.358 \mathrm{E}-17$ & $1.163 \mathrm{E}-29$ & $2.542 \mathrm{E}-30$ & $6.504 \mathrm{E}-33$ & $0.000 \mathrm{E}+00$ \\
\hline hf182 & $9.995 \mathrm{E}-16$ & $9.995 \mathrm{E}-16$ & $9.995 \mathrm{E}-16$ & $9.995 \mathrm{E}-16$ & $9.995 \mathrm{E}-16$ \\
\hline hg196 & $2.578 \mathrm{E}-14$ & $2.578 \mathrm{E}-14$ & $2.578 \mathrm{E}-14$ & $2.578 \mathrm{E}-14$ & $2.578 \mathrm{E}-14$ \\
\hline hg198 & $6.626 \mathrm{E}-13$ & $6.626 \mathrm{E}-13$ & $6.626 \mathrm{E}-13$ & $6.626 \mathrm{E}-13$ & $6.626 \mathrm{E}-13$ \\
\hline hg199 & $1.134 \mathrm{E}-13$ & $1.134 \mathrm{E}-13$ & $1.134 \mathrm{E}-13$ & $1.134 \mathrm{E}-13$ & $1.134 \mathrm{E}-13$ \\
\hline
\end{tabular}




\begin{tabular}{|c|c|c|c|c|c|}
\hline \multicolumn{6}{|c|}{ Table C-4. Continued } \\
\hline & $1 / 22 / 2009$ & $10 / 21 / 2013$ & $1 / 22 / 2014$ & $1 / 22 / 2015$ & $1 / 22 / 2016$ \\
\hline hg200 & $7.688 \mathrm{E}-13$ & $7.688 \mathrm{E}-13$ & $7.688 \mathrm{E}-13$ & $7.688 \mathrm{E}-13$ & $7.688 \mathrm{E}-13$ \\
\hline hg201 & $3.517 \mathrm{E}-13$ & $3.517 \mathrm{E}-13$ & $3.517 \mathrm{E}-13$ & $3.517 \mathrm{E}-13$ & $3.517 \mathrm{E}-13$ \\
\hline hg202 & $3.789 \mathrm{E}-13$ & $3.789 \mathrm{E}-13$ & $3.789 \mathrm{E}-13$ & $3.789 \mathrm{E}-13$ & $3.789 \mathrm{E}-13$ \\
\hline hg204 & $3.685 \mathrm{E}-13$ & $3.685 \mathrm{E}-13$ & $3.685 \mathrm{E}-13$ & $3.685 \mathrm{E}-13$ & $3.685 \mathrm{E}-13$ \\
\hline ho163 & $2.454 \mathrm{E}-07$ & $2.452 \mathrm{E}-07$ & $2.452 \mathrm{E}-07$ & $2.452 \mathrm{E}-07$ & $2.452 \mathrm{E}-07$ \\
\hline ho165 & $2.646 \mathrm{E}-01$ & $2.646 \mathrm{E}-01$ & $2.646 \mathrm{E}-01$ & $2.646 \mathrm{E}-01$ & $2.646 \mathrm{E}-01$ \\
\hline ho166m & $6.880 \mathrm{E}-04$ & $6.860 \mathrm{E}-04$ & $6.860 \mathrm{E}-04$ & $6.853 \mathrm{E}-04$ & $6.853 \mathrm{E}-04$ \\
\hline i125 & 1.172E-09 & $1.933 \mathrm{E}-18$ & $6.531 \mathrm{E}-19$ & $9.232 \mathrm{E}-21$ & $1.304 \mathrm{E}-22$ \\
\hline i126 & $2.017 \mathrm{E}-11$ & $0.000 \mathrm{E}+00$ & $0.000 \mathrm{E}+00$ & $0.000 \mathrm{E}+00$ & $0.000 \mathrm{E}+00$ \\
\hline i127 & $1.010 \mathrm{E}+02$ & $1.013 \mathrm{E}+02$ & $1.013 \mathrm{E}+02$ & $1.013 \mathrm{E}+02$ & $1.013 \mathrm{E}+02$ \\
\hline i129 & $3.182 \mathrm{E}+02$ & $3.182 \mathrm{E}+02$ & $3.182 \mathrm{E}+02$ & $3.182 \mathrm{E}+02$ & $3.182 \mathrm{E}+02$ \\
\hline i131 & $1.310 \mathrm{E}-09$ & $0.000 \mathrm{E}+00$ & $0.000 \mathrm{E}+00$ & $0.000 \mathrm{E}+00$ & $0.000 \mathrm{E}+00$ \\
\hline in113 & $9.484 \mathrm{E}-04$ & $2.109 \mathrm{E}-03$ & $2.164 \mathrm{E}-03$ & $2.373 \mathrm{E}-03$ & $2.572 \mathrm{E}-03$ \\
\hline in113m & $4.587 \mathrm{E}-14$ & $1.345 \mathrm{E}-18$ & 7.682E-19 & $8.523 \mathrm{E}-20$ & $9.464 \mathrm{E}-21$ \\
\hline in114 & 5.199E-12 & $1.510 \mathrm{E}-22$ & 4.107E-23 & $2.478 \mathrm{E}-25$ & $1.496 \mathrm{E}-27$ \\
\hline in114m & $3.197 \mathrm{E}-07$ & $9.285 \mathrm{E}-18$ & $2.525 \mathrm{E}-18$ & $1.524 \mathrm{E}-20$ & $9.199 \mathrm{E}-23$ \\
\hline in115 & $3.565 \mathrm{E}+00$ & $3.566 \mathrm{E}+00$ & $3.566 \mathrm{E}+00$ & $3.566 \mathrm{E}+00$ & $3.566 \mathrm{E}+00$ \\
\hline in115m & $3.180 \mathrm{E}-10$ & $6.235 \mathrm{E}-22$ & $1.467 \mathrm{E}-22$ & $5.021 \mathrm{E}-25$ & $1.717 \mathrm{E}-27$ \\
\hline ir191 & $1.074 \mathrm{E}-14$ & $1.074 \mathrm{E}-14$ & $1.074 \mathrm{E}-14$ & $1.074 \mathrm{E}-14$ & $1.074 \mathrm{E}-14$ \\
\hline ir192 & 2.303E-16 & $1.977 \mathrm{E}-23$ & $8.258 \mathrm{E}-24$ & $2.682 \mathrm{E}-25$ & $8.715 \mathrm{E}-27$ \\
\hline ir193 & $1.762 \mathrm{E}-13$ & $1.774 \mathrm{E}-13$ & $1.775 \mathrm{E}-13$ & $1.778 \mathrm{E}-13$ & $1.780 \mathrm{E}-13$ \\
\hline k 39 & 7.039E-14 & $7.039 \mathrm{E}-14$ & 7.039E-14 & $7.039 \mathrm{E}-14$ & 7.039E-14 \\
\hline k 40 & $6.979 \mathrm{E}-14$ & $6.979 \mathrm{E}-14$ & $6.979 \mathrm{E}-14$ & $6.979 \mathrm{E}-14$ & $6.979 \mathrm{E}-14$ \\
\hline k 41 & $7.675 \mathrm{E}-14$ & $7.675 \mathrm{E}-14$ & $7.675 \mathrm{E}-14$ & $7.675 \mathrm{E}-14$ & $7.675 \mathrm{E}-14$ \\
\hline kr 78 & $2.029 \mathrm{E}-13$ & $2.029 \mathrm{E}-13$ & $2.029 \mathrm{E}-13$ & $2.029 \mathrm{E}-13$ & $2.029 \mathrm{E}-13$ \\
\hline kr 80 & $5.535 \mathrm{E}-04$ & $5.535 \mathrm{E}-04$ & $5.535 \mathrm{E}-04$ & $5.535 \mathrm{E}-04$ & $5.535 \mathrm{E}-04$ \\
\hline kr 81 & $4.751 \mathrm{E}-05$ & $4.751 \mathrm{E}-05$ & $4.751 \mathrm{E}-05$ & $4.751 \mathrm{E}-05$ & $4.751 \mathrm{E}-05$ \\
\hline kr 82 & $1.023 \mathrm{E}+00$ & $1.023 \mathrm{E}+00$ & $1.023 \mathrm{E}+00$ & $1.023 \mathrm{E}+00$ & $1.023 \mathrm{E}+00$ \\
\hline kr 83 & $4.223 \mathrm{E}+01$ & $4.223 \mathrm{E}+01$ & $4.223 \mathrm{E}+01$ & $4.223 \mathrm{E}+01$ & $4.223 \mathrm{E}+01$ \\
\hline kr 83m & $4.775 \mathrm{E}-11$ & $4.236 \mathrm{E}-17$ & $2.005 \mathrm{E}-17$ & $1.065 \mathrm{E}-18$ & $5.660 \mathrm{E}-20$ \\
\hline kr 84 & $9.551 \mathrm{E}+01$ & $9.551 \mathrm{E}+01$ & $9.551 \mathrm{E}+01$ & $9.551 \mathrm{E}+01$ & $9.551 \mathrm{E}+01$ \\
\hline kr 85 & $1.976 \mathrm{E}+01$ & $1.456 \mathrm{E}+01$ & $1.432 \mathrm{E}+01$ & $1.343 \mathrm{E}+01$ & $1.259 \mathrm{E}+01$ \\
\hline kr 86 & $1.426 \mathrm{E}+02$ & $1.426 \mathrm{E}+02$ & $1.426 \mathrm{E}+02$ & $1.426 \mathrm{E}+02$ & $1.426 \mathrm{E}+02$ \\
\hline la137 & $5.518 \mathrm{E}-04$ & $5.518 \mathrm{E}-04$ & $5.518 \mathrm{E}-04$ & $5.518 \mathrm{E}-04$ & $5.518 \mathrm{E}-04$ \\
\hline la138 & $3.028 \mathrm{E}-02$ & $3.028 \mathrm{E}-02$ & $3.028 \mathrm{E}-02$ & $3.028 \mathrm{E}-02$ & $3.028 \mathrm{E}-02$ \\
\hline la139 & $1.599 \mathrm{E}+03$ & $1.599 \mathrm{E}+03$ & $1.599 \mathrm{E}+03$ & $1.599 \mathrm{E}+03$ & $1.599 \mathrm{E}+03$ \\
\hline la140 & $2.333 \mathrm{E}-06$ & $0.000 \mathrm{E}+00$ & $0.000 \mathrm{E}+00$ & $0.000 \mathrm{E}+00$ & $0.000 \mathrm{E}+00$ \\
\hline li6 & $2.552 \mathrm{E}-10$ & $2.552 \mathrm{E}-10$ & $2.552 \mathrm{E}-10$ & $2.552 \mathrm{E}-10$ & $2.552 \mathrm{E}-10$ \\
\hline
\end{tabular}




\begin{tabular}{|c|c|c|c|c|c|}
\hline \multicolumn{6}{|c|}{ Table C-4. Continued } \\
\hline & $1 / 22 / 2009$ & $10 / 21 / 2013$ & $1 / 22 / 2014$ & $1 / 22 / 2015$ & $1 / 22 / 2016$ \\
\hline li7 & $2.439 \mathrm{E}-11$ & $2.439 \mathrm{E}-11$ & $2.439 \mathrm{E}-11$ & $2.439 \mathrm{E}-11$ & $2.439 \mathrm{E}-11$ \\
\hline lu175 & $2.977 \mathrm{E}-13$ & $2.987 \mathrm{E}-13$ & $2.987 \mathrm{E}-13$ & $2.987 \mathrm{E}-13$ & $2.987 \mathrm{E}-13$ \\
\hline lu176 & $7.251 \mathrm{E}-15$ & $7.251 \mathrm{E}-15$ & $7.251 \mathrm{E}-15$ & $7.251 \mathrm{E}-15$ & $7.251 \mathrm{E}-15$ \\
\hline mg 24 & $4.354 \mathrm{E}-14$ & 4.354E-14 & 4.354E-14 & $4.354 \mathrm{E}-14$ & $4.354 \mathrm{E}-14$ \\
\hline mg 25 & $4.531 \mathrm{E}-14$ & $4.531 \mathrm{E}-14$ & $4.531 \mathrm{E}-14$ & $4.531 \mathrm{E}-14$ & $4.531 \mathrm{E}-14$ \\
\hline mg 26 & $4.715 \mathrm{E}-14$ & $4.715 \mathrm{E}-14$ & 4.715E-14 & $4.715 \mathrm{E}-14$ & $4.715 \mathrm{E}-14$ \\
\hline mn 54 & $2.254 \mathrm{E}-17$ & 4.799E-19 & $3.904 \mathrm{E}-19$ & $1.735 \mathrm{E}-19$ & $7.715 \mathrm{E}-20$ \\
\hline mn 55 & $9.763 \mathrm{E}-14$ & $9.776 \mathrm{E}-14$ & $9.776 \mathrm{E}-14$ & $9.776 \mathrm{E}-14$ & $9.776 \mathrm{E}-14$ \\
\hline mo 92 & $6.793 \mathrm{E}-12$ & $6.793 \mathrm{E}-12$ & $6.793 \mathrm{E}-12$ & $6.793 \mathrm{E}-12$ & $6.793 \mathrm{E}-12$ \\
\hline mo 93 & 4.749E-07 & 4.745E-07 & 4.745E-07 & $4.745 \mathrm{E}-07$ & 4.743E-07 \\
\hline mo 94 & $1.581 \mathrm{E}-02$ & $1.581 \mathrm{E}-02$ & $1.581 \mathrm{E}-02$ & $1.581 \mathrm{E}-02$ & $1.581 \mathrm{E}-02$ \\
\hline mo 95 & $8.629 \mathrm{E}+02$ & $8.722 \mathrm{E}+02$ & $8.722 \mathrm{E}+02$ & $8.722 \mathrm{E}+02$ & $8.722 \mathrm{E}+02$ \\
\hline mo 96 & $4.751 \mathrm{E}+01$ & $4.751 \mathrm{E}+01$ & $4.751 \mathrm{E}+01$ & $4.751 \mathrm{E}+01$ & $4.751 \mathrm{E}+01$ \\
\hline mo 97 & $1.047 \mathrm{E}+03$ & $1.047 \mathrm{E}+03$ & $1.047 \mathrm{E}+03$ & $1.047 \mathrm{E}+03$ & $1.047 \mathrm{E}+03$ \\
\hline mo 98 & $1.146 \mathrm{E}+03$ & $1.146 \mathrm{E}+03$ & $1.146 \mathrm{E}+03$ & $1.146 \mathrm{E}+03$ & $1.146 \mathrm{E}+03$ \\
\hline mo100 & $1.351 \mathrm{E}+03$ & $1.351 \mathrm{E}+03$ & $1.351 \mathrm{E}+03$ & $1.351 \mathrm{E}+03$ & $1.351 \mathrm{E}+03$ \\
\hline n 14 & 4.707E-06 & $4.921 \mathrm{E}-06$ & 4.933E-06 & $4.978 \mathrm{E}-06$ & $5.023 \mathrm{E}-06$ \\
\hline n 15 & $1.131 \mathrm{E}-02$ & $1.131 \mathrm{E}-02$ & $1.131 \mathrm{E}-02$ & $1.131 \mathrm{E}-02$ & $1.131 \mathrm{E}-02$ \\
\hline na 23 & 4.167E-14 & 4.167E-14 & 4.167E-14 & 4.167E-14 & 4.167E-14 \\
\hline nb 91 & $1.153 \mathrm{E}-08$ & $1.148 \mathrm{E}-08$ & $1.147 \mathrm{E}-08$ & $1.146 \mathrm{E}-08$ & $1.145 \mathrm{E}-08$ \\
\hline nb 91m & $7.569 \mathrm{E}-15$ & $2.027 \mathrm{E}-23$ & 7.032E-24 & $1.100 \mathrm{E}-25$ & $1.723 \mathrm{E}-27$ \\
\hline nb 92 & $1.002 \mathrm{E}-06$ & $1.002 \mathrm{E}-06$ & $1.002 \mathrm{E}-06$ & $1.002 \mathrm{E}-06$ & $1.002 \mathrm{E}-06$ \\
\hline nb 92m & $1.896 \mathrm{E}-18$ & $0.000 \mathrm{E}+00$ & $0.000 \mathrm{E}+00$ & $0.000 \mathrm{E}+00$ & $0.000 \mathrm{E}+00$ \\
\hline nb 93 & $1.180 \mathrm{E}-04$ & 4.252E-04 & 4.486E-04 & $5.464 \mathrm{E}-04$ & $6.537 \mathrm{E}-04$ \\
\hline nb 93m & $6.800 \mathrm{E}-04$ & $1.915 \mathrm{E}-03$ & $1.974 \mathrm{E}-03$ & $2.201 \mathrm{E}-03$ & $2.418 \mathrm{E}-03$ \\
\hline nb 94 & $3.605 \mathrm{E}-03$ & $3.605 \mathrm{E}-03$ & $3.605 \mathrm{E}-03$ & $3.605 \mathrm{E}-03$ & $3.605 \mathrm{E}-03$ \\
\hline nb 95 & $4.982 \mathrm{E}+00$ & $3.750 \mathrm{E}-08$ & $1.368 \mathrm{E}-08$ & $2.713 \mathrm{E}-10$ & $5.223 \mathrm{E}-12$ \\
\hline nb 95m & $2.813 \mathrm{E}-03$ & $2.004 \mathrm{E}-11$ & $7.324 \mathrm{E}-12$ & $1.408 \mathrm{E}-13$ & $2.709 \mathrm{E}-15$ \\
\hline nd142 & $2.157 \mathrm{E}+01$ & $2.157 \mathrm{E}+01$ & $2.157 \mathrm{E}+01$ & $2.157 \mathrm{E}+01$ & $2.157 \mathrm{E}+01$ \\
\hline nd143 & $1.066 \mathrm{E}+03$ & $1.066 \mathrm{E}+03$ & $1.066 \mathrm{E}+03$ & $1.066 \mathrm{E}+03$ & $1.066 \mathrm{E}+03$ \\
\hline nd144 & $1.161 \mathrm{E}+03$ & $1.368 \mathrm{E}+03$ & $1.369 \mathrm{E}+03$ & $1.370 \mathrm{E}+03$ & $1.371 \mathrm{E}+03$ \\
\hline nd145 & $8.020 \mathrm{E}+02$ & $8.020 \mathrm{E}+02$ & $8.020 \mathrm{E}+02$ & $8.020 \mathrm{E}+02$ & $8.020 \mathrm{E}+02$ \\
\hline nd146 & $8.762 \mathrm{E}+02$ & $8.762 \mathrm{E}+02$ & $8.762 \mathrm{E}+02$ & $8.762 \mathrm{E}+02$ & $8.762 \mathrm{E}+02$ \\
\hline nd147 & $5.259 \mathrm{E}-07$ & $0.000 \mathrm{E}+00$ & $0.000 \mathrm{E}+00$ & $0.000 \mathrm{E}+00$ & $0.000 \mathrm{E}+00$ \\
\hline nd148 & $5.164 \mathrm{E}+02$ & $5.164 \mathrm{E}+02$ & $5.164 \mathrm{E}+02$ & $5.164 \mathrm{E}+02$ & $5.164 \mathrm{E}+02$ \\
\hline nd150 & $3.046 \mathrm{E}+02$ & $3.046 \mathrm{E}+02$ & $3.046 \mathrm{E}+02$ & $3.046 \mathrm{E}+02$ & $3.046 \mathrm{E}+02$ \\
\hline ni 58 & $1.044 \mathrm{E}-13$ & $1.044 \mathrm{E}-13$ & $1.044 \mathrm{E}-13$ & $1.044 \mathrm{E}-13$ & $1.044 \mathrm{E}-13$ \\
\hline ni 59 & $8.470 \mathrm{E}-14$ & $8.470 \mathrm{E}-14$ & $8.470 \mathrm{E}-14$ & $8.470 \mathrm{E}-14$ & $8.470 \mathrm{E}-14$ \\
\hline
\end{tabular}




\begin{tabular}{|c|c|c|c|c|c|}
\hline \multicolumn{6}{|c|}{ Table C-4. Continued } \\
\hline & $1 / 22 / 2009$ & $10 / 21 / 2013$ & $1 / 22 / 2014$ & $1 / 22 / 2015$ & $1 / 22 / 2016$ \\
\hline ni 60 & $1.309 \mathrm{E}-13$ & $1.355 \mathrm{E}-13$ & $1.357 \mathrm{E}-13$ & $1.363 \mathrm{E}-13$ & $1.369 \mathrm{E}-13$ \\
\hline ni 61 & $1.106 \mathrm{E}-13$ & $1.106 \mathrm{E}-13$ & $1.106 \mathrm{E}-13$ & $1.106 \mathrm{E}-13$ & $1.106 \mathrm{E}-13$ \\
\hline ni 62 & $1.109 \mathrm{E}-13$ & $1.109 \mathrm{E}-13$ & $1.109 \mathrm{E}-13$ & $1.109 \mathrm{E}-13$ & $1.109 \mathrm{E}-13$ \\
\hline ni 63 & $1.862 \mathrm{E}-15$ & $1.802 \mathrm{E}-15$ & $1.799 \mathrm{E}-15$ & $1.787 \mathrm{E}-15$ & $1.774 \mathrm{E}-15$ \\
\hline ni 64 & $1.165 \mathrm{E}-13$ & $1.165 \mathrm{E}-13$ & $1.165 \mathrm{E}-13$ & $1.165 \mathrm{E}-13$ & $1.165 \mathrm{E}-13$ \\
\hline np235 & $2.251 \mathrm{E}-06$ & $1.085 \mathrm{E}-07$ & $9.219 \mathrm{E}-08$ & $4.869 \mathrm{E}-08$ & $2.572 \mathrm{E}-08$ \\
\hline np236 & $3.541 \mathrm{E}-04$ & $3.541 \mathrm{E}-04$ & $3.541 \mathrm{E}-04$ & $3.541 \mathrm{E}-04$ & $3.541 \mathrm{E}-04$ \\
\hline np237 & $1.813 \mathrm{E}+02$ & $1.886 \mathrm{E}+02$ & $1.892 \mathrm{E}+02$ & $1.918 \mathrm{E}+02$ & $1.947 \mathrm{E}+02$ \\
\hline np238 & 8.066E-07 & $7.880 \mathrm{E}-07$ & $7.874 \mathrm{E}-07$ & $7.834 \mathrm{E}-07$ & $7.794 \mathrm{E}-07$ \\
\hline np239 & $3.336 \mathrm{E}-04$ & $3.334 \mathrm{E}-04$ & $3.334 \mathrm{E}-04$ & $3.334 \mathrm{E}-04$ & $3.334 \mathrm{E}-04$ \\
\hline np240 & $1.921 \mathrm{E}-16$ & $1.921 \mathrm{E}-16$ & $1.921 \mathrm{E}-16$ & $1.921 \mathrm{E}-16$ & $1.921 \mathrm{E}-16$ \\
\hline np240m & $1.868 \mathrm{E}-14$ & $1.868 \mathrm{E}-14$ & $1.868 \mathrm{E}-14$ & $1.868 \mathrm{E}-14$ & $1.868 \mathrm{E}-14$ \\
\hline о 16 & $1.343 \mathrm{E}+05$ & $1.343 \mathrm{E}+05$ & $1.343 \mathrm{E}+05$ & $1.343 \mathrm{E}+05$ & $1.343 \mathrm{E}+05$ \\
\hline о 17 & $3.187 \mathrm{E}-02$ & $3.187 \mathrm{E}-02$ & $3.187 \mathrm{E}-02$ & $3.187 \mathrm{E}-02$ & $3.187 \mathrm{E}-02$ \\
\hline о 18 & $1.241 \mathrm{E}-07$ & $1.241 \mathrm{E}-07$ & $1.241 \mathrm{E}-07$ & $1.241 \mathrm{E}-07$ & $1.241 \mathrm{E}-07$ \\
\hline os186 & $2.122 \mathrm{E}-13$ & $2.122 \mathrm{E}-13$ & $2.122 \mathrm{E}-13$ & $2.122 \mathrm{E}-13$ & $2.122 \mathrm{E}-13$ \\
\hline os187 & $3.350 \mathrm{E}-14$ & $3.350 \mathrm{E}-14$ & $3.350 \mathrm{E}-14$ & $3.350 \mathrm{E}-14$ & $3.350 \mathrm{E}-14$ \\
\hline os188 & $1.323 \mathrm{E}-13$ & $1.323 \mathrm{E}-13$ & $1.323 \mathrm{E}-13$ & $1.323 \mathrm{E}-13$ & $1.323 \mathrm{E}-13$ \\
\hline os189 & $8.616 \mathrm{E}-15$ & $8.616 \mathrm{E}-15$ & $8.616 \mathrm{E}-15$ & $8.616 \mathrm{E}-15$ & $8.616 \mathrm{E}-15$ \\
\hline os190 & $2.234 \mathrm{E}-15$ & $2.234 \mathrm{E}-15$ & $2.234 \mathrm{E}-15$ & $2.234 \mathrm{E}-15$ & $2.234 \mathrm{E}-15$ \\
\hline os192 & $9.140 \mathrm{E}-15$ & $9.153 \mathrm{E}-15$ & $9.153 \mathrm{E}-15$ & $9.153 \mathrm{E}-15$ & $9.153 \mathrm{E}-15$ \\
\hline p 31 & $5.623 \mathrm{E}-14$ & $5.623 \mathrm{E}-14$ & $5.623 \mathrm{E}-14$ & $5.623 \mathrm{E}-14$ & $5.623 \mathrm{E}-14$ \\
\hline pa230 & $1.110 \mathrm{E}-15$ & $0.000 \mathrm{E}+00$ & $0.000 \mathrm{E}+00$ & $0.000 \mathrm{E}+00$ & $0.000 \mathrm{E}+00$ \\
\hline pa231 & $2.487 \mathrm{E}-05$ & $2.933 \mathrm{E}-05$ & $2.958 \mathrm{E}-05$ & $3.051 \mathrm{E}-05$ & $3.146 \mathrm{E}-05$ \\
\hline pa232 & $1.645 \mathrm{E}-14$ & $1.644 \mathrm{E}-14$ & $1.644 \mathrm{E}-14$ & $1.644 \mathrm{E}-14$ & $1.644 \mathrm{E}-14$ \\
\hline pa233 & $6.136 \mathrm{E}-06$ & $6.379 \mathrm{E}-06$ & $6.399 \mathrm{E}-06$ & $6.495 \mathrm{E}-06$ & $6.593 \mathrm{E}-06$ \\
\hline pa234 & $2.466 \mathrm{E}-10$ & $2.468 \mathrm{E}-10$ & $2.466 \mathrm{E}-10$ & $2.466 \mathrm{E}-10$ & $2.466 \mathrm{E}-10$ \\
\hline pa234m & 4.444E-10 & 4.446E-10 & 4.444E-10 & $4.444 \mathrm{E}-10$ & $4.444 \mathrm{E}-10$ \\
\hline pb204 & $1.689 \mathrm{E}-17$ & $4.698 \mathrm{E}-17$ & 4.797E-17 & $5.144 \mathrm{E}-17$ & $5.433 \mathrm{E}-17$ \\
\hline pb204 & $3.686 \mathrm{E}-13$ & $3.686 \mathrm{E}-13$ & $3.686 \mathrm{E}-13$ & $3.686 \mathrm{E}-13$ & $3.686 \mathrm{E}-13$ \\
\hline pb205 & $1.196 \mathrm{E}-15$ & $1.197 \mathrm{E}-15$ & $1.197 \mathrm{E}-15$ & $1.197 \mathrm{E}-15$ & $1.198 \mathrm{E}-15$ \\
\hline pb206 & $1.905 \mathrm{E}-12$ & $9.358 \mathrm{E}-12$ & $9.776 \mathrm{E}-12$ & $1.144 \mathrm{E}-11$ & $1.319 \mathrm{E}-11$ \\
\hline pb207 & $6.426 \mathrm{E}-11$ & $3.250 \mathrm{E}-10$ & $3.482 \mathrm{E}-10$ & 4.491E-10 & $5.651 \mathrm{E}-10$ \\
\hline pb208 & $8.556 \mathrm{E}-07$ & $1.976 \mathrm{E}-05$ & 2.152E-05 & $2.896 \mathrm{E}-05$ & $3.721 \mathrm{E}-05$ \\
\hline pb209 & $1.478 \mathrm{E}-14$ & $1.536 \mathrm{E}-14$ & $1.538 \mathrm{E}-14$ & $1.556 \mathrm{E}-14$ & $1.574 \mathrm{E}-14$ \\
\hline pb210 & $5.270 \mathrm{E}-11$ & $5.370 \mathrm{E}-11$ & $5.424 \mathrm{E}-11$ & $5.701 \mathrm{E}-11$ & $6.096 \mathrm{E}-11$ \\
\hline pb211 & $2.005 \mathrm{E}-15$ & $9.166 \mathrm{E}-15$ & $9.358 \mathrm{E}-15$ & $1.105 \mathrm{E}-14$ & $1.257 \mathrm{E}-14$ \\
\hline pb212 & $1.918 \mathrm{E}-09$ & $1.209 \mathrm{E}-08$ & $1.254 \mathrm{E}-08$ & $1.407 \mathrm{E}-08$ & $1.536 \mathrm{E}-08$ \\
\hline
\end{tabular}




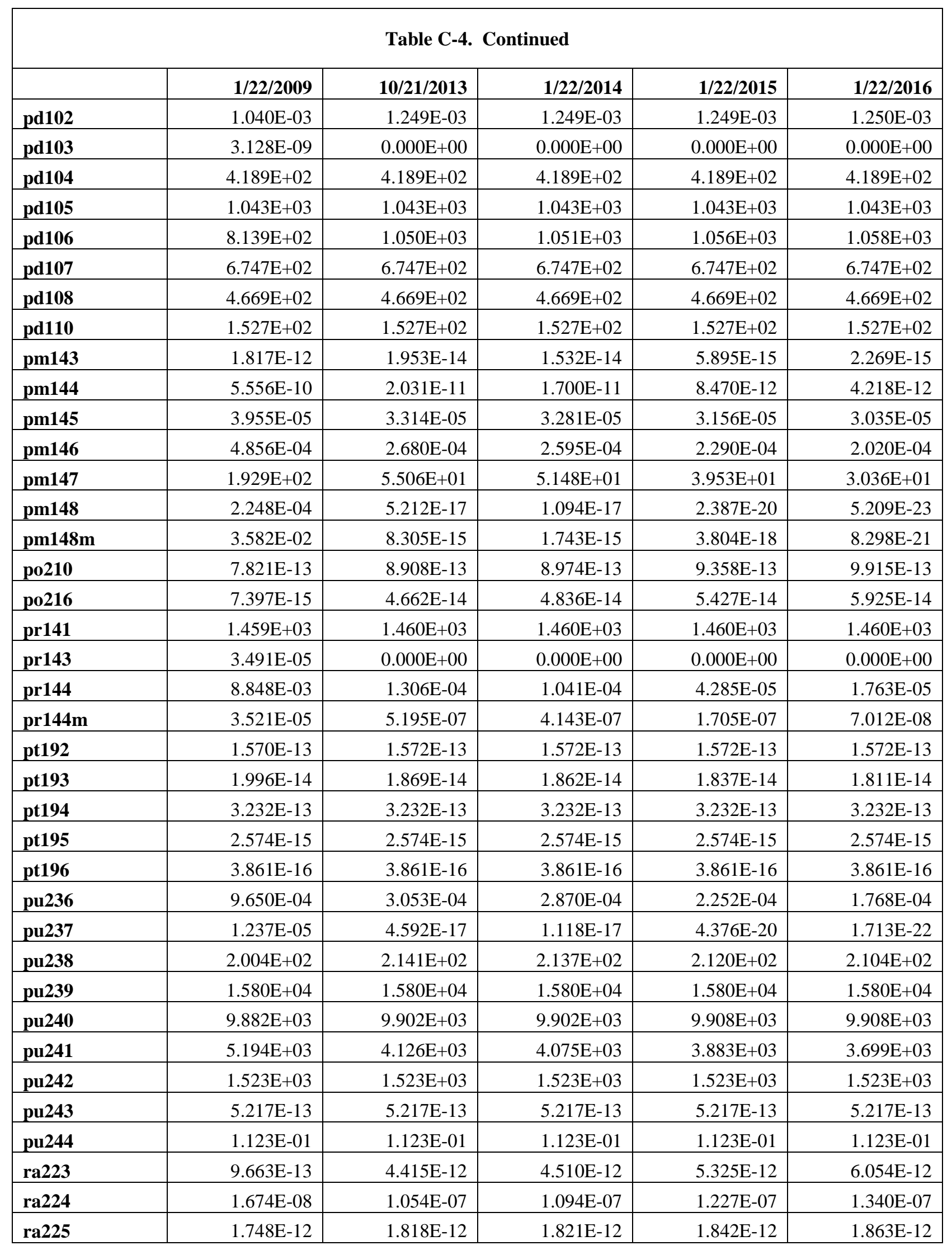




\begin{tabular}{|c|c|c|c|c|c|}
\hline \multicolumn{6}{|c|}{ Table C-4. Continued } \\
\hline & $1 / 22 / 2009$ & $10 / 21 / 2013$ & $1 / 22 / 2014$ & $1 / 22 / 2015$ & $1 / 22 / 2016$ \\
\hline ra226 & $1.464 \mathrm{E}-09$ & $9.093 \mathrm{E}-09$ & $9.769 \mathrm{E}-09$ & $1.272 \mathrm{E}-08$ & $1.620 \mathrm{E}-08$ \\
\hline ra228 & $3.209 \mathrm{E}-14$ & $5.711 \mathrm{E}-14$ & 5.833E-14 & $6.302 \mathrm{E}-14$ & $6.760 \mathrm{E}-14$ \\
\hline rb 83 & 7.264E-08 & $6.442 \mathrm{E}-14$ & $3.049 \mathrm{E}-14$ & $1.620 \mathrm{E}-15$ & 8.609E-17 \\
\hline rb 84 & 7.615E-08 & $9.690 \mathrm{E}-24$ & $1.359 \mathrm{E}-24$ & $6.103 \mathrm{E}-28$ & $2.739 \mathrm{E}-31$ \\
\hline rb 85 & $8.033 \mathrm{E}+01$ & $8.550 \mathrm{E}+01$ & $8.576 \mathrm{E}+01$ & $8.662 \mathrm{E}+01$ & $8.749 \mathrm{E}+01$ \\
\hline rb 86 & $1.428 \mathrm{E}-06$ & $1.424 \mathrm{E}-34$ & $0.000 \mathrm{E}+00$ & $0.000 \mathrm{E}+00$ & $0.000 \mathrm{E}+00$ \\
\hline rb 87 & $1.871 \mathrm{E}+02$ & $1.871 \mathrm{E}+02$ & $1.871 \mathrm{E}+02$ & $1.871 \mathrm{E}+02$ & $1.871 \mathrm{E}+02$ \\
\hline re185 & $6.727 \mathrm{E}-14$ & $6.740 \mathrm{E}-14$ & $6.740 \mathrm{E}-14$ & $6.740 \mathrm{E}-14$ & $6.740 \mathrm{E}-14$ \\
\hline re186m & $1.996 \mathrm{E}-16$ & $1.996 \mathrm{E}-16$ & $1.996 \mathrm{E}-16$ & $1.996 \mathrm{E}-16$ & $1.996 \mathrm{E}-16$ \\
\hline re187 & $3.550 \mathrm{E}-13$ & $3.550 \mathrm{E}-13$ & $3.550 \mathrm{E}-13$ & $3.550 \mathrm{E}-13$ & $3.550 \mathrm{E}-13$ \\
\hline rh101 & $6.366 \mathrm{E}-07$ & $2.350 \mathrm{E}-07$ & $2.228 \mathrm{E}-07$ & $1.805 \mathrm{E}-07$ & $1.464 \mathrm{E}-07$ \\
\hline rh102 & $9.504 \mathrm{E}-04$ & $3.279 \mathrm{E}-06$ & $2.489 \mathrm{E}-06$ & $9.332 \mathrm{E}-07$ & 4.409E-07 \\
\hline rh102m & $2.250 \mathrm{E}-03$ & $9.345 \mathrm{E}-04$ & $8.914 \mathrm{E}-04$ & $7.410 \mathrm{E}-04$ & $6.156 \mathrm{E}-04$ \\
\hline rh103 & $1.002 \mathrm{E}+03$ & $1.003 \mathrm{E}+03$ & $1.003 \mathrm{E}+03$ & $1.003 \mathrm{E}+03$ & $1.003 \mathrm{E}+03$ \\
\hline rh103m & $6.840 \mathrm{E}-04$ & $3.490 \mathrm{E}-17$ & $6.754 \mathrm{E}-18$ & $1.071 \mathrm{E}-20$ & $1.699 \mathrm{E}-23$ \\
\hline rh106 & $2.304 \mathrm{E}-04$ & $9.107 \mathrm{E}-06$ & $7.655 \mathrm{E}-06$ & $3.876 \mathrm{E}-06$ & $1.962 \mathrm{E}-06$ \\
\hline rn220 & $2.890 \mathrm{E}-12$ & $1.821 \mathrm{E}-11$ & $1.889 \mathrm{E}-11$ & $2.120 \mathrm{E}-11$ & $2.314 \mathrm{E}-11$ \\
\hline rn222 & 9.332E-15 & $5.846 \mathrm{E}-14$ & $6.278 \mathrm{E}-14$ & $8.179 \mathrm{E}-14$ & $1.041 \mathrm{E}-13$ \\
\hline ru 96 & $1.726 \mathrm{E}-13$ & $1.726 \mathrm{E}-13$ & $1.726 \mathrm{E}-13$ & $1.726 \mathrm{E}-13$ & $1.726 \mathrm{E}-13$ \\
\hline ru 98 & $1.926 \mathrm{E}-06$ & $1.935 \mathrm{E}-06$ & $1.936 \mathrm{E}-06$ & $1.938 \mathrm{E}-06$ & $1.940 \mathrm{E}-06$ \\
\hline ru 99 & $4.561 \mathrm{E}-02$ & $6.240 \mathrm{E}-02$ & $6.330 \mathrm{E}-02$ & $6.681 \mathrm{E}-02$ & 7.039E-02 \\
\hline ru100 & $1.696 \mathrm{E}+02$ & $1.696 \mathrm{E}+02$ & $1.696 \mathrm{E}+02$ & $1.696 \mathrm{E}+02$ & $1.696 \mathrm{E}+02$ \\
\hline ru101 & $1.168 \mathrm{E}+03$ & $1.168 \mathrm{E}+03$ & $1.168 \mathrm{E}+03$ & $1.168 \mathrm{E}+03$ & $1.168 \mathrm{E}+03$ \\
\hline ru102 & $1.347 \mathrm{E}+03$ & $1.347 \mathrm{E}+03$ & $1.347 \mathrm{E}+03$ & $1.347 \mathrm{E}+03$ & $1.347 \mathrm{E}+03$ \\
\hline ru103 & $6.966 \mathrm{E}-01$ & $3.553 \mathrm{E}-14$ & $6.873 \mathrm{E}-15$ & $1.090 \mathrm{E}-17$ & $1.730 \mathrm{E}-20$ \\
\hline ru104 & $1.275 \mathrm{E}+03$ & $1.275 \mathrm{E}+03$ & $1.275 \mathrm{E}+03$ & $1.275 \mathrm{E}+03$ & $1.275 \mathrm{E}+03$ \\
\hline ru106 & $2.461 \mathrm{E}+02$ & $9.723 \mathrm{E}+00$ & $8.179 \mathrm{E}+00$ & $4.140 \mathrm{E}+00$ & $2.096 \mathrm{E}+00$ \\
\hline s 32 & 5.794E-14 & $5.794 \mathrm{E}-14$ & $5.794 \mathrm{E}-14$ & $5.794 \mathrm{E}-14$ & $5.794 \mathrm{E}-14$ \\
\hline s 33 & 5.954E-14 & $5.954 \mathrm{E}-14$ & $5.954 \mathrm{E}-14$ & $5.954 \mathrm{E}-14$ & $5.954 \mathrm{E}-14$ \\
\hline s 34 & $6.168 \mathrm{E}-14$ & $6.168 \mathrm{E}-14$ & $6.168 \mathrm{E}-14$ & $6.168 \mathrm{E}-14$ & $6.168 \mathrm{E}-14$ \\
\hline s 36 & $6.525 \mathrm{E}-14$ & $6.525 \mathrm{E}-14$ & $6.525 \mathrm{E}-14$ & $6.525 \mathrm{E}-14$ & $6.525 \mathrm{E}-14$ \\
\hline sb121 & $8.894 \mathrm{E}+00$ & $8.954 \mathrm{E}+00$ & $8.954 \mathrm{E}+00$ & $8.967 \mathrm{E}+00$ & $8.981 \mathrm{E}+00$ \\
\hline sb123 & $1.172 \mathrm{E}+01$ & $1.181 \mathrm{E}+01$ & $1.181 \mathrm{E}+01$ & $1.181 \mathrm{E}+01$ & $1.181 \mathrm{E}+01$ \\
\hline sb124 & $4.124 \mathrm{E}-03$ & $8.901 \mathrm{E}-12$ & $3.051 \mathrm{E}-12$ & $4.563 \mathrm{E}-14$ & $6.827 \mathrm{E}-16$ \\
\hline sb125 & $1.558 \mathrm{E}+01$ & $4.728 \mathrm{E}+00$ & $4.435 \mathrm{E}+00$ & $3.450 \mathrm{E}+00$ & $2.684 \mathrm{E}+00$ \\
\hline sb126 & $9.630 \mathrm{E}-07$ & $9.577 \mathrm{E}-07$ & $9.577 \mathrm{E}-07$ & $9.577 \mathrm{E}-07$ & $9.577 \mathrm{E}-07$ \\
\hline sb126m & $7.370 \mathrm{E}-09$ & 7.370E-09 & 7.370E-09 & $7.370 \mathrm{E}-09$ & $7.370 \mathrm{E}-09$ \\
\hline sc 45 & 7.907E-14 & 7.907E-14 & 7.907E-14 & $7.907 \mathrm{E}-14$ & 7.907E-14 \\
\hline
\end{tabular}




\begin{tabular}{|c|c|c|c|c|c|}
\hline \multicolumn{6}{|c|}{ Table C-4. Continued } \\
\hline & $1 / 22 / 2009$ & $10 / 21 / 2013$ & $1 / 22 / 2014$ & $1 / 22 / 2015$ & $1 / 22 / 2016$ \\
\hline sc 46 & $4.065 \mathrm{E}-17$ & $2.415 \mathrm{E}-23$ & $1.119 \mathrm{E}-23$ & $5.464 \mathrm{E}-25$ & $2.668 \mathrm{E}-26$ \\
\hline se 74 & $4.360 \mathrm{E}-07$ & $4.360 \mathrm{E}-07$ & $4.360 \mathrm{E}-07$ & $4.360 \mathrm{E}-07$ & $4.360 \mathrm{E}-07$ \\
\hline se 75 & $9.471 \mathrm{E}-09$ & $4.183 \mathrm{E}-13$ & $2.442 \mathrm{E}-13$ & $2.955 \mathrm{E}-14$ & $3.575 \mathrm{E}-15$ \\
\hline se 76 & $8.868 \mathrm{E}-03$ & $8.868 \mathrm{E}-03$ & $8.868 \mathrm{E}-03$ & $8.868 \mathrm{E}-03$ & $8.868 \mathrm{E}-03$ \\
\hline se 77 & $1.005 \mathrm{E}+00$ & $1.005 \mathrm{E}+00$ & $1.005 \mathrm{E}+00$ & $1.005 \mathrm{E}+00$ & $1.005 \mathrm{E}+00$ \\
\hline se 78 & $2.857 \mathrm{E}+00$ & $2.857 \mathrm{E}+00$ & $2.857 \mathrm{E}+00$ & $2.857 \mathrm{E}+00$ & $2.857 \mathrm{E}+00$ \\
\hline se 79 & $6.143 \mathrm{E}+00$ & $6.143 \mathrm{E}+00$ & $6.143 \mathrm{E}+00$ & $6.143 \mathrm{E}+00$ & $6.143 \mathrm{E}+00$ \\
\hline se 80 & $1.404 \mathrm{E}+01$ & $1.404 \mathrm{E}+01$ & $1.404 \mathrm{E}+01$ & $1.404 \mathrm{E}+01$ & $1.404 \mathrm{E}+01$ \\
\hline se 82 & $3.663 \mathrm{E}+01$ & $3.663 \mathrm{E}+01$ & $3.663 \mathrm{E}+01$ & $3.663 \mathrm{E}+01$ & $3.663 \mathrm{E}+01$ \\
\hline si 28 & $5.077 \mathrm{E}-14$ & $5.077 \mathrm{E}-14$ & $5.077 \mathrm{E}-14$ & $5.077 \mathrm{E}-14$ & $5.077 \mathrm{E}-14$ \\
\hline si 29 & $5.264 \mathrm{E}-14$ & $5.264 \mathrm{E}-14$ & $5.264 \mathrm{E}-14$ & $5.264 \mathrm{E}-14$ & $5.264 \mathrm{E}-14$ \\
\hline si 30 & $5.469 \mathrm{E}-14$ & $5.469 \mathrm{E}-14$ & $5.469 \mathrm{E}-14$ & $5.469 \mathrm{E}-14$ & $5.469 \mathrm{E}-14$ \\
\hline sm144 & $6.476 \mathrm{E}-11$ & $6.476 \mathrm{E}-11$ & $6.476 \mathrm{E}-11$ & $6.476 \mathrm{E}-11$ & $6.476 \mathrm{E}-11$ \\
\hline sm145 & $3.547 \mathrm{E}-07$ & $1.037 \mathrm{E}-08$ & $8.570 \mathrm{E}-09$ & 4.073E-09 & $1.935 \mathrm{E}-09$ \\
\hline sm146 & $3.670 \mathrm{E}-03$ & $3.745 \mathrm{E}-03$ & $3.747 \mathrm{E}-03$ & $3.758 \mathrm{E}-03$ & $3.767 \mathrm{E}-03$ \\
\hline sm147 & $1.279 \mathrm{E}+02$ & $2.657 \mathrm{E}+02$ & $2.693 \mathrm{E}+02$ & $2.812 \mathrm{E}+02$ & $2.904 \mathrm{E}+02$ \\
\hline sm148 & $2.082 \mathrm{E}+02$ & $2.082 \mathrm{E}+02$ & $2.082 \mathrm{E}+02$ & $2.082 \mathrm{E}+02$ & $2.082 \mathrm{E}+02$ \\
\hline sm149 & $6.576 \mathrm{E}+00$ & $6.576 \mathrm{E}+00$ & $6.576 \mathrm{E}+00$ & $6.576 \mathrm{E}+00$ & $6.576 \mathrm{E}+00$ \\
\hline sm150 & $4.358 \mathrm{E}+02$ & $4.358 \mathrm{E}+02$ & $4.358 \mathrm{E}+02$ & $4.358 \mathrm{E}+02$ & $4.358 \mathrm{E}+02$ \\
\hline sm151 & $2.788 \mathrm{E}+01$ & $2.688 \mathrm{E}+01$ & $2.683 \mathrm{E}+01$ & $2.662 \mathrm{E}+01$ & $2.642 \mathrm{E}+01$ \\
\hline sm152 & $1.681 \mathrm{E}+02$ & $1.681 \mathrm{E}+02$ & $1.681 \mathrm{E}+02$ & $1.681 \mathrm{E}+02$ & $1.681 \mathrm{E}+02$ \\
\hline sm154 & $8.662 \mathrm{E}+01$ & $8.662 \mathrm{E}+01$ & $8.662 \mathrm{E}+01$ & $8.662 \mathrm{E}+01$ & $8.662 \mathrm{E}+01$ \\
\hline sn112 & $3.639 \mathrm{E}-09$ & 3.639E-09 & $3.639 \mathrm{E}-09$ & $3.639 \mathrm{E}-09$ & 3.639E-09 \\
\hline sn113 & $7.635 \mathrm{E}-11$ & $2.240 \mathrm{E}-15$ & $1.279 \mathrm{E}-15$ & $1.420 \mathrm{E}-16$ & $1.575 \mathrm{E}-17$ \\
\hline sn114 & $1.858 \mathrm{E}-04$ & $1.861 \mathrm{E}-04$ & $1.861 \mathrm{E}-04$ & $1.861 \mathrm{E}-04$ & $1.861 \mathrm{E}-04$ \\
\hline sn115 & $5.304 \mathrm{E}-01$ & $5.304 \mathrm{E}-01$ & $5.304 \mathrm{E}-01$ & $5.304 \mathrm{E}-01$ & $5.304 \mathrm{E}-01$ \\
\hline sn116 & $4.348 \mathrm{E}+00$ & $4.348 \mathrm{E}+00$ & $4.348 \mathrm{E}+00$ & $4.348 \mathrm{E}+00$ & $4.348 \mathrm{E}+00$ \\
\hline sn117 & $1.085 \mathrm{E}+01$ & $1.085 \mathrm{E}+01$ & $1.085 \mathrm{E}+01$ & $1.085 \mathrm{E}+01$ & $1.085 \mathrm{E}+01$ \\
\hline sn117m & $2.908 \mathrm{E}-09$ & $0.000 \mathrm{E}+00$ & $0.000 \mathrm{E}+00$ & $0.000 \mathrm{E}+00$ & $0.000 \mathrm{E}+00$ \\
\hline sn118 & $9.405 \mathrm{E}+00$ & $9.405 \mathrm{E}+00$ & $9.405 \mathrm{E}+00$ & $9.405 \mathrm{E}+00$ & $9.405 \mathrm{E}+00$ \\
\hline sn119 & $8.974 \mathrm{E}+00$ & $9.040 \mathrm{E}+00$ & $9.040 \mathrm{E}+00$ & $9.040 \mathrm{E}+00$ & $9.040 \mathrm{E}+00$ \\
\hline sn119m & $7.065 \mathrm{E}-02$ & $1.171 \mathrm{E}-03$ & $9.398 \mathrm{E}-04$ & $3.962 \mathrm{E}-04$ & $1.671 \mathrm{E}-04$ \\
\hline sn120 & $9.040 \mathrm{E}+00$ & $9.040 \mathrm{E}+00$ & $9.040 \mathrm{E}+00$ & $9.040 \mathrm{E}+00$ & $9.040 \mathrm{E}+00$ \\
\hline sn121 & 4.422E-05 & $4.103 \mathrm{E}-05$ & $4.087 \mathrm{E}-05$ & 4.022E-05 & $3.959 \mathrm{E}-05$ \\
\hline sn121m & $8.112 \mathrm{E}-01$ & $7.529 \mathrm{E}-01$ & $7.496 \mathrm{E}-01$ & 7.377E-01 & 7.264E-01 \\
\hline sn122 & $1.213 \mathrm{E}+01$ & $1.213 \mathrm{E}+01$ & $1.213 \mathrm{E}+01$ & $1.213 \mathrm{E}+01$ & $1.213 \mathrm{E}+01$ \\
\hline sn123 & $8.583 \mathrm{E}-02$ & 7.867E-06 & 4.777E-06 & $6.740 \mathrm{E}-07$ & $9.511 \mathrm{E}-08$ \\
\hline sn124 & $1.966 \mathrm{E}+01$ & $1.966 \mathrm{E}+01$ & $1.966 \mathrm{E}+01$ & $1.966 \mathrm{E}+01$ & $1.966 \mathrm{E}+01$ \\
\hline
\end{tabular}




\begin{tabular}{|c|c|c|c|c|c|}
\hline \multicolumn{6}{|c|}{ Table C-4. Continued } \\
\hline & $1 / 22 / 2009$ & $10 / 21 / 2013$ & $1 / 22 / 2014$ & $1 / 22 / 2015$ & $1 / 22 / 2016$ \\
\hline sn125 & $8.218 \mathrm{E}-10$ & $0.000 \mathrm{E}+00$ & $0.000 \mathrm{E}+00$ & $0.000 \mathrm{E}+00$ & $0.000 \mathrm{E}+00$ \\
\hline sn126 & 4.654E+01 & $4.654 \mathrm{E}+01$ & $4.654 \mathrm{E}+01$ & $4.654 \mathrm{E}+01$ & 4.654E+01 \\
\hline sr 84 & $8.822 \mathrm{E}-06$ & $8.822 \mathrm{E}-06$ & $8.822 \mathrm{E}-06$ & $8.822 \mathrm{E}-06$ & $8.822 \mathrm{E}-06$ \\
\hline sr 85 & $1.571 \mathrm{E}-08$ & $1.414 \mathrm{E}-16$ & $5.231 \mathrm{E}-17$ & $1.056 \mathrm{E}-18$ & $2.135 \mathrm{E}-20$ \\
\hline sr 86 & $5.128 \mathrm{E}-01$ & $5.128 \mathrm{E}-01$ & $5.128 \mathrm{E}-01$ & $5.128 \mathrm{E}-01$ & $5.128 \mathrm{E}-01$ \\
\hline sr 87 & $5.741 \mathrm{E}-03$ & $5.741 \mathrm{E}-03$ & $5.741 \mathrm{E}-03$ & $5.741 \mathrm{E}-03$ & $5.741 \mathrm{E}-03$ \\
\hline sr 88 & $2.487 \mathrm{E}+02$ & $2.487 \mathrm{E}+02$ & $2.487 \mathrm{E}+02$ & $2.487 \mathrm{E}+02$ & $2.487 \mathrm{E}+02$ \\
\hline sr 89 & $5.670 \mathrm{E}-01$ & $2.687 \mathrm{E}-11$ & $7.503 \mathrm{E}-12$ & $5.020 \mathrm{E}-14$ & $3.359 \mathrm{E}-16$ \\
\hline sr 90 & $3.820 \mathrm{E}+02$ & $3.408 \mathrm{E}+02$ & $3.387 \mathrm{E}+02$ & $3.307 \mathrm{E}+02$ & $3.228 \mathrm{E}+02$ \\
\hline ta180m & $2.015 \mathrm{E}-17$ & $2.015 \mathrm{E}-17$ & $2.015 \mathrm{E}-17$ & $2.015 \mathrm{E}-17$ & $2.015 \mathrm{E}-17$ \\
\hline ta181 & $1.815 \mathrm{E}-13$ & $1.815 \mathrm{E}-13$ & $1.815 \mathrm{E}-13$ & $1.815 \mathrm{E}-13$ & $1.815 \mathrm{E}-13$ \\
\hline ta182 & $7.019 \mathrm{E}-16$ & $1.996 \mathrm{E}-20$ & $1.139 \mathrm{E}-20$ & $1.288 \mathrm{E}-21$ & $1.734 \mathrm{E}-22$ \\
\hline tb157 & $9.113 \mathrm{E}-07$ & $8.702 \mathrm{E}-07$ & 8.682E-07 & $8.596 \mathrm{E}-07$ & $8.510 \mathrm{E}-07$ \\
\hline tb158 & $1.171 \mathrm{E}-04$ & $1.150 \mathrm{E}-04$ & $1.149 \mathrm{E}-04$ & $1.145 \mathrm{E}-04$ & $1.140 \mathrm{E}-04$ \\
\hline tb159 & $7.489 \mathrm{E}+00$ & $7.489 \mathrm{E}+00$ & $7.489 \mathrm{E}+00$ & $7.489 \mathrm{E}+00$ & $7.489 \mathrm{E}+00$ \\
\hline tb160 & $1.879 \mathrm{E}-02$ & $1.144 \mathrm{E}-09$ & $4.689 \mathrm{E}-10$ & $1.417 \mathrm{E}-11$ & $4.282 \mathrm{E}-13$ \\
\hline tb161 & 7.914E-14 & $0.000 \mathrm{E}+00$ & $0.000 \mathrm{E}+00$ & $0.000 \mathrm{E}+00$ & $0.000 \mathrm{E}+00$ \\
\hline tc 95 & $6.188 \mathrm{E}-18$ & $1.734 \mathrm{E}-26$ & $6.029 \mathrm{E}-27$ & $9.531 \mathrm{E}-29$ & $0.000 \mathrm{E}+00$ \\
\hline tc $95 \mathrm{~m}$ & $1.151 \mathrm{E}-14$ & $3.228 \mathrm{E}-23$ & $1.122 \mathrm{E}-23$ & $1.773 \mathrm{E}-25$ & $2.802 \mathrm{E}-27$ \\
\hline tc 97 & $1.145 \mathrm{E}-05$ & $1.155 \mathrm{E}-05$ & $1.155 \mathrm{E}-05$ & $1.155 \mathrm{E}-05$ & $1.155 \mathrm{E}-05$ \\
\hline tc $97 \mathrm{~m}$ & $1.043 \mathrm{E}-07$ & $1.929 \mathrm{E}-13$ & $9.498 \mathrm{E}-14$ & $5.892 \mathrm{E}-15$ & $3.655 \mathrm{E}-16$ \\
\hline tc 98 & $1.180 \mathrm{E}-02$ & $1.180 \mathrm{E}-02$ & $1.180 \mathrm{E}-02$ & $1.180 \mathrm{E}-02$ & $1.180 \mathrm{E}-02$ \\
\hline tc 99 & $1.078 \mathrm{E}+03$ & $1.078 \mathrm{E}+03$ & $1.078 \mathrm{E}+03$ & $1.078 \mathrm{E}+03$ & $1.078 \mathrm{E}+03$ \\
\hline te120 & $2.598 \mathrm{E}-09$ & $2.598 \mathrm{E}-09$ & $2.598 \mathrm{E}-09$ & $2.598 \mathrm{E}-09$ & $2.598 \mathrm{E}-09$ \\
\hline te121 & $3.472 \mathrm{E}-08$ & $2.309 \mathrm{E}-11$ & $1.559 \mathrm{E}-11$ & $3.340 \mathrm{E}-12$ & $7.151 \mathrm{E}-13$ \\
\hline te121m & $2.964 \mathrm{E}-07$ & $1.971 \mathrm{E}-10$ & $1.332 \mathrm{E}-10$ & $2.851 \mathrm{E}-11$ & $6.108 \mathrm{E}-12$ \\
\hline te122 & 7.894E-01 & 7.894E-01 & 7.894E-01 & 7.894E-01 & 7.894E-01 \\
\hline te123 & $9.670 \mathrm{E}-03$ & $1.012 \mathrm{E}-02$ & $1.012 \mathrm{E}-02$ & $1.012 \mathrm{E}-02$ & $1.012 \mathrm{E}-02$ \\
\hline te123m & 4.543E-04 & $1.909 \mathrm{E}-08$ & $1.111 \mathrm{E}-08$ & $1.331 \mathrm{E}-09$ & $1.593 \mathrm{E}-10$ \\
\hline te124 & $5.658 \mathrm{E}-01$ & $5.700 \mathrm{E}-01$ & $5.700 \mathrm{E}-01$ & $5.700 \mathrm{E}-01$ & $5.700 \mathrm{E}-01$ \\
\hline te125 & $1.084 \mathrm{E}+01$ & $2.185 \mathrm{E}+01$ & $2.214 \mathrm{E}+01$ & $2.314 \mathrm{E}+01$ & $2.391 \mathrm{E}+01$ \\
\hline te125m & $2.163 \mathrm{E}-01$ & $6.596 \mathrm{E}-02$ & $6.187 \mathrm{E}-02$ & 4.813E-02 & $3.745 \mathrm{E}-02$ \\
\hline te126 & $1.127 \mathrm{E}+00$ & $1.127 \mathrm{E}+00$ & $1.127 \mathrm{E}+00$ & $1.127 \mathrm{E}+00$ & $1.127 \mathrm{E}+00$ \\
\hline te127 & $1.045 \mathrm{E}-03$ & $1.709 \mathrm{E}-08$ & $9.464 \mathrm{E}-09$ & $9.292 \mathrm{E}-10$ & $9.120 \mathrm{E}-11$ \\
\hline te127m & $2.984 \mathrm{E}-01$ & $4.883 \mathrm{E}-06$ & $2.703 \mathrm{E}-06$ & $2.653 \mathrm{E}-07$ & $2.605 \mathrm{E}-08$ \\
\hline te128 & $1.797 \mathrm{E}+02$ & $1.797 \mathrm{E}+02$ & $1.797 \mathrm{E}+02$ & $1.797 \mathrm{E}+02$ & $1.797 \mathrm{E}+02$ \\
\hline te129 & $7.960 \mathrm{E}-06$ & $2.367 \mathrm{E}-21$ & $3.476 \mathrm{E}-22$ & $1.866 \mathrm{E}-25$ & $1.002 \mathrm{E}-28$ \\
\hline te129m & $8.768 \mathrm{E}-03$ & $2.609 \mathrm{E}-18$ & $3.830 \mathrm{E}-19$ & $2.056 \mathrm{E}-22$ & $1.104 \mathrm{E}-25$ \\
\hline
\end{tabular}




\begin{tabular}{|c|c|c|c|c|c|}
\hline \multicolumn{6}{|c|}{ Table C-4. Continued } \\
\hline & $1 / 22 / 2009$ & $10 / 21 / 2013$ & $1 / 22 / 2014$ & $1 / 22 / 2015$ & $1 / 22 / 2016$ \\
\hline te130 & $5.991 \mathrm{E}+02$ & $5.991 \mathrm{E}+02$ & $5.991 \mathrm{E}+02$ & $5.991 \mathrm{E}+02$ & $5.991 \mathrm{E}+02$ \\
\hline th227 & $1.622 \mathrm{E}-12$ & $7.244 \mathrm{E}-12$ & $7.450 \mathrm{E}-12$ & $8.735 \mathrm{E}-12$ & $9.935 \mathrm{E}-12$ \\
\hline th228 & $3.295 \mathrm{E}-06$ & $2.041 \mathrm{E}-05$ & $2.114 \mathrm{E}-05$ & $2.376 \mathrm{E}-05$ & $2.593 \mathrm{E}-05$ \\
\hline th229 & $3.201 \mathrm{E}-07$ & $3.329 \mathrm{E}-07$ & $3.338 \mathrm{E}-07$ & 3.373E-07 & $3.411 \mathrm{E}-07$ \\
\hline th230 & $8.782 \mathrm{E}-05$ & $2.861 \mathrm{E}-04$ & $2.998 \mathrm{E}-04$ & $3.560 \mathrm{E}-04$ & $4.169 \mathrm{E}-04$ \\
\hline th231 & $3.920 \mathrm{E}-09$ & 3.929E-09 & 3.929E-09 & 3.931E-09 & 3.933E-09 \\
\hline th232 & $2.003 \mathrm{E}-04$ & $2.411 \mathrm{E}-04$ & $2.434 \mathrm{E}-04$ & $2.521 \mathrm{E}-04$ & $2.608 \mathrm{E}-04$ \\
\hline th234 & $1.331 \mathrm{E}-05$ & $1.331 \mathrm{E}-05$ & $1.331 \mathrm{E}-05$ & $1.331 \mathrm{E}-05$ & $1.331 \mathrm{E}-05$ \\
\hline ti 46 & $8.590 \mathrm{E}-14$ & $8.596 \mathrm{E}-14$ & $8.596 \mathrm{E}-14$ & $8.596 \mathrm{E}-14$ & $8.596 \mathrm{E}-14$ \\
\hline ti 47 & $8.510 \mathrm{E}-14$ & $8.510 \mathrm{E}-14$ & $8.510 \mathrm{E}-14$ & $8.510 \mathrm{E}-14$ & $8.510 \mathrm{E}-14$ \\
\hline ti 48 & $8.649 \mathrm{E}-14$ & $8.649 \mathrm{E}-14$ & 8.649E-14 & $8.649 \mathrm{E}-14$ & $8.649 \mathrm{E}-14$ \\
\hline ti 49 & $8.961 \mathrm{E}-14$ & 8.961E-14 & $8.961 \mathrm{E}-14$ & $8.961 \mathrm{E}-14$ & $8.961 \mathrm{E}-14$ \\
\hline ti 50 & $9.087 \mathrm{E}-14$ & $9.087 \mathrm{E}-14$ & $9.087 \mathrm{E}-14$ & 9.087E-14 & $9.087 \mathrm{E}-14$ \\
\hline tl203 & $2.776 \mathrm{E}-15$ & $2.781 \mathrm{E}-15$ & $2.781 \mathrm{E}-15$ & $2.781 \mathrm{E}-15$ & $2.781 \mathrm{E}-15$ \\
\hline tl204 & $5.336 \mathrm{E}-17$ & $2.237 \mathrm{E}-17$ & $2.135 \mathrm{E}-17$ & $1.778 \mathrm{E}-17$ & $1.481 \mathrm{E}-17$ \\
\hline tl205 & $1.325 \mathrm{E}-15$ & $1.325 \mathrm{E}-15$ & $1.325 \mathrm{E}-15$ & $1.325 \mathrm{E}-15$ & $1.325 \mathrm{E}-15$ \\
\hline tl207 & $2.591 \mathrm{E}-16$ & $1.184 \mathrm{E}-15$ & $1.210 \mathrm{E}-15$ & $1.428 \mathrm{E}-15$ & $1.624 \mathrm{E}-15$ \\
\hline t1208 & $3.235 \mathrm{E}-12$ & $2.039 \mathrm{E}-11$ & $2.115 \mathrm{E}-11$ & $2.373 \mathrm{E}-11$ & $2.590 \mathrm{E}-11$ \\
\hline tm168 & $1.055 \mathrm{E}-09$ & $2.630 \mathrm{E}-15$ & $1.316 \mathrm{E}-15$ & $8.689 \mathrm{E}-17$ & $5.738 \mathrm{E}-18$ \\
\hline $\operatorname{tm} 169$ & $1.017 \mathrm{E}-03$ & $1.017 \mathrm{E}-03$ & $1.017 \mathrm{E}-03$ & $1.017 \mathrm{E}-03$ & $1.017 \mathrm{E}-03$ \\
\hline $\operatorname{tm} 170$ & $5.542 \mathrm{E}-05$ & 4.864E-09 & $2.947 \mathrm{E}-09$ & $4.120 \mathrm{E}-10$ & $5.761 \mathrm{E}-11$ \\
\hline $\operatorname{tm171}$ & $1.748 \mathrm{E}-04$ & $3.152 \mathrm{E}-05$ & $2.876 \mathrm{E}-05$ & $2.005 \mathrm{E}-05$ & $1.398 \mathrm{E}-05$ \\
\hline $\mathbf{u 2 3 0}$ & $2.977 \mathrm{E}-15$ & $0.000 \mathrm{E}+00$ & $0.000 \mathrm{E}+00$ & $0.000 \mathrm{E}+00$ & $0.000 \mathrm{E}+00$ \\
\hline $\mathbf{u} 232$ & $4.383 \mathrm{E}-04$ & $1.049 \mathrm{E}-03$ & $1.064 \mathrm{E}-03$ & $1.113 \mathrm{E}-03$ & $1.149 \mathrm{E}-03$ \\
\hline $\mathbf{u} 233$ & $4.875 \mathrm{E}-04$ & $7.841 \mathrm{E}-04$ & $7.993 \mathrm{E}-04$ & 8.662E-04 & $9.338 \mathrm{E}-04$ \\
\hline $\mathbf{u} 234$ & $1.111 \mathrm{E}+01$ & $1.904 \mathrm{E}+01$ & $1.947 \mathrm{E}+01$ & $2.112 \mathrm{E}+01$ & $2.276 \mathrm{E}+01$ \\
\hline $\mathbf{u} 235$ & $9.643 \mathrm{E}+02$ & $9.663 \mathrm{E}+02$ & $9.663 \mathrm{E}+02$ & $9.670 \mathrm{E}+02$ & $9.670 \mathrm{E}+02$ \\
\hline u236 & $2.935 \mathrm{E}+02$ & $2.984 \mathrm{E}+02$ & $2.986 \mathrm{E}+02$ & $2.997 \mathrm{E}+02$ & $3.007 \mathrm{E}+02$ \\
\hline $\mathbf{u 2 3 7}$ & $1.620 \mathrm{E}-04$ & $1.287 \mathrm{E}-04$ & $1.272 \mathrm{E}-04$ & $1.212 \mathrm{E}-04$ & $1.154 \mathrm{E}-04$ \\
\hline $\mathbf{u} 238$ & $9.166 \mathrm{E}+05$ & $9.166 \mathrm{E}+05$ & $9.166 \mathrm{E}+05$ & $9.166 \mathrm{E}+05$ & $9.166 \mathrm{E}+05$ \\
\hline $\mathbf{u 2 4 0}$ & $2.188 \mathrm{E}-12$ & $2.188 \mathrm{E}-12$ & $2.188 \mathrm{E}-12$ & $2.188 \mathrm{E}-12$ & $2.188 \mathrm{E}-12$ \\
\hline v 50 & $5.799 \mathrm{E}-17$ & $5.799 \mathrm{E}-17$ & $5.799 \mathrm{E}-17$ & $5.799 \mathrm{E}-17$ & $5.799 \mathrm{E}-17$ \\
\hline v 51 & $1.786 \mathrm{E}-15$ & $1.786 \mathrm{E}-15$ & $1.786 \mathrm{E}-15$ & $1.786 \mathrm{E}-15$ & $1.786 \mathrm{E}-15$ \\
\hline w182 & $2.812 \mathrm{E}-13$ & $2.819 \mathrm{E}-13$ & $2.819 \mathrm{E}-13$ & $2.819 \mathrm{E}-13$ & $2.819 \mathrm{E}-13$ \\
\hline w183 & $6.473 \mathrm{E}-13$ & $6.473 \mathrm{E}-13$ & $6.473 \mathrm{E}-13$ & $6.473 \mathrm{E}-13$ & $6.473 \mathrm{E}-13$ \\
\hline w184 & $5.571 \mathrm{E}-13$ & $5.571 \mathrm{E}-13$ & $5.571 \mathrm{E}-13$ & $5.571 \mathrm{E}-13$ & $5.571 \mathrm{E}-13$ \\
\hline w185 & $1.169 \mathrm{E}-16$ & $1.322 \mathrm{E}-23$ & $5.602 \mathrm{E}-24$ & $1.929 \mathrm{E}-25$ & $6.641 \mathrm{E}-27$ \\
\hline w186 & $2.135 \mathrm{E}-13$ & $2.135 \mathrm{E}-13$ & $2.135 \mathrm{E}-13$ & $2.135 \mathrm{E}-13$ & $2.135 \mathrm{E}-13$ \\
\hline
\end{tabular}




\begin{tabular}{|c|c|c|c|c|c|}
\hline \multicolumn{6}{|c|}{ Table C-4. Continued } \\
\hline & $1 / 22 / 2009$ & $10 / 21 / 2013$ & $1 / 22 / 2014$ & $1 / 22 / 2015$ & $1 / 22 / 2016$ \\
\hline xe124 & $2.367 \mathrm{E}-13$ & $2.367 \mathrm{E}-13$ & $2.367 \mathrm{E}-13$ & $2.367 \mathrm{E}-13$ & $2.367 \mathrm{E}-13$ \\
\hline xe126 & $3.735 \mathrm{E}-04$ & $3.735 \mathrm{E}-04$ & $3.735 \mathrm{E}-04$ & $3.735 \mathrm{E}-04$ & $3.735 \mathrm{E}-04$ \\
\hline xe127 & $5.583 \mathrm{E}-08$ & $2.599 \mathrm{E}-22$ & $4.422 \mathrm{E}-23$ & $4.237 \mathrm{E}-26$ & $4.059 \mathrm{E}-29$ \\
\hline xe128 & $8.477 \mathrm{E}+00$ & $8.477 \mathrm{E}+00$ & $8.477 \mathrm{E}+00$ & $8.477 \mathrm{E}+00$ & $8.477 \mathrm{E}+00$ \\
\hline xe129 & $5.492 \mathrm{E}-02$ & $5.498 \mathrm{E}-02$ & $5.498 \mathrm{E}-02$ & $5.500 \mathrm{E}-02$ & $5.501 \mathrm{E}-02$ \\
\hline xe129m & $2.841 \mathrm{E}-13$ & $0.000 \mathrm{E}+00$ & $0.000 \mathrm{E}+00$ & $0.000 \mathrm{E}+00$ & $0.000 \mathrm{E}+00$ \\
\hline xe130 & $1.093 \mathrm{E}+01$ & $1.093 \mathrm{E}+01$ & $1.093 \mathrm{E}+01$ & $1.093 \mathrm{E}+01$ & $1.093 \mathrm{E}+01$ \\
\hline xe131 & $6.853 \mathrm{E}+02$ & $6.853 \mathrm{E}+02$ & $6.853 \mathrm{E}+02$ & $6.853 \mathrm{E}+02$ & $6.853 \mathrm{E}+02$ \\
\hline xe131m & $1.047 \mathrm{E}-07$ & $0.000 \mathrm{E}+00$ & $0.000 \mathrm{E}+00$ & $0.000 \mathrm{E}+00$ & $0.000 \mathrm{E}+00$ \\
\hline xe132 & $1.709 \mathrm{E}+03$ & $1.709 \mathrm{E}+03$ & $1.709 \mathrm{E}+03$ & $1.709 \mathrm{E}+03$ & $1.709 \mathrm{E}+03$ \\
\hline xe133 & $1.066 \mathrm{E}-14$ & $0.000 \mathrm{E}+00$ & $0.000 \mathrm{E}+00$ & $0.000 \mathrm{E}+00$ & $0.000 \mathrm{E}+00$ \\
\hline xe134 & $2.092 \mathrm{E}+03$ & $2.092 \mathrm{E}+03$ & $2.092 \mathrm{E}+03$ & $2.092 \mathrm{E}+03$ & $2.092 \mathrm{E}+03$ \\
\hline xe136 & $3.112 \mathrm{E}+03$ & $3.112 \mathrm{E}+03$ & $3.112 \mathrm{E}+03$ & $3.112 \mathrm{E}+03$ & $3.112 \mathrm{E}+03$ \\
\hline y 88 & $2.979 \mathrm{E}-05$ & $3.816 \mathrm{E}-10$ & $2.084 \mathrm{E}-10$ & $1.943 \mathrm{E}-11$ & $1.812 \mathrm{E}-12$ \\
\hline y 89 & $3.271 \mathrm{E}+02$ & $3.277 \mathrm{E}+02$ & $3.277 \mathrm{E}+02$ & $3.277 \mathrm{E}+02$ & $3.277 \mathrm{E}+02$ \\
\hline y 89m & $1.961 \mathrm{E}-10$ & $9.292 \mathrm{E}-21$ & $2.595 \mathrm{E}-21$ & $1.736 \mathrm{E}-23$ & $1.162 \mathrm{E}-25$ \\
\hline y 90 & $9.690 \mathrm{E}-02$ & 8.643E-02 & $8.590 \mathrm{E}-02$ & 8.391E-02 & $8.185 \mathrm{E}-02$ \\
\hline y 91 & $1.589 \mathrm{E}+00$ & $1.927 \mathrm{E}-09$ & $6.401 \mathrm{E}-10$ & $8.477 \mathrm{E}-12$ & $1.123 \mathrm{E}-13$ \\
\hline yb168 & $2.158 \mathrm{E}-12$ & $2.263 \mathrm{E}-12$ & $2.263 \mathrm{E}-12$ & $2.263 \mathrm{E}-12$ & 2.263E-12 \\
\hline yb169 & $3.487 \mathrm{E}-12$ & $1.774 \mathrm{E}-28$ & $2.368 \mathrm{E}-29$ & $8.762 \mathrm{E}-33$ & $0.000 \mathrm{E}+00$ \\
\hline yb170 & $6.999 \mathrm{E}-04$ & $7.556 \mathrm{E}-04$ & $7.556 \mathrm{E}-04$ & $7.556 \mathrm{E}-04$ & $7.556 \mathrm{E}-04$ \\
\hline yb171 & $2.157 \mathrm{E}-04$ & $3.590 \mathrm{E}-04$ & $3.618 \mathrm{E}-04$ & 3.705E-04 & $3.766 \mathrm{E}-04$ \\
\hline yb172 & 4.384E-04 & 4.384E-04 & 4.384E-04 & 4.384E-04 & 4.384E-04 \\
\hline yb176 & $1.145 \mathrm{E}-16$ & $1.145 \mathrm{E}-16$ & $1.145 \mathrm{E}-16$ & $1.145 \mathrm{E}-16$ & $1.145 \mathrm{E}-16$ \\
\hline zn 64 & 4.177E-16 & 4.177E-16 & 4.177E-16 & 4.177E-16 & 4.177E-16 \\
\hline zn 66 & $1.489 \mathrm{E}-04$ & $1.489 \mathrm{E}-04$ & $1.489 \mathrm{E}-04$ & $1.489 \mathrm{E}-04$ & $1.489 \mathrm{E}-04$ \\
\hline zn 67 & $4.158 \mathrm{E}-04$ & $4.158 \mathrm{E}-04$ & $4.158 \mathrm{E}-04$ & $4.158 \mathrm{E}-04$ & 4.158E-04 \\
\hline zn 68 & 8.324E-04 & 8.324E-04 & $8.324 \mathrm{E}-04$ & 8.324E-04 & 8.324E-04 \\
\hline zn 70 & 4.106E-03 & 4.106E-03 & $4.106 \mathrm{E}-03$ & 4.106E-03 & 4.106E-03 \\
\hline zr 88 & $7.138 \mathrm{E}-12$ & 3.967E-18 & $1.831 \mathrm{E}-18$ & $8.815 \mathrm{E}-20$ & 4.244E-21 \\
\hline $\operatorname{zr} 90$ & $2.124 \mathrm{E}+01$ & $6.249 \mathrm{E}+01$ & $6.457 \mathrm{E}+01$ & $7.264 \mathrm{E}+01$ & $8.053 \mathrm{E}+01$ \\
\hline $\operatorname{zr} 91$ & $4.771 \mathrm{E}+02$ & $4.787 \mathrm{E}+02$ & $4.787 \mathrm{E}+02$ & $4.787 \mathrm{E}+02$ & $4.787 \mathrm{E}+02$ \\
\hline $\operatorname{zr} 92$ & $5.797 \mathrm{E}+02$ & $5.797 \mathrm{E}+02$ & $5.797 \mathrm{E}+02$ & $5.797 \mathrm{E}+02$ & $5.797 \mathrm{E}+02$ \\
\hline $\operatorname{zr} 93$ & $7.171 \mathrm{E}+02$ & $7.171 \mathrm{E}+02$ & $7.171 \mathrm{E}+02$ & $7.171 \mathrm{E}+02$ & $7.171 \mathrm{E}+02$ \\
\hline zr 94 & $8.238 \mathrm{E}+02$ & $8.238 \mathrm{E}+02$ & $8.238 \mathrm{E}+02$ & $8.238 \mathrm{E}+02$ & $8.238 \mathrm{E}+02$ \\
\hline $\operatorname{zr} 95$ & $4.359 \mathrm{E}+00$ & $3.105 \mathrm{E}-08$ & $1.135 \mathrm{E}-08$ & $2.182 \mathrm{E}-10$ & 4.197E-12 \\
\hline zr 96 & $9.610 \mathrm{E}+02$ & $9.610 \mathrm{E}+02$ & $9.610 \mathrm{E}+02$ & $9.610 \mathrm{E}+02$ & $9.610 \mathrm{E}+02$ \\
\hline Total & $1.1343 E+06$ & $1.1343 E+06$ & $1.1343 E+06$ & $1.1343 \mathrm{E}+06$ & $1.1343 E+06$ \\
\hline
\end{tabular}


Table C-5. MOX Fuel Rod K5 (high Pu): Nuclide mass in grams per MTIHM

\begin{tabular}{|c|c|c|c|c|c|}
\hline & $1 / 22 / 2009$ & $10 / 21 / 2013$ & $1 / 22 / 2014$ & $1 / 22 / 2015$ & $1 / 22 / 2016$ \\
\hline ac225 & 7.679E-13 & $8.117 \mathrm{E}-13$ & $8.137 \mathrm{E}-13$ & $8.263 \mathrm{E}-13$ & $8.396 \mathrm{E}-13$ \\
\hline ac227 & $7.620 \mathrm{E}-10$ & $3.238 \mathrm{E}-09$ & $3.375 \mathrm{E}-09$ & $3.915 \mathrm{E}-09$ & 4.462E-09 \\
\hline ag105 & $6.982 \mathrm{E}-15$ & $1.619 \mathrm{E}-27$ & $3.398 \mathrm{E}-28$ & $7.414 \mathrm{E}-31$ & $1.618 \mathrm{E}-33$ \\
\hline $\operatorname{ag} 107$ & $1.295 \mathrm{E}-04$ & 4.242E-04 & 4.400E-04 & $5.020 \mathrm{E}-04$ & 5.641E-04 \\
\hline $\operatorname{ag108}$ & $5.547 \mathrm{E}-13$ & $5.506 \mathrm{E}-13$ & $5.503 \mathrm{E}-13$ & $5.494 \mathrm{E}-13$ & $5.486 \mathrm{E}-13$ \\
\hline ag108m & $6.166 \mathrm{E}-04$ & $6.120 \mathrm{E}-04$ & $6.118 \mathrm{E}-04$ & $6.108 \mathrm{E}-04$ & $6.098 \mathrm{E}-04$ \\
\hline ag109 & $2.052 \mathrm{E}+02$ & $2.052 \mathrm{E}+02$ & $2.052 \mathrm{E}+02$ & $2.052 \mathrm{E}+02$ & $2.052 \mathrm{E}+02$ \\
\hline ag109m & $1.476 \mathrm{E}-10$ & $1.092 \mathrm{E}-11$ & $9.498 \mathrm{E}-12$ & $5.489 \mathrm{E}-12$ & $3.172 \mathrm{E}-12$ \\
\hline $\operatorname{ag110}$ & $1.843 \mathrm{E}-08$ & $1.502 \mathrm{E}-10$ & $1.160 \mathrm{E}-10$ & $4.213 \mathrm{E}-11$ & $1.530 \mathrm{E}-11$ \\
\hline ag110m & $1.188 \mathrm{E}+00$ & $9.684 \mathrm{E}-03$ & $7.480 \mathrm{E}-03$ & $2.717 \mathrm{E}-03$ & $9.870 \mathrm{E}-04$ \\
\hline ag111 & $1.570 \mathrm{E}-11$ & $0.000 \mathrm{E}+00$ & $0.000 \mathrm{E}+00$ & $0.000 \mathrm{E}+00$ & $0.000 \mathrm{E}+00$ \\
\hline al 27 & 4.901E-14 & $4.901 \mathrm{E}-14$ & $4.901 \mathrm{E}-14$ & $4.901 \mathrm{E}-14$ & $4.901 \mathrm{E}-14$ \\
\hline am241 & $4.091 \mathrm{E}+02$ & $1.421 \mathrm{E}+03$ & $1.469 \mathrm{E}+03$ & $1.650 \mathrm{E}+03$ & $1.823 \mathrm{E}+03$ \\
\hline $\operatorname{am} 242$ & $5.285 \mathrm{E}-05$ & $5.164 \mathrm{E}-05$ & $5.157 \mathrm{E}-05$ & 5.132E-05 & $5.107 \mathrm{E}-05$ \\
\hline am242m & $4.097 \mathrm{E}+00$ & $4.002 \mathrm{E}+00$ & $3.997 \mathrm{E}+00$ & $3.978 \mathrm{E}+00$ & $3.958 \mathrm{E}+00$ \\
\hline am243 & $2.719 \mathrm{E}+02$ & $2.718 \mathrm{E}+02$ & $2.718 \mathrm{E}+02$ & $2.717 \mathrm{E}+02$ & $2.717 \mathrm{E}+02$ \\
\hline $\operatorname{am} 244$ & $6.989 \mathrm{E}-16$ & $4.849 \mathrm{E}-16$ & $4.754 \mathrm{E}-16$ & $4.402 \mathrm{E}-16$ & $4.075 \mathrm{E}-16$ \\
\hline am245 & $8.854 \mathrm{E}-15$ & $2.075 \mathrm{E}-16$ & $1.696 \mathrm{E}-16$ & $7.693 \mathrm{E}-17$ & $3.489 \mathrm{E}-17$ \\
\hline ar 36 & $6.487 \mathrm{E}-14$ & $6.487 \mathrm{E}-14$ & $6.487 \mathrm{E}-14$ & $6.487 \mathrm{E}-14$ & $6.487 \mathrm{E}-14$ \\
\hline ar 38 & $6.896 \mathrm{E}-14$ & $6.896 \mathrm{E}-14$ & $6.896 \mathrm{E}-14$ & $6.896 \mathrm{E}-14$ & $6.896 \mathrm{E}-14$ \\
\hline ar 39 & $1.075 \mathrm{E}-16$ & $1.062 \mathrm{E}-16$ & $1.061 \mathrm{E}-16$ & $1.059 \mathrm{E}-16$ & $1.055 \mathrm{E}-16$ \\
\hline ar 40 & $7.288 \mathrm{E}-14$ & $7.288 \mathrm{E}-14$ & $7.288 \mathrm{E}-14$ & $7.288 \mathrm{E}-14$ & $7.288 \mathrm{E}-14$ \\
\hline as 73 & $1.553 \mathrm{E}-10$ & $4.949 \mathrm{E}-17$ & $2.218 \mathrm{E}-17$ & $9.498 \mathrm{E}-19$ & $4.066 \mathrm{E}-20$ \\
\hline as 74 & $1.663 \mathrm{E}-12$ & $0.000 \mathrm{E}+00$ & $0.000 \mathrm{E}+00$ & $0.000 \mathrm{E}+00$ & $0.000 \mathrm{E}+00$ \\
\hline as 75 & $1.669 \mathrm{E}-01$ & $1.669 \mathrm{E}-01$ & $1.669 \mathrm{E}-01$ & $1.669 \mathrm{E}-01$ & $1.669 \mathrm{E}-01$ \\
\hline au197 & $2.654 \mathrm{E}-13$ & $2.654 \mathrm{E}-13$ & $2.654 \mathrm{E}-13$ & $2.654 \mathrm{E}-13$ & $2.654 \mathrm{E}-13$ \\
\hline b 10 & $4.441 \mathrm{E}-11$ & $2.345 \mathrm{E}-10$ & $2.447 \mathrm{E}-10$ & $2.847 \mathrm{E}-10$ & $3.248 \mathrm{E}-10$ \\
\hline b 11 & 4.939E-08 & 4.939E-08 & 4.939E-08 & 4.939E-08 & $4.939 \mathrm{E}-08$ \\
\hline ba130 & $1.971 \mathrm{E}-13$ & $1.971 \mathrm{E}-13$ & $1.971 \mathrm{E}-13$ & $1.971 \mathrm{E}-13$ & $1.971 \mathrm{E}-13$ \\
\hline ba131 & $3.151 \mathrm{E}-18$ & $0.000 \mathrm{E}+00$ & $0.000 \mathrm{E}+00$ & $0.000 \mathrm{E}+00$ & $0.000 \mathrm{E}+00$ \\
\hline ba132 & $2.433 \mathrm{E}-04$ & $2.433 \mathrm{E}-04$ & $2.433 \mathrm{E}-04$ & $2.433 \mathrm{E}-04$ & $2.433 \mathrm{E}-04$ \\
\hline ba133 & $1.285 \mathrm{E}-04$ & $9.398 \mathrm{E}-05$ & $9.239 \mathrm{E}-05$ & 8.655E-05 & $8.104 \mathrm{E}-05$ \\
\hline ba134 & $8.682 \mathrm{E}+01$ & $1.853 \mathrm{E}+02$ & $1.874 \mathrm{E}+02$ & $1.939 \mathrm{E}+02$ & $1.987 \mathrm{E}+02$ \\
\hline ba135 & $3.224 \mathrm{E}-01$ & $3.236 \mathrm{E}-01$ & $3.236 \mathrm{E}-01$ & 3.239E-01 & $3.241 \mathrm{E}-01$ \\
\hline ba136 & $4.354 \mathrm{E}+01$ & $4.354 \mathrm{E}+01$ & $4.354 \mathrm{E}+01$ & $4.354 \mathrm{E}+01$ & $4.354 \mathrm{E}+01$ \\
\hline ba136m & $3.235 \mathrm{E}-14$ & $0.000 \mathrm{E}+00$ & $0.000 \mathrm{E}+00$ & $0.000 \mathrm{E}+00$ & $0.000 \mathrm{E}+00$ \\
\hline ba137 & $7.633 \mathrm{E}+01$ & $2.309 \mathrm{E}+02$ & $2.387 \mathrm{E}+02$ & $2.690 \mathrm{E}+02$ & $2.986 \mathrm{E}+02$ \\
\hline
\end{tabular}




\begin{tabular}{|c|c|c|c|c|c|}
\hline \multicolumn{6}{|c|}{ Table C-5. Continued } \\
\hline & $1 / 22 / 2009$ & $10 / 21 / 2013$ & $1 / 22 / 2014$ & $1 / 22 / 2015$ & $1 / 22 / 2016$ \\
\hline ba137m & $2.280 \mathrm{E}-04$ & $2.044 \mathrm{E}-04$ & $2.032 \mathrm{E}-04$ & $1.986 \mathrm{E}-04$ & $1.941 \mathrm{E}-04$ \\
\hline ba138 & $1.485 \mathrm{E}+03$ & $1.485 \mathrm{E}+03$ & $1.485 \mathrm{E}+03$ & $1.485 \mathrm{E}+03$ & $1.485 \mathrm{E}+03$ \\
\hline ba140 & $1.458 \mathrm{E}-05$ & $0.000 \mathrm{E}+00$ & $0.000 \mathrm{E}+00$ & $0.000 \mathrm{E}+00$ & $0.000 \mathrm{E}+00$ \\
\hline be 9 & $1.945 \mathrm{E}-06$ & $1.945 \mathrm{E}-06$ & $1.945 \mathrm{E}-06$ & $1.945 \mathrm{E}-06$ & $1.945 \mathrm{E}-06$ \\
\hline be 10 & $8.728 \mathrm{E}-05$ & $8.728 \mathrm{E}-05$ & $8.728 \mathrm{E}-05$ & $8.728 \mathrm{E}-05$ & $8.728 \mathrm{E}-05$ \\
\hline bi208 & $6.416 \mathrm{E}-16$ & $6.416 \mathrm{E}-16$ & $6.416 \mathrm{E}-16$ & $6.416 \mathrm{E}-16$ & $6.416 \mathrm{E}-16$ \\
\hline bi209 & $6.509 \mathrm{E}-11$ & $1.530 \mathrm{E}-10$ & $1.579 \mathrm{E}-10$ & $1.771 \mathrm{E}-10$ & $1.967 \mathrm{E}-10$ \\
\hline bi210 & $2.808 \mathrm{E}-14$ & $2.964 \mathrm{E}-14$ & $3.002 \mathrm{E}-14$ & $3.193 \mathrm{E}-14$ & $3.456 \mathrm{E}-14$ \\
\hline bi210m & $8.509 \mathrm{E}-16$ & $8.509 \mathrm{E}-16$ & $8.509 \mathrm{E}-16$ & $8.509 \mathrm{E}-16$ & $8.509 \mathrm{E}-16$ \\
\hline bi211 & $1.231 \mathrm{E}-16$ & $5.638 \mathrm{E}-16$ & $5.759 \mathrm{E}-16$ & $6.816 \mathrm{E}-16$ & $7.766 \mathrm{E}-16$ \\
\hline bi212 & $1.341 \mathrm{E}-10$ & $8.735 \mathrm{E}-10$ & $9.067 \mathrm{E}-10$ & $1.018 \mathrm{E}-09$ & $1.112 \mathrm{E}-09$ \\
\hline bi213 & $2.302 \mathrm{E}-15$ & $2.433 \mathrm{E}-15$ & $2.438 \mathrm{E}-15$ & $2.478 \mathrm{E}-15$ & $2.516 \mathrm{E}-15$ \\
\hline bk247 & $1.312 \mathrm{E}-13$ & $1.309 \mathrm{E}-13$ & $1.309 \mathrm{E}-13$ & $1.308 \mathrm{E}-13$ & $1.308 \mathrm{E}-13$ \\
\hline bk248 & $5.544 \mathrm{E}-12$ & $3.846 \mathrm{E}-12$ & $3.771 \mathrm{E}-12$ & $3.492 \mathrm{E}-12$ & 3.233E-12 \\
\hline bk249 & $2.325 \mathrm{E}-06$ & $5.447 \mathrm{E}-08$ & 4.453E-08 & $2.020 \mathrm{E}-08$ & $9.159 \mathrm{E}-09$ \\
\hline bk250 & $4.029 \mathrm{E}-16$ & $5.259 \mathrm{E}-18$ & 4.182E-18 & $1.728 \mathrm{E}-18$ & $7.474 \mathrm{E}-19$ \\
\hline br 79 & $2.961 \mathrm{E}-03$ & $3.021 \mathrm{E}-03$ & $3.024 \mathrm{E}-03$ & $3.037 \mathrm{E}-03$ & $3.050 \mathrm{E}-03$ \\
\hline br 81 & $2.275 \mathrm{E}+01$ & $2.275 \mathrm{E}+01$ & $2.275 \mathrm{E}+01$ & $2.275 \mathrm{E}+01$ & $2.275 \mathrm{E}+01$ \\
\hline c 12 & $6.443 \mathrm{E}-02$ & $6.443 \mathrm{E}-02$ & $6.443 \mathrm{E}-02$ & $6.443 \mathrm{E}-02$ & $6.443 \mathrm{E}-02$ \\
\hline c 13 & $1.021 \mathrm{E}+01$ & $1.021 \mathrm{E}+01$ & $1.021 \mathrm{E}+01$ & $1.021 \mathrm{E}+01$ & $1.021 \mathrm{E}+01$ \\
\hline c 14 & $3.053 \mathrm{E}-04$ & $3.051 \mathrm{E}-04$ & $3.051 \mathrm{E}-04$ & $3.051 \mathrm{E}-04$ & $3.050 \mathrm{E}-04$ \\
\hline ca 40 & $7.248 \mathrm{E}-14$ & $7.248 \mathrm{E}-14$ & $7.248 \mathrm{E}-14$ & $7.248 \mathrm{E}-14$ & $7.248 \mathrm{E}-14$ \\
\hline ca 41 & $3.343 \mathrm{E}-17$ & $3.343 \mathrm{E}-17$ & $3.343 \mathrm{E}-17$ & $3.343 \mathrm{E}-17$ & $3.343 \mathrm{E}-17$ \\
\hline ca 42 & $7.640 \mathrm{E}-14$ & $7.640 \mathrm{E}-14$ & $7.640 \mathrm{E}-14$ & $7.640 \mathrm{E}-14$ & $7.640 \mathrm{E}-14$ \\
\hline ca 43 & $7.719 \mathrm{E}-14$ & $7.719 \mathrm{E}-14$ & 7.719E-14 & 7.719E-14 & $7.719 \mathrm{E}-14$ \\
\hline ca 44 & $8.078 \mathrm{E}-14$ & $8.078 \mathrm{E}-14$ & $8.078 \mathrm{E}-14$ & $8.078 \mathrm{E}-14$ & $8.078 \mathrm{E}-14$ \\
\hline ca 46 & $8.343 \mathrm{E}-14$ & $8.343 \mathrm{E}-14$ & $8.343 \mathrm{E}-14$ & $8.343 \mathrm{E}-14$ & $8.343 \mathrm{E}-14$ \\
\hline ca 48 & $8.708 \mathrm{E}-14$ & $8.708 \mathrm{E}-14$ & $8.708 \mathrm{E}-14$ & $8.708 \mathrm{E}-14$ & $8.708 \mathrm{E}-14$ \\
\hline cd106 & $2.653 \mathrm{E}-13$ & $2.653 \mathrm{E}-13$ & $2.653 \mathrm{E}-13$ & $2.653 \mathrm{E}-13$ & $2.653 \mathrm{E}-13$ \\
\hline cd108 & $1.207 \mathrm{E}-03$ & $1.207 \mathrm{E}-03$ & $1.208 \mathrm{E}-03$ & $1.208 \mathrm{E}-03$ & $1.208 \mathrm{E}-03$ \\
\hline cd109 & $1.485 \mathrm{E}-04$ & $1.100 \mathrm{E}-05$ & $9.564 \mathrm{E}-06$ & $5.526 \mathrm{E}-06$ & $3.193 \mathrm{E}-06$ \\
\hline cd110 & $1.146 \mathrm{E}+02$ & $1.158 \mathrm{E}+02$ & $1.158 \mathrm{E}+02$ & $1.158 \mathrm{E}+02$ & $1.158 \mathrm{E}+02$ \\
\hline cd111 & $6.118 \mathrm{E}+01$ & $6.118 \mathrm{E}+01$ & $6.118 \mathrm{E}+01$ & $6.118 \mathrm{E}+01$ & $6.118 \mathrm{E}+01$ \\
\hline cd112 & $2.879 \mathrm{E}+01$ & $2.879 \mathrm{E}+01$ & $2.879 \mathrm{E}+01$ & $2.879 \mathrm{E}+01$ & $2.879 \mathrm{E}+01$ \\
\hline cd113 & $4.198 \mathrm{E}-01$ & $4.198 \mathrm{E}-01$ & 4.198E-01 & 4.198E-01 & 4.198E-01 \\
\hline cd113m & $5.719 \mathrm{E}-03$ & $4.529 \mathrm{E}-03$ & 4.473E-03 & $4.258 \mathrm{E}-03$ & $4.054 \mathrm{E}-03$ \\
\hline cd114 & $3.100 \mathrm{E}+01$ & $3.100 \mathrm{E}+01$ & $3.100 \mathrm{E}+01$ & $3.100 \mathrm{E}+01$ & $3.100 \mathrm{E}+01$ \\
\hline cd115m & $6.586 \mathrm{E}-04$ & $1.292 \mathrm{E}-15$ & $3.040 \mathrm{E}-16$ & $1.040 \mathrm{E}-18$ & $3.558 \mathrm{E}-21$ \\
\hline
\end{tabular}




\begin{tabular}{|c|c|c|c|c|c|}
\hline \multicolumn{6}{|c|}{ Table C-5. Continued } \\
\hline & $1 / 22 / 2009$ & $10 / 21 / 2013$ & $1 / 22 / 2014$ & $1 / 22 / 2015$ & $1 / 22 / 2016$ \\
\hline cd116 & $1.056 \mathrm{E}+01$ & $1.056 \mathrm{E}+01$ & $1.056 \mathrm{E}+01$ & $1.056 \mathrm{E}+01$ & $1.056 \mathrm{E}+01$ \\
\hline ce136 & $2.310 \mathrm{E}-13$ & $2.310 \mathrm{E}-13$ & $2.310 \mathrm{E}-13$ & $2.310 \mathrm{E}-13$ & $2.310 \mathrm{E}-13$ \\
\hline ce138 & $8.761 \mathrm{E}-06$ & $8.761 \mathrm{E}-06$ & 8.761E-06 & $8.761 \mathrm{E}-06$ & $8.761 \mathrm{E}-06$ \\
\hline ce139 & $1.262 \mathrm{E}-03$ & $2.046 \mathrm{E}-07$ & $1.281 \mathrm{E}-07$ & $2.038 \mathrm{E}-08$ & 3.242E-09 \\
\hline ce140 & $1.405 \mathrm{E}+03$ & $1.405 \mathrm{E}+03$ & $1.405 \mathrm{E}+03$ & $1.405 \mathrm{E}+03$ & $1.405 \mathrm{E}+03$ \\
\hline ce141 & $2.164 \mathrm{E}-01$ & $1.937 \mathrm{E}-17$ & $2.667 \mathrm{E}-18$ & $1.112 \mathrm{E}-21$ & $4.635 \mathrm{E}-25$ \\
\hline ce142 & $1.225 \mathrm{E}+03$ & $1.225 \mathrm{E}+03$ & $1.225 \mathrm{E}+03$ & $1.225 \mathrm{E}+03$ & $1.225 \mathrm{E}+03$ \\
\hline ce144 & $1.908 \mathrm{E}+02$ & $2.814 \mathrm{E}+00$ & $2.244 \mathrm{E}+00$ & $9.232 \mathrm{E}-01$ & $3.800 \mathrm{E}-01$ \\
\hline cf248 & $3.467 \mathrm{E}-12$ & $9.458 \mathrm{E}-14$ & 7.792E-14 & $3.650 \mathrm{E}-14$ & $1.709 \mathrm{E}-14$ \\
\hline cf249 & $2.467 \mathrm{E}-06$ & 4.699E-06 & 4.706E-06 & $4.721 \mathrm{E}-06$ & 4.723E-06 \\
\hline cf250 & $1.045 \mathrm{E}-06$ & $8.131 \mathrm{E}-07$ & $8.018 \mathrm{E}-07$ & $7.606 \mathrm{E}-07$ & $7.215 \mathrm{E}-07$ \\
\hline cf251 & 4.843E-07 & $4.825 \mathrm{E}-07$ & 4.823E-07 & $4.820 \mathrm{E}-07$ & $4.816 \mathrm{E}-07$ \\
\hline cf252 & $1.206 \mathrm{E}-07$ & $3.479 \mathrm{E}-08$ & $3.254 \mathrm{E}-08$ & $2.504 \mathrm{E}-08$ & $1.927 \mathrm{E}-08$ \\
\hline cf253 & $6.211 \mathrm{E}-15$ & $0.000 \mathrm{E}+00$ & $0.000 \mathrm{E}+00$ & $0.000 \mathrm{E}+00$ & $0.000 \mathrm{E}+00$ \\
\hline cf254 & $2.666 \mathrm{E}-13$ & $6.351 \mathrm{E}-22$ & $2.188 \mathrm{E}-22$ & $3.341 \mathrm{E}-24$ & $5.103 \mathrm{E}-26$ \\
\hline cl 35 & $6.102 \mathrm{E}-14$ & $6.102 \mathrm{E}-14$ & $6.102 \mathrm{E}-14$ & $6.102 \mathrm{E}-14$ & $6.102 \mathrm{E}-14$ \\
\hline cl 36 & $2.729 \mathrm{E}-15$ & $2.729 \mathrm{E}-15$ & $2.729 \mathrm{E}-15$ & $2.729 \mathrm{E}-15$ & $2.729 \mathrm{E}-15$ \\
\hline cl 37 & $6.763 \mathrm{E}-14$ & $6.763 \mathrm{E}-14$ & $6.763 \mathrm{E}-14$ & $6.763 \mathrm{E}-14$ & $6.763 \mathrm{E}-14$ \\
\hline cm240 & $1.432 \mathrm{E}-11$ & $6.823 \mathrm{E}-31$ & $6.268 \mathrm{E}-32$ & $0.000 \mathrm{E}+00$ & $0.000 \mathrm{E}+00$ \\
\hline $\mathrm{cm} 241$ & $2.085 \mathrm{E}-08$ & $2.595 \mathrm{E}-24$ & $3.635 \mathrm{E}-25$ & $1.624 \mathrm{E}-28$ & $7.255 \mathrm{E}-32$ \\
\hline cm242 & $1.798 \mathrm{E}+01$ & $2.173 \mathrm{E}-02$ & $1.803 \mathrm{E}-02$ & $1.199 \mathrm{E}-02$ & $1.067 \mathrm{E}-02$ \\
\hline cm243 & $1.361 \mathrm{E}+00$ & $1.215 \mathrm{E}+00$ & $1.208 \mathrm{E}+00$ & $1.179 \mathrm{E}+00$ & $1.152 \mathrm{E}+00$ \\
\hline cm244 & $8.914 \mathrm{E}+01$ & $7.434 \mathrm{E}+01$ & $7.361 \mathrm{E}+01$ & $7.089 \mathrm{E}+01$ & $6.823 \mathrm{E}+01$ \\
\hline cm245 & $7.460 \mathrm{E}+00$ & $7.460 \mathrm{E}+00$ & $7.460 \mathrm{E}+00$ & $7.460 \mathrm{E}+00$ & $7.454 \mathrm{E}+00$ \\
\hline cm246 & $3.981 \mathrm{E}-01$ & $3.978 \mathrm{E}-01$ & $3.978 \mathrm{E}-01$ & $3.978 \mathrm{E}-01$ & $3.977 \mathrm{E}-01$ \\
\hline cm247 & $5.214 \mathrm{E}-03$ & $5.214 \mathrm{E}-03$ & $5.214 \mathrm{E}-03$ & $5.214 \mathrm{E}-03$ & $5.214 \mathrm{E}-03$ \\
\hline $\mathrm{cm} 248$ & $2.829 \mathrm{E}-04$ & $2.830 \mathrm{E}-04$ & $2.830 \mathrm{E}-04$ & $2.830 \mathrm{E}-04$ & $2.830 \mathrm{E}-04$ \\
\hline cm250 & $2.696 \mathrm{E}-11$ & $2.695 \mathrm{E}-11$ & $2.695 \mathrm{E}-11$ & $2.695 \mathrm{E}-11$ & $2.695 \mathrm{E}-11$ \\
\hline co 59 & $1.071 \mathrm{E}-13$ & $1.071 \mathrm{E}-13$ & $1.071 \mathrm{E}-13$ & $1.071 \mathrm{E}-13$ & $1.071 \mathrm{E}-13$ \\
\hline co 60 & $8.277 \mathrm{E}-15$ & $4.435 \mathrm{E}-15$ & 4.289E-15 & $3.761 \mathrm{E}-15$ & $3.297 \mathrm{E}-15$ \\
\hline cr 50 & $8.927 \mathrm{E}-14$ & 8.927E-14 & 8.927E-14 & $8.927 \mathrm{E}-14$ & 8.927E-14 \\
\hline cr 52 & $9.432 \mathrm{E}-14$ & $9.432 \mathrm{E}-14$ & $9.432 \mathrm{E}-14$ & $9.432 \mathrm{E}-14$ & $9.432 \mathrm{E}-14$ \\
\hline cr 53 & $9.458 \mathrm{E}-14$ & $9.458 \mathrm{E}-14$ & $9.458 \mathrm{E}-14$ & $9.458 \mathrm{E}-14$ & $9.458 \mathrm{E}-14$ \\
\hline cr 54 & $9.982 \mathrm{E}-14$ & $9.982 \mathrm{E}-14$ & $9.982 \mathrm{E}-14$ & $9.982 \mathrm{E}-14$ & $9.982 \mathrm{E}-14$ \\
\hline $\operatorname{cs} 131$ & $1.659 \mathrm{E}-15$ & $0.000 \mathrm{E}+00$ & $0.000 \mathrm{E}+00$ & $0.000 \mathrm{E}+00$ & $0.000 \mathrm{E}+00$ \\
\hline $\operatorname{cs} 132$ & $1.409 \mathrm{E}-16$ & $0.000 \mathrm{E}+00$ & $0.000 \mathrm{E}+00$ & $0.000 \mathrm{E}+00$ & $0.000 \mathrm{E}+00$ \\
\hline cs133 & $2.182 \mathrm{E}-17$ & $2.231 \mathrm{E}-17$ & $2.233 \mathrm{E}-17$ & $2.241 \mathrm{E}-17$ & $2.249 \mathrm{E}-17$ \\
\hline $\operatorname{cs} 133$ & $1.402 \mathrm{E}+03$ & $1.402 \mathrm{E}+03$ & $1.402 \mathrm{E}+03$ & $1.402 \mathrm{E}+03$ & $1.402 \mathrm{E}+03$ \\
\hline
\end{tabular}




\begin{tabular}{|c|c|c|c|c|c|}
\hline \multicolumn{6}{|c|}{ Table C-5. Continued } \\
\hline & $1 / 22 / 2009$ & $10 / 21 / 2013$ & $1 / 22 / 2014$ & $1 / 22 / 2015$ & $1 / 22 / 2016$ \\
\hline cs134 & $1.237 \mathrm{E}+02$ & $2.515 \mathrm{E}+01$ & $2.309 \mathrm{E}+01$ & $1.651 \mathrm{E}+01$ & $1.181 \mathrm{E}+01$ \\
\hline $\operatorname{cs} 135$ & $8.403 \mathrm{E}+02$ & $8.403 \mathrm{E}+02$ & $8.403 \mathrm{E}+02$ & $8.403 \mathrm{E}+02$ & $8.403 \mathrm{E}+02$ \\
\hline $\operatorname{cs} 136$ & $1.077 \mathrm{E}-06$ & $0.000 \mathrm{E}+00$ & $0.000 \mathrm{E}+00$ & $0.000 \mathrm{E}+00$ & $0.000 \mathrm{E}+00$ \\
\hline $\operatorname{cs137}$ & $1.493 \mathrm{E}+03$ & $1.338 \mathrm{E}+03$ & $1.330 \mathrm{E}+03$ & $1.300 \mathrm{E}+03$ & $1.270 \mathrm{E}+03$ \\
\hline cu 63 & $1.135 \mathrm{E}-13$ & $1.136 \mathrm{E}-13$ & $1.136 \mathrm{E}-13$ & $1.136 \mathrm{E}-13$ & $1.136 \mathrm{E}-13$ \\
\hline cu 65 & $1.951 \mathrm{E}-16$ & $1.953 \mathrm{E}-16$ & $1.953 \mathrm{E}-16$ & $1.953 \mathrm{E}-16$ & $1.953 \mathrm{E}-16$ \\
\hline cu 65 & 3.729E-07 & $3.729 \mathrm{E}-07$ & 3.729E-07 & $3.729 \mathrm{E}-07$ & 3.729E-07 \\
\hline dy156 & $3.076 \mathrm{E}-12$ & $3.076 \mathrm{E}-12$ & $3.076 \mathrm{E}-12$ & $3.076 \mathrm{E}-12$ & $3.076 \mathrm{E}-12$ \\
\hline dy158 & $1.164 \mathrm{E}-07$ & $4.003 \mathrm{E}-07$ & 4.154E-07 & 4.745E-07 & $5.333 \mathrm{E}-07$ \\
\hline dy159 & $2.131 \mathrm{E}-07$ & $5.197 \mathrm{E}-11$ & $3.326 \mathrm{E}-11$ & $5.767 \mathrm{E}-12$ & $1.000 \mathrm{E}-12$ \\
\hline dy160 & $7.201 \mathrm{E}-01$ & $7.341 \mathrm{E}-01$ & 7.341E-01 & $7.341 \mathrm{E}-01$ & $7.341 \mathrm{E}-01$ \\
\hline dy161 & $1.102 \mathrm{E}+00$ & $1.102 \mathrm{E}+00$ & $1.102 \mathrm{E}+00$ & $1.102 \mathrm{E}+00$ & $1.102 \mathrm{E}+00$ \\
\hline dy162 & 6.664E-01 & $6.664 \mathrm{E}-01$ & 6.664E-01 & $6.664 \mathrm{E}-01$ & $6.664 \mathrm{E}-01$ \\
\hline dy163 & 5.379E-01 & $5.379 \mathrm{E}-01$ & 5.379E-01 & $5.379 \mathrm{E}-01$ & $5.379 \mathrm{E}-01$ \\
\hline dy164 & $2.160 \mathrm{E}-01$ & $2.160 \mathrm{E}-01$ & $2.160 \mathrm{E}-01$ & $2.160 \mathrm{E}-01$ & $2.160 \mathrm{E}-01$ \\
\hline er162 & $3.549 \mathrm{E}-12$ & $3.549 \mathrm{E}-12$ & $3.549 \mathrm{E}-12$ & $3.549 \mathrm{E}-12$ & $3.549 \mathrm{E}-12$ \\
\hline er164 & 2.222E-06 & $2.222 \mathrm{E}-06$ & $2.222 \mathrm{E}-06$ & $2.222 \mathrm{E}-06$ & $2.222 \mathrm{E}-06$ \\
\hline er166 & $5.790 \mathrm{E}-02$ & $5.790 \mathrm{E}-02$ & $5.790 \mathrm{E}-02$ & $5.790 \mathrm{E}-02$ & $5.790 \mathrm{E}-02$ \\
\hline er167 & $3.371 \mathrm{E}-03$ & $3.371 \mathrm{E}-03$ & $3.371 \mathrm{E}-03$ & $3.371 \mathrm{E}-03$ & $3.371 \mathrm{E}-03$ \\
\hline er168 & $1.008 \mathrm{E}-02$ & $1.008 \mathrm{E}-02$ & $1.008 \mathrm{E}-02$ & $1.008 \mathrm{E}-02$ & $1.008 \mathrm{E}-02$ \\
\hline er169 & $9.724 \mathrm{E}-14$ & $0.000 \mathrm{E}+00$ & $0.000 \mathrm{E}+00$ & $0.000 \mathrm{E}+00$ & $0.000 \mathrm{E}+00$ \\
\hline er170 & 5.459E-04 & $5.459 \mathrm{E}-04$ & $5.459 \mathrm{E}-04$ & $5.459 \mathrm{E}-04$ & $5.459 \mathrm{E}-04$ \\
\hline es252 & $2.406 \mathrm{E}-16$ & $1.885 \mathrm{E}-17$ & $1.644 \mathrm{E}-17$ & $9.617 \mathrm{E}-18$ & 5.623E-18 \\
\hline es 253 & $1.495 \mathrm{E}-13$ & $0.000 \mathrm{E}+00$ & $0.000 \mathrm{E}+00$ & $0.000 \mathrm{E}+00$ & $0.000 \mathrm{E}+00$ \\
\hline es254 & $8.429 \mathrm{E}-13$ & $1.080 \mathrm{E}-14$ & $8.549 \mathrm{E}-15$ & $3.415 \mathrm{E}-15$ & $1.364 \mathrm{E}-15$ \\
\hline es 255 & $1.219 \mathrm{E}-15$ & $9.505 \mathrm{E}-29$ & $1.882 \mathrm{E}-29$ & $3.266 \mathrm{E}-32$ & $0.000 \mathrm{E}+00$ \\
\hline eu147 & $2.448 \mathrm{E}-18$ & $0.000 \mathrm{E}+00$ & $0.000 \mathrm{E}+00$ & $0.000 \mathrm{E}+00$ & $0.000 \mathrm{E}+00$ \\
\hline eu149 & $8.197 \mathrm{E}-11$ & $2.042 \mathrm{E}-16$ & $1.021 \mathrm{E}-16$ & $6.750 \mathrm{E}-18$ & $4.456 \mathrm{E}-19$ \\
\hline eu151 & $2.105 \mathrm{E}-01$ & $1.223 \mathrm{E}+00$ & $1.276 \mathrm{E}+00$ & $1.484 \mathrm{E}+00$ & $1.691 \mathrm{E}+00$ \\
\hline eu152 & 4.898E-02 & $3.842 \mathrm{E}-02$ & 3.792E-02 & $3.603 \mathrm{E}-02$ & $3.424 \mathrm{E}-02$ \\
\hline eu153 & $1.870 \mathrm{E}+02$ & $1.870 \mathrm{E}+02$ & $1.870 \mathrm{E}+02$ & $1.870 \mathrm{E}+02$ & $1.870 \mathrm{E}+02$ \\
\hline eu154 & $5.042 \mathrm{E}+01$ & $3.440 \mathrm{E}+01$ & $3.370 \mathrm{E}+01$ & $3.110 \mathrm{E}+01$ & $2.869 \mathrm{E}+01$ \\
\hline eu155 & $1.231 \mathrm{E}+01$ & $6.164 \mathrm{E}+00$ & $5.939 \mathrm{E}+00$ & $5.134 \mathrm{E}+00$ & $4.438 \mathrm{E}+00$ \\
\hline eu156 & $3.184 \mathrm{E}-05$ & $0.000 \mathrm{E}+00$ & $0.000 \mathrm{E}+00$ & $0.000 \mathrm{E}+00$ & $0.000 \mathrm{E}+00$ \\
\hline f 19 & $6.790 \mathrm{E}-14$ & $6.790 \mathrm{E}-14$ & $6.790 \mathrm{E}-14$ & $6.790 \mathrm{E}-14$ & $6.790 \mathrm{E}-14$ \\
\hline fe 54 & $9.770 \mathrm{E}-14$ & $9.770 \mathrm{E}-14$ & $9.770 \mathrm{E}-14$ & $9.770 \mathrm{E}-14$ & $9.770 \mathrm{E}-14$ \\
\hline fe 55 & $1.567 \mathrm{E}-16$ & $4.726 \mathrm{E}-17$ & $4.432 \mathrm{E}-17$ & $3.443 \mathrm{E}-17$ & $2.675 \mathrm{E}-17$ \\
\hline fe 56 & $1.059 \mathrm{E}-13$ & $1.059 \mathrm{E}-13$ & $1.059 \mathrm{E}-13$ & $1.059 \mathrm{E}-13$ & $1.059 \mathrm{E}-13$ \\
\hline
\end{tabular}




\begin{tabular}{|c|c|c|c|c|c|}
\hline \multicolumn{6}{|c|}{ Table C-5. Continued } \\
\hline & $1 / 22 / 2009$ & $10 / 21 / 2013$ & $1 / 22 / 2014$ & $1 / 22 / 2015$ & $1 / 22 / 2016$ \\
\hline fe 57 & $1.035 \mathrm{E}-13$ & $1.035 \mathrm{E}-13$ & $1.035 \mathrm{E}-13$ & $1.035 \mathrm{E}-13$ & $1.035 \mathrm{E}-13$ \\
\hline fe 58 & $3.064 \mathrm{E}-13$ & $3.064 \mathrm{E}-13$ & $3.064 \mathrm{E}-13$ & $3.064 \mathrm{E}-13$ & $3.064 \mathrm{E}-13$ \\
\hline fr221 & $2.567 \mathrm{E}-16$ & $2.713 \mathrm{E}-16$ & $2.719 \mathrm{E}-16$ & $2.763 \mathrm{E}-16$ & $2.806 \mathrm{E}-16$ \\
\hline ga 69 & $1.444 \mathrm{E}-03$ & $1.444 \mathrm{E}-03$ & $1.444 \mathrm{E}-03$ & $1.444 \mathrm{E}-03$ & $1.444 \mathrm{E}-03$ \\
\hline ga 71 & $6.572 \mathrm{E}-03$ & $6.572 \mathrm{E}-03$ & $6.572 \mathrm{E}-03$ & $6.572 \mathrm{E}-03$ & $6.572 \mathrm{E}-03$ \\
\hline gd151 & $8.582 \mathrm{E}-08$ & $5.326 \mathrm{E}-12$ & $3.167 \mathrm{E}-12$ & 4.117E-13 & $5.352 \mathrm{E}-14$ \\
\hline gd152 & $8.814 \mathrm{E}-02$ & $9.113 \mathrm{E}-02$ & $9.126 \mathrm{E}-02$ & $9.179 \mathrm{E}-02$ & $9.226 \mathrm{E}-02$ \\
\hline gd153 & $3.435 \mathrm{E}-03$ & $2.322 \mathrm{E}-05$ & $1.775 \mathrm{E}-05$ & 6.199E-06 & $2.164 \mathrm{E}-06$ \\
\hline gd154 & $7.082 \mathrm{E}+00$ & $2.310 \mathrm{E}+01$ & $2.380 \mathrm{E}+01$ & $2.641 \mathrm{E}+01$ & $2.881 \mathrm{E}+01$ \\
\hline gd155 & $1.580 \mathrm{E}+00$ & $7.732 \mathrm{E}+00$ & $7.951 \mathrm{E}+00$ & $8.761 \mathrm{E}+00$ & $9.458 \mathrm{E}+00$ \\
\hline gd156 & $1.226 \mathrm{E}+02$ & $1.226 \mathrm{E}+02$ & $1.226 \mathrm{E}+02$ & $1.226 \mathrm{E}+02$ & $1.226 \mathrm{E}+02$ \\
\hline gd157 & $3.585 \mathrm{E}-01$ & $3.585 \mathrm{E}-01$ & $3.585 \mathrm{E}-01$ & $3.585 \mathrm{E}-01$ & $3.585 \mathrm{E}-01$ \\
\hline gd158 & $3.977 \mathrm{E}+01$ & $3.977 \mathrm{E}+01$ & $3.977 \mathrm{E}+01$ & $3.977 \mathrm{E}+01$ & $3.977 \mathrm{E}+01$ \\
\hline gd160 & $2.893 \mathrm{E}+00$ & $2.893 \mathrm{E}+00$ & $2.893 \mathrm{E}+00$ & $2.893 \mathrm{E}+00$ & $2.893 \mathrm{E}+00$ \\
\hline ge 70 & $1.443 \mathrm{E}-05$ & $1.443 \mathrm{E}-05$ & 1.443E-05 & $1.443 \mathrm{E}-05$ & $1.443 \mathrm{E}-05$ \\
\hline ge 71 & $3.573 \mathrm{E}-16$ & $0.000 \mathrm{E}+00$ & $0.000 \mathrm{E}+00$ & $0.000 \mathrm{E}+00$ & $0.000 \mathrm{E}+00$ \\
\hline ge 72 & $1.688 \mathrm{E}-02$ & $1.688 \mathrm{E}-02$ & $1.688 \mathrm{E}-02$ & $1.688 \mathrm{E}-02$ & $1.688 \mathrm{E}-02$ \\
\hline ge 73 & $3.491 \mathrm{E}-02$ & $3.491 \mathrm{E}-02$ & $3.491 \mathrm{E}-02$ & $3.491 \mathrm{E}-02$ & $3.491 \mathrm{E}-02$ \\
\hline ge $73 m$ & $1.117 \mathrm{E}-17$ & $3.560 \mathrm{E}-24$ & $1.595 \mathrm{E}-24$ & $6.830 \mathrm{E}-26$ & $2.924 \mathrm{E}-27$ \\
\hline ge 74 & $8.303 \mathrm{E}-02$ & 8.303E-02 & $8.303 \mathrm{E}-02$ & 8.303E-02 & $8.303 \mathrm{E}-02$ \\
\hline ge 76 & $3.735 \mathrm{E}-01$ & $3.735 \mathrm{E}-01$ & $3.735 \mathrm{E}-01$ & $3.735 \mathrm{E}-01$ & $3.735 \mathrm{E}-01$ \\
\hline h1 & $5.405 \mathrm{E}-03$ & $5.405 \mathrm{E}-03$ & $5.405 \mathrm{E}-03$ & $5.405 \mathrm{E}-03$ & $5.405 \mathrm{E}-03$ \\
\hline h2 & $1.154 \mathrm{E}-03$ & $1.154 \mathrm{E}-03$ & $1.154 \mathrm{E}-03$ & $1.154 \mathrm{E}-03$ & $1.154 \mathrm{E}-03$ \\
\hline h3 & 7.013E-02 & $5.368 \mathrm{E}-02$ & $5.291 \mathrm{E}-02$ & $5.002 \mathrm{E}-02$ & $4.729 \mathrm{E}-02$ \\
\hline he3 & 4.444E-03 & $2.087 \mathrm{E}-02$ & $2.163 \mathrm{E}-02$ & $2.452 \mathrm{E}-02$ & $2.726 \mathrm{E}-02$ \\
\hline he4 & $6.746 \mathrm{E}+00$ & $7.641 \mathrm{E}+00$ & $7.675 \mathrm{E}+00$ & $7.812 \mathrm{E}+00$ & $7.951 \mathrm{E}+00$ \\
\hline hf174 & $1.673 \mathrm{E}-13$ & $1.673 \mathrm{E}-13$ & $1.673 \mathrm{E}-13$ & $1.673 \mathrm{E}-13$ & $1.673 \mathrm{E}-13$ \\
\hline hf175 & $9.730 \mathrm{E}-16$ & $3.430 \mathrm{E}-23$ & $1.366 \mathrm{E}-23$ & $3.679 \mathrm{E}-25$ & $9.909 \mathrm{E}-27$ \\
\hline hf176 & $3.297 \mathrm{E}-13$ & $3.297 \mathrm{E}-13$ & $3.297 \mathrm{E}-13$ & $3.297 \mathrm{E}-13$ & 3.297E-13 \\
\hline hf177 & $6.421 \mathrm{E}-14$ & $6.422 \mathrm{E}-14$ & $6.422 \mathrm{E}-14$ & $6.422 \mathrm{E}-14$ & $6.422 \mathrm{E}-14$ \\
\hline hf178 & $3.956 \mathrm{E}-13$ & $3.956 \mathrm{E}-13$ & $3.956 \mathrm{E}-13$ & $3.956 \mathrm{E}-13$ & $3.956 \mathrm{E}-13$ \\
\hline hf179 & $7.268 \mathrm{E}-13$ & $7.268 \mathrm{E}-13$ & $7.268 \mathrm{E}-13$ & $7.268 \mathrm{E}-13$ & $7.268 \mathrm{E}-13$ \\
\hline hf180 & $5.382 \mathrm{E}-13$ & $5.382 \mathrm{E}-13$ & $5.382 \mathrm{E}-13$ & $5.382 \mathrm{E}-13$ & $5.382 \mathrm{E}-13$ \\
\hline hf181 & $1.928 \mathrm{E}-17$ & $9.511 \mathrm{E}-30$ & $2.079 \mathrm{E}-30$ & $5.318 \mathrm{E}-33$ & $0.000 \mathrm{E}+00$ \\
\hline hf182 & $6.916 \mathrm{E}-16$ & $6.916 \mathrm{E}-16$ & $6.916 \mathrm{E}-16$ & $6.916 \mathrm{E}-16$ & $6.916 \mathrm{E}-16$ \\
\hline hg196 & $4.202 \mathrm{E}-14$ & 4.202E-14 & 4.202E-14 & 4.202E-14 & 4.202E-14 \\
\hline hg198 & $6.457 \mathrm{E}-13$ & $6.457 \mathrm{E}-13$ & $6.457 \mathrm{E}-13$ & $6.457 \mathrm{E}-13$ & $6.457 \mathrm{E}-13$ \\
\hline hg199 & $1.310 \mathrm{E}-13$ & $1.310 \mathrm{E}-13$ & $1.310 \mathrm{E}-13$ & $1.310 \mathrm{E}-13$ & $1.310 \mathrm{E}-13$ \\
\hline
\end{tabular}




\begin{tabular}{|c|c|c|c|c|c|}
\hline \multicolumn{6}{|c|}{ Table C-5. Continued } \\
\hline & $1 / 22 / 2009$ & $10 / 21 / 2013$ & $1 / 22 / 2014$ & $1 / 22 / 2015$ & $1 / 22 / 2016$ \\
\hline hg200 & $7.175 \mathrm{E}-13$ & $7.175 \mathrm{E}-13$ & $7.175 \mathrm{E}-13$ & $7.175 \mathrm{E}-13$ & $7.175 \mathrm{E}-13$ \\
\hline hg201 & $3.541 \mathrm{E}-13$ & $3.541 \mathrm{E}-13$ & $3.541 \mathrm{E}-13$ & $3.541 \mathrm{E}-13$ & $3.541 \mathrm{E}-13$ \\
\hline hg202 & $3.775 \mathrm{E}-13$ & $3.775 \mathrm{E}-13$ & $3.775 \mathrm{E}-13$ & $3.775 \mathrm{E}-13$ & $3.775 \mathrm{E}-13$ \\
\hline hg204 & 3.692E-13 & $3.692 \mathrm{E}-13$ & 3.692E-13 & $3.692 \mathrm{E}-13$ & $3.692 \mathrm{E}-13$ \\
\hline ho163 & 2.203E-07 & $2.202 \mathrm{E}-07$ & 2.202E-07 & $2.201 \mathrm{E}-07$ & $2.201 \mathrm{E}-07$ \\
\hline ho165 & $1.839 \mathrm{E}-01$ & $1.839 \mathrm{E}-01$ & $1.839 \mathrm{E}-01$ & $1.839 \mathrm{E}-01$ & $1.839 \mathrm{E}-01$ \\
\hline ho166m & 4.904E-04 & 4.890E-04 & 4.889E-04 & $4.886 \mathrm{E}-04$ & 4.884E-04 \\
\hline i125 & $1.006 \mathrm{E}-09$ & $1.659 \mathrm{E}-18$ & 5.605E-19 & $7.918 \mathrm{E}-21$ & $1.120 \mathrm{E}-22$ \\
\hline i126 & $1.674 \mathrm{E}-11$ & $0.000 \mathrm{E}+00$ & $0.000 \mathrm{E}+00$ & $0.000 \mathrm{E}+00$ & $0.000 \mathrm{E}+00$ \\
\hline i127 & $9.000 \mathrm{E}+01$ & $9.027 \mathrm{E}+01$ & $9.027 \mathrm{E}+01$ & $9.027 \mathrm{E}+01$ & $9.027 \mathrm{E}+01$ \\
\hline i129 & $2.784 \mathrm{E}+02$ & $2.784 \mathrm{E}+02$ & $2.784 \mathrm{E}+02$ & $2.784 \mathrm{E}+02$ & $2.784 \mathrm{E}+02$ \\
\hline i131 & $1.255 \mathrm{E}-09$ & $0.000 \mathrm{E}+00$ & $0.000 \mathrm{E}+00$ & $0.000 \mathrm{E}+00$ & $0.000 \mathrm{E}+00$ \\
\hline in113 & $9.830 \mathrm{E}-04$ & $2.171 \mathrm{E}-03$ & $2.227 \mathrm{E}-03$ & $2.442 \mathrm{E}-03$ & $2.646 \mathrm{E}-03$ \\
\hline in113m & $3.522 \mathrm{E}-14$ & $1.033 \mathrm{E}-18$ & 5.899E-19 & $6.547 \mathrm{E}-20$ & $7.268 \mathrm{E}-21$ \\
\hline in114 & $4.858 \mathrm{E}-12$ & $1.410 \mathrm{E}-22$ & $3.836 \mathrm{E}-23$ & $2.316 \mathrm{E}-25$ & $1.398 \mathrm{E}-27$ \\
\hline in114m & $2.987 \mathrm{E}-07$ & $8.675 \mathrm{E}-18$ & $2.359 \mathrm{E}-18$ & $1.424 \mathrm{E}-20$ & $8.595 \mathrm{E}-23$ \\
\hline in115 & $3.497 \mathrm{E}+00$ & $3.497 \mathrm{E}+00$ & $3.497 \mathrm{E}+00$ & $3.497 \mathrm{E}+00$ & $3.497 \mathrm{E}+00$ \\
\hline in115m & $2.936 \mathrm{E}-10$ & $5.756 \mathrm{E}-22$ & $1.355 \mathrm{E}-22$ & $4.635 \mathrm{E}-25$ & $1.586 \mathrm{E}-27$ \\
\hline ir191 & $1.881 \mathrm{E}-14$ & $1.881 \mathrm{E}-14$ & $1.881 \mathrm{E}-14$ & $1.881 \mathrm{E}-14$ & $1.881 \mathrm{E}-14$ \\
\hline ir192 & $3.793 \mathrm{E}-16$ & $3.255 \mathrm{E}-23$ & $1.359 \mathrm{E}-23$ & $4.416 \mathrm{E}-25$ & $1.434 \mathrm{E}-26$ \\
\hline ir193 & $1.966 \mathrm{E}-13$ & $1.977 \mathrm{E}-13$ & $1.978 \mathrm{E}-13$ & $1.980 \mathrm{E}-13$ & $1.982 \mathrm{E}-13$ \\
\hline k 39 & $7.055 \mathrm{E}-14$ & $7.055 \mathrm{E}-14$ & $7.055 \mathrm{E}-14$ & $7.055 \mathrm{E}-14$ & $7.055 \mathrm{E}-14$ \\
\hline k 40 & $7.036 \mathrm{E}-14$ & $7.036 \mathrm{E}-14$ & $7.036 \mathrm{E}-14$ & $7.036 \mathrm{E}-14$ & $7.036 \mathrm{E}-14$ \\
\hline k 41 & $7.640 \mathrm{E}-14$ & $7.640 \mathrm{E}-14$ & $7.640 \mathrm{E}-14$ & $7.640 \mathrm{E}-14$ & $7.640 \mathrm{E}-14$ \\
\hline kr 78 & $1.863 \mathrm{E}-13$ & $1.863 \mathrm{E}-13$ & $1.863 \mathrm{E}-13$ & $1.863 \mathrm{E}-13$ & $1.863 \mathrm{E}-13$ \\
\hline kr 80 & $4.318 \mathrm{E}-04$ & $4.318 \mathrm{E}-04$ & $4.318 \mathrm{E}-04$ & $4.318 \mathrm{E}-04$ & $4.318 \mathrm{E}-04$ \\
\hline kr 81 & $3.781 \mathrm{E}-05$ & $3.781 \mathrm{E}-05$ & $3.781 \mathrm{E}-05$ & $3.781 \mathrm{E}-05$ & $3.781 \mathrm{E}-05$ \\
\hline kr 82 & 7.779E-01 & $7.779 \mathrm{E}-01$ & 7.779E-01 & $7.779 \mathrm{E}-01$ & 7.779E-01 \\
\hline kr 83 & $3.781 \mathrm{E}+01$ & $3.781 \mathrm{E}+01$ & $3.781 \mathrm{E}+01$ & $3.781 \mathrm{E}+01$ & $3.781 \mathrm{E}+01$ \\
\hline kr 83m & 4.779E-11 & $4.239 \mathrm{E}-17$ & $2.006 \mathrm{E}-17$ & $1.066 \mathrm{E}-18$ & $5.664 \mathrm{E}-20$ \\
\hline kr 84 & $8.137 \mathrm{E}+01$ & $8.137 \mathrm{E}+01$ & $8.137 \mathrm{E}+01$ & $8.137 \mathrm{E}+01$ & $8.137 \mathrm{E}+01$ \\
\hline kr 85 & $1.722 \mathrm{E}+01$ & $1.268 \mathrm{E}+01$ & $1.248 \mathrm{E}+01$ & $1.170 \mathrm{E}+01$ & $1.097 \mathrm{E}+01$ \\
\hline kr 86 & $1.233 \mathrm{E}+02$ & $1.233 \mathrm{E}+02$ & $1.233 \mathrm{E}+02$ & $1.233 \mathrm{E}+02$ & $1.233 \mathrm{E}+02$ \\
\hline la137 & $1.465 \mathrm{E}-14$ & $1.465 \mathrm{E}-14$ & $1.465 \mathrm{E}-14$ & $1.465 \mathrm{E}-14$ & $1.465 \mathrm{E}-14$ \\
\hline la137 & 4.947E-04 & 4.947E-04 & 4.947E-04 & 4.947E-04 & 4.947E-04 \\
\hline la138 & $1.191 \mathrm{E}-15$ & $1.191 \mathrm{E}-15$ & $1.191 \mathrm{E}-15$ & $1.191 \mathrm{E}-15$ & $1.191 \mathrm{E}-15$ \\
\hline la138 & $2.416 \mathrm{E}-02$ & $2.416 \mathrm{E}-02$ & $2.416 \mathrm{E}-02$ & $2.416 \mathrm{E}-02$ & $2.416 \mathrm{E}-02$ \\
\hline la139 & $1.316 \mathrm{E}-16$ & $1.316 \mathrm{E}-16$ & $1.316 \mathrm{E}-16$ & $1.316 \mathrm{E}-16$ & $1.316 \mathrm{E}-16$ \\
\hline
\end{tabular}




\begin{tabular}{|c|c|c|c|c|c|}
\hline \multicolumn{6}{|c|}{ Table C-5. Continued } \\
\hline & $1 / 22 / 2009$ & $10 / 21 / 2013$ & $1 / 22 / 2014$ & $1 / 22 / 2015$ & $1 / 22 / 2016$ \\
\hline la139 & $1.377 \mathrm{E}+03$ & $1.377 \mathrm{E}+03$ & $1.377 \mathrm{E}+03$ & $1.377 \mathrm{E}+03$ & $1.377 \mathrm{E}+03$ \\
\hline la140 & $2.214 \mathrm{E}-06$ & $0.000 \mathrm{E}+00$ & $0.000 \mathrm{E}+00$ & $0.000 \mathrm{E}+00$ & $0.000 \mathrm{E}+00$ \\
\hline li 6 & $1.882 \mathrm{E}-10$ & $1.882 \mathrm{E}-10$ & $1.882 \mathrm{E}-10$ & $1.882 \mathrm{E}-10$ & $1.882 \mathrm{E}-10$ \\
\hline li 7 & $1.495 \mathrm{E}-11$ & $1.495 \mathrm{E}-11$ & $1.495 \mathrm{E}-11$ & $1.495 \mathrm{E}-11$ & $1.495 \mathrm{E}-11$ \\
\hline lu175 & $3.054 \mathrm{E}-13$ & $3.064 \mathrm{E}-13$ & $3.064 \mathrm{E}-13$ & $3.064 \mathrm{E}-13$ & $3.064 \mathrm{E}-13$ \\
\hline lu176 & $8.522 \mathrm{E}-15$ & $8.522 \mathrm{E}-15$ & $8.522 \mathrm{E}-15$ & $8.522 \mathrm{E}-15$ & $8.522 \mathrm{E}-15$ \\
\hline mg 24 & $4.359 \mathrm{E}-14$ & $4.359 \mathrm{E}-14$ & $4.359 \mathrm{E}-14$ & $4.359 \mathrm{E}-14$ & $4.359 \mathrm{E}-14$ \\
\hline mg 25 & $4.537 \mathrm{E}-14$ & 4.537E-14 & $4.537 \mathrm{E}-14$ & $4.537 \mathrm{E}-14$ & 4.537E-14 \\
\hline mg 26 & 4.722E-14 & $4.722 \mathrm{E}-14$ & 4.722E-14 & 4.722E-14 & 4.722E-14 \\
\hline mn 54 & $1.984 \mathrm{E}-17$ & $4.224 \mathrm{E}-19$ & $3.435 \mathrm{E}-19$ & $1.527 \mathrm{E}-19$ & $6.790 \mathrm{E}-20$ \\
\hline mn 55 & $9.810 \mathrm{E}-14$ & $9.823 \mathrm{E}-14$ & $9.823 \mathrm{E}-14$ & $9.823 \mathrm{E}-14$ & $9.823 \mathrm{E}-14$ \\
\hline mo 92 & $4.300 \mathrm{E}-12$ & $4.300 \mathrm{E}-12$ & $4.300 \mathrm{E}-12$ & $4.300 \mathrm{E}-12$ & $4.300 \mathrm{E}-12$ \\
\hline mo 93 & 3.432E-07 & $3.429 \mathrm{E}-07$ & 3.429E-07 & $3.428 \mathrm{E}-07$ & $3.427 \mathrm{E}-07$ \\
\hline mo 94 & $1.143 \mathrm{E}-02$ & $1.144 \mathrm{E}-02$ & $1.144 \mathrm{E}-02$ & $1.144 \mathrm{E}-02$ & $1.144 \mathrm{E}-02$ \\
\hline mo 95 & $7.520 \mathrm{E}+02$ & $7.606 \mathrm{E}+02$ & $7.606 \mathrm{E}+02$ & $7.606 \mathrm{E}+02$ & $7.606 \mathrm{E}+02$ \\
\hline mo 96 & $3.449 \mathrm{E}+01$ & $3.449 \mathrm{E}+01$ & $3.449 \mathrm{E}+01$ & $3.449 \mathrm{E}+01$ & $3.449 \mathrm{E}+01$ \\
\hline mo 97 & $9.053 \mathrm{E}+02$ & $9.053 \mathrm{E}+02$ & $9.053 \mathrm{E}+02$ & $9.053 \mathrm{E}+02$ & $9.053 \mathrm{E}+02$ \\
\hline mo 98 & $9.890 \mathrm{E}+02$ & $9.890 \mathrm{E}+02$ & $9.890 \mathrm{E}+02$ & $9.890 \mathrm{E}+02$ & $9.890 \mathrm{E}+02$ \\
\hline mo100 & $1.167 \mathrm{E}+03$ & $1.167 \mathrm{E}+03$ & $1.167 \mathrm{E}+03$ & $1.167 \mathrm{E}+03$ & $1.167 \mathrm{E}+03$ \\
\hline n 14 & $4.028 \mathrm{E}-06$ & 4.203E-06 & 4.213E-06 & 4.250E-06 & 4.287E-06 \\
\hline n 15 & $9.511 \mathrm{E}-03$ & $9.511 \mathrm{E}-03$ & $9.511 \mathrm{E}-03$ & $9.511 \mathrm{E}-03$ & $9.511 \mathrm{E}-03$ \\
\hline na 23 & 4.174E-14 & $4.174 \mathrm{E}-14$ & 4.174E-14 & 4.174E-14 & 4.174E-14 \\
\hline nb 91 & $1.017 \mathrm{E}-08$ & $1.013 \mathrm{E}-08$ & $1.013 \mathrm{E}-08$ & $1.012 \mathrm{E}-08$ & $1.010 \mathrm{E}-08$ \\
\hline nb 91m & $5.989 \mathrm{E}-15$ & $1.605 \mathrm{E}-23$ & $5.565 \mathrm{E}-24$ & $8.708 \mathrm{E}-26$ & $1.363 \mathrm{E}-27$ \\
\hline nb 92 & 8.887E-07 & 8.887E-07 & 8.887E-07 & 8.887E-07 & 8.887E-07 \\
\hline nb 92m & $1.420 \mathrm{E}-18$ & $0.000 \mathrm{E}+00$ & $0.000 \mathrm{E}+00$ & $0.000 \mathrm{E}+00$ & $0.000 \mathrm{E}+00$ \\
\hline nb 93 & $1.009 \mathrm{E}-04$ & $3.641 \mathrm{E}-04$ & $3.841 \mathrm{E}-04$ & $4.683 \mathrm{E}-04$ & $5.607 \mathrm{E}-04$ \\
\hline nb 93m & $5.717 \mathrm{E}-04$ & $1.645 \mathrm{E}-03$ & $1.696 \mathrm{E}-03$ & $1.894 \mathrm{E}-03$ & $2.083 \mathrm{E}-03$ \\
\hline nb 94 & $2.997 \mathrm{E}-03$ & $2.997 \mathrm{E}-03$ & $2.996 \mathrm{E}-03$ & $2.996 \mathrm{E}-03$ & $2.996 \mathrm{E}-03$ \\
\hline nb 95 & $4.766 \mathrm{E}+00$ & $3.587 \mathrm{E}-08$ & $1.309 \mathrm{E}-08$ & $2.595 \mathrm{E}-10$ & 4.997E-12 \\
\hline nb 95m & $2.691 \mathrm{E}-03$ & $1.917 \mathrm{E}-11$ & 7.002E-12 & $1.347 \mathrm{E}-13$ & $2.591 \mathrm{E}-15$ \\
\hline nd142 & $1.539 \mathrm{E}+01$ & $1.539 \mathrm{E}+01$ & $1.539 \mathrm{E}+01$ & $1.539 \mathrm{E}+01$ & $1.539 \mathrm{E}+01$ \\
\hline nd143 & $9.525 \mathrm{E}+02$ & $9.525 \mathrm{E}+02$ & $9.525 \mathrm{E}+02$ & $9.525 \mathrm{E}+02$ & $9.525 \mathrm{E}+02$ \\
\hline nd144 & $9.544 \mathrm{E}+02$ & $1.142 \mathrm{E}+03$ & $1.143 \mathrm{E}+03$ & $1.144 \mathrm{E}+03$ & $1.145 \mathrm{E}+03$ \\
\hline nd145 & $7.042 \mathrm{E}+02$ & $7.042 \mathrm{E}+02$ & $7.042 \mathrm{E}+02$ & $7.042 \mathrm{E}+02$ & $7.042 \mathrm{E}+02$ \\
\hline nd146 & $7.387 \mathrm{E}+02$ & $7.387 \mathrm{E}+02$ & $7.387 \mathrm{E}+02$ & $7.387 \mathrm{E}+02$ & $7.387 \mathrm{E}+02$ \\
\hline nd147 & $4.976 \mathrm{E}-07$ & $0.000 \mathrm{E}+00$ & $0.000 \mathrm{E}+00$ & $0.000 \mathrm{E}+00$ & $0.000 \mathrm{E}+00$ \\
\hline nd148 & $4.432 \mathrm{E}+02$ & $4.432 \mathrm{E}+02$ & $4.432 \mathrm{E}+02$ & $4.432 \mathrm{E}+02$ & $4.432 \mathrm{E}+02$ \\
\hline
\end{tabular}




\begin{tabular}{|c|c|c|c|c|c|}
\hline \multicolumn{6}{|c|}{ Table C-5. Continued } \\
\hline & $1 / 22 / 2009$ & $10 / 21 / 2013$ & $1 / 22 / 2014$ & $1 / 22 / 2015$ & $1 / 22 / 2016$ \\
\hline nd150 & $2.617 \mathrm{E}+02$ & $2.617 \mathrm{E}+02$ & $2.617 \mathrm{E}+02$ & $2.617 \mathrm{E}+02$ & $2.617 \mathrm{E}+02$ \\
\hline ni 58 & $1.047 \mathrm{E}-13$ & $1.047 \mathrm{E}-13$ & $1.047 \mathrm{E}-13$ & $1.047 \mathrm{E}-13$ & $1.047 \mathrm{E}-13$ \\
\hline ni 59 & $8.821 \mathrm{E}-14$ & $8.821 \mathrm{E}-14$ & $8.821 \mathrm{E}-14$ & $8.821 \mathrm{E}-14$ & $8.821 \mathrm{E}-14$ \\
\hline ni 60 & $1.276 \mathrm{E}-13$ & $1.314 \mathrm{E}-13$ & $1.316 \mathrm{E}-13$ & $1.321 \mathrm{E}-13$ & $1.326 \mathrm{E}-13$ \\
\hline ni 61 & $1.108 \mathrm{E}-13$ & $1.108 \mathrm{E}-13$ & $1.108 \mathrm{E}-13$ & $1.108 \mathrm{E}-13$ & $1.108 \mathrm{E}-13$ \\
\hline ni 62 & $1.114 \mathrm{E}-13$ & $1.114 \mathrm{E}-13$ & $1.114 \mathrm{E}-13$ & $1.114 \mathrm{E}-13$ & $1.114 \mathrm{E}-13$ \\
\hline ni 63 & $1.539 \mathrm{E}-15$ & $1.490 \mathrm{E}-15$ & $1.487 \mathrm{E}-15$ & $1.477 \mathrm{E}-15$ & $1.467 \mathrm{E}-15$ \\
\hline ni 64 & $1.166 \mathrm{E}-13$ & $1.166 \mathrm{E}-13$ & $1.166 \mathrm{E}-13$ & $1.166 \mathrm{E}-13$ & $1.166 \mathrm{E}-13$ \\
\hline np235 & $1.746 \mathrm{E}-06$ & $8.423 \mathrm{E}-08$ & $7.155 \mathrm{E}-08$ & $3.778 \mathrm{E}-08$ & $1.995 \mathrm{E}-08$ \\
\hline np236 & $3.017 \mathrm{E}-04$ & 3.017E-04 & 3.017E-04 & $3.017 \mathrm{E}-04$ & 3.017E-04 \\
\hline np237 & $1.598 \mathrm{E}+02$ & $1.667 \mathrm{E}+02$ & $1.673 \mathrm{E}+02$ & $1.698 \mathrm{E}+02$ & $1.726 \mathrm{E}+02$ \\
\hline np238 & $7.600 \mathrm{E}-07$ & $7.427 \mathrm{E}-07$ & $7.420 \mathrm{E}-07$ & 7.381E-07 & 7.347E-07 \\
\hline np239 & $2.340 \mathrm{E}-04$ & $2.340 \mathrm{E}-04$ & $2.340 \mathrm{E}-04$ & $2.340 \mathrm{E}-04$ & $2.339 \mathrm{E}-04$ \\
\hline np240 & $1.124 \mathrm{E}-16$ & $1.124 \mathrm{E}-16$ & $1.124 \mathrm{E}-16$ & $1.124 \mathrm{E}-16$ & $1.124 \mathrm{E}-16$ \\
\hline np240m & $1.092 \mathrm{E}-14$ & $1.092 \mathrm{E}-14$ & $1.092 \mathrm{E}-14$ & $1.092 \mathrm{E}-14$ & $1.092 \mathrm{E}-14$ \\
\hline o 16 & $1.344 \mathrm{E}+05$ & $1.344 \mathrm{E}+05$ & $1.344 \mathrm{E}+05$ & $1.344 \mathrm{E}+05$ & $1.344 \mathrm{E}+05$ \\
\hline o 17 & $2.620 \mathrm{E}-02$ & $2.620 \mathrm{E}-02$ & $2.620 \mathrm{E}-02$ & $2.620 \mathrm{E}-02$ & $2.620 \mathrm{E}-02$ \\
\hline o 18 & 8.482E-08 & $8.482 \mathrm{E}-08$ & 8.482E-08 & 8.482E-08 & $8.482 \mathrm{E}-08$ \\
\hline os186 & $2.052 \mathrm{E}-13$ & $2.052 \mathrm{E}-13$ & $2.052 \mathrm{E}-13$ & $2.052 \mathrm{E}-13$ & $2.052 \mathrm{E}-13$ \\
\hline os187 & $2.715 \mathrm{E}-14$ & $2.715 \mathrm{E}-14$ & $2.715 \mathrm{E}-14$ & $2.715 \mathrm{E}-14$ & $2.715 \mathrm{E}-14$ \\
\hline os188 & $1.101 \mathrm{E}-13$ & $1.102 \mathrm{E}-13$ & $1.102 \mathrm{E}-13$ & $1.102 \mathrm{E}-13$ & $1.102 \mathrm{E}-13$ \\
\hline os189 & $6.269 \mathrm{E}-15$ & $6.269 \mathrm{E}-15$ & $6.269 \mathrm{E}-15$ & $6.269 \mathrm{E}-15$ & $6.269 \mathrm{E}-15$ \\
\hline os190 & $1.366 \mathrm{E}-15$ & $1.366 \mathrm{E}-15$ & $1.366 \mathrm{E}-15$ & $1.366 \mathrm{E}-15$ & $1.366 \mathrm{E}-15$ \\
\hline os192 & $9.823 \mathrm{E}-15$ & $9.843 \mathrm{E}-15$ & $9.843 \mathrm{E}-15$ & $9.843 \mathrm{E}-15$ & $9.843 \mathrm{E}-15$ \\
\hline p 31 & $5.630 \mathrm{E}-14$ & $5.630 \mathrm{E}-14$ & $5.630 \mathrm{E}-14$ & $5.630 \mathrm{E}-14$ & $5.630 \mathrm{E}-14$ \\
\hline pa230 & $1.039 \mathrm{E}-15$ & $0.000 \mathrm{E}+00$ & $0.000 \mathrm{E}+00$ & $0.000 \mathrm{E}+00$ & $0.000 \mathrm{E}+00$ \\
\hline pa231 & $2.553 \mathrm{E}-05$ & $3.075 \mathrm{E}-05$ & $3.104 \mathrm{E}-05$ & $3.214 \mathrm{E}-05$ & $3.324 \mathrm{E}-05$ \\
\hline pa232 & $1.401 \mathrm{E}-14$ & $1.401 \mathrm{E}-14$ & $1.401 \mathrm{E}-14$ & $1.401 \mathrm{E}-14$ & $1.401 \mathrm{E}-14$ \\
\hline pa233 & $5.408 \mathrm{E}-06$ & $5.640 \mathrm{E}-06$ & $5.659 \mathrm{E}-06$ & 5.751E-06 & $5.843 \mathrm{E}-06$ \\
\hline pa234 & $2.480 \mathrm{E}-10$ & $2.481 \mathrm{E}-10$ & $2.480 \mathrm{E}-10$ & $2.480 \mathrm{E}-10$ & $2.480 \mathrm{E}-10$ \\
\hline pa234m & $4.469 \mathrm{E}-10$ & $4.470 \mathrm{E}-10$ & $4.469 \mathrm{E}-10$ & $4.469 \mathrm{E}-10$ & $4.469 \mathrm{E}-10$ \\
\hline pb204 & $3.694 \mathrm{E}-13$ & 3.694E-13 & 3.694E-13 & $3.694 \mathrm{E}-13$ & $3.694 \mathrm{E}-13$ \\
\hline pb205 & $1.001 \mathrm{E}-15$ & $1.001 \mathrm{E}-15$ & $1.001 \mathrm{E}-15$ & $1.001 \mathrm{E}-15$ & $1.001 \mathrm{E}-15$ \\
\hline pb206 & $1.627 \mathrm{E}-12$ & 8.117E-12 & $8.482 \mathrm{E}-12$ & $9.969 \mathrm{E}-12$ & $1.156 \mathrm{E}-11$ \\
\hline pb207 & 4.872E-11 & $3.185 \mathrm{E}-10$ & $3.426 \mathrm{E}-10$ & 4.474E-10 & $5.682 \mathrm{E}-10$ \\
\hline pb208 & $6.114 \mathrm{E}-07$ & $1.493 \mathrm{E}-05$ & $1.626 \mathrm{E}-05$ & 2.194E-05 & $2.824 \mathrm{E}-05$ \\
\hline pb209 & $9.671 \mathrm{E}-15$ & $1.022 \mathrm{E}-14$ & $1.024 \mathrm{E}-14$ & $1.041 \mathrm{E}-14$ & $1.057 \mathrm{E}-14$ \\
\hline pb210 & $4.540 \mathrm{E}-11$ & 4.791E-11 & $4.854 \mathrm{E}-11$ & $5.162 \mathrm{E}-11$ & $5.588 \mathrm{E}-11$ \\
\hline
\end{tabular}




\begin{tabular}{|c|c|c|c|c|c|}
\hline \multicolumn{6}{|c|}{ Table C-5. Continued } \\
\hline & $1 / 22 / 2009$ & $10 / 21 / 2013$ & $1 / 22 / 2014$ & $1 / 22 / 2015$ & $1 / 22 / 2016$ \\
\hline pb211 & $2.075 \mathrm{E}-15$ & $9.511 \mathrm{E}-15$ & $9.717 \mathrm{E}-15$ & $1.150 \mathrm{E}-14$ & $1.310 \mathrm{E}-14$ \\
\hline pb212 & $1.413 \mathrm{E}-09$ & $9.206 \mathrm{E}-09$ & $9.558 \mathrm{E}-09$ & $1.074 \mathrm{E}-08$ & $1.173 \mathrm{E}-08$ \\
\hline pd102 & $7.786 \mathrm{E}-04$ & $9.451 \mathrm{E}-04$ & $9.458 \mathrm{E}-04$ & $9.458 \mathrm{E}-04$ & $9.458 \mathrm{E}-04$ \\
\hline pd103 & 2.112E-09 & $0.000 \mathrm{E}+00$ & $0.000 \mathrm{E}+00$ & $0.000 \mathrm{E}+00$ & $0.000 \mathrm{E}+00$ \\
\hline pd104 & $3.150 \mathrm{E}+02$ & $3.150 \mathrm{E}+02$ & $3.150 \mathrm{E}+02$ & $3.150 \mathrm{E}+02$ & $3.150 \mathrm{E}+02$ \\
\hline pd105 & $9.133 \mathrm{E}+02$ & $9.133 \mathrm{E}+02$ & $9.133 \mathrm{E}+02$ & $9.133 \mathrm{E}+02$ & $9.133 \mathrm{E}+02$ \\
\hline pd106 & $6.704 \mathrm{E}+02$ & $8.814 \mathrm{E}+02$ & $8.828 \mathrm{E}+02$ & $8.861 \mathrm{E}+02$ & $8.881 \mathrm{E}+02$ \\
\hline pd107 & $5.824 \mathrm{E}+02$ & $5.824 \mathrm{E}+02$ & $5.824 \mathrm{E}+02$ & $5.824 \mathrm{E}+02$ & $5.824 \mathrm{E}+02$ \\
\hline pd108 & $3.987 \mathrm{E}+02$ & $3.987 \mathrm{E}+02$ & $3.987 \mathrm{E}+02$ & $3.987 \mathrm{E}+02$ & $3.987 \mathrm{E}+02$ \\
\hline pd110 & $1.292 \mathrm{E}+02$ & $1.292 \mathrm{E}+02$ & $1.292 \mathrm{E}+02$ & $1.292 \mathrm{E}+02$ & $1.292 \mathrm{E}+02$ \\
\hline pm143 & $1.280 \mathrm{E}-12$ & $1.376 \mathrm{E}-14$ & $1.079 \mathrm{E}-14$ & $4.153 \mathrm{E}-15$ & $1.598 \mathrm{E}-15$ \\
\hline pm144 & $3.946 \mathrm{E}-10$ & $1.442 \mathrm{E}-11$ & $1.207 \mathrm{E}-11$ & $6.014 \mathrm{E}-12$ & $2.995 \mathrm{E}-12$ \\
\hline pm145 & $3.094 \mathrm{E}-05$ & $2.594 \mathrm{E}-05$ & $2.569 \mathrm{E}-05$ & $2.470 \mathrm{E}-05$ & $2.375 \mathrm{E}-05$ \\
\hline pm146 & $4.588 \mathrm{E}-04$ & $2.531 \mathrm{E}-04$ & $2.452 \mathrm{E}-04$ & $2.163 \mathrm{E}-04$ & $1.909 \mathrm{E}-04$ \\
\hline pm147 & $1.831 \mathrm{E}+02$ & $5.228 \mathrm{E}+01$ & $4.888 \mathrm{E}+01$ & $3.753 \mathrm{E}+01$ & $2.883 \mathrm{E}+01$ \\
\hline pm148 & $2.167 \mathrm{E}-04$ & $5.024 \mathrm{E}-17$ & $1.055 \mathrm{E}-17$ & $2.301 \mathrm{E}-20$ & $5.022 \mathrm{E}-23$ \\
\hline pm148m & $3.453 \mathrm{E}-02$ & $8.005 \mathrm{E}-15$ & $1.680 \mathrm{E}-15$ & $3.666 \mathrm{E}-18$ & $7.998 \mathrm{E}-21$ \\
\hline po210 & $6.677 \mathrm{E}-13$ & $7.905 \mathrm{E}-13$ & $7.985 \mathrm{E}-13$ & $8.423 \mathrm{E}-13$ & $9.033 \mathrm{E}-13$ \\
\hline po216 & $5.451 \mathrm{E}-15$ & $3.551 \mathrm{E}-14$ & $3.686 \mathrm{E}-14$ & 4.142E-14 & $4.525 \mathrm{E}-14$ \\
\hline pr141 & $1.264 \mathrm{E}+03$ & $1.264 \mathrm{E}+03$ & $1.264 \mathrm{E}+03$ & $1.264 \mathrm{E}+03$ & $1.264 \mathrm{E}+03$ \\
\hline pr143 & $3.317 \mathrm{E}-05$ & $0.000 \mathrm{E}+00$ & $0.000 \mathrm{E}+00$ & $0.000 \mathrm{E}+00$ & $0.000 \mathrm{E}+00$ \\
\hline pr144 & $8.031 \mathrm{E}-03$ & $1.185 \mathrm{E}-04$ & $9.451 \mathrm{E}-05$ & $3.890 \mathrm{E}-05$ & $1.601 \mathrm{E}-05$ \\
\hline pr144m & 3.196E-05 & $4.716 \mathrm{E}-07$ & $3.761 \mathrm{E}-07$ & $1.547 \mathrm{E}-07$ & 6.367E-08 \\
\hline pt192 & $1.730 \mathrm{E}-13$ & $1.734 \mathrm{E}-13$ & $1.734 \mathrm{E}-13$ & $1.734 \mathrm{E}-13$ & $1.734 \mathrm{E}-13$ \\
\hline pt193 & $1.784 \mathrm{E}-14$ & $1.670 \mathrm{E}-14$ & $1.665 \mathrm{E}-14$ & $1.641 \mathrm{E}-14$ & $1.619 \mathrm{E}-14$ \\
\hline pt194 & $2.818 \mathrm{E}-13$ & $2.818 \mathrm{E}-13$ & $2.818 \mathrm{E}-13$ & $2.818 \mathrm{E}-13$ & $2.818 \mathrm{E}-13$ \\
\hline pt195 & $1.890 \mathrm{E}-15$ & $1.891 \mathrm{E}-15$ & $1.891 \mathrm{E}-15$ & $1.891 \mathrm{E}-15$ & $1.891 \mathrm{E}-15$ \\
\hline pt196 & $2.395 \mathrm{E}-16$ & $2.395 \mathrm{E}-16$ & $2.395 \mathrm{E}-16$ & $2.395 \mathrm{E}-16$ & $2.395 \mathrm{E}-16$ \\
\hline pu236 & 7.454E-04 & $2.359 \mathrm{E}-04$ & $2.218 \mathrm{E}-04$ & $1.740 \mathrm{E}-04$ & $1.366 \mathrm{E}-04$ \\
\hline pu237 & $9.485 \mathrm{E}-06$ & $3.520 \mathrm{E}-17$ & $8.575 \mathrm{E}-18$ & $3.355 \mathrm{E}-20$ & $1.313 \mathrm{E}-22$ \\
\hline pu238 & $1.571 \mathrm{E}+02$ & $1.685 \mathrm{E}+02$ & $1.681 \mathrm{E}+02$ & $1.669 \mathrm{E}+02$ & $1.655 \mathrm{E}+02$ \\
\hline pu239 & $1.834 \mathrm{E}+04$ & $1.834 \mathrm{E}+04$ & $1.834 \mathrm{E}+04$ & $1.834 \mathrm{E}+04$ & $1.834 \mathrm{E}+04$ \\
\hline pu240 & $9.863 \mathrm{E}+03$ & $9.876 \mathrm{E}+03$ & $9.876 \mathrm{E}+03$ & $9.876 \mathrm{E}+03$ & $9.876 \mathrm{E}+03$ \\
\hline pu241 & $4.956 \mathrm{E}+03$ & $3.937 \mathrm{E}+03$ & $3.889 \mathrm{E}+03$ & $3.705 \mathrm{E}+03$ & $3.529 \mathrm{E}+03$ \\
\hline pu242 & $1.164 \mathrm{E}+03$ & $1.164 \mathrm{E}+03$ & $1.164 \mathrm{E}+03$ & $1.164 \mathrm{E}+03$ & $1.164 \mathrm{E}+03$ \\
\hline pu243 & $1.859 \mathrm{E}-13$ & $1.859 \mathrm{E}-13$ & $1.859 \mathrm{E}-13$ & $1.859 \mathrm{E}-13$ & $1.859 \mathrm{E}-13$ \\
\hline pu244 & $6.570 \mathrm{E}-02$ & $6.570 \mathrm{E}-02$ & $6.570 \mathrm{E}-02$ & $6.570 \mathrm{E}-02$ & $6.570 \mathrm{E}-02$ \\
\hline ra223 & $1.000 \mathrm{E}-12$ & $4.582 \mathrm{E}-12$ & $4.681 \mathrm{E}-12$ & $5.539 \mathrm{E}-12$ & $6.312 \mathrm{E}-12$ \\
\hline
\end{tabular}




\begin{tabular}{|c|c|c|c|c|c|}
\hline \multicolumn{6}{|c|}{ Table C-5. Continued } \\
\hline & $1 / 22 / 2009$ & $10 / 21 / 2013$ & $1 / 22 / 2014$ & $1 / 22 / 2015$ & $1 / 22 / 2016$ \\
\hline ra224 & $1.233 \mathrm{E}-08$ & $8.031 \mathrm{E}-08$ & $8.336 \mathrm{E}-08$ & $9.365 \mathrm{E}-08$ & $1.023 \mathrm{E}-07$ \\
\hline ra225 & $1.144 \mathrm{E}-12$ & $1.209 \mathrm{E}-12$ & $1.213 \mathrm{E}-12$ & $1.232 \mathrm{E}-12$ & $1.251 \mathrm{E}-12$ \\
\hline ra226 & $1.594 \mathrm{E}-09$ & $9.485 \mathrm{E}-09$ & $1.016 \mathrm{E}-08$ & $1.309 \mathrm{E}-08$ & $1.651 \mathrm{E}-08$ \\
\hline ra228 & $2.600 \mathrm{E}-14$ & $4.945 \mathrm{E}-14$ & $5.059 \mathrm{E}-14$ & $5.496 \mathrm{E}-14$ & $5.920 \mathrm{E}-14$ \\
\hline rb 83 & $7.268 \mathrm{E}-08$ & $6.447 \mathrm{E}-14$ & $3.052 \mathrm{E}-14$ & $1.621 \mathrm{E}-15$ & $8.615 \mathrm{E}-17$ \\
\hline rb 84 & 5.954E-08 & $7.573 \mathrm{E}-24$ & $1.063 \mathrm{E}-24$ & $4.769 \mathrm{E}-28$ & $2.141 \mathrm{E}-31$ \\
\hline rb 85 & $6.956 \mathrm{E}+01$ & $7.407 \mathrm{E}+01$ & $7.434 \mathrm{E}+01$ & $7.507 \mathrm{E}+01$ & $7.580 \mathrm{E}+01$ \\
\hline rb 86 & $1.116 \mathrm{E}-06$ & $1.114 \mathrm{E}-34$ & $0.000 \mathrm{E}+00$ & $0.000 \mathrm{E}+00$ & $0.000 \mathrm{E}+00$ \\
\hline rb 87 & $1.619 \mathrm{E}+02$ & $1.619 \mathrm{E}+02$ & $1.619 \mathrm{E}+02$ & $1.619 \mathrm{E}+02$ & $1.619 \mathrm{E}+02$ \\
\hline re185 & $8.476 \mathrm{E}-14$ & $8.482 \mathrm{E}-14$ & $8.482 \mathrm{E}-14$ & $8.482 \mathrm{E}-14$ & $8.482 \mathrm{E}-14$ \\
\hline re186m & $2.123 \mathrm{E}-16$ & $2.123 \mathrm{E}-16$ & $2.123 \mathrm{E}-16$ & $2.123 \mathrm{E}-16$ & $2.123 \mathrm{E}-16$ \\
\hline re187 & $3.581 \mathrm{E}-13$ & $3.581 \mathrm{E}-13$ & $3.581 \mathrm{E}-13$ & $3.581 \mathrm{E}-13$ & $3.581 \mathrm{E}-13$ \\
\hline rh101 & 4.947E-07 & $1.826 \mathrm{E}-07$ & $1.731 \mathrm{E}-07$ & $1.403 \mathrm{E}-07$ & $1.138 \mathrm{E}-07$ \\
\hline rh102 & 7.593E-04 & $2.613 \mathrm{E}-06$ & $1.982 \mathrm{E}-06$ & $7.401 \mathrm{E}-07$ & $3.471 \mathrm{E}-07$ \\
\hline rh102m & $1.753 \mathrm{E}-03$ & $7.274 \mathrm{E}-04$ & $6.943 \mathrm{E}-04$ & $5.769 \mathrm{E}-04$ & 4.794E-04 \\
\hline rh103 & $9.106 \mathrm{E}+02$ & $9.113 \mathrm{E}+02$ & $9.113 \mathrm{E}+02$ & $9.113 \mathrm{E}+02$ & $9.113 \mathrm{E}+02$ \\
\hline rh103m & $6.504 \mathrm{E}-04$ & $3.317 \mathrm{E}-17$ & $6.418 \mathrm{E}-18$ & $1.018 \mathrm{E}-20$ & $1.615 \mathrm{E}-23$ \\
\hline rh106 & $2.056 \mathrm{E}-04$ & $8.124 \mathrm{E}-06$ & $6.830 \mathrm{E}-06$ & $3.459 \mathrm{E}-06$ & $1.752 \mathrm{E}-06$ \\
\hline rn220 & $2.129 \mathrm{E}-12$ & $1.387 \mathrm{E}-11$ & $1.440 \mathrm{E}-11$ & $1.618 \mathrm{E}-11$ & $1.767 \mathrm{E}-11$ \\
\hline rn222 & $1.016 \mathrm{E}-14$ & $6.094 \mathrm{E}-14$ & $6.528 \mathrm{E}-14$ & $8.416 \mathrm{E}-14$ & $1.061 \mathrm{E}-13$ \\
\hline ru 96 & $1.730 \mathrm{E}-13$ & $1.730 \mathrm{E}-13$ & $1.730 \mathrm{E}-13$ & $1.730 \mathrm{E}-13$ & $1.730 \mathrm{E}-13$ \\
\hline ru 98 & $1.355 \mathrm{E}-06$ & $1.362 \mathrm{E}-06$ & $1.363 \mathrm{E}-06$ & $1.364 \mathrm{E}-06$ & $1.365 \mathrm{E}-06$ \\
\hline ru 99 & $3.943 \mathrm{E}-02$ & $5.421 \mathrm{E}-02$ & $5.500 \mathrm{E}-02$ & $5.812 \mathrm{E}-02$ & $6.123 \mathrm{E}-02$ \\
\hline ru100 & $1.260 \mathrm{E}+02$ & $1.260 \mathrm{E}+02$ & $1.260 \mathrm{E}+02$ & $1.260 \mathrm{E}+02$ & $1.260 \mathrm{E}+02$ \\
\hline ru101 & $1.016 \mathrm{E}+03$ & $1.016 \mathrm{E}+03$ & $1.016 \mathrm{E}+03$ & $1.016 \mathrm{E}+03$ & $1.016 \mathrm{E}+03$ \\
\hline ru102 & $1.149 \mathrm{E}+03$ & $1.149 \mathrm{E}+03$ & $1.149 \mathrm{E}+03$ & $1.149 \mathrm{E}+03$ & $1.149 \mathrm{E}+03$ \\
\hline ru103 & $6.621 \mathrm{E}-01$ & $3.376 \mathrm{E}-14$ & $6.534 \mathrm{E}-15$ & $1.036 \mathrm{E}-17$ & $1.644 \mathrm{E}-20$ \\
\hline ru104 & $1.096 \mathrm{E}+03$ & $1.096 \mathrm{E}+03$ & $1.096 \mathrm{E}+03$ & $1.096 \mathrm{E}+03$ & $1.096 \mathrm{E}+03$ \\
\hline ru106 & $2.196 \mathrm{E}+02$ & $8.682 \mathrm{E}+00$ & $7.294 \mathrm{E}+00$ & $3.695 \mathrm{E}+00$ & $1.871 \mathrm{E}+00$ \\
\hline s 32 & 5.804E-14 & $5.804 \mathrm{E}-14$ & 5.804E-14 & $5.804 \mathrm{E}-14$ & $5.804 \mathrm{E}-14$ \\
\hline s 33 & 5.967E-14 & $5.967 \mathrm{E}-14$ & $5.967 \mathrm{E}-14$ & $5.967 \mathrm{E}-14$ & 5.967E-14 \\
\hline s 34 & $6.177 \mathrm{E}-14$ & $6.177 \mathrm{E}-14$ & $6.177 \mathrm{E}-14$ & $6.177 \mathrm{E}-14$ & $6.177 \mathrm{E}-14$ \\
\hline s 36 & $6.535 \mathrm{E}-14$ & $6.535 \mathrm{E}-14$ & $6.535 \mathrm{E}-14$ & $6.535 \mathrm{E}-14$ & $6.535 \mathrm{E}-14$ \\
\hline sb121 & $7.779 \mathrm{E}+00$ & $7.832 \mathrm{E}+00$ & $7.832 \mathrm{E}+00$ & $7.845 \mathrm{E}+00$ & $7.852 \mathrm{E}+00$ \\
\hline sb123 & $1.019 \mathrm{E}+01$ & $1.028 \mathrm{E}+01$ & $1.028 \mathrm{E}+01$ & $1.028 \mathrm{E}+01$ & $1.028 \mathrm{E}+01$ \\
\hline sb124 & $3.232 \mathrm{E}-03$ & $6.976 \mathrm{E}-12$ & $2.391 \mathrm{E}-12$ & $3.575 \mathrm{E}-14$ & $5.347 \mathrm{E}-16$ \\
\hline sb125 & $1.387 \mathrm{E}+01$ & $4.209 \mathrm{E}+00$ & $3.948 \mathrm{E}+00$ & $3.071 \mathrm{E}+00$ & $2.389 \mathrm{E}+00$ \\
\hline sb126 & $8.390 \mathrm{E}-07$ & $8.343 \mathrm{E}-07$ & $8.343 \mathrm{E}-07$ & 8.343E-07 & 8.343E-07 \\
\hline
\end{tabular}




\begin{tabular}{|c|c|c|c|c|c|}
\hline \multicolumn{6}{|c|}{ Table C-5. Continued } \\
\hline & $1 / 22 / 2009$ & $10 / 21 / 2013$ & $1 / 22 / 2014$ & $1 / 22 / 2015$ & $1 / 22 / 2016$ \\
\hline sb126m & $6.419 \mathrm{E}-09$ & $6.419 \mathrm{E}-09$ & $6.419 \mathrm{E}-09$ & $6.419 \mathrm{E}-09$ & $6.419 \mathrm{E}-09$ \\
\hline sc 45 & $7.965 \mathrm{E}-14$ & 7.965E-14 & $7.965 \mathrm{E}-14$ & $7.965 \mathrm{E}-14$ & $7.965 \mathrm{E}-14$ \\
\hline sc 46 & $3.576 \mathrm{E}-17$ & $2.125 \mathrm{E}-23$ & $9.843 \mathrm{E}-24$ & $4.807 \mathrm{E}-25$ & $2.347 \mathrm{E}-26$ \\
\hline se 74 & $3.631 \mathrm{E}-07$ & $3.631 \mathrm{E}-07$ & $3.631 \mathrm{E}-07$ & $3.631 \mathrm{E}-07$ & $3.631 \mathrm{E}-07$ \\
\hline se 75 & 7.049E-09 & $3.111 \mathrm{E}-13$ & $1.817 \mathrm{E}-13$ & $2.198 \mathrm{E}-14$ & $2.659 \mathrm{E}-15$ \\
\hline se 76 & $6.595 \mathrm{E}-03$ & $6.595 \mathrm{E}-03$ & $6.595 \mathrm{E}-03$ & $6.595 \mathrm{E}-03$ & $6.595 \mathrm{E}-03$ \\
\hline se 77 & $8.848 \mathrm{E}-01$ & $8.848 \mathrm{E}-01$ & $8.848 \mathrm{E}-01$ & $8.848 \mathrm{E}-01$ & $8.848 \mathrm{E}-01$ \\
\hline se 78 & $2.476 \mathrm{E}+00$ & $2.476 \mathrm{E}+00$ & $2.476 \mathrm{E}+00$ & $2.476 \mathrm{E}+00$ & $2.476 \mathrm{E}+00$ \\
\hline se 79 & $5.416 \mathrm{E}+00$ & $5.416 \mathrm{E}+00$ & $5.416 \mathrm{E}+00$ & $5.416 \mathrm{E}+00$ & $5.416 \mathrm{E}+00$ \\
\hline se 80 & $1.222 \mathrm{E}+01$ & $1.222 \mathrm{E}+01$ & $1.222 \mathrm{E}+01$ & $1.222 \mathrm{E}+01$ & $1.222 \mathrm{E}+01$ \\
\hline se 82 & $3.181 \mathrm{E}+01$ & $3.181 \mathrm{E}+01$ & $3.181 \mathrm{E}+01$ & $3.181 \mathrm{E}+01$ & $3.181 \mathrm{E}+01$ \\
\hline si 28 & $5.084 \mathrm{E}-14$ & $5.084 \mathrm{E}-14$ & $5.084 \mathrm{E}-14$ & $5.084 \mathrm{E}-14$ & $5.084 \mathrm{E}-14$ \\
\hline si 29 & $5.270 \mathrm{E}-14$ & $5.270 \mathrm{E}-14$ & $5.270 \mathrm{E}-14$ & $5.270 \mathrm{E}-14$ & $5.270 \mathrm{E}-14$ \\
\hline si 30 & $5.472 \mathrm{E}-14$ & $5.472 \mathrm{E}-14$ & $5.472 \mathrm{E}-14$ & $5.472 \mathrm{E}-14$ & $5.472 \mathrm{E}-14$ \\
\hline sm144 & $4.377 \mathrm{E}-11$ & $4.377 \mathrm{E}-11$ & $4.377 \mathrm{E}-11$ & $4.377 \mathrm{E}-11$ & $4.377 \mathrm{E}-11$ \\
\hline sm145 & $2.884 \mathrm{E}-07$ & $8.423 \mathrm{E}-09$ & $6.969 \mathrm{E}-09$ & $3.312 \mathrm{E}-09$ & $1.574 \mathrm{E}-09$ \\
\hline sm146 & $2.867 \mathrm{E}-03$ & $2.936 \mathrm{E}-03$ & $2.939 \mathrm{E}-03$ & $2.949 \mathrm{E}-03$ & $2.958 \mathrm{E}-03$ \\
\hline sm147 & $1.185 \mathrm{E}+02$ & $2.494 \mathrm{E}+02$ & $2.528 \mathrm{E}+02$ & $2.642 \mathrm{E}+02$ & $2.729 \mathrm{E}+02$ \\
\hline sm148 & $1.663 \mathrm{E}+02$ & $1.663 \mathrm{E}+02$ & $1.663 \mathrm{E}+02$ & $1.663 \mathrm{E}+02$ & $1.663 \mathrm{E}+02$ \\
\hline sm149 & $6.690 \mathrm{E}+00$ & $6.690 \mathrm{E}+00$ & $6.690 \mathrm{E}+00$ & $6.690 \mathrm{E}+00$ & $6.690 \mathrm{E}+00$ \\
\hline sm150 & $3.664 \mathrm{E}+02$ & $3.664 \mathrm{E}+02$ & $3.664 \mathrm{E}+02$ & $3.664 \mathrm{E}+02$ & $3.664 \mathrm{E}+02$ \\
\hline sm151 & $2.821 \mathrm{E}+01$ & $2.719 \mathrm{E}+01$ & $2.714 \mathrm{E}+01$ & $2.693 \mathrm{E}+01$ & $2.673 \mathrm{E}+01$ \\
\hline sm152 & $1.583 \mathrm{E}+02$ & $1.583 \mathrm{E}+02$ & $1.583 \mathrm{E}+02$ & $1.583 \mathrm{E}+02$ & $1.583 \mathrm{E}+02$ \\
\hline sm154 & $7.341 \mathrm{E}+01$ & $7.341 \mathrm{E}+01$ & $7.341 \mathrm{E}+01$ & $7.341 \mathrm{E}+01$ & $7.341 \mathrm{E}+01$ \\
\hline sn112 & 3.072E-09 & $3.072 \mathrm{E}-09$ & $3.072 \mathrm{E}-09$ & 3.072E-09 & $3.072 \mathrm{E}-09$ \\
\hline sn113 & $5.865 \mathrm{E}-11$ & $1.720 \mathrm{E}-15$ & $9.823 \mathrm{E}-16$ & $1.090 \mathrm{E}-16$ & $1.210 \mathrm{E}-17$ \\
\hline sn114 & $1.598 \mathrm{E}-04$ & $1.602 \mathrm{E}-04$ & $1.602 \mathrm{E}-04$ & $1.602 \mathrm{E}-04$ & $1.602 \mathrm{E}-04$ \\
\hline sn115 & $4.571 \mathrm{E}-01$ & $4.571 \mathrm{E}-01$ & $4.571 \mathrm{E}-01$ & $4.571 \mathrm{E}-01$ & $4.571 \mathrm{E}-01$ \\
\hline sn116 & $3.467 \mathrm{E}+00$ & $3.467 \mathrm{E}+00$ & $3.467 \mathrm{E}+00$ & $3.467 \mathrm{E}+00$ & $3.467 \mathrm{E}+00$ \\
\hline sn117 & $9.412 \mathrm{E}+00$ & $9.412 \mathrm{E}+00$ & $9.412 \mathrm{E}+00$ & $9.412 \mathrm{E}+00$ & $9.412 \mathrm{E}+00$ \\
\hline sn117m & 2.367E-09 & $0.000 \mathrm{E}+00$ & $0.000 \mathrm{E}+00$ & $0.000 \mathrm{E}+00$ & $0.000 \mathrm{E}+00$ \\
\hline sn118 & $8.091 \mathrm{E}+00$ & $8.091 \mathrm{E}+00$ & $8.091 \mathrm{E}+00$ & $8.091 \mathrm{E}+00$ & $8.091 \mathrm{E}+00$ \\
\hline sn119 & $7.739 \mathrm{E}+00$ & $7.799 \mathrm{E}+00$ & $7.799 \mathrm{E}+00$ & $7.799 \mathrm{E}+00$ & $7.799 \mathrm{E}+00$ \\
\hline sn119m & $6.227 \mathrm{E}-02$ & $1.032 \mathrm{E}-03$ & 8.283E-04 & $3.493 \mathrm{E}-04$ & $1.473 \mathrm{E}-04$ \\
\hline sn120 & $7.772 \mathrm{E}+00$ & $7.772 \mathrm{E}+00$ & $7.772 \mathrm{E}+00$ & $7.772 \mathrm{E}+00$ & $7.772 \mathrm{E}+00$ \\
\hline sn121 & $3.865 \mathrm{E}-05$ & $3.585 \mathrm{E}-05$ & $3.572 \mathrm{E}-05$ & $3.515 \mathrm{E}-05$ & $3.460 \mathrm{E}-05$ \\
\hline sn121m & $7.089 \mathrm{E}-01$ & $6.578 \mathrm{E}-01$ & $6.551 \mathrm{E}-01$ & $6.449 \mathrm{E}-01$ & $6.348 \mathrm{E}-01$ \\
\hline sn122 & $1.049 \mathrm{E}+01$ & $1.049 \mathrm{E}+01$ & $1.049 \mathrm{E}+01$ & $1.049 \mathrm{E}+01$ & $1.049 \mathrm{E}+01$ \\
\hline
\end{tabular}




\begin{tabular}{|c|c|c|c|c|c|}
\hline \multicolumn{6}{|c|}{ Table C-5. Continued } \\
\hline & $1 / 22 / 2009$ & $10 / 21 / 2013$ & $1 / 22 / 2014$ & $1 / 22 / 2015$ & $1 / 22 / 2016$ \\
\hline sn123 & $8.363 \mathrm{E}-02$ & $7.666 \mathrm{E}-06$ & $4.655 \mathrm{E}-06$ & $6.568 \mathrm{E}-07$ & $9.266 \mathrm{E}-08$ \\
\hline sn124 & $1.710 \mathrm{E}+01$ & $1.710 \mathrm{E}+01$ & $1.710 \mathrm{E}+01$ & $1.710 \mathrm{E}+01$ & $1.710 \mathrm{E}+01$ \\
\hline sn125 & $8.051 \mathrm{E}-10$ & $0.000 \mathrm{E}+00$ & $0.000 \mathrm{E}+00$ & $0.000 \mathrm{E}+00$ & $0.000 \mathrm{E}+00$ \\
\hline sn126 & $4.055 \mathrm{E}+01$ & $4.055 \mathrm{E}+01$ & $4.055 \mathrm{E}+01$ & $4.055 \mathrm{E}+01$ & $4.055 \mathrm{E}+01$ \\
\hline sr 84 & $6.424 \mathrm{E}-06$ & $6.426 \mathrm{E}-06$ & $6.426 \mathrm{E}-06$ & $6.426 \mathrm{E}-06$ & $6.426 \mathrm{E}-06$ \\
\hline sr 85 & $1.227 \mathrm{E}-08$ & $1.104 \mathrm{E}-16$ & $4.086 \mathrm{E}-17$ & $8.257 \mathrm{E}-19$ & $1.668 \mathrm{E}-20$ \\
\hline sr 86 & $3.733 \mathrm{E}-01$ & $3.733 \mathrm{E}-01$ & $3.733 \mathrm{E}-01$ & 3.733E-01 & 3.733E-01 \\
\hline sr 87 & 4.660E-03 & $4.660 \mathrm{E}-03$ & 4.660E-03 & $4.660 \mathrm{E}-03$ & 4.660E-03 \\
\hline sr 88 & $2.152 \mathrm{E}+02$ & $2.152 \mathrm{E}+02$ & $2.152 \mathrm{E}+02$ & $2.152 \mathrm{E}+02$ & $2.152 \mathrm{E}+02$ \\
\hline sr 89 & $5.441 \mathrm{E}-01$ & $2.579 \mathrm{E}-11$ & $7.201 \mathrm{E}-12$ & 4.817E-14 & $3.224 \mathrm{E}-16$ \\
\hline sr 90 & $3.314 \mathrm{E}+02$ & $2.956 \mathrm{E}+02$ & $2.938 \mathrm{E}+02$ & $2.868 \mathrm{E}+02$ & $2.800 \mathrm{E}+02$ \\
\hline ta180m & $1.823 \mathrm{E}-17$ & $1.823 \mathrm{E}-17$ & $1.823 \mathrm{E}-17$ & $1.823 \mathrm{E}-17$ & $1.823 \mathrm{E}-17$ \\
\hline ta181 & $1.971 \mathrm{E}-13$ & $1.971 \mathrm{E}-13$ & $1.971 \mathrm{E}-13$ & $1.971 \mathrm{E}-13$ & $1.971 \mathrm{E}-13$ \\
\hline ta182 & $7.865 \mathrm{E}-16$ & $2.235 \mathrm{E}-20$ & $1.276 \mathrm{E}-20$ & $1.428 \mathrm{E}-21$ & $1.792 \mathrm{E}-22$ \\
\hline tb157 & 8.801E-07 & 8.403E-07 & 8.383E-07 & 8.303E-07 & $8.224 \mathrm{E}-07$ \\
\hline tb158 & $9.451 \mathrm{E}-05$ & $9.279 \mathrm{E}-05$ & $9.266 \mathrm{E}-05$ & $9.232 \mathrm{E}-05$ & $9.199 \mathrm{E}-05$ \\
\hline tb159 & $6.281 \mathrm{E}+00$ & $6.281 \mathrm{E}+00$ & $6.281 \mathrm{E}+00$ & $6.281 \mathrm{E}+00$ & $6.281 \mathrm{E}+00$ \\
\hline tb160 & $1.438 \mathrm{E}-02$ & $8.748 \mathrm{E}-10$ & $3.588 \mathrm{E}-10$ & $1.084 \mathrm{E}-11$ & $3.276 \mathrm{E}-13$ \\
\hline tb161 & $6.690 \mathrm{E}-14$ & $0.000 \mathrm{E}+00$ & $0.000 \mathrm{E}+00$ & $0.000 \mathrm{E}+00$ & $0.000 \mathrm{E}+00$ \\
\hline tc 95 & $4.507 \mathrm{E}-18$ & $1.264 \mathrm{E}-26$ & $4.392 \mathrm{E}-27$ & $6.943 \mathrm{E}-29$ & $0.000 \mathrm{E}+00$ \\
\hline tc $95 \mathrm{~m}$ & $8.390 \mathrm{E}-15$ & $2.352 \mathrm{E}-23$ & $8.170 \mathrm{E}-24$ & $1.292 \mathrm{E}-25$ & $2.041 \mathrm{E}-27$ \\
\hline tc 97 & 8.662E-06 & $8.741 \mathrm{E}-06$ & 8.741E-06 & 8.741E-06 & $8.741 \mathrm{E}-06$ \\
\hline tc $97 \mathrm{~m}$ & $8.436 \mathrm{E}-08$ & $1.560 \mathrm{E}-13$ & $7.686 \mathrm{E}-14$ & $4.766 \mathrm{E}-15$ & $2.956 \mathrm{E}-16$ \\
\hline tc 98 & 8.794E-03 & $8.794 \mathrm{E}-03$ & 8.794E-03 & 8.794E-03 & $8.794 \mathrm{E}-03$ \\
\hline tc 99 & $9.491 \mathrm{E}+02$ & $9.491 \mathrm{E}+02$ & $9.491 \mathrm{E}+02$ & $9.491 \mathrm{E}+02$ & $9.491 \mathrm{E}+02$ \\
\hline te120 & $1.758 \mathrm{E}-09$ & $1.758 \mathrm{E}-09$ & $1.758 \mathrm{E}-09$ & $1.758 \mathrm{E}-09$ & $1.758 \mathrm{E}-09$ \\
\hline te121 & $2.251 \mathrm{E}-08$ & $1.497 \mathrm{E}-11$ & $1.011 \mathrm{E}-11$ & $2.165 \mathrm{E}-12$ & $4.638 \mathrm{E}-13$ \\
\hline te121m & $1.922 \mathrm{E}-07$ & $1.278 \mathrm{E}-10$ & $8.628 \mathrm{E}-11$ & $1.849 \mathrm{E}-11$ & $3.960 \mathrm{E}-12$ \\
\hline te122 & $5.816 \mathrm{E}-01$ & $5.816 \mathrm{E}-01$ & $5.816 \mathrm{E}-01$ & 5.816E-01 & $5.816 \mathrm{E}-01$ \\
\hline te123 & $2.175 \mathrm{E}-15$ & $2.175 \mathrm{E}-15$ & $2.175 \mathrm{E}-15$ & $2.175 \mathrm{E}-15$ & $2.175 \mathrm{E}-15$ \\
\hline te123 & $6.633 \mathrm{E}-03$ & $6.943 \mathrm{E}-03$ & $6.943 \mathrm{E}-03$ & $6.943 \mathrm{E}-03$ & $6.943 \mathrm{E}-03$ \\
\hline te123m & $3.090 \mathrm{E}-04$ & $1.298 \mathrm{E}-08$ & $7.560 \mathrm{E}-09$ & $9.053 \mathrm{E}-10$ & $1.084 \mathrm{E}-10$ \\
\hline te124 & $2.212 \mathrm{E}-13$ & $2.212 \mathrm{E}-13$ & $2.212 \mathrm{E}-13$ & $2.212 \mathrm{E}-13$ & $2.212 \mathrm{E}-13$ \\
\hline te124 & $4.116 \mathrm{E}-01$ & 4.149E-01 & 4.149E-01 & 4.149E-01 & 4.149E-01 \\
\hline te125 & $1.738 \mathrm{E}-15$ & $1.738 \mathrm{E}-15$ & $1.738 \mathrm{E}-15$ & $1.738 \mathrm{E}-15$ & $1.738 \mathrm{E}-15$ \\
\hline te125 & $9.219 \mathrm{E}+00$ & $1.901 \mathrm{E}+01$ & $1.927 \mathrm{E}+01$ & $2.016 \mathrm{E}+01$ & $2.085 \mathrm{E}+01$ \\
\hline te $125 \mathrm{~m}$ & $1.923 \mathrm{E}-01$ & $5.871 \mathrm{E}-02$ & $5.507 \mathrm{E}-02$ & 4.284E-02 & $3.333 \mathrm{E}-02$ \\
\hline te126 & $9.378 \mathrm{E}-01$ & $9.378 \mathrm{E}-01$ & $9.385 \mathrm{E}-01$ & $9.385 \mathrm{E}-01$ & $9.385 \mathrm{E}-01$ \\
\hline
\end{tabular}




\begin{tabular}{|c|c|c|c|c|c|}
\hline \multicolumn{6}{|c|}{ Table C-5. Continued } \\
\hline & $1 / 22 / 2009$ & $10 / 21 / 2013$ & $1 / 22 / 2014$ & $1 / 22 / 2015$ & $1 / 22 / 2016$ \\
\hline te127 & $1.068 \mathrm{E}-03$ & $1.748 \mathrm{E}-08$ & 9.671E-09 & $9.498 \mathrm{E}-10$ & $9.319 \mathrm{E}-11$ \\
\hline te127m & $3.050 \mathrm{E}-01$ & 4.992E-06 & $2.763 \mathrm{E}-06$ & $2.713 \mathrm{E}-07$ & $2.663 \mathrm{E}-08$ \\
\hline te128 & $1.555 \mathrm{E}+02$ & $1.555 \mathrm{E}+02$ & $1.555 \mathrm{E}+02$ & $1.555 \mathrm{E}+02$ & $1.555 \mathrm{E}+02$ \\
\hline te129 & 7.772E-06 & $2.312 \mathrm{E}-21$ & $3.394 \mathrm{E}-22$ & $1.822 \mathrm{E}-25$ & $9.783 \mathrm{E}-29$ \\
\hline te129m & $8.562 \mathrm{E}-03$ & $2.547 \mathrm{E}-18$ & $3.739 \mathrm{E}-19$ & $2.008 \mathrm{E}-22$ & $1.078 \mathrm{E}-25$ \\
\hline te130 & $5.180 \mathrm{E}+02$ & $5.180 \mathrm{E}+02$ & $5.180 \mathrm{E}+02$ & $5.180 \mathrm{E}+02$ & $5.180 \mathrm{E}+02$ \\
\hline th227 & $1.679 \mathrm{E}-12$ & $7.520 \mathrm{E}-12$ & $7.732 \mathrm{E}-12$ & $9.086 \mathrm{E}-12$ & $1.035 \mathrm{E}-11$ \\
\hline th228 & $2.428 \mathrm{E}-06$ & $1.554 \mathrm{E}-05$ & $1.612 \mathrm{E}-05$ & $1.813 \mathrm{E}-05$ & $1.981 \mathrm{E}-05$ \\
\hline th229 & $2.095 \mathrm{E}-07$ & $2.215 \mathrm{E}-07$ & $2.223 \mathrm{E}-07$ & $2.255 \mathrm{E}-07$ & $2.291 \mathrm{E}-07$ \\
\hline th230 & $9.511 \mathrm{E}-05$ & $2.872 \mathrm{E}-04$ & 2.999E-04 & $3.517 \mathrm{E}-04$ & 4.072E-04 \\
\hline th231 & 4.593E-09 & 4.603E-09 & 4.604E-09 & 4.606E-09 & 4.608E-09 \\
\hline th232 & $1.781 \mathrm{E}-04$ & $2.154 \mathrm{E}-04$ & $2.175 \mathrm{E}-04$ & $2.255 \mathrm{E}-04$ & $2.334 \mathrm{E}-04$ \\
\hline th234 & $1.338 \mathrm{E}-05$ & $1.339 \mathrm{E}-05$ & $1.338 \mathrm{E}-05$ & $1.338 \mathrm{E}-05$ & $1.338 \mathrm{E}-05$ \\
\hline ti 46 & $8.555 \mathrm{E}-14$ & $8.562 \mathrm{E}-14$ & $8.562 \mathrm{E}-14$ & $8.562 \mathrm{E}-14$ & $8.562 \mathrm{E}-14$ \\
\hline ti 47 & $8.522 \mathrm{E}-14$ & $8.522 \mathrm{E}-14$ & $8.522 \mathrm{E}-14$ & $8.522 \mathrm{E}-14$ & $8.522 \mathrm{E}-14$ \\
\hline ti 48 & $8.668 \mathrm{E}-14$ & $8.668 \mathrm{E}-14$ & $8.668 \mathrm{E}-14$ & $8.668 \mathrm{E}-14$ & $8.668 \mathrm{E}-14$ \\
\hline ti 49 & $8.960 \mathrm{E}-14$ & $8.960 \mathrm{E}-14$ & $8.960 \mathrm{E}-14$ & $8.960 \mathrm{E}-14$ & $8.960 \mathrm{E}-14$ \\
\hline ti 50 & $9.093 \mathrm{E}-14$ & $9.093 \mathrm{E}-14$ & $9.093 \mathrm{E}-14$ & $9.093 \mathrm{E}-14$ & $9.093 \mathrm{E}-14$ \\
\hline tl203 & $2.299 \mathrm{E}-15$ & $2.303 \mathrm{E}-15$ & $2.303 \mathrm{E}-15$ & $2.303 \mathrm{E}-15$ & $2.303 \mathrm{E}-15$ \\
\hline tl204 & $3.747 \mathrm{E}-17$ & $1.570 \mathrm{E}-17$ & $1.499 \mathrm{E}-17$ & $1.248 \mathrm{E}-17$ & $1.039 \mathrm{E}-17$ \\
\hline tl205 & $1.112 \mathrm{E}-15$ & $1.112 \mathrm{E}-15$ & $1.112 \mathrm{E}-15$ & $1.112 \mathrm{E}-15$ & $1.112 \mathrm{E}-15$ \\
\hline tl207 & $2.683 \mathrm{E}-16$ & $1.229 \mathrm{E}-15$ & $1.256 \mathrm{E}-15$ & $1.486 \mathrm{E}-15$ & $1.693 \mathrm{E}-15$ \\
\hline tl208 & $2.383 \mathrm{E}-12$ & $1.552 \mathrm{E}-11$ & $1.612 \mathrm{E}-11$ & $1.811 \mathrm{E}-11$ & $1.979 \mathrm{E}-11$ \\
\hline $\operatorname{tm} 168$ & $8.555 \mathrm{E}-10$ & $2.131 \mathrm{E}-15$ & $1.067 \mathrm{E}-15$ & $7.042 \mathrm{E}-17$ & $4.651 \mathrm{E}-18$ \\
\hline $\operatorname{tm} 169$ & 8.867E-04 & $8.867 \mathrm{E}-04$ & $8.867 \mathrm{E}-04$ & 8.867E-04 & $8.867 \mathrm{E}-04$ \\
\hline $\operatorname{tm} 170$ & 4.412E-05 & $3.872 \mathrm{E}-09$ & $2.346 \mathrm{E}-09$ & $3.279 \mathrm{E}-10$ & $4.586 \mathrm{E}-11$ \\
\hline $\operatorname{tm} 171$ & $1.443 \mathrm{E}-04$ & $2.602 \mathrm{E}-05$ & $2.374 \mathrm{E}-05$ & $1.655 \mathrm{E}-05$ & $1.154 \mathrm{E}-05$ \\
\hline u230 & $2.785 \mathrm{E}-15$ & $0.000 \mathrm{E}+00$ & $0.000 \mathrm{E}+00$ & $0.000 \mathrm{E}+00$ & $0.000 \mathrm{E}+00$ \\
\hline u232 & 3.293E-04 & 8.011E-04 & $8.131 \mathrm{E}-04$ & 8.516E-04 & 8.794E-04 \\
\hline u233 & 4.636E-04 & $7.255 \mathrm{E}-04$ & $7.387 \mathrm{E}-04$ & $7.978 \mathrm{E}-04$ & $8.582 \mathrm{E}-04$ \\
\hline u234 & $1.148 \mathrm{E}+01$ & $1.772 \mathrm{E}+01$ & $1.806 \mathrm{E}+01$ & $1.936 \mathrm{E}+01$ & $2.065 \mathrm{E}+01$ \\
\hline u235 & $1.130 \mathrm{E}+03$ & $1.132 \mathrm{E}+03$ & $1.132 \mathrm{E}+03$ & $1.133 \mathrm{E}+03$ & $1.133 \mathrm{E}+03$ \\
\hline u236 & $2.683 \mathrm{E}+02$ & $2.732 \mathrm{E}+02$ & $2.735 \mathrm{E}+02$ & $2.745 \mathrm{E}+02$ & $2.754 \mathrm{E}+02$ \\
\hline u237 & $1.546 \mathrm{E}-04$ & $1.229 \mathrm{E}-04$ & $1.213 \mathrm{E}-04$ & $1.156 \mathrm{E}-04$ & $1.101 \mathrm{E}-04$ \\
\hline u238 & $9.213 \mathrm{E}+05$ & $9.213 \mathrm{E}+05$ & $9.213 \mathrm{E}+05$ & $9.213 \mathrm{E}+05$ & $9.213 \mathrm{E}+05$ \\
\hline u240 & $1.280 \mathrm{E}-12$ & $1.280 \mathrm{E}-12$ & $1.280 \mathrm{E}-12$ & $1.280 \mathrm{E}-12$ & $1.280 \mathrm{E}-12$ \\
\hline v 50 & $4.941 \mathrm{E}-17$ & $4.941 \mathrm{E}-17$ & $4.941 \mathrm{E}-17$ & $4.941 \mathrm{E}-17$ & $4.941 \mathrm{E}-17$ \\
\hline v 51 & $1.473 \mathrm{E}-15$ & $1.473 \mathrm{E}-15$ & $1.473 \mathrm{E}-15$ & $1.473 \mathrm{E}-15$ & $1.473 \mathrm{E}-15$ \\
\hline
\end{tabular}




\begin{tabular}{|c|c|c|c|c|c|}
\hline \multicolumn{6}{|c|}{ Table C-5. Continued } \\
\hline & $1 / 22 / 2009$ & $10 / 21 / 2013$ & $1 / 22 / 2014$ & $1 / 22 / 2015$ & $1 / 22 / 2016$ \\
\hline w182 & $3.135 \mathrm{E}-13$ & $3.143 \mathrm{E}-13$ & $3.143 \mathrm{E}-13$ & $3.143 \mathrm{E}-13$ & $3.143 \mathrm{E}-13$ \\
\hline w183 & $6.390 \mathrm{E}-13$ & $6.390 \mathrm{E}-13$ & $6.390 \mathrm{E}-13$ & $6.390 \mathrm{E}-13$ & $6.390 \mathrm{E}-13$ \\
\hline w184 & $5.173 \mathrm{E}-13$ & $5.173 \mathrm{E}-13$ & $5.173 \mathrm{E}-13$ & $5.173 \mathrm{E}-13$ & $5.173 \mathrm{E}-13$ \\
\hline w185 & $9.803 \mathrm{E}-17$ & $1.109 \mathrm{E}-23$ & $4.700 \mathrm{E}-24$ & $1.618 \mathrm{E}-25$ & $5.571 \mathrm{E}-27$ \\
\hline w186 & $2.308 \mathrm{E}-13$ & $2.308 \mathrm{E}-13$ & $2.308 \mathrm{E}-13$ & $2.308 \mathrm{E}-13$ & $2.308 \mathrm{E}-13$ \\
\hline xe124 & $1.822 \mathrm{E}-13$ & $1.822 \mathrm{E}-13$ & $1.822 \mathrm{E}-13$ & $1.822 \mathrm{E}-13$ & $1.822 \mathrm{E}-13$ \\
\hline xe126 & 2.893E-04 & $2.893 \mathrm{E}-04$ & 2.893E-04 & $2.893 \mathrm{E}-04$ & $2.893 \mathrm{E}-04$ \\
\hline xe127 & $3.717 \mathrm{E}-08$ & $1.730 \mathrm{E}-22$ & $2.944 \mathrm{E}-23$ & $2.821 \mathrm{E}-26$ & $2.703 \mathrm{E}-29$ \\
\hline xe128 & $6.262 \mathrm{E}+00$ & $6.262 \mathrm{E}+00$ & $6.262 \mathrm{E}+00$ & $6.262 \mathrm{E}+00$ & $6.262 \mathrm{E}+00$ \\
\hline xe129 & $3.391 \mathrm{E}-02$ & $3.397 \mathrm{E}-02$ & $3.397 \mathrm{E}-02$ & $3.398 \mathrm{E}-02$ & $3.400 \mathrm{E}-02$ \\
\hline xe129m & $1.866 \mathrm{E}-13$ & $0.000 \mathrm{E}+00$ & $0.000 \mathrm{E}+00$ & $0.000 \mathrm{E}+00$ & $0.000 \mathrm{E}+00$ \\
\hline xe130 & $7.958 \mathrm{E}+00$ & $7.958 \mathrm{E}+00$ & $7.958 \mathrm{E}+00$ & $7.958 \mathrm{E}+00$ & $7.958 \mathrm{E}+00$ \\
\hline xe131 & $6.277 \mathrm{E}+02$ & $6.277 \mathrm{E}+02$ & $6.277 \mathrm{E}+02$ & $6.277 \mathrm{E}+02$ & $6.277 \mathrm{E}+02$ \\
\hline xe131m & 9.963E-08 & $0.000 \mathrm{E}+00$ & $0.000 \mathrm{E}+00$ & $0.000 \mathrm{E}+00$ & $0.000 \mathrm{E}+00$ \\
\hline xe132 & $9.929 \mathrm{E}-15$ & $9.929 \mathrm{E}-15$ & $9.929 \mathrm{E}-15$ & $9.929 \mathrm{E}-15$ & $9.929 \mathrm{E}-15$ \\
\hline xe132 & $1.444 \mathrm{E}+03$ & $1.444 \mathrm{E}+03$ & $1.444 \mathrm{E}+03$ & $1.444 \mathrm{E}+03$ & $1.444 \mathrm{E}+03$ \\
\hline xe133 & $1.015 \mathrm{E}-14$ & $0.000 \mathrm{E}+00$ & $0.000 \mathrm{E}+00$ & $0.000 \mathrm{E}+00$ & $0.000 \mathrm{E}+00$ \\
\hline xe134 & $1.802 \mathrm{E}+03$ & $1.802 \mathrm{E}+03$ & $1.802 \mathrm{E}+03$ & $1.802 \mathrm{E}+03$ & $1.802 \mathrm{E}+03$ \\
\hline xe136 & $2.591 \mathrm{E}+03$ & $2.591 \mathrm{E}+03$ & $2.591 \mathrm{E}+03$ & $2.591 \mathrm{E}+03$ & $2.591 \mathrm{E}+03$ \\
\hline y 88 & $2.283 \mathrm{E}-05$ & $2.923 \mathrm{E}-10$ & $1.597 \mathrm{E}-10$ & $1.489 \mathrm{E}-11$ & $1.388 \mathrm{E}-12$ \\
\hline y 89 & $2.833 \mathrm{E}+02$ & $2.838 \mathrm{E}+02$ & $2.838 \mathrm{E}+02$ & $2.838 \mathrm{E}+02$ & $2.838 \mathrm{E}+02$ \\
\hline y 89m & $1.882 \mathrm{E}-10$ & $8.921 \mathrm{E}-21$ & $2.490 \mathrm{E}-21$ & $1.666 \mathrm{E}-23$ & $1.115 \mathrm{E}-25$ \\
\hline у 90 & 8.403E-02 & $7.500 \mathrm{E}-02$ & 7.454E-02 & 7.274E-02 & 7.102E-02 \\
\hline у 91 & $1.522 \mathrm{E}+00$ & $1.845 \mathrm{E}-09$ & $6.132 \mathrm{E}-10$ & $8.124 \mathrm{E}-12$ & $1.076 \mathrm{E}-13$ \\
\hline yb168 & $1.711 \mathrm{E}-12$ & $1.797 \mathrm{E}-12$ & $1.797 \mathrm{E}-12$ & $1.797 \mathrm{E}-12$ & $1.797 \mathrm{E}-12$ \\
\hline yb169 & $2.366 \mathrm{E}-12$ & $1.203 \mathrm{E}-28$ & $1.607 \mathrm{E}-29$ & $5.946 \mathrm{E}-33$ & $0.000 \mathrm{E}+00$ \\
\hline yb170 & $5.288 \mathrm{E}-04$ & $5.729 \mathrm{E}-04$ & 5.729E-04 & $5.729 \mathrm{E}-04$ & $5.729 \mathrm{E}-04$ \\
\hline yb171 & $1.662 \mathrm{E}-04$ & $2.845 \mathrm{E}-04$ & $2.867 \mathrm{E}-04$ & $2.940 \mathrm{E}-04$ & $2.989 \mathrm{E}-04$ \\
\hline yb172 & $3.556 \mathrm{E}-04$ & $3.556 \mathrm{E}-04$ & $3.556 \mathrm{E}-04$ & $3.556 \mathrm{E}-04$ & $3.556 \mathrm{E}-04$ \\
\hline yb176 & $9.830 \mathrm{E}-17$ & $9.830 \mathrm{E}-17$ & $9.830 \mathrm{E}-17$ & $9.830 \mathrm{E}-17$ & $9.830 \mathrm{E}-17$ \\
\hline zn 64 & $3.477 \mathrm{E}-16$ & $3.477 \mathrm{E}-16$ & $3.477 \mathrm{E}-16$ & $3.477 \mathrm{E}-16$ & $3.477 \mathrm{E}-16$ \\
\hline zn 66 & $1.264 \mathrm{E}-04$ & $1.264 \mathrm{E}-04$ & $1.264 \mathrm{E}-04$ & $1.264 \mathrm{E}-04$ & $1.264 \mathrm{E}-04$ \\
\hline zn 67 & $3.535 \mathrm{E}-04$ & $3.535 \mathrm{E}-04$ & $3.535 \mathrm{E}-04$ & $3.535 \mathrm{E}-04$ & $3.535 \mathrm{E}-04$ \\
\hline zn 68 & $7.075 \mathrm{E}-04$ & $7.075 \mathrm{E}-04$ & 7.075E-04 & $7.075 \mathrm{E}-04$ & 7.075E-04 \\
\hline zn 70 & $3.534 \mathrm{E}-03$ & $3.534 \mathrm{E}-03$ & $3.534 \mathrm{E}-03$ & $3.534 \mathrm{E}-03$ & $3.534 \mathrm{E}-03$ \\
\hline zr 88 & $7.089 \mathrm{E}-12$ & $3.938 \mathrm{E}-18$ & $1.818 \mathrm{E}-18$ & $8.755 \mathrm{E}-20$ & $4.214 \mathrm{E}-21$ \\
\hline $\operatorname{zr} 90$ & $1.778 \mathrm{E}+01$ & $5.356 \mathrm{E}+01$ & $5.537 \mathrm{E}+01$ & $6.235 \mathrm{E}+01$ & $6.916 \mathrm{E}+01$ \\
\hline zr 91 & $4.130 \mathrm{E}+02$ & $4.145 \mathrm{E}+02$ & $4.145 \mathrm{E}+02$ & $4.145 \mathrm{E}+02$ & $4.145 \mathrm{E}+02$ \\
\hline
\end{tabular}




\begin{tabular}{|l|r|r|r|r|r|}
\hline \multicolumn{7}{|c|}{ Table C-5. Continued } \\
\hline & $\mathbf{1 / 2 2 / 2 0 0 9}$ & $\mathbf{1 0 / 2 1 / 2 0 1 3}$ & $\mathbf{1 / 2 2 / 2 0 1 4}$ & $\mathbf{1 / 2 2 / 2 0 1 5}$ & $\mathbf{1 / 2 2 / 2 0 1 6}$ \\
\hline zr 92 & $5.014 \mathrm{E}+02$ & $5.014 \mathrm{E}+02$ & $5.014 \mathrm{E}+02$ & $5.014 \mathrm{E}+02$ & $5.014 \mathrm{E}+02$ \\
\hline zr 93 & $6.217 \mathrm{E}+02$ & $6.217 \mathrm{E}+02$ & $6.217 \mathrm{E}+02$ & $6.217 \mathrm{E}+02$ & $6.217 \mathrm{E}+02$ \\
\hline zr 94 & $7.115 \mathrm{E}+02$ & $7.115 \mathrm{E}+02$ & $7.115 \mathrm{E}+02$ & $7.115 \mathrm{E}+02$ & $7.115 \mathrm{E}+02$ \\
\hline zr 95 & $4.170 \mathrm{E}+00$ & $2.970 \mathrm{E}-08$ & $1.085 \mathrm{E}-08$ & $2.087 \mathrm{E}-10$ & $4.015 \mathrm{E}-12$ \\
\hline zr 96 & $8.303 \mathrm{E}+02$ & $8.303 \mathrm{E}+02$ & $8.303 \mathrm{E}+02$ & $8.303 \mathrm{E}+02$ & $8.303 \mathrm{E}+02$ \\
\hline Total & $\mathbf{1 . 1 3 4 1 E + 0 6}$ & $\mathbf{1 . 1 3 4 1 E + 0 6}$ & $\mathbf{1 . 1 3 4 1 E}+\mathbf{0 6}$ & $\mathbf{1 . 1 3 4 1 E + 0 6}$ & $\mathbf{1 . 1 3 4 1 E + 0 6}$ \\
\hline
\end{tabular}


Table C-6. Activity in Fuel Rod B4 (high Pu loading) in Ci per MTIHM

\begin{tabular}{|c|c|c|c|c|c|}
\hline & $1 / 22 / 2009$ & $10 / 21 / 2013$ & $1 / 22 / 2014$ & $1 / 22 / 2015$ & $1 / 22 / 2016$ \\
\hline ac225 & $6.790 \mathrm{E}-08$ & 7.054E-08 & 7.067E-08 & 7.153E-08 & $7.232 \mathrm{E}-08$ \\
\hline ac226 & $1.246 \mathrm{E}-15$ & $0.000 \mathrm{E}+00$ & $0.000 \mathrm{E}+00$ & $0.000 \mathrm{E}+00$ & $0.000 \mathrm{E}+00$ \\
\hline ac227 & $5.339 \mathrm{E}-08$ & $2.261 \mathrm{E}-07$ & $2.356 \mathrm{E}-07$ & $2.728 \mathrm{E}-07$ & $3.102 \mathrm{E}-07$ \\
\hline ac228 & $8.712 \mathrm{E}-12$ & $1.552 \mathrm{E}-11$ & $1.585 \mathrm{E}-11$ & $1.713 \mathrm{E}-11$ & $1.837 \mathrm{E}-11$ \\
\hline $\operatorname{ag} 105$ & $2.859 \mathrm{E}-10$ & $6.631 \mathrm{E}-23$ & $1.392 \mathrm{E}-23$ & $3.036 \mathrm{E}-26$ & $6.624 \mathrm{E}-29$ \\
\hline $\operatorname{ag} 106 \mathrm{~m}$ & $1.066 \mathrm{E}-15$ & $0.000 \mathrm{E}+00$ & $0.000 \mathrm{E}+00$ & $0.000 \mathrm{E}+00$ & $0.000 \mathrm{E}+00$ \\
\hline $\operatorname{ag} 108$ & $5.270 \mathrm{E}-04$ & $5.231 \mathrm{E}-04$ & $5.229 \mathrm{E}-04$ & $5.220 \mathrm{E}-04$ & $5.212 \mathrm{E}-04$ \\
\hline $\operatorname{ag} 108 m$ & $6.058 \mathrm{E}-03$ & $6.013 \mathrm{E}-03$ & $6.010 \mathrm{E}-03$ & $6.001 \mathrm{E}-03$ & $5.991 \mathrm{E}-03$ \\
\hline $\operatorname{ag} 109 m$ & $5.738 \mathrm{E}-01$ & 4.247E-02 & $3.693 \mathrm{E}-02$ & $2.134 \mathrm{E}-02$ & $1.233 \mathrm{E}-02$ \\
\hline $\operatorname{ag} 110$ & $9.709 \mathrm{E}+01$ & 7.912E-01 & $6.112 \mathrm{E}-01$ & $2.220 \mathrm{E}-01$ & $8.058 \mathrm{E}-02$ \\
\hline $\operatorname{ag} 110 \mathrm{~m}$ & $7.140 \mathrm{E}+03$ & $5.817 \mathrm{E}+01$ & $4.494 \mathrm{E}+01$ & $1.632 \mathrm{E}+01$ & $5.926 \mathrm{E}+00$ \\
\hline ag111 & $2.698 \mathrm{E}-06$ & $0.000 \mathrm{E}+00$ & $0.000 \mathrm{E}+00$ & $0.000 \mathrm{E}+00$ & $0.000 \mathrm{E}+00$ \\
\hline am241 & $1.477 \mathrm{E}+03$ & $5.128 \mathrm{E}+03$ & $5.300 \mathrm{E}+03$ & $5.955 \mathrm{E}+03$ & $6.578 \mathrm{E}+03$ \\
\hline $\operatorname{am} 242$ & $4.557 \mathrm{E}+01$ & $4.452 \mathrm{E}+01$ & $4.446 \mathrm{E}+01$ & $4.424 \mathrm{E}+01$ & $4.403 \mathrm{E}+01$ \\
\hline am242m & $4.578 \mathrm{E}+01$ & $4.473 \mathrm{E}+01$ & $4.467 \mathrm{E}+01$ & $4.445 \mathrm{E}+01$ & $4.423 \mathrm{E}+01$ \\
\hline am243 & $7.688 \mathrm{E}+01$ & $7.681 \mathrm{E}+01$ & $7.681 \mathrm{E}+01$ & $7.681 \mathrm{E}+01$ & $7.681 \mathrm{E}+01$ \\
\hline am244 & $3.712 \mathrm{E}-09$ & $2.576 \mathrm{E}-09$ & $2.526 \mathrm{E}-09$ & $2.339 \mathrm{E}-09$ & $2.165 \mathrm{E}-09$ \\
\hline am245 & $1.968 \mathrm{E}-07$ & 4.611E-09 & $3.770 \mathrm{E}-09$ & $1.710 \mathrm{E}-09$ & $7.754 \mathrm{E}-10$ \\
\hline am246m & $3.481 \mathrm{E}-12$ & $3.478 \mathrm{E}-12$ & $3.478 \mathrm{E}-12$ & $3.478 \mathrm{E}-12$ & $3.477 \mathrm{E}-12$ \\
\hline ar 37 & $1.224 \mathrm{E}-14$ & $1.582 \mathrm{E}-29$ & $0.000 \mathrm{E}+00$ & $0.000 \mathrm{E}+00$ & $0.000 \mathrm{E}+00$ \\
\hline ar 39 & $4.098 \mathrm{E}-15$ & $4.048 \mathrm{E}-15$ & $4.045 \mathrm{E}-15$ & $4.035 \mathrm{E}-15$ & $4.025 \mathrm{E}-15$ \\
\hline $\operatorname{ar} 42$ & $6.730 \mathrm{E}-21$ & $6.088 \mathrm{E}-21$ & $6.056 \mathrm{E}-21$ & $5.930 \mathrm{E}-21$ & $5.806 \mathrm{E}-21$ \\
\hline as 73 & $3.489 \mathrm{E}-06$ & $1.112 \mathrm{E}-12$ & $4.981 \mathrm{E}-13$ & $2.133 \mathrm{E}-14$ & $9.134 \mathrm{E}-16$ \\
\hline as 74 & $1.949 \mathrm{E}-07$ & $0.000 \mathrm{E}+00$ & $0.000 \mathrm{E}+00$ & $0.000 \mathrm{E}+00$ & $0.000 \mathrm{E}+00$ \\
\hline at217 & $6.790 \mathrm{E}-08$ & $7.054 \mathrm{E}-08$ & 7.067E-08 & 7.153E-08 & 7.232E-08 \\
\hline at218 & $2.880 \mathrm{E}-13$ & $1.802 \mathrm{E}-12$ & $1.935 \mathrm{E}-12$ & $2.519 \mathrm{E}-12$ & $3.208 \mathrm{E}-12$ \\
\hline au194 & $8.394 \mathrm{E}-21$ & $8.328 \mathrm{E}-21$ & $8.322 \mathrm{E}-21$ & $8.309 \mathrm{E}-21$ & $8.295 \mathrm{E}-21$ \\
\hline au195 & $8.480 \mathrm{E}-16$ & $1.334 \mathrm{E}-18$ & $9.431 \mathrm{E}-19$ & $2.423 \mathrm{E}-19$ & $6.221 \mathrm{E}-20$ \\
\hline ba131 & 7.767E-18 & $0.000 \mathrm{E}+00$ & $0.000 \mathrm{E}+00$ & $0.000 \mathrm{E}+00$ & $0.000 \mathrm{E}+00$ \\
\hline ba131 & $4.131 \mathrm{E}-13$ & $0.000 \mathrm{E}+00$ & $0.000 \mathrm{E}+00$ & $0.000 \mathrm{E}+00$ & $0.000 \mathrm{E}+00$ \\
\hline ba133 & $5.380 \mathrm{E}-02$ & $3.935 \mathrm{E}-02$ & $3.870 \mathrm{E}-02$ & $3.623 \mathrm{E}-02$ & 3.392E-02 \\
\hline ba136m & $9.788 \mathrm{E}-03$ & $0.000 \mathrm{E}+00$ & $0.000 \mathrm{E}+00$ & $0.000 \mathrm{E}+00$ & $0.000 \mathrm{E}+00$ \\
\hline ba137m & $1.422 \mathrm{E}+05$ & $1.275 \mathrm{E}+05$ & $1.267 \mathrm{E}+05$ & $1.238 \mathrm{E}+05$ & $1.210 \mathrm{E}+05$ \\
\hline ba140 & $1.125 \mathrm{E}+00$ & $0.000 \mathrm{E}+00$ & $0.000 \mathrm{E}+00$ & $0.000 \mathrm{E}+00$ & $0.000 \mathrm{E}+00$ \\
\hline be 10 & $2.904 \mathrm{E}-06$ & $2.904 \mathrm{E}-06$ & $2.904 \mathrm{E}-06$ & $2.904 \mathrm{E}-06$ & $2.904 \mathrm{E}-06$ \\
\hline bi210 & $4.037 \mathrm{E}-09$ & 4.117E-09 & $4.158 \mathrm{E}-09$ & 4.371E-09 & 4.675E-09 \\
\hline bi211 & $4.960 \mathrm{E}-08$ & $2.266 \mathrm{E}-07$ & $2.314 \mathrm{E}-07$ & $2.733 \mathrm{E}-07$ & $3.107 \mathrm{E}-07$ \\
\hline bi212 & $2.661 \mathrm{E}-03$ & $1.676 \mathrm{E}-02$ & $1.738 \mathrm{E}-02$ & $1.951 \mathrm{E}-02$ & $2.129 \mathrm{E}-02$ \\
\hline
\end{tabular}




\begin{tabular}{|c|c|c|c|c|c|}
\hline \multicolumn{6}{|c|}{ Table C-6. Continued } \\
\hline & $1 / 22 / 2009$ & $10 / 21 / 2013$ & $1 / 22 / 2014$ & $1 / 22 / 2015$ & $1 / 22 / 2016$ \\
\hline bi213 & $6.790 \mathrm{E}-08$ & 7.054E-08 & 7.067E-08 & $7.146 \mathrm{E}-08$ & 7.232E-08 \\
\hline bi214 & $1.440 \mathrm{E}-09$ & 9.009E-09 & $9.676 \mathrm{E}-09$ & $1.260 \mathrm{E}-08$ & $1.604 \mathrm{E}-08$ \\
\hline bk247 & $5.867 \mathrm{E}-13$ & $5.853 \mathrm{E}-13$ & $5.852 \mathrm{E}-13$ & $5.849 \mathrm{E}-13$ & $5.846 \mathrm{E}-13$ \\
\hline bk248 & 3.712E-09 & $2.575 \mathrm{E}-09$ & $2.525 \mathrm{E}-09$ & $2.338 \mathrm{E}-09$ & $2.165 \mathrm{E}-09$ \\
\hline bk249 & $1.357 \mathrm{E}-02$ & $3.179 \mathrm{E}-04$ & $2.600 \mathrm{E}-04$ & $1.179 \mathrm{E}-04$ & $5.348 \mathrm{E}-05$ \\
\hline bk250 & $1.059 \mathrm{E}-08$ & $1.372 \mathrm{E}-10$ & $1.090 \mathrm{E}-10$ & $4.445 \mathrm{E}-11$ & $1.868 \mathrm{E}-11$ \\
\hline bk251 & $9.550 \mathrm{E}-12$ & $0.000 \mathrm{E}+00$ & $0.000 \mathrm{E}+00$ & $0.000 \mathrm{E}+00$ & $0.000 \mathrm{E}+00$ \\
\hline c 14 & $1.661 \mathrm{E}-03$ & $1.660 \mathrm{E}-03$ & $1.660 \mathrm{E}-03$ & $1.660 \mathrm{E}-03$ & $1.660 \mathrm{E}-03$ \\
\hline ca 41 & $3.428 \mathrm{E}-18$ & $3.428 \mathrm{E}-18$ & $3.428 \mathrm{E}-18$ & $3.428 \mathrm{E}-18$ & $3.428 \mathrm{E}-18$ \\
\hline ca 45 & $1.645 \mathrm{E}-13$ & $1.018 \mathrm{E}-16$ & $6.849 \mathrm{E}-17$ & $1.446 \mathrm{E}-17$ & $3.051 \mathrm{E}-18$ \\
\hline cd109 & $5.738 \mathrm{E}-01$ & 4.247E-02 & $3.693 \mathrm{E}-02$ & $2.134 \mathrm{E}-02$ & $1.233 \mathrm{E}-02$ \\
\hline cd113 & $1.561 \mathrm{E}-13$ & $1.561 \mathrm{E}-13$ & $1.561 \mathrm{E}-13$ & $1.561 \mathrm{E}-13$ & $1.561 \mathrm{E}-13$ \\
\hline cd113m & $1.261 \mathrm{E}+00$ & 9.993E-01 & 9.867E-01 & 9.392E-01 & 8.943E-01 \\
\hline cd115m & $1.818 \mathrm{E}+01$ & $3.566 \mathrm{E}-11$ & 8.394E-12 & $2.871 \mathrm{E}-14$ & $9.821 \mathrm{E}-17$ \\
\hline cd116 & $1.213 \mathrm{E}-15$ & $1.213 \mathrm{E}-15$ & $1.213 \mathrm{E}-15$ & $1.213 \mathrm{E}-15$ & $1.213 \mathrm{E}-15$ \\
\hline ce139 & $1.121 \mathrm{E}+01$ & $1.816 \mathrm{E}-03$ & $1.137 \mathrm{E}-03$ & $1.809 \mathrm{E}-04$ & $2.879 \mathrm{E}-05$ \\
\hline ce141 & $6.470 \mathrm{E}+03$ & $5.793 \mathrm{E}-13$ & 7.972E-14 & $3.324 \mathrm{E}-17$ & $1.386 \mathrm{E}-20$ \\
\hline ce144 & $6.690 \mathrm{E}+05$ & $9.874 \mathrm{E}+03$ & $7.873 \mathrm{E}+03$ & $3.240 \mathrm{E}+03$ & $1.333 \mathrm{E}+03$ \\
\hline cf248 & $2.355 \mathrm{E}-08$ & $6.423 \mathrm{E}-10$ & $5.294 \mathrm{E}-10$ & $2.479 \mathrm{E}-10$ & $1.161 \mathrm{E}-10$ \\
\hline cf249 & $3.619 \mathrm{E}-05$ & $6.869 \mathrm{E}-05$ & $6.882 \mathrm{E}-05$ & $6.902 \mathrm{E}-05$ & $6.902 \mathrm{E}-05$ \\
\hline cf 250 & 4.601E-04 & $3.578 \mathrm{E}-04$ & $3.531 \mathrm{E}-04$ & 3.349E-04 & $3.176 \mathrm{E}-04$ \\
\hline cf251 & $3.306 \mathrm{E}-06$ & $3.294 \mathrm{E}-06$ & $3.293 \mathrm{E}-06$ & $3.290 \mathrm{E}-06$ & $3.288 \mathrm{E}-06$ \\
\hline cf 252 & $3.410 \mathrm{E}-04$ & $9.834 \mathrm{E}-05$ & $9.200 \mathrm{E}-05$ & $7.080 \mathrm{E}-05$ & $5.449 \mathrm{E}-05$ \\
\hline cf 253 & $1.071 \mathrm{E}-09$ & $0.000 \mathrm{E}+00$ & $0.000 \mathrm{E}+00$ & $0.000 \mathrm{E}+00$ & $0.000 \mathrm{E}+00$ \\
\hline cf 254 & $1.578 \mathrm{E}-08$ & $3.760 \mathrm{E}-17$ & $1.295 \mathrm{E}-17$ & $1.978 \mathrm{E}-19$ & $3.021 \mathrm{E}-21$ \\
\hline cl 36 & $1.077 \mathrm{E}-16$ & $1.077 \mathrm{E}-16$ & $1.077 \mathrm{E}-16$ & $1.077 \mathrm{E}-16$ & $1.077 \mathrm{E}-16$ \\
\hline cm240 & $3.951 \mathrm{E}-07$ & $1.883 \mathrm{E}-26$ & $1.730 \mathrm{E}-27$ & $0.000 \mathrm{E}+00$ & $0.000 \mathrm{E}+00$ \\
\hline cm241 & $4.618 \mathrm{E}-04$ & $5.745 \mathrm{E}-20$ & $8.051 \mathrm{E}-21$ & $3.596 \mathrm{E}-24$ & $1.607 \mathrm{E}-27$ \\
\hline cm242 & $7.285 \mathrm{E}+04$ & $8.262 \mathrm{E}+01$ & $6.763 \mathrm{E}+01$ & $4.317 \mathrm{E}+01$ & $3.785 \mathrm{E}+01$ \\
\hline cm243 & $9.398 \mathrm{E}+01$ & $8.394 \mathrm{E}+01$ & $8.348 \mathrm{E}+01$ & $8.150 \mathrm{E}+01$ & $7.959 \mathrm{E}+01$ \\
\hline cm244 & $1.200 \mathrm{E}+04$ & $1.001 \mathrm{E}+04$ & $9.914 \mathrm{E}+03$ & $9.537 \mathrm{E}+03$ & $9.180 \mathrm{E}+03$ \\
\hline cm245 & $2.423 \mathrm{E}+00$ & $2.423 \mathrm{E}+00$ & $2.423 \mathrm{E}+00$ & $2.422 \mathrm{E}+00$ & $2.422 \mathrm{E}+00$ \\
\hline cm246 & $2.870 \mathrm{E}-01$ & $2.868 \mathrm{E}-01$ & $2.868 \mathrm{E}-01$ & $2.868 \mathrm{E}-01$ & $2.868 \mathrm{E}-01$ \\
\hline cm247 & $1.326 \mathrm{E}-06$ & $1.326 \mathrm{E}-06$ & $1.326 \mathrm{E}-06$ & $1.326 \mathrm{E}-06$ & $1.326 \mathrm{E}-06$ \\
\hline cm248 & $3.789 \mathrm{E}-06$ & $3.791 \mathrm{E}-06$ & $3.791 \mathrm{E}-06$ & 3.791E-06 & $3.791 \mathrm{E}-06$ \\
\hline cm249 & $3.327 \mathrm{E}-12$ & $0.000 \mathrm{E}+00$ & $0.000 \mathrm{E}+00$ & $0.000 \mathrm{E}+00$ & $0.000 \mathrm{E}+00$ \\
\hline cm250 & $1.933 \mathrm{E}-11$ & $1.933 \mathrm{E}-11$ & $1.933 \mathrm{E}-11$ & $1.932 \mathrm{E}-11$ & $1.932 \mathrm{E}-11$ \\
\hline co 56 & $4.004 \mathrm{E}-21$ & $7.041 \mathrm{E}-28$ & $3.057 \mathrm{E}-28$ & $1.155 \mathrm{E}-29$ & $0.000 \mathrm{E}+00$ \\
\hline
\end{tabular}




\begin{tabular}{|c|c|c|c|c|c|}
\hline \multicolumn{6}{|c|}{ Table C-6. Continued } \\
\hline & $1 / 22 / 2009$ & $10 / 21 / 2013$ & $1 / 22 / 2014$ & $1 / 22 / 2015$ & $1 / 22 / 2016$ \\
\hline $\cos 57$ & $9.246 \mathrm{E}-16$ & $1.112 \mathrm{E}-17$ & $8.771 \mathrm{E}-18$ & $3.458 \mathrm{E}-18$ & $1.363 \mathrm{E}-18$ \\
\hline $\cos 58$ & $1.933 \mathrm{E}-14$ & $8.394 \mathrm{E}-22$ & $3.380 \mathrm{E}-22$ & $9.511 \mathrm{E}-24$ & $2.677 \mathrm{E}-25$ \\
\hline $\cos 60$ & $1.112 \mathrm{E}-11$ & $5.960 \mathrm{E}-12$ & $5.764 \mathrm{E}-12$ & $5.055 \mathrm{E}-12$ & 4.432E-12 \\
\hline $\operatorname{co~} 60 \mathrm{~m}$ & $3.088 \mathrm{E}-21$ & $3.088 \mathrm{E}-21$ & $3.088 \mathrm{E}-21$ & $3.088 \mathrm{E}-21$ & $3.088 \mathrm{E}-21$ \\
\hline cr 51 & $1.034 \mathrm{E}-14$ & $0.000 \mathrm{E}+00$ & $0.000 \mathrm{E}+00$ & $0.000 \mathrm{E}+00$ & $0.000 \mathrm{E}+00$ \\
\hline cs131 & $2.098 \mathrm{E}-10$ & $0.000 \mathrm{E}+00$ & $0.000 \mathrm{E}+00$ & $0.000 \mathrm{E}+00$ & $0.000 \mathrm{E}+00$ \\
\hline cs132 & $2.698 \mathrm{E}-11$ & $0.000 \mathrm{E}+00$ & $0.000 \mathrm{E}+00$ & $0.000 \mathrm{E}+00$ & $0.000 \mathrm{E}+00$ \\
\hline $\operatorname{cs134}$ & $2.080 \mathrm{E}+05$ & $4.231 \mathrm{E}+04$ & $3.884 \mathrm{E}+04$ & $2.777 \mathrm{E}+04$ & $1.986 \mathrm{E}+04$ \\
\hline cs135 & $1.009 \mathrm{E}+00$ & $1.009 \mathrm{E}+00$ & $1.009 \mathrm{E}+00$ & $1.009 \mathrm{E}+00$ & $1.009 \mathrm{E}+00$ \\
\hline cs136 & 8.837E-02 & $0.000 \mathrm{E}+00$ & $0.000 \mathrm{E}+00$ & $0.000 \mathrm{E}+00$ & $0.000 \mathrm{E}+00$ \\
\hline $\operatorname{cs137}$ & $1.501 \mathrm{E}+05$ & $1.346 \mathrm{E}+05$ & $1.338 \mathrm{E}+05$ & $1.308 \mathrm{E}+05$ & $1.278 \mathrm{E}+05$ \\
\hline dy159 & $1.675 \mathrm{E}-03$ & $4.086 \mathrm{E}-07$ & $2.615 \mathrm{E}-07$ & 4.534E-08 & $7.860 \mathrm{E}-09$ \\
\hline er169 & $9.643 \mathrm{E}-09$ & $0.000 \mathrm{E}+00$ & $0.000 \mathrm{E}+00$ & $0.000 \mathrm{E}+00$ & $0.000 \mathrm{E}+00$ \\
\hline es252 & $1.752 \mathrm{E}-12$ & $1.372 \mathrm{E}-13$ & $1.197 \mathrm{E}-13$ & $7.001 \mathrm{E}-14$ & 4.094E-14 \\
\hline es253 & $2.241 \mathrm{E}-08$ & $0.000 \mathrm{E}+00$ & $0.000 \mathrm{E}+00$ & $0.000 \mathrm{E}+00$ & $0.000 \mathrm{E}+00$ \\
\hline es254 & $1.058 \mathrm{E}-08$ & $1.357 \mathrm{E}-10$ & $1.073 \mathrm{E}-10$ & $4.288 \mathrm{E}-11$ & $1.713 \mathrm{E}-11$ \\
\hline es255 & $1.193 \mathrm{E}-10$ & $9.306 \mathrm{E}-24$ & $1.843 \mathrm{E}-24$ & $3.197 \mathrm{E}-27$ & $5.546 \mathrm{E}-30$ \\
\hline eu147 & $1.207 \mathrm{E}-13$ & $0.000 \mathrm{E}+00$ & $0.000 \mathrm{E}+00$ & $0.000 \mathrm{E}+00$ & $0.000 \mathrm{E}+00$ \\
\hline eu149 & $7.470 \mathrm{E}-07$ & $1.861 \mathrm{E}-12$ & $9.313 \mathrm{E}-13$ & $6.150 \mathrm{E}-14$ & $4.061 \mathrm{E}-15$ \\
\hline eu152 & $7.292 \mathrm{E}+00$ & $5.716 \mathrm{E}+00$ & $5.642 \mathrm{E}+00$ & $5.361 \mathrm{E}+00$ & $5.093 \mathrm{E}+00$ \\
\hline eu154 & $1.691 \mathrm{E}+04$ & $1.154 \mathrm{E}+04$ & $1.131 \mathrm{E}+04$ & $1.044 \mathrm{E}+04$ & $9.623 \mathrm{E}+03$ \\
\hline eu155 & $7.377 \mathrm{E}+03$ & $3.693 \mathrm{E}+03$ & $3.558 \mathrm{E}+03$ & $3.076 \mathrm{E}+03$ & $2.658 \mathrm{E}+03$ \\
\hline eu156 & $2.333 \mathrm{E}+00$ & $0.000 \mathrm{E}+00$ & $0.000 \mathrm{E}+00$ & $0.000 \mathrm{E}+00$ & $0.000 \mathrm{E}+00$ \\
\hline fe 55 & $4.394 \mathrm{E}-13$ & $1.326 \mathrm{E}-13$ & $1.243 \mathrm{E}-13$ & $9.656 \mathrm{E}-14$ & $7.503 \mathrm{E}-14$ \\
\hline fe 59 & $4.748 \mathrm{E}-14$ & $8.949 \mathrm{E}-26$ & $2.102 \mathrm{E}-26$ & $7.133 \mathrm{E}-29$ & $0.000 \mathrm{E}+00$ \\
\hline fe 60 & $3.088 \mathrm{E}-21$ & $3.088 \mathrm{E}-21$ & $3.088 \mathrm{E}-21$ & $3.088 \mathrm{E}-21$ & $3.088 \mathrm{E}-21$ \\
\hline fr221 & $6.790 \mathrm{E}-08$ & 7.054E-08 & 7.067E-08 & 7.153E-08 & 7.232E-08 \\
\hline fr 223 & $7.371 \mathrm{E}-10$ & $3.121 \mathrm{E}-09$ & $3.251 \mathrm{E}-09$ & $3.764 \mathrm{E}-09$ & $4.280 \mathrm{E}-09$ \\
\hline ga 68 & $1.557 \mathrm{E}-15$ & $1.849 \mathrm{E}-17$ & $1.458 \mathrm{E}-17$ & $5.730 \mathrm{E}-18$ & $2.252 \mathrm{E}-18$ \\
\hline gd151 & $6.426 \mathrm{E}-04$ & $3.988 \mathrm{E}-08$ & $2.371 \mathrm{E}-08$ & 3.082E-09 & $4.006 \mathrm{E}-10$ \\
\hline gd152 & $1.865 \mathrm{E}-12$ & $1.920 \mathrm{E}-12$ & $1.923 \mathrm{E}-12$ & $1.933 \mathrm{E}-12$ & $1.942 \mathrm{E}-12$ \\
\hline gd153 & $1.227 \mathrm{E}+01$ & $8.295 \mathrm{E}-02$ & $6.344 \mathrm{E}-02$ & $2.215 \mathrm{E}-02$ & $7.734 \mathrm{E}-03$ \\
\hline ge 68 & $1.557 \mathrm{E}-15$ & $1.849 \mathrm{E}-17$ & $1.458 \mathrm{E}-17$ & $5.729 \mathrm{E}-18$ & $2.252 \mathrm{E}-18$ \\
\hline ge 71 & $7.860 \mathrm{E}-11$ & $0.000 \mathrm{E}+00$ & $0.000 \mathrm{E}+00$ & $0.000 \mathrm{E}+00$ & $0.000 \mathrm{E}+00$ \\
\hline ge 73m & $3.489 \mathrm{E}-06$ & $1.112 \mathrm{E}-12$ & $4.981 \mathrm{E}-13$ & $2.133 \mathrm{E}-14$ & $9.134 \mathrm{E}-16$ \\
\hline h3 & $7.885 \mathrm{E}+02$ & $6.037 \mathrm{E}+02$ & $5.951 \mathrm{E}+02$ & $5.626 \mathrm{E}+02$ & $5.318 \mathrm{E}+02$ \\
\hline hf172 & $1.642 \mathrm{E}-19$ & $2.829 \mathrm{E}-20$ & $2.574 \mathrm{E}-20$ & $1.777 \mathrm{E}-20$ & $1.227 \mathrm{E}-20$ \\
\hline hf175 & $1.029 \mathrm{E}-11$ & 3.627E-19 & $1.444 \mathrm{E}-19$ & $3.890 \mathrm{E}-21$ & $1.048 \mathrm{E}-22$ \\
\hline
\end{tabular}




\begin{tabular}{|c|c|c|c|c|c|}
\hline \multicolumn{6}{|c|}{ Table C-6. Continued } \\
\hline & $1 / 22 / 2009$ & $10 / 21 / 2013$ & $1 / 22 / 2014$ & $1 / 22 / 2015$ & $1 / 22 / 2016$ \\
\hline hf177m & $2.821 \mathrm{E}-14$ & $1.580 \mathrm{E}-17$ & $1.057 \mathrm{E}-17$ & $2.185 \mathrm{E}-18$ & $4.515 \mathrm{E}-19$ \\
\hline hf181 & $3.975 \mathrm{E}-13$ & $1.961 \mathrm{E}-25$ & $4.286 \mathrm{E}-26$ & $1.096 \mathrm{E}-28$ & $0.000 \mathrm{E}+00$ \\
\hline hf182 & $2.181 \mathrm{E}-19$ & $2.181 \mathrm{E}-19$ & $2.181 \mathrm{E}-19$ & $2.181 \mathrm{E}-19$ & $2.181 \mathrm{E}-19$ \\
\hline hg194 & $8.388 \mathrm{E}-21$ & $8.328 \mathrm{E}-21$ & $8.322 \mathrm{E}-21$ & $8.309 \mathrm{E}-21$ & $8.295 \mathrm{E}-21$ \\
\hline hg203 & $6.021 \mathrm{E}-14$ & $3.830 \mathrm{E}-25$ & $9.603 \mathrm{E}-26$ & 4.209E-28 & $0.000 \mathrm{E}+00$ \\
\hline hg206 & $7.668 \mathrm{E}-17$ & $7.820 \mathrm{E}-17$ & $7.899 \mathrm{E}-17$ & $8.302 \mathrm{E}-17$ & $8.877 \mathrm{E}-17$ \\
\hline ho163 & $1.180 \mathrm{E}-07$ & $1.180 \mathrm{E}-07$ & $1.180 \mathrm{E}-07$ & $1.180 \mathrm{E}-07$ & $1.179 \mathrm{E}-07$ \\
\hline ho166m & $1.234 \mathrm{E}-03$ & $1.230 \mathrm{E}-03$ & $1.230 \mathrm{E}-03$ & $1.229 \mathrm{E}-03$ & $1.228 \mathrm{E}-03$ \\
\hline i125 & $2.065 \mathrm{E}-05$ & $3.407 \mathrm{E}-14$ & $1.151 \mathrm{E}-14$ & $1.627 \mathrm{E}-16$ & $2.298 \mathrm{E}-18$ \\
\hline i126 & $1.619 \mathrm{E}-06$ & $0.000 \mathrm{E}+00$ & $0.000 \mathrm{E}+00$ & $0.000 \mathrm{E}+00$ & $0.000 \mathrm{E}+00$ \\
\hline i129 & $5.625 \mathrm{E}-02$ & $5.625 \mathrm{E}-02$ & $5.625 \mathrm{E}-02$ & $5.625 \mathrm{E}-02$ & $5.625 \mathrm{E}-02$ \\
\hline i131 & $1.629 \mathrm{E}-04$ & $0.000 \mathrm{E}+00$ & $0.000 \mathrm{E}+00$ & $0.000 \mathrm{E}+00$ & $0.000 \mathrm{E}+00$ \\
\hline in113m & $7.675 \mathrm{E}-07$ & $2.250 \mathrm{E}-11$ & $1.285 \mathrm{E}-11$ & $1.426 \mathrm{E}-12$ & $1.583 \mathrm{E}-13$ \\
\hline in114 & $7.186 \mathrm{E}-03$ & $2.086 \mathrm{E}-13$ & $5.674 \mathrm{E}-14$ & $3.424 \mathrm{E}-16$ & $2.067 \mathrm{E}-18$ \\
\hline in $114 m$ & $7.424 \mathrm{E}-03$ & $2.156 \mathrm{E}-13$ & $5.864 \mathrm{E}-14$ & $3.539 \mathrm{E}-16$ & $2.136 \mathrm{E}-18$ \\
\hline in115 & $2.522 \mathrm{E}-11$ & $2.522 \mathrm{E}-11$ & $2.522 \mathrm{E}-11$ & $2.522 \mathrm{E}-11$ & $2.522 \mathrm{E}-11$ \\
\hline in115m & $1.932 \mathrm{E}-03$ & $3.788 \mathrm{E}-15$ & $8.916 \mathrm{E}-16$ & $3.050 \mathrm{E}-18$ & $1.044 \mathrm{E}-20$ \\
\hline ir190 & $1.312 \mathrm{E}-22$ & $0.000 \mathrm{E}+00$ & $0.000 \mathrm{E}+00$ & $0.000 \mathrm{E}+00$ & $0.000 \mathrm{E}+00$ \\
\hline ir191m & $6.548 \mathrm{E}-19$ & $0.000 \mathrm{E}+00$ & $0.000 \mathrm{E}+00$ & $0.000 \mathrm{E}+00$ & $0.000 \mathrm{E}+00$ \\
\hline ir192 & $2.143 \mathrm{E}-12$ & $1.839 \mathrm{E}-19$ & $7.681 \mathrm{E}-20$ & $2.496 \mathrm{E}-21$ & $8.110 \mathrm{E}-23$ \\
\hline ir193m & $1.077 \mathrm{E}-19$ & $0.000 \mathrm{E}+00$ & $0.000 \mathrm{E}+00$ & $0.000 \mathrm{E}+00$ & $0.000 \mathrm{E}+00$ \\
\hline ir194 & $1.501 \mathrm{E}-18$ & 8.672E-19 & $8.421 \mathrm{E}-19$ & $7.503 \mathrm{E}-19$ & $6.684 \mathrm{E}-19$ \\
\hline k 40 & $4.982 \mathrm{E}-19$ & $4.982 \mathrm{E}-19$ & $4.982 \mathrm{E}-19$ & 4.982E-19 & $4.982 \mathrm{E}-19$ \\
\hline k 42 & $6.730 \mathrm{E}-21$ & $6.089 \mathrm{E}-21$ & $6.056 \mathrm{E}-21$ & $5.930 \mathrm{E}-21$ & $5.806 \mathrm{E}-21$ \\
\hline kr 81 & $9.154 \mathrm{E}-07$ & $9.154 \mathrm{E}-07$ & $9.154 \mathrm{E}-07$ & $9.154 \mathrm{E}-07$ & $9.154 \mathrm{E}-07$ \\
\hline kr 83m & $9.881 \mathrm{E}-04$ & $8.764 \mathrm{E}-10$ & $4.148 \mathrm{E}-10$ & $2.204 \mathrm{E}-11$ & $1.171 \mathrm{E}-12$ \\
\hline kr 85 & $7.734 \mathrm{E}+03$ & $5.698 \mathrm{E}+03$ & $5.605 \mathrm{E}+03$ & $5.256 \mathrm{E}+03$ & $4.928 \mathrm{E}+03$ \\
\hline la137 & $2.406 \mathrm{E}-05$ & $2.405 \mathrm{E}-05$ & $2.405 \mathrm{E}-05$ & $2.405 \mathrm{E}-05$ & $2.405 \mathrm{E}-05$ \\
\hline la138 & $7.694 \mathrm{E}-10$ & 7.694E-10 & $7.694 \mathrm{E}-10$ & $7.694 \mathrm{E}-10$ & 7.694E-10 \\
\hline la140 & $1.297 \mathrm{E}+00$ & $0.000 \mathrm{E}+00$ & $0.000 \mathrm{E}+00$ & $0.000 \mathrm{E}+00$ & $0.000 \mathrm{E}+00$ \\
\hline lu172 & $1.656 \mathrm{E}-19$ & $2.829 \mathrm{E}-20$ & $2.574 \mathrm{E}-20$ & $1.777 \mathrm{E}-20$ & $1.227 \mathrm{E}-20$ \\
\hline lu172m & $1.642 \mathrm{E}-19$ & $2.829 \mathrm{E}-20$ & $2.574 \mathrm{E}-20$ & $1.777 \mathrm{E}-20$ & $1.227 \mathrm{E}-20$ \\
\hline lu173 & $5.154 \mathrm{E}-15$ & 4.673E-16 & $4.108 \mathrm{E}-16$ & $2.478 \mathrm{E}-16$ & $1.495 \mathrm{E}-16$ \\
\hline lu174 & $7.391 \mathrm{E}-15$ & $2.832 \mathrm{E}-15$ & $2.685 \mathrm{E}-15$ & $2.178 \mathrm{E}-15$ & $1.767 \mathrm{E}-15$ \\
\hline lu174m & $1.971 \mathrm{E}-15$ & 4.177E-19 & $2.653 \mathrm{E}-19$ & 4.466E-20 & $7.516 \mathrm{E}-21$ \\
\hline lu176 & $3.928 \mathrm{E}-22$ & $3.928 \mathrm{E}-22$ & $3.928 \mathrm{E}-22$ & $3.928 \mathrm{E}-22$ & $3.928 \mathrm{E}-22$ \\
\hline lu177 & $8.011 \mathrm{E}-15$ & $4.490 \mathrm{E}-18$ & $3.004 \mathrm{E}-18$ & $6.207 \mathrm{E}-19$ & $1.283 \mathrm{E}-19$ \\
\hline lu177m & $3.590 \mathrm{E}-14$ & $2.011 \mathrm{E}-17$ & $1.345 \mathrm{E}-17$ & $2.780 \mathrm{E}-18$ & $5.744 \mathrm{E}-19$ \\
\hline
\end{tabular}




\begin{tabular}{|c|c|c|c|c|c|}
\hline \multicolumn{6}{|c|}{ Table C-6. Continued } \\
\hline & $1 / 22 / 2009$ & $10 / 21 / 2013$ & $1 / 22 / 2014$ & $1 / 22 / 2015$ & $1 / 22 / 2016$ \\
\hline mn 53 & $6.569 \mathrm{E}-22$ & $6.569 \mathrm{E}-22$ & $6.569 \mathrm{E}-22$ & $6.569 \mathrm{E}-22$ & $6.569 \mathrm{E}-22$ \\
\hline mn 54 & $1.738 \mathrm{E}-13$ & $3.700 \mathrm{E}-15$ & $3.009 \mathrm{E}-15$ & $1.337 \mathrm{E}-15$ & $5.946 \mathrm{E}-16$ \\
\hline mo 93 & $4.559 \mathrm{E}-07$ & $4.555 \mathrm{E}-07$ & $4.555 \mathrm{E}-07$ & $4.554 \mathrm{E}-07$ & 4.553E-07 \\
\hline mo100 & $6.624 \mathrm{E}-13$ & $6.624 \mathrm{E}-13$ & $6.624 \mathrm{E}-13$ & $6.624 \mathrm{E}-13$ & $6.624 \mathrm{E}-13$ \\
\hline na 22 & $5.360 \mathrm{E}-19$ & $1.515 \mathrm{E}-19$ & $1.416 \mathrm{E}-19$ & $1.085 \mathrm{E}-19$ & $8.315 \mathrm{E}-20$ \\
\hline nb 91 & $6.677 \mathrm{E}-08$ & $6.644 \mathrm{E}-08$ & $6.644 \mathrm{E}-08$ & $6.638 \mathrm{E}-08$ & $6.631 \mathrm{E}-08$ \\
\hline nb 91m & $1.783 \mathrm{E}-10$ & 4.779E-19 & $1.657 \mathrm{E}-19$ & $2.594 \mathrm{E}-21$ & $4.060 \mathrm{E}-23$ \\
\hline nb 92 & $1.125 \mathrm{E}-10$ & $1.125 \mathrm{E}-10$ & $1.125 \mathrm{E}-10$ & $1.125 \mathrm{E}-10$ & $1.125 \mathrm{E}-10$ \\
\hline nb 92m & $2.647 \mathrm{E}-13$ & $0.000 \mathrm{E}+00$ & $0.000 \mathrm{E}+00$ & $0.000 \mathrm{E}+00$ & $0.000 \mathrm{E}+00$ \\
\hline nb 93m & $1.621 \mathrm{E}-01$ & 4.567E-01 & $4.708 \mathrm{E}-01$ & $5.250 \mathrm{E}-01$ & $5.768 \mathrm{E}-01$ \\
\hline nb 94 & $6.763 \mathrm{E}-04$ & $6.763 \mathrm{E}-04$ & $6.763 \mathrm{E}-04$ & $6.763 \mathrm{E}-04$ & $6.763 \mathrm{E}-04$ \\
\hline nb 95 & $1.959 \mathrm{E}+05$ & $1.475 \mathrm{E}-03$ & $5.379 \mathrm{E}-04$ & $1.067 \mathrm{E}-05$ & $2.054 \mathrm{E}-07$ \\
\hline nb 95m & $1.072 \mathrm{E}+03$ & $7.635 \mathrm{E}-06$ & $2.791 \mathrm{E}-06$ & $5.368 \mathrm{E}-08$ & $1.032 \mathrm{E}-09$ \\
\hline nd144 & $1.258 \mathrm{E}-09$ & $1.483 \mathrm{E}-09$ & $1.483 \mathrm{E}-09$ & $1.485 \mathrm{E}-09$ & $1.486 \mathrm{E}-09$ \\
\hline nd147 & $4.258 \mathrm{E}-02$ & $0.000 \mathrm{E}+00$ & $0.000 \mathrm{E}+00$ & $0.000 \mathrm{E}+00$ & $0.000 \mathrm{E}+00$ \\
\hline nd150 & $9.194 \mathrm{E}-14$ & $9.194 \mathrm{E}-14$ & $9.194 \mathrm{E}-14$ & $9.194 \mathrm{E}-14$ & $9.194 \mathrm{E}-14$ \\
\hline ni 59 & $6.743 \mathrm{E}-15$ & $6.743 \mathrm{E}-15$ & $6.743 \mathrm{E}-15$ & $6.743 \mathrm{E}-15$ & $6.743 \mathrm{E}-15$ \\
\hline ni 63 & $1.034 \mathrm{E}-13$ & $1.001 \mathrm{E}-13$ & $9.993 \mathrm{E}-14$ & $9.927 \mathrm{E}-14$ & $9.854 \mathrm{E}-14$ \\
\hline np235 & $3.148 \mathrm{E}-03$ & $1.518 \mathrm{E}-04$ & $1.290 \mathrm{E}-04$ & $6.809 \mathrm{E}-05$ & 3.597E-05 \\
\hline np236 & $3.512 \mathrm{E}-06$ & $3.512 \mathrm{E}-06$ & $3.512 \mathrm{E}-06$ & $3.512 \mathrm{E}-06$ & $3.512 \mathrm{E}-06$ \\
\hline np237 & $1.274 \mathrm{E}-01$ & $1.326 \mathrm{E}-01$ & $1.330 \mathrm{E}-01$ & $1.349 \mathrm{E}-01$ & $1.369 \mathrm{E}-01$ \\
\hline np238 & $2.102 \mathrm{E}-01$ & $2.053 \mathrm{E}-01$ & $2.050 \mathrm{E}-01$ & $2.040 \mathrm{E}-01$ & $2.030 \mathrm{E}-01$ \\
\hline np239 & $7.688 \mathrm{E}+01$ & $7.681 \mathrm{E}+01$ & $7.681 \mathrm{E}+01$ & $7.681 \mathrm{E}+01$ & $7.681 \mathrm{E}+01$ \\
\hline np240 & $2.404 \mathrm{E}-09$ & $2.404 \mathrm{E}-09$ & $2.404 \mathrm{E}-09$ & $2.404 \mathrm{E}-09$ & $2.404 \mathrm{E}-09$ \\
\hline np240m & $2.003 \mathrm{E}-06$ & $2.003 \mathrm{E}-06$ & $2.003 \mathrm{E}-06$ & 2.003E-06 & $2.003 \mathrm{E}-06$ \\
\hline os185 & $1.565 \mathrm{E}-15$ & $4.177 \mathrm{E}-21$ & $2.098 \mathrm{E}-21$ & $1.405 \mathrm{E}-22$ & $9.418 \mathrm{E}-24$ \\
\hline os191 & $6.548 \mathrm{E}-19$ & $0.000 \mathrm{E}+00$ & $0.000 \mathrm{E}+00$ & $0.000 \mathrm{E}+00$ & $0.000 \mathrm{E}+00$ \\
\hline os194 & $1.500 \mathrm{E}-18$ & $8.672 \mathrm{E}-19$ & $8.421 \mathrm{E}-19$ & $7.503 \mathrm{E}-19$ & $6.684 \mathrm{E}-19$ \\
\hline p 32 & $1.836 \mathrm{E}-18$ & $3.570 \mathrm{E}-19$ & $3.566 \mathrm{E}-19$ & $3.550 \mathrm{E}-19$ & $3.533 \mathrm{E}-19$ \\
\hline p 33 & $2.144 \mathrm{E}-16$ & $0.000 \mathrm{E}+00$ & $0.000 \mathrm{E}+00$ & $0.000 \mathrm{E}+00$ & $0.000 \mathrm{E}+00$ \\
\hline pa230 & $3.620 \mathrm{E}-11$ & $0.000 \mathrm{E}+00$ & $0.000 \mathrm{E}+00$ & $0.000 \mathrm{E}+00$ & $0.000 \mathrm{E}+00$ \\
\hline pa231 & $1.177 \mathrm{E}-06$ & $1.388 \mathrm{E}-06$ & $1.400 \mathrm{E}-06$ & $1.444 \mathrm{E}-06$ & $1.489 \mathrm{E}-06$ \\
\hline pa232 & 7.027E-09 & 7.027E-09 & 7.027E-09 & 7.027E-09 & 7.027E-09 \\
\hline pa233 & $1.273 \mathrm{E}-01$ & $1.324 \mathrm{E}-01$ & $1.329 \mathrm{E}-01$ & $1.349 \mathrm{E}-01$ & $1.369 \mathrm{E}-01$ \\
\hline pa234 & 4.932E-04 & $4.934 \mathrm{E}-04$ & 4.932E-04 & 4.932E-04 & 4.932E-04 \\
\hline pa234m & $3.083 \mathrm{E}-01$ & $3.084 \mathrm{E}-01$ & $3.083 \mathrm{E}-01$ & $3.083 \mathrm{E}-01$ & $3.083 \mathrm{E}-01$ \\
\hline pb209 & $6.790 \mathrm{E}-08$ & 7.054E-08 & 7.067E-08 & $7.153 \mathrm{E}-08$ & 7.232E-08 \\
\hline pb210 & 4.035E-09 & 4.115E-09 & 4.156E-09 & 4.368E-09 & 4.672E-09 \\
\hline
\end{tabular}




\begin{tabular}{|c|c|c|c|c|c|}
\hline \multicolumn{6}{|c|}{ Table C-6. Continued } \\
\hline & $1 / 22 / 2009$ & $10 / 21 / 2013$ & $1 / 22 / 2014$ & $1 / 22 / 2015$ & $1 / 22 / 2016$ \\
\hline pb211 & $4.960 \mathrm{E}-08$ & $2.266 \mathrm{E}-07$ & $2.314 \mathrm{E}-07$ & $2.733 \mathrm{E}-07$ & 3.107E-07 \\
\hline pb212 & $2.661 \mathrm{E}-03$ & $1.676 \mathrm{E}-02$ & $1.738 \mathrm{E}-02$ & $1.951 \mathrm{E}-02$ & $2.129 \mathrm{E}-02$ \\
\hline pb214 & $1.439 \mathrm{E}-09$ & $9.009 \mathrm{E}-09$ & $9.676 \mathrm{E}-09$ & $1.260 \mathrm{E}-08$ & $1.604 \mathrm{E}-08$ \\
\hline pd103 & $2.322 \mathrm{E}-04$ & $0.000 \mathrm{E}+00$ & $0.000 \mathrm{E}+00$ & $0.000 \mathrm{E}+00$ & $0.000 \mathrm{E}+00$ \\
\hline pd107 & $3.471 \mathrm{E}-01$ & $3.471 \mathrm{E}-01$ & $3.471 \mathrm{E}-01$ & $3.471 \mathrm{E}-01$ & $3.471 \mathrm{E}-01$ \\
\hline pm143 & $6.225 \mathrm{E}-09$ & $6.690 \mathrm{E}-11$ & $5.246 \mathrm{E}-11$ & $2.019 \mathrm{E}-11$ & $7.774 \mathrm{E}-12$ \\
\hline pm144 & $1.385 \mathrm{E}-06$ & 5.062E-08 & $4.239 \mathrm{E}-08$ & $2.111 \mathrm{E}-08$ & $1.051 \mathrm{E}-08$ \\
\hline pm145 & $5.511 \mathrm{E}-03$ & 4.619E-03 & $4.573 \mathrm{E}-03$ & 4.398E-03 & $4.230 \mathrm{E}-03$ \\
\hline pm146 & $2.165 \mathrm{E}-01$ & $1.195 \mathrm{E}-01$ & $1.157 \mathrm{E}-01$ & $1.021 \mathrm{E}-01$ & 9.009E-02 \\
\hline pm147 & $1.793 \mathrm{E}+05$ & $5.118 \mathrm{E}+04$ & $4.785 E+04$ & $3.675 \mathrm{E}+04$ & $2.822 \mathrm{E}+04$ \\
\hline pm148 & $3.709 \mathrm{E}+01$ & $8.599 \mathrm{E}-12$ & $1.805 \mathrm{E}-12$ & $3.939 \mathrm{E}-15$ & 8.593E-18 \\
\hline pm148m & $7.681 \mathrm{E}+02$ & $1.781 \mathrm{E}-10$ & $3.739 \mathrm{E}-11$ & $8.157 \mathrm{E}-14$ & $1.781 \mathrm{E}-16$ \\
\hline po209 & $2.582 \mathrm{E}-16$ & $2.501 \mathrm{E}-16$ & $2.497 \mathrm{E}-16$ & $2.479 \mathrm{E}-16$ & $2.463 \mathrm{E}-16$ \\
\hline po210 & $3.510 \mathrm{E}-09$ & $4.000 \mathrm{E}-09$ & $4.029 \mathrm{E}-09$ & 4.201E-09 & 4.453E-09 \\
\hline po211 & $1.369 \mathrm{E}-10$ & $6.255 \mathrm{E}-10$ & $6.388 \mathrm{E}-10$ & 7.542E-10 & $8.579 \mathrm{E}-10$ \\
\hline po212 & $1.705 \mathrm{E}-03$ & $1.073 \mathrm{E}-02$ & $1.114 \mathrm{E}-02$ & $1.250 \mathrm{E}-02$ & $1.364 \mathrm{E}-02$ \\
\hline po213 & $6.638 \mathrm{E}-08$ & $6.902 \mathrm{E}-08$ & $6.915 \mathrm{E}-08$ & 6.994E-08 & $7.074 \mathrm{E}-08$ \\
\hline po214 & $1.520 \mathrm{E}-09$ & $9.009 \mathrm{E}-09$ & $9.676 \mathrm{E}-09$ & $1.260 \mathrm{E}-08$ & $1.604 \mathrm{E}-08$ \\
\hline po215 & $4.960 \mathrm{E}-08$ & $2.266 \mathrm{E}-07$ & $2.314 \mathrm{E}-07$ & $2.733 \mathrm{E}-07$ & 3.107E-07 \\
\hline po216 & $2.661 \mathrm{E}-03$ & $1.676 \mathrm{E}-02$ & $1.738 \mathrm{E}-02$ & $1.951 \mathrm{E}-02$ & $2.129 \mathrm{E}-02$ \\
\hline po218 & $1.440 \mathrm{E}-09$ & $9.009 \mathrm{E}-09$ & $9.676 \mathrm{E}-09$ & $1.260 \mathrm{E}-08$ & $1.604 \mathrm{E}-08$ \\
\hline pr143 & $2.351 \mathrm{E}+00$ & $0.000 \mathrm{E}+00$ & $0.000 \mathrm{E}+00$ & $0.000 \mathrm{E}+00$ & $0.000 \mathrm{E}+00$ \\
\hline pr144 & $6.690 \mathrm{E}+05$ & $9.874 \mathrm{E}+03$ & $7.873 \mathrm{E}+03$ & $3.240 \mathrm{E}+03$ & $1.333 \mathrm{E}+03$ \\
\hline pr144m & $6.389 \mathrm{E}+03$ & $9.425 \mathrm{E}+01$ & $7.516 \mathrm{E}+01$ & $3.094 \mathrm{E}+01$ & $1.273 \mathrm{E}+01$ \\
\hline pt193 & $7.358 \mathrm{E}-13$ & $6.889 \mathrm{E}-13$ & $6.862 \mathrm{E}-13$ & $6.770 \mathrm{E}-13$ & $6.677 \mathrm{E}-13$ \\
\hline pu236 & $5.103 \mathrm{E}-01$ & $1.614 \mathrm{E}-01$ & $1.518 \mathrm{E}-01$ & $1.191 \mathrm{E}-01$ & $9.346 \mathrm{E}-02$ \\
\hline pu237 & $1.489 \mathrm{E}-01$ & $5.528 \mathrm{E}-13$ & $1.347 \mathrm{E}-13$ & $5.269 \mathrm{E}-16$ & $2.062 \mathrm{E}-18$ \\
\hline pu238 & $3.421 \mathrm{E}+03$ & $3.654 \mathrm{E}+03$ & $3.647 \mathrm{E}+03$ & $3.619 \mathrm{E}+03$ & $3.591 \mathrm{E}+03$ \\
\hline pu239 & $9.900 \mathrm{E}+02$ & $9.900 \mathrm{E}+02$ & $9.900 \mathrm{E}+02$ & $9.900 \mathrm{E}+02$ & $9.900 \mathrm{E}+02$ \\
\hline pu240 & $2.261 \mathrm{E}+03$ & $2.266 \mathrm{E}+03$ & $2.266 \mathrm{E}+03$ & $2.267 \mathrm{E}+03$ & $2.268 \mathrm{E}+03$ \\
\hline pu241 & $5.413 \mathrm{E}+05$ & $4.300 \mathrm{E}+05$ & $4.247 \mathrm{E}+05$ & $4.047 \mathrm{E}+05$ & $3.855 \mathrm{E}+05$ \\
\hline pu242 & $6.001 \mathrm{E}+00$ & $6.001 \mathrm{E}+00$ & $6.001 \mathrm{E}+00$ & $6.001 \mathrm{E}+00$ & $6.001 \mathrm{E}+00$ \\
\hline pu243 & $1.326 \mathrm{E}-06$ & $1.326 \mathrm{E}-06$ & $1.326 \mathrm{E}-06$ & $1.326 \mathrm{E}-06$ & $1.326 \mathrm{E}-06$ \\
\hline pu244 & $2.006 \mathrm{E}-06$ & $2.006 \mathrm{E}-06$ & $2.006 \mathrm{E}-06$ & $2.006 \mathrm{E}-06$ & $2.006 \mathrm{E}-06$ \\
\hline pu246 & $3.479 \mathrm{E}-12$ & $3.478 \mathrm{E}-12$ & $3.478 \mathrm{E}-12$ & $3.478 \mathrm{E}-12$ & $3.477 \mathrm{E}-12$ \\
\hline ra222 & $8.130 \mathrm{E}-11$ & $0.000 \mathrm{E}+00$ & $0.000 \mathrm{E}+00$ & $0.000 \mathrm{E}+00$ & $0.000 \mathrm{E}+00$ \\
\hline ra223 & $4.960 \mathrm{E}-08$ & $2.266 \mathrm{E}-07$ & $2.314 \mathrm{E}-07$ & $2.733 \mathrm{E}-07$ & $3.107 \mathrm{E}-07$ \\
\hline ra224 & $2.661 \mathrm{E}-03$ & $1.676 \mathrm{E}-02$ & $1.738 \mathrm{E}-02$ & $1.951 \mathrm{E}-02$ & $2.129 \mathrm{E}-02$ \\
\hline
\end{tabular}




\begin{tabular}{|c|c|c|c|c|c|}
\hline \multicolumn{6}{|c|}{ Table C-6. Continued } \\
\hline & $1 / 22 / 2009$ & $10 / 21 / 2013$ & $1 / 22 / 2014$ & $1 / 22 / 2015$ & $1 / 22 / 2016$ \\
\hline ra225 & $6.783 \mathrm{E}-08$ & 7.054E-08 & 7.074E-08 & 7.153E-08 & 7.232E- 08 \\
\hline ra226 & $1.451 \mathrm{E}-09$ & $9.009 \mathrm{E}-09$ & $9.676 \mathrm{E}-09$ & $1.260 \mathrm{E}-08$ & $1.604 \mathrm{E}-08$ \\
\hline ra228 & $8.712 \mathrm{E}-12$ & $1.552 \mathrm{E}-11$ & $1.585 \mathrm{E}-11$ & $1.713 \mathrm{E}-11$ & $1.837 \mathrm{E}-11$ \\
\hline rb 83 & $1.329 \mathrm{E}-03$ & $1.179 \mathrm{E}-09$ & $5.581 \mathrm{E}-10$ & $2.965 \mathrm{E}-11$ & $1.575 \mathrm{E}-12$ \\
\hline rb 84 & 3.603E-03 & $4.584 \mathrm{E}-19$ & $6.431 \mathrm{E}-20$ & $2.887 \mathrm{E}-23$ & $1.296 \mathrm{E}-26$ \\
\hline rb 86 & $1.161 \mathrm{E}-01$ & $1.158 \mathrm{E}-29$ & $0.000 \mathrm{E}+00$ & $0.000 \mathrm{E}+00$ & $0.000 \mathrm{E}+00$ \\
\hline rb 87 & $1.600 \mathrm{E}-05$ & $1.600 \mathrm{E}-05$ & $1.600 \mathrm{E}-05$ & $1.600 \mathrm{E}-05$ & $1.600 \mathrm{E}-05$ \\
\hline re183 & $7.430 \mathrm{E}-19$ & $2.619 \mathrm{E}-26$ & $1.043 \mathrm{E}-26$ & $2.809 \mathrm{E}-28$ & $7.569 \mathrm{E}-30$ \\
\hline re184 & $1.292 \mathrm{E}-16$ & $9.828 \mathrm{E}-20$ & $6.710 \mathrm{E}-20$ & $1.507 \mathrm{E}-20$ & 3.372E-21 \\
\hline re184m & $1.273 \mathrm{E}-16$ & $1.043 \mathrm{E}-19$ & $7.120 \mathrm{E}-20$ & $1.593 \mathrm{E}-20$ & $3.566 \mathrm{E}-21$ \\
\hline re186 & $1.915 \mathrm{E}-18$ & $1.915 \mathrm{E}-18$ & $1.915 \mathrm{E}-18$ & $1.915 \mathrm{E}-18$ & $1.915 \mathrm{E}-18$ \\
\hline re186m & $1.915 \mathrm{E}-18$ & $1.915 \mathrm{E}-18$ & $1.915 \mathrm{E}-18$ & $1.915 \mathrm{E}-18$ & $1.915 \mathrm{E}-18$ \\
\hline re187 & $1.563 \mathrm{E}-20$ & $1.563 \mathrm{E}-20$ & $1.563 \mathrm{E}-20$ & $1.563 \mathrm{E}-20$ & $1.563 \mathrm{E}-20$ \\
\hline re188 & $1.915 \mathrm{E}-14$ & $6.399 \mathrm{E}-22$ & $2.540 \mathrm{E}-22$ & $0.000 \mathrm{E}+00$ & $0.000 \mathrm{E}+00$ \\
\hline rh 99 & $6.961 \mathrm{E}-15$ & $0.000 \mathrm{E}+00$ & $0.000 \mathrm{E}+00$ & $0.000 \mathrm{E}+00$ & $0.000 \mathrm{E}+00$ \\
\hline rh101 & $6.829 \mathrm{E}-04$ & $2.522 \mathrm{E}-04$ & $2.390 \mathrm{E}-04$ & $1.938 \mathrm{E}-04$ & $1.571 \mathrm{E}-04$ \\
\hline rh102 & $5.873 \mathrm{E}+00$ & $2.026 \mathrm{E}-02$ & $1.538 \mathrm{E}-02$ & $5.768 \mathrm{E}-03$ & $2.724 \mathrm{E}-03$ \\
\hline rh102m & $2.109 \mathrm{E}+00$ & $8.758 \mathrm{E}-01$ & $8.355 \mathrm{E}-01$ & $6.941 \mathrm{E}-01$ & $5.770 \mathrm{E}-01$ \\
\hline rh103m & $2.228 \mathrm{E}+04$ & $1.137 \mathrm{E}-09$ & $2.199 \mathrm{E}-10$ & $3.488 \mathrm{E}-13$ & $5.533 \mathrm{E}-16$ \\
\hline rh106 & $8.157 \mathrm{E}+05$ & $3.224 \mathrm{E}+04$ & $2.711 \mathrm{E}+04$ & $1.373 \mathrm{E}+04$ & $6.948 \mathrm{E}+03$ \\
\hline rn217 & $4.751 \mathrm{E}-12$ & $4.940 \mathrm{E}-12$ & $4.948 \mathrm{E}-12$ & $5.005 \mathrm{E}-12$ & $5.062 \mathrm{E}-12$ \\
\hline rn218 & $8.130 \mathrm{E}-11$ & $1.802 \mathrm{E}-15$ & $1.935 \mathrm{E}-15$ & $2.519 \mathrm{E}-15$ & $3.208 \mathrm{E}-15$ \\
\hline rn219 & $4.960 \mathrm{E}-08$ & $2.266 \mathrm{E}-07$ & $2.314 \mathrm{E}-07$ & $2.733 \mathrm{E}-07$ & $3.107 \mathrm{E}-07$ \\
\hline rn220 & $2.661 \mathrm{E}-03$ & $1.676 \mathrm{E}-02$ & $1.738 \mathrm{E}-02$ & $1.951 \mathrm{E}-02$ & $2.129 \mathrm{E}-02$ \\
\hline rn222 & $1.440 \mathrm{E}-09$ & $9.009 \mathrm{E}-09$ & $9.676 \mathrm{E}-09$ & $1.260 \mathrm{E}-08$ & $1.604 \mathrm{E}-08$ \\
\hline ru103 & $2.252 \mathrm{E}+04$ & $1.149 \mathrm{E}-09$ & $2.222 \mathrm{E}-10$ & $3.526 \mathrm{E}-13$ & $5.593 \mathrm{E}-16$ \\
\hline ru106 & $8.157 \mathrm{E}+05$ & $3.224 \mathrm{E}+04$ & $2.711 \mathrm{E}+04$ & $1.373 \mathrm{E}+04$ & $6.948 \mathrm{E}+03$ \\
\hline s 35 & $1.243 \mathrm{E}-13$ & $1.358 \mathrm{E}-19$ & $6.501 \mathrm{E}-20$ & $3.609 \mathrm{E}-21$ & $2.003 \mathrm{E}-22$ \\
\hline sb120m & $1.566 \mathrm{E}-15$ & $0.000 \mathrm{E}+00$ & $0.000 \mathrm{E}+00$ & $0.000 \mathrm{E}+00$ & $0.000 \mathrm{E}+00$ \\
\hline sb124 & $7.199 \mathrm{E}+01$ & $1.553 \mathrm{E}-07$ & 5.323E-08 & $7.959 \mathrm{E}-10$ & $1.191 \mathrm{E}-11$ \\
\hline sb125 & $1.617 \mathrm{E}+04$ & $4.909 \mathrm{E}+03$ & $4.605 \mathrm{E}+03$ & $3.582 \mathrm{E}+03$ & $2.787 \mathrm{E}+03$ \\
\hline sb126 & 8.097E-02 & $8.051 \mathrm{E}-02$ & $8.051 \mathrm{E}-02$ & $8.051 \mathrm{E}-02$ & $8.051 \mathrm{E}-02$ \\
\hline sb126m & $5.750 \mathrm{E}-01$ & $5.749 \mathrm{E}-01$ & $5.749 \mathrm{E}-01$ & 5.749E-01 & $5.749 \mathrm{E}-01$ \\
\hline sb127 & $3.306 \mathrm{E}-16$ & $0.000 \mathrm{E}+00$ & $0.000 \mathrm{E}+00$ & $0.000 \mathrm{E}+00$ & $0.000 \mathrm{E}+00$ \\
\hline sc $45 \mathrm{~m}$ & $3.126 \mathrm{E}-18$ & $1.935 \mathrm{E}-21$ & $1.302 \mathrm{E}-21$ & $2.747 \mathrm{E}-22$ & $5.796 \mathrm{E}-23$ \\
\hline sc 46 & $1.365 \mathrm{E}-12$ & 8.104E-19 & $3.755 \mathrm{E}-19$ & $1.833 \mathrm{E}-20$ & $8.956 \mathrm{E}-22$ \\
\hline se 75 & $1.374 \mathrm{E}-04$ & $6.068 \mathrm{E}-09$ & 3.543E-09 & $4.286 \mathrm{E}-10$ & $5.187 \mathrm{E}-11$ \\
\hline se 79 & $9.438 \mathrm{E}-02$ & $9.438 \mathrm{E}-02$ & $9.438 \mathrm{E}-02$ & $9.438 \mathrm{E}-02$ & $9.438 \mathrm{E}-02$ \\
\hline
\end{tabular}




\begin{tabular}{|c|c|c|c|c|c|}
\hline \multicolumn{6}{|c|}{ Table C-6. Continued } \\
\hline & $1 / 22 / 2009$ & $10 / 21 / 2013$ & $1 / 22 / 2014$ & $1 / 22 / 2015$ & $1 / 22 / 2016$ \\
\hline si 32 & $3.646 \mathrm{E}-19$ & $3.569 \mathrm{E}-19$ & $3.565 \mathrm{E}-19$ & $3.549 \mathrm{E}-19$ & 3.533E-19 \\
\hline sm145 & $9.405 \mathrm{E}-04$ & $2.748 \mathrm{E}-05$ & $2.273 \mathrm{E}-05$ & $1.080 \mathrm{E}-05$ & 5.132E-06 \\
\hline sm146 & $8.731 \mathrm{E}-08$ & $8.910 \mathrm{E}-08$ & 8.916E-08 & $8.936 \mathrm{E}-08$ & $8.962 \mathrm{E}-08$ \\
\hline sm147 & $2.943 \mathrm{E}-06$ & $6.114 \mathrm{E}-06$ & $6.196 \mathrm{E}-06$ & $6.471 \mathrm{E}-06$ & $6.684 \mathrm{E}-06$ \\
\hline sm148 & $7.186 \mathrm{E}-11$ & $7.186 \mathrm{E}-11$ & $7.186 \mathrm{E}-11$ & $7.186 \mathrm{E}-11$ & $7.186 \mathrm{E}-11$ \\
\hline sm151 & $7.377 \mathrm{E}+02$ & $7.113 \mathrm{E}+02$ & $7.100 \mathrm{E}+02$ & $7.047 \mathrm{E}+02$ & $6.988 \mathrm{E}+02$ \\
\hline sn113 & $7.668 \mathrm{E}-07$ & $2.248 \mathrm{E}-11$ & $1.284 \mathrm{E}-11$ & $1.425 \mathrm{E}-12$ & $1.582 \mathrm{E}-13$ \\
\hline sn117m & $2.384 \mathrm{E}-04$ & $0.000 \mathrm{E}+00$ & $0.000 \mathrm{E}+00$ & $0.000 \mathrm{E}+00$ & $0.000 \mathrm{E}+00$ \\
\hline sn119m & $2.646 \mathrm{E}+02$ & $4.387 \mathrm{E}+00$ & $3.520 \mathrm{E}+00$ & $1.485 \mathrm{E}+00$ & $6.261 \mathrm{E}-01$ \\
\hline sn121 & $4.243 \mathrm{E}+01$ & $3.936 \mathrm{E}+01$ & $3.921 \mathrm{E}+01$ & $3.859 \mathrm{E}+01$ & $3.799 \mathrm{E}+01$ \\
\hline sn121m & $5.467 \mathrm{E}+01$ & $5.072 \mathrm{E}+01$ & $5.052 \mathrm{E}+01$ & $4.973 \mathrm{E}+01$ & $4.895 \mathrm{E}+01$ \\
\hline $\operatorname{sn} 123$ & $1.139 \mathrm{E}-22$ & $1.044 \mathrm{E}-26$ & $6.340 \mathrm{E}-27$ & $8.949 \mathrm{E}-28$ & $1.262 \mathrm{E}-28$ \\
\hline sn123 & $7.067 \mathrm{E}+02$ & $6.479 \mathrm{E}-02$ & $3.934 \mathrm{E}-02$ & $5.551 \mathrm{E}-03$ & 7.833E-04 \\
\hline sn125 & 8.923E-05 & $0.000 \mathrm{E}+00$ & $0.000 \mathrm{E}+00$ & $0.000 \mathrm{E}+00$ & $0.000 \mathrm{E}+00$ \\
\hline sn126 & $5.750 \mathrm{E}-01$ & 5.749E-01 & $5.749 \mathrm{E}-01$ & $5.749 \mathrm{E}-01$ & $5.749 \mathrm{E}-01$ \\
\hline sr 85 & $3.716 \mathrm{E}-04$ & $3.344 \mathrm{E}-12$ & $1.237 \mathrm{E}-12$ & $2.500 \mathrm{E}-14$ & $5.050 \mathrm{E}-16$ \\
\hline sr 89 & $1.648 \mathrm{E}+04$ & $7.807 \mathrm{E}-07$ & $2.180 \mathrm{E}-07$ & $1.459 \mathrm{E}-09$ & $9.762 \mathrm{E}-12$ \\
\hline sr 90 & $5.275 \mathrm{E}+04$ & $4.706 \mathrm{E}+04$ & $4.677 \mathrm{E}+04$ & $4.566 \mathrm{E}+04$ & $4.457 \mathrm{E}+04$ \\
\hline ta179 & $2.833 \mathrm{E}-17$ & $4.650 \mathrm{E}-18$ & 4.220E-18 & $2.884 \mathrm{E}-18$ & $1.971 \mathrm{E}-18$ \\
\hline ta182 & 4.391E-12 & $1.249 \mathrm{E}-16$ & 7.133E-17 & $8.058 \mathrm{E}-18$ & $1.082 \mathrm{E}-18$ \\
\hline tb157 & $2.938 \mathrm{E}-05$ & $2.805 \mathrm{E}-05$ & $2.798 \mathrm{E}-05$ & $2.771 \mathrm{E}-05$ & $2.744 \mathrm{E}-05$ \\
\hline tb158 & $1.475 \mathrm{E}-03$ & $1.448 \mathrm{E}-03$ & $1.446 \mathrm{E}-03$ & $1.441 \mathrm{E}-03$ & $1.435 \mathrm{E}-03$ \\
\hline tb160 & $2.115 \mathrm{E}+02$ & $1.287 \mathrm{E}-05$ & $5.278 \mathrm{E}-06$ & $1.595 \mathrm{E}-07$ & $4.820 \mathrm{E}-09$ \\
\hline tb161 & $9.280 \mathrm{E}-09$ & $0.000 \mathrm{E}+00$ & $0.000 \mathrm{E}+00$ & $0.000 \mathrm{E}+00$ & $0.000 \mathrm{E}+00$ \\
\hline tc 95 & $1.014 \mathrm{E}-11$ & $2.844 \mathrm{E}-20$ & $9.887 \mathrm{E}-21$ & $1.562 \mathrm{E}-22$ & $0.000 \mathrm{E}+00$ \\
\hline tc $95 \mathrm{~m}$ & $2.579 \mathrm{E}-10$ & $7.232 \mathrm{E}-19$ & $2.513 \mathrm{E}-19$ & $3.971 \mathrm{E}-21$ & $6.276 \mathrm{E}-23$ \\
\hline tc 97 & $1.002 \mathrm{E}-08$ & $1.011 \mathrm{E}-08$ & $1.011 \mathrm{E}-08$ & $1.011 \mathrm{E}-08$ & $1.011 \mathrm{E}-08$ \\
\hline tc $97 \mathrm{~m}$ & $1.542 \mathrm{E}-03$ & $2.853 \mathrm{E}-09$ & $1.405 \mathrm{E}-09$ & $8.712 \mathrm{E}-11$ & $5.403 \mathrm{E}-12$ \\
\hline tc 98 & $1.024 \mathrm{E}-05$ & $1.024 \mathrm{E}-05$ & $1.024 \mathrm{E}-05$ & $1.024 \mathrm{E}-05$ & $1.024 \mathrm{E}-05$ \\
\hline tc 99 & $1.847 \mathrm{E}+01$ & $1.847 \mathrm{E}+01$ & $1.847 \mathrm{E}+01$ & $1.847 \mathrm{E}+01$ & $1.847 \mathrm{E}+01$ \\
\hline te121 & $1.946 \mathrm{E}-03$ & $1.294 \mathrm{E}-06$ & $8.738 \mathrm{E}-07$ & $1.871 \mathrm{E}-07$ & $4.008 \mathrm{E}-08$ \\
\hline te121m & $1.939 \mathrm{E}-03$ & $1.289 \mathrm{E}-06$ & $8.705 \mathrm{E}-07$ & $1.865 \mathrm{E}-07$ & $3.996 \mathrm{E}-08$ \\
\hline te123m & $4.028 \mathrm{E}+00$ & 1.692E-04 & $9.854 \mathrm{E}-05$ & $1.180 \mathrm{E}-05$ & $1.413 \mathrm{E}-06$ \\
\hline te125m & $3.942 \mathrm{E}+03$ & $1.202 \mathrm{E}+03$ & $1.127 \mathrm{E}+03$ & $8.771 \mathrm{E}+02$ & $6.823 \mathrm{E}+02$ \\
\hline te127 & $2.769 \mathrm{E}+03$ & $4.531 \mathrm{E}-02$ & $2.508 \mathrm{E}-02$ & $2.462 \mathrm{E}-03$ & $2.417 \mathrm{E}-04$ \\
\hline te127m & $2.827 \mathrm{E}+03$ & $4.626 \mathrm{E}-02$ & $2.561 \mathrm{E}-02$ & $2.514 \mathrm{E}-03$ & $2.467 \mathrm{E}-04$ \\
\hline te128 & $5.709 \mathrm{E}-14$ & $5.709 \mathrm{E}-14$ & $5.709 \mathrm{E}-14$ & $5.709 \mathrm{E}-14$ & $5.709 \mathrm{E}-14$ \\
\hline te129 & $1.670 \mathrm{E}+02$ & $4.968 \mathrm{E}-14$ & $7.292 \mathrm{E}-15$ & $3.916 \mathrm{E}-18$ & $2.102 \mathrm{E}-21$ \\
\hline
\end{tabular}




\begin{tabular}{|c|c|c|c|c|c|}
\hline \multicolumn{6}{|c|}{ Table C-6. Continued } \\
\hline & $1 / 22 / 2009$ & $10 / 21 / 2013$ & $1 / 22 / 2014$ & $1 / 22 / 2015$ & $1 / 22 / 2016$ \\
\hline te129m & $2.640 \mathrm{E}-22$ & $0.000 \mathrm{E}+00$ & $0.000 \mathrm{E}+00$ & $0.000 \mathrm{E}+00$ & $0.000 \mathrm{E}+00$ \\
\hline te129m & $2.646 \mathrm{E}+02$ & 7.873E-14 & $1.156 \mathrm{E}-14$ & $6.206 \mathrm{E}-18$ & 3.332E-21 \\
\hline th226 & $8.130 \mathrm{E}-11$ & $0.000 \mathrm{E}+00$ & $0.000 \mathrm{E}+00$ & $0.000 \mathrm{E}+00$ & $0.000 \mathrm{E}+00$ \\
\hline th227 & $5.006 \mathrm{E}-08$ & $2.235 \mathrm{E}-07$ & $2.297 \mathrm{E}-07$ & $2.695 \mathrm{E}-07$ & $3.065 \mathrm{E}-07$ \\
\hline th228 & $2.697 \mathrm{E}-03$ & $1.670 \mathrm{E}-02$ & $1.730 \mathrm{E}-02$ & $1.944 \mathrm{E}-02$ & $2.123 \mathrm{E}-02$ \\
\hline th229 & $6.783 \mathrm{E}-08$ & 7.054E-08 & 7.074E-08 & 7.153E-08 & $7.232 \mathrm{E}-08$ \\
\hline th230 & $1.816 \mathrm{E}-06$ & 5.907E-06 & $6.187 \mathrm{E}-06$ & $7.344 \mathrm{E}-06$ & $8.599 \mathrm{E}-06$ \\
\hline th231 & $2.096 \mathrm{E}-03$ & $2.100 \mathrm{E}-03$ & $2.101 \mathrm{E}-03$ & $2.102 \mathrm{E}-03$ & $2.103 \mathrm{E}-03$ \\
\hline th232 & $2.189 \mathrm{E}-11$ & $2.637 \mathrm{E}-11$ & $2.662 \mathrm{E}-11$ & $2.757 \mathrm{E}-11$ & $2.853 \mathrm{E}-11$ \\
\hline th234 & $3.083 \mathrm{E}-01$ & 3.084E-01 & $3.083 \mathrm{E}-01$ & 3.083E-01 & 3.083E-01 \\
\hline t1202 & $5.280 \mathrm{E}-23$ & $0.000 \mathrm{E}+00$ & $1.191 \mathrm{E}-27$ & $0.000 \mathrm{E}+00$ & $0.000 \mathrm{E}+00$ \\
\hline tl204 & $2.442 \mathrm{E}-14$ & $1.024 \mathrm{E}-14$ & $9.775 \mathrm{E}-15$ & $8.137 \mathrm{E}-15$ & $6.776 \mathrm{E}-15$ \\
\hline t1206 & $5.407 \mathrm{E}-15$ & $5.514 \mathrm{E}-15$ & $5.569 \mathrm{E}-15$ & $5.854 \mathrm{E}-15$ & $6.261 \mathrm{E}-15$ \\
\hline tl207 & 4.946E-08 & $2.260 \mathrm{E}-07$ & $2.308 \mathrm{E}-07$ & $2.725 \mathrm{E}-07$ & $3.099 \mathrm{E}-07$ \\
\hline tl208 & $9.564 \mathrm{E}-04$ & 6.023E-03 & $6.248 \mathrm{E}-03$ & $7.014 \mathrm{E}-03$ & $7.655 \mathrm{E}-03$ \\
\hline t1209 & $1.493 \mathrm{E}-09$ & $1.552 \mathrm{E}-09$ & $1.555 \mathrm{E}-09$ & $1.573 \mathrm{E}-09$ & $1.591 \mathrm{E}-09$ \\
\hline tl210 & $3.023 \mathrm{E}-13$ & $1.892 \mathrm{E}-12$ & $2.032 \mathrm{E}-12$ & $2.645 \mathrm{E}-12$ & $3.368 \mathrm{E}-12$ \\
\hline $\operatorname{tm} 167$ & $2.534 \mathrm{E}-15$ & $0.000 \mathrm{E}+00$ & $0.000 \mathrm{E}+00$ & $0.000 \mathrm{E}+00$ & $0.000 \mathrm{E}+00$ \\
\hline $\operatorname{tm} 168$ & $8.811 \mathrm{E}-06$ & $2.195 \mathrm{E}-11$ & $1.098 \mathrm{E}-11$ & $7.258 \mathrm{E}-13$ & $4.791 \mathrm{E}-14$ \\
\hline $\operatorname{tm} 170$ & $3.303 \mathrm{E}-01$ & $2.899 \mathrm{E}-05$ & $1.756 \mathrm{E}-05$ & $2.456 \mathrm{E}-06$ & $3.433 \mathrm{E}-07$ \\
\hline $\operatorname{tm} 171$ & $1.900 \mathrm{E}-01$ & $3.427 \mathrm{E}-02$ & $3.126 \mathrm{E}-02$ & $2.180 \mathrm{E}-02$ & $1.519 \mathrm{E}-02$ \\
\hline $\mathbf{u} 230$ & $8.124 \mathrm{E}-11$ & $0.000 \mathrm{E}+00$ & $0.000 \mathrm{E}+00$ & $0.000 \mathrm{E}+00$ & $0.000 \mathrm{E}+00$ \\
\hline $\mathbf{u} 232$ & $9.781 \mathrm{E}-03$ & $2.339 \mathrm{E}-02$ & 2.374E-02 & $2.485 \mathrm{E}-02$ & $2.565 \mathrm{E}-02$ \\
\hline $\mathbf{u} 233$ & $4.708 \mathrm{E}-06$ & $7.562 \mathrm{E}-06$ & $7.708 \mathrm{E}-06$ & $8.355 \mathrm{E}-06$ & $9.009 \mathrm{E}-06$ \\
\hline $\mathbf{u} 234$ & $6.922 \mathrm{E}-02$ & $1.184 \mathrm{E}-01$ & $1.211 \mathrm{E}-01$ & $1.313 \mathrm{E}-01$ & $1.415 \mathrm{E}-01$ \\
\hline $\mathbf{u} 235$ & $2.096 \mathrm{E}-03$ & $2.100 \mathrm{E}-03$ & $2.101 \mathrm{E}-03$ & $2.102 \mathrm{E}-03$ & $2.103 \mathrm{E}-03$ \\
\hline u236 & $1.896 \mathrm{E}-02$ & $1.928 \mathrm{E}-02$ & $1.929 \mathrm{E}-02$ & $1.936 \mathrm{E}-02$ & $1.943 \mathrm{E}-02$ \\
\hline $\mathbf{u} 237$ & $1.328 \mathrm{E}+01$ & $1.055 \mathrm{E}+01$ & $1.042 \mathrm{E}+01$ & $9.927 \mathrm{E}+00$ & $9.458 \mathrm{E}+00$ \\
\hline $\mathbf{u} 238$ & $3.083 \mathrm{E}-01$ & $3.083 \mathrm{E}-01$ & $3.083 \mathrm{E}-01$ & $3.083 \mathrm{E}-01$ & $3.083 \mathrm{E}-01$ \\
\hline $\mathrm{u} 240$ & $2.003 \mathrm{E}-06$ & $2.003 \mathrm{E}-06$ & 2.003E-06 & 2.003E-06 & $2.003 \mathrm{E}-06$ \\
\hline v 49 & $4.221 \mathrm{E}-16$ & $1.108 \mathrm{E}-17$ & $9.114 \mathrm{E}-18$ & 4.234E-18 & $1.967 \mathrm{E}-18$ \\
\hline w181 & $4.149 \mathrm{E}-15$ & $2.059 \mathrm{E}-19$ & $1.209 \mathrm{E}-19$ & $1.500 \mathrm{E}-20$ & $1.860 \mathrm{E}-21$ \\
\hline w185 & $1.090 \mathrm{E}-12$ & $1.232 \mathrm{E}-19$ & $5.224 \mathrm{E}-20$ & $1.798 \mathrm{E}-21$ & $6.192 \mathrm{E}-23$ \\
\hline w188 & $1.896 \mathrm{E}-14$ & 6.334E-22 & $2.514 \mathrm{E}-22$ & 6.697E-24 & $1.783 \mathrm{E}-25$ \\
\hline xe127 & $1.571 \mathrm{E}-03$ & $7.311 \mathrm{E}-18$ & $1.244 \mathrm{E}-18$ & $1.192 \mathrm{E}-21$ & $1.143 \mathrm{E}-24$ \\
\hline xe129m & $3.221 \mathrm{E}-08$ & $0.000 \mathrm{E}+00$ & $0.000 \mathrm{E}+00$ & $0.000 \mathrm{E}+00$ & $0.000 \mathrm{E}+00$ \\
\hline xe131m & $8.824 \mathrm{E}-03$ & $0.000 \mathrm{E}+00$ & $0.000 \mathrm{E}+00$ & $0.000 \mathrm{E}+00$ & $0.000 \mathrm{E}+00$ \\
\hline xe133 & $1.999 \mathrm{E}-09$ & $0.000 \mathrm{E}+00$ & $0.000 \mathrm{E}+00$ & $0.000 \mathrm{E}+00$ & $0.000 \mathrm{E}+00$ \\
\hline
\end{tabular}




\begin{tabular}{|c|c|c|c|c|c|}
\hline \multicolumn{6}{|c|}{ Table C-6. Continued } \\
\hline & $1 / 22 / 2009$ & $10 / 22 / 2013$ & $1 / 22 / 2014$ & $1 / 22 / 2015$ & $1 / 22 / 2016$ \\
\hline y 88 & 4.139E-01 & $5.302 \mathrm{E}-06$ & $2.896 \mathrm{E}-06$ & $2.700 \mathrm{E}-07$ & $2.518 \mathrm{E}-08$ \\
\hline y $89 \mathrm{~m}$ & $1.588 \mathrm{E}+00$ & $7.529 \mathrm{E}-11$ & $2.102 \mathrm{E}-11$ & $1.407 \mathrm{E}-13$ & $9.412 \mathrm{E}-16$ \\
\hline у 90 & $5.276 \mathrm{E}+04$ & 4.707E+04 & $4.678 \mathrm{E}+04$ & $4.567 \mathrm{E}+04$ & 4.459E+04 \\
\hline y 91 & $3.899 \mathrm{E}+04$ & 4.729E-05 & $1.571 \mathrm{E}-05$ & $2.081 \mathrm{E}-07$ & $2.757 \mathrm{E}-09$ \\
\hline yb169 & 8.388E-08 & $4.267 \mathrm{E}-24$ & $5.698 \mathrm{E}-25$ & $2.109 \mathrm{E}-28$ & $0.000 \mathrm{E}+00$ \\
\hline $\operatorname{zn} 65$ & $9.544 \mathrm{E}-16$ & $6.935 \mathrm{E}-18$ & $5.326 \mathrm{E}-18$ & $1.888 \mathrm{E}-18$ & $6.690 \mathrm{E}-19$ \\
\hline zr 88 & $1.273 \mathrm{E}-07$ & $7.074 \mathrm{E}-14$ & $3.266 \mathrm{E}-14$ & $1.573 \mathrm{E}-15$ & $7.569 \mathrm{E}-17$ \\
\hline $\operatorname{zr} 93$ & $1.804 \mathrm{E}+00$ & $1.804 \mathrm{E}+00$ & $1.804 \mathrm{E}+00$ & $1.804 \mathrm{E}+00$ & $1.804 \mathrm{E}+00$ \\
\hline zr 95 & $9.365 \mathrm{E}+04$ & $6.671 \mathrm{E}-04$ & $2.438 \mathrm{E}-04$ & 4.689E-06 & 9.015E-08 \\
\hline zr 96 & $1.792 \mathrm{E}-13$ & $1.792 \mathrm{E}-13$ & $1.792 \mathrm{E}-13$ & $1.792 \mathrm{E}-13$ & $1.792 \mathrm{E}-13$ \\
\hline Total & $4.862 \mathrm{E}+06$ & $1.015 E+06$ & $9.857 \mathrm{E}+05$ & 8.965E+05 & $8.331 \mathrm{E}+05$ \\
\hline
\end{tabular}


Table C-7. Activity in Fuel Rod Al (low Pu loading) in Ci per MTIHM

\begin{tabular}{|c|c|c|c|c|c|}
\hline & $1 / 22 / 2009$ & $10 / 21 / 2013$ & $1 / 22 / 2014$ & $1 / 22 / 2015$ & $1 / 22 / 2016$ \\
\hline ac225 & $8.608 \mathrm{E}-08$ & $8.858 \mathrm{E}-08$ & 8.872E-08 & 8.951E-08 & $9.030 \mathrm{E}-08$ \\
\hline ac226 & $1.070 \mathrm{E}-15$ & $0.000 \mathrm{E}+00$ & $0.000 \mathrm{E}+00$ & $0.000 \mathrm{E}+00$ & $0.000 \mathrm{E}+00$ \\
\hline ac227 & 4.893E-08 & $2.058 \mathrm{E}-07$ & $2.142 \mathrm{E}-07$ & $2.473 \mathrm{E}-07$ & $2.804 \mathrm{E}-07$ \\
\hline ac228 & $1.171 \mathrm{E}-11$ & $1.947 \mathrm{E}-11$ & $1.985 \mathrm{E}-11$ & $2.129 \mathrm{E}-11$ & $2.269 \mathrm{E}-11$ \\
\hline ag105 & $2.202 \mathrm{E}-10$ & $5.107 \mathrm{E}-23$ & $1.072 \mathrm{E}-23$ & $2.339 \mathrm{E}-26$ & $5.104 \mathrm{E}-29$ \\
\hline ag106m & $8.093 \mathrm{E}-16$ & $0.000 \mathrm{E}+00$ & $0.000 \mathrm{E}+00$ & $0.000 \mathrm{E}+00$ & $0.000 \mathrm{E}+00$ \\
\hline $\operatorname{ag} 108$ & $4.268 \mathrm{E}-04$ & 4.236E-04 & $4.235 \mathrm{E}-04$ & $4.228 \mathrm{E}-04$ & $4.221 \mathrm{E}-04$ \\
\hline ag108m & $4.906 \mathrm{E}-03$ & $4.869 \mathrm{E}-03$ & $4.867 \mathrm{E}-03$ & 4.859E-03 & $4.852 \mathrm{E}-03$ \\
\hline $\operatorname{ag} 109 m$ & $5.252 \mathrm{E}-01$ & $3.888 \mathrm{E}-02$ & $3.380 \mathrm{E}-02$ & $1.954 \mathrm{E}-02$ & $1.129 \mathrm{E}-02$ \\
\hline ag110 & $8.298 \mathrm{E}+01$ & $6.767 \mathrm{E}-01$ & $5.227 \mathrm{E}-01$ & $1.898 \mathrm{E}-01$ & $6.893 \mathrm{E}-02$ \\
\hline $\operatorname{ag} 110 \mathrm{~m}$ & $6.103 \mathrm{E}+03$ & $4.975 \mathrm{E}+01$ & $3.843 \mathrm{E}+01$ & $1.396 \mathrm{E}+01$ & $5.068 \mathrm{E}+00$ \\
\hline ag111 & $2.056 \mathrm{E}-06$ & $0.000 \mathrm{E}+00$ & $0.000 \mathrm{E}+00$ & $0.000 \mathrm{E}+00$ & $0.000 \mathrm{E}+00$ \\
\hline am241 & $9.815 \mathrm{E}+02$ & $3.444 \mathrm{E}+03$ & $3.560 \mathrm{E}+03$ & $4.002 \mathrm{E}+03$ & $4.421 \mathrm{E}+03$ \\
\hline am242 & $2.937 \mathrm{E}+01$ & $2.869 \mathrm{E}+01$ & $2.866 \mathrm{E}+01$ & $2.851 \mathrm{E}+01$ & $2.838 \mathrm{E}+01$ \\
\hline $\operatorname{am} 242 \mathrm{~m}$ & $2.950 \mathrm{E}+01$ & $2.882 \mathrm{E}+01$ & $2.879 \mathrm{E}+01$ & $2.865 \mathrm{E}+01$ & $2.851 \mathrm{E}+01$ \\
\hline $\operatorname{am} 243$ & $8.331 \mathrm{E}+01$ & $8.324 \mathrm{E}+01$ & $8.324 \mathrm{E}+01$ & $8.324 \mathrm{E}+01$ & $8.324 \mathrm{E}+01$ \\
\hline am244 & $1.169 \mathrm{E}-08$ & 8.106E-09 & $7.948 \mathrm{E}-09$ & 7.361E-09 & $6.814 \mathrm{E}-09$ \\
\hline $\operatorname{am} 245$ & $5.806 \mathrm{E}-07$ & $1.360 \mathrm{E}-08$ & $1.112 \mathrm{E}-08$ & 5.044E-09 & $2.288 \mathrm{E}-09$ \\
\hline am246m & $1.177 \mathrm{E}-11$ & $1.176 \mathrm{E}-11$ & $1.176 \mathrm{E}-11$ & $1.176 \mathrm{E}-11$ & $1.176 \mathrm{E}-11$ \\
\hline ar 37 & $1.338 \mathrm{E}-14$ & $1.729 \mathrm{E}-29$ & $0.000 \mathrm{E}+00$ & $0.000 \mathrm{E}+00$ & $0.000 \mathrm{E}+00$ \\
\hline ar 39 & $4.368 \mathrm{E}-15$ & $4.316 \mathrm{E}-15$ & $4.312 \mathrm{E}-15$ & $4.302 \mathrm{E}-15$ & $4.291 \mathrm{E}-15$ \\
\hline $\operatorname{ar} 42$ & $9.267 \mathrm{E}-21$ & 8.383E-21 & 8.344E-21 & $8.166 \mathrm{E}-21$ & 7.994E-21 \\
\hline as 73 & $2.527 \mathrm{E}-06$ & $8.054 \mathrm{E}-13$ & $3.607 \mathrm{E}-13$ & $1.545 \mathrm{E}-14$ & $6.616 \mathrm{E}-16$ \\
\hline as 74 & $1.472 \mathrm{E}-07$ & $0.000 \mathrm{E}+00$ & $0.000 \mathrm{E}+00$ & $0.000 \mathrm{E}+00$ & $0.000 \mathrm{E}+00$ \\
\hline at217 & $8.608 \mathrm{E}-08$ & $8.858 \mathrm{E}-08$ & 8.872E-08 & 8.951E-08 & $9.030 \mathrm{E}-08$ \\
\hline at218 & $2.647 \mathrm{E}-13$ & $1.673 \mathrm{E}-12$ & $1.797 \mathrm{E}-12$ & $2.343 \mathrm{E}-12$ & $2.987 \mathrm{E}-12$ \\
\hline au194 & $6.267 \mathrm{E}-21$ & $6.218 \mathrm{E}-21$ & $6.215 \mathrm{E}-21$ & $6.205 \mathrm{E}-21$ & $6.196 \mathrm{E}-21$ \\
\hline au195 & $3.941 \mathrm{E}-16$ & $6.199 \mathrm{E}-19$ & 4.384E-19 & $1.126 \mathrm{E}-19$ & $2.891 \mathrm{E}-20$ \\
\hline ba131 & $3.359 \mathrm{E}-13$ & $0.000 \mathrm{E}+00$ & $0.000 \mathrm{E}+00$ & $0.000 \mathrm{E}+00$ & $0.000 \mathrm{E}+00$ \\
\hline ba133 & $5.547 \mathrm{E}-02$ & $4.056 \mathrm{E}-02$ & $3.989 \mathrm{E}-02$ & $3.735 \mathrm{E}-02$ & $3.497 \mathrm{E}-02$ \\
\hline ba136m & $6.622 \mathrm{E}-03$ & $0.000 \mathrm{E}+00$ & $0.000 \mathrm{E}+00$ & $0.000 \mathrm{E}+00$ & $0.000 \mathrm{E}+00$ \\
\hline ba137m & $1.181 \mathrm{E}+05$ & $1.059 \mathrm{E}+05$ & $1.053 \mathrm{E}+05$ & $1.028 \mathrm{E}+05$ & $1.005 \mathrm{E}+05$ \\
\hline ba140 & $8.575 \mathrm{E}-01$ & $0.000 \mathrm{E}+00$ & $0.000 \mathrm{E}+00$ & $0.000 \mathrm{E}+00$ & $0.000 \mathrm{E}+00$ \\
\hline be 10 & $3.362 \mathrm{E}-06$ & $3.362 \mathrm{E}-06$ & 3.362E-06 & 3.362E-06 & $3.362 \mathrm{E}-06$ \\
\hline bi207 & $7.084 \mathrm{E}-17$ & $6.384 \mathrm{E}-17$ & $6.349 \mathrm{E}-17$ & $6.211 \mathrm{E}-17$ & $6.075 \mathrm{E}-17$ \\
\hline bi210 & $4.038 \mathrm{E}-09$ & 4.070E-09 & 4.107E-09 & 4.297E-09 & 4.572E-09 \\
\hline bi211 & $4.543 \mathrm{E}-08$ & $2.063 \mathrm{E}-07$ & $2.105 \mathrm{E}-07$ & $2.478 \mathrm{E}-07$ & $2.809 \mathrm{E}-07$ \\
\hline
\end{tabular}




\begin{tabular}{|c|c|c|c|c|c|}
\hline \multicolumn{6}{|c|}{ Table C-7. Continued } \\
\hline & $1 / 22 / 2009$ & $10 / 21 / 2013$ & $1 / 22 / 2014$ & $1 / 22 / 2015$ & $1 / 22 / 2016$ \\
\hline bi212 & $3.115 \mathrm{E}-03$ & $1.858 \mathrm{E}-02$ & $1.926 \mathrm{E}-02$ & $2.157 \mathrm{E}-02$ & $2.350 \mathrm{E}-02$ \\
\hline bi213 & $8.608 \mathrm{E}-08$ & $8.858 \mathrm{E}-08$ & 8.872E-08 & 8.951E-08 & $9.030 \mathrm{E}-08$ \\
\hline bi214 & $1.324 \mathrm{E}-09$ & 8.364E-09 & 8.984E-09 & $1.171 \mathrm{E}-08$ & $1.494 \mathrm{E}-08$ \\
\hline bk247 & $1.913 \mathrm{E}-12$ & $1.909 \mathrm{E}-12$ & $1.909 \mathrm{E}-12$ & $1.908 \mathrm{E}-12$ & $1.907 \mathrm{E}-12$ \\
\hline bk248 & $1.169 \mathrm{E}-08$ & $8.106 \mathrm{E}-09$ & $7.948 \mathrm{E}-09$ & 7.361E-09 & $6.814 \mathrm{E}-09$ \\
\hline bk249 & 4.004E-02 & $9.379 \mathrm{E}-04$ & 7.664E-04 & $3.478 \mathrm{E}-04$ & $1.577 \mathrm{E}-04$ \\
\hline bk250 & $6.518 \mathrm{E}-08$ & $8.403 \mathrm{E}-10$ & 6.662E-10 & $2.693 \mathrm{E}-10$ & $1.107 \mathrm{E}-10$ \\
\hline bk251 & $6.343 \mathrm{E}-11$ & $0.000 \mathrm{E}+00$ & $0.000 \mathrm{E}+00$ & $0.000 \mathrm{E}+00$ & $0.000 \mathrm{E}+00$ \\
\hline c 14 & $1.882 \mathrm{E}-03$ & $1.881 \mathrm{E}-03$ & $1.881 \mathrm{E}-03$ & $1.880 \mathrm{E}-03$ & $1.880 \mathrm{E}-03$ \\
\hline ca 41 & $4.661 \mathrm{E}-18$ & $4.661 \mathrm{E}-18$ & $4.661 \mathrm{E}-18$ & $4.661 \mathrm{E}-18$ & 4.661E-18 \\
\hline ca 45 & $1.899 \mathrm{E}-13$ & $1.175 \mathrm{E}-16$ & $7.909 \mathrm{E}-17$ & $1.669 \mathrm{E}-17$ & $3.521 \mathrm{E}-18$ \\
\hline cd109 & $5.252 \mathrm{E}-01$ & $3.888 \mathrm{E}-02$ & $3.380 \mathrm{E}-02$ & $1.954 \mathrm{E}-02$ & $1.129 \mathrm{E}-02$ \\
\hline cd113 & $1.059 \mathrm{E}-13$ & $1.059 \mathrm{E}-13$ & $1.059 \mathrm{E}-13$ & $1.059 \mathrm{E}-13$ & $1.059 \mathrm{E}-13$ \\
\hline cd113m & $8.766 \mathrm{E}-01$ & $6.945 \mathrm{E}-01$ & $6.853 \mathrm{E}-01$ & $6.527 \mathrm{E}-01$ & $6.214 \mathrm{E}-01$ \\
\hline cd115m & $1.451 \mathrm{E}+01$ & $2.845 \mathrm{E}-11$ & $6.695 \mathrm{E}-12$ & $2.291 \mathrm{E}-14$ & $7.836 \mathrm{E}-17$ \\
\hline cd116 & $1.020 \mathrm{E}-15$ & $1.020 \mathrm{E}-15$ & $1.020 \mathrm{E}-15$ & $1.020 \mathrm{E}-15$ & $1.020 \mathrm{E}-15$ \\
\hline ce139 & $9.050 \mathrm{E}+00$ & $1.467 \mathrm{E}-03$ & $9.182 \mathrm{E}-04$ & $1.461 \mathrm{E}-04$ & $2.325 \mathrm{E}-05$ \\
\hline ce141 & $4.923 \mathrm{E}+03$ & $4.408 \mathrm{E}-13$ & $6.068 \mathrm{E}-14$ & $2.530 \mathrm{E}-17$ & $1.055 \mathrm{E}-20$ \\
\hline ce144 & $5.365 \mathrm{E}+05$ & $7.915 \mathrm{E}+03$ & $6.313 \mathrm{E}+03$ & $2.597 \mathrm{E}+03$ & $1.069 \mathrm{E}+03$ \\
\hline cf 248 & $7.796 \mathrm{E}-08$ & $2.126 \mathrm{E}-09$ & $1.753 \mathrm{E}-09$ & $8.205 \mathrm{E}-10$ & $3.843 \mathrm{E}-10$ \\
\hline cf249 & $1.090 \mathrm{E}-04$ & $2.049 \mathrm{E}-04$ & $2.052 \mathrm{E}-04$ & $2.059 \mathrm{E}-04$ & $2.059 \mathrm{E}-04$ \\
\hline cf250 & $1.625 \mathrm{E}-03$ & $1.263 \mathrm{E}-03$ & $1.247 \mathrm{E}-03$ & $1.182 \mathrm{E}-03$ & $1.121 \mathrm{E}-03$ \\
\hline cf251 & $1.221 \mathrm{E}-05$ & $1.216 \mathrm{E}-05$ & $1.216 \mathrm{E}-05$ & $1.215 \mathrm{E}-05$ & $1.214 \mathrm{E}-05$ \\
\hline cf 252 & $1.684 \mathrm{E}-03$ & $4.857 \mathrm{E}-04$ & $4.543 \mathrm{E}-04$ & $3.496 \mathrm{E}-04$ & $2.691 \mathrm{E}-04$ \\
\hline cf253 & $5.635 \mathrm{E}-09$ & $0.000 \mathrm{E}+00$ & $0.000 \mathrm{E}+00$ & $0.000 \mathrm{E}+00$ & $0.000 \mathrm{E}+00$ \\
\hline cf254 & $1.001 \mathrm{E}-07$ & $2.385 \mathrm{E}-16$ & $8.219 \mathrm{E}-17$ & $1.255 \mathrm{E}-18$ & $1.916 \mathrm{E}-20$ \\
\hline cl 36 & $1.477 \mathrm{E}-16$ & $1.477 \mathrm{E}-16$ & $1.477 \mathrm{E}-16$ & $1.477 \mathrm{E}-16$ & $1.477 \mathrm{E}-16$ \\
\hline cm240 & $2.953 \mathrm{E}-07$ & $1.408 \mathrm{E}-26$ & $1.293 \mathrm{E}-27$ & $0.000 \mathrm{E}+00$ & $0.000 \mathrm{E}+00$ \\
\hline cm241 & $3.369 \mathrm{E}-04$ & 4.191E-20 & 5.872E-21 & $2.624 \mathrm{E}-24$ & $1.172 \mathrm{E}-27$ \\
\hline cm242 & $5.684 \mathrm{E}+04$ & $5.948 \mathrm{E}+01$ & $4.777 \mathrm{E}+01$ & $2.871 \mathrm{E}+01$ & $2.458 \mathrm{E}+01$ \\
\hline cm243 & $8.212 \mathrm{E}+01$ & $7.335 \mathrm{E}+01$ & $7.288 \mathrm{E}+01$ & $7.117 \mathrm{E}+01$ & $6.952 \mathrm{E}+01$ \\
\hline cm244 & $1.580 \mathrm{E}+04$ & $1.318 \mathrm{E}+04$ & $1.305 \mathrm{E}+04$ & $1.256 \mathrm{E}+04$ & $1.209 \mathrm{E}+04$ \\
\hline cm245 & $3.467 \mathrm{E}+00$ & $3.465 \mathrm{E}+00$ & $3.465 \mathrm{E}+00$ & $3.465 \mathrm{E}+00$ & $3.465 \mathrm{E}+00$ \\
\hline cm246 & $5.668 \mathrm{E}-01$ & $5.665 \mathrm{E}-01$ & $5.664 \mathrm{E}-01$ & $5.663 \mathrm{E}-01$ & $5.663 \mathrm{E}-01$ \\
\hline cm247 & $3.037 \mathrm{E}-06$ & $3.037 \mathrm{E}-06$ & $3.037 \mathrm{E}-06$ & $3.037 \mathrm{E}-06$ & $3.037 \mathrm{E}-06$ \\
\hline cm248 & $1.083 \mathrm{E}-05$ & $1.084 \mathrm{E}-05$ & $1.084 \mathrm{E}-05$ & $1.084 \mathrm{E}-05$ & $1.084 \mathrm{E}-05$ \\
\hline cm249 & $1.751 \mathrm{E}-11$ & $0.000 \mathrm{E}+00$ & $0.000 \mathrm{E}+00$ & $0.000 \mathrm{E}+00$ & $0.000 \mathrm{E}+00$ \\
\hline cm250 & $6.536 \mathrm{E}-11$ & $6.534 \mathrm{E}-11$ & $6.534 \mathrm{E}-11$ & $6.533 \mathrm{E}-11$ & $6.533 \mathrm{E}-11$ \\
\hline
\end{tabular}




\begin{tabular}{|c|c|c|c|c|c|}
\hline \multicolumn{6}{|c|}{ Table C-7. Continued } \\
\hline & $1 / 22 / 2009$ & $10 / 21 / 2013$ & $1 / 22 / 2014$ & $1 / 22 / 2015$ & $1 / 22 / 2016$ \\
\hline $\cos 56$ & $3.803 \mathrm{E}-21$ & $6.688 \mathrm{E}-28$ & $2.904 \mathrm{E}-28$ & $1.097 \mathrm{E}-29$ & $0.000 \mathrm{E}+00$ \\
\hline $\cos 57$ & $9.221 \mathrm{E}-16$ & $1.109 \mathrm{E}-17$ & $8.746 \mathrm{E}-18$ & $3.447 \mathrm{E}-18$ & $1.359 \mathrm{E}-18$ \\
\hline $\cos 58$ & $1.760 \mathrm{E}-14$ & $7.638 \mathrm{E}-22$ & $3.076 \mathrm{E}-22$ & $8.660 \mathrm{E}-24$ & $2.437 \mathrm{E}-25$ \\
\hline co 60 & $1.400 \mathrm{E}-11$ & $7.500 \mathrm{E}-12$ & $7.256 \mathrm{E}-12$ & $6.362 \mathrm{E}-12$ & $5.578 \mathrm{E}-12$ \\
\hline co $60 \mathrm{~m}$ & $5.333 \mathrm{E}-21$ & $5.333 \mathrm{E}-21$ & $5.333 \mathrm{E}-21$ & $5.333 \mathrm{E}-21$ & $5.333 \mathrm{E}-21$ \\
\hline cr 51 & $1.152 \mathrm{E}-14$ & $0.000 \mathrm{E}+00$ & $0.000 \mathrm{E}+00$ & $0.000 \mathrm{E}+00$ & $0.000 \mathrm{E}+00$ \\
\hline $\operatorname{cs} 131$ & $1.574 \mathrm{E}-10$ & $0.000 \mathrm{E}+00$ & $0.000 \mathrm{E}+00$ & $0.000 \mathrm{E}+00$ & $0.000 \mathrm{E}+00$ \\
\hline $\operatorname{cs} 132$ & $2.037 \mathrm{E}-11$ & $0.000 \mathrm{E}+00$ & $0.000 \mathrm{E}+00$ & $0.000 \mathrm{E}+00$ & $0.000 \mathrm{E}+00$ \\
\hline cs134 & $1.888 \mathrm{E}+05$ & $3.839 \mathrm{E}+04$ & $3.525 \mathrm{E}+04$ & $2.520 \mathrm{E}+04$ & $1.803 \mathrm{E}+04$ \\
\hline $\operatorname{cs} 135$ & $6.547 \mathrm{E}-01$ & $6.547 \mathrm{E}-01$ & $6.547 \mathrm{E}-01$ & $6.547 \mathrm{E}-01$ & $6.547 \mathrm{E}-01$ \\
\hline $\operatorname{cs} 136$ & $5.979 \mathrm{E}-02$ & $0.000 \mathrm{E}+00$ & $0.000 \mathrm{E}+00$ & $0.000 \mathrm{E}+00$ & $0.000 \mathrm{E}+00$ \\
\hline $\operatorname{cs137}$ & $1.247 \mathrm{E}+05$ & $1.118 \mathrm{E}+05$ & $1.111 \mathrm{E}+05$ & $1.086 \mathrm{E}+05$ & $1.061 \mathrm{E}+05$ \\
\hline dy159 & $1.323 \mathrm{E}-03$ & $3.226 \mathrm{E}-07$ & $2.065 \mathrm{E}-07$ & $3.580 \mathrm{E}-08$ & $6.209 \mathrm{E}-09$ \\
\hline er169 & 8.654E-09 & $0.000 \mathrm{E}+00$ & $0.000 \mathrm{E}+00$ & $0.000 \mathrm{E}+00$ & $0.000 \mathrm{E}+00$ \\
\hline es 252 & $9.459 \mathrm{E}-12$ & 7.407E-13 & $6.463 \mathrm{E}-13$ & $3.780 \mathrm{E}-13$ & $2.211 \mathrm{E}-13$ \\
\hline es 253 & $1.183 \mathrm{E}-07$ & $0.000 \mathrm{E}+00$ & $0.000 \mathrm{E}+00$ & $0.000 \mathrm{E}+00$ & $0.000 \mathrm{E}+00$ \\
\hline es254 & $6.514 \mathrm{E}-08$ & $8.350 \mathrm{E}-10$ & $6.609 \mathrm{E}-10$ & $2.640 \mathrm{E}-10$ & $1.055 \mathrm{E}-10$ \\
\hline es 255 & $7.922 \mathrm{E}-10$ & $6.180 \mathrm{E}-23$ & $1.224 \mathrm{E}-23$ & $2.123 \mathrm{E}-26$ & $3.682 \mathrm{E}-29$ \\
\hline eu147 & $8.911 \mathrm{E}-14$ & $0.000 \mathrm{E}+00$ & $0.000 \mathrm{E}+00$ & $0.000 \mathrm{E}+00$ & $0.000 \mathrm{E}+00$ \\
\hline eu149 & 4.102E-07 & $1.022 \mathrm{E}-12$ & $5.114 \mathrm{E}-13$ & 3.377E-14 & $2.230 \mathrm{E}-15$ \\
\hline eu152 & $3.979 \mathrm{E}+00$ & $3.121 \mathrm{E}+00$ & $3.080 \mathrm{E}+00$ & $2.927 \mathrm{E}+00$ & $2.781 \mathrm{E}+00$ \\
\hline eu154 & $1.383 \mathrm{E}+04$ & $9.432 \mathrm{E}+03$ & $9.241 \mathrm{E}+03$ & $8.529 \mathrm{E}+03$ & $7.869 \mathrm{E}+03$ \\
\hline eu155 & $6.312 \mathrm{E}+03$ & $3.159 \mathrm{E}+03$ & $3.045 \mathrm{E}+03$ & $2.632 \mathrm{E}+03$ & $2.275 \mathrm{E}+03$ \\
\hline eu156 & $2.075 \mathrm{E}+00$ & $0.000 \mathrm{E}+00$ & $0.000 \mathrm{E}+00$ & $0.000 \mathrm{E}+00$ & $0.000 \mathrm{E}+00$ \\
\hline fe 55 & $5.760 \mathrm{E}-13$ & $1.737 \mathrm{E}-13$ & $1.629 \mathrm{E}-13$ & $1.266 \mathrm{E}-13$ & $9.835 \mathrm{E}-14$ \\
\hline fe 59 & $4.988 \mathrm{E}-14$ & $9.406 \mathrm{E}-26$ & $2.208 \mathrm{E}-26$ & $7.493 \mathrm{E}-29$ & $0.000 \mathrm{E}+00$ \\
\hline fe 60 & $5.333 \mathrm{E}-21$ & $5.333 \mathrm{E}-21$ & $5.333 \mathrm{E}-21$ & $5.333 \mathrm{E}-21$ & $5.333 \mathrm{E}-21$ \\
\hline fr221 & $8.608 \mathrm{E}-08$ & $8.858 \mathrm{E}-08$ & $8.872 \mathrm{E}-08$ & $8.951 \mathrm{E}-08$ & $9.030 \mathrm{E}-08$ \\
\hline fr223 & $6.754 \mathrm{E}-10$ & $2.840 \mathrm{E}-09$ & $2.956 \mathrm{E}-09$ & $3.413 \mathrm{E}-09$ & $3.869 \mathrm{E}-09$ \\
\hline ga 68 & $1.910 \mathrm{E}-15$ & $2.268 \mathrm{E}-17$ & $1.788 \mathrm{E}-17$ & $7.031 \mathrm{E}-18$ & $2.763 \mathrm{E}-18$ \\
\hline gd151 & $3.432 \mathrm{E}-04$ & $2.130 \mathrm{E}-08$ & $1.266 \mathrm{E}-08$ & $1.646 \mathrm{E}-09$ & $2.140 \mathrm{E}-10$ \\
\hline gd152 & $1.075 \mathrm{E}-12$ & $1.105 \mathrm{E}-12$ & $1.107 \mathrm{E}-12$ & $1.112 \mathrm{E}-12$ & $1.117 \mathrm{E}-12$ \\
\hline gd153 & $6.853 \mathrm{E}+00$ & $4.630 \mathrm{E}-02$ & $3.541 \mathrm{E}-02$ & $1.236 \mathrm{E}-02$ & $4.316 \mathrm{E}-03$ \\
\hline ge 68 & $1.910 \mathrm{E}-15$ & $2.268 \mathrm{E}-17$ & $1.787 \mathrm{E}-17$ & $7.025 \mathrm{E}-18$ & $2.762 \mathrm{E}-18$ \\
\hline ge 71 & $7.559 \mathrm{E}-11$ & $0.000 \mathrm{E}+00$ & $0.000 \mathrm{E}+00$ & $0.000 \mathrm{E}+00$ & $0.000 \mathrm{E}+00$ \\
\hline ge $73 m$ & $2.527 \mathrm{E}-06$ & $8.054 \mathrm{E}-13$ & $3.607 \mathrm{E}-13$ & $1.545 \mathrm{E}-14$ & $6.616 \mathrm{E}-16$ \\
\hline h3 & $6.512 \mathrm{E}+02$ & $4.986 \mathrm{E}+02$ & $4.915 \mathrm{E}+02$ & $4.647 \mathrm{E}+02$ & $4.392 \mathrm{E}+02$ \\
\hline hf172 & $1.464 \mathrm{E}-19$ & $2.522 \mathrm{E}-20$ & $2.295 \mathrm{E}-20$ & $1.584 \mathrm{E}-20$ & $1.094 \mathrm{E}-20$ \\
\hline
\end{tabular}




\begin{tabular}{|c|c|c|c|c|c|}
\hline \multicolumn{6}{|c|}{ Table C-7. Continued } \\
\hline & $1 / 22 / 2009$ & $10 / 21 / 2013$ & $1 / 22 / 2014$ & $1 / 22 / 2015$ & $1 / 22 / 2016$ \\
\hline hf175 & $8.964 \mathrm{E}-12$ & $3.161 \mathrm{E}-19$ & $1.259 \mathrm{E}-19$ & $3.390 \mathrm{E}-21$ & $9.135 \mathrm{E}-23$ \\
\hline hf177m & $2.403 \mathrm{E}-14$ & $1.346 \mathrm{E}-17$ & $9.010 \mathrm{E}-18$ & $1.861 \mathrm{E}-18$ & $3.845 \mathrm{E}-19$ \\
\hline hf181 & $4.653 \mathrm{E}-13$ & $2.295 \mathrm{E}-25$ & $5.016 \mathrm{E}-26$ & $1.284 \mathrm{E}-28$ & $0.000 \mathrm{E}+00$ \\
\hline hf182 & $3.297 \mathrm{E}-19$ & 3.297E-19 & $3.297 \mathrm{E}-19$ & $3.297 \mathrm{E}-19$ & 3.297E-19 \\
\hline hg194 & $6.264 \mathrm{E}-21$ & $6.218 \mathrm{E}-21$ & $6.215 \mathrm{E}-21$ & $6.205 \mathrm{E}-21$ & $6.196 \mathrm{E}-21$ \\
\hline hg203 & $6.675 \mathrm{E}-14$ & $4.246 \mathrm{E}-25$ & $1.065 \mathrm{E}-25$ & 4.667E-28 & $0.000 \mathrm{E}+00$ \\
\hline hg206 & $7.671 \mathrm{E}-17$ & $7.730 \mathrm{E}-17$ & $7.796 \mathrm{E}-17$ & $8.159 \mathrm{E}-17$ & $8.680 \mathrm{E}-17$ \\
\hline ho163 & $8.568 \mathrm{E}-08$ & 8.561E-08 & $8.561 \mathrm{E}-08$ & $8.561 \mathrm{E}-08$ & 8.561E-08 \\
\hline ho166m & $1.109 \mathrm{E}-03$ & $1.106 \mathrm{E}-03$ & $1.106 \mathrm{E}-03$ & $1.105 \mathrm{E}-03$ & $1.105 \mathrm{E}-03$ \\
\hline i125 & $1.497 \mathrm{E}-05$ & $2.468 \mathrm{E}-14$ & 8.337E-15 & $1.179 \mathrm{E}-16$ & $1.665 \mathrm{E}-18$ \\
\hline i126 & $1.165 \mathrm{E}-06$ & $0.000 \mathrm{E}+00$ & $0.000 \mathrm{E}+00$ & $0.000 \mathrm{E}+00$ & $0.000 \mathrm{E}+00$ \\
\hline i129 & $4.529 \mathrm{E}-02$ & $4.529 \mathrm{E}-02$ & $4.529 \mathrm{E}-02$ & $4.529 \mathrm{E}-02$ & $4.529 \mathrm{E}-02$ \\
\hline i131 & $1.236 \mathrm{E}-04$ & $0.000 \mathrm{E}+00$ & $0.000 \mathrm{E}+00$ & $0.000 \mathrm{E}+00$ & $0.000 \mathrm{E}+00$ \\
\hline in113m & $5.607 \mathrm{E}-07$ & $1.644 \mathrm{E}-11$ & $9.386 \mathrm{E}-12$ & $1.042 \mathrm{E}-12$ & $1.157 \mathrm{E}-13$ \\
\hline in114 & $4.516 \mathrm{E}-03$ & $1.311 \mathrm{E}-13$ & $3.566 \mathrm{E}-14$ & $2.153 \mathrm{E}-16$ & $1.299 \mathrm{E}-18$ \\
\hline in114m & $4.668 \mathrm{E}-03$ & $1.355 \mathrm{E}-13$ & $3.686 \mathrm{E}-14$ & $2.225 \mathrm{E}-16$ & $1.343 \mathrm{E}-18$ \\
\hline in115 & $1.942 \mathrm{E}-11$ & $1.942 \mathrm{E}-11$ & $1.942 \mathrm{E}-11$ & $1.942 \mathrm{E}-11$ & $1.942 \mathrm{E}-11$ \\
\hline in115m & $1.541 \mathrm{E}-03$ & $3.022 \mathrm{E}-15$ & $7.110 \mathrm{E}-16$ & $2.434 \mathrm{E}-18$ & 8.324E-21 \\
\hline ir190 & $5.021 \mathrm{E}-23$ & $0.000 \mathrm{E}+00$ & $0.000 \mathrm{E}+00$ & $0.000 \mathrm{E}+00$ & $0.000 \mathrm{E}+00$ \\
\hline ir191m & $1.103 \mathrm{E}-18$ & $0.000 \mathrm{E}+00$ & $0.000 \mathrm{E}+00$ & $0.000 \mathrm{E}+00$ & $0.000 \mathrm{E}+00$ \\
\hline ir192 & $8.951 \mathrm{E}-13$ & $7.684 \mathrm{E}-20$ & $3.208 \mathrm{E}-20$ & $1.042 \mathrm{E}-21$ & $3.386 \mathrm{E}-23$ \\
\hline ir193m & $5.382 \mathrm{E}-20$ & $0.000 \mathrm{E}+00$ & $0.000 \mathrm{E}+00$ & $0.000 \mathrm{E}+00$ & $0.000 \mathrm{E}+00$ \\
\hline ir194 & $2.070 \mathrm{E}-18$ & $1.196 \mathrm{E}-18$ & $1.162 \mathrm{E}-18$ & $1.036 \mathrm{E}-18$ & $9.221 \mathrm{E}-19$ \\
\hline k 40 & $4.901 \mathrm{E}-19$ & $4.901 \mathrm{E}-19$ & 4.901E-19 & 4.901E-19 & $4.901 \mathrm{E}-19$ \\
\hline k 42 & $9.267 \mathrm{E}-21$ & 8.383E-21 & 8.344E-21 & $8.166 \mathrm{E}-21$ & 7.994E-21 \\
\hline kr 81 & $7.856 \mathrm{E}-07$ & $7.856 \mathrm{E}-07$ & $7.856 \mathrm{E}-07$ & $7.856 \mathrm{E}-07$ & $7.856 \mathrm{E}-07$ \\
\hline kr 83m & $7.124 \mathrm{E}-04$ & $6.316 \mathrm{E}-10$ & $2.990 \mathrm{E}-10$ & $1.588 \mathrm{E}-11$ & $8.443 \mathrm{E}-13$ \\
\hline kr 85 & $6.502 \mathrm{E}+03$ & $4.789 \mathrm{E}+03$ & $4.711 \mathrm{E}+03$ & $4.418 \mathrm{E}+03$ & $4.142 \mathrm{E}+03$ \\
\hline la137 & $1.828 \mathrm{E}-05$ & $1.828 \mathrm{E}-05$ & $1.828 \mathrm{E}-05$ & $1.828 \mathrm{E}-05$ & $1.828 \mathrm{E}-05$ \\
\hline la138 & $6.389 \mathrm{E}-10$ & $6.389 \mathrm{E}-10$ & $6.389 \mathrm{E}-10$ & $6.389 \mathrm{E}-10$ & $6.389 \mathrm{E}-10$ \\
\hline la140 & $9.894 \mathrm{E}-01$ & $0.000 \mathrm{E}+00$ & $0.000 \mathrm{E}+00$ & $0.000 \mathrm{E}+00$ & $0.000 \mathrm{E}+00$ \\
\hline lu172 & $1.477 \mathrm{E}-19$ & $2.522 \mathrm{E}-20$ & $2.295 \mathrm{E}-20$ & $1.584 \mathrm{E}-20$ & $1.094 \mathrm{E}-20$ \\
\hline lu172m & $1.464 \mathrm{E}-19$ & $2.522 \mathrm{E}-20$ & $2.295 \mathrm{E}-20$ & $1.584 \mathrm{E}-20$ & $1.094 \mathrm{E}-20$ \\
\hline lu173 & $4.272 \mathrm{E}-15$ & 3.873E-16 & $3.405 \mathrm{E}-16$ & $2.054 \mathrm{E}-16$ & $1.239 \mathrm{E}-16$ \\
\hline lu174 & $7.295 \mathrm{E}-15$ & $2.792 \mathrm{E}-15$ & $2.647 \mathrm{E}-15$ & $2.148 \mathrm{E}-15$ & $1.742 \mathrm{E}-15$ \\
\hline lu174m & $1.871 \mathrm{E}-15$ & $3.965 \mathrm{E}-19$ & $2.518 \mathrm{E}-19$ & 4.239E-20 & $7.137 \mathrm{E}-21$ \\
\hline lu176 & $3.284 \mathrm{E}-22$ & $3.284 \mathrm{E}-22$ & $3.284 \mathrm{E}-22$ & $3.284 \mathrm{E}-22$ & $3.284 \mathrm{E}-22$ \\
\hline lu177 & $6.827 \mathrm{E}-15$ & $3.824 \mathrm{E}-18$ & $2.559 \mathrm{E}-18$ & $5.286 \mathrm{E}-19$ & $1.092 \mathrm{E}-19$ \\
\hline
\end{tabular}




\begin{tabular}{|c|c|c|c|c|c|}
\hline \multicolumn{6}{|c|}{ Table C-7. Continued } \\
\hline & $1 / 22 / 2009$ & $10 / 21 / 2013$ & $1 / 22 / 2014$ & $1 / 22 / 2015$ & $1 / 22 / 2016$ \\
\hline lu177m & $3.057 \mathrm{E}-14$ & $1.713 \mathrm{E}-17$ & $1.146 \mathrm{E}-17$ & $2.368 \mathrm{E}-18$ & 4.892E-19 \\
\hline mn 53 & $6.972 \mathrm{E}-22$ & $6.972 \mathrm{E}-22$ & $6.972 \mathrm{E}-22$ & $6.972 \mathrm{E}-22$ & $6.972 \mathrm{E}-22$ \\
\hline mn 54 & $1.755 \mathrm{E}-13$ & $3.737 \mathrm{E}-15$ & $3.039 \mathrm{E}-15$ & $1.351 \mathrm{E}-15$ & $6.006 \mathrm{E}-16$ \\
\hline mo 93 & $3.987 \mathrm{E}-07$ & 3.984E-07 & $3.984 \mathrm{E}-07$ & $3.983 \mathrm{E}-07$ & 3.983E-07 \\
\hline mo100 & $5.508 \mathrm{E}-13$ & $5.508 \mathrm{E}-13$ & $5.508 \mathrm{E}-13$ & $5.508 \mathrm{E}-13$ & $5.508 \mathrm{E}-13$ \\
\hline na 22 & $4.506 \mathrm{E}-19$ & $1.274 \mathrm{E}-19$ & $1.190 \mathrm{E}-19$ & $9.122 \mathrm{E}-20$ & $6.992 \mathrm{E}-20$ \\
\hline nb 91 & $5.151 \mathrm{E}-08$ & 5.127E-08 & $5.126 \mathrm{E}-08$ & $5.120 \mathrm{E}-08$ & $5.115 \mathrm{E}-08$ \\
\hline nb 91m & $1.307 \mathrm{E}-10$ & $3.502 \mathrm{E}-19$ & $1.214 \mathrm{E}-19$ & $1.901 \mathrm{E}-21$ & $2.975 \mathrm{E}-23$ \\
\hline nb 92 & $8.641 \mathrm{E}-11$ & $8.641 \mathrm{E}-11$ & $8.641 \mathrm{E}-11$ & $8.641 \mathrm{E}-11$ & $8.641 \mathrm{E}-11$ \\
\hline nb 92m & $2.136 \mathrm{E}-13$ & $0.000 \mathrm{E}+00$ & $0.000 \mathrm{E}+00$ & $0.000 \mathrm{E}+00$ & $0.000 \mathrm{E}+00$ \\
\hline nb 93m & $1.397 \mathrm{E}-01$ & $3.868 \mathrm{E}-01$ & $3.987 \mathrm{E}-01$ & $4.440 \mathrm{E}-01$ & 4.876E-01 \\
\hline nb 94 & $5.514 \mathrm{E}-04$ & $5.513 \mathrm{E}-04$ & $5.513 \mathrm{E}-04$ & $5.513 \mathrm{E}-04$ & $5.512 \mathrm{E}-04$ \\
\hline nb 95 & $1.491 \mathrm{E}+05$ & $1.123 \mathrm{E}-03$ & 4.095E-04 & $8.120 \mathrm{E}-06$ & $1.563 \mathrm{E}-07$ \\
\hline nb 95m & $8.159 \mathrm{E}+02$ & $5.814 \mathrm{E}-06$ & $2.125 \mathrm{E}-06$ & $4.086 \mathrm{E}-08$ & $7.856 \mathrm{E}-10$ \\
\hline nd144 & $1.132 \mathrm{E}-09$ & $1.312 \mathrm{E}-09$ & $1.313 \mathrm{E}-09$ & $1.314 \mathrm{E}-09$ & $1.314 \mathrm{E}-09$ \\
\hline nd147 & $3.263 \mathrm{E}-02$ & $0.000 \mathrm{E}+00$ & $0.000 \mathrm{E}+00$ & $0.000 \mathrm{E}+00$ & $0.000 \mathrm{E}+00$ \\
\hline nd150 & $7.737 \mathrm{E}-14$ & $7.737 \mathrm{E}-14$ & 7.737E-14 & $7.737 \mathrm{E}-14$ & $7.737 \mathrm{E}-14$ \\
\hline ni 59 & $6.335 \mathrm{E}-15$ & $6.335 \mathrm{E}-15$ & $6.335 \mathrm{E}-15$ & $6.335 \mathrm{E}-15$ & $6.334 \mathrm{E}-15$ \\
\hline ni 63 & $1.427 \mathrm{E}-13$ & $1.381 \mathrm{E}-13$ & $1.379 \mathrm{E}-13$ & $1.369 \mathrm{E}-13$ & $1.359 \mathrm{E}-13$ \\
\hline np235 & $3.377 \mathrm{E}-03$ & $1.629 \mathrm{E}-04$ & $1.384 \mathrm{E}-04$ & $7.308 \mathrm{E}-05$ & $3.859 \mathrm{E}-05$ \\
\hline np236 & $3.264 \mathrm{E}-06$ & $3.264 \mathrm{E}-06$ & $3.264 \mathrm{E}-06$ & $3.264 \mathrm{E}-06$ & $3.264 \mathrm{E}-06$ \\
\hline np237 & $1.357 \mathrm{E}-01$ & $1.392 \mathrm{E}-01$ & $1.395 \mathrm{E}-01$ & $1.408 \mathrm{E}-01$ & $1.421 \mathrm{E}-01$ \\
\hline np238 & $1.354 \mathrm{E}-01$ & $1.323 \mathrm{E}-01$ & $1.321 \mathrm{E}-01$ & $1.315 \mathrm{E}-01$ & $1.309 \mathrm{E}-01$ \\
\hline np239 & $8.331 \mathrm{E}+01$ & $8.324 \mathrm{E}+01$ & $8.324 \mathrm{E}+01$ & $8.324 \mathrm{E}+01$ & $8.324 \mathrm{E}+01$ \\
\hline np240 & $3.240 \mathrm{E}-09$ & $3.240 \mathrm{E}-09$ & $3.240 \mathrm{E}-09$ & $3.240 \mathrm{E}-09$ & $3.240 \mathrm{E}-09$ \\
\hline np240m & $2.700 \mathrm{E}-06$ & $2.700 \mathrm{E}-06$ & $2.700 \mathrm{E}-06$ & $2.700 \mathrm{E}-06$ & $2.700 \mathrm{E}-06$ \\
\hline os185 & $1.522 \mathrm{E}-15$ & $4.062 \mathrm{E}-21$ & $2.040 \mathrm{E}-21$ & $1.367 \mathrm{E}-22$ & $9.162 \mathrm{E}-24$ \\
\hline os191 & $1.103 \mathrm{E}-18$ & $0.000 \mathrm{E}+00$ & $0.000 \mathrm{E}+00$ & $0.000 \mathrm{E}+00$ & $0.000 \mathrm{E}+00$ \\
\hline os194 & $2.070 \mathrm{E}-18$ & $1.196 \mathrm{E}-18$ & $1.162 \mathrm{E}-18$ & $1.035 \mathrm{E}-18$ & $9.221 \mathrm{E}-19$ \\
\hline p 32 & $1.824 \mathrm{E}-18$ & $3.989 \mathrm{E}-19$ & $3.984 \mathrm{E}-19$ & $3.966 \mathrm{E}-19$ & $3.948 \mathrm{E}-19$ \\
\hline p 33 & $2.024 \mathrm{E}-16$ & $0.000 \mathrm{E}+00$ & $0.000 \mathrm{E}+00$ & $0.000 \mathrm{E}+00$ & $0.000 \mathrm{E}+00$ \\
\hline pa230 & $3.109 \mathrm{E}-11$ & $0.000 \mathrm{E}+00$ & $0.000 \mathrm{E}+00$ & $0.000 \mathrm{E}+00$ & $0.000 \mathrm{E}+00$ \\
\hline pa231 & $1.086 \mathrm{E}-06$ & $1.245 \mathrm{E}-06$ & $1.253 \mathrm{E}-06$ & $1.287 \mathrm{E}-06$ & $1.321 \mathrm{E}-06$ \\
\hline pa232 & $6.529 \mathrm{E}-09$ & $6.529 \mathrm{E}-09$ & $6.529 \mathrm{E}-09$ & $6.529 \mathrm{E}-09$ & $6.529 \mathrm{E}-09$ \\
\hline pa233 & $1.357 \mathrm{E}-01$ & $1.391 \mathrm{E}-01$ & $1.394 \mathrm{E}-01$ & $1.408 \mathrm{E}-01$ & $1.421 \mathrm{E}-01$ \\
\hline pa234 & $5.038 \mathrm{E}-04$ & $5.040 \mathrm{E}-04$ & $5.038 \mathrm{E}-04$ & $5.038 \mathrm{E}-04$ & $5.038 \mathrm{E}-04$ \\
\hline pa234m & $3.149 \mathrm{E}-01$ & $3.150 \mathrm{E}-01$ & $3.149 \mathrm{E}-01$ & $3.149 \mathrm{E}-01$ & $3.149 \mathrm{E}-01$ \\
\hline pb209 & $8.608 \mathrm{E}-08$ & $8.858 \mathrm{E}-08$ & 8.872E-08 & $8.951 \mathrm{E}-08$ & $9.030 \mathrm{E}-08$ \\
\hline
\end{tabular}




\begin{tabular}{|c|c|c|c|c|c|}
\hline \multicolumn{6}{|c|}{ Table C-7. Continued } \\
\hline & $1 / 22 / 2009$ & $10 / 21 / 2013$ & $1 / 22 / 2014$ & $1 / 22 / 2015$ & $1 / 22 / 2016$ \\
\hline pb210 & $4.036 \mathrm{E}-09$ & $4.068 \mathrm{E}-09$ & 4.104E-09 & 4.295E-09 & $4.570 \mathrm{E}-09$ \\
\hline pb211 & $4.543 \mathrm{E}-08$ & $2.063 \mathrm{E}-07$ & $2.105 \mathrm{E}-07$ & $2.478 \mathrm{E}-07$ & $2.809 \mathrm{E}-07$ \\
\hline pb212 & $3.115 \mathrm{E}-03$ & $1.858 \mathrm{E}-02$ & $1.926 \mathrm{E}-02$ & $2.157 \mathrm{E}-02$ & $2.350 \mathrm{E}-02$ \\
\hline pb214 & $1.323 \mathrm{E}-09$ & 8.364E-09 & 8.984E-09 & $1.171 \mathrm{E}-08$ & $1.493 \mathrm{E}-08$ \\
\hline pd103 & $2.118 \mathrm{E}-04$ & $0.000 \mathrm{E}+00$ & $0.000 \mathrm{E}+00$ & $0.000 \mathrm{E}+00$ & $0.000 \mathrm{E}+00$ \\
\hline pd107 & $2.834 \mathrm{E}-01$ & $2.834 \mathrm{E}-01$ & $2.834 \mathrm{E}-01$ & $2.834 \mathrm{E}-01$ & $2.834 \mathrm{E}-01$ \\
\hline pm143 & $5.136 \mathrm{E}-09$ & $5.521 \mathrm{E}-11$ & $4.329 \mathrm{E}-11$ & $1.666 \mathrm{E}-11$ & $6.414 \mathrm{E}-12$ \\
\hline pm144 & $1.137 \mathrm{E}-06$ & 4.155E-08 & $3.479 \mathrm{E}-08$ & $1.733 \mathrm{E}-08$ & 8.634E-09 \\
\hline pm145 & $4.513 \mathrm{E}-03$ & 3.779E-03 & $3.742 \mathrm{E}-03$ & $3.599 \mathrm{E}-03$ & 3.461E-03 \\
\hline pm146 & $1.345 \mathrm{E}-01$ & $7.420 \mathrm{E}-02$ & $7.183 \mathrm{E}-02$ & $6.339 \mathrm{E}-02$ & $5.593 \mathrm{E}-02$ \\
\hline pm147 & $1.371 \mathrm{E}+05$ & $3.912 \mathrm{E}+04$ & $3.657 \mathrm{E}+04$ & $2.809 \mathrm{E}+04$ & $2.157 \mathrm{E}+04$ \\
\hline pm148 & $2.623 \mathrm{E}+01$ & $6.082 \mathrm{E}-12$ & $1.276 \mathrm{E}-12$ & $2.785 \mathrm{E}-15$ & $6.079 \mathrm{E}-18$ \\
\hline pm148m & $5.433 \mathrm{E}+02$ & $1.260 \mathrm{E}-10$ & $2.644 \mathrm{E}-11$ & $5.770 \mathrm{E}-14$ & $1.259 \mathrm{E}-16$ \\
\hline po209 & $2.852 \mathrm{E}-16$ & $2.762 \mathrm{E}-16$ & $2.757 \mathrm{E}-16$ & $2.739 \mathrm{E}-16$ & $2.720 \mathrm{E}-16$ \\
\hline po210 & $3.555 \mathrm{E}-09$ & 3.962E-09 & 3.987E-09 & 4.140E-09 & 4.367E-09 \\
\hline po211 & $1.254 \mathrm{E}-10$ & $5.692 \mathrm{E}-10$ & $5.811 \mathrm{E}-10$ & $6.840 \mathrm{E}-10$ & $7.757 \mathrm{E}-10$ \\
\hline po212 & $1.995 \mathrm{E}-03$ & $1.190 \mathrm{E}-02$ & $1.234 \mathrm{E}-02$ & $1.382 \mathrm{E}-02$ & $1.506 \mathrm{E}-02$ \\
\hline po213 & $8.416 \mathrm{E}-08$ & 8.667E-08 & 8.680E-08 & $8.753 \mathrm{E}-08$ & 8.832E-08 \\
\hline po214 & $1.393 \mathrm{E}-09$ & 8.364E-09 & 8.984E-09 & $1.171 \mathrm{E}-08$ & $1.493 \mathrm{E}-08$ \\
\hline po215 & $4.543 \mathrm{E}-08$ & $2.063 \mathrm{E}-07$ & $2.105 \mathrm{E}-07$ & $2.478 \mathrm{E}-07$ & $2.809 \mathrm{E}-07$ \\
\hline po216 & $3.115 \mathrm{E}-03$ & $1.858 \mathrm{E}-02$ & $1.926 \mathrm{E}-02$ & $2.157 \mathrm{E}-02$ & $2.350 \mathrm{E}-02$ \\
\hline po218 & $1.324 \mathrm{E}-09$ & 8.364E-09 & 8.984E-09 & $1.171 \mathrm{E}-08$ & $1.494 \mathrm{E}-08$ \\
\hline pr143 & $1.794 \mathrm{E}+00$ & $0.000 \mathrm{E}+00$ & $0.000 \mathrm{E}+00$ & $0.000 \mathrm{E}+00$ & $0.000 \mathrm{E}+00$ \\
\hline pr144 & $5.365 \mathrm{E}+05$ & $7.915 \mathrm{E}+03$ & $6.313 \mathrm{E}+03$ & $2.597 \mathrm{E}+03$ & $1.069 \mathrm{E}+03$ \\
\hline pr144m & $5.122 \mathrm{E}+03$ & $7.559 \mathrm{E}+01$ & $6.027 \mathrm{E}+01$ & $2.480 \mathrm{E}+01$ & $1.020 \mathrm{E}+01$ \\
\hline pt193 & $7.381 \mathrm{E}-13$ & $6.913 \mathrm{E}-13$ & $6.886 \mathrm{E}-13$ & $6.794 \mathrm{E}-13$ & $6.701 \mathrm{E}-13$ \\
\hline pu236 & $5.518 \mathrm{E}-01$ & $1.746 \mathrm{E}-01$ & $1.642 \mathrm{E}-01$ & $1.288 \mathrm{E}-01$ & $1.011 \mathrm{E}-01$ \\
\hline pu237 & $1.332 \mathrm{E}-01$ & $4.942 \mathrm{E}-13$ & $1.204 \mathrm{E}-13$ & $4.710 \mathrm{E}-16$ & $1.843 \mathrm{E}-18$ \\
\hline pu238 & $3.304 \mathrm{E}+03$ & $3.463 \mathrm{E}+03$ & $3.456 \mathrm{E}+03$ & $3.429 \mathrm{E}+03$ & $3.403 \mathrm{E}+03$ \\
\hline pu239 & $6.374 \mathrm{E}+02$ & $6.374 \mathrm{E}+02$ & $6.374 \mathrm{E}+02$ & $6.374 \mathrm{E}+02$ & $6.374 \mathrm{E}+02$ \\
\hline pu240 & $1.285 \mathrm{E}+03$ & $1.291 \mathrm{E}+03$ & $1.291 \mathrm{E}+03$ & $1.293 \mathrm{E}+03$ & $1.294 \mathrm{E}+03$ \\
\hline pu241 & $3.651 \mathrm{E}+05$ & $2.900 \mathrm{E}+05$ & $2.865 \mathrm{E}+05$ & $2.729 \mathrm{E}+05$ & $2.600 \mathrm{E}+05$ \\
\hline pu242 & $5.723 \mathrm{E}+00$ & $5.723 \mathrm{E}+00$ & $5.723 \mathrm{E}+00$ & $5.723 \mathrm{E}+00$ & $5.723 \mathrm{E}+00$ \\
\hline pu243 & $3.037 \mathrm{E}-06$ & 3.037E-06 & $3.037 \mathrm{E}-06$ & $3.037 \mathrm{E}-06$ & $3.037 \mathrm{E}-06$ \\
\hline pu244 & $2.703 \mathrm{E}-06$ & $2.703 \mathrm{E}-06$ & $2.703 \mathrm{E}-06$ & 2.703E-06 & $2.703 \mathrm{E}-06$ \\
\hline pu246 & $1.177 \mathrm{E}-11$ & $1.176 \mathrm{E}-11$ & $1.176 \mathrm{E}-11$ & $1.176 \mathrm{E}-11$ & $1.176 \mathrm{E}-11$ \\
\hline ra222 & $6.998 \mathrm{E}-11$ & $0.000 \mathrm{E}+00$ & $0.000 \mathrm{E}+00$ & $0.000 \mathrm{E}+00$ & $0.000 \mathrm{E}+00$ \\
\hline ra223 & 4.543E-08 & $2.063 \mathrm{E}-07$ & $2.105 \mathrm{E}-07$ & $2.478 \mathrm{E}-07$ & $2.809 \mathrm{E}-07$ \\
\hline
\end{tabular}




\begin{tabular}{|c|c|c|c|c|c|}
\hline \multicolumn{6}{|c|}{ Table C-7. Continued } \\
\hline & $1 / 22 / 2009$ & $10 / 21 / 2013$ & $1 / 22 / 2014$ & $1 / 22 / 2015$ & $1 / 22 / 2016$ \\
\hline ra224 & $3.115 \mathrm{E}-03$ & $1.858 \mathrm{E}-02$ & $1.926 \mathrm{E}-02$ & $2.157 \mathrm{E}-02$ & $2.350 \mathrm{E}-02$ \\
\hline ra225 & $8.601 \mathrm{E}-08$ & $8.858 \mathrm{E}-08$ & 8.878E-08 & 8.951E-08 & $9.030 \mathrm{E}-08$ \\
\hline ra226 & $1.334 \mathrm{E}-09$ & 8.364E-09 & 8.984E-09 & $1.171 \mathrm{E}-08$ & $1.494 \mathrm{E}-08$ \\
\hline ra228 & $1.171 \mathrm{E}-11$ & $1.947 \mathrm{E}-11$ & $1.985 \mathrm{E}-11$ & $2.129 \mathrm{E}-11$ & $2.269 \mathrm{E}-11$ \\
\hline rb 83 & $9.577 \mathrm{E}-04$ & $8.496 \mathrm{E}-10$ & $4.023 \mathrm{E}-10$ & $2.137 \mathrm{E}-11$ & $1.135 \mathrm{E}-12$ \\
\hline rb 84 & $2.874 \mathrm{E}-03$ & 3.657E-19 & $5.130 \mathrm{E}-20$ & $2.303 \mathrm{E}-23$ & $1.034 \mathrm{E}-26$ \\
\hline rb 86 & $9.722 \mathrm{E}-02$ & $9.696 \mathrm{E}-30$ & $0.000 \mathrm{E}+00$ & $0.000 \mathrm{E}+00$ & $0.000 \mathrm{E}+00$ \\
\hline rb 87 & $1.369 \mathrm{E}-05$ & $1.369 \mathrm{E}-05$ & $1.369 \mathrm{E}-05$ & $1.369 \mathrm{E}-05$ & $1.369 \mathrm{E}-05$ \\
\hline re183 & $5.520 \mathrm{E}-19$ & $1.946 \mathrm{E}-26$ & $7.750 \mathrm{E}-27$ & $2.088 \mathrm{E}-28$ & $5.623 \mathrm{E}-30$ \\
\hline re184 & $8.443 \mathrm{E}-17$ & $6.414 \mathrm{E}-20$ & $4.380 \mathrm{E}-20$ & $9.835 \mathrm{E}-21$ & $2.201 \mathrm{E}-21$ \\
\hline re184m & $8.311 \mathrm{E}-17$ & $6.807 \mathrm{E}-20$ & 4.647E-20 & $1.040 \mathrm{E}-20$ & $2.328 \mathrm{E}-21$ \\
\hline re186 & $1.746 \mathrm{E}-18$ & $1.746 \mathrm{E}-18$ & $1.746 \mathrm{E}-18$ & $1.746 \mathrm{E}-18$ & $1.746 \mathrm{E}-18$ \\
\hline re186m & $1.746 \mathrm{E}-18$ & $1.746 \mathrm{E}-18$ & $1.746 \mathrm{E}-18$ & $1.746 \mathrm{E}-18$ & $1.746 \mathrm{E}-18$ \\
\hline re187 & $1.524 \mathrm{E}-20$ & $1.524 \mathrm{E}-20$ & $1.524 \mathrm{E}-20$ & $1.524 \mathrm{E}-20$ & $1.524 \mathrm{E}-20$ \\
\hline re188 & $1.806 \mathrm{E}-14$ & $6.033 \mathrm{E}-22$ & $2.395 \mathrm{E}-22$ & $0.000 \mathrm{E}+00$ & $0.000 \mathrm{E}+00$ \\
\hline rh 99 & $5.208 \mathrm{E}-15$ & $0.000 \mathrm{E}+00$ & $0.000 \mathrm{E}+00$ & $0.000 \mathrm{E}+00$ & $0.000 \mathrm{E}+00$ \\
\hline rh101 & $5.417 \mathrm{E}-04$ & $2.000 \mathrm{E}-04$ & $1.896 \mathrm{E}-04$ & $1.537 \mathrm{E}-04$ & $1.246 \mathrm{E}-04$ \\
\hline rh102 & $4.430 \mathrm{E}+00$ & $1.536 \mathrm{E}-02$ & $1.167 \mathrm{E}-02$ & 4.412E-03 & $2.105 \mathrm{E}-03$ \\
\hline rh102m & $1.659 \mathrm{E}+00$ & $6.886 \mathrm{E}-01$ & $6.570 \mathrm{E}-01$ & $5.460 \mathrm{E}-01$ & 4.537E- 01 \\
\hline rh103m & $1.689 \mathrm{E}+04$ & $8.614 \mathrm{E}-10$ & $1.667 \mathrm{E}-10$ & $2.644 \mathrm{E}-13$ & 4.194E-16 \\
\hline rh106 & $6.484 \mathrm{E}+05$ & $2.563 \mathrm{E}+04$ & $2.154 \mathrm{E}+04$ & $1.091 \mathrm{E}+04$ & $5.524 \mathrm{E}+03$ \\
\hline rn217 & $6.025 \mathrm{E}-12$ & $6.203 \mathrm{E}-12$ & $6.211 \mathrm{E}-12$ & $6.267 \mathrm{E}-12$ & $6.322 \mathrm{E}-12$ \\
\hline rn218 & $6.998 \mathrm{E}-11$ & $1.673 \mathrm{E}-15$ & $1.797 \mathrm{E}-15$ & $2.343 \mathrm{E}-15$ & $2.987 \mathrm{E}-15$ \\
\hline rn219 & 4.543E-08 & $2.063 \mathrm{E}-07$ & $2.105 \mathrm{E}-07$ & $2.478 \mathrm{E}-07$ & 2.809E-07 \\
\hline rn220 & $3.115 \mathrm{E}-03$ & $1.858 \mathrm{E}-02$ & $1.926 \mathrm{E}-02$ & $2.157 \mathrm{E}-02$ & $2.350 \mathrm{E}-02$ \\
\hline rn222 & $1.323 \mathrm{E}-09$ & $8.364 \mathrm{E}-09$ & 8.984E-09 & $1.171 \mathrm{E}-08$ & $1.494 \mathrm{E}-08$ \\
\hline ru103 & $1.708 \mathrm{E}+04$ & 8.707E-10 & $1.685 \mathrm{E}-10$ & $2.673 \mathrm{E}-13$ & $4.240 \mathrm{E}-16$ \\
\hline ru106 & $6.484 \mathrm{E}+05$ & $2.563 \mathrm{E}+04$ & $2.154 \mathrm{E}+04$ & $1.091 \mathrm{E}+04$ & $5.524 \mathrm{E}+03$ \\
\hline s 35 & $1.253 \mathrm{E}-13$ & $1.369 \mathrm{E}-19$ & $6.555 \mathrm{E}-20$ & 3.639E-21 & $2.020 \mathrm{E}-22$ \\
\hline sb120m & $1.239 \mathrm{E}-15$ & $0.000 \mathrm{E}+00$ & $0.000 \mathrm{E}+00$ & $0.000 \mathrm{E}+00$ & $0.000 \mathrm{E}+00$ \\
\hline sb124 & $6.154 \mathrm{E}+01$ & $1.328 \mathrm{E}-07$ & $4.552 \mathrm{E}-08$ & $6.807 \mathrm{E}-10$ & $1.018 \mathrm{E}-11$ \\
\hline sb125 & $1.309 \mathrm{E}+04$ & $3.973 \mathrm{E}+03$ & $3.727 \mathrm{E}+03$ & $2.900 \mathrm{E}+03$ & $2.256 \mathrm{E}+03$ \\
\hline sb126 & $6.562 \mathrm{E}-02$ & $6.527 \mathrm{E}-02$ & $6.527 \mathrm{E}-02$ & $6.527 \mathrm{E}-02$ & $6.526 \mathrm{E}-02$ \\
\hline sb126m & $4.662 \mathrm{E}-01$ & 4.662E-01 & 4.662E-01 & $4.662 \mathrm{E}-01$ & 4.661E-01 \\
\hline sb127 & $2.490 \mathrm{E}-16$ & $0.000 \mathrm{E}+00$ & $0.000 \mathrm{E}+00$ & $0.000 \mathrm{E}+00$ & $0.000 \mathrm{E}+00$ \\
\hline sc $45 \mathrm{~m}$ & $3.608 \mathrm{E}-18$ & $2.233 \mathrm{E}-21$ & $1.503 \mathrm{E}-21$ & $3.171 \mathrm{E}-22$ & $6.688 \mathrm{E}-23$ \\
\hline sc 46 & $1.559 \mathrm{E}-12$ & $9.261 \mathrm{E}-19$ & 4.292E-19 & $2.096 \mathrm{E}-20$ & $1.023 \mathrm{E}-21$ \\
\hline se 75 & $1.135 \mathrm{E}-04$ & $5.012 \mathrm{E}-09$ & $2.926 \mathrm{E}-09$ & $3.540 \mathrm{E}-10$ & $4.283 \mathrm{E}-11$ \\
\hline
\end{tabular}




\begin{tabular}{|c|c|c|c|c|c|}
\hline \multicolumn{6}{|c|}{ Table C-7. Continued } \\
\hline & $1 / 22 / 2009$ & $10 / 21 / 2013$ & $1 / 22 / 2014$ & $1 / 22 / 2015$ & $1 / 22 / 2016$ \\
\hline se 79 & $7.612 \mathrm{E}-02$ & 7.612E-02 & 7.612E-02 & 7.612E-02 & 7.612E-02 \\
\hline si 32 & $4.074 \mathrm{E}-19$ & $3.988 \mathrm{E}-19$ & 3.983E-19 & $3.965 \mathrm{E}-19$ & 3.947E-19 \\
\hline sm145 & $7.130 \mathrm{E}-04$ & $2.083 \mathrm{E}-05$ & $1.723 \mathrm{E}-05$ & $8.186 \mathrm{E}-06$ & $3.890 \mathrm{E}-06$ \\
\hline $\operatorname{sm} 146$ & $6.959 \mathrm{E}-08$ & 7.064E-08 & $7.071 \mathrm{E}-08$ & 7.084E-08 & 7.097E-08 \\
\hline sm147 & $2.287 \mathrm{E}-06$ & 4.711E-06 & 4.774E-06 & $4.984 \mathrm{E}-06$ & $5.145 \mathrm{E}-06$ \\
\hline $\operatorname{sm} 148$ & $6.254 \mathrm{E}-11$ & $6.254 \mathrm{E}-11$ & $6.254 \mathrm{E}-11$ & $6.254 \mathrm{E}-11$ & $6.254 \mathrm{E}-11$ \\
\hline sm151 & $5.166 \mathrm{E}+02$ & $4.981 \mathrm{E}+02$ & $4.971 \mathrm{E}+02$ & $4.932 \mathrm{E}+02$ & $4.895 \mathrm{E}+02$ \\
\hline sn113 & $5.603 \mathrm{E}-07$ & $1.643 \mathrm{E}-11$ & $9.386 \mathrm{E}-12$ & $1.041 \mathrm{E}-12$ & $1.156 \mathrm{E}-13$ \\
\hline sn117m & $1.910 \mathrm{E}-04$ & $0.000 \mathrm{E}+00$ & $0.000 \mathrm{E}+00$ & $0.000 \mathrm{E}+00$ & $0.000 \mathrm{E}+00$ \\
\hline sn119m & $2.198 \mathrm{E}+02$ & $3.644 \mathrm{E}+00$ & $2.924 \mathrm{E}+00$ & $1.233 \mathrm{E}+00$ & $5.200 \mathrm{E}-01$ \\
\hline sn121 & $3.516 \mathrm{E}+01$ & $3.262 \mathrm{E}+01$ & $3.249 \mathrm{E}+01$ & $3.198 \mathrm{E}+01$ & $3.148 \mathrm{E}+01$ \\
\hline sn121m & $4.530 \mathrm{E}+01$ & $4.204 \mathrm{E}+01$ & $4.186 \mathrm{E}+01$ & $4.121 \mathrm{E}+01$ & $4.056 \mathrm{E}+01$ \\
\hline sn123 & $5.157 \mathrm{E}+02$ & 4.727E-02 & $2.870 \mathrm{E}-02$ & $4.050 \mathrm{E}-03$ & $5.715 \mathrm{E}-04$ \\
\hline sn125 & 6.682E-05 & $0.000 \mathrm{E}+00$ & $0.000 \mathrm{E}+00$ & $0.000 \mathrm{E}+00$ & $0.000 \mathrm{E}+00$ \\
\hline sn126 & $4.662 \mathrm{E}-01$ & 4.662E-01 & 4.662E-01 & $4.662 \mathrm{E}-01$ & 4.661E-01 \\
\hline sr 85 & $3.165 \mathrm{E}-04$ & $2.849 \mathrm{E}-12$ & $1.054 \mathrm{E}-12$ & $2.130 \mathrm{E}-14$ & 4.303E-16 \\
\hline sr 89 & $1.274 \mathrm{E}+04$ & $6.034 \mathrm{E}-07$ & $1.685 \mathrm{E}-07$ & $1.127 \mathrm{E}-09$ & $7.546 \mathrm{E}-12$ \\
\hline sr 90 & $4.506 \mathrm{E}+04$ & $4.020 \mathrm{E}+04$ & $3.995 \mathrm{E}+04$ & $3.900 \mathrm{E}+04$ & $3.807 \mathrm{E}+04$ \\
\hline ta179 & $2.640 \mathrm{E}-17$ & 4.332E-18 & 3.932E-18 & 2.687E-18 & $1.836 \mathrm{E}-18$ \\
\hline ta182 & $3.596 \mathrm{E}-12$ & $1.024 \mathrm{E}-16$ & $5.856 \mathrm{E}-17$ & $6.748 \mathrm{E}-18$ & $1.038 \mathrm{E}-18$ \\
\hline tb157 & $1.972 \mathrm{E}-05$ & $1.882 \mathrm{E}-05$ & $1.878 \mathrm{E}-05$ & $1.860 \mathrm{E}-05$ & $1.842 \mathrm{E}-05$ \\
\hline tb158 & $1.131 \mathrm{E}-03$ & $1.110 \mathrm{E}-03$ & $1.109 \mathrm{E}-03$ & $1.104 \mathrm{E}-03$ & $1.100 \mathrm{E}-03$ \\
\hline tb160 & $1.832 \mathrm{E}+02$ & $1.115 \mathrm{E}-05$ & 4.572E-06 & $1.381 \mathrm{E}-07$ & $4.175 \mathrm{E}-09$ \\
\hline tb161 & 7.434E-09 & $0.000 \mathrm{E}+00$ & $0.000 \mathrm{E}+00$ & $0.000 \mathrm{E}+00$ & $0.000 \mathrm{E}+00$ \\
\hline tc 95 & $7.928 \mathrm{E}-12$ & $2.222 \mathrm{E}-20$ & $7.724 \mathrm{E}-21$ & $1.221 \mathrm{E}-22$ & $0.000 \mathrm{E}+00$ \\
\hline tc $95 \mathrm{~m}$ & $2.015 \mathrm{E}-10$ & $5.649 \mathrm{E}-19$ & $1.964 \mathrm{E}-19$ & 3.103E-21 & $4.903 \mathrm{E}-23$ \\
\hline tc 97 & 8.561E-09 & 8.627E-09 & 8.627E-09 & 8.627E-09 & $8.627 \mathrm{E}-09$ \\
\hline tc $97 \mathrm{~m}$ & $1.198 \mathrm{E}-03$ & $2.217 \mathrm{E}-09$ & $1.092 \mathrm{E}-09$ & $6.767 \mathrm{E}-11$ & $4.200 \mathrm{E}-12$ \\
\hline tc 98 & 8.799E-06 & $8.799 \mathrm{E}-06$ & 8.799E-06 & 8.799E-06 & $8.799 \mathrm{E}-06$ \\
\hline tc 99 & $1.508 \mathrm{E}+01$ & $1.508 \mathrm{E}+01$ & $1.508 \mathrm{E}+01$ & $1.508 \mathrm{E}+01$ & $1.508 \mathrm{E}+01$ \\
\hline te121 & $1.880 \mathrm{E}-03$ & $1.251 \mathrm{E}-06$ & $8.443 \mathrm{E}-07$ & $1.809 \mathrm{E}-07$ & $3.874 \mathrm{E}-08$ \\
\hline te121m & $1.874 \mathrm{E}-03$ & $1.247 \mathrm{E}-06$ & $8.416 \mathrm{E}-07$ & $1.803 \mathrm{E}-07$ & $3.862 \mathrm{E}-08$ \\
\hline te123m & $4.014 \mathrm{E}+00$ & $1.687 \mathrm{E}-04$ & $9.821 \mathrm{E}-05$ & $1.176 \mathrm{E}-05$ & $1.408 \mathrm{E}-06$ \\
\hline te $125 \mathrm{~m}$ & $3.191 \mathrm{E}+03$ & $9.729 \mathrm{E}+02$ & $9.129 \mathrm{E}+02$ & $7.097 \mathrm{E}+02$ & $5.523 \mathrm{E}+02$ \\
\hline te127 & $1.945 \mathrm{E}+03$ & $3.183 \mathrm{E}-02$ & $1.762 \mathrm{E}-02$ & $1.729 \mathrm{E}-03$ & $1.698 \mathrm{E}-04$ \\
\hline te127m & $1.986 \mathrm{E}+03$ & $3.250 \mathrm{E}-02$ & $1.799 \mathrm{E}-02$ & $1.766 \mathrm{E}-03$ & $1.733 \mathrm{E}-04$ \\
\hline te128 & $4.660 \mathrm{E}-14$ & $4.660 \mathrm{E}-14$ & $4.660 \mathrm{E}-14$ & $4.660 \mathrm{E}-14$ & $4.660 \mathrm{E}-14$ \\
\hline te129 & $1.245 \mathrm{E}+02$ & $3.705 \mathrm{E}-14$ & $5.440 \mathrm{E}-15$ & $2.921 \mathrm{E}-18$ & $1.568 \mathrm{E}-21$ \\
\hline
\end{tabular}




\begin{tabular}{|c|c|c|c|c|c|}
\hline \multicolumn{6}{|c|}{ Table C-7. Continued } \\
\hline & $1 / 22 / 2009$ & $10 / 21 / 2013$ & $1 / 22 / 2014$ & $1 / 22 / 2015$ & $1 / 22 / 2016$ \\
\hline te129m & $1.973 \mathrm{E}+02$ & $5.873 \mathrm{E}-14$ & $8.621 \mathrm{E}-15$ & $4.629 \mathrm{E}-18$ & $2.485 \mathrm{E}-21$ \\
\hline th226 & $6.998 \mathrm{E}-11$ & $0.000 \mathrm{E}+00$ & $0.000 \mathrm{E}+00$ & $0.000 \mathrm{E}+00$ & $0.000 \mathrm{E}+00$ \\
\hline th227 & $4.585 \mathrm{E}-08$ & $2.034 \mathrm{E}-07$ & $2.090 \mathrm{E}-07$ & $2.444 \mathrm{E}-07$ & $2.771 \mathrm{E}-07$ \\
\hline th228 & $3.155 \mathrm{E}-03$ & $1.851 \mathrm{E}-02$ & $1.917 \mathrm{E}-02$ & $2.150 \mathrm{E}-02$ & 2.342E-02 \\
\hline th229 & $8.601 \mathrm{E}-08$ & $8.858 \mathrm{E}-08$ & $8.878 \mathrm{E}-08$ & 8.951E-08 & $9.030 \mathrm{E}-08$ \\
\hline th230 & $1.673 \mathrm{E}-06$ & $5.516 \mathrm{E}-06$ & $5.779 \mathrm{E}-06$ & $6.873 \mathrm{E}-06$ & $8.054 \mathrm{E}-06$ \\
\hline th231 & $1.569 \mathrm{E}-03$ & $1.572 \mathrm{E}-03$ & $1.572 \mathrm{E}-03$ & $1.573 \mathrm{E}-03$ & $1.574 \mathrm{E}-03$ \\
\hline th232 & $2.683 \mathrm{E}-11$ & $3.177 \mathrm{E}-11$ & $3.203 \mathrm{E}-11$ & $3.308 \mathrm{E}-11$ & 3.413E-11 \\
\hline th234 & $3.149 \mathrm{E}-01$ & $3.150 \mathrm{E}-01$ & $3.149 \mathrm{E}-01$ & 3.149E-01 & 3.149E-01 \\
\hline tl202 & $6.655 \mathrm{E}-23$ & $0.000 \mathrm{E}+00$ & $2.158 \mathrm{E}-27$ & $0.000 \mathrm{E}+00$ & $0.000 \mathrm{E}+00$ \\
\hline tl204 & $3.876 \mathrm{E}-14$ & $1.625 \mathrm{E}-14$ & $1.551 \mathrm{E}-14$ & $1.291 \mathrm{E}-14$ & $1.075 \mathrm{E}-14$ \\
\hline tl206 & $5.408 \mathrm{E}-15$ & $5.452 \mathrm{E}-15$ & $5.500 \mathrm{E}-15$ & $5.754 \mathrm{E}-15$ & $6.124 \mathrm{E}-15$ \\
\hline tl207 & $4.530 \mathrm{E}-08$ & $2.057 \mathrm{E}-07$ & $2.099 \mathrm{E}-07$ & $2.471 \mathrm{E}-07$ & $2.801 \mathrm{E}-07$ \\
\hline tl208 & $1.119 \mathrm{E}-03$ & $6.675 \mathrm{E}-03$ & $6.919 \mathrm{E}-03$ & $7.750 \mathrm{E}-03$ & 8.449E-03 \\
\hline tl209 & $1.894 \mathrm{E}-09$ & $1.949 \mathrm{E}-09$ & $1.952 \mathrm{E}-09$ & $1.970 \mathrm{E}-09$ & $1.987 \mathrm{E}-09$ \\
\hline tl210 & $2.780 \mathrm{E}-13$ & $1.756 \mathrm{E}-12$ & $1.886 \mathrm{E}-12$ & $2.460 \mathrm{E}-12$ & $3.137 \mathrm{E}-12$ \\
\hline $\operatorname{tm} 167$ & $1.503 \mathrm{E}-15$ & $0.000 \mathrm{E}+00$ & $0.000 \mathrm{E}+00$ & $0.000 \mathrm{E}+00$ & $0.000 \mathrm{E}+00$ \\
\hline $\operatorname{tm} 168$ & 7.203E-06 & $1.795 \mathrm{E}-11$ & $8.984 \mathrm{E}-12$ & $5.932 \mathrm{E}-13$ & 3.917E-14 \\
\hline $\operatorname{tm} 170$ & $3.049 \mathrm{E}-01$ & $2.676 \mathrm{E}-05$ & $1.621 \mathrm{E}-05$ & $2.266 \mathrm{E}-06$ & $3.169 \mathrm{E}-07$ \\
\hline $\operatorname{tm} 171$ & $1.900 \mathrm{E}-01$ & $3.427 \mathrm{E}-02$ & $3.126 \mathrm{E}-02$ & $2.179 \mathrm{E}-02$ & $1.519 \mathrm{E}-02$ \\
\hline $\mathbf{u 2 3 0}$ & $6.992 \mathrm{E}-11$ & $0.000 \mathrm{E}+00$ & $0.000 \mathrm{E}+00$ & $0.000 \mathrm{E}+00$ & $0.000 \mathrm{E}+00$ \\
\hline $\mathbf{u} 232$ & $1.109 \mathrm{E}-02$ & $2.579 \mathrm{E}-02$ & $2.616 \mathrm{E}-02$ & $2.735 \mathrm{E}-02$ & $2.822 \mathrm{E}-02$ \\
\hline $\mathbf{u} 233$ & 4.319E-06 & 7.341E-06 & $7.500 \mathrm{E}-06$ & 8.172E-06 & $8.852 \mathrm{E}-06$ \\
\hline u234 & $6.475 \mathrm{E}-02$ & $1.115 \mathrm{E}-01$ & $1.140 \mathrm{E}-01$ & $1.237 \mathrm{E}-01$ & $1.333 \mathrm{E}-01$ \\
\hline u235 & $1.569 \mathrm{E}-03$ & $1.572 \mathrm{E}-03$ & $1.572 \mathrm{E}-03$ & $1.573 \mathrm{E}-03$ & $1.574 \mathrm{E}-03$ \\
\hline $\mathrm{u} 236$ & $2.098 \mathrm{E}-02$ & $2.117 \mathrm{E}-02$ & $2.117 \mathrm{E}-02$ & $2.121 \mathrm{E}-02$ & $2.125 \mathrm{E}-02$ \\
\hline u237 & $8.957 \mathrm{E}+00$ & $7.117 \mathrm{E}+00$ & $7.031 \mathrm{E}+00$ & $6.695 \mathrm{E}+00$ & $6.378 \mathrm{E}+00$ \\
\hline u238 & $3.149 \mathrm{E}-01$ & $3.149 \mathrm{E}-01$ & $3.149 \mathrm{E}-01$ & 3.149E-01 & 3.149E-01 \\
\hline u240 & $2.700 \mathrm{E}-06$ & $2.700 \mathrm{E}-06$ & $2.700 \mathrm{E}-06$ & $2.700 \mathrm{E}-06$ & $2.700 \mathrm{E}-06$ \\
\hline v 49 & 4.277E-16 & $1.123 \mathrm{E}-17$ & $9.234 \mathrm{E}-18$ & 4.291E-18 & $1.993 \mathrm{E}-18$ \\
\hline w181 & $3.415 \mathrm{E}-15$ & $1.694 \mathrm{E}-19$ & $9.953 \mathrm{E}-20$ & $1.235 \mathrm{E}-20$ & $1.531 \mathrm{E}-21$ \\
\hline w185 & $1.202 \mathrm{E}-12$ & $1.359 \mathrm{E}-19$ & $5.762 \mathrm{E}-20$ & $1.984 \mathrm{E}-21$ & $6.833 \mathrm{E}-23$ \\
\hline w188 & $1.787 \mathrm{E}-14$ & $5.972 \mathrm{E}-22$ & $2.371 \mathrm{E}-22$ & $6.314 \mathrm{E}-24$ & $1.681 \mathrm{E}-25$ \\
\hline xe127 & $1.322 \mathrm{E}-03$ & $6.155 \mathrm{E}-18$ & $1.047 \mathrm{E}-18$ & $1.003 \mathrm{E}-21$ & 9.617E-25 \\
\hline xe129m & $2.962 \mathrm{E}-08$ & $0.000 \mathrm{E}+00$ & $0.000 \mathrm{E}+00$ & $0.000 \mathrm{E}+00$ & $0.000 \mathrm{E}+00$ \\
\hline xe131m & $6.682 \mathrm{E}-03$ & $0.000 \mathrm{E}+00$ & $0.000 \mathrm{E}+00$ & $0.000 \mathrm{E}+00$ & $0.000 \mathrm{E}+00$ \\
\hline xe133 & $1.524 \mathrm{E}-09$ & $0.000 \mathrm{E}+00$ & $0.000 \mathrm{E}+00$ & $0.000 \mathrm{E}+00$ & $0.000 \mathrm{E}+00$ \\
\hline y 88 & $3.454 \mathrm{E}-01$ & 4.424E-06 & $2.417 \mathrm{E}-06$ & $2.253 \mathrm{E}-07$ & $2.101 \mathrm{E}-08$ \\
\hline
\end{tabular}




\begin{tabular}{|l|r|r|r|r|r|}
\hline \multicolumn{7}{|c|}{ Table C-7. Continued } \\
\hline & $\mathbf{1 / 2 2 / 2 0 0 9}$ & $\mathbf{1 0 / 2 1 / 2 0 1 3}$ & $\mathbf{1 / 2 2 / 2 0 1 4}$ & $\mathbf{1 / 2 2 / 2 0 1 5}$ & $\mathbf{1 / 2 2 / 2 0 1 6}$ \\
\hline y 89m & $1.227 \mathrm{E}+00$ & $5.817 \mathrm{E}-11$ & $1.624 \mathrm{E}-11$ & $1.087 \mathrm{E}-13$ & $7.275 \mathrm{E}-16$ \\
\hline $\mathbf{y} \mathbf{9 0}$ & $4.508 \mathrm{E}+04$ & $4.021 \mathrm{E}+04$ & $3.996 \mathrm{E}+04$ & $3.901 \mathrm{E}+04$ & $3.808 \mathrm{E}+04$ \\
\hline $\mathbf{y ~ 9 1}$ & $3.009 \mathrm{E}+04$ & $3.650 \mathrm{E}-05$ & $1.213 \mathrm{E}-05$ & $1.606 \mathrm{E}-07$ & $2.128 \mathrm{E}-09$ \\
\hline yb169 & $8.021 \mathrm{E}-08$ & $4.081 \mathrm{E}-24$ & $5.450 \mathrm{E}-25$ & $2.016 \mathrm{E}-28$ & $0.000 \mathrm{E}+00$ \\
\hline zn 65 & $1.384 \mathrm{E}-15$ & $1.007 \mathrm{E}-17$ & $7.724 \mathrm{E}-18$ & $2.739 \mathrm{E}-18$ & $9.709 \mathrm{E}-19$ \\
\hline zr 88 & $9.182 \mathrm{E}-08$ & $5.101 \mathrm{E}-14$ & $2.355 \mathrm{E}-14$ & $1.134 \mathrm{E}-15$ & $5.457 \mathrm{E}-17$ \\
\hline zr 93 & $1.517 \mathrm{E}+00$ & $1.517 \mathrm{E}+00$ & $1.517 \mathrm{E}+00$ & $1.517 \mathrm{E}+00$ & $1.517 \mathrm{E}+00$ \\
\hline zr 95 & $7.130 \mathrm{E}+04$ & $5.079 \mathrm{E}-04$ & $1.856 \mathrm{E}-04$ & $3.569 \mathrm{E}-06$ & $6.866 \mathrm{E}-08$ \\
\hline zr 96 & $1.504 \mathrm{E}-13$ & $1.504 \mathrm{E}-13$ & $1.504 \mathrm{E}-13$ & $1.504 \mathrm{E}-13$ & $1.504 \mathrm{E}-13$ \\
\hline Total & $\mathbf{3 . 8 3 7 3 E}+\mathbf{0 6}$ & $\mathbf{7 . 7 8 6 3 E}+\mathbf{0 5}$ & $\mathbf{7 . 5 5 5 0 E}+\mathbf{0 5}$ & $\mathbf{6 . 8 5 2 7 E}+\mathbf{0 5}$ & $\mathbf{6 . 3 5 9 1 E}+\mathbf{0 5}$ \\
\hline
\end{tabular}


Table C-8. Activity in Fuel Rod Cl (medium Pu loading) in Ci per MTIHM

\begin{tabular}{|c|c|c|c|c|c|}
\hline & $1 / 22 / 2009$ & $10 / 21 / 2013$ & $1 / 22 / 2014$ & $1 / 22 / 2015$ & $1 / 22 / 2016$ \\
\hline ac225 & 7.657E-08 & 7.917E-08 & 7.930E-08 & $8.010 \mathrm{E}-08$ & $8.089 \mathrm{E}-08$ \\
\hline ac226 & $1.151 \mathrm{E}-15$ & $0.000 \mathrm{E}+00$ & $0.000 \mathrm{E}+00$ & $0.000 \mathrm{E}+00$ & $0.000 \mathrm{E}+00$ \\
\hline ac227 & $5.128 \mathrm{E}-08$ & $2.165 \mathrm{E}-07$ & $2.254 \mathrm{E}-07$ & $2.606 \mathrm{E}-07$ & 2.959E-07 \\
\hline ac228 & $1.025 \mathrm{E}-11$ & $1.763 \mathrm{E}-11$ & $1.799 \mathrm{E}-11$ & $1.937 \mathrm{E}-11$ & $2.071 \mathrm{E}-11$ \\
\hline $\operatorname{ag} 105$ & $2.452 \mathrm{E}-10$ & $5.685 \mathrm{E}-23$ & $1.193 \mathrm{E}-23$ & $2.604 \mathrm{E}-26$ & $5.683 \mathrm{E}-29$ \\
\hline ag106m & $8.940 \mathrm{E}-16$ & $0.000 \mathrm{E}+00$ & $0.000 \mathrm{E}+00$ & $0.000 \mathrm{E}+00$ & $0.000 \mathrm{E}+00$ \\
\hline $\operatorname{ag} 108$ & $4.578 \mathrm{E}-04$ & 4.544E-04 & 4.542E-04 & $4.535 \mathrm{E}-04$ & $4.528 \mathrm{E}-04$ \\
\hline ag108m & $5.262 \mathrm{E}-03$ & $5.223 \mathrm{E}-03$ & $5.221 \mathrm{E}-03$ & 5.213E-03 & 5.204E-03 \\
\hline ag109m & $5.350 \mathrm{E}-01$ & $3.960 \mathrm{E}-02$ & $3.443 \mathrm{E}-02$ & $1.989 \mathrm{E}-02$ & $1.150 \mathrm{E}-02$ \\
\hline $\operatorname{ag} 110$ & $8.774 \mathrm{E}+01$ & $7.152 \mathrm{E}-01$ & $5.526 \mathrm{E}-01$ & $2.007 \mathrm{E}-01$ & $7.285 \mathrm{E}-02$ \\
\hline ag110m & $6.453 \mathrm{E}+03$ & $5.260 \mathrm{E}+01$ & $4.063 \mathrm{E}+01$ & $1.476 \mathrm{E}+01$ & $5.359 \mathrm{E}+00$ \\
\hline $\operatorname{ag} 111$ & $2.278 \mathrm{E}-06$ & $0.000 \mathrm{E}+00$ & $0.000 \mathrm{E}+00$ & $0.000 \mathrm{E}+00$ & $0.000 \mathrm{E}+00$ \\
\hline am241 & $1.169 \mathrm{E}+03$ & $4.079 \mathrm{E}+03$ & $4.217 \mathrm{E}+03$ & $4.739 \mathrm{E}+03$ & $5.235 \mathrm{E}+03$ \\
\hline am242 & $3.571 \mathrm{E}+01$ & $3.489 \mathrm{E}+01$ & $3.484 \mathrm{E}+01$ & $3.468 \mathrm{E}+01$ & $3.450 \mathrm{E}+01$ \\
\hline $\operatorname{am} 242 m$ & $3.587 \mathrm{E}+01$ & $3.505 \mathrm{E}+01$ & $3.500 \mathrm{E}+01$ & $3.484 \mathrm{E}+01$ & $3.466 \mathrm{E}+01$ \\
\hline $\operatorname{am} 243$ & $7.996 \mathrm{E}+01$ & $7.996 \mathrm{E}+01$ & $7.996 \mathrm{E}+01$ & $7.996 \mathrm{E}+01$ & $7.996 \mathrm{E}+01$ \\
\hline am244 & $6.933 \mathrm{E}-09$ & 4.810E-09 & 4.716E-09 & 4.367E-09 & 4.043E-09 \\
\hline am245 & $3.577 \mathrm{E}-07$ & $8.382 \mathrm{E}-09$ & $6.853 \mathrm{E}-09$ & $3.107 \mathrm{E}-09$ & 1.409E-09 \\
\hline am246m & $6.833 \mathrm{E}-12$ & $6.827 \mathrm{E}-12$ & $6.827 \mathrm{E}-12$ & $6.827 \mathrm{E}-12$ & $6.827 \mathrm{E}-12$ \\
\hline ar 37 & $1.299 \mathrm{E}-14$ & $1.680 \mathrm{E}-29$ & $0.000 \mathrm{E}+00$ & $0.000 \mathrm{E}+00$ & $0.000 \mathrm{E}+00$ \\
\hline ar 39 & $4.264 \mathrm{E}-15$ & $4.212 \mathrm{E}-15$ & $4.210 \mathrm{E}-15$ & 4.199E-15 & $4.188 \mathrm{E}-15$ \\
\hline $\operatorname{ar} 42$ & 7.957E-21 & $7.199 \mathrm{E}-21$ & 7.159E-21 & 7.013E-21 & $6.866 \mathrm{E}-21$ \\
\hline as 73 & $2.832 \mathrm{E}-06$ & $9.020 \mathrm{E}-13$ & 4.042E-13 & $1.731 \mathrm{E}-14$ & $7.411 \mathrm{E}-16$ \\
\hline as 74 & $1.626 \mathrm{E}-07$ & $0.000 \mathrm{E}+00$ & $0.000 \mathrm{E}+00$ & $0.000 \mathrm{E}+00$ & $0.000 \mathrm{E}+00$ \\
\hline at217 & 7.657E-08 & 7.917E-08 & $7.930 \mathrm{E}-08$ & $8.010 \mathrm{E}-08$ & $8.089 \mathrm{E}-08$ \\
\hline at218 & $2.761 \mathrm{E}-13$ & $1.732 \mathrm{E}-12$ & $1.861 \mathrm{E}-12$ & $2.424 \mathrm{E}-12$ & $3.087 \mathrm{E}-12$ \\
\hline au194 & 7.252E-21 & $7.199 \mathrm{E}-21$ & 7.192E-21 & 7.179E-21 & 7.172E-21 \\
\hline au195 & $5.749 \mathrm{E}-16$ & $9.040 \mathrm{E}-19$ & 6.396E-19 & $1.642 \mathrm{E}-19$ & $4.218 \mathrm{E}-20$ \\
\hline ba131 & $3.584 \mathrm{E}-13$ & $0.000 \mathrm{E}+00$ & $0.000 \mathrm{E}+00$ & $0.000 \mathrm{E}+00$ & $0.000 \mathrm{E}+00$ \\
\hline ba133 & $5.363 \mathrm{E}-02$ & $3.922 \mathrm{E}-02$ & $3.857 \mathrm{E}-02$ & $3.611 \mathrm{E}-02$ & $3.381 \mathrm{E}-02$ \\
\hline ba136m & 7.797E-03 & $0.000 \mathrm{E}+00$ & $0.000 \mathrm{E}+00$ & $0.000 \mathrm{E}+00$ & $0.000 \mathrm{E}+00$ \\
\hline ba137m & $1.258 \mathrm{E}+05$ & $1.127 \mathrm{E}+05$ & $1.121 \mathrm{E}+05$ & $1.095 \mathrm{E}+05$ & $1.070 \mathrm{E}+05$ \\
\hline ba140 & $9.485 \mathrm{E}-01$ & $0.000 \mathrm{E}+00$ & $0.000 \mathrm{E}+00$ & $0.000 \mathrm{E}+00$ & $0.000 \mathrm{E}+00$ \\
\hline be 10 & $3.104 \mathrm{E}-06$ & $3.104 \mathrm{E}-06$ & $3.104 \mathrm{E}-06$ & $3.104 \mathrm{E}-06$ & $3.104 \mathrm{E}-06$ \\
\hline bi207 & $6.105 \mathrm{E}-17$ & $5.501 \mathrm{E}-17$ & $5.471 \mathrm{E}-17$ & 5.352E-17 & $5.235 \mathrm{E}-17$ \\
\hline bi210 & 4.041E-09 & 4.096E-09 & 4.134E-09 & 4.335E-09 & 4.624E-09 \\
\hline bi211 & 4.762E-08 & $2.170 \mathrm{E}-07$ & $2.215 \mathrm{E}-07$ & $2.611 \mathrm{E}-07$ & $2.965 \mathrm{E}-07$ \\
\hline bi212 & $2.869 \mathrm{E}-03$ & $1.756 \mathrm{E}-02$ & $1.821 \mathrm{E}-02$ & $2.041 \mathrm{E}-02$ & $2.226 \mathrm{E}-02$ \\
\hline bi213 & 7.657E-08 & 7.917E-08 & 7.923E-08 & 8.010E-08 & $8.089 \mathrm{E}-08$ \\
\hline
\end{tabular}




\begin{tabular}{|c|c|c|c|c|c|}
\hline \multicolumn{6}{|c|}{ Table C-8. Continued } \\
\hline & $1 / 22 / 2009$ & $10 / 21 / 2013$ & $1 / 22 / 2014$ & $1 / 22 / 2015$ & $1 / 22 / 2016$ \\
\hline bi214 & $1.380 \mathrm{E}-09$ & 8.661E-09 & $9.306 \mathrm{E}-09$ & $1.212 \mathrm{E}-08$ & $1.543 \mathrm{E}-08$ \\
\hline bk247 & $1.117 \mathrm{E}-12$ & $1.114 \mathrm{E}-12$ & $1.114 \mathrm{E}-12$ & $1.113 \mathrm{E}-12$ & $1.113 \mathrm{E}-12$ \\
\hline bk248 & $6.933 \mathrm{E}-09$ & 4.809E-09 & 4.716E-09 & 4.366E-09 & 4.043E-09 \\
\hline bk249 & $2.466 \mathrm{E}-02$ & $5.777 \mathrm{E}-04$ & 4.723E-04 & $2.142 \mathrm{E}-04$ & $9.718 \mathrm{E}-05$ \\
\hline bk250 & $2.912 \mathrm{E}-08$ & $3.762 \mathrm{E}-10$ & $2.985 \mathrm{E}-10$ & $1.210 \mathrm{E}-10$ & $5.017 \mathrm{E}-11$ \\
\hline bk251 & $2.754 \mathrm{E}-11$ & $0.000 \mathrm{E}+00$ & $0.000 \mathrm{E}+00$ & $0.000 \mathrm{E}+00$ & $0.000 \mathrm{E}+00$ \\
\hline c 14 & $1.765 \mathrm{E}-03$ & $1.763 \mathrm{E}-03$ & $1.763 \mathrm{E}-03$ & $1.763 \mathrm{E}-03$ & $1.763 \mathrm{E}-03$ \\
\hline ca 41 & $4.077 \mathrm{E}-18$ & $4.077 \mathrm{E}-18$ & $4.077 \mathrm{E}-18$ & $4.077 \mathrm{E}-18$ & $4.077 \mathrm{E}-18$ \\
\hline ca 45 & $1.795 \mathrm{E}-13$ & $1.111 \mathrm{E}-16$ & $7.478 \mathrm{E}-17$ & $1.577 \mathrm{E}-17$ & $3.329 \mathrm{E}-18$ \\
\hline cd109 & $5.350 \mathrm{E}-01$ & $3.960 \mathrm{E}-02$ & $3.443 \mathrm{E}-02$ & $1.989 \mathrm{E}-02$ & $1.150 \mathrm{E}-02$ \\
\hline cd113 & $1.232 \mathrm{E}-13$ & $1.232 \mathrm{E}-13$ & $1.232 \mathrm{E}-13$ & $1.232 \mathrm{E}-13$ & $1.232 \mathrm{E}-13$ \\
\hline cd113m & $1.009 \mathrm{E}+00$ & $7.990 \mathrm{E}-01$ & $7.890 \mathrm{E}-01$ & $7.511 \mathrm{E}-01$ & 7.152E-01 \\
\hline cd115m & $1.574 \mathrm{E}+01$ & $3.086 \mathrm{E}-11$ & $7.265 \mathrm{E}-12$ & $2.485 \mathrm{E}-14$ & $8.502 \mathrm{E}-17$ \\
\hline cd116 & $1.080 \mathrm{E}-15$ & $1.080 \mathrm{E}-15$ & $1.080 \mathrm{E}-15$ & $1.080 \mathrm{E}-15$ & $1.080 \mathrm{E}-15$ \\
\hline ce139 & $6.095 \mathrm{E}-18$ & $9.877 \mathrm{E}-22$ & $6.184 \mathrm{E}-22$ & $9.838 \mathrm{E}-23$ & $1.566 \mathrm{E}-23$ \\
\hline ce139 & $9.738 \mathrm{E}+00$ & $1.578 \mathrm{E}-03$ & $9.877 \mathrm{E}-04$ & $1.572 \mathrm{E}-04$ & $2.501 \mathrm{E}-05$ \\
\hline ce141 & $5.447 \mathrm{E}+03$ & $4.876 \mathrm{E}-13$ & $6.714 \mathrm{E}-14$ & $2.798 \mathrm{E}-17$ & $1.167 \mathrm{E}-20$ \\
\hline ce144 & $5.805 \mathrm{E}+05$ & $8.568 \mathrm{E}+03$ & $6.833 \mathrm{E}+03$ & $2.811 \mathrm{E}+03$ & $1.157 \mathrm{E}+03$ \\
\hline cf248 & $4.510 \mathrm{E}-08$ & $1.230 \mathrm{E}-09$ & $1.014 \mathrm{E}-09$ & $4.747 \mathrm{E}-10$ & $2.223 \mathrm{E}-10$ \\
\hline cf249 & 6.642E-05 & $1.255 \mathrm{E}-04$ & $1.257 \mathrm{E}-04$ & $1.261 \mathrm{E}-04$ & $1.261 \mathrm{E}-04$ \\
\hline cf 250 & $9.246 \mathrm{E}-04$ & 7.192E-04 & $7.092 \mathrm{E}-04$ & $6.727 \mathrm{E}-04$ & 6.382E-04 \\
\hline cf 251 & $6.827 \mathrm{E}-06$ & $6.800 \mathrm{E}-06$ & $6.800 \mathrm{E}-06$ & $6.793 \mathrm{E}-06$ & $6.787 \mathrm{E}-06$ \\
\hline cf252 & $8.256 \mathrm{E}-04$ & $2.382 \mathrm{E}-04$ & $2.228 \mathrm{E}-04$ & $1.715 \mathrm{E}-04$ & $1.319 \mathrm{E}-04$ \\
\hline cf 253 & $2.695 \mathrm{E}-09$ & $0.000 \mathrm{E}+00$ & $0.000 \mathrm{E}+00$ & $0.000 \mathrm{E}+00$ & $0.000 \mathrm{E}+00$ \\
\hline cf254 & 4.420E-08 & $1.053 \mathrm{E}-16$ & $3.629 \mathrm{E}-17$ & $5.541 \mathrm{E}-19$ & $8.462 \mathrm{E}-21$ \\
\hline cl 36 & $1.288 \mathrm{E}-16$ & $1.288 \mathrm{E}-16$ & $1.288 \mathrm{E}-16$ & $1.288 \mathrm{E}-16$ & $1.288 \mathrm{E}-16$ \\
\hline cm240 & $3.365 \mathrm{E}-07$ & $1.604 \mathrm{E}-26$ & $1.473 \mathrm{E}-27$ & $0.000 \mathrm{E}+00$ & $0.000 \mathrm{E}+00$ \\
\hline cm241 & $3.875 \mathrm{E}-04$ & $4.821 \mathrm{E}-20$ & $6.753 \mathrm{E}-21$ & $3.018 \mathrm{E}-24$ & $1.349 \mathrm{E}-27$ \\
\hline cm242 & $6.367 \mathrm{E}+04$ & $6.886 \mathrm{E}+01$ & $5.579 \mathrm{E}+01$ & $3.442 \mathrm{E}+01$ & $2.979 \mathrm{E}+01$ \\
\hline cm243 & $8.741 \mathrm{E}+01$ & $7.804 \mathrm{E}+01$ & $7.757 \mathrm{E}+01$ & $7.578 \mathrm{E}+01$ & $7.398 \mathrm{E}+01$ \\
\hline cm244 & $1.391 \mathrm{E}+04$ & $1.160 \mathrm{E}+04$ & $1.149 \mathrm{E}+04$ & $1.105 \mathrm{E}+04$ & $1.064 \mathrm{E}+04$ \\
\hline cm245 & $2.957 \mathrm{E}+00$ & $2.955 \mathrm{E}+00$ & $2.955 \mathrm{E}+00$ & $2.955 \mathrm{E}+00$ & $2.955 \mathrm{E}+00$ \\
\hline $\mathrm{cm} 246$ & 4.166E-01 & 4.163E-01 & 4.163E-01 & 4.162E-01 & 4.162E-01 \\
\hline cm247 & $2.087 \mathrm{E}-06$ & $2.087 \mathrm{E}-06$ & $2.087 \mathrm{E}-06$ & $2.087 \mathrm{E}-06$ & $2.087 \mathrm{E}-06$ \\
\hline cm248 & $6.747 \mathrm{E}-06$ & $6.753 \mathrm{E}-06$ & $6.753 \mathrm{E}-06$ & $6.753 \mathrm{E}-06$ & $6.753 \mathrm{E}-06$ \\
\hline cm249 & $8.375 \mathrm{E}-12$ & $0.000 \mathrm{E}+00$ & $0.000 \mathrm{E}+00$ & $0.000 \mathrm{E}+00$ & $0.000 \mathrm{E}+00$ \\
\hline cm250 & $3.793 \mathrm{E}-11$ & $3.791 \mathrm{E}-11$ & 3.791E-11 & $3.791 \mathrm{E}-11$ & $3.791 \mathrm{E}-11$ \\
\hline co 56 & $3.910 \mathrm{E}-21$ & $6.880 \mathrm{E}-28$ & $2.985 \mathrm{E}-28$ & $1.128 \mathrm{E}-29$ & $0.000 \mathrm{E}+00$ \\
\hline
\end{tabular}




\begin{tabular}{|c|c|c|c|c|c|}
\hline \multicolumn{6}{|c|}{ Table C-8. Continued } \\
\hline & $1 / 22 / 2009$ & $10 / 22 / 2013$ & $1 / 22 / 2014$ & $1 / 22 / 2015$ & $1 / 22 / 2016$ \\
\hline $\cos 57$ & $9.259 \mathrm{E}-16$ & $1.114 \mathrm{E}-17$ & $8.787 \mathrm{E}-18$ & $3.462 \mathrm{E}-18$ & $1.365 \mathrm{E}-18$ \\
\hline $\cos 58$ & $1.838 \mathrm{E}-14$ & $7.983 \mathrm{E}-22$ & $3.214 \mathrm{E}-22$ & $9.047 \mathrm{E}-24$ & $2.546 \mathrm{E}-25$ \\
\hline $\operatorname{co} 60$ & $1.268 \mathrm{E}-11$ & $6.793 \mathrm{E}-12$ & $6.572 \mathrm{E}-12$ & $5.762 \mathrm{E}-12$ & $5.053 \mathrm{E}-12$ \\
\hline $\operatorname{co~} 60 \mathrm{~m}$ & $4.168 \mathrm{E}-21$ & $4.168 \mathrm{E}-21$ & $4.168 \mathrm{E}-21$ & $4.168 \mathrm{E}-21$ & $4.168 \mathrm{E}-21$ \\
\hline cr 51 & $1.111 \mathrm{E}-14$ & $0.000 \mathrm{E}+00$ & $0.000 \mathrm{E}+00$ & $0.000 \mathrm{E}+00$ & $0.000 \mathrm{E}+00$ \\
\hline $\operatorname{cs} 131$ & $1.748 \mathrm{E}-10$ & $0.000 \mathrm{E}+00$ & $0.000 \mathrm{E}+00$ & $0.000 \mathrm{E}+00$ & $0.000 \mathrm{E}+00$ \\
\hline $\operatorname{cs} 132$ & $2.256 \mathrm{E}-11$ & $0.000 \mathrm{E}+00$ & $0.000 \mathrm{E}+00$ & $0.000 \mathrm{E}+00$ & $0.000 \mathrm{E}+00$ \\
\hline $\operatorname{cs134}$ & $1.944 \mathrm{E}+05$ & $3.954 \mathrm{E}+04$ & $3.629 \mathrm{E}+04$ & $2.595 \mathrm{E}+04$ & $1.856 \mathrm{E}+04$ \\
\hline $\operatorname{cs} 135$ & 7.844E-01 & 7.844E-01 & 7.844E-01 & 7.844E-01 & $7.844 \mathrm{E}-01$ \\
\hline $\operatorname{cs} 136$ & 7.039E-02 & $0.000 \mathrm{E}+00$ & $0.000 \mathrm{E}+00$ & $0.000 \mathrm{E}+00$ & $0.000 \mathrm{E}+00$ \\
\hline $\operatorname{cs} 137$ & $1.328 \mathrm{E}+05$ & $1.190 \mathrm{E}+05$ & $1.184 \mathrm{E}+05$ & $1.157 \mathrm{E}+05$ & $1.130 \mathrm{E}+05$ \\
\hline dy159 & $1.430 \mathrm{E}-03$ & $3.489 \mathrm{E}-07$ & $2.233 \mathrm{E}-07$ & $3.872 \mathrm{E}-08$ & $6.714 \mathrm{E}-09$ \\
\hline er169 & 8.914E-09 & $0.000 \mathrm{E}+00$ & $0.000 \mathrm{E}+00$ & $0.000 \mathrm{E}+00$ & $0.000 \mathrm{E}+00$ \\
\hline es252 & 4.445E-12 & $3.482 \mathrm{E}-13$ & 3.037E-13 & $1.776 \mathrm{E}-13$ & $1.039 \mathrm{E}-13$ \\
\hline es 253 & $5.649 \mathrm{E}-08$ & $0.000 \mathrm{E}+00$ & $0.000 \mathrm{E}+00$ & $0.000 \mathrm{E}+00$ & $0.000 \mathrm{E}+00$ \\
\hline es 254 & $2.911 \mathrm{E}-08$ & $3.730 \mathrm{E}-10$ & $2.953 \mathrm{E}-10$ & $1.179 \mathrm{E}-10$ & $4.711 \mathrm{E}-11$ \\
\hline es255 & $3.439 \mathrm{E}-10$ & $2.683 \mathrm{E}-23$ & $5.312 \mathrm{E}-24$ & $9.219 \mathrm{E}-27$ & $1.599 \mathrm{E}-29$ \\
\hline eu147 & $1.013 \mathrm{E}-13$ & $0.000 \mathrm{E}+00$ & $0.000 \mathrm{E}+00$ & $0.000 \mathrm{E}+00$ & $0.000 \mathrm{E}+00$ \\
\hline eu149 & $5.164 \mathrm{E}-07$ & $1.287 \mathrm{E}-12$ & $6.438 \mathrm{E}-13$ & $4.251 \mathrm{E}-14$ & $2.808 \mathrm{E}-15$ \\
\hline eu152 & $5.031 \mathrm{E}+00$ & $3.946 \mathrm{E}+00$ & $3.894 \mathrm{E}+00$ & $3.700 \mathrm{E}+00$ & $3.516 \mathrm{E}+00$ \\
\hline eu154 & $1.496 \mathrm{E}+04$ & $1.021 \mathrm{E}+04$ & $9.997 \mathrm{E}+03$ & $9.226 \mathrm{E}+03$ & $8.515 \mathrm{E}+03$ \\
\hline eu155 & $6.674 \mathrm{E}+03$ & $3.341 \mathrm{E}+03$ & $3.220 \mathrm{E}+03$ & $2.783 \mathrm{E}+03$ & $2.406 \mathrm{E}+03$ \\
\hline eu156 & $2.164 \mathrm{E}+00$ & $0.000 \mathrm{E}+00$ & $0.000 \mathrm{E}+00$ & $0.000 \mathrm{E}+00$ & $0.000 \mathrm{E}+00$ \\
\hline fe 55 & $5.127 \mathrm{E}-13$ & $1.547 \mathrm{E}-13$ & $1.450 \mathrm{E}-13$ & $1.127 \mathrm{E}-13$ & $8.754 \mathrm{E}-14$ \\
\hline fe 59 & $4.940 \mathrm{E}-14$ & $9.312 \mathrm{E}-26$ & $2.188 \mathrm{E}-26$ & $7.418 \mathrm{E}-29$ & $0.000 \mathrm{E}+00$ \\
\hline fe 60 & $4.168 \mathrm{E}-21$ & $4.168 \mathrm{E}-21$ & $4.168 \mathrm{E}-21$ & $4.168 \mathrm{E}-21$ & $4.168 \mathrm{E}-21$ \\
\hline fr221 & 7.657E-08 & 7.917E-08 & $7.930 \mathrm{E}-08$ & 8.010E-08 & $8.089 \mathrm{E}-08$ \\
\hline fr 223 & $7.079 \mathrm{E}-10$ & $2.987 \mathrm{E}-09$ & $3.111 \mathrm{E}-09$ & $3.596 \mathrm{E}-09$ & $4.083 \mathrm{E}-09$ \\
\hline ga 68 & $1.712 \mathrm{E}-15$ & $2.033 \mathrm{E}-17$ & $1.603 \mathrm{E}-17$ & $6.301 \mathrm{E}-18$ & $2.477 \mathrm{E}-18$ \\
\hline gd151 & 4.458E-04 & $2.766 \mathrm{E}-08$ & $1.645 \mathrm{E}-08$ & $2.138 \mathrm{E}-09$ & $2.779 \mathrm{E}-10$ \\
\hline gd152 & $1.349 \mathrm{E}-12$ & $1.387 \mathrm{E}-12$ & $1.389 \mathrm{E}-12$ & $1.395 \mathrm{E}-12$ & $1.402 \mathrm{E}-12$ \\
\hline gd153 & $8.754 \mathrm{E}+00$ & $5.919 \mathrm{E}-02$ & $4.527 \mathrm{E}-02$ & $1.581 \mathrm{E}-02$ & $5.517 \mathrm{E}-03$ \\
\hline ge 68 & $1.712 \mathrm{E}-15$ & $2.033 \mathrm{E}-17$ & $1.603 \mathrm{E}-17$ & $6.299 \mathrm{E}-18$ & $2.476 \mathrm{E}-18$ \\
\hline ge 71 & $7.518 \mathrm{E}-11$ & $0.000 \mathrm{E}+00$ & $0.000 \mathrm{E}+00$ & $0.000 \mathrm{E}+00$ & $0.000 \mathrm{E}+00$ \\
\hline ge 73m & $2.832 \mathrm{E}-06$ & $9.020 \mathrm{E}-13$ & $4.042 \mathrm{E}-13$ & $1.731 \mathrm{E}-14$ & $7.411 \mathrm{E}-16$ \\
\hline h3 & $6.952 \mathrm{E}+02$ & $5.323 \mathrm{E}+02$ & $5.247 \mathrm{E}+02$ & $4.960 \mathrm{E}+02$ & $4.689 \mathrm{E}+02$ \\
\hline hf172 & $1.555 \mathrm{E}-19$ & $2.679 \mathrm{E}-20$ & $2.438 \mathrm{E}-20$ & $1.683 \mathrm{E}-20$ & $1.163 \mathrm{E}-20$ \\
\hline hf175 & $9.758 \mathrm{E}-12$ & $3.441 \mathrm{E}-19$ & $1.370 \mathrm{E}-19$ & $3.690 \mathrm{E}-21$ & $9.937 \mathrm{E}-23$ \\
\hline
\end{tabular}




\begin{tabular}{|c|c|c|c|c|c|}
\hline \multicolumn{6}{|c|}{ Table C-8. Continued } \\
\hline & $1 / 22 / 2009$ & $10 / 22 / 2013$ & $1 / 22 / 2014$ & $1 / 22 / 2015$ & $1 / 22 / 2016$ \\
\hline hf177m & $2.565 \mathrm{E}-14$ & $1.437 \mathrm{E}-17$ & $9.618 \mathrm{E}-18$ & $1.987 \mathrm{E}-18$ & 4.105E-19 \\
\hline hf181 & $4.388 \mathrm{E}-13$ & $2.165 \mathrm{E}-25$ & $4.731 \mathrm{E}-26$ & $1.210 \mathrm{E}-28$ & $0.000 \mathrm{E}+00$ \\
\hline hf182 & $2.753 \mathrm{E}-19$ & $2.753 \mathrm{E}-19$ & $2.753 \mathrm{E}-19$ & $2.753 \mathrm{E}-19$ & $2.753 \mathrm{E}-19$ \\
\hline hg194 & $7.252 \mathrm{E}-21$ & $7.199 \mathrm{E}-21$ & $7.192 \mathrm{E}-21$ & $7.179 \mathrm{E}-21$ & 7.172E-21 \\
\hline hg203 & $6.446 \mathrm{E}-14$ & $4.100 \mathrm{E}-25$ & $1.028 \mathrm{E}-25$ & $4.505 \mathrm{E}-28$ & $0.000 \mathrm{E}+00$ \\
\hline hg206 & 7.677E-17 & 7.777E-17 & $7.850 \mathrm{E}-17$ & $8.229 \mathrm{E}-17$ & $8.781 \mathrm{E}-17$ \\
\hline ho163 & $9.525 \mathrm{E}-08$ & $9.519 \mathrm{E}-08$ & $9.519 \mathrm{E}-08$ & $9.519 \mathrm{E}-08$ & 9.519E-08 \\
\hline ho166m & $1.137 \mathrm{E}-03$ & $1.134 \mathrm{E}-03$ & $1.133 \mathrm{E}-03$ & $1.133 \mathrm{E}-03$ & $1.132 \mathrm{E}-03$ \\
\hline i125 & $1.682 \mathrm{E}-05$ & $2.774 \mathrm{E}-14$ & $9.372 \mathrm{E}-15$ & $1.325 \mathrm{E}-16$ & $1.872 \mathrm{E}-18$ \\
\hline i126 & $1.314 \mathrm{E}-06$ & $0.000 \mathrm{E}+00$ & $0.000 \mathrm{E}+00$ & $0.000 \mathrm{E}+00$ & $0.000 \mathrm{E}+00$ \\
\hline i129 & $4.890 \mathrm{E}-02$ & $4.890 \mathrm{E}-02$ & 4.890E-02 & 4.890E-02 & $4.890 \mathrm{E}-02$ \\
\hline i131 & $1.367 \mathrm{E}-04$ & $0.000 \mathrm{E}+00$ & $0.000 \mathrm{E}+00$ & $0.000 \mathrm{E}+00$ & $0.000 \mathrm{E}+00$ \\
\hline in113m & $6.301 \mathrm{E}-07$ & $1.847 \mathrm{E}-11$ & $1.055 \mathrm{E}-11$ & $1.171 \mathrm{E}-12$ & $1.300 \mathrm{E}-13$ \\
\hline in114 & $5.443 \mathrm{E}-03$ & $1.581 \mathrm{E}-13$ & $4.299 \mathrm{E}-14$ & $2.594 \mathrm{E}-16$ & $1.566 \mathrm{E}-18$ \\
\hline in $114 m$ & $5.625 \mathrm{E}-03$ & $1.633 \mathrm{E}-13$ & 4.443E-14 & $2.681 \mathrm{E}-16$ & $1.619 \mathrm{E}-18$ \\
\hline in115 & $2.132 \mathrm{E}-11$ & $2.132 \mathrm{E}-11$ & $2.132 \mathrm{E}-11$ & $2.132 \mathrm{E}-11$ & $2.132 \mathrm{E}-11$ \\
\hline in $115 m$ & $1.672 \mathrm{E}-03$ & $3.279 \mathrm{E}-15$ & 7.717E-16 & $2.640 \mathrm{E}-18$ & $9.033 \mathrm{E}-21$ \\
\hline ir190 & $7.970 \mathrm{E}-23$ & $0.000 \mathrm{E}+00$ & $0.000 \mathrm{E}+00$ & $0.000 \mathrm{E}+00$ & $0.000 \mathrm{E}+00$ \\
\hline ir191m & 8.834E-19 & $0.000 \mathrm{E}+00$ & $0.000 \mathrm{E}+00$ & $0.000 \mathrm{E}+00$ & $0.000 \mathrm{E}+00$ \\
\hline ir192 & $1.375 \mathrm{E}-12$ & $1.181 \mathrm{E}-19$ & $4.929 \mathrm{E}-20$ & $1.601 \mathrm{E}-21$ & 5.202E-23 \\
\hline ir193m & $7.591 \mathrm{E}-20$ & $0.000 \mathrm{E}+00$ & $0.000 \mathrm{E}+00$ & $0.000 \mathrm{E}+00$ & $0.000 \mathrm{E}+00$ \\
\hline ir194 & $1.811 \mathrm{E}-18$ & $1.046 \mathrm{E}-18$ & $1.016 \mathrm{E}-18$ & $9.053 \mathrm{E}-19$ & $8.070 \mathrm{E}-19$ \\
\hline k 40 & 4.977E-19 & 4.977E-19 & 4.977E-19 & 4.977E-19 & 4.977E-19 \\
\hline k 42 & $7.957 \mathrm{E}-21$ & $7.199 \mathrm{E}-21$ & $7.166 \mathrm{E}-21$ & $7.013 \mathrm{E}-21$ & $6.866 \mathrm{E}-21$ \\
\hline kr 81 & $8.229 \mathrm{E}-07$ & $8.229 \mathrm{E}-07$ & $8.229 \mathrm{E}-07$ & $8.229 \mathrm{E}-07$ & $8.229 \mathrm{E}-07$ \\
\hline kr 83m & $7.996 \mathrm{E}-04$ & $7.092 \mathrm{E}-10$ & $3.356 \mathrm{E}-10$ & $1.783 \mathrm{E}-11$ & $9.472 \mathrm{E}-13$ \\
\hline kr 85 & $6.886 \mathrm{E}+03$ & $5.074 \mathrm{E}+03$ & $4.991 \mathrm{E}+03$ & $4.680 \mathrm{E}+03$ & $4.388 \mathrm{E}+03$ \\
\hline la137 & $2.021 \mathrm{E}-05$ & $2.021 \mathrm{E}-05$ & $2.021 \mathrm{E}-05$ & $2.021 \mathrm{E}-05$ & $2.021 \mathrm{E}-05$ \\
\hline la138 & $6.780 \mathrm{E}-10$ & $6.780 \mathrm{E}-10$ & $6.780 \mathrm{E}-10$ & $6.780 \mathrm{E}-10$ & $6.780 \mathrm{E}-10$ \\
\hline la140 & $1.095 \mathrm{E}+00$ & $0.000 \mathrm{E}+00$ & $0.000 \mathrm{E}+00$ & $0.000 \mathrm{E}+00$ & $0.000 \mathrm{E}+00$ \\
\hline lu172 & $1.569 \mathrm{E}-19$ & $2.679 \mathrm{E}-20$ & $2.438 \mathrm{E}-20$ & $1.683 \mathrm{E}-20$ & $1.163 \mathrm{E}-20$ \\
\hline lu172m & $1.555 \mathrm{E}-19$ & $2.679 \mathrm{E}-20$ & $2.438 \mathrm{E}-20$ & $1.683 \mathrm{E}-20$ & $1.163 \mathrm{E}-20$ \\
\hline lu173 & 4.697E-15 & $4.259 \mathrm{E}-16$ & $3.744 \mathrm{E}-16$ & $2.258 \mathrm{E}-16$ & $1.362 \mathrm{E}-16$ \\
\hline lu174 & $7.345 \mathrm{E}-15$ & $2.814 \mathrm{E}-15$ & $2.668 \mathrm{E}-15$ & $2.164 \mathrm{E}-15$ & $1.755 \mathrm{E}-15$ \\
\hline lu174m & $1.922 \mathrm{E}-15$ & 4.072E-19 & $2.586 \mathrm{E}-19$ & $4.354 \mathrm{E}-20$ & 7.332E-21 \\
\hline lu176 & $3.561 \mathrm{E}-22$ & $3.561 \mathrm{E}-22$ & $3.561 \mathrm{E}-22$ & $3.561 \mathrm{E}-22$ & $3.561 \mathrm{E}-22$ \\
\hline lu177 & $7.285 \mathrm{E}-15$ & $4.082 \mathrm{E}-18$ & $2.731 \mathrm{E}-18$ & $5.643 \mathrm{E}-19$ & $1.166 \mathrm{E}-19$ \\
\hline lu177m & $3.264 \mathrm{E}-14$ & $1.829 \mathrm{E}-17$ & $1.224 \mathrm{E}-17$ & $2.528 \mathrm{E}-18$ & 5.223E-19 \\
\hline
\end{tabular}




\begin{tabular}{|c|c|c|c|c|c|}
\hline \multicolumn{6}{|c|}{ Table C-8. Continued } \\
\hline & $1 / 22 / 2009$ & $10 / 21 / 2013$ & $1 / 22 / 2014$ & $1 / 22 / 2015$ & $1 / 22 / 2016$ \\
\hline mn 53 & $6.793 \mathrm{E}-22$ & $6.793 \mathrm{E}-22$ & $6.793 \mathrm{E}-22$ & $6.793 \mathrm{E}-22$ & $6.793 \mathrm{E}-22$ \\
\hline mn 54 & $1.751 \mathrm{E}-13$ & $3.728 \mathrm{E}-15$ & $3.032 \mathrm{E}-15$ & $1.348 \mathrm{E}-15$ & 5.992E-16 \\
\hline mo 93 & 4.135E-07 & 4.132E-07 & 4.132E-07 & 4.130E-07 & $4.130 \mathrm{E}-07$ \\
\hline mo100 & $5.863 \mathrm{E}-13$ & $5.863 \mathrm{E}-13$ & $5.863 \mathrm{E}-13$ & $5.863 \mathrm{E}-13$ & 5.863E-13 \\
\hline na 22 & 4.873E-19 & $1.377 \mathrm{E}-19$ & $1.287 \mathrm{E}-19$ & $9.864 \mathrm{E}-20$ & $7.558 \mathrm{E}-20$ \\
\hline nb 91 & 5.662E-08 & $5.635 \mathrm{E}-08$ & $5.633 \mathrm{E}-08$ & $5.627 \mathrm{E}-08$ & 5.622E-08 \\
\hline nb 91m & $1.467 \mathrm{E}-10$ & $3.930 \mathrm{E}-19$ & $1.363 \mathrm{E}-19$ & $2.133 \mathrm{E}-21$ & $3.339 \mathrm{E}-23$ \\
\hline nb 92 & $9.512 \mathrm{E}-11$ & $9.512 \mathrm{E}-11$ & $9.512 \mathrm{E}-11$ & $9.512 \mathrm{E}-11$ & $9.512 \mathrm{E}-11$ \\
\hline nb 92m & $2.299 \mathrm{E}-13$ & $0.000 \mathrm{E}+00$ & $0.000 \mathrm{E}+00$ & $0.000 \mathrm{E}+00$ & $0.000 \mathrm{E}+00$ \\
\hline nb 93m & $1.464 \mathrm{E}-01$ & 4.084E-01 & 4.210E-01 & 4.692E-01 & $5.153 \mathrm{E}-01$ \\
\hline nb 94 & $5.892 \mathrm{E}-04$ & 5.891E-04 & 5.891E-04 & $5.891 \mathrm{E}-04$ & $5.891 \mathrm{E}-04$ \\
\hline nb 95 & $1.649 \mathrm{E}+05$ & $1.241 \mathrm{E}-03$ & $4.528 \mathrm{E}-04$ & $8.980 \mathrm{E}-06$ & $1.729 \mathrm{E}-07$ \\
\hline nb 95m & $9.027 \mathrm{E}+02$ & $6.429 \mathrm{E}-06$ & $2.349 \mathrm{E}-06$ & $4.518 \mathrm{E}-08$ & $8.688 \mathrm{E}-10$ \\
\hline nd144 & $1.163 \mathrm{E}-09$ & $1.358 \mathrm{E}-09$ & $1.359 \mathrm{E}-09$ & $1.360 \mathrm{E}-09$ & $1.361 \mathrm{E}-09$ \\
\hline nd147 & $3.603 \mathrm{E}-02$ & $0.000 \mathrm{E}+00$ & $0.000 \mathrm{E}+00$ & $0.000 \mathrm{E}+00$ & $0.000 \mathrm{E}+00$ \\
\hline nd150 & $8.196 \mathrm{E}-14$ & $8.196 \mathrm{E}-14$ & $8.196 \mathrm{E}-14$ & $8.196 \mathrm{E}-14$ & $8.196 \mathrm{E}-14$ \\
\hline ni 59 & $6.575 \mathrm{E}-15$ & $6.575 \mathrm{E}-15$ & $6.575 \mathrm{E}-15$ & $6.575 \mathrm{E}-15$ & $6.575 \mathrm{E}-15$ \\
\hline ni 63 & $1.240 \mathrm{E}-13$ & $1.200 \mathrm{E}-13$ & $1.198 \mathrm{E}-13$ & $1.190 \mathrm{E}-13$ & $1.182 \mathrm{E}-13$ \\
\hline np235 & $3.248 \mathrm{E}-03$ & $1.566 \mathrm{E}-04$ & $1.331 \mathrm{E}-04$ & $7.026 \mathrm{E}-05$ & $3.711 \mathrm{E}-05$ \\
\hline np236 & $3.346 \mathrm{E}-06$ & $3.346 \mathrm{E}-06$ & $3.346 \mathrm{E}-06$ & $3.346 \mathrm{E}-06$ & $3.346 \mathrm{E}-06$ \\
\hline np237 & $1.316 \mathrm{E}-01$ & $1.357 \mathrm{E}-01$ & $1.361 \mathrm{E}-01$ & $1.375 \mathrm{E}-01$ & $1.391 \mathrm{E}-01$ \\
\hline np238 & $1.647 \mathrm{E}-01$ & $1.609 \mathrm{E}-01$ & $1.607 \mathrm{E}-01$ & $1.599 \mathrm{E}-01$ & $1.591 \mathrm{E}-01$ \\
\hline np239 & $7.996 \mathrm{E}+01$ & $7.996 \mathrm{E}+01$ & $7.996 \mathrm{E}+01$ & $7.996 \mathrm{E}+01$ & $7.996 \mathrm{E}+01$ \\
\hline np240 & $2.814 \mathrm{E}-09$ & $2.814 \mathrm{E}-09$ & $2.814 \mathrm{E}-09$ & $2.814 \mathrm{E}-09$ & $2.814 \mathrm{E}-09$ \\
\hline np240m & $2.345 \mathrm{E}-06$ & $2.345 \mathrm{E}-06$ & $2.345 \mathrm{E}-06$ & $2.345 \mathrm{E}-06$ & $2.345 \mathrm{E}-06$ \\
\hline os185 & $1.550 \mathrm{E}-15$ & $4.138 \mathrm{E}-21$ & $2.079 \mathrm{E}-21$ & $1.393 \mathrm{E}-22$ & $9.332 \mathrm{E}-24$ \\
\hline os191 & 8.834E-19 & $0.000 \mathrm{E}+00$ & $0.000 \mathrm{E}+00$ & $0.000 \mathrm{E}+00$ & $0.000 \mathrm{E}+00$ \\
\hline os194 & $1.810 \mathrm{E}-18$ & $1.046 \mathrm{E}-18$ & $1.016 \mathrm{E}-18$ & $9.053 \mathrm{E}-19$ & $8.063 \mathrm{E}-19$ \\
\hline p 32 & $1.835 \mathrm{E}-18$ & $3.787 \mathrm{E}-19$ & $3.783 \mathrm{E}-19$ & $3.766 \mathrm{E}-19$ & $3.749 \mathrm{E}-19$ \\
\hline p 33 & $2.087 \mathrm{E}-16$ & $0.000 \mathrm{E}+00$ & $0.000 \mathrm{E}+00$ & $0.000 \mathrm{E}+00$ & $0.000 \mathrm{E}+00$ \\
\hline pa230 & $3.341 \mathrm{E}-11$ & $0.000 \mathrm{E}+00$ & $0.000 \mathrm{E}+00$ & $0.000 \mathrm{E}+00$ & $0.000 \mathrm{E}+00$ \\
\hline pa231 & $1.135 \mathrm{E}-06$ & $1.319 \mathrm{E}-06$ & $1.329 \mathrm{E}-06$ & $1.368 \mathrm{E}-06$ & $1.407 \mathrm{E}-06$ \\
\hline pa232 & $6.694 \mathrm{E}-09$ & $6.694 \mathrm{E}-09$ & 6.694E-09 & 6.694E-09 & $6.694 \mathrm{E}-09$ \\
\hline pa233 & $1.316 \mathrm{E}-01$ & $1.356 \mathrm{E}-01$ & $1.359 \mathrm{E}-01$ & $1.375 \mathrm{E}-01$ & 1.392E-01 \\
\hline pa234 & $5.004 \mathrm{E}-04$ & $5.006 \mathrm{E}-04$ & $5.004 \mathrm{E}-04$ & $5.004 \mathrm{E}-04$ & $5.004 \mathrm{E}-04$ \\
\hline pa234m & $3.127 \mathrm{E}-01$ & $3.129 \mathrm{E}-01$ & $3.127 \mathrm{E}-01$ & $3.127 \mathrm{E}-01$ & $3.127 \mathrm{E}-01$ \\
\hline pb209 & 7.657E-08 & 7.917E-08 & $7.930 \mathrm{E}-08$ & $8.010 \mathrm{E}-08$ & $8.089 \mathrm{E}-08$ \\
\hline pb210 & 4.040E-09 & 4.094E-09 & 4.132E-09 & 4.333E-09 & 4.621E-09 \\
\hline
\end{tabular}




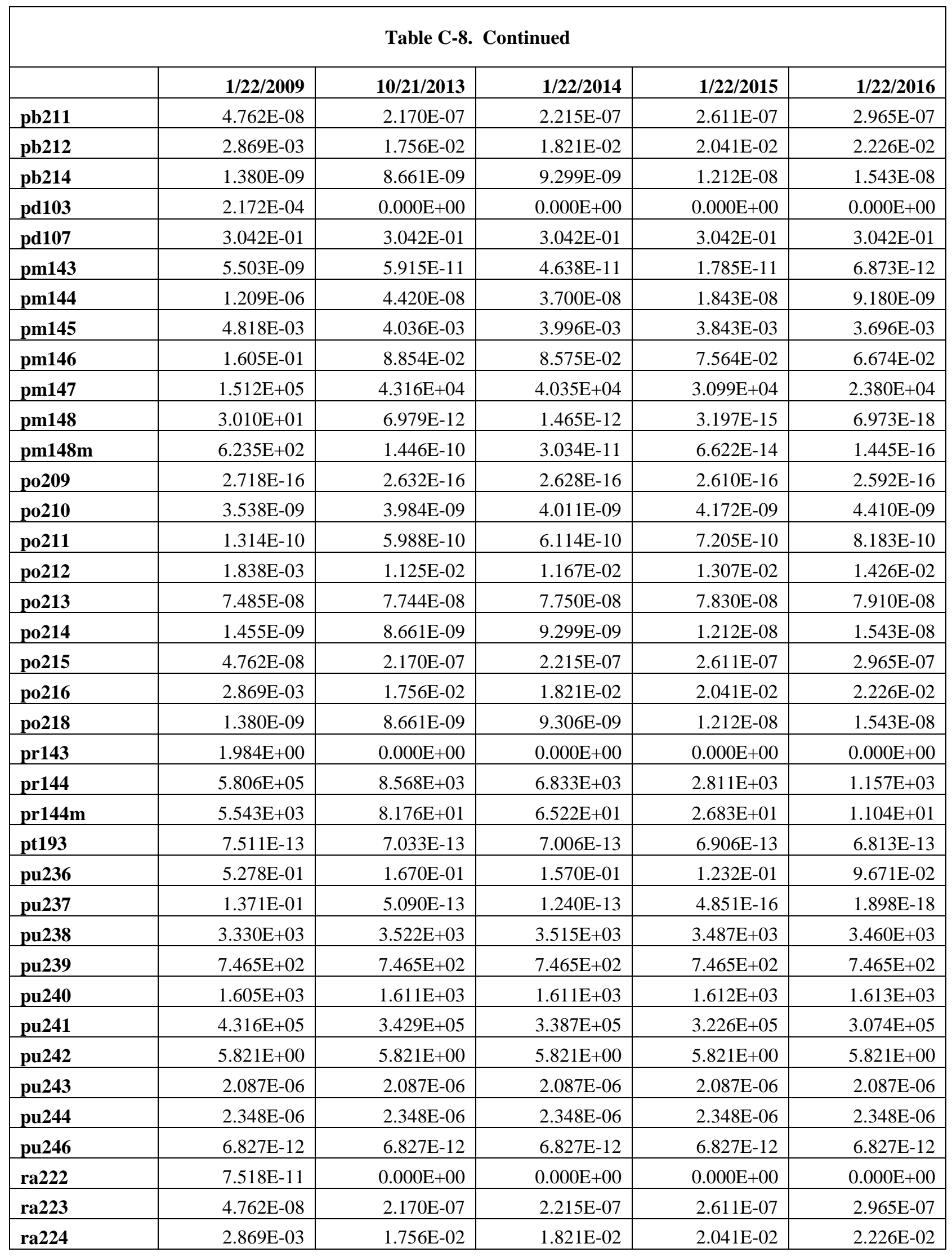




\begin{tabular}{|c|c|c|c|c|c|}
\hline \multicolumn{6}{|c|}{ Table C-8. Continued } \\
\hline & $1 / 22 / 2009$ & $10 / 21 / 2013$ & $1 / 22 / 2014$ & $1 / 22 / 2015$ & $1 / 22 / 2016$ \\
\hline ra225 & 7.651E-08 & 7.917E-08 & $7.930 \mathrm{E}-08$ & $8.010 \mathrm{E}-08$ & $8.089 \mathrm{E}-08$ \\
\hline ra226 & $1.391 \mathrm{E}-09$ & $8.661 \mathrm{E}-09$ & $9.306 \mathrm{E}-09$ & $1.212 \mathrm{E}-08$ & $1.543 \mathrm{E}-08$ \\
\hline ra228 & $1.025 \mathrm{E}-11$ & $1.763 \mathrm{E}-11$ & $1.799 \mathrm{E}-11$ & $1.937 \mathrm{E}-11$ & $2.071 \mathrm{E}-11$ \\
\hline rb 83 & $1.075 \mathrm{E}-03$ & $9.538 \mathrm{E}-10$ & $4.515 \mathrm{E}-10$ & $2.399 \mathrm{E}-11$ & $1.274 \mathrm{E}-12$ \\
\hline rb 84 & $3.109 \mathrm{E}-03$ & $3.955 \mathrm{E}-19$ & $5.548 \mathrm{E}-20$ & $2.490 \mathrm{E}-23$ & $1.118 \mathrm{E}-26$ \\
\hline rb 86 & $1.037 \mathrm{E}-01$ & $1.034 \mathrm{E}-29$ & $0.000 \mathrm{E}+00$ & $0.000 \mathrm{E}+00$ & $0.000 \mathrm{E}+00$ \\
\hline rb 87 & $1.439 \mathrm{E}-05$ & $1.439 \mathrm{E}-05$ & $1.439 \mathrm{E}-05$ & $1.439 \mathrm{E}-05$ & $1.439 \mathrm{E}-05$ \\
\hline re183 & $6.370 \mathrm{E}-19$ & $2.246 \mathrm{E}-26$ & $8.940 \mathrm{E}-27$ & $2.409 \mathrm{E}-28$ & $6.488 \mathrm{E}-30$ \\
\hline re184 & $1.027 \mathrm{E}-16$ & $7.810 \mathrm{E}-20$ & $5.333 \mathrm{E}-20$ & $1.198 \mathrm{E}-20$ & $2.680 \mathrm{E}-21$ \\
\hline re184m & $1.012 \mathrm{E}-16$ & $8.289 \mathrm{E}-20$ & $5.659 \mathrm{E}-20$ & $1.266 \mathrm{E}-20$ & $2.834 \mathrm{E}-21$ \\
\hline re186 & $1.838 \mathrm{E}-18$ & $1.838 \mathrm{E}-18$ & $1.838 \mathrm{E}-18$ & $1.838 \mathrm{E}-18$ & $1.838 \mathrm{E}-18$ \\
\hline re186m & $1.838 \mathrm{E}-18$ & $1.838 \mathrm{E}-18$ & $1.838 \mathrm{E}-18$ & $1.838 \mathrm{E}-18$ & $1.838 \mathrm{E}-18$ \\
\hline re187 & $1.555 \mathrm{E}-20$ & $1.555 \mathrm{E}-20$ & $1.555 \mathrm{E}-20$ & $1.555 \mathrm{E}-20$ & $1.555 \mathrm{E}-20$ \\
\hline re188 & $1.889 \mathrm{E}-14$ & $6.310 \mathrm{E}-22$ & $2.505 \mathrm{E}-22$ & $0.000 \mathrm{E}+00$ & $0.000 \mathrm{E}+00$ \\
\hline rh 99 & $5.904 \mathrm{E}-15$ & $0.000 \mathrm{E}+00$ & $0.000 \mathrm{E}+00$ & $0.000 \mathrm{E}+00$ & $0.000 \mathrm{E}+00$ \\
\hline rh101 & $5.872 \mathrm{E}-04$ & $2.168 \mathrm{E}-04$ & $2.055 \mathrm{E}-04$ & $1.666 \mathrm{E}-04$ & $1.350 \mathrm{E}-04$ \\
\hline rh102 & $4.908 \mathrm{E}+00$ & $1.698 \mathrm{E}-02$ & $1.290 \mathrm{E}-02$ & $4.858 \mathrm{E}-03$ & $2.307 \mathrm{E}-03$ \\
\hline rh102m & $1.803 \mathrm{E}+00$ & $7.491 \mathrm{E}-01$ & $7.146 \mathrm{E}-01$ & $5.936 \mathrm{E}-01$ & 4.933E-01 \\
\hline rh103m & $1.870 \mathrm{E}+04$ & $9.538 \mathrm{E}-10$ & $1.846 \mathrm{E}-10$ & $2.928 \mathrm{E}-13$ & 4.645E-16 \\
\hline rh106 & $7.039 \mathrm{E}+05$ & $2.782 \mathrm{E}+04$ & $2.339 \mathrm{E}+04$ & $1.185 \mathrm{E}+04$ & $5.998 \mathrm{E}+03$ \\
\hline rn217 & $5.359 \mathrm{E}-12$ & $5.541 \mathrm{E}-12$ & $5.549 \mathrm{E}-12$ & $5.605 \mathrm{E}-12$ & $5.662 \mathrm{E}-12$ \\
\hline rn218 & $7.518 \mathrm{E}-11$ & $1.732 \mathrm{E}-15$ & $1.861 \mathrm{E}-15$ & $2.424 \mathrm{E}-15$ & $3.087 \mathrm{E}-15$ \\
\hline rn219 & $4.762 \mathrm{E}-08$ & $2.170 \mathrm{E}-07$ & $2.215 \mathrm{E}-07$ & $2.611 \mathrm{E}-07$ & $2.965 \mathrm{E}-07$ \\
\hline rn220 & $2.869 \mathrm{E}-03$ & $1.756 \mathrm{E}-02$ & $1.821 \mathrm{E}-02$ & $2.041 \mathrm{E}-02$ & $2.226 \mathrm{E}-02$ \\
\hline rn222 & $1.380 \mathrm{E}-09$ & 8.661E-09 & $9.306 \mathrm{E}-09$ & $1.212 \mathrm{E}-08$ & $1.543 \mathrm{E}-08$ \\
\hline ru103 & $1.891 \mathrm{E}+04$ & $9.645 \mathrm{E}-10$ & $1.866 \mathrm{E}-10$ & $2.960 \mathrm{E}-13$ & $4.695 \mathrm{E}-16$ \\
\hline ru106 & $7.039 \mathrm{E}+05$ & $2.782 \mathrm{E}+04$ & $2.339 \mathrm{E}+04$ & $1.185 \mathrm{E}+04$ & $5.998 \mathrm{E}+03$ \\
\hline s 35 & $1.258 \mathrm{E}-13$ & $1.375 \mathrm{E}-19$ & $6.582 \mathrm{E}-20$ & $3.654 \mathrm{E}-21$ & $2.029 \mathrm{E}-22$ \\
\hline sb120m & $1.344 \mathrm{E}-15$ & $0.000 \mathrm{E}+00$ & $0.000 \mathrm{E}+00$ & $0.000 \mathrm{E}+00$ & $0.000 \mathrm{E}+00$ \\
\hline sb124 & $6.510 \mathrm{E}+01$ & $1.405 \mathrm{E}-07$ & 4.815E-08 & 7.199E-10 & $1.077 \mathrm{E}-11$ \\
\hline sb125 & $1.409 \mathrm{E}+04$ & $4.275 \mathrm{E}+03$ & $4.010 \mathrm{E}+03$ & $3.119 \mathrm{E}+03$ & $2.427 \mathrm{E}+03$ \\
\hline sb126 & $7.059 \mathrm{E}-02$ & 7.019E-02 & 7.019E-02 & 7.019E-02 & 7.019E-02 \\
\hline sb126m & $5.014 \mathrm{E}-01$ & $5.014 \mathrm{E}-01$ & $5.014 \mathrm{E}-01$ & $5.014 \mathrm{E}-01$ & $5.014 \mathrm{E}-01$ \\
\hline sb127 & $2.756 \mathrm{E}-16$ & $0.000 \mathrm{E}+00$ & $0.000 \mathrm{E}+00$ & $0.000 \mathrm{E}+00$ & $0.000 \mathrm{E}+00$ \\
\hline sc $45 \mathrm{~m}$ & $3.411 \mathrm{E}-18$ & $2.112 \mathrm{E}-21$ & $1.420 \mathrm{E}-21$ & $2.997 \mathrm{E}-22$ & $6.325 \mathrm{E}-23$ \\
\hline sc 46 & $1.486 \mathrm{E}-12$ & 8.834E-19 & $4.092 \mathrm{E}-19$ & $1.998 \mathrm{E}-20$ & $9.758 \mathrm{E}-22$ \\
\hline se 75 & $1.213 \mathrm{E}-04$ & 5.355E-09 & $3.127 \mathrm{E}-09$ & $3.783 \mathrm{E}-10$ & $4.576 \mathrm{E}-11$ \\
\hline se 79 & $8.209 \mathrm{E}-02$ & $8.209 \mathrm{E}-02$ & $8.209 \mathrm{E}-02$ & 8.209E-02 & 8.209E-02 \\
\hline
\end{tabular}




\begin{tabular}{|c|c|c|c|c|c|}
\hline \multicolumn{6}{|c|}{ Table C-8. Continued } \\
\hline & $1 / 22 / 2009$ & $10 / 21 / 2013$ & $1 / 22 / 2014$ & $1 / 22 / 2015$ & $1 / 22 / 2016$ \\
\hline si 32 & $3.869 \mathrm{E}-19$ & $3.786 \mathrm{E}-19$ & $3.781 \mathrm{E}-19$ & $3.765 \mathrm{E}-19$ & $3.748 \mathrm{E}-19$ \\
\hline sm145 & 7.903E-04 & $2.309 \mathrm{E}-05$ & $1.910 \mathrm{E}-05$ & $9.080 \mathrm{E}-06$ & 4.313E-06 \\
\hline sm146 & $7.538 \mathrm{E}-08$ & $7.671 \mathrm{E}-08$ & $7.671 \mathrm{E}-08$ & 7.691E-08 & $7.711 \mathrm{E}-08$ \\
\hline $\operatorname{sm} 147$ & $2.509 \mathrm{E}-06$ & 5.183E-06 & 5.252E-06 & 5.484E-06 & $5.663 \mathrm{E}-06$ \\
\hline $\operatorname{sm} 148$ & $6.551 \mathrm{E}-11$ & $6.553 \mathrm{E}-11$ & $6.553 \mathrm{E}-11$ & $6.553 \mathrm{E}-11$ & $6.553 \mathrm{E}-11$ \\
\hline sm151 & $5.913 \mathrm{E}+02$ & $5.701 \mathrm{E}+02$ & $5.690 \mathrm{E}+02$ & $5.647 \mathrm{E}+02$ & $5.603 \mathrm{E}+02$ \\
\hline sn113 & $6.297 \mathrm{E}-07$ & $1.847 \mathrm{E}-11$ & $1.054 \mathrm{E}-11$ & $1.171 \mathrm{E}-12$ & $1.299 \mathrm{E}-13$ \\
\hline sn117m & $2.066 \mathrm{E}-04$ & $0.000 \mathrm{E}+00$ & $0.000 \mathrm{E}+00$ & $0.000 \mathrm{E}+00$ & $0.000 \mathrm{E}+00$ \\
\hline sn119m & $2.340 \mathrm{E}+02$ & $3.879 \mathrm{E}+00$ & $3.113 \mathrm{E}+00$ & $1.313 \mathrm{E}+00$ & $5.535 \mathrm{E}-01$ \\
\hline sn121 & $3.747 \mathrm{E}+01$ & $3.476 \mathrm{E}+01$ & $3.462 \mathrm{E}+01$ & $3.409 \mathrm{E}+01$ & $3.355 \mathrm{E}+01$ \\
\hline sn121m & $4.828 \mathrm{E}+01$ & $4.479 \mathrm{E}+01$ & $4.461 \mathrm{E}+01$ & $4.392 \mathrm{E}+01$ & $4.323 \mathrm{E}+01$ \\
\hline sn123 & $5.774 \mathrm{E}+02$ & 5.292E-02 & 3.213E-02 & 4.534E-03 & $6.398 \mathrm{E}-04$ \\
\hline sn125 & $7.405 \mathrm{E}-05$ & $0.000 \mathrm{E}+00$ & $0.000 \mathrm{E}+00$ & $0.000 \mathrm{E}+00$ & $0.000 \mathrm{E}+00$ \\
\hline sn126 & $5.014 \mathrm{E}-01$ & 5.014E-01 & 5.014E-01 & 5.014E-01 & $5.014 \mathrm{E}-01$ \\
\hline sr 85 & $3.314 \mathrm{E}-04$ & $2.983 \mathrm{E}-12$ & $1.103 \mathrm{E}-12$ & $2.229 \mathrm{E}-14$ & $4.505 \mathrm{E}-16$ \\
\hline sr 89 & $1.401 \mathrm{E}+04$ & $6.638 \mathrm{E}-07$ & $1.854 \mathrm{E}-07$ & $1.240 \mathrm{E}-09$ & $8.302 \mathrm{E}-12$ \\
\hline sr 90 & $4.741 \mathrm{E}+04$ & $4.230 \mathrm{E}+04$ & $4.204 \mathrm{E}+04$ & $4.104 \mathrm{E}+04$ & $4.006 \mathrm{E}+04$ \\
\hline ta179 & $2.733 \mathrm{E}-17$ & $4.485 \mathrm{E}-18$ & $4.071 \mathrm{E}-18$ & $2.782 \mathrm{E}-18$ & $1.901 \mathrm{E}-18$ \\
\hline ta182 & $3.974 \mathrm{E}-12$ & $1.131 \mathrm{E}-16$ & $6.462 \mathrm{E}-17$ & 7.372E-18 & $1.058 \mathrm{E}-18$ \\
\hline tb157 & $2.283 \mathrm{E}-05$ & $2.179 \mathrm{E}-05$ & $2.174 \mathrm{E}-05$ & 2.152E-05 & $2.132 \mathrm{E}-05$ \\
\hline tb158 & $1.240 \mathrm{E}-03$ & $1.218 \mathrm{E}-03$ & $1.216 \mathrm{E}-03$ & $1.212 \mathrm{E}-03$ & $1.207 \mathrm{E}-03$ \\
\hline tb160 & $1.925 \mathrm{E}+02$ & $1.172 \mathrm{E}-05$ & 4.804E-06 & $1.452 \mathrm{E}-07$ & 4.387E-09 \\
\hline tb161 & 8.070E-09 & $0.000 \mathrm{E}+00$ & $0.000 \mathrm{E}+00$ & $0.000 \mathrm{E}+00$ & $0.000 \mathrm{E}+00$ \\
\hline tc 95 & $8.814 \mathrm{E}-12$ & $2.471 \mathrm{E}-20$ & $8.588 \mathrm{E}-21$ & $1.357 \mathrm{E}-22$ & $0.000 \mathrm{E}+00$ \\
\hline tc $95 \mathrm{~m}$ & $2.241 \mathrm{E}-10$ & $6.282 \mathrm{E}-19$ & $2.184 \mathrm{E}-19$ & $3.450 \mathrm{E}-21$ & $5.453 \mathrm{E}-23$ \\
\hline tc 97 & 8.967E-09 & $9.040 \mathrm{E}-09$ & $9.040 \mathrm{E}-09$ & $9.040 \mathrm{E}-09$ & $9.040 \mathrm{E}-09$ \\
\hline tc $97 \mathrm{~m}$ & $1.310 \mathrm{E}-03$ & $2.424 \mathrm{E}-09$ & 1.194E-09 & $7.405 \mathrm{E}-11$ & $4.592 \mathrm{E}-12$ \\
\hline tc 98 & $9.186 \mathrm{E}-06$ & $9.186 \mathrm{E}-06$ & $9.186 \mathrm{E}-06$ & $9.186 \mathrm{E}-06$ & $9.186 \mathrm{E}-06$ \\
\hline tc 99 & $1.617 \mathrm{E}+01$ & $1.617 \mathrm{E}+01$ & $1.617 \mathrm{E}+01$ & $1.617 \mathrm{E}+01$ & $1.617 \mathrm{E}+01$ \\
\hline te121 & $1.873 \mathrm{E}-03$ & $1.246 \mathrm{E}-06$ & $8.409 \mathrm{E}-07$ & $1.801 \mathrm{E}-07$ & $3.859 \mathrm{E}-08$ \\
\hline te121m & $1.866 \mathrm{E}-03$ & $1.241 \mathrm{E}-06$ & 8.382E-07 & $1.795 \mathrm{E}-07$ & $3.847 \mathrm{E}-08$ \\
\hline te123m & $3.975 \mathrm{E}+00$ & $1.670 \mathrm{E}-04$ & $9.725 \mathrm{E}-05$ & $1.165 \mathrm{E}-05$ & $1.394 \mathrm{E}-06$ \\
\hline te $125 \mathrm{~m}$ & $3.433 \mathrm{E}+03$ & $1.047 \mathrm{E}+03$ & $9.818 \mathrm{E}+02$ & $7.637 \mathrm{E}+02$ & $5.942 \mathrm{E}+02$ \\
\hline te127 & $2.217 \mathrm{E}+03$ & $3.628 \mathrm{E}-02$ & $2.008 \mathrm{E}-02$ & 1.972E-03 & $1.935 \mathrm{E}-04$ \\
\hline te127m & $2.263 \mathrm{E}+03$ & $3.704 \mathrm{E}-02$ & $2.050 \mathrm{E}-02$ & $2.013 \mathrm{E}-03$ & $1.975 \mathrm{E}-04$ \\
\hline te128 & 4.997E-14 & 4.997E-14 & 4.997E-14 & 4.997E-14 & 4.997E-14 \\
\hline te129 & $1.386 \mathrm{E}+02$ & 4.124E-14 & $6.054 \mathrm{E}-15$ & $3.250 \mathrm{E}-18$ & $1.745 \mathrm{E}-21$ \\
\hline te129m & $2.197 \mathrm{E}+02$ & $6.536 \mathrm{E}-14$ & $9.598 \mathrm{E}-15$ & 5.151E-18 & $2.766 \mathrm{E}-21$ \\
\hline
\end{tabular}




\begin{tabular}{|c|c|c|c|c|c|}
\hline \multicolumn{6}{|c|}{ Table C-8. Continued } \\
\hline & $1 / 22 / 2009$ & $10 / 21 / 2013$ & $1 / 22 / 2014$ & $1 / 22 / 2015$ & $1 / 22 / 2016$ \\
\hline th226 & $7.518 \mathrm{E}-11$ & $0.000 \mathrm{E}+00$ & $0.000 \mathrm{E}+00$ & $0.000 \mathrm{E}+00$ & $0.000 \mathrm{E}+00$ \\
\hline th227 & 4.806E-08 & $2.140 \mathrm{E}-07$ & $2.199 \mathrm{E}-07$ & $2.575 \mathrm{E}-07$ & $2.924 \mathrm{E}-07$ \\
\hline th228 & $2.907 \mathrm{E}-03$ & $1.750 \mathrm{E}-02$ & $1.813 \mathrm{E}-02$ & $2.035 \mathrm{E}-02$ & $2.219 \mathrm{E}-02$ \\
\hline th229 & 7.651E-08 & 7.917E-08 & 7.937E-08 & $8.010 \mathrm{E}-08$ & $8.089 \mathrm{E}-08$ \\
\hline th230 & $1.741 \mathrm{E}-06$ & $5.689 \mathrm{E}-06$ & $5.960 \mathrm{E}-06$ & $7.079 \mathrm{E}-06$ & $8.289 \mathrm{E}-06$ \\
\hline th231 & $1.819 \mathrm{E}-03$ & $1.822 \mathrm{E}-03$ & $1.822 \mathrm{E}-03$ & $1.823 \mathrm{E}-03$ & $1.823 \mathrm{E}-03$ \\
\hline th232 & $2.461 \mathrm{E}-11$ & $2.935 \mathrm{E}-11$ & $2.961 \mathrm{E}-11$ & $3.062 \mathrm{E}-11$ & 3.163E-11 \\
\hline th234 & $3.127 \mathrm{E}-01$ & $3.129 \mathrm{E}-01$ & $3.127 \mathrm{E}-01$ & $3.127 \mathrm{E}-01$ & 3.127E-01 \\
\hline $\mathrm{tl202}$ & $6.001 \mathrm{E}-23$ & $0.000 \mathrm{E}+00$ & $1.644 \mathrm{E}-27$ & $0.000 \mathrm{E}+00$ & $0.000 \mathrm{E}+00$ \\
\hline tl204 & $3.163 \mathrm{E}-14$ & $1.326 \mathrm{E}-14$ & $1.266 \mathrm{E}-14$ & $1.054 \mathrm{E}-14$ & $8.774 \mathrm{E}-15$ \\
\hline tl206 & $5.413 \mathrm{E}-15$ & $5.486 \mathrm{E}-15$ & $5.538 \mathrm{E}-15$ & $5.806 \mathrm{E}-15$ & $6.192 \mathrm{E}-15$ \\
\hline tl207 & 4.749E-08 & $2.164 \mathrm{E}-07$ & $2.209 \mathrm{E}-07$ & 2.604E-07 & $2.957 \mathrm{E}-07$ \\
\hline $\mathrm{t} \mathbf{2 0 8}$ & $1.031 \mathrm{E}-03$ & $6.312 \mathrm{E}-03$ & $6.545 \mathrm{E}-03$ & $7.338 \mathrm{E}-03$ & 8.003E-03 \\
\hline tl209 & $1.684 \mathrm{E}-09$ & $1.742 \mathrm{E}-09$ & $1.744 \mathrm{E}-09$ & $1.761 \mathrm{E}-09$ & $1.779 \mathrm{E}-09$ \\
\hline tl210 & $2.899 \mathrm{E}-13$ & $1.819 \mathrm{E}-12$ & $1.954 \mathrm{E}-12$ & $2.545 \mathrm{E}-12$ & $3.242 \mathrm{E}-12$ \\
\hline $\operatorname{tm} 167$ & $1.779 \mathrm{E}-15$ & $0.000 \mathrm{E}+00$ & $0.000 \mathrm{E}+00$ & $0.000 \mathrm{E}+00$ & $0.000 \mathrm{E}+00$ \\
\hline $\operatorname{tm} 168$ & $7.671 \mathrm{E}-06$ & $1.910 \mathrm{E}-11$ & $9.558 \mathrm{E}-12$ & $6.313 \mathrm{E}-13$ & $4.168 \mathrm{E}-14$ \\
\hline $\operatorname{tm} 170$ & $3.109 \mathrm{E}-01$ & $2.729 \mathrm{E}-05$ & $1.653 \mathrm{E}-05$ & $2.311 \mathrm{E}-06$ & $3.232 \mathrm{E}-07$ \\
\hline $\operatorname{tm} 171$ & $1.872 \mathrm{E}-01$ & $3.377 \mathrm{E}-02$ & $3.081 \mathrm{E}-02$ & $2.148 \mathrm{E}-02$ & $1.497 \mathrm{E}-02$ \\
\hline $\mathbf{u} 230$ & $7.505 \mathrm{E}-11$ & $0.000 \mathrm{E}+00$ & $0.000 \mathrm{E}+00$ & $0.000 \mathrm{E}+00$ & $0.000 \mathrm{E}+00$ \\
\hline $\mathrm{u} 232$ & $1.038 \mathrm{E}-02$ & $2.444 \mathrm{E}-02$ & $2.479 \mathrm{E}-02$ & $2.594 \mathrm{E}-02$ & $2.677 \mathrm{E}-02$ \\
\hline $\mathbf{u} 233$ & $4.489 \mathrm{E}-06$ & $7.425 \mathrm{E}-06$ & $7.578 \mathrm{E}-06$ & $8.236 \mathrm{E}-06$ & $8.900 \mathrm{E}-06$ \\
\hline $\mathbf{u} 234$ & $6.680 \mathrm{E}-02$ & $1.143 \mathrm{E}-01$ & $1.169 \mathrm{E}-01$ & $1.267 \mathrm{E}-01$ & $1.365 \mathrm{E}-01$ \\
\hline $\mathbf{u} 235$ & $1.818 \mathrm{E}-03$ & $1.822 \mathrm{E}-03$ & $1.822 \mathrm{E}-03$ & $1.823 \mathrm{E}-03$ & $1.823 \mathrm{E}-03$ \\
\hline u236 & $2.017 \mathrm{E}-02$ & $2.039 \mathrm{E}-02$ & $2.041 \mathrm{E}-02$ & $2.045 \mathrm{E}-02$ & $2.051 \mathrm{E}-02$ \\
\hline u237 & $1.059 \mathrm{E}+01$ & $8.409 \mathrm{E}+00$ & $8.309 \mathrm{E}+00$ & $7.917 \mathrm{E}+00$ & $7.538 \mathrm{E}+00$ \\
\hline u238 & $3.127 \mathrm{E}-01$ & $3.127 \mathrm{E}-01$ & $3.127 \mathrm{E}-01$ & $3.127 \mathrm{E}-01$ & $3.127 \mathrm{E}-01$ \\
\hline u240 & $2.345 \mathrm{E}-06$ & $2.345 \mathrm{E}-06$ & $2.345 \mathrm{E}-06$ & $2.345 \mathrm{E}-06$ & $2.345 \mathrm{E}-06$ \\
\hline v 49 & 4.263E-16 & $1.119 \mathrm{E}-17$ & $9.206 \mathrm{E}-18$ & $4.276 \mathrm{E}-18$ & $1.986 \mathrm{E}-18$ \\
\hline w181 & $3.744 \mathrm{E}-15$ & $1.858 \mathrm{E}-19$ & $1.091 \mathrm{E}-19$ & $1.353 \mathrm{E}-20$ & $1.678 \mathrm{E}-21$ \\
\hline w185 & $1.164 \mathrm{E}-12$ & $1.317 \mathrm{E}-19$ & $5.580 \mathrm{E}-20$ & $1.921 \mathrm{E}-21$ & $6.614 \mathrm{E}-23$ \\
\hline w188 & $1.870 \mathrm{E}-14$ & $6.246 \mathrm{E}-22$ & $2.479 \mathrm{E}-22$ & 6.603E-24 & $1.759 \mathrm{E}-25$ \\
\hline xe127 & $1.403 \mathrm{E}-03$ & $6.527 \mathrm{E}-18$ & $1.111 \mathrm{E}-18$ & $1.064 \mathrm{E}-21$ & $1.020 \mathrm{E}-24$ \\
\hline xe129m & $3.038 \mathrm{E}-08$ & $0.000 \mathrm{E}+00$ & $0.000 \mathrm{E}+00$ & $0.000 \mathrm{E}+00$ & $0.000 \mathrm{E}+00$ \\
\hline xe131m & $7.398 \mathrm{E}-03$ & $0.000 \mathrm{E}+00$ & $0.000 \mathrm{E}+00$ & $0.000 \mathrm{E}+00$ & $0.000 \mathrm{E}+00$ \\
\hline xe133 & $1.685 \mathrm{E}-09$ & $0.000 \mathrm{E}+00$ & $0.000 \mathrm{E}+00$ & $0.000 \mathrm{E}+00$ & $0.000 \mathrm{E}+00$ \\
\hline y 88 & 3.663E-01 & 4.692E-06 & $2.564 \mathrm{E}-06$ & $2.390 \mathrm{E}-07$ & $2.228 \mathrm{E}-08$ \\
\hline y $89 \mathrm{~m}$ & $1.351 \mathrm{E}+00$ & $6.399 \mathrm{E}-11$ & $1.787 \mathrm{E}-11$ & $1.196 \mathrm{E}-13$ & $8.003 \mathrm{E}-16$ \\
\hline
\end{tabular}




\begin{tabular}{|l|r|r|r|r|r|}
\hline \multicolumn{7}{|c|}{ Table C-8. Continued } \\
\hline & $\mathbf{1 / 2 2 / 2 0 0 9}$ & $\mathbf{1 0 / 2 1 / 2 0 1 3}$ & $\mathbf{1 / 2 2 / 2 0 1 4}$ & $\mathbf{1 / 2 2 / 2 0 1 5}$ & $\mathbf{1 / 2 2 / 2 0 1 6}$ \\
\hline y 90 & $4.743 \mathrm{E}+04$ & $4.231 \mathrm{E}+04$ & $4.205 \mathrm{E}+04$ & $4.105 \mathrm{E}+04$ & $4.007 \mathrm{E}+04$ \\
\hline y 91 & $3.312 \mathrm{E}+04$ & $4.016 \mathrm{E}-05$ & $1.335 \mathrm{E}-05$ & $1.767 \mathrm{E}-07$ & $2.342 \mathrm{E}-09$ \\
\hline yb169 & $8.003 \mathrm{E}-08$ & $4.071 \mathrm{E}-24$ & $5.436 \mathrm{E}-25$ & $2.011 \mathrm{E}-28$ & $0.000 \mathrm{E}+00$ \\
\hline zn 65 & $1.180 \mathrm{E}-15$ & $8.575 \mathrm{E}-18$ & $6.581 \mathrm{E}-18$ & $2.332 \mathrm{E}-18$ & $8.269 \mathrm{E}-19$ \\
\hline zr 88 & $1.031 \mathrm{E}-07$ & $5.727 \mathrm{E}-14$ & $2.644 \mathrm{E}-14$ & $1.273 \mathrm{E}-15$ & $6.128 \mathrm{E}-17$ \\
\hline zr 93 & $1.607 \mathrm{E}+00$ & $1.607 \mathrm{E}+00$ & $1.607 \mathrm{E}+00$ & $1.607 \mathrm{E}+00$ & $1.607 \mathrm{E}+00$ \\
\hline zr 95 & $7.883 \mathrm{E}+04$ & $5.616 \mathrm{E}-04$ & $2.053 \mathrm{E}-04$ & $3.947 \mathrm{E}-06$ & $7.591 \mathrm{E}-08$ \\
\hline zr 96 & $1.595 \mathrm{E}-13$ & $1.595 \mathrm{E}-13$ & $1.595 \mathrm{E}-13$ & $1.595 \mathrm{E}-13$ & $1.595 \mathrm{E}-13$ \\
\hline Total & $\mathbf{4 . 1 8 5 2 E + 0 6}$ & $\mathbf{8 . 6 1 9 5 E + 0 5}$ & $\mathbf{8 . 3 6 7 7 E + 0 5}$ & $\mathbf{7 . 5 9 8 9 E}+\mathbf{0 5}$ & $\mathbf{7 . 0 5 6 9 E}+\mathbf{0 5}$ \\
\hline
\end{tabular}


Table C-9. Activity in Fuel Rod B14 (high Pu loading) in Ci per MTIHM

\begin{tabular}{|c|c|c|c|c|c|}
\hline & $1 / 22 / 2009$ & $10 / 21 / 2013$ & $1 / 22 / 2014$ & $1 / 22 / 2015$ & $1 / 22 / 2016$ \\
\hline ac225 & $6.813 \mathrm{E}-08$ & 7.085E-08 & 7.092E-08 & 7.178E-08 & 7.257E-08 \\
\hline ac226 & $1.247 \mathrm{E}-15$ & $0.000 \mathrm{E}+00$ & $0.000 \mathrm{E}+00$ & $0.000 \mathrm{E}+00$ & $0.000 \mathrm{E}+00$ \\
\hline ac227 & $5.329 \mathrm{E}-08$ & $2.258 \mathrm{E}-07$ & $2.352 \mathrm{E}-07$ & $2.723 \mathrm{E}-07$ & 3.097E-07 \\
\hline ac228 & $8.755 \mathrm{E}-12$ & $1.558 \mathrm{E}-11$ & $1.591 \mathrm{E}-11$ & $1.719 \mathrm{E}-11$ & $1.844 \mathrm{E}-11$ \\
\hline $\operatorname{ag} 105$ & $2.876 \mathrm{E}-10$ & $6.668 \mathrm{E}-23$ & $1.400 \mathrm{E}-23$ & $3.055 \mathrm{E}-26$ & $6.668 \mathrm{E}-29$ \\
\hline ag106m & $1.067 \mathrm{E}-15$ & $0.000 \mathrm{E}+00$ & $0.000 \mathrm{E}+00$ & $0.000 \mathrm{E}+00$ & $0.000 \mathrm{E}+00$ \\
\hline $\operatorname{ag} 108$ & $5.272 \mathrm{E}-04$ & 5.233E-04 & $5.231 \mathrm{E}-04$ & $5.223 \mathrm{E}-04$ & $5.214 \mathrm{E}-04$ \\
\hline $\operatorname{ag} 108 m$ & $6.060 \mathrm{E}-03$ & $6.015 \mathrm{E}-03$ & $6.012 \mathrm{E}-03$ & $6.003 \mathrm{E}-03$ & $5.993 \mathrm{E}-03$ \\
\hline $\operatorname{ag} 109 m$ & $5.758 \mathrm{E}-01$ & 4.262E-02 & $3.706 \mathrm{E}-02$ & $2.141 \mathrm{E}-02$ & $1.237 \mathrm{E}-02$ \\
\hline $\operatorname{ag} 110$ & $9.730 \mathrm{E}+01$ & 7.927E-01 & $6.126 \mathrm{E}-01$ & $2.224 \mathrm{E}-01$ & 8.079E-02 \\
\hline $\operatorname{ag} 110 \mathrm{~m}$ & $7.151 \mathrm{E}+03$ & $5.830 \mathrm{E}+01$ & $4.504 \mathrm{E}+01$ & $1.636 \mathrm{E}+01$ & $5.940 \mathrm{E}+00$ \\
\hline $\operatorname{ag} 111$ & $2.699 \mathrm{E}-06$ & $0.000 \mathrm{E}+00$ & $0.000 \mathrm{E}+00$ & $0.000 \mathrm{E}+00$ & $0.000 \mathrm{E}+00$ \\
\hline am241 & $1.471 \mathrm{E}+03$ & $5.109 \mathrm{E}+03$ & $5.280 \mathrm{E}+03$ & $5.933 \mathrm{E}+03$ & $6.554 \mathrm{E}+03$ \\
\hline am242 & $4.537 \mathrm{E}+01$ & $4.432 \mathrm{E}+01$ & 4.427E+01 & $4.405 \mathrm{E}+01$ & $4.383 \mathrm{E}+01$ \\
\hline am242m & $4.557 \mathrm{E}+01$ & $4.453 \mathrm{E}+01$ & 4.447E+01 & $4.425 \mathrm{E}+01$ & $4.403 \mathrm{E}+01$ \\
\hline am243 & $7.741 \mathrm{E}+01$ & $7.735 \mathrm{E}+01$ & $7.735 \mathrm{E}+01$ & $7.735 \mathrm{E}+01$ & $7.735 \mathrm{E}+01$ \\
\hline am244 & $3.835 \mathrm{E}-09$ & $2.660 \mathrm{E}-09$ & $2.609 \mathrm{E}-09$ & $2.415 \mathrm{E}-09$ & $2.236 \mathrm{E}-09$ \\
\hline am245 & $2.029 \mathrm{E}-07$ & 4.753E-09 & $3.886 \mathrm{E}-09$ & $1.762 \mathrm{E}-09$ & 7.993E-10 \\
\hline am246m & $3.609 \mathrm{E}-12$ & $3.605 \mathrm{E}-12$ & $3.605 \mathrm{E}-12$ & $3.605 \mathrm{E}-12$ & $3.605 \mathrm{E}-12$ \\
\hline ar 37 & $1.235 \mathrm{E}-14$ & $1.597 \mathrm{E}-29$ & $0.000 \mathrm{E}+00$ & $0.000 \mathrm{E}+00$ & $0.000 \mathrm{E}+00$ \\
\hline ar 39 & $4.119 \mathrm{E}-15$ & $4.069 \mathrm{E}-15$ & $4.067 \mathrm{E}-15$ & $4.056 \mathrm{E}-15$ & $4.046 \mathrm{E}-15$ \\
\hline $\operatorname{ar} 42$ & $6.793 \mathrm{E}-21$ & $6.149 \mathrm{E}-21$ & $6.116 \mathrm{E}-21$ & $5.988 \mathrm{E}-21$ & $5.864 \mathrm{E}-21$ \\
\hline as 73 & $3.482 \mathrm{E}-06$ & $1.109 \mathrm{E}-12$ & $4.970 \mathrm{E}-13$ & $2.128 \mathrm{E}-14$ & $9.113 \mathrm{E}-16$ \\
\hline as 74 & $1.950 \mathrm{E}-07$ & $0.000 \mathrm{E}+00$ & $0.000 \mathrm{E}+00$ & $0.000 \mathrm{E}+00$ & $0.000 \mathrm{E}+00$ \\
\hline at217 & $6.813 \mathrm{E}-08$ & $7.085 \mathrm{E}-08$ & $7.092 \mathrm{E}-08$ & $7.178 \mathrm{E}-08$ & 7.257E-08 \\
\hline at218 & $2.873 \mathrm{E}-13$ & $1.799 \mathrm{E}-12$ & $1.933 \mathrm{E}-12$ & $2.517 \mathrm{E}-12$ & $3.205 \mathrm{E}-12$ \\
\hline au194 & $8.397 \mathrm{E}-21$ & $8.331 \mathrm{E}-21$ & $8.324 \mathrm{E}-21$ & $8.311 \mathrm{E}-21$ & $8.298 \mathrm{E}-21$ \\
\hline au195 & $8.430 \mathrm{E}-16$ & $1.326 \mathrm{E}-18$ & $9.378 \mathrm{E}-19$ & $2.409 \mathrm{E}-19$ & $6.186 \mathrm{E}-20$ \\
\hline ba131 & $7.814 \mathrm{E}-18$ & $0.000 \mathrm{E}+00$ & $0.000 \mathrm{E}+00$ & $0.000 \mathrm{E}+00$ & $0.000 \mathrm{E}+00$ \\
\hline ba131 & $4.146 \mathrm{E}-13$ & $0.000 \mathrm{E}+00$ & $0.000 \mathrm{E}+00$ & $0.000 \mathrm{E}+00$ & $0.000 \mathrm{E}+00$ \\
\hline ba133 & $7.721 \mathrm{E}-16$ & $5.649 \mathrm{E}-16$ & $5.555 \mathrm{E}-16$ & $5.201 \mathrm{E}-16$ & $4.869 \mathrm{E}-16$ \\
\hline ba133 & $5.408 \mathrm{E}-02$ & $3.955 \mathrm{E}-02$ & $3.889 \mathrm{E}-02$ & 3.641E-02 & $3.409 \mathrm{E}-02$ \\
\hline ba136m & $9.776 \mathrm{E}-03$ & $0.000 \mathrm{E}+00$ & $0.000 \mathrm{E}+00$ & $0.000 \mathrm{E}+00$ & $0.000 \mathrm{E}+00$ \\
\hline ba137m & $1.307 \mathrm{E}-22$ & $1.172 \mathrm{E}-22$ & $1.164 \mathrm{E}-22$ & $1.138 \mathrm{E}-22$ & $1.112 \mathrm{E}-22$ \\
\hline ba137m & $1.421 \mathrm{E}+05$ & $1.274 \mathrm{E}+05$ & $1.267 \mathrm{E}+05$ & $1.237 \mathrm{E}+05$ & $1.210 \mathrm{E}+05$ \\
\hline ba140 & $1.124 \mathrm{E}+00$ & $0.000 \mathrm{E}+00$ & $0.000 \mathrm{E}+00$ & $0.000 \mathrm{E}+00$ & $0.000 \mathrm{E}+00$ \\
\hline be 10 & $2.915 \mathrm{E}-06$ & $2.915 \mathrm{E}-06$ & $2.915 \mathrm{E}-06$ & $2.915 \mathrm{E}-06$ & $2.915 \mathrm{E}-06$ \\
\hline bi210 & $4.045 \mathrm{E}-09$ & $4.122 \mathrm{E}-09$ & 4.164E-09 & 4.376E-09 & $4.679 \mathrm{E}-09$ \\
\hline
\end{tabular}




\begin{tabular}{|c|c|c|c|c|c|}
\hline \multicolumn{6}{|c|}{ Table C-9. Continued } \\
\hline & $1 / 22 / 2009$ & $10 / 21 / 2013$ & $1 / 22 / 2014$ & $1 / 22 / 2015$ & $1 / 22 / 2016$ \\
\hline bi211 & 4.951E-08 & $2.263 \mathrm{E}-07$ & $2.311 \mathrm{E}-07$ & $2.729 \mathrm{E}-07$ & $3.103 \mathrm{E}-07$ \\
\hline bi212 & $2.666 \mathrm{E}-03$ & $1.680 \mathrm{E}-02$ & $1.743 \mathrm{E}-02$ & $1.956 \mathrm{E}-02$ & $2.135 \mathrm{E}-02$ \\
\hline bi213 & $6.813 \mathrm{E}-08$ & $7.085 \mathrm{E}-08$ & $7.092 \mathrm{E}-08$ & $7.178 \mathrm{E}-08$ & 7.257E-08 \\
\hline bi214 & 1.437E-09 & 8.994E-09 & $9.663 \mathrm{E}-09$ & $1.258 \mathrm{E}-08$ & $1.603 \mathrm{E}-08$ \\
\hline bk247 & $6.064 \mathrm{E}-13$ & $6.050 \mathrm{E}-13$ & $6.049 \mathrm{E}-13$ & $6.046 \mathrm{E}-13$ & $6.043 \mathrm{E}-13$ \\
\hline bk248 & 3.834E-09 & $2.660 \mathrm{E}-09$ & $2.609 \mathrm{E}-09$ & $2.415 \mathrm{E}-09$ & $2.236 \mathrm{E}-09$ \\
\hline bk249 & $1.399 \mathrm{E}-02$ & $3.277 \mathrm{E}-04$ & $2.679 \mathrm{E}-04$ & $1.215 \mathrm{E}-04$ & 5.512E-05 \\
\hline bk250 & $1.113 \mathrm{E}-08$ & $1.442 \mathrm{E}-10$ & $1.145 \mathrm{E}-10$ & $4.670 \mathrm{E}-11$ & $1.962 \mathrm{E}-11$ \\
\hline bk251 & $1.011 \mathrm{E}-11$ & $0.000 \mathrm{E}+00$ & $0.000 \mathrm{E}+00$ & $0.000 \mathrm{E}+00$ & $0.000 \mathrm{E}+00$ \\
\hline c 14 & $1.666 \mathrm{E}-03$ & $1.665 \mathrm{E}-03$ & $1.664 \mathrm{E}-03$ & $1.664 \mathrm{E}-03$ & $1.664 \mathrm{E}-03$ \\
\hline ca 41 & $3.460 \mathrm{E}-18$ & $3.460 \mathrm{E}-18$ & $3.460 \mathrm{E}-18$ & $3.460 \mathrm{E}-18$ & $3.460 \mathrm{E}-18$ \\
\hline ca 45 & $1.660 \mathrm{E}-13$ & $1.028 \mathrm{E}-16$ & $6.913 \mathrm{E}-17$ & $1.459 \mathrm{E}-17$ & $3.079 \mathrm{E}-18$ \\
\hline cd109 & $5.758 \mathrm{E}-01$ & $4.262 \mathrm{E}-02$ & $3.706 \mathrm{E}-02$ & $2.141 \mathrm{E}-02$ & $1.237 \mathrm{E}-02$ \\
\hline cd113 & $1.551 \mathrm{E}-13$ & $1.551 \mathrm{E}-13$ & $1.551 \mathrm{E}-13$ & $1.551 \mathrm{E}-13$ & $1.551 \mathrm{E}-13$ \\
\hline cd113m & $1.254 \mathrm{E}+00$ & $9.928 \mathrm{E}-01$ & $9.809 \mathrm{E}-01$ & $9.338 \mathrm{E}-01$ & 8.888E-01 \\
\hline cd115m & $1.819 \mathrm{E}+01$ & $3.566 \mathrm{E}-11$ & $8.391 \mathrm{E}-12$ & $2.872 \mathrm{E}-14$ & $9.822 \mathrm{E}-17$ \\
\hline cd116 & $1.212 \mathrm{E}-15$ & $1.212 \mathrm{E}-15$ & $1.212 \mathrm{E}-15$ & $1.212 \mathrm{E}-15$ & $1.212 \mathrm{E}-15$ \\
\hline ce139 & $1.122 \mathrm{E}+01$ & $1.819 \mathrm{E}-03$ & $1.139 \mathrm{E}-03$ & $1.812 \mathrm{E}-04$ & $2.883 \mathrm{E}-05$ \\
\hline ce141 & $6.467 \mathrm{E}+03$ & $5.789 \mathrm{E}-13$ & 7.967E-14 & $3.322 \mathrm{E}-17$ & $1.385 \mathrm{E}-20$ \\
\hline ce144 & $6.687 \mathrm{E}+05$ & $9.869 \mathrm{E}+03$ & $7.874 \mathrm{E}+03$ & $3.239 \mathrm{E}+03$ & $1.333 \mathrm{E}+03$ \\
\hline cf 248 & $2.432 \mathrm{E}-08$ & $6.634 \mathrm{E}-10$ & $5.467 \mathrm{E}-10$ & $2.560 \mathrm{E}-10$ & $1.199 \mathrm{E}-10$ \\
\hline cf249 & $3.728 \mathrm{E}-05$ & $7.078 \mathrm{E}-05$ & 7.092E-05 & $7.112 \mathrm{E}-05$ & $7.118 \mathrm{E}-05$ \\
\hline cf250 & 4.765E-04 & $3.706 \mathrm{E}-04$ & $3.656 \mathrm{E}-04$ & $3.467 \mathrm{E}-04$ & $3.288 \mathrm{E}-04$ \\
\hline cf 251 & $3.427 \mathrm{E}-06$ & $3.415 \mathrm{E}-06$ & $3.414 \mathrm{E}-06$ & $3.411 \mathrm{E}-06$ & $3.409 \mathrm{E}-06$ \\
\hline cf252 & $3.561 \mathrm{E}-04$ & $1.027 \mathrm{E}-04$ & $9.604 \mathrm{E}-05$ & $7.390 \mathrm{E}-05$ & $5.689 \mathrm{E}-05$ \\
\hline cf 253 & $1.122 \mathrm{E}-09$ & $0.000 \mathrm{E}+00$ & $0.000 \mathrm{E}+00$ & $0.000 \mathrm{E}+00$ & $0.000 \mathrm{E}+00$ \\
\hline cf 254 & $1.664 \mathrm{E}-08$ & $3.966 \mathrm{E}-17$ & $1.367 \mathrm{E}-17$ & $2.086 \mathrm{E}-19$ & $3.187 \mathrm{E}-21$ \\
\hline cl 36 & $1.088 \mathrm{E}-16$ & $1.088 \mathrm{E}-16$ & $1.088 \mathrm{E}-16$ & $1.088 \mathrm{E}-16$ & $1.088 \mathrm{E}-16$ \\
\hline cm240 & $3.975 \mathrm{E}-07$ & $1.894 \mathrm{E}-26$ & $1.740 \mathrm{E}-27$ & $0.000 \mathrm{E}+00$ & $0.000 \mathrm{E}+00$ \\
\hline cm241 & $4.640 \mathrm{E}-04$ & 5.773E-20 & $8.086 \mathrm{E}-21$ & 3.613E-24 & $1.615 \mathrm{E}-27$ \\
\hline cm242 & $7.310 \mathrm{E}+04$ & $8.258 \mathrm{E}+01$ & $6.754 \mathrm{E}+01$ & $4.303 \mathrm{E}+01$ & $3.769 \mathrm{E}+01$ \\
\hline cm243 & $9.478 \mathrm{E}+01$ & $8.470 \mathrm{E}+01$ & $8.417 \mathrm{E}+01$ & $8.218 \mathrm{E}+01$ & $8.026 \mathrm{E}+01$ \\
\hline cm244 & $1.214 \mathrm{E}+04$ & $1.012 \mathrm{E}+04$ & $1.002 \mathrm{E}+04$ & $9.650 \mathrm{E}+03$ & $9.285 \mathrm{E}+03$ \\
\hline cm245 & $2.456 \mathrm{E}+00$ & $2.454 \mathrm{E}+00$ & $2.454 \mathrm{E}+00$ & $2.454 \mathrm{E}+00$ & $2.454 \mathrm{E}+00$ \\
\hline $\mathrm{cm} 246$ & $2.930 \mathrm{E}-01$ & $2.928 \mathrm{E}-01$ & $2.928 \mathrm{E}-01$ & $2.928 \mathrm{E}-01$ & $2.927 \mathrm{E}-01$ \\
\hline cm247 & $1.358 \mathrm{E}-06$ & $1.358 \mathrm{E}-06$ & $1.358 \mathrm{E}-06$ & $1.358 \mathrm{E}-06$ & $1.358 \mathrm{E}-06$ \\
\hline $\mathrm{cm} 248$ & $3.900 \mathrm{E}-06$ & $3.902 \mathrm{E}-06$ & $3.902 \mathrm{E}-06$ & $3.902 \mathrm{E}-06$ & 3.902E-06 \\
\hline cm249 & $3.488 \mathrm{E}-12$ & $0.000 \mathrm{E}+00$ & $0.000 \mathrm{E}+00$ & $0.000 \mathrm{E}+00$ & $0.000 \mathrm{E}+00$ \\
\hline
\end{tabular}




\begin{tabular}{|c|c|c|c|c|c|}
\hline \multicolumn{6}{|c|}{ Table C-9. Continued } \\
\hline & $1 / 22 / 2009$ & $10 / 21 / 2013$ & $1 / 22 / 2014$ & $1 / 22 / 2015$ & $1 / 22 / 2016$ \\
\hline cm250 & $2.004 \mathrm{E}-11$ & $2.003 \mathrm{E}-11$ & $2.003 \mathrm{E}-11$ & $2.003 \mathrm{E}-11$ & $2.003 \mathrm{E}-11$ \\
\hline $\cos 56$ & $4.028 \mathrm{E}-21$ & $7.085 \mathrm{E}-28$ & $3.075 \mathrm{E}-28$ & $1.162 \mathrm{E}-29$ & $0.000 \mathrm{E}+00$ \\
\hline $\cos 57$ & $9.299 \mathrm{E}-16$ & $1.119 \mathrm{E}-17$ & $8.822 \mathrm{E}-18$ & $3.478 \mathrm{E}-18$ & $1.371 \mathrm{E}-18$ \\
\hline $\cos 58$ & $1.939 \mathrm{E}-14$ & $8.417 \mathrm{E}-22$ & $3.390 \mathrm{E}-22$ & $9.544 \mathrm{E}-24$ & $2.686 \mathrm{E}-25$ \\
\hline co 60 & $1.121 \mathrm{E}-11$ & $6.008 \mathrm{E}-12$ & $5.811 \mathrm{E}-12$ & $5.095 \mathrm{E}-12$ & $4.468 \mathrm{E}-12$ \\
\hline co $60 \mathrm{~m}$ & $3.132 \mathrm{E}-21$ & 3.132E-21 & $3.132 \mathrm{E}-21$ & $3.132 \mathrm{E}-21$ & $3.132 \mathrm{E}-21$ \\
\hline cr 51 & $1.045 \mathrm{E}-14$ & $0.000 \mathrm{E}+00$ & $0.000 \mathrm{E}+00$ & $0.000 \mathrm{E}+00$ & $0.000 \mathrm{E}+00$ \\
\hline $\operatorname{cs} 131$ & $2.100 \mathrm{E}-10$ & $0.000 \mathrm{E}+00$ & $0.000 \mathrm{E}+00$ & $0.000 \mathrm{E}+00$ & $0.000 \mathrm{E}+00$ \\
\hline $\operatorname{cs} 132$ & $2.701 \mathrm{E}-11$ & $0.000 \mathrm{E}+00$ & $0.000 \mathrm{E}+00$ & $0.000 \mathrm{E}+00$ & $0.000 \mathrm{E}+00$ \\
\hline $\operatorname{cs} 134$ & $2.086 \mathrm{E}+05$ & $4.242 \mathrm{E}+04$ & $3.895 \mathrm{E}+04$ & $2.785 \mathrm{E}+04$ & $1.992 \mathrm{E}+04$ \\
\hline $\operatorname{cs} 135$ & $1.004 \mathrm{E}+00$ & $1.004 \mathrm{E}+00$ & $1.004 \mathrm{E}+00$ & $1.004 \mathrm{E}+00$ & $1.004 \mathrm{E}+00$ \\
\hline $\operatorname{cs} 136$ & $8.828 \mathrm{E}-02$ & $0.000 \mathrm{E}+00$ & $0.000 \mathrm{E}+00$ & $0.000 \mathrm{E}+00$ & $0.000 \mathrm{E}+00$ \\
\hline $\operatorname{cs} 137$ & $1.501 \mathrm{E}+05$ & $1.345 \mathrm{E}+05$ & $1.337 \mathrm{E}+05$ & $1.307 \mathrm{E}+05$ & $1.277 \mathrm{E}+05$ \\
\hline dy159 & $1.674 \mathrm{E}-03$ & $4.082 \mathrm{E}-07$ & $2.612 \mathrm{E}-07$ & $4.529 \mathrm{E}-08$ & $7.854 \mathrm{E}-09$ \\
\hline er169 & $9.670 \mathrm{E}-09$ & $0.000 \mathrm{E}+00$ & $0.000 \mathrm{E}+00$ & $0.000 \mathrm{E}+00$ & $0.000 \mathrm{E}+00$ \\
\hline es252 & $1.836 \mathrm{E}-12$ & $1.438 \mathrm{E}-13$ & $1.255 \mathrm{E}-13$ & 7.337E-14 & $4.291 \mathrm{E}-14$ \\
\hline es 253 & $2.349 \mathrm{E}-08$ & $0.000 \mathrm{E}+00$ & $0.000 \mathrm{E}+00$ & $0.000 \mathrm{E}+00$ & $0.000 \mathrm{E}+00$ \\
\hline es254 & $1.112 \mathrm{E}-08$ & $1.426 \mathrm{E}-10$ & $1.129 \mathrm{E}-10$ & $4.508 \mathrm{E}-11$ & $1.801 \mathrm{E}-11$ \\
\hline es 255 & $1.263 \mathrm{E}-10$ & $9.849 \mathrm{E}-24$ & $1.950 \mathrm{E}-24$ & $3.383 \mathrm{E}-27$ & $5.870 \mathrm{E}-30$ \\
\hline eu147 & $1.214 \mathrm{E}-13$ & $0.000 \mathrm{E}+00$ & $0.000 \mathrm{E}+00$ & $0.000 \mathrm{E}+00$ & $0.000 \mathrm{E}+00$ \\
\hline eu149 & 7.397E-07 & $1.844 \mathrm{E}-12$ & $9.226 \mathrm{E}-13$ & $6.092 \mathrm{E}-14$ & $4.023 \mathrm{E}-15$ \\
\hline eu152 & $7.191 \mathrm{E}+00$ & $5.638 \mathrm{E}+00$ & $5.565 \mathrm{E}+00$ & $5.288 \mathrm{E}+00$ & $5.024 \mathrm{E}+00$ \\
\hline eu154 & $1.691 \mathrm{E}+04$ & $1.154 \mathrm{E}+04$ & $1.131 \mathrm{E}+04$ & $1.043 \mathrm{E}+04$ & $9.623 \mathrm{E}+03$ \\
\hline eu155 & $7.383 \mathrm{E}+03$ & $3.696 \mathrm{E}+03$ & $3.561 \mathrm{E}+03$ & $3.079 \mathrm{E}+03$ & $2.661 \mathrm{E}+03$ \\
\hline eu156 & $2.344 \mathrm{E}+00$ & $0.000 \mathrm{E}+00$ & $0.000 \mathrm{E}+00$ & $0.000 \mathrm{E}+00$ & $0.000 \mathrm{E}+00$ \\
\hline fe 55 & $4.435 \mathrm{E}-13$ & $1.337 \mathrm{E}-13$ & $1.255 \mathrm{E}-13$ & $9.743 \mathrm{E}-14$ & $7.569 \mathrm{E}-14$ \\
\hline fe 59 & $4.787 \mathrm{E}-14$ & $9.027 \mathrm{E}-26$ & $2.120 \mathrm{E}-26$ & $7.191 \mathrm{E}-29$ & $0.000 \mathrm{E}+00$ \\
\hline fe 60 & $3.132 \mathrm{E}-21$ & $3.132 \mathrm{E}-21$ & $3.132 \mathrm{E}-21$ & $3.132 \mathrm{E}-21$ & $3.132 \mathrm{E}-21$ \\
\hline fr221 & $6.813 \mathrm{E}-08$ & $7.085 \mathrm{E}-08$ & 7.092E-08 & $7.178 \mathrm{E}-08$ & 7.257E-08 \\
\hline fr223 & 7.357E-10 & $3.116 \mathrm{E}-09$ & $3.246 \mathrm{E}-09$ & 3.759E-09 & 4.274E-09 \\
\hline ga 68 & $1.569 \mathrm{E}-15$ & $1.862 \mathrm{E}-17$ & $1.468 \mathrm{E}-17$ & $5.771 \mathrm{E}-18$ & $2.268 \mathrm{E}-18$ \\
\hline gd151 & $6.387 \mathrm{E}-04$ & 3.964E-08 & $2.357 \mathrm{E}-08$ & 3.064E-09 & $3.983 \mathrm{E}-10$ \\
\hline gd152 & $1.850 \mathrm{E}-12$ & $1.904 \mathrm{E}-12$ & $1.907 \mathrm{E}-12$ & $1.917 \mathrm{E}-12$ & $1.926 \mathrm{E}-12$ \\
\hline gd153 & $1.218 \mathrm{E}+01$ & $8.232 \mathrm{E}-02$ & $6.298 \mathrm{E}-02$ & $2.198 \mathrm{E}-02$ & 7.675E-03 \\
\hline ge 68 & $1.568 \mathrm{E}-15$ & $1.862 \mathrm{E}-17$ & $1.468 \mathrm{E}-17$ & 5.769E-18 & $2.268 \mathrm{E}-18$ \\
\hline ge 71 & $7.894 \mathrm{E}-11$ & $0.000 \mathrm{E}+00$ & $0.000 \mathrm{E}+00$ & $0.000 \mathrm{E}+00$ & $0.000 \mathrm{E}+00$ \\
\hline ge 73m & $3.482 \mathrm{E}-06$ & $1.109 \mathrm{E}-12$ & $4.970 \mathrm{E}-13$ & $2.128 \mathrm{E}-14$ & $9.113 \mathrm{E}-16$ \\
\hline h3 & $7.879 \mathrm{E}+02$ & $6.034 \mathrm{E}+02$ & $5.948 \mathrm{E}+02$ & $5.623 \mathrm{E}+02$ & $5.315 \mathrm{E}+02$ \\
\hline
\end{tabular}




\begin{tabular}{|c|c|c|c|c|c|}
\hline \multicolumn{6}{|c|}{ Table C-9. Continued } \\
\hline & $1 / 22 / 2009$ & $10 / 21 / 2013$ & $1 / 22 / 2014$ & $1 / 22 / 2015$ & $1 / 22 / 2016$ \\
\hline hf172 & $1.647 \mathrm{E}-19$ & $2.837 \mathrm{E}-20$ & $2.582 \mathrm{E}-20$ & $1.783 \mathrm{E}-20$ & $1.231 \mathrm{E}-20$ \\
\hline hf175 & $1.034 \mathrm{E}-11$ & $3.647 \mathrm{E}-19$ & $1.452 \mathrm{E}-19$ & $3.911 \mathrm{E}-21$ & $1.053 \mathrm{E}-22$ \\
\hline hf177m & $2.826 \mathrm{E}-14$ & $1.583 \mathrm{E}-17$ & $1.060 \mathrm{E}-17$ & $2.189 \mathrm{E}-18$ & $4.523 \mathrm{E}-19$ \\
\hline hf181 & $4.015 \mathrm{E}-13$ & $1.980 \mathrm{E}-25$ & $4.329 \mathrm{E}-26$ & $1.107 \mathrm{E}-28$ & $0.000 \mathrm{E}+00$ \\
\hline hf182 & $2.207 \mathrm{E}-19$ & $2.207 \mathrm{E}-19$ & $2.207 \mathrm{E}-19$ & $2.207 \mathrm{E}-19$ & $2.207 \mathrm{E}-19$ \\
\hline hg194 & $8.391 \mathrm{E}-21$ & $8.331 \mathrm{E}-21$ & $8.324 \mathrm{E}-21$ & $8.311 \mathrm{E}-21$ & $8.298 \mathrm{E}-21$ \\
\hline hg203 & $6.078 \mathrm{E}-14$ & $3.866 \mathrm{E}-25$ & $9.690 \mathrm{E}-26$ & $4.248 \mathrm{E}-28$ & $0.000 \mathrm{E}+00$ \\
\hline hg206 & $7.682 \mathrm{E}-17$ & 7.827E-17 & 7.907E-17 & $8.311 \mathrm{E}-17$ & $8.888 \mathrm{E}-17$ \\
\hline ho163 & $1.178 \mathrm{E}-07$ & $1.178 \mathrm{E}-07$ & $1.178 \mathrm{E}-07$ & $1.177 \mathrm{E}-07$ & $1.177 \mathrm{E}-07$ \\
\hline ho166m & $1.235 \mathrm{E}-03$ & $1.232 \mathrm{E}-03$ & $1.231 \mathrm{E}-03$ & $1.231 \mathrm{E}-03$ & $1.230 \mathrm{E}-03$ \\
\hline i125 & $2.062 \mathrm{E}-05$ & $3.401 \mathrm{E}-14$ & $1.149 \mathrm{E}-14$ & $1.624 \mathrm{E}-16$ & $2.295 \mathrm{E}-18$ \\
\hline i126 & $1.617 \mathrm{E}-06$ & $0.000 \mathrm{E}+00$ & $0.000 \mathrm{E}+00$ & $0.000 \mathrm{E}+00$ & $0.000 \mathrm{E}+00$ \\
\hline i129 & $5.619 \mathrm{E}-02$ & $5.619 \mathrm{E}-02$ & $5.619 \mathrm{E}-02$ & $5.619 \mathrm{E}-02$ & $5.619 \mathrm{E}-02$ \\
\hline i131 & $1.627 \mathrm{E}-04$ & $0.000 \mathrm{E}+00$ & $0.000 \mathrm{E}+00$ & $0.000 \mathrm{E}+00$ & $0.000 \mathrm{E}+00$ \\
\hline in113m & $7.675 \mathrm{E}-07$ & $2.251 \mathrm{E}-11$ & $1.285 \mathrm{E}-11$ & $1.427 \mathrm{E}-12$ & $1.583 \mathrm{E}-13$ \\
\hline in114 & $7.158 \mathrm{E}-03$ & $2.079 \mathrm{E}-13$ & $5.654 \mathrm{E}-14$ & $3.413 \mathrm{E}-16$ & $2.060 \mathrm{E}-18$ \\
\hline in114m & $7.403 \mathrm{E}-03$ & $2.149 \mathrm{E}-13$ & $5.844 \mathrm{E}-14$ & $3.527 \mathrm{E}-16$ & $2.129 \mathrm{E}-18$ \\
\hline in115 & $2.514 \mathrm{E}-11$ & $2.515 \mathrm{E}-11$ & $2.515 \mathrm{E}-11$ & $2.515 \mathrm{E}-11$ & $2.515 \mathrm{E}-11$ \\
\hline in115m & $1.933 \mathrm{E}-03$ & $3.789 \mathrm{E}-15$ & $8.914 \mathrm{E}-16$ & $3.051 \mathrm{E}-18$ & $1.044 \mathrm{E}-20$ \\
\hline ir190 & $1.299 \mathrm{E}-22$ & $0.000 \mathrm{E}+00$ & $0.000 \mathrm{E}+00$ & $0.000 \mathrm{E}+00$ & $0.000 \mathrm{E}+00$ \\
\hline ir191m & $6.668 \mathrm{E}-19$ & $0.000 \mathrm{E}+00$ & $0.000 \mathrm{E}+00$ & $0.000 \mathrm{E}+00$ & $0.000 \mathrm{E}+00$ \\
\hline ir192 & $2.123 \mathrm{E}-12$ & $1.822 \mathrm{E}-19$ & $7.609 \mathrm{E}-20$ & $2.472 \mathrm{E}-21$ & $8.033 \mathrm{E}-23$ \\
\hline ir193m & $1.074 \mathrm{E}-19$ & $0.000 \mathrm{E}+00$ & $0.000 \mathrm{E}+00$ & $0.000 \mathrm{E}+00$ & $0.000 \mathrm{E}+00$ \\
\hline ir194 & $1.520 \mathrm{E}-18$ & $8.782 \mathrm{E}-19$ & $8.530 \mathrm{E}-19$ & $7.602 \mathrm{E}-19$ & $6.774 \mathrm{E}-19$ \\
\hline k 40 & $4.999 \mathrm{E}-19$ & $4.999 \mathrm{E}-19$ & $4.999 \mathrm{E}-19$ & $4.999 \mathrm{E}-19$ & $4.999 \mathrm{E}-19$ \\
\hline k 42 & $6.793 \mathrm{E}-21$ & $6.149 \mathrm{E}-21$ & $6.116 \mathrm{E}-21$ & $5.989 \mathrm{E}-21$ & $5.864 \mathrm{E}-21$ \\
\hline kr 81 & $9.160 \mathrm{E}-07$ & $9.160 \mathrm{E}-07$ & $9.160 \mathrm{E}-07$ & $9.160 \mathrm{E}-07$ & $9.160 \mathrm{E}-07$ \\
\hline kr 83m & $9.855 \mathrm{E}-04$ & $8.742 \mathrm{E}-10$ & $4.139 \mathrm{E}-10$ & $2.199 \mathrm{E}-11$ & $1.168 \mathrm{E}-12$ \\
\hline kr 85 & $7.735 \mathrm{E}+03$ & $5.695 \mathrm{E}+03$ & $5.602 \mathrm{E}+03$ & $5.253 \mathrm{E}+03$ & $4.926 \mathrm{E}+03$ \\
\hline la137 & $2.401 \mathrm{E}-05$ & $2.401 \mathrm{E}-05$ & $2.401 \mathrm{E}-05$ & $2.401 \mathrm{E}-05$ & $2.401 \mathrm{E}-05$ \\
\hline la138 & $7.695 \mathrm{E}-10$ & $7.695 \mathrm{E}-10$ & $7.695 \mathrm{E}-10$ & $7.695 \mathrm{E}-10$ & $7.695 \mathrm{E}-10$ \\
\hline la140 & $1.297 \mathrm{E}+00$ & $0.000 \mathrm{E}+00$ & $0.000 \mathrm{E}+00$ & $0.000 \mathrm{E}+00$ & $0.000 \mathrm{E}+00$ \\
\hline lu172 & $1.662 \mathrm{E}-19$ & $2.837 \mathrm{E}-20$ & $2.582 \mathrm{E}-20$ & $1.783 \mathrm{E}-20$ & $1.231 \mathrm{E}-20$ \\
\hline lu172m & $1.647 \mathrm{E}-19$ & $2.837 \mathrm{E}-20$ & $2.582 \mathrm{E}-20$ & $1.783 \mathrm{E}-20$ & $1.231 \mathrm{E}-20$ \\
\hline lu173 & $5.165 \mathrm{E}-15$ & 4.682E-16 & $4.116 \mathrm{E}-16$ & $2.483 \mathrm{E}-16$ & $1.498 \mathrm{E}-16$ \\
\hline lu174 & $7.423 \mathrm{E}-15$ & $2.844 \mathrm{E}-15$ & $2.696 \mathrm{E}-15$ & $2.187 \mathrm{E}-15$ & $1.774 \mathrm{E}-15$ \\
\hline lu174m & $1.982 \mathrm{E}-15$ & $4.199 \mathrm{E}-19$ & $2.666 \mathrm{E}-19$ & $4.490 \mathrm{E}-20$ & $7.556 \mathrm{E}-21$ \\
\hline lu176 & $3.920 \mathrm{E}-22$ & $3.920 \mathrm{E}-22$ & $3.920 \mathrm{E}-22$ & $3.920 \mathrm{E}-22$ & $3.920 \mathrm{E}-22$ \\
\hline
\end{tabular}




\begin{tabular}{|c|c|c|c|c|c|}
\hline \multicolumn{6}{|c|}{ Table C-9. Continued } \\
\hline & $1 / 22 / 2009$ & $10 / 21 / 2013$ & $1 / 22 / 2014$ & $1 / 22 / 2015$ & $1 / 22 / 2016$ \\
\hline lu177 & $8.026 \mathrm{E}-15$ & $4.498 \mathrm{E}-18$ & $3.010 \mathrm{E}-18$ & $6.217 \mathrm{E}-19$ & $1.284 \mathrm{E}-19$ \\
\hline lu177m & $3.596 \mathrm{E}-14$ & $2.015 \mathrm{E}-17$ & $1.348 \mathrm{E}-17$ & $2.785 \mathrm{E}-18$ & $5.754 \mathrm{E}-19$ \\
\hline mn 53 & $6.605 \mathrm{E}-22$ & $6.605 \mathrm{E}-22$ & $6.605 \mathrm{E}-22$ & $6.605 \mathrm{E}-22$ & $6.605 \mathrm{E}-22$ \\
\hline mn 54 & $1.748 \mathrm{E}-13$ & $3.721 \mathrm{E}-15$ & $3.026 \mathrm{E}-15$ & $1.345 \mathrm{E}-15$ & $5.980 \mathrm{E}-16$ \\
\hline mo 93 & 4.567E-07 & $4.563 \mathrm{E}-07$ & $4.563 \mathrm{E}-07$ & $4.562 \mathrm{E}-07$ & $4.561 \mathrm{E}-07$ \\
\hline mo100 & $6.622 \mathrm{E}-13$ & $6.622 \mathrm{E}-13$ & $6.622 \mathrm{E}-13$ & $6.622 \mathrm{E}-13$ & $6.622 \mathrm{E}-13$ \\
\hline na 22 & $5.359 \mathrm{E}-19$ & $1.514 \mathrm{E}-19$ & $1.416 \mathrm{E}-19$ & $1.085 \mathrm{E}-19$ & $8.311 \mathrm{E}-20$ \\
\hline nb 91 & $6.668 \mathrm{E}-08$ & 6.634E-08 & $6.634 \mathrm{E}-08$ & $6.625 \mathrm{E}-08$ & $6.618 \mathrm{E}-08$ \\
\hline nb $91 \mathrm{~m}$ & $1.785 \mathrm{E}-10$ & $4.782 \mathrm{E}-19$ & $1.658 \mathrm{E}-19$ & $2.595 \mathrm{E}-21$ & $4.063 \mathrm{E}-23$ \\
\hline nb 92 & $1.123 \mathrm{E}-10$ & $1.123 \mathrm{E}-10$ & $1.123 \mathrm{E}-10$ & $1.123 \mathrm{E}-10$ & $1.123 \mathrm{E}-10$ \\
\hline nb 92m & $2.652 \mathrm{E}-13$ & $0.000 \mathrm{E}+00$ & $0.000 \mathrm{E}+00$ & $0.000 \mathrm{E}+00$ & $0.000 \mathrm{E}+00$ \\
\hline nb 93m & $1.620 \mathrm{E}-01$ & $4.565 \mathrm{E}-01$ & $4.706 \mathrm{E}-01$ & $5.247 \mathrm{E}-01$ & $5.765 \mathrm{E}-01$ \\
\hline nb 94 & $6.760 \mathrm{E}-04$ & $6.760 \mathrm{E}-04$ & $6.760 \mathrm{E}-04$ & $6.754 \mathrm{E}-04$ & $6.754 \mathrm{E}-04$ \\
\hline nb 95 & $1.958 \mathrm{E}+05$ & $1.473 \mathrm{E}-03$ & $5.376 \mathrm{E}-04$ & $1.066 \mathrm{E}-05$ & $2.053 \mathrm{E}-07$ \\
\hline nb 95m & $1.072 \mathrm{E}+03$ & 7.635E-06 & $2.789 \mathrm{E}-06$ & 5.364E-08 & $1.032 \mathrm{E}-09$ \\
\hline nd144 & $1.259 \mathrm{E}-09$ & $1.484 \mathrm{E}-09$ & $1.485 \mathrm{E}-09$ & $1.486 \mathrm{E}-09$ & $1.487 \mathrm{E}-09$ \\
\hline nd147 & $4.256 \mathrm{E}-02$ & $0.000 \mathrm{E}+00$ & $0.000 \mathrm{E}+00$ & $0.000 \mathrm{E}+00$ & $0.000 \mathrm{E}+00$ \\
\hline nd150 & $9.193 \mathrm{E}-14$ & $9.193 \mathrm{E}-14$ & $9.193 \mathrm{E}-14$ & $9.193 \mathrm{E}-14$ & $9.193 \mathrm{E}-14$ \\
\hline ni 59 & $6.760 \mathrm{E}-15$ & $6.760 \mathrm{E}-15$ & $6.760 \mathrm{E}-15$ & $6.760 \mathrm{E}-15$ & $6.760 \mathrm{E}-15$ \\
\hline ni 63 & $1.045 \mathrm{E}-13$ & $1.011 \mathrm{E}-13$ & $1.009 \mathrm{E}-13$ & $1.002 \mathrm{E}-13$ & $9.955 \mathrm{E}-14$ \\
\hline np235 & $3.157 \mathrm{E}-03$ & $1.522 \mathrm{E}-04$ & $1.294 \mathrm{E}-04$ & $6.833 \mathrm{E}-05$ & 3.607E-05 \\
\hline np236 & $3.508 \mathrm{E}-06$ & $3.508 \mathrm{E}-06$ & $3.508 \mathrm{E}-06$ & $3.508 \mathrm{E}-06$ & $3.508 \mathrm{E}-06$ \\
\hline np237 & $1.276 \mathrm{E}-01$ & $1.328 \mathrm{E}-01$ & $1.332 \mathrm{E}-01$ & $1.350 \mathrm{E}-01$ & $1.370 \mathrm{E}-01$ \\
\hline np238 & $2.092 \mathrm{E}-01$ & $2.044 \mathrm{E}-01$ & $2.041 \mathrm{E}-01$ & $2.031 \mathrm{E}-01$ & $2.021 \mathrm{E}-01$ \\
\hline np239 & $7.741 \mathrm{E}+01$ & $7.735 \mathrm{E}+01$ & $7.735 \mathrm{E}+01$ & $7.735 \mathrm{E}+01$ & $7.735 \mathrm{E}+01$ \\
\hline np240 & $2.433 \mathrm{E}-09$ & $2.433 \mathrm{E}-09$ & $2.433 \mathrm{E}-09$ & $2.433 \mathrm{E}-09$ & $2.433 \mathrm{E}-09$ \\
\hline np240m & $2.027 \mathrm{E}-06$ & $2.027 \mathrm{E}-06$ & $2.027 \mathrm{E}-06$ & $2.027 \mathrm{E}-06$ & $2.027 \mathrm{E}-06$ \\
\hline os185 & $1.575 \mathrm{E}-15$ & 4.203E-21 & $2.111 \mathrm{E}-21$ & $1.414 \mathrm{E}-22$ & $9.478 \mathrm{E}-24$ \\
\hline os191 & $6.668 \mathrm{E}-19$ & $0.000 \mathrm{E}+00$ & $0.000 \mathrm{E}+00$ & $0.000 \mathrm{E}+00$ & $0.000 \mathrm{E}+00$ \\
\hline os194 & $1.519 \mathrm{E}-18$ & 8.782E-19 & 8.523E-19 & $7.595 \mathrm{E}-19$ & $6.767 \mathrm{E}-19$ \\
\hline p 32 & $1.848 \mathrm{E}-18$ & $3.593 \mathrm{E}-19$ & $3.589 \mathrm{E}-19$ & $3.572 \mathrm{E}-19$ & $3.556 \mathrm{E}-19$ \\
\hline p 33 & $2.157 \mathrm{E}-16$ & $0.000 \mathrm{E}+00$ & $0.000 \mathrm{E}+00$ & $0.000 \mathrm{E}+00$ & $0.000 \mathrm{E}+00$ \\
\hline pa230 & $3.623 \mathrm{E}-11$ & $0.000 \mathrm{E}+00$ & $0.000 \mathrm{E}+00$ & $0.000 \mathrm{E}+00$ & $0.000 \mathrm{E}+00$ \\
\hline pa231 & $1.176 \mathrm{E}-06$ & $1.387 \mathrm{E}-06$ & $1.398 \mathrm{E}-06$ & $1.442 \mathrm{E}-06$ & $1.487 \mathrm{E}-06$ \\
\hline pa232 & 7.019E-09 & 7.019E-09 & 7.019E-09 & 7.019E-09 & 7.019E-09 \\
\hline pa233 & $1.275 \mathrm{E}-01$ & $1.326 \mathrm{E}-01$ & $1.330 \mathrm{E}-01$ & $1.350 \mathrm{E}-01$ & $1.370 \mathrm{E}-01$ \\
\hline pa234 & 4.932E-04 & 4.934E-04 & 4.932E-04 & 4.932E-04 & 4.932E-04 \\
\hline pa234m & $3.083 \mathrm{E}-01$ & $3.083 \mathrm{E}-01$ & $3.083 \mathrm{E}-01$ & $3.083 \mathrm{E}-01$ & $3.083 \mathrm{E}-01$ \\
\hline
\end{tabular}




\begin{tabular}{|c|c|c|c|c|c|}
\hline \multicolumn{6}{|c|}{ Table C-9. Continued } \\
\hline & $1 / 22 / 2009$ & $10 / 21 / 2013$ & $1 / 22 / 2014$ & $1 / 22 / 2015$ & $1 / 22 / 2016$ \\
\hline pb209 & $6.813 \mathrm{E}-08$ & $7.085 \mathrm{E}-08$ & $7.092 \mathrm{E}-08$ & $7.178 \mathrm{E}-08$ & 7.257E-08 \\
\hline pb210 & 4.043E-09 & 4.120E-09 & 4.162E-09 & 4.374E-09 & 4.677E-09 \\
\hline pb211 & 4.951E-08 & $2.263 \mathrm{E}-07$ & $2.311 \mathrm{E}-07$ & $2.729 \mathrm{E}-07$ & 3.103E-07 \\
\hline pb212 & $2.666 \mathrm{E}-03$ & $1.680 \mathrm{E}-02$ & $1.743 \mathrm{E}-02$ & $1.956 \mathrm{E}-02$ & $2.135 \mathrm{E}-02$ \\
\hline pb214 & $1.436 \mathrm{E}-09$ & 8.994E-09 & $9.663 \mathrm{E}-09$ & $1.258 \mathrm{E}-08$ & $1.602 \mathrm{E}-08$ \\
\hline pd103 & $2.334 \mathrm{E}-04$ & $0.000 \mathrm{E}+00$ & $0.000 \mathrm{E}+00$ & $0.000 \mathrm{E}+00$ & $0.000 \mathrm{E}+00$ \\
\hline pd107 & $3.470 \mathrm{E}-01$ & $3.470 \mathrm{E}-01$ & $3.470 \mathrm{E}-01$ & $3.470 \mathrm{E}-01$ & $3.470 \mathrm{E}-01$ \\
\hline pm143 & $6.264 \mathrm{E}-09$ & $6.734 \mathrm{E}-11$ & $5.279 \mathrm{E}-11$ & $2.032 \mathrm{E}-11$ & $7.821 \mathrm{E}-12$ \\
\hline pm144 & $1.389 \mathrm{E}-06$ & $5.074 \mathrm{E}-08$ & 4.249E-08 & $2.116 \mathrm{E}-08$ & $1.054 \mathrm{E}-08$ \\
\hline pm145 & $5.511 \mathrm{E}-03$ & $4.618 \mathrm{E}-03$ & $4.572 \mathrm{E}-03$ & 4.398E-03 & $4.229 \mathrm{E}-03$ \\
\hline pm146 & $2.151 \mathrm{E}-01$ & $1.187 \mathrm{E}-01$ & $1.150 \mathrm{E}-01$ & $1.014 \mathrm{E}-01$ & 8.947E-02 \\
\hline pm147 & $1.789 \mathrm{E}+05$ & $5.106 \mathrm{E}+04$ & 4.774E+04 & $3.666 \mathrm{E}+04$ & $2.815 \mathrm{E}+04$ \\
\hline pm148 & $3.696 \mathrm{E}+01$ & $8.570 \mathrm{E}-12$ & $1.799 \mathrm{E}-12$ & $3.926 \mathrm{E}-15$ & 8.563E-18 \\
\hline pm148m & $7.655 \mathrm{E}+02$ & $1.776 \mathrm{E}-10$ & $3.726 \mathrm{E}-11$ & $8.132 \mathrm{E}-14$ & $1.774 \mathrm{E}-16$ \\
\hline po209 & $2.595 \mathrm{E}-16$ & $2.513 \mathrm{E}-16$ & $2.508 \mathrm{E}-16$ & $2.491 \mathrm{E}-16$ & $2.474 \mathrm{E}-16$ \\
\hline po210 & $3.517 \mathrm{E}-09$ & 4.005E-09 & 4.034E-09 & 4.207E-09 & $4.458 \mathrm{E}-09$ \\
\hline po211 & $1.367 \mathrm{E}-10$ & $6.246 \mathrm{E}-10$ & $6.379 \mathrm{E}-10$ & $7.529 \mathrm{E}-10$ & $8.563 \mathrm{E}-10$ \\
\hline po212 & $1.708 \mathrm{E}-03$ & $1.076 \mathrm{E}-02$ & $1.117 \mathrm{E}-02$ & $1.253 \mathrm{E}-02$ & $1.367 \mathrm{E}-02$ \\
\hline po213 & $6.668 \mathrm{E}-08$ & $6.926 \mathrm{E}-08$ & $6.939 \mathrm{E}-08$ & $7.019 \mathrm{E}-08$ & $7.098 \mathrm{E}-08$ \\
\hline po214 & $1.518 \mathrm{E}-09$ & 8.994E-09 & $9.663 \mathrm{E}-09$ & $1.258 \mathrm{E}-08$ & $1.602 \mathrm{E}-08$ \\
\hline po215 & $4.951 \mathrm{E}-08$ & $2.263 \mathrm{E}-07$ & $2.311 \mathrm{E}-07$ & $2.729 \mathrm{E}-07$ & 3.103E-07 \\
\hline po216 & $2.666 \mathrm{E}-03$ & $1.680 \mathrm{E}-02$ & $1.743 \mathrm{E}-02$ & $1.956 \mathrm{E}-02$ & $2.135 \mathrm{E}-02$ \\
\hline po218 & $1.437 \mathrm{E}-09$ & 8.994E-09 & $9.663 \mathrm{E}-09$ & $1.258 \mathrm{E}-08$ & $1.603 \mathrm{E}-08$ \\
\hline pr143 & $2.351 \mathrm{E}+00$ & $0.000 \mathrm{E}+00$ & $0.000 \mathrm{E}+00$ & $0.000 \mathrm{E}+00$ & $0.000 \mathrm{E}+00$ \\
\hline pr144 & $6.687 \mathrm{E}+05$ & $9.869 \mathrm{E}+03$ & $7.874 \mathrm{E}+03$ & $3.239 \mathrm{E}+03$ & $1.333 \mathrm{E}+03$ \\
\hline pr144m & $6.388 \mathrm{E}+03$ & $9.425 \mathrm{E}+01$ & $7.516 \mathrm{E}+01$ & $3.093 \mathrm{E}+01$ & $1.273 \mathrm{E}+01$ \\
\hline pt193 & 7.397E-13 & $6.926 \mathrm{E}-13$ & $6.899 \mathrm{E}-13$ & $6.807 \mathrm{E}-13$ & $6.714 \mathrm{E}-13$ \\
\hline pu236 & $5.116 \mathrm{E}-01$ & $1.618 \mathrm{E}-01$ & $1.522 \mathrm{E}-01$ & $1.194 \mathrm{E}-01$ & 9.372E-02 \\
\hline pu237 & $1.495 \mathrm{E}-01$ & $5.546 \mathrm{E}-13$ & $1.351 \mathrm{E}-13$ & $5.286 \mathrm{E}-16$ & $2.069 \mathrm{E}-18$ \\
\hline pu238 & $3.434 \mathrm{E}+03$ & $3.668 \mathrm{E}+03$ & $3.661 \mathrm{E}+03$ & $3.633 \mathrm{E}+03$ & $3.604 \mathrm{E}+03$ \\
\hline pu239 & $9.809 \mathrm{E}+02$ & $9.809 \mathrm{E}+02$ & $9.809 \mathrm{E}+02$ & $9.809 \mathrm{E}+02$ & $9.809 \mathrm{E}+02$ \\
\hline pu240 & $2.245 \mathrm{E}+03$ & $2.249 \mathrm{E}+03$ & $2.249 \mathrm{E}+03$ & $2.250 \mathrm{E}+03$ & $2.251 \mathrm{E}+03$ \\
\hline pu241 & $5.394 \mathrm{E}+05$ & $4.285 \mathrm{E}+05$ & $4.232 \mathrm{E}+05$ & $4.032 \mathrm{E}+05$ & $3.841 \mathrm{E}+05$ \\
\hline pu242 & $6.028 \mathrm{E}+00$ & $6.028 \mathrm{E}+00$ & $6.028 \mathrm{E}+00$ & $6.028 \mathrm{E}+00$ & $6.028 \mathrm{E}+00$ \\
\hline pu243 & $1.358 \mathrm{E}-06$ & $1.358 \mathrm{E}-06$ & $1.358 \mathrm{E}-06$ & $1.358 \mathrm{E}-06$ & $1.358 \mathrm{E}-06$ \\
\hline pu244 & $2.030 \mathrm{E}-06$ & $2.030 \mathrm{E}-06$ & $2.030 \mathrm{E}-06$ & $2.030 \mathrm{E}-06$ & $2.030 \mathrm{E}-06$ \\
\hline pu246 & $3.607 \mathrm{E}-12$ & $3.605 \mathrm{E}-12$ & $3.605 \mathrm{E}-12$ & $3.605 \mathrm{E}-12$ & $3.605 \mathrm{E}-12$ \\
\hline $\mathrm{ra222}$ & $8.139 \mathrm{E}-11$ & $0.000 \mathrm{E}+00$ & $0.000 \mathrm{E}+00$ & $0.000 \mathrm{E}+00$ & $0.000 \mathrm{E}+00$ \\
\hline
\end{tabular}




\begin{tabular}{|c|c|c|c|c|c|}
\hline \multicolumn{6}{|c|}{ Table C-9. Continued } \\
\hline & $1 / 22 / 2009$ & $10 / 21 / 2013$ & $1 / 22 / 2014$ & $1 / 22 / 2015$ & $1 / 22 / 2016$ \\
\hline ra223 & 4.951E-08 & $2.263 \mathrm{E}-07$ & $2.311 \mathrm{E}-07$ & $2.729 \mathrm{E}-07$ & $3.103 \mathrm{E}-07$ \\
\hline ra224 & $2.666 \mathrm{E}-03$ & $1.680 \mathrm{E}-02$ & $1.743 \mathrm{E}-02$ & $1.956 \mathrm{E}-02$ & $2.135 \mathrm{E}-02$ \\
\hline ra225 & $6.813 \mathrm{E}-08$ & $7.085 \mathrm{E}-08$ & $7.098 \mathrm{E}-08$ & $7.178 \mathrm{E}-08$ & $7.257 \mathrm{E}-08$ \\
\hline ra226 & $1.448 \mathrm{E}-09$ & 8.994E-09 & $9.663 \mathrm{E}-09$ & $1.258 \mathrm{E}-08$ & $1.603 \mathrm{E}-08$ \\
\hline ra228 & $8.755 \mathrm{E}-12$ & $1.558 \mathrm{E}-11$ & $1.591 \mathrm{E}-11$ & $1.719 \mathrm{E}-11$ & $1.844 \mathrm{E}-11$ \\
\hline rb 83 & $1.326 \mathrm{E}-03$ & $1.176 \mathrm{E}-09$ & $5.568 \mathrm{E}-10$ & $2.958 \mathrm{E}-11$ & $1.571 \mathrm{E}-12$ \\
\hline rb 84 & $3.610 \mathrm{E}-03$ & $4.592 \mathrm{E}-19$ & $6.442 \mathrm{E}-20$ & $2.892 \mathrm{E}-23$ & $1.298 \mathrm{E}-26$ \\
\hline rb 86 & $1.164 \mathrm{E}-01$ & $1.161 \mathrm{E}-29$ & $0.000 \mathrm{E}+00$ & $0.000 \mathrm{E}+00$ & $0.000 \mathrm{E}+00$ \\
\hline rb 87 & $1.599 \mathrm{E}-05$ & $1.599 \mathrm{E}-05$ & $1.599 \mathrm{E}-05$ & $1.599 \mathrm{E}-05$ & $1.599 \mathrm{E}-05$ \\
\hline re183 & $7.436 \mathrm{E}-19$ & $2.623 \mathrm{E}-26$ & $1.045 \mathrm{E}-26$ & $2.813 \mathrm{E}-28$ & $7.576 \mathrm{E}-30$ \\
\hline re184 & $1.286 \mathrm{E}-16$ & $9.783 \mathrm{E}-20$ & $6.681 \mathrm{E}-20$ & $1.501 \mathrm{E}-20$ & $3.358 \mathrm{E}-21$ \\
\hline re184m & $1.269 \mathrm{E}-16$ & $1.038 \mathrm{E}-19$ & $7.092 \mathrm{E}-20$ & $1.587 \mathrm{E}-20$ & $3.550 \mathrm{E}-21$ \\
\hline re186 & $1.919 \mathrm{E}-18$ & $1.919 \mathrm{E}-18$ & $1.919 \mathrm{E}-18$ & $1.919 \mathrm{E}-18$ & $1.919 \mathrm{E}-18$ \\
\hline re186m & $1.919 \mathrm{E}-18$ & $1.919 \mathrm{E}-18$ & $1.919 \mathrm{E}-18$ & $1.919 \mathrm{E}-18$ & $1.919 \mathrm{E}-18$ \\
\hline re187 & $1.568 \mathrm{E}-20$ & $1.568 \mathrm{E}-20$ & $1.568 \mathrm{E}-20$ & $1.568 \mathrm{E}-20$ & $1.568 \mathrm{E}-20$ \\
\hline re188 & $1.933 \mathrm{E}-14$ & $6.457 \mathrm{E}-22$ & $2.563 \mathrm{E}-22$ & $0.000 \mathrm{E}+00$ & $0.000 \mathrm{E}+00$ \\
\hline rh 99 & $6.999 \mathrm{E}-15$ & $0.000 \mathrm{E}+00$ & $0.000 \mathrm{E}+00$ & $0.000 \mathrm{E}+00$ & $0.000 \mathrm{E}+00$ \\
\hline rh101 & $6.833 \mathrm{E}-04$ & $2.521 \mathrm{E}-04$ & $2.390 \mathrm{E}-04$ & $1.937 \mathrm{E}-04$ & $1.571 \mathrm{E}-04$ \\
\hline rh102 & $5.872 \mathrm{E}+00$ & $2.026 \mathrm{E}-02$ & $1.538 \mathrm{E}-02$ & $5.767 \mathrm{E}-03$ & $2.723 \mathrm{E}-03$ \\
\hline rh102m & $2.109 \mathrm{E}+00$ & $8.755 \mathrm{E}-01$ & $8.351 \mathrm{E}-01$ & $6.939 \mathrm{E}-01$ & $5.768 \mathrm{E}-01$ \\
\hline $\operatorname{rh103m}$ & $2.227 \mathrm{E}+04$ & $1.136 \mathrm{E}-09$ & $2.198 \mathrm{E}-10$ & $3.486 \mathrm{E}-13$ & $5.530 \mathrm{E}-16$ \\
\hline rh106 & $8.159 \mathrm{E}+05$ & $3.224 \mathrm{E}+04$ & $2.710 \mathrm{E}+04$ & $1.373 \mathrm{E}+04$ & $6.946 \mathrm{E}+03$ \\
\hline rn217 & $4.770 \mathrm{E}-12$ & $4.959 \mathrm{E}-12$ & $4.966 \mathrm{E}-12$ & $5.024 \mathrm{E}-12$ & $5.081 \mathrm{E}-12$ \\
\hline rn218 & $8.139 \mathrm{E}-11$ & $1.799 \mathrm{E}-15$ & $1.933 \mathrm{E}-15$ & $2.517 \mathrm{E}-15$ & $3.205 \mathrm{E}-15$ \\
\hline rn219 & 4.951E-08 & $2.263 \mathrm{E}-07$ & $2.311 \mathrm{E}-07$ & $2.729 \mathrm{E}-07$ & $3.103 \mathrm{E}-07$ \\
\hline rn220 & $2.666 \mathrm{E}-03$ & $1.680 \mathrm{E}-02$ & $1.743 \mathrm{E}-02$ & $1.956 \mathrm{E}-02$ & $2.135 \mathrm{E}-02$ \\
\hline rn222 & $1.437 \mathrm{E}-09$ & 8.994E-09 & $9.663 \mathrm{E}-09$ & $1.258 \mathrm{E}-08$ & $1.603 \mathrm{E}-08$ \\
\hline ru103 & $2.251 \mathrm{E}+04$ & $1.148 \mathrm{E}-09$ & $2.222 \mathrm{E}-10$ & $3.524 \mathrm{E}-13$ & $5.590 \mathrm{E}-16$ \\
\hline ru106 & $8.159 \mathrm{E}+05$ & $3.224 \mathrm{E}+04$ & $2.710 \mathrm{E}+04$ & $1.373 \mathrm{E}+04$ & $6.946 \mathrm{E}+03$ \\
\hline s 35 & $1.251 \mathrm{E}-13$ & $1.367 \mathrm{E}-19$ & $6.546 \mathrm{E}-20$ & $3.634 \mathrm{E}-21$ & $2.017 \mathrm{E}-22$ \\
\hline sb120m & $1.568 \mathrm{E}-15$ & $0.000 \mathrm{E}+00$ & $0.000 \mathrm{E}+00$ & $0.000 \mathrm{E}+00$ & $0.000 \mathrm{E}+00$ \\
\hline sb124 & $7.218 \mathrm{E}+01$ & $1.558 \mathrm{E}-07$ & 5.339E-08 & $7.986 \mathrm{E}-10$ & $1.194 \mathrm{E}-11$ \\
\hline sb125 & $1.616 \mathrm{E}+04$ & $4.905 \mathrm{E}+03$ & $4.600 \mathrm{E}+03$ & $3.579 \mathrm{E}+03$ & $2.784 \mathrm{E}+03$ \\
\hline sb126 & $8.086 \mathrm{E}-02$ & 8.039E-02 & $8.039 \mathrm{E}-02$ & $8.039 \mathrm{E}-02$ & 8.039E-02 \\
\hline sb126m & $5.744 \mathrm{E}-01$ & $5.744 \mathrm{E}-01$ & $5.744 \mathrm{E}-01$ & $5.744 \mathrm{E}-01$ & $5.744 \mathrm{E}-01$ \\
\hline sb127 & $3.301 \mathrm{E}-16$ & $0.000 \mathrm{E}+00$ & $0.000 \mathrm{E}+00$ & $0.000 \mathrm{E}+00$ & $0.000 \mathrm{E}+00$ \\
\hline sc $45 \mathrm{~m}$ & $3.154 \mathrm{E}-18$ & $1.953 \mathrm{E}-21$ & $1.314 \mathrm{E}-21$ & $2.772 \mathrm{E}-22$ & $5.849 \mathrm{E}-23$ \\
\hline sc 46 & $1.378 \mathrm{E}-12$ & $8.185 \mathrm{E}-19$ & $3.792 \mathrm{E}-19$ & $1.852 \mathrm{E}-20$ & $9.040 \mathrm{E}-22$ \\
\hline
\end{tabular}




\begin{tabular}{|c|c|c|c|c|c|}
\hline \multicolumn{6}{|c|}{ Table C-9. Continued } \\
\hline & $1 / 22 / 2009$ & $10 / 21 / 2013$ & $1 / 22 / 2014$ & $1 / 22 / 2015$ & $1 / 22 / 2016$ \\
\hline se 75 & $1.377 \mathrm{E}-04$ & $6.082 \mathrm{E}-09$ & $3.550 \mathrm{E}-09$ & $4.296 \mathrm{E}-10$ & $5.197 \mathrm{E}-11$ \\
\hline se 79 & $9.425 \mathrm{E}-02$ & $9.425 \mathrm{E}-02$ & $9.425 \mathrm{E}-02$ & $9.425 \mathrm{E}-02$ & $9.425 \mathrm{E}-02$ \\
\hline si 32 & $3.670 \mathrm{E}-19$ & $3.592 \mathrm{E}-19$ & $3.588 \mathrm{E}-19$ & $3.572 \mathrm{E}-19$ & $3.555 \mathrm{E}-19$ \\
\hline sm145 & $9.398 \mathrm{E}-04$ & $2.746 \mathrm{E}-05$ & $2.271 \mathrm{E}-05$ & $1.080 \mathrm{E}-05$ & $5.129 \mathrm{E}-06$ \\
\hline sm146 & 8.729E-08 & 8.908E-08 & 8.914E-08 & 8.941E-08 & $8.961 \mathrm{E}-08$ \\
\hline sm147 & $2.935 \mathrm{E}-06$ & $6.099 \mathrm{E}-06$ & $6.181 \mathrm{E}-06$ & $6.455 \mathrm{E}-06$ & $6.668 \mathrm{E}-06$ \\
\hline $\operatorname{sm} 148$ & 7.191E-11 & 7.191E-11 & 7.191E-11 & 7.191E-11 & $7.191 \mathrm{E}-11$ \\
\hline sm151 & $7.337 \mathrm{E}+02$ & $7.072 \mathrm{E}+02$ & $7.059 \mathrm{E}+02$ & $7.006 \mathrm{E}+02$ & $6.952 \mathrm{E}+02$ \\
\hline sn113 & $7.675 \mathrm{E}-07$ & $2.249 \mathrm{E}-11$ & $1.284 \mathrm{E}-11$ & $1.426 \mathrm{E}-12$ & $1.583 \mathrm{E}-13$ \\
\hline sn117m & $2.387 \mathrm{E}-04$ & $0.000 \mathrm{E}+00$ & $0.000 \mathrm{E}+00$ & $0.000 \mathrm{E}+00$ & $0.000 \mathrm{E}+00$ \\
\hline sn119m & $2.646 \mathrm{E}+02$ & $4.387 \mathrm{E}+00$ & $3.520 \mathrm{E}+00$ & $1.485 \mathrm{E}+00$ & $6.260 \mathrm{E}-01$ \\
\hline sn121 & $4.239 \mathrm{E}+01$ & $3.933 \mathrm{E}+01$ & $3.917 \mathrm{E}+01$ & $3.856 \mathrm{E}+01$ & $3.796 \mathrm{E}+01$ \\
\hline sn121m & $5.462 \mathrm{E}+01$ & $5.068 \mathrm{E}+01$ & $5.048 \mathrm{E}+01$ & $4.968 \mathrm{E}+01$ & $4.891 \mathrm{E}+01$ \\
\hline sn123 & $7.059 \mathrm{E}+02$ & $6.467 \mathrm{E}-02$ & 3.927E-02 & $5.541 \mathrm{E}-03$ & $7.821 \mathrm{E}-04$ \\
\hline sn125 & 8.914E-05 & $0.000 \mathrm{E}+00$ & $0.000 \mathrm{E}+00$ & $0.000 \mathrm{E}+00$ & $0.000 \mathrm{E}+00$ \\
\hline sn126 & 5.744E-01 & 5.744E-01 & 5.744E-01 & 5.744E-01 & $5.744 \mathrm{E}-01$ \\
\hline sr 85 & $3.723 \mathrm{E}-04$ & $3.350 \mathrm{E}-12$ & $1.240 \mathrm{E}-12$ & $2.505 \mathrm{E}-14$ & $5.060 \mathrm{E}-16$ \\
\hline sr 89 & $1.647 \mathrm{E}+04$ & 7.807E-07 & 2.179E-07 & $1.458 \mathrm{E}-09$ & $9.756 \mathrm{E}-12$ \\
\hline sr 90 & $5.274 \mathrm{E}+04$ & $4.704 \mathrm{E}+04$ & $4.675 \mathrm{E}+04$ & $4.565 \mathrm{E}+04$ & $4.456 \mathrm{E}+04$ \\
\hline ta179 & $2.843 \mathrm{E}-17$ & $4.665 \mathrm{E}-18$ & 4.234E-18 & $2.894 \mathrm{E}-18$ & $1.978 \mathrm{E}-18$ \\
\hline ta182 & 4.389E-12 & $1.249 \mathrm{E}-16$ & $7.125 \mathrm{E}-17$ & 8.053E-18 & $1.084 \mathrm{E}-18$ \\
\hline tb157 & $2.924 \mathrm{E}-05$ & 2.792E-05 & $2.785 \mathrm{E}-05$ & $2.758 \mathrm{E}-05$ & $2.731 \mathrm{E}-05$ \\
\hline tb158 & $1.473 \mathrm{E}-03$ & $1.446 \mathrm{E}-03$ & $1.445 \mathrm{E}-03$ & 1.439E-03 & $1.434 \mathrm{E}-03$ \\
\hline tb160 & $2.122 \mathrm{E}+02$ & $1.292 \mathrm{E}-05$ & 5.295E-06 & $1.600 \mathrm{E}-07$ & $4.835 \mathrm{E}-09$ \\
\hline tb161 & 9.299E-09 & $0.000 \mathrm{E}+00$ & $0.000 \mathrm{E}+00$ & $0.000 \mathrm{E}+00$ & $0.000 \mathrm{E}+00$ \\
\hline tc 95 & $1.021 \mathrm{E}-11$ & $2.863 \mathrm{E}-20$ & $9.948 \mathrm{E}-21$ & $1.572 \mathrm{E}-22$ & $0.000 \mathrm{E}+00$ \\
\hline tc $95 \mathrm{~m}$ & $2.595 \mathrm{E}-10$ & 7.277E-19 & $2.529 \mathrm{E}-19$ & $3.997 \mathrm{E}-21$ & $6.316 \mathrm{E}-23$ \\
\hline tc 97 & $1.003 \mathrm{E}-08$ & $1.011 \mathrm{E}-08$ & $1.011 \mathrm{E}-08$ & $1.011 \mathrm{E}-08$ & $1.011 \mathrm{E}-08$ \\
\hline tc $97 \mathrm{~m}$ & $1.544 \mathrm{E}-03$ & $2.855 \mathrm{E}-09$ & $1.406 \mathrm{E}-09$ & $8.722 \mathrm{E}-11$ & $5.408 \mathrm{E}-12$ \\
\hline tc 98 & $1.025 \mathrm{E}-05$ & $1.025 \mathrm{E}-05$ & $1.025 \mathrm{E}-05$ & $1.025 \mathrm{E}-05$ & $1.025 \mathrm{E}-05$ \\
\hline tc 99 & $1.845 \mathrm{E}+01$ & $1.844 \mathrm{E}+01$ & $1.844 \mathrm{E}+01$ & $1.844 \mathrm{E}+01$ & $1.844 \mathrm{E}+01$ \\
\hline te121 & $2.409 \mathrm{E}-20$ & $1.601 \mathrm{E}-23$ & $1.081 \mathrm{E}-23$ & $2.006 \mathrm{E}-29$ & $0.000 \mathrm{E}+00$ \\
\hline te121 & $1.956 \mathrm{E}-03$ & $1.300 \mathrm{E}-06$ & 8.782E-07 & $1.881 \mathrm{E}-07$ & $4.029 \mathrm{E}-08$ \\
\hline te121m & $1.949 \mathrm{E}-03$ & $1.296 \mathrm{E}-06$ & $8.755 \mathrm{E}-07$ & $1.875 \mathrm{E}-07$ & $4.016 \mathrm{E}-08$ \\
\hline te123m & $4.048 \mathrm{E}+00$ & $1.701 \mathrm{E}-04$ & 9.902E-05 & $1.186 \mathrm{E}-05$ & $1.420 \mathrm{E}-06$ \\
\hline te $125 \mathrm{~m}$ & $3.938 \mathrm{E}+03$ & $1.201 \mathrm{E}+03$ & $1.127 \mathrm{E}+03$ & $8.762 \mathrm{E}+02$ & $6.820 \mathrm{E}+02$ \\
\hline te127 & $2.758 \mathrm{E}+03$ & 4.513E-02 & $2.499 \mathrm{E}-02$ & $2.452 \mathrm{E}-03$ & $2.407 \mathrm{E}-04$ \\
\hline te127m & $2.816 \mathrm{E}+03$ & 4.608E-02 & $2.550 \mathrm{E}-02$ & $2.504 \mathrm{E}-03$ & $2.458 \mathrm{E}-04$ \\
\hline
\end{tabular}




\begin{tabular}{|c|c|c|c|c|c|}
\hline \multicolumn{6}{|c|}{ Table C-9. Continued } \\
\hline & $1 / 22 / 2009$ & $10 / 21 / 2013$ & $1 / 22 / 2014$ & $1 / 22 / 2015$ & $1 / 22 / 2016$ \\
\hline te128 & $5.704 \mathrm{E}-14$ & $5.704 \mathrm{E}-14$ & $5.704 \mathrm{E}-14$ & $5.704 \mathrm{E}-14$ & 5.704E-14 \\
\hline te129 & $1.667 \mathrm{E}+02$ & $4.960 \mathrm{E}-14$ & $7.284 \mathrm{E}-15$ & $3.910 \mathrm{E}-18$ & $2.099 \mathrm{E}-21$ \\
\hline te129m & $2.642 \mathrm{E}+02$ & $7.860 \mathrm{E}-14$ & $1.155 \mathrm{E}-14$ & $6.197 \mathrm{E}-18$ & $3.327 \mathrm{E}-21$ \\
\hline th226 & $8.139 \mathrm{E}-11$ & $0.000 \mathrm{E}+00$ & $0.000 \mathrm{E}+00$ & $0.000 \mathrm{E}+00$ & $0.000 \mathrm{E}+00$ \\
\hline th227 & 4.997E-08 & $2.232 \mathrm{E}-07$ & $2.294 \mathrm{E}-07$ & $2.692 \mathrm{E}-07$ & $3.060 \mathrm{E}-07$ \\
\hline th228 & $2.703 \mathrm{E}-03$ & $1.674 \mathrm{E}-02$ & $1.734 \mathrm{E}-02$ & $1.949 \mathrm{E}-02$ & $2.128 \mathrm{E}-02$ \\
\hline th229 & $6.813 \mathrm{E}-08$ & $7.085 \mathrm{E}-08$ & $7.105 \mathrm{E}-08$ & $7.178 \mathrm{E}-08$ & $7.257 \mathrm{E}-08$ \\
\hline th230 & $1.812 \mathrm{E}-06$ & 5.903E-06 & $6.184 \mathrm{E}-06$ & 7.344E-06 & $8.603 \mathrm{E}-06$ \\
\hline th231 & $2.085 \mathrm{E}-03$ & $2.090 \mathrm{E}-03$ & $2.090 \mathrm{E}-03$ & $2.090 \mathrm{E}-03$ & $2.092 \mathrm{E}-03$ \\
\hline th232 & $2.198 \mathrm{E}-11$ & $2.646 \mathrm{E}-11$ & $2.670 \mathrm{E}-11$ & $2.766 \mathrm{E}-11$ & $2.861 \mathrm{E}-11$ \\
\hline th234 & $3.083 \mathrm{E}-01$ & $3.083 \mathrm{E}-01$ & $3.083 \mathrm{E}-01$ & $3.083 \mathrm{E}-01$ & $3.083 \mathrm{E}-01$ \\
\hline tl202 & $5.340 \mathrm{E}-23$ & $0.000 \mathrm{E}+00$ & $1.208 \mathrm{E}-27$ & $0.000 \mathrm{E}+00$ & $0.000 \mathrm{E}+00$ \\
\hline tl204 & $2.473 \mathrm{E}-14$ & $1.037 \mathrm{E}-14$ & $9.895 \mathrm{E}-15$ & $8.238 \mathrm{E}-15$ & $6.860 \mathrm{E}-15$ \\
\hline tl206 & 5.417E-15 & $5.521 \mathrm{E}-15$ & 5.577E-15 & $5.860 \mathrm{E}-15$ & $6.266 \mathrm{E}-15$ \\
\hline tl207 & 4.937E-08 & $2.257 \mathrm{E}-07$ & $2.305 \mathrm{E}-07$ & $2.721 \mathrm{E}-07$ & $3.094 \mathrm{E}-07$ \\
\hline tl208 & $9.584 \mathrm{E}-04$ & $6.038 \mathrm{E}-03$ & $6.264 \mathrm{E}-03$ & $7.032 \mathrm{E}-03$ & $7.675 \mathrm{E}-03$ \\
\hline tl209 & $1.499 \mathrm{E}-09$ & $1.558 \mathrm{E}-09$ & $1.561 \mathrm{E}-09$ & $1.579 \mathrm{E}-09$ & $1.597 \mathrm{E}-09$ \\
\hline tl210 & $3.017 \mathrm{E}-13$ & $1.890 \mathrm{E}-12$ & $2.029 \mathrm{E}-12$ & $2.642 \mathrm{E}-12$ & $3.365 \mathrm{E}-12$ \\
\hline $\operatorname{tm} 167$ & $2.530 \mathrm{E}-15$ & $0.000 \mathrm{E}+00$ & $0.000 \mathrm{E}+00$ & $0.000 \mathrm{E}+00$ & $0.000 \mathrm{E}+00$ \\
\hline $\operatorname{tm} 168$ & $8.815 \mathrm{E}-06$ & $2.196 \mathrm{E}-11$ & $1.099 \mathrm{E}-11$ & 7.257E-13 & $4.793 \mathrm{E}-14$ \\
\hline $\operatorname{tm} 170$ & $5.704 \mathrm{E}-23$ & $5.006 \mathrm{E}-27$ & $3.032 \mathrm{E}-27$ & 4.240E-28 & $5.929 \mathrm{E}-29$ \\
\hline $\operatorname{tm170}$ & 3.312E-01 & $2.906 \mathrm{E}-05$ & $1.761 \mathrm{E}-05$ & $2.462 \mathrm{E}-06$ & $3.442 \mathrm{E}-07$ \\
\hline $\operatorname{tm} 171$ & $1.904 \mathrm{E}-01$ & $3.434 \mathrm{E}-02$ & $3.132 \mathrm{E}-02$ & $2.184 \mathrm{E}-02$ & $1.522 \mathrm{E}-02$ \\
\hline $\mathbf{u} 230$ & $8.132 \mathrm{E}-11$ & $0.000 \mathrm{E}+00$ & $0.000 \mathrm{E}+00$ & $0.000 \mathrm{E}+00$ & $0.000 \mathrm{E}+00$ \\
\hline $\mathbf{u} 232$ & $9.809 \mathrm{E}-03$ & $2.346 \mathrm{E}-02$ & $2.379 \mathrm{E}-02$ & $2.491 \mathrm{E}-02$ & $2.572 \mathrm{E}-02$ \\
\hline $\mathbf{u} 233$ & $4.700 \mathrm{E}-06$ & $7.556 \mathrm{E}-06$ & $7.708 \mathrm{E}-06$ & $8.351 \mathrm{E}-06$ & $9.007 \mathrm{E}-06$ \\
\hline u234 & $6.919 \mathrm{E}-02$ & $1.186 \mathrm{E}-01$ & $1.212 \mathrm{E}-01$ & $1.315 \mathrm{E}-01$ & $1.417 \mathrm{E}-01$ \\
\hline $\mathbf{u} 235$ & $2.085 \mathrm{E}-03$ & $2.090 \mathrm{E}-03$ & $2.090 \mathrm{E}-03$ & $2.090 \mathrm{E}-03$ & $2.092 \mathrm{E}-03$ \\
\hline $\mathbf{u} 236$ & $1.900 \mathrm{E}-02$ & $1.931 \mathrm{E}-02$ & $1.933 \mathrm{E}-02$ & $1.939 \mathrm{E}-02$ & $1.946 \mathrm{E}-02$ \\
\hline $\mathbf{u} 237$ & $1.324 \mathrm{E}+01$ & $1.051 \mathrm{E}+01$ & $1.039 \mathrm{E}+01$ & $9.889 \mathrm{E}+00$ & $9.425 \mathrm{E}+00$ \\
\hline $\mathbf{u} 238$ & $3.083 \mathrm{E}-01$ & $3.083 \mathrm{E}-01$ & $3.083 \mathrm{E}-01$ & $3.083 \mathrm{E}-01$ & $3.083 \mathrm{E}-01$ \\
\hline $\mathbf{u} 240$ & $2.027 \mathrm{E}-06$ & $2.027 \mathrm{E}-06$ & $2.027 \mathrm{E}-06$ & $2.027 \mathrm{E}-06$ & $2.027 \mathrm{E}-06$ \\
\hline v 49 & $4.246 \mathrm{E}-16$ & $1.114 \mathrm{E}-17$ & $9.166 \mathrm{E}-18$ & $4.258 \mathrm{E}-18$ & $1.978 \mathrm{E}-18$ \\
\hline w181 & $4.162 \mathrm{E}-15$ & $2.065 \mathrm{E}-19$ & $1.214 \mathrm{E}-19$ & $1.504 \mathrm{E}-20$ & $1.866 \mathrm{E}-21$ \\
\hline w185 & $1.099 \mathrm{E}-12$ & $1.243 \mathrm{E}-19$ & $5.268 \mathrm{E}-20$ & $1.813 \mathrm{E}-21$ & $6.244 \mathrm{E}-23$ \\
\hline w188 & $1.913 \mathrm{E}-14$ & $6.391 \mathrm{E}-22$ & $2.537 \mathrm{E}-22$ & $6.754 \mathrm{E}-24$ & $1.799 \mathrm{E}-25$ \\
\hline xe127 & $1.577 \mathrm{E}-03$ & 7.344E-18 & $1.249 \mathrm{E}-18$ & $1.197 \mathrm{E}-21$ & $1.147 \mathrm{E}-24$ \\
\hline xe129m & $3.240 \mathrm{E}-08$ & $0.000 \mathrm{E}+00$ & $0.000 \mathrm{E}+00$ & $0.000 \mathrm{E}+00$ & $0.000 \mathrm{E}+00$ \\
\hline
\end{tabular}




\begin{tabular}{|c|c|c|c|c|c|}
\hline \multicolumn{6}{|c|}{ Table C-9. Continued } \\
\hline & $1 / 22 / 2009$ & $10 / 21 / 2013$ & $1 / 22 / 2014$ & $1 / 22 / 2015$ & $1 / 22 / 2016$ \\
\hline xe131m & $8.822 \mathrm{E}-03$ & $0.000 \mathrm{E}+00$ & $0.000 \mathrm{E}+00$ & $0.000 \mathrm{E}+00$ & $0.000 \mathrm{E}+00$ \\
\hline xe133 & $1.998 \mathrm{E}-09$ & $0.000 \mathrm{E}+00$ & $0.000 \mathrm{E}+00$ & $0.000 \mathrm{E}+00$ & $0.000 \mathrm{E}+00$ \\
\hline y 88 & 4.148E-01 & $5.312 \mathrm{E}-06$ & $2.902 \mathrm{E}-06$ & $2.705 \mathrm{E}-07$ & $2.523 \mathrm{E}-08$ \\
\hline y 89m & $1.588 \mathrm{E}+00$ & $7.522 \mathrm{E}-11$ & $2.101 \mathrm{E}-11$ & $1.406 \mathrm{E}-13$ & $9.405 \mathrm{E}-16$ \\
\hline y 90 & $5.275 \mathrm{E}+04$ & $4.705 \mathrm{E}+04$ & $4.677 \mathrm{E}+04$ & $4.565 \mathrm{E}+04$ & $4.457 \mathrm{E}+04$ \\
\hline y 91 & $3.897 \mathrm{E}+04$ & $4.726 \mathrm{E}-05$ & $1.571 \mathrm{E}-05$ & $2.080 \mathrm{E}-07$ & $2.756 \mathrm{E}-09$ \\
\hline yb169 & $8.417 \mathrm{E}-08$ & $4.282 \mathrm{E}-24$ & $5.717 \mathrm{E}-25$ & $2.116 \mathrm{E}-28$ & $0.000 \mathrm{E}+00$ \\
\hline zn 65 & $9.663 \mathrm{E}-16$ & $7.025 \mathrm{E}-18$ & $5.392 \mathrm{E}-18$ & $1.911 \mathrm{E}-18$ & $6.774 \mathrm{E}-19$ \\
\hline zr 88 & $1.271 \mathrm{E}-07$ & $7.059 \mathrm{E}-14$ & $3.260 \mathrm{E}-14$ & $1.569 \mathrm{E}-15$ & $7.556 \mathrm{E}-17$ \\
\hline zr 93 & $1.803 \mathrm{E}+00$ & $1.803 \mathrm{E}+00$ & $1.803 \mathrm{E}+00$ & $1.803 \mathrm{E}+00$ & $1.803 \mathrm{E}+00$ \\
\hline zr 95 & $9.358 \mathrm{E}+04$ & $6.668 \mathrm{E}-04$ & $2.436 \mathrm{E}-04$ & $4.686 \mathrm{E}-06$ & $9.014 \mathrm{E}-08$ \\
\hline zr 96 & $1.791 \mathrm{E}-13$ & $1.791 \mathrm{E}-13$ & $1.791 \mathrm{E}-13$ & $1.791 \mathrm{E}-13$ & $1.791 \mathrm{E}-13$ \\
\hline Total & $4.8602 \mathrm{E}+06$ & $1.0134 \mathrm{E}+06$ & $9.8416 \mathrm{E}+05$ & 8.9490E+05 & 8.3167E+05 \\
\hline
\end{tabular}


Table C-10. Activity in Fuel Rod K5 (high Pu loading) in Ci per MTIHM

\begin{tabular}{|c|c|c|c|c|c|}
\hline & $1 / 22 / 2009$ & $10 / 21 / 2013$ & $1 / 22 / 2014$ & $1 / 22 / 2015$ & $1 / 22 / 2016$ \\
\hline ac225 & $4.458 \mathrm{E}-08$ & $4.712 \mathrm{E}-08$ & $4.723 \mathrm{E}-08$ & $4.799 \mathrm{E}-08$ & 4.874E-08 \\
\hline ac226 & $1.167 \mathrm{E}-15$ & $0.000 \mathrm{E}+00$ & $0.000 \mathrm{E}+00$ & $0.000 \mathrm{E}+00$ & $0.000 \mathrm{E}+00$ \\
\hline ac227 & $5.515 \mathrm{E}-08$ & $2.344 \mathrm{E}-07$ & $2.443 \mathrm{E}-07$ & $2.833 \mathrm{E}-07$ & $3.229 \mathrm{E}-07$ \\
\hline ac228 & $7.095 \mathrm{E}-12$ & $1.349 \mathrm{E}-11$ & $1.381 \mathrm{E}-11$ & $1.499 \mathrm{E}-11$ & $1.616 \mathrm{E}-11$ \\
\hline ag105 & $2.104 \mathrm{E}-10$ & $4.878 \mathrm{E}-23$ & $1.024 \mathrm{E}-23$ & $2.234 \mathrm{E}-26$ & $4.876 \mathrm{E}-29$ \\
\hline ag106m & $9.093 \mathrm{E}-16$ & $0.000 \mathrm{E}+00$ & $0.000 \mathrm{E}+00$ & $0.000 \mathrm{E}+00$ & $0.000 \mathrm{E}+00$ \\
\hline ag108 & 4.056E-04 & $4.026 \mathrm{E}-04$ & $4.024 \mathrm{E}-04$ & 4.018E-04 & 4.011E-04 \\
\hline ag108m & 4.662E-03 & 4.627E-03 & $4.625 \mathrm{E}-03$ & $4.618 \mathrm{E}-03$ & $4.610 \mathrm{E}-03$ \\
\hline $\operatorname{ag109m}$ & $3.860 \mathrm{E}-01$ & $2.856 \mathrm{E}-02$ & $2.484 \mathrm{E}-02$ & $1.436 \mathrm{E}-02$ & $8.297 \mathrm{E}-03$ \\
\hline $\operatorname{ag} 110$ & $7.686 \mathrm{E}+01$ & $6.264 \mathrm{E}-01$ & $4.839 \mathrm{E}-01$ & $1.758 \mathrm{E}-01$ & $6.381 \mathrm{E}-02$ \\
\hline ag110m & $5.650 \mathrm{E}+03$ & $4.606 \mathrm{E}+01$ & $3.558 \mathrm{E}+01$ & $1.292 \mathrm{E}+01$ & $4.692 \mathrm{E}+00$ \\
\hline ag111 & $2.480 \mathrm{E}-06$ & $0.000 \mathrm{E}+00$ & $0.000 \mathrm{E}+00$ & $0.000 \mathrm{E}+00$ & $0.000 \mathrm{E}+00$ \\
\hline am241 & $1.403 \mathrm{E}+03$ & $4.874 \mathrm{E}+03$ & $5.038 \mathrm{E}+03$ & $5.660 \mathrm{E}+03$ & $6.252 \mathrm{E}+03$ \\
\hline am242 & $4.274 \mathrm{E}+01$ & $4.176 \mathrm{E}+01$ & $4.171 \mathrm{E}+01$ & $4.150 \mathrm{E}+01$ & $4.130 \mathrm{E}+01$ \\
\hline am242m & $4.294 \mathrm{E}+01$ & $4.195 \mathrm{E}+01$ & $4.189 \mathrm{E}+01$ & $4.170 \mathrm{E}+01$ & $4.149 \mathrm{E}+01$ \\
\hline am243 & $5.430 \mathrm{E}+01$ & $5.428 \mathrm{E}+01$ & $5.427 \mathrm{E}+01$ & $5.427 \mathrm{E}+01$ & $5.427 \mathrm{E}+01$ \\
\hline $\operatorname{am} 244$ & $8.894 \mathrm{E}-10$ & $6.168 \mathrm{E}-10$ & $6.048 \mathrm{E}-10$ & $5.600 \mathrm{E}-10$ & $5.184 \mathrm{E}-10$ \\
\hline am245 & $5.528 \mathrm{E}-08$ & $1.295 \mathrm{E}-09$ & $1.059 \mathrm{E}-09$ & $4.803 \mathrm{E}-10$ & $2.178 \mathrm{E}-10$ \\
\hline am246m & $8.370 \mathrm{E}-13$ & $8.363 \mathrm{E}-13$ & $8.363 \mathrm{E}-13$ & 8.363E-13 & $8.363 \mathrm{E}-13$ \\
\hline $\operatorname{ar} 37$ & $1.103 \mathrm{E}-14$ & $1.426 \mathrm{E}-29$ & $0.000 \mathrm{E}+00$ & $0.000 \mathrm{E}+00$ & $0.000 \mathrm{E}+00$ \\
\hline $\operatorname{ar} 39$ & 3.664E-15 & $3.620 \mathrm{E}-15$ & $3.617 \mathrm{E}-15$ & $3.608 \mathrm{E}-15$ & $3.599 \mathrm{E}-15$ \\
\hline $\operatorname{ar} 42$ & $5.100 \mathrm{E}-21$ & 4.615E-21 & $4.590 \mathrm{E}-21$ & $4.495 \mathrm{E}-21$ & 4.401E-21 \\
\hline as 73 & $3.461 \mathrm{E}-06$ & $1.103 \mathrm{E}-12$ & $4.941 \mathrm{E}-13$ & $2.116 \mathrm{E}-14$ & $9.060 \mathrm{E}-16$ \\
\hline as 74 & $1.652 \mathrm{E}-07$ & $0.000 \mathrm{E}+00$ & $0.000 \mathrm{E}+00$ & $0.000 \mathrm{E}+00$ & $0.000 \mathrm{E}+00$ \\
\hline at217 & 4.459E-08 & 4.712E-08 & $4.723 \mathrm{E}-08$ & 4.799E-08 & $4.874 \mathrm{E}-08$ \\
\hline at218 & $3.127 \mathrm{E}-13$ & $1.876 \mathrm{E}-12$ & $2.009 \mathrm{E}-12$ & $2.590 \mathrm{E}-12$ & $3.266 \mathrm{E}-12$ \\
\hline au194 & $8.217 \mathrm{E}-21$ & $8.157 \mathrm{E}-21$ & $8.151 \mathrm{E}-21$ & $8.137 \mathrm{E}-21$ & $8.124 \mathrm{E}-21$ \\
\hline au195 & $1.029 \mathrm{E}-15$ & 1.619E-18 & $1.145 \mathrm{E}-18$ & 2.940E-19 & $7.553 \mathrm{E}-20$ \\
\hline ba131 & $2.733 \mathrm{E}-13$ & $0.000 \mathrm{E}+00$ & $0.000 \mathrm{E}+00$ & $0.000 \mathrm{E}+00$ & $0.000 \mathrm{E}+00$ \\
\hline ba133 & $3.286 \mathrm{E}-02$ & 2.403E-02 & $2.364 \mathrm{E}-02$ & 2.213E-02 & $2.071 \mathrm{E}-02$ \\
\hline ba136m & $8.708 \mathrm{E}-03$ & $0.000 \mathrm{E}+00$ & $0.000 \mathrm{E}+00$ & $0.000 \mathrm{E}+00$ & $0.000 \mathrm{E}+00$ \\
\hline ba137m & $1.227 \mathrm{E}+05$ & $1.100 \mathrm{E}+05$ & $1.093 \mathrm{E}+05$ & $1.069 \mathrm{E}+05$ & $1.044 \mathrm{E}+05$ \\
\hline ba140 & $1.067 \mathrm{E}+00$ & $0.000 \mathrm{E}+00$ & $0.000 \mathrm{E}+00$ & $0.000 \mathrm{E}+00$ & $0.000 \mathrm{E}+00$ \\
\hline be 10 & $2.068 \mathrm{E}-06$ & $2.068 \mathrm{E}-06$ & $2.068 \mathrm{E}-06$ & $2.068 \mathrm{E}-06$ & $2.068 \mathrm{E}-06$ \\
\hline bi210 & $3.485 \mathrm{E}-09$ & $3.678 \mathrm{E}-09$ & $3.726 \mathrm{E}-09$ & $3.963 \mathrm{E}-09$ & 4.289E-09 \\
\hline bi211 & $5.126 \mathrm{E}-08$ & $2.348 \mathrm{E}-07$ & $2.399 \mathrm{E}-07$ & $2.839 \mathrm{E}-07$ & $3.235 \mathrm{E}-07$ \\
\hline
\end{tabular}


Table C-10. Continued

\begin{tabular}{|c|c|c|c|c|c|}
\hline & $1 / 22 / 2009$ & $10 / 21 / 2013$ & $1 / 22 / 2014$ & $1 / 22 / 2015$ & $1 / 22 / 2016$ \\
\hline bi212 & $1.965 \mathrm{E}-03$ & $1.280 \mathrm{E}-02$ & $1.328 \mathrm{E}-02$ & $1.493 \mathrm{E}-02$ & $1.631 \mathrm{E}-02$ \\
\hline bi213 & $4.458 \mathrm{E}-08$ & 4.712E-08 & 4.723E-08 & 4.799E-08 & $4.874 \mathrm{E}-08$ \\
\hline bi214 & $1.563 \mathrm{E}-09$ & $9.378 \mathrm{E}-09$ & $1.005 \mathrm{E}-08$ & $1.295 \mathrm{E}-08$ & $1.633 \mathrm{E}-08$ \\
\hline bk247 & $1.377 \mathrm{E}-13$ & $1.373 \mathrm{E}-13$ & $1.373 \mathrm{E}-13$ & $1.373 \mathrm{E}-13$ & $1.372 \mathrm{E}-13$ \\
\hline bk248 & $8.887 \mathrm{E}-10$ & $6.167 \mathrm{E}-10$ & $6.047 \mathrm{E}-10$ & $5.599 \mathrm{E}-10$ & $5.184 \mathrm{E}-10$ \\
\hline bk249 & $3.812 \mathrm{E}-03$ & 8.927E-05 & $7.301 \mathrm{E}-05$ & $3.311 \mathrm{E}-05$ & $1.502 \mathrm{E}-05$ \\
\hline bk250 & $1.573 \mathrm{E}-09$ & $2.053 \mathrm{E}-11$ & $1.633 \mathrm{E}-11$ & $6.743 \mathrm{E}-12$ & $2.918 \mathrm{E}-12$ \\
\hline bk251 & $1.256 \mathrm{E}-12$ & $0.000 \mathrm{E}+00$ & $0.000 \mathrm{E}+00$ & $0.000 \mathrm{E}+00$ & $0.000 \mathrm{E}+00$ \\
\hline c 14 & $1.369 \mathrm{E}-03$ & $1.368 \mathrm{E}-03$ & $1.367 \mathrm{E}-03$ & $1.367 \mathrm{E}-03$ & $1.367 \mathrm{E}-03$ \\
\hline ca 41 & $2.859 \mathrm{E}-18$ & $2.859 \mathrm{E}-18$ & $2.859 \mathrm{E}-18$ & $2.859 \mathrm{E}-18$ & $2.859 \mathrm{E}-18$ \\
\hline ca 45 & $1.451 \mathrm{E}-13$ & $8.980 \mathrm{E}-17$ & $6.043 \mathrm{E}-17$ & $1.275 \mathrm{E}-17$ & $2.690 \mathrm{E}-18$ \\
\hline cd109 & $3.860 \mathrm{E}-01$ & $2.856 \mathrm{E}-02$ & $2.484 \mathrm{E}-02$ & $1.436 \mathrm{E}-02$ & 8.297E-03 \\
\hline cd113 & $1.653 \mathrm{E}-13$ & $1.653 \mathrm{E}-13$ & $1.653 \mathrm{E}-13$ & $1.653 \mathrm{E}-13$ & $1.653 \mathrm{E}-13$ \\
\hline cd113m & $1.284 \mathrm{E}+00$ & $1.017 \mathrm{E}+00$ & $1.004 \mathrm{E}+00$ & $9.558 \mathrm{E}-01$ & $9.100 \mathrm{E}-01$ \\
\hline cd115m & $1.679 \mathrm{E}+01$ & $3.292 \mathrm{E}-11$ & $7.746 \mathrm{E}-12$ & $2.651 \mathrm{E}-14$ & $9.067 \mathrm{E}-17$ \\
\hline cd116 & $1.050 \mathrm{E}-15$ & $1.050 \mathrm{E}-15$ & $1.050 \mathrm{E}-15$ & $1.050 \mathrm{E}-15$ & $1.050 \mathrm{E}-15$ \\
\hline ce139 & $8.615 \mathrm{E}+00$ & $1.397 \mathrm{E}-03$ & $8.748 \mathrm{E}-04$ & $1.391 \mathrm{E}-04$ & $2.214 \mathrm{E}-05$ \\
\hline ce141 & $6.167 \mathrm{E}+03$ & $5.522 \mathrm{E}-13$ & $7.600 \mathrm{E}-14$ & $3.169 \mathrm{E}-17$ & $1.321 \mathrm{E}-20$ \\
\hline ce144 & $6.073 \mathrm{E}+05$ & $8.960 \mathrm{E}+03$ & $7.148 \mathrm{E}+03$ & $2.940 \mathrm{E}+03$ & $1.210 \mathrm{E}+03$ \\
\hline cf248 & $5.476 \mathrm{E}-09$ & $1.493 \mathrm{E}-10$ & $1.231 \mathrm{E}-10$ & $5.765 \mathrm{E}-11$ & $2.700 \mathrm{E}-11$ \\
\hline cf249 & $1.010 \mathrm{E}-05$ & $1.923 \mathrm{E}-05$ & $1.926 \mathrm{E}-05$ & $1.932 \mathrm{E}-05$ & $1.933 \mathrm{E}-05$ \\
\hline cf250 & $1.144 \mathrm{E}-04$ & 8.894E-05 & $8.774 \mathrm{E}-05$ & $8.317 \mathrm{E}-05$ & $7.892 \mathrm{E}-05$ \\
\hline cf251 & $7.686 \mathrm{E}-07$ & 7.653E-07 & $7.653 \mathrm{E}-07$ & $7.646 \mathrm{E}-07$ & $7.640 \mathrm{E}-07$ \\
\hline cf 252 & $6.473 \mathrm{E}-05$ & $1.866 \mathrm{E}-05$ & $1.746 \mathrm{E}-05$ & $1.344 \mathrm{E}-05$ & $1.034 \mathrm{E}-05$ \\
\hline cf 253 & $1.801 \mathrm{E}-10$ & $0.000 \mathrm{E}+00$ & $0.000 \mathrm{E}+00$ & $0.000 \mathrm{E}+00$ & $0.000 \mathrm{E}+00$ \\
\hline cf 254 & $2.266 \mathrm{E}-09$ & $5.399 \mathrm{E}-18$ & $1.860 \mathrm{E}-18$ & $2.841 \mathrm{E}-20$ & 4.337E-22 \\
\hline cl 36 & $9.007 \mathrm{E}-17$ & $9.007 \mathrm{E}-17$ & $9.007 \mathrm{E}-17$ & $9.007 \mathrm{E}-17$ & $9.007 \mathrm{E}-17$ \\
\hline cm240 & $2.886 \mathrm{E}-07$ & $1.376 \mathrm{E}-26$ & $1.264 \mathrm{E}-27$ & $0.000 \mathrm{E}+00$ & $0.000 \mathrm{E}+00$ \\
\hline cm241 & $3.447 \mathrm{E}-04$ & $4.288 \mathrm{E}-20$ & $6.007 \mathrm{E}-21$ & $2.684 \mathrm{E}-24$ & $1.199 \mathrm{E}-27$ \\
\hline cm242 & $5.956 \mathrm{E}+04$ & $7.201 \mathrm{E}+01$ & $5.973 \mathrm{E}+01$ & $3.970 \mathrm{E}+01$ & $3.534 \mathrm{E}+01$ \\
\hline cm243 & $6.883 \mathrm{E}+01$ & $6.147 \mathrm{E}+01$ & $6.110 \mathrm{E}+01$ & $5.966 \mathrm{E}+01$ & $5.826 \mathrm{E}+01$ \\
\hline cm244 & $7.215 \mathrm{E}+03$ & $6.019 \mathrm{E}+03$ & $5.960 \mathrm{E}+03$ & $5.737 \mathrm{E}+03$ & $5.522 \mathrm{E}+03$ \\
\hline cm245 & $1.282 \mathrm{E}+00$ & $1.281 \mathrm{E}+00$ & $1.281 \mathrm{E}+00$ & $1.281 \mathrm{E}+00$ & $1.280 \mathrm{E}+00$ \\
\hline cm246 & $1.216 \mathrm{E}-01$ & $1.215 \mathrm{E}-01$ & $1.215 \mathrm{E}-01$ & $1.215 \mathrm{E}-01$ & $1.215 \mathrm{E}-01$ \\
\hline cm247 & $4.839 \mathrm{E}-07$ & 4.839E-07 & $4.839 \mathrm{E}-07$ & 4.839E-07 & 4.839E-07 \\
\hline cm248 & $1.172 \mathrm{E}-06$ & $1.173 \mathrm{E}-06$ & $1.173 \mathrm{E}-06$ & $1.173 \mathrm{E}-06$ & $1.173 \mathrm{E}-06$ \\
\hline
\end{tabular}


Table C-10. Continued

\begin{tabular}{|c|c|c|c|c|c|}
\hline & 1/22/2009 & $10 / 21 / 2013$ & $1 / 22 / 2014$ & $1 / 22 / 2015$ & $1 / 22 / 2016$ \\
\hline cm249 & $5.596 \mathrm{E}-13$ & $0.000 \mathrm{E}+00$ & $0.000 \mathrm{E}+00$ & $0.000 \mathrm{E}+00$ & $0.000 \mathrm{E}+00$ \\
\hline cm250 & $4.647 \mathrm{E}-12$ & 4.645E-12 & $4.645 \mathrm{E}-12$ & $4.645 \mathrm{E}-12$ & $4.644 \mathrm{E}-12$ \\
\hline co 56 & $3.641 \mathrm{E}-21$ & $6.405 \mathrm{E}-28$ & $2.780 \mathrm{E}-28$ & $1.051 \mathrm{E}-29$ & $0.000 \mathrm{E}+00$ \\
\hline co 57 & $8.224 \mathrm{E}-16$ & $9.896 \mathrm{E}-18$ & $7.805 \mathrm{E}-18$ & $3.076 \mathrm{E}-18$ & $1.213 \mathrm{E}-18$ \\
\hline $\cos 58$ & $1.861 \mathrm{E}-14$ & $8.084 \mathrm{E}-22$ & $3.254 \mathrm{E}-22$ & $9.159 \mathrm{E}-24$ & $2.578 \mathrm{E}-25$ \\
\hline co 60 & $9.359 \mathrm{E}-12$ & $5.015 \mathrm{E}-12$ & $4.850 \mathrm{E}-12$ & $4.253 \mathrm{E}-12$ & $3.729 \mathrm{E}-12$ \\
\hline co 60m & $2.178 \mathrm{E}-21$ & $2.178 \mathrm{E}-21$ & $2.178 \mathrm{E}-21$ & $2.178 \mathrm{E}-21$ & $2.178 \mathrm{E}-21$ \\
\hline cr 51 & $9.186 \mathrm{E}-15$ & $0.000 \mathrm{E}+00$ & $0.000 \mathrm{E}+00$ & $0.000 \mathrm{E}+00$ & $0.000 \mathrm{E}+00$ \\
\hline $\operatorname{cs} 131$ & $1.707 \mathrm{E}-10$ & $0.000 \mathrm{E}+00$ & $0.000 \mathrm{E}+00$ & $0.000 \mathrm{E}+00$ & $0.000 \mathrm{E}+00$ \\
\hline $\operatorname{cs} 132$ & $2.152 \mathrm{E}-11$ & $0.000 \mathrm{E}+00$ & $0.000 \mathrm{E}+00$ & $0.000 \mathrm{E}+00$ & $0.000 \mathrm{E}+00$ \\
\hline cs134 & $1.598 \mathrm{E}+05$ & $3.250 \mathrm{E}+04$ & $2.984 \mathrm{E}+04$ & $2.134 \mathrm{E}+04$ & $1.526 \mathrm{E}+04$ \\
\hline cs135 & $9.677 \mathrm{E}-01$ & $9.677 \mathrm{E}-01$ & $9.677 \mathrm{E}-01$ & $9.677 \mathrm{E}-01$ & $9.677 \mathrm{E}-01$ \\
\hline cs136 & $7.859 \mathrm{E}-02$ & $0.000 \mathrm{E}+00$ & $0.000 \mathrm{E}+00$ & $0.000 \mathrm{E}+00$ & $0.000 \mathrm{E}+00$ \\
\hline cs137 & $1.296 \mathrm{E}+05$ & $1.162 \mathrm{E}+05$ & $1.154 \mathrm{E}+05$ & $1.128 \mathrm{E}+05$ & $1.102 \mathrm{E}+05$ \\
\hline dy159 & $1.213 \mathrm{E}-03$ & $2.957 \mathrm{E}-07$ & $1.892 \mathrm{E}-07$ & $3.281 \mathrm{E}-08$ & $5.690 \mathrm{E}-09$ \\
\hline er169 & $7.998 \mathrm{E}-09$ & $0.000 \mathrm{E}+00$ & $0.000 \mathrm{E}+00$ & $0.000 \mathrm{E}+00$ & $0.000 \mathrm{E}+00$ \\
\hline es252 & $2.644 \mathrm{E}-13$ & $2.071 \mathrm{E}-14$ & $1.807 \mathrm{E}-14$ & $1.057 \mathrm{E}-14$ & $6.180 \mathrm{E}-15$ \\
\hline es 253 & $3.771 \mathrm{E}-09$ & $0.000 \mathrm{E}+00$ & $0.000 \mathrm{E}+00$ & $0.000 \mathrm{E}+00$ & $0.000 \mathrm{E}+00$ \\
\hline es254 & $1.572 \mathrm{E}-09$ & $2.015 \mathrm{E}-11$ & $1.595 \mathrm{E}-11$ & $6.370 \mathrm{E}-12$ & $2.545 \mathrm{E}-12$ \\
\hline es255 & $1.568 \mathrm{E}-11$ & $1.224 \mathrm{E}-24$ & $2.423 \mathrm{E}-25$ & 4.203E-28 & $0.000 \mathrm{E}+00$ \\
\hline eu147 & $9.027 \mathrm{E}-14$ & $0.000 \mathrm{E}+00$ & $0.000 \mathrm{E}+00$ & $0.000 \mathrm{E}+00$ & $0.000 \mathrm{E}+00$ \\
\hline eu149 & $7.719 \mathrm{E}-07$ & $1.923 \mathrm{E}-12$ & $9.624 \mathrm{E}-13$ & $6.354 \mathrm{E}-14$ & $4.196 \mathrm{E}-15$ \\
\hline eu152 & $8.516 \mathrm{E}+00$ & $6.677 \mathrm{E}+00$ & $6.591 \mathrm{E}+00$ & $6.263 \mathrm{E}+00$ & $5.950 \mathrm{E}+00$ \\
\hline eu154 & $1.361 \mathrm{E}+04$ & $9.286 \mathrm{E}+03$ & $9.100 \mathrm{E}+03$ & $8.396 \mathrm{E}+03$ & $7.746 \mathrm{E}+03$ \\
\hline eu155 & $5.978 \mathrm{E}+03$ & $2.993 \mathrm{E}+03$ & $2.883 \mathrm{E}+03$ & $2.492 \mathrm{E}+03$ & $2.154 \mathrm{E}+03$ \\
\hline eu156 & $1.756 \mathrm{E}+00$ & $0.000 \mathrm{E}+00$ & $0.000 \mathrm{E}+00$ & $0.000 \mathrm{E}+00$ & $0.000 \mathrm{E}+00$ \\
\hline fe 55 & $3.714 \mathrm{E}-13$ & $1.120 \mathrm{E}-13$ & $1.050 \mathrm{E}-13$ & $8.157 \mathrm{E}-14$ & $6.339 \mathrm{E}-14$ \\
\hline fe 59 & $4.284 \mathrm{E}-14$ & $8.078 \mathrm{E}-26$ & $1.896 \mathrm{E}-26$ & $6.435 \mathrm{E}-29$ & $0.000 \mathrm{E}+00$ \\
\hline fe 60 & $2.178 \mathrm{E}-21$ & $2.178 \mathrm{E}-21$ & $2.178 \mathrm{E}-21$ & $2.178 \mathrm{E}-21$ & $2.178 \mathrm{E}-21$ \\
\hline fr221 & $4.459 \mathrm{E}-08$ & 4.712E-08 & $4.723 \mathrm{E}-08$ & 4.799E-08 & 4.874E-08 \\
\hline fr223 & $7.613 \mathrm{E}-10$ & $3.234 \mathrm{E}-09$ & $3.370 \mathrm{E}-09$ & $3.911 \mathrm{E}-09$ & 4.456E-09 \\
\hline ga 68 & $9.478 \mathrm{E}-16$ & $1.126 \mathrm{E}-17$ & $8.874 \mathrm{E}-18$ & $3.488 \mathrm{E}-18$ & $1.371 \mathrm{E}-18$ \\
\hline gd151 & $5.988 \mathrm{E}-04$ & $3.716 \mathrm{E}-08$ & $2.210 \mathrm{E}-08$ & 2.872E-09 & $3.733 \mathrm{E}-10$ \\
\hline gd152 & $1.921 \mathrm{E}-12$ & $1.985 \mathrm{E}-12$ & $1.988 \mathrm{E}-12$ & $1.999 \mathrm{E}-12$ & $2.010 \mathrm{E}-12$ \\
\hline gd153 & $1.220 \mathrm{E}+01$ & 8.244E-02 & $6.307 \mathrm{E}-02$ & 2.202E-02 & $7.686 \mathrm{E}-03$ \\
\hline ge 68 & $9.478 \mathrm{E}-16$ & $1.126 \mathrm{E}-17$ & $8.874 \mathrm{E}-18$ & $3.487 \mathrm{E}-18$ & $1.371 \mathrm{E}-18$ \\
\hline
\end{tabular}


Table C-10. Continued

\begin{tabular}{|c|c|c|c|c|c|}
\hline & $1 / 22 / 2009$ & $10 / 21 / 2013$ & $1 / 22 / 2014$ & $1 / 22 / 2015$ & $1 / 22 / 2016$ \\
\hline ge 71 & $5.751 \mathrm{E}-11$ & $0.000 \mathrm{E}+00$ & $0.000 \mathrm{E}+00$ & $0.000 \mathrm{E}+00$ & $0.000 \mathrm{E}+00$ \\
\hline ge 73m & $3.461 \mathrm{E}-06$ & $1.103 \mathrm{E}-12$ & $4.941 \mathrm{E}-13$ & $2.116 \mathrm{E}-14$ & $9.060 \mathrm{E}-16$ \\
\hline h3 & $6.781 \mathrm{E}+02$ & $5.194 \mathrm{E}+02$ & $5.121 \mathrm{E}+02$ & $4.840 \mathrm{E}+02$ & $4.576 \mathrm{E}+02$ \\
\hline hf172 & $1.549 \mathrm{E}-19$ & $2.669 \mathrm{E}-20$ & $2.428 \mathrm{E}-20$ & $1.677 \mathrm{E}-20$ & $1.158 \mathrm{E}-20$ \\
\hline hf175 & $1.037 \mathrm{E}-11$ & $3.658 \mathrm{E}-19$ & $1.456 \mathrm{E}-19$ & $3.923 \mathrm{E}-21$ & $1.057 \mathrm{E}-22$ \\
\hline hf177m & $3.179 \mathrm{E}-14$ & $1.781 \mathrm{E}-17$ & $1.192 \mathrm{E}-17$ & $2.462 \mathrm{E}-18$ & $5.088 \mathrm{E}-19$ \\
\hline hf181 & $3.283 \mathrm{E}-13$ & $1.619 \mathrm{E}-25$ & $3.540 \mathrm{E}-26$ & $9.053 \mathrm{E}-29$ & $0.000 \mathrm{E}+00$ \\
\hline hf182 & $1.528 \mathrm{E}-19$ & $1.528 \mathrm{E}-19$ & $1.528 \mathrm{E}-19$ & $1.528 \mathrm{E}-19$ & $1.528 \mathrm{E}-19$ \\
\hline hg194 & $8.217 \mathrm{E}-21$ & $8.157 \mathrm{E}-21$ & $8.151 \mathrm{E}-21$ & $8.137 \mathrm{E}-21$ & $8.124 \mathrm{E}-21$ \\
\hline hg203 & $5.330 \mathrm{E}-14$ & $3.390 \mathrm{E}-25$ & $8.502 \mathrm{E}-26$ & $3.726 \mathrm{E}-28$ & $0.000 \mathrm{E}+00$ \\
\hline hg206 & $6.618 \mathrm{E}-17$ & $6.982 \mathrm{E}-17$ & $7.075 \mathrm{E}-17$ & $7.527 \mathrm{E}-17$ & $8.144 \mathrm{E}-17$ \\
\hline ho163 & $1.058 \mathrm{E}-07$ & $1.057 \mathrm{E}-07$ & $1.057 \mathrm{E}-07$ & $1.057 \mathrm{E}-07$ & $1.057 \mathrm{E}-07$ \\
\hline ho166m & 8.801E-04 & $8.781 \mathrm{E}-04$ & $8.781 \mathrm{E}-04$ & $8.774 \mathrm{E}-04$ & $8.768 \mathrm{E}-04$ \\
\hline i125 & $1.770 \mathrm{E}-05$ & $2.919 \mathrm{E}-14$ & $9.863 \mathrm{E}-15$ & $1.394 \mathrm{E}-16$ & $1.969 \mathrm{E}-18$ \\
\hline i126 & $1.342 \mathrm{E}-06$ & $0.000 \mathrm{E}+00$ & $0.000 \mathrm{E}+00$ & $0.000 \mathrm{E}+00$ & $0.000 \mathrm{E}+00$ \\
\hline i129 & $4.916 \mathrm{E}-02$ & $4.917 \mathrm{E}-02$ & 4.917E-02 & $4.917 \mathrm{E}-02$ & 4.917E-02 \\
\hline i131 & $1.560 \mathrm{E}-04$ & $0.000 \mathrm{E}+00$ & $0.000 \mathrm{E}+00$ & $0.000 \mathrm{E}+00$ & $0.000 \mathrm{E}+00$ \\
\hline in113m & $5.895 \mathrm{E}-07$ & $1.728 \mathrm{E}-11$ & $9.870 \mathrm{E}-12$ & $1.096 \mathrm{E}-12$ & $1.216 \mathrm{E}-13$ \\
\hline in114 & $6.690 \mathrm{E}-03$ & $1.942 \mathrm{E}-13$ & $5.283 \mathrm{E}-14$ & $3.189 \mathrm{E}-16$ & $1.924 \mathrm{E}-18$ \\
\hline in114m & $6.916 \mathrm{E}-03$ & $2.008 \mathrm{E}-13$ & $5.460 \mathrm{E}-14$ & $3.295 \mathrm{E}-16$ & $1.989 \mathrm{E}-18$ \\
\hline in115 & $2.466 \mathrm{E}-11$ & $2.466 \mathrm{E}-11$ & $2.466 \mathrm{E}-11$ & $2.466 \mathrm{E}-11$ & $2.466 \mathrm{E}-11$ \\
\hline in115m & $1.784 \mathrm{E}-03$ & $3.498 \mathrm{E}-15$ & $8.230 \mathrm{E}-16$ & $2.816 \mathrm{E}-18$ & $9.637 \mathrm{E}-21$ \\
\hline ir190 & $2.041 \mathrm{E}-22$ & $0.000 \mathrm{E}+00$ & $0.000 \mathrm{E}+00$ & $0.000 \mathrm{E}+00$ & $0.000 \mathrm{E}+00$ \\
\hline ir191m & $3.646 \mathrm{E}-19$ & $0.000 \mathrm{E}+00$ & $0.000 \mathrm{E}+00$ & $0.000 \mathrm{E}+00$ & $0.000 \mathrm{E}+00$ \\
\hline ir192 & $3.495 \mathrm{E}-12$ & $2.999 \mathrm{E}-19$ & $1.252 \mathrm{E}-19$ & $4.070 \mathrm{E}-21$ & $1.322 \mathrm{E}-22$ \\
\hline ir193m & $1.495 \mathrm{E}-19$ & $0.000 \mathrm{E}+00$ & $0.000 \mathrm{E}+00$ & $0.000 \mathrm{E}+00$ & $0.000 \mathrm{E}+00$ \\
\hline ir194 & $1.127 \mathrm{E}-18$ & $6.513 \mathrm{E}-19$ & $6.324 \mathrm{E}-19$ & $5.634 \mathrm{E}-19$ & $5.020 \mathrm{E}-19$ \\
\hline k 40 & $5.040 \mathrm{E}-19$ & $5.040 \mathrm{E}-19$ & $5.040 \mathrm{E}-19$ & $5.040 \mathrm{E}-19$ & $5.040 \mathrm{E}-19$ \\
\hline k 42 & $5.101 \mathrm{E}-21$ & $4.616 \mathrm{E}-21$ & $4.590 \mathrm{E}-21$ & $4.495 \mathrm{E}-21$ & $4.401 \mathrm{E}-21$ \\
\hline kr 81 & $7.288 \mathrm{E}-07$ & $7.288 \mathrm{E}-07$ & $7.288 \mathrm{E}-07$ & $7.288 \mathrm{E}-07$ & $7.288 \mathrm{E}-07$ \\
\hline kr 83m & $9.863 \mathrm{E}-04$ & $8.748 \mathrm{E}-10$ & $4.142 \mathrm{E}-10$ & $2.201 \mathrm{E}-11$ & $1.169 \mathrm{E}-12$ \\
\hline kr 85 & $6.737 \mathrm{E}+03$ & $4.962 \mathrm{E}+03$ & $4.881 \mathrm{E}+03$ & $4.576 \mathrm{E}+03$ & $4.291 \mathrm{E}+03$ \\
\hline la137 & $2.152 \mathrm{E}-05$ & $2.152 \mathrm{E}-05$ & $2.152 \mathrm{E}-05$ & $2.152 \mathrm{E}-05$ & $2.152 \mathrm{E}-05$ \\
\hline la138 & $6.138 \mathrm{E}-10$ & $6.138 \mathrm{E}-10$ & $6.138 \mathrm{E}-10$ & $6.138 \mathrm{E}-10$ & $6.138 \mathrm{E}-10$ \\
\hline la140 & $1.231 \mathrm{E}+00$ & $0.000 \mathrm{E}+00$ & $0.000 \mathrm{E}+00$ & $0.000 \mathrm{E}+00$ & $0.000 \mathrm{E}+00$ \\
\hline lu172 & $1.563 \mathrm{E}-19$ & $2.669 \mathrm{E}-20$ & $2.429 \mathrm{E}-20$ & $1.677 \mathrm{E}-20$ & $1.158 \mathrm{E}-20$ \\
\hline
\end{tabular}


Table C-10. Continued

\begin{tabular}{|c|c|c|c|c|c|}
\hline & $1 / 22 / 2009$ & $10 / 21 / 2013$ & $1 / 22 / 2014$ & $1 / 22 / 2015$ & $1 / 22 / 2016$ \\
\hline lu172m & $1.549 \mathrm{E}-19$ & $2.669 \mathrm{E}-20$ & $2.429 \mathrm{E}-20$ & $1.677 \mathrm{E}-20$ & $1.158 \mathrm{E}-20$ \\
\hline lu173 & $5.101 \mathrm{E}-15$ & $4.624 \mathrm{E}-16$ & $4.065 \mathrm{E}-16$ & $2.452 \mathrm{E}-16$ & $1.479 \mathrm{E}-16$ \\
\hline lu174 & $6.797 \mathrm{E}-15$ & $2.607 \mathrm{E}-15$ & $2.472 \mathrm{E}-15$ & $2.005 \mathrm{E}-15$ & $1.626 \mathrm{E}-15$ \\
\hline lu174m & $1.849 \mathrm{E}-15$ & $3.919 \mathrm{E}-19$ & $2.489 \mathrm{E}-19$ & $4.190 \mathrm{E}-20$ & $7.055 \mathrm{E}-21$ \\
\hline lu176 & $4.605 \mathrm{E}-22$ & $4.605 \mathrm{E}-22$ & 4.605E-22 & $4.605 \mathrm{E}-22$ & $4.605 \mathrm{E}-22$ \\
\hline lu177 & $9.033 \mathrm{E}-15$ & $5.060 \mathrm{E}-18$ & $3.386 \mathrm{E}-18$ & $6.996 \mathrm{E}-19$ & $1.445 \mathrm{E}-19$ \\
\hline lu177m & $4.045 \mathrm{E}-14$ & $2.266 \mathrm{E}-17$ & $1.517 \mathrm{E}-17$ & $3.133 \mathrm{E}-18$ & $6.473 \mathrm{E}-19$ \\
\hline mn 53 & $5.609 \mathrm{E}-22$ & $5.609 \mathrm{E}-22$ & $5.609 \mathrm{E}-22$ & $5.609 \mathrm{E}-22$ & $5.609 \mathrm{E}-22$ \\
\hline mn 54 & $1.538 \mathrm{E}-13$ & $3.274 \mathrm{E}-15$ & $2.664 \mathrm{E}-15$ & $1.184 \mathrm{E}-15$ & $5.263 \mathrm{E}-16$ \\
\hline mo 93 & $3.299 \mathrm{E}-07$ & $3.297 \mathrm{E}-07$ & $3.297 \mathrm{E}-07$ & $3.296 \mathrm{E}-07$ & $3.295 \mathrm{E}-07$ \\
\hline mo100 & $5.717 \mathrm{E}-13$ & $5.717 \mathrm{E}-13$ & $5.717 \mathrm{E}-13$ & $5.717 \mathrm{E}-13$ & $5.717 \mathrm{E}-13$ \\
\hline na 22 & $5.494 \mathrm{E}-19$ & $1.552 \mathrm{E}-19$ & $1.451 \mathrm{E}-19$ & $1.112 \mathrm{E}-19$ & $8.522 \mathrm{E}-20$ \\
\hline nb 91 & 5.882E-08 & $5.854 \mathrm{E}-08$ & $5.852 \mathrm{E}-08$ & $5.846 \mathrm{E}-08$ & $5.840 \mathrm{E}-08$ \\
\hline nb 91m & $1.413 \mathrm{E}-10$ & $3.785 \mathrm{E}-19$ & $1.312 \mathrm{E}-19$ & $2.054 \mathrm{E}-21$ & $3.216 \mathrm{E}-23$ \\
\hline nb 92 & $9.956 \mathrm{E}-11$ & $9.956 \mathrm{E}-11$ & $9.956 \mathrm{E}-11$ & $9.956 \mathrm{E}-11$ & $9.956 \mathrm{E}-11$ \\
\hline nb 92m & $1.987 \mathrm{E}-13$ & $0.000 \mathrm{E}+00$ & $0.000 \mathrm{E}+00$ & $0.000 \mathrm{E}+00$ & $0.000 \mathrm{E}+00$ \\
\hline nb 93m & $1.363 \mathrm{E}-01$ & $3.922 \mathrm{E}-01$ & $4.045 \mathrm{E}-01$ & $4.515 \mathrm{E}-01$ & $4.966 \mathrm{E}-01$ \\
\hline nb 94 & $5.617 \mathrm{E}-04$ & $5.616 \mathrm{E}-04$ & $5.616 \mathrm{E}-04$ & $5.616 \mathrm{E}-04$ & $5.616 \mathrm{E}-04$ \\
\hline nb 95 & $1.873 \mathrm{E}+05$ & $1.410 \mathrm{E}-03$ & $5.143 \mathrm{E}-04$ & $1.020 \mathrm{E}-05$ & $1.963 \mathrm{E}-07$ \\
\hline nb 95m & $1.025 \mathrm{E}+03$ & $7.301 \mathrm{E}-06$ & $2.668 \mathrm{E}-06$ & $5.132 \mathrm{E}-08$ & $9.870 \mathrm{E}-10$ \\
\hline nd144 & $1.035 \mathrm{E}-09$ & $1.239 \mathrm{E}-09$ & $1.240 \mathrm{E}-09$ & $1.241 \mathrm{E}-09$ & $1.242 \mathrm{E}-09$ \\
\hline nd147 & $4.028 \mathrm{E}-02$ & $0.000 \mathrm{E}+00$ & $0.000 \mathrm{E}+00$ & $0.000 \mathrm{E}+00$ & $0.000 \mathrm{E}+00$ \\
\hline nd150 & $7.898 \mathrm{E}-14$ & $7.898 \mathrm{E}-14$ & $7.898 \mathrm{E}-14$ & $7.898 \mathrm{E}-14$ & $7.898 \mathrm{E}-14$ \\
\hline ni 59 & $7.036 \mathrm{E}-15$ & $7.036 \mathrm{E}-15$ & $7.036 \mathrm{E}-15$ & $7.036 \mathrm{E}-15$ & $7.036 \mathrm{E}-15$ \\
\hline ni 63 & $8.635 \mathrm{E}-14$ & $8.356 \mathrm{E}-14$ & 8.343E-14 & $8.283 \mathrm{E}-14$ & $8.230 \mathrm{E}-14$ \\
\hline np235 & $2.450 \mathrm{E}-03$ & $1.181 \mathrm{E}-04$ & $1.004 \mathrm{E}-04$ & $5.301 \mathrm{E}-05$ & $2.799 \mathrm{E}-05$ \\
\hline np236 & $2.989 \mathrm{E}-06$ & $2.989 \mathrm{E}-06$ & $2.989 \mathrm{E}-06$ & $2.989 \mathrm{E}-06$ & $2.989 \mathrm{E}-06$ \\
\hline np237 & $1.124 \mathrm{E}-01$ & $1.173 \mathrm{E}-01$ & $1.178 \mathrm{E}-01$ & $1.195 \mathrm{E}-01$ & $1.215 \mathrm{E}-01$ \\
\hline np238 & $1.971 \mathrm{E}-01$ & $1.925 \mathrm{E}-01$ & $1.923 \mathrm{E}-01$ & $1.914 \mathrm{E}-01$ & $1.904 \mathrm{E}-01$ \\
\hline np239 & $5.430 \mathrm{E}+01$ & $5.428 \mathrm{E}+01$ & $5.427 \mathrm{E}+01$ & $5.427 \mathrm{E}+01$ & $5.427 \mathrm{E}+01$ \\
\hline np240 & $1.423 \mathrm{E}-09$ & $1.423 \mathrm{E}-09$ & $1.423 \mathrm{E}-09$ & $1.423 \mathrm{E}-09$ & $1.423 \mathrm{E}-09$ \\
\hline np240m & $1.186 \mathrm{E}-06$ & $1.186 \mathrm{E}-06$ & $1.186 \mathrm{E}-06$ & $1.186 \mathrm{E}-06$ & $1.186 \mathrm{E}-06$ \\
\hline os185 & $1.357 \mathrm{E}-15$ & $3.624 \mathrm{E}-21$ & $1.820 \mathrm{E}-21$ & $1.219 \mathrm{E}-22$ & $8.170 \mathrm{E}-24$ \\
\hline os191 & $3.646 \mathrm{E}-19$ & $0.000 \mathrm{E}+00$ & $0.000 \mathrm{E}+00$ & $0.000 \mathrm{E}+00$ & $0.000 \mathrm{E}+00$ \\
\hline os194 & $1.126 \mathrm{E}-18$ & $6.511 \mathrm{E}-19$ & $6.322 \mathrm{E}-19$ & $5.632 \mathrm{E}-19$ & $5.018 \mathrm{E}-19$ \\
\hline p 32 & $1.635 \mathrm{E}-18$ & $2.928 \mathrm{E}-19$ & $2.925 \mathrm{E}-19$ & $2.912 \mathrm{E}-19$ & $2.899 \mathrm{E}-19$ \\
\hline
\end{tabular}


Table C-10. Continued

\begin{tabular}{|c|c|c|c|c|c|}
\hline & $1 / 22 / 2009$ & $10 / 21 / 2013$ & $1 / 22 / 2014$ & $1 / 22 / 2015$ & $1 / 22 / 2016$ \\
\hline p 33 & $1.952 \mathrm{E}-16$ & $0.000 \mathrm{E}+00$ & $0.000 \mathrm{E}+00$ & $0.000 \mathrm{E}+00$ & $0.000 \mathrm{E}+00$ \\
\hline pa230 & $3.392 \mathrm{E}-11$ & $0.000 \mathrm{E}+00$ & $0.000 \mathrm{E}+00$ & $0.000 \mathrm{E}+00$ & $0.000 \mathrm{E}+00$ \\
\hline pa231 & $1.207 \mathrm{E}-06$ & $1.454 \mathrm{E}-06$ & $1.467 \mathrm{E}-06$ & $1.519 \mathrm{E}-06$ & $1.571 \mathrm{E}-06$ \\
\hline pa232 & $5.978 \mathrm{E}-09$ & $5.977 \mathrm{E}-09$ & $5.977 \mathrm{E}-09$ & $5.977 \mathrm{E}-09$ & 5.977E-09 \\
\hline pa233 & $1.124 \mathrm{E}-01$ & $1.172 \mathrm{E}-01$ & $1.176 \mathrm{E}-01$ & $1.195 \mathrm{E}-01$ & $1.215 \mathrm{E}-01$ \\
\hline pa234 & 4.959E-04 & $4.961 \mathrm{E}-04$ & $4.959 \mathrm{E}-04$ & $4.959 \mathrm{E}-04$ & 4.959E-04 \\
\hline pa234m & $3.100 \mathrm{E}-01$ & $3.100 \mathrm{E}-01$ & $3.100 \mathrm{E}-01$ & $3.100 \mathrm{E}-01$ & $3.100 \mathrm{E}-01$ \\
\hline pb209 & 4.459E-08 & $4.712 \mathrm{E}-08$ & $4.723 \mathrm{E}-08$ & $4.799 \mathrm{E}-08$ & 4.874E-08 \\
\hline pb210 & $3.483 \mathrm{E}-09$ & $3.676 \mathrm{E}-09$ & $3.724 \mathrm{E}-09$ & $3.960 \mathrm{E}-09$ & $4.287 \mathrm{E}-09$ \\
\hline pb211 & $5.126 \mathrm{E}-08$ & $2.348 \mathrm{E}-07$ & $2.399 \mathrm{E}-07$ & $2.839 \mathrm{E}-07$ & $3.235 \mathrm{E}-07$ \\
\hline pb212 & $1.965 \mathrm{E}-03$ & $1.280 \mathrm{E}-02$ & $1.328 \mathrm{E}-02$ & $1.493 \mathrm{E}-02$ & $1.631 \mathrm{E}-02$ \\
\hline pb214 & $1.563 \mathrm{E}-09$ & $9.378 \mathrm{E}-09$ & $1.004 \mathrm{E}-08$ & $1.294 \mathrm{E}-08$ & $1.633 \mathrm{E}-08$ \\
\hline pd103 & $1.576 \mathrm{E}-04$ & $0.000 \mathrm{E}+00$ & $0.000 \mathrm{E}+00$ & $0.000 \mathrm{E}+00$ & $0.000 \mathrm{E}+00$ \\
\hline pd107 & $2.995 \mathrm{E}-01$ & $2.995 \mathrm{E}-01$ & $2.995 \mathrm{E}-01$ & $2.995 \mathrm{E}-01$ & $2.995 \mathrm{E}-01$ \\
\hline pm143 & 4.413E-09 & 4.744E-11 & $3.719 \mathrm{E}-11$ & $1.432 \mathrm{E}-11$ & $5.510 \mathrm{E}-12$ \\
\hline pm144 & $9.863 \mathrm{E}-07$ & $3.604 \mathrm{E}-08$ & $3.017 \mathrm{E}-08$ & $1.503 \mathrm{E}-08$ & 7.487E-09 \\
\hline pm145 & $4.312 \mathrm{E}-03$ & $3.615 \mathrm{E}-03$ & $3.579 \mathrm{E}-03$ & $3.442 \mathrm{E}-03$ & $3.311 \mathrm{E}-03$ \\
\hline pm146 & $2.032 \mathrm{E}-01$ & $1.122 \mathrm{E}-01$ & $1.087 \mathrm{E}-01$ & $9.584 \mathrm{E}-02$ & $8.456 \mathrm{E}-02$ \\
\hline pm147 & $1.698 \mathrm{E}+05$ & $4.849 \mathrm{E}+04$ & $4.533 \mathrm{E}+04$ & $3.481 \mathrm{E}+04$ & $2.673 \mathrm{E}+04$ \\
\hline pm148 & $3.563 \mathrm{E}+01$ & $8.263 \mathrm{E}-12$ & $1.734 \mathrm{E}-12$ & $3.784 \mathrm{E}-15$ & $8.257 \mathrm{E}-18$ \\
\hline pm148m & $7.381 \mathrm{E}+02$ & $1.711 \mathrm{E}-10$ & $3.591 \mathrm{E}-11$ & $7.839 \mathrm{E}-14$ & $1.710 \mathrm{E}-16$ \\
\hline po209 & $1.832 \mathrm{E}-16$ & $1.774 \mathrm{E}-16$ & $1.771 \mathrm{E}-16$ & $1.759 \mathrm{E}-16$ & $1.747 \mathrm{E}-16$ \\
\hline po210 & $3.003 \mathrm{E}-09$ & $3.553 \mathrm{E}-09$ & $3.589 \mathrm{E}-09$ & $3.785 \mathrm{E}-09$ & $4.060 \mathrm{E}-09$ \\
\hline po211 & $1.415 \mathrm{E}-10$ & $6.482 \mathrm{E}-10$ & $6.622 \mathrm{E}-10$ & $7.839 \mathrm{E}-10$ & $8.927 \mathrm{E}-10$ \\
\hline po212 & $1.258 \mathrm{E}-03$ & $8.197 \mathrm{E}-03$ & $8.509 \mathrm{E}-03$ & $9.558 \mathrm{E}-03$ & $1.045 \mathrm{E}-02$ \\
\hline po213 & $4.361 \mathrm{E}-08$ & $4.609 \mathrm{E}-08$ & $4.619 \mathrm{E}-08$ & 4.694E-08 & 4.767E-08 \\
\hline po214 & $1.639 \mathrm{E}-09$ & $9.378 \mathrm{E}-09$ & $1.004 \mathrm{E}-08$ & $1.294 \mathrm{E}-08$ & $1.633 \mathrm{E}-08$ \\
\hline po215 & $5.126 \mathrm{E}-08$ & $2.348 \mathrm{E}-07$ & $2.399 \mathrm{E}-07$ & $2.839 \mathrm{E}-07$ & $3.235 \mathrm{E}-07$ \\
\hline po216 & $1.965 \mathrm{E}-03$ & $1.280 \mathrm{E}-02$ & $1.328 \mathrm{E}-02$ & $1.493 \mathrm{E}-02$ & $1.631 \mathrm{E}-02$ \\
\hline po218 & $1.563 \mathrm{E}-09$ & $9.378 \mathrm{E}-09$ & $1.005 \mathrm{E}-08$ & $1.295 \mathrm{E}-08$ & $1.633 \mathrm{E}-08$ \\
\hline pr143 & $2.233 \mathrm{E}+00$ & $0.000 \mathrm{E}+00$ & $0.000 \mathrm{E}+00$ & $0.000 \mathrm{E}+00$ & $0.000 \mathrm{E}+00$ \\
\hline pr144 & $6.073 \mathrm{E}+05$ & $8.960 \mathrm{E}+03$ & $7.148 \mathrm{E}+03$ & $2.940 \mathrm{E}+03$ & $1.210 \mathrm{E}+03$ \\
\hline pr144m & $5.798 \mathrm{E}+03$ & $8.555 \mathrm{E}+01$ & $6.823 \mathrm{E}+01$ & $2.808 \mathrm{E}+01$ & $1.155 \mathrm{E}+01$ \\
\hline pt193 & $6.611 \mathrm{E}-13$ & $6.191 \mathrm{E}-13$ & $6.169 \mathrm{E}-13$ & $6.084 \mathrm{E}-13$ & $6.000 \mathrm{E}-13$ \\
\hline pu236 & $3.954 \mathrm{E}-01$ & $1.251 \mathrm{E}-01$ & $1.176 \mathrm{E}-01$ & $9.226 \mathrm{E}-02$ & $7.241 \mathrm{E}-02$ \\
\hline pu237 & $1.146 \mathrm{E}-01$ & $4.251 \mathrm{E}-13$ & $1.035 \mathrm{E}-13$ & $4.052 \mathrm{E}-16$ & $1.586 \mathrm{E}-18$ \\
\hline
\end{tabular}


Table C-10. Continued

\begin{tabular}{|c|c|c|c|c|c|}
\hline & $1 / 22 / 2009$ & $10 / 21 / 2013$ & $1 / 22 / 2014$ & $1 / 22 / 2015$ & $1 / 22 / 2016$ \\
\hline pu238 & $2.691 \mathrm{E}+03$ & $2.887 \mathrm{E}+03$ & $2.881 \mathrm{E}+03$ & $2.859 \mathrm{E}+03$ & $2.837 \mathrm{E}+03$ \\
\hline pu239 & $1.138 \mathrm{E}+03$ & $1.138 \mathrm{E}+03$ & $1.138 \mathrm{E}+03$ & $1.138 \mathrm{E}+03$ & $1.138 \mathrm{E}+03$ \\
\hline pu240 & $2.241 \mathrm{E}+03$ & $2.243 \mathrm{E}+03$ & $2.243 \mathrm{E}+03$ & $2.243 \mathrm{E}+03$ & $2.243 \mathrm{E}+03$ \\
\hline pu241 & $5.147 \mathrm{E}+05$ & $4.089 \mathrm{E}+05$ & $4.039 \mathrm{E}+05$ & $3.848 \mathrm{E}+05$ & $3.665 \mathrm{E}+05$ \\
\hline pu242 & $4.605 \mathrm{E}+00$ & $4.605 \mathrm{E}+00$ & $4.605 \mathrm{E}+00$ & $4.605 \mathrm{E}+00$ & $4.605 \mathrm{E}+00$ \\
\hline pu243 & 4.839E-07 & $4.839 \mathrm{E}-07$ & $4.839 \mathrm{E}-07$ & $4.839 \mathrm{E}-07$ & $4.839 \mathrm{E}-07$ \\
\hline pu244 & $1.187 \mathrm{E}-06$ & $1.187 \mathrm{E}-06$ & $1.187 \mathrm{E}-06$ & $1.187 \mathrm{E}-06$ & $1.187 \mathrm{E}-06$ \\
\hline pu246 & $8.363 \mathrm{E}-13$ & $8.363 \mathrm{E}-13$ & $8.363 \mathrm{E}-13$ & $8.363 \mathrm{E}-13$ & $8.363 \mathrm{E}-13$ \\
\hline ra222 & $7.613 \mathrm{E}-11$ & $0.000 \mathrm{E}+00$ & $0.000 \mathrm{E}+00$ & $0.000 \mathrm{E}+00$ & $0.000 \mathrm{E}+00$ \\
\hline ra223 & $5.126 \mathrm{E}-08$ & $2.348 \mathrm{E}-07$ & $2.399 \mathrm{E}-07$ & $2.839 \mathrm{E}-07$ & $3.235 \mathrm{E}-07$ \\
\hline ra224 & $1.965 \mathrm{E}-03$ & $1.280 \mathrm{E}-02$ & $1.328 \mathrm{E}-02$ & $1.493 \mathrm{E}-02$ & $1.631 \mathrm{E}-02$ \\
\hline ra225 & 4.457E-08 & $4.712 \mathrm{E}-08$ & $4.726 \mathrm{E}-08$ & 4.799E-08 & $4.874 \mathrm{E}-08$ \\
\hline ra226 & $1.576 \mathrm{E}-09$ & $9.378 \mathrm{E}-09$ & $1.005 \mathrm{E}-08$ & $1.295 \mathrm{E}-08$ & $1.633 \mathrm{E}-08$ \\
\hline ra228 & $7.095 \mathrm{E}-12$ & $1.349 \mathrm{E}-11$ & $1.380 \mathrm{E}-11$ & $1.499 \mathrm{E}-11$ & $1.615 \mathrm{E}-11$ \\
\hline rb 83 & $1.327 \mathrm{E}-03$ & $1.177 \mathrm{E}-09$ & $5.573 \mathrm{E}-10$ & $2.961 \mathrm{E}-11$ & $1.573 \mathrm{E}-12$ \\
\hline rb 84 & $2.821 \mathrm{E}-03$ & $3.589 \mathrm{E}-19$ & $5.034 \mathrm{E}-20$ & $2.260 \mathrm{E}-23$ & $1.014 \mathrm{E}-26$ \\
\hline rb 86 & $9.106 \mathrm{E}-02$ & $9.080 \mathrm{E}-30$ & $0.000 \mathrm{E}+00$ & $0.000 \mathrm{E}+00$ & $0.000 \mathrm{E}+00$ \\
\hline rb 87 & $1.384 \mathrm{E}-05$ & $1.384 \mathrm{E}-05$ & $1.384 \mathrm{E}-05$ & $1.384 \mathrm{E}-05$ & $1.384 \mathrm{E}-05$ \\
\hline re183 & $8.509 \mathrm{E}-19$ & $2.999 \mathrm{E}-26$ & $1.194 \mathrm{E}-26$ & $3.216 \mathrm{E}-28$ & $8.662 \mathrm{E}-30$ \\
\hline re184 & $1.629 \mathrm{E}-16$ & $1.242 \mathrm{E}-19$ & $8.482 \mathrm{E}-20$ & $1.906 \mathrm{E}-20$ & 4.264E-21 \\
\hline re184m & $1.611 \mathrm{E}-16$ & $1.318 \mathrm{E}-19$ & $9.007 \mathrm{E}-20$ & $2.015 \mathrm{E}-20$ & $4.509 \mathrm{E}-21$ \\
\hline re186 & $2.041 \mathrm{E}-18$ & $2.041 \mathrm{E}-18$ & $2.041 \mathrm{E}-18$ & $2.041 \mathrm{E}-18$ & $2.041 \mathrm{E}-18$ \\
\hline re186m & $2.041 \mathrm{E}-18$ & $2.041 \mathrm{E}-18$ & $2.041 \mathrm{E}-18$ & $2.041 \mathrm{E}-18$ & $2.041 \mathrm{E}-18$ \\
\hline re187 & $1.582 \mathrm{E}-20$ & $1.582 \mathrm{E}-20$ & $1.582 \mathrm{E}-20$ & $1.582 \mathrm{E}-20$ & $1.582 \mathrm{E}-20$ \\
\hline re188 & $1.726 \mathrm{E}-14$ & $5.766 \mathrm{E}-22$ & $2.289 \mathrm{E}-22$ & $0.000 \mathrm{E}+00$ & $0.000 \mathrm{E}+00$ \\
\hline rh 99 & $5.158 \mathrm{E}-15$ & $0.000 \mathrm{E}+00$ & $0.000 \mathrm{E}+00$ & $0.000 \mathrm{E}+00$ & $0.000 \mathrm{E}+00$ \\
\hline rh101 & $5.308 \mathrm{E}-04$ & $1.959 \mathrm{E}-04$ & $1.857 \mathrm{E}-04$ & $1.506 \mathrm{E}-04$ & $1.221 \mathrm{E}-04$ \\
\hline rh102 & $4.693 \mathrm{E}+00$ & $1.614 \mathrm{E}-02$ & $1.225 \mathrm{E}-02$ & $4.570 \mathrm{E}-03$ & $2.145 \mathrm{E}-03$ \\
\hline rh102m & $1.642 \mathrm{E}+00$ & $6.816 \mathrm{E}-01$ & $6.505 \mathrm{E}-01$ & $5.406 \mathrm{E}-01$ & 4.493E-01 \\
\hline rh103m & $2.117 \mathrm{E}+04$ & $1.079 \mathrm{E}-09$ & $2.089 \mathrm{E}-10$ & $3.313 \mathrm{E}-13$ & $5.255 \mathrm{E}-16$ \\
\hline rh106 & $7.281 \mathrm{E}+05$ & $2.877 \mathrm{E}+04$ & $2.419 \mathrm{E}+04$ & $1.225 \mathrm{E}+04$ & $6.202 \mathrm{E}+03$ \\
\hline rn217 & $3.121 \mathrm{E}-12$ & $3.299 \mathrm{E}-12$ & $3.306 \mathrm{E}-12$ & $3.359 \mathrm{E}-12$ & $3.412 \mathrm{E}-12$ \\
\hline rn218 & $7.613 \mathrm{E}-11$ & $1.876 \mathrm{E}-15$ & $2.009 \mathrm{E}-15$ & $2.590 \mathrm{E}-15$ & $3.266 \mathrm{E}-15$ \\
\hline rn219 & $5.126 \mathrm{E}-08$ & $2.348 \mathrm{E}-07$ & $2.399 \mathrm{E}-07$ & $2.839 \mathrm{E}-07$ & $3.235 \mathrm{E}-07$ \\
\hline rn220 & $1.965 \mathrm{E}-03$ & $1.280 \mathrm{E}-02$ & $1.328 \mathrm{E}-02$ & $1.493 \mathrm{E}-02$ & $1.631 \mathrm{E}-02$ \\
\hline rn222 & $1.563 \mathrm{E}-09$ & $9.378 \mathrm{E}-09$ & $1.005 \mathrm{E}-08$ & $1.295 \mathrm{E}-08$ & $1.633 \mathrm{E}-08$ \\
\hline
\end{tabular}


Table C-10. Continued

\begin{tabular}{|c|c|c|c|c|c|}
\hline & $1 / 22 / 2009$ & $10 / 21 / 2013$ & $1 / 22 / 2014$ & $1 / 22 / 2015$ & $1 / 22 / 2016$ \\
\hline ru103 & $2.140 \mathrm{E}+04$ & $1.091 \mathrm{E}-09$ & $2.111 \mathrm{E}-10$ & $3.349 \mathrm{E}-13$ & $5.312 \mathrm{E}-16$ \\
\hline ru106 & $7.281 \mathrm{E}+05$ & $2.877 \mathrm{E}+04$ & $2.419 \mathrm{E}+04$ & $1.225 \mathrm{E}+04$ & $6.202 \mathrm{E}+03$ \\
\hline s 35 & $1.129 \mathrm{E}-13$ & $1.234 \mathrm{E}-19$ & $5.907 \mathrm{E}-20$ & $3.279 \mathrm{E}-21$ & $1.821 \mathrm{E}-22$ \\
\hline sb120m & $1.254 \mathrm{E}-15$ & $0.000 \mathrm{E}+00$ & $0.000 \mathrm{E}+00$ & $0.000 \mathrm{E}+00$ & $0.000 \mathrm{E}+00$ \\
\hline sb124 & $5.656 \mathrm{E}+01$ & $1.221 \mathrm{E}-07$ & 4.183E-08 & $6.256 \mathrm{E}-10$ & $9.359 \mathrm{E}-12$ \\
\hline sb125 & $1.438 \mathrm{E}+04$ & $4.365 \mathrm{E}+03$ & $4.095 \mathrm{E}+03$ & $3.185 \mathrm{E}+03$ & $2.478 \mathrm{E}+03$ \\
\hline sb126 & 7.042E-02 & $7.009 \mathrm{E}-02$ & 7.009E-02 & $7.009 \mathrm{E}-02$ & $7.002 \mathrm{E}-02$ \\
\hline sb126m & $5.004 \mathrm{E}-01$ & $5.004 \mathrm{E}-01$ & $5.004 \mathrm{E}-01$ & $5.004 \mathrm{E}-01$ & $5.004 \mathrm{E}-01$ \\
\hline sb127 & $3.208 \mathrm{E}-16$ & $0.000 \mathrm{E}+00$ & $0.000 \mathrm{E}+00$ & $0.000 \mathrm{E}+00$ & $0.000 \mathrm{E}+00$ \\
\hline sc 45m & $2.756 \mathrm{E}-18$ & $1.706 \mathrm{E}-21$ & $1.148 \mathrm{E}-21$ & $2.423 \mathrm{E}-22$ & $5.111 \mathrm{E}-23$ \\
\hline sc 46 & $1.212 \mathrm{E}-12$ & $7.201 \mathrm{E}-19$ & 3.337E-19 & $1.629 \mathrm{E}-20$ & $7.951 \mathrm{E}-22$ \\
\hline se 75 & $1.025 \mathrm{E}-04$ & $4.523 \mathrm{E}-09$ & $2.641 \mathrm{E}-09$ & $3.195 \mathrm{E}-10$ & $3.866 \mathrm{E}-11$ \\
\hline se 79 & $8.310 \mathrm{E}-02$ & $8.310 \mathrm{E}-02$ & $8.310 \mathrm{E}-02$ & $8.310 \mathrm{E}-02$ & $8.310 \mathrm{E}-02$ \\
\hline si 32 & $2.991 \mathrm{E}-19$ & $2.928 \mathrm{E}-19$ & $2.924 \mathrm{E}-19$ & $2.911 \mathrm{E}-19$ & $2.898 \mathrm{E}-19$ \\
\hline sm145 & $7.640 \mathrm{E}-04$ & $2.232 \mathrm{E}-05$ & $1.847 \mathrm{E}-05$ & $8.774 \mathrm{E}-06$ & $4.170 \mathrm{E}-06$ \\
\hline sm146 & $6.816 \mathrm{E}-08$ & $6.982 \mathrm{E}-08$ & $6.989 \mathrm{E}-08$ & $7.016 \mathrm{E}-08$ & $7.036 \mathrm{E}-08$ \\
\hline sm147 & $2.721 \mathrm{E}-06$ & $5.725 \mathrm{E}-06$ & $5.803 \mathrm{E}-06$ & $6.063 \mathrm{E}-06$ & $6.263 \mathrm{E}-06$ \\
\hline sm148 & $5.740 \mathrm{E}-11$ & $5.741 \mathrm{E}-11$ & $5.741 \mathrm{E}-11$ & $5.741 \mathrm{E}-11$ & $5.741 \mathrm{E}-11$ \\
\hline sm151 & $7.420 \mathrm{E}+02$ & $7.155 \mathrm{E}+02$ & $7.142 \mathrm{E}+02$ & $7.089 \mathrm{E}+02$ & $7.036 \mathrm{E}+02$ \\
\hline sn113 & 5.891E-07 & $1.727 \mathrm{E}-11$ & $9.863 \mathrm{E}-12$ & $1.095 \mathrm{E}-12$ & $1.215 \mathrm{E}-13$ \\
\hline sn117m & $1.943 \mathrm{E}-04$ & $0.000 \mathrm{E}+00$ & $0.000 \mathrm{E}+00$ & $0.000 \mathrm{E}+00$ & $0.000 \mathrm{E}+00$ \\
\hline sn119m & $2.333 \mathrm{E}+02$ & $3.868 \mathrm{E}+00$ & $3.104 \mathrm{E}+00$ & $1.309 \mathrm{E}+00$ & $5.519 \mathrm{E}-01$ \\
\hline sn121 & $3.704 \mathrm{E}+01$ & $3.437 \mathrm{E}+01$ & $3.423 \mathrm{E}+01$ & $3.370 \mathrm{E}+01$ & $3.317 \mathrm{E}+01$ \\
\hline sn121m & $4.774 \mathrm{E}+01$ & $4.429 \mathrm{E}+01$ & $4.411 \mathrm{E}+01$ & $4.342 \mathrm{E}+01$ & $4.274 \mathrm{E}+01$ \\
\hline sn123 & $6.876 \mathrm{E}+02$ & $6.302 \mathrm{E}-02$ & $3.826 \mathrm{E}-02$ & $5.399 \mathrm{E}-03$ & $7.620 \mathrm{E}-04$ \\
\hline sn125 & $8.735 \mathrm{E}-05$ & $0.000 \mathrm{E}+00$ & $0.000 \mathrm{E}+00$ & $0.000 \mathrm{E}+00$ & $0.000 \mathrm{E}+00$ \\
\hline sn126 & $5.004 \mathrm{E}-01$ & $5.004 \mathrm{E}-01$ & $5.004 \mathrm{E}-01$ & $5.004 \mathrm{E}-01$ & $5.004 \mathrm{E}-01$ \\
\hline sr 85 & $2.908 \mathrm{E}-04$ & $2.617 \mathrm{E}-12$ & $9.684 \mathrm{E}-13$ & $1.957 \mathrm{E}-14$ & $3.953 \mathrm{E}-16$ \\
\hline sr 89 & $1.580 \mathrm{E}+04$ & $7.487 \mathrm{E}-07$ & $2.091 \mathrm{E}-07$ & $1.399 \mathrm{E}-09$ & $9.365 \mathrm{E}-12$ \\
\hline $\operatorname{sr} 90$ & $4.574 \mathrm{E}+04$ & $4.080 \mathrm{E}+04$ & $4.055 \mathrm{E}+04$ & $3.959 \mathrm{E}+04$ & $3.865 \mathrm{E}+04$ \\
\hline ta179 & $2.735 \mathrm{E}-17$ & $4.488 \mathrm{E}-18$ & $4.073 \mathrm{E}-18$ & $2.784 \mathrm{E}-18$ & $1.903 \mathrm{E}-18$ \\
\hline ta182 & $4.919 \mathrm{E}-12$ & $1.398 \mathrm{E}-16$ & $7.978 \mathrm{E}-17$ & $8.934 \mathrm{E}-18$ & $1.121 \mathrm{E}-18$ \\
\hline tb157 & $2.825 \mathrm{E}-05$ & $2.697 \mathrm{E}-05$ & $2.690 \mathrm{E}-05$ & $2.664 \mathrm{E}-05$ & $2.638 \mathrm{E}-05$ \\
\hline tb158 & $1.188 \mathrm{E}-03$ & $1.167 \mathrm{E}-03$ & $1.166 \mathrm{E}-03$ & $1.161 \mathrm{E}-03$ & $1.157 \mathrm{E}-03$ \\
\hline tb160 & $1.623 \mathrm{E}+02$ & $9.883 \mathrm{E}-06$ & $4.051 \mathrm{E}-06$ & $1.225 \mathrm{E}-07$ & $3.700 \mathrm{E}-09$ \\
\hline tb161 & $7.859 \mathrm{E}-09$ & $0.000 \mathrm{E}+00$ & $0.000 \mathrm{E}+00$ & $0.000 \mathrm{E}+00$ & $0.000 \mathrm{E}+00$ \\
\hline
\end{tabular}


Table C-10. Continued

\begin{tabular}{|c|c|c|c|c|c|}
\hline & $1 / 22 / 2009$ & $10 / 21 / 2013$ & $1 / 22 / 2014$ & $1 / 22 / 2015$ & $1 / 22 / 2016$ \\
\hline tc 95 & $7.440 \mathrm{E}-12$ & $2.085 \mathrm{E}-20$ & $7.248 \mathrm{E}-21$ & $1.146 \mathrm{E}-22$ & $0.000 \mathrm{E}+00$ \\
\hline tc $95 \mathrm{~m}$ & $1.891 \mathrm{E}-10$ & $5.301 \mathrm{E}-19$ & $1.843 \mathrm{E}-19$ & $2.912 \mathrm{E}-21$ & $4.601 \mathrm{E}-23$ \\
\hline tc 97 & $7.586 \mathrm{E}-09$ & $7.659 \mathrm{E}-09$ & $7.659 \mathrm{E}-09$ & $7.659 \mathrm{E}-09$ & $7.659 \mathrm{E}-09$ \\
\hline tc $97 \mathrm{~m}$ & $1.248 \mathrm{E}-03$ & $2.309 \mathrm{E}-09$ & $1.137 \mathrm{E}-09$ & $7.055 \mathrm{E}-11$ & $4.375 \mathrm{E}-12$ \\
\hline tc 98 & $7.646 \mathrm{E}-06$ & $7.646 \mathrm{E}-06$ & $7.646 \mathrm{E}-06$ & $7.646 \mathrm{E}-06$ & $7.646 \mathrm{E}-06$ \\
\hline tc 99 & $1.624 \mathrm{E}+01$ & $1.624 \mathrm{E}+01$ & $1.624 \mathrm{E}+01$ & $1.624 \mathrm{E}+01$ & $1.624 \mathrm{E}+01$ \\
\hline te121 & $2.571 \mathrm{E}-20$ & $1.710 \mathrm{E}-23$ & $1.154 \mathrm{E}-23$ & $2.141 \mathrm{E}-29$ & $0.000 \mathrm{E}+00$ \\
\hline te121 & $1.268 \mathrm{E}-03$ & $8.429 \mathrm{E}-07$ & $5.693 \mathrm{E}-07$ & $1.219 \mathrm{E}-07$ & $2.612 \mathrm{E}-08$ \\
\hline te121m & $1.264 \mathrm{E}-03$ & $8.403 \mathrm{E}-07$ & $5.675 \mathrm{E}-07$ & $1.216 \mathrm{E}-07$ & $2.604 \mathrm{E}-08$ \\
\hline te123m & $2.752 \mathrm{E}+00$ & $1.157 \mathrm{E}-04$ & $6.737 \mathrm{E}-05$ & $8.064 \mathrm{E}-06$ & $9.657 \mathrm{E}-07$ \\
\hline te125m & $3.502 \mathrm{E}+03$ & $1.069 \mathrm{E}+03$ & $1.003 \mathrm{E}+03$ & $7.799 \mathrm{E}+02$ & $6.068 \mathrm{E}+02$ \\
\hline te127 & $2.820 \mathrm{E}+03$ & 4.614E-02 & $2.554 \mathrm{E}-02$ & $2.507 \mathrm{E}-03$ & $2.461 \mathrm{E}-04$ \\
\hline te127m & $2.879 \mathrm{E}+03$ & $4.710 \mathrm{E}-02$ & $2.608 \mathrm{E}-02$ & $2.559 \mathrm{E}-03$ & $2.513 \mathrm{E}-04$ \\
\hline te128 & $4.938 \mathrm{E}-14$ & $4.938 \mathrm{E}-14$ & $4.938 \mathrm{E}-14$ & $4.938 \mathrm{E}-14$ & $4.938 \mathrm{E}-14$ \\
\hline te129 & $1.627 \mathrm{E}+02$ & $4.843 \mathrm{E}-14$ & $7.109 \mathrm{E}-15$ & $3.817 \mathrm{E}-18$ & $2.050 \mathrm{E}-21$ \\
\hline te129m & $2.580 \mathrm{E}+02$ & $7.679 \mathrm{E}-14$ & $1.127 \mathrm{E}-14$ & $6.051 \mathrm{E}-18$ & $3.248 \mathrm{E}-21$ \\
\hline $\operatorname{th} 226$ & $7.613 \mathrm{E}-11$ & $0.000 \mathrm{E}+00$ & $0.000 \mathrm{E}+00$ & $0.000 \mathrm{E}+00$ & $0.000 \mathrm{E}+00$ \\
\hline th227 & 5.172E-08 & $2.316 \mathrm{E}-07$ & $2.381 \mathrm{E}-07$ & $2.800 \mathrm{E}-07$ & $3.191 \mathrm{E}-07$ \\
\hline th228 & $1.992 \mathrm{E}-03$ & $1.275 \mathrm{E}-02$ & $1.322 \mathrm{E}-02$ & 1.487E-02 & $1.625 \mathrm{E}-02$ \\
\hline th229 & $4.458 \mathrm{E}-08$ & $4.712 \mathrm{E}-08$ & $4.730 \mathrm{E}-08$ & $4.799 \mathrm{E}-08$ & $4.874 \mathrm{E}-08$ \\
\hline th230 & $1.961 \mathrm{E}-06$ & $5.925 \mathrm{E}-06$ & $6.186 \mathrm{E}-06$ & $7.255 \mathrm{E}-06$ & $8.403 \mathrm{E}-06$ \\
\hline th231 & $2.443 \mathrm{E}-03$ & $2.448 \mathrm{E}-03$ & $2.448 \mathrm{E}-03$ & $2.450 \mathrm{E}-03$ & $2.450 \mathrm{E}-03$ \\
\hline th232 & $1.954 \mathrm{E}-11$ & $2.364 \mathrm{E}-11$ & $2.387 \mathrm{E}-11$ & $2.474 \mathrm{E}-11$ & $2.561 \mathrm{E}-11$ \\
\hline th234 & $3.099 \mathrm{E}-01$ & $3.100 \mathrm{E}-01$ & $3.100 \mathrm{E}-01$ & $3.100 \mathrm{E}-01$ & $3.100 \mathrm{E}-01$ \\
\hline tl202 & $3.961 \mathrm{E}-23$ & $0.000 \mathrm{E}+00$ & $7.049 \mathrm{E}-28$ & $0.000 \mathrm{E}+00$ & $0.000 \mathrm{E}+00$ \\
\hline tl204 & $1.736 \mathrm{E}-14$ & $7.281 \mathrm{E}-15$ & $6.949 \mathrm{E}-15$ & $5.785 \mathrm{E}-15$ & $4.817 \mathrm{E}-15$ \\
\hline tl206 & $4.666 \mathrm{E}-15$ & $4.926 \mathrm{E}-15$ & $4.989 \mathrm{E}-15$ & $5.307 \mathrm{E}-15$ & $5.744 \mathrm{E}-15$ \\
\hline tl207 & 5.112E-08 & $2.342 \mathrm{E}-07$ & $2.393 \mathrm{E}-07$ & $2.831 \mathrm{E}-07$ & $3.226 \mathrm{E}-07$ \\
\hline tl208 & 7.062E-04 & $4.600 \mathrm{E}-03$ & $4.774 \mathrm{E}-03$ & $5.364 \mathrm{E}-03$ & $5.860 \mathrm{E}-03$ \\
\hline t1209 & $9.810 \mathrm{E}-10$ & $1.037 \mathrm{E}-09$ & $1.039 \mathrm{E}-09$ & $1.056 \mathrm{E}-09$ & $1.073 \mathrm{E}-09$ \\
\hline$t 1210$ & $3.283 \mathrm{E}-13$ & $1.970 \mathrm{E}-12$ & $2.110 \mathrm{E}-12$ & $2.719 \mathrm{E}-12$ & $3.429 \mathrm{E}-12$ \\
\hline $\operatorname{tm} 167$ & $2.071 \mathrm{E}-15$ & $0.000 \mathrm{E}+00$ & $0.000 \mathrm{E}+00$ & $0.000 \mathrm{E}+00$ & $0.000 \mathrm{E}+00$ \\
\hline $\operatorname{tm} 168$ & 7.142E-06 & $1.780 \mathrm{E}-11$ & $8.907 \mathrm{E}-12$ & $5.882 \mathrm{E}-13$ & $3.884 \mathrm{E}-14$ \\
\hline $\operatorname{tm} 170$ & $2.636 \mathrm{E}-01$ & $2.314 \mathrm{E}-05$ & $1.402 \mathrm{E}-05$ & $1.960 \mathrm{E}-06$ & $2.740 \mathrm{E}-07$ \\
\hline $\operatorname{tm} 171$ & $1.572 \mathrm{E}-01$ & $2.835 \mathrm{E}-02$ & $2.587 \mathrm{E}-02$ & $1.803 \mathrm{E}-02$ & $1.257 \mathrm{E}-02$ \\
\hline $\mathbf{u} 230$ & 7.606E-11 & $0.000 \mathrm{E}+00$ & $0.000 \mathrm{E}+00$ & $0.000 \mathrm{E}+00$ & $0.000 \mathrm{E}+00$ \\
\hline
\end{tabular}


Table C-10. Continued

\begin{tabular}{|c|c|c|c|c|c|}
\hline & $1 / 22 / 2009$ & $10 / 21 / 2013$ & $1 / 22 / 2014$ & $1 / 22 / 2015$ & $1 / 22 / 2016$ \\
\hline u232 & 7.367E-03 & $1.792 \mathrm{E}-02$ & $1.819 \mathrm{E}-02$ & $1.905 \mathrm{E}-02$ & $1.968 \mathrm{E}-02$ \\
\hline $\mathbf{u} 233$ & $4.470 \mathrm{E}-06$ & $6.996 \mathrm{E}-06$ & $7.122 \mathrm{E}-06$ & 7.693E-06 & $8.270 \mathrm{E}-06$ \\
\hline u234 & $7.148 \mathrm{E}-02$ & $1.104 \mathrm{E}-01$ & $1.124 \mathrm{E}-01$ & $1.205 \mathrm{E}-01$ & $1.286 \mathrm{E}-01$ \\
\hline u235 & $2.443 \mathrm{E}-03$ & $2.448 \mathrm{E}-03$ & $2.448 \mathrm{E}-03$ & $2.450 \mathrm{E}-03$ & $2.450 \mathrm{E}-03$ \\
\hline u236 & $1.736 \mathrm{E}-02$ & $1.768 \mathrm{E}-02$ & $1.770 \mathrm{E}-02$ & $1.776 \mathrm{E}-02$ & $1.783 \mathrm{E}-02$ \\
\hline u237 & $1.262 \mathrm{E}+01$ & $1.003 \mathrm{E}+01$ & $9.909 \mathrm{E}+00$ & $9.438 \mathrm{E}+00$ & $8.994 \mathrm{E}+00$ \\
\hline u238 & $3.099 \mathrm{E}-01$ & $3.099 \mathrm{E}-01$ & $3.099 \mathrm{E}-01$ & $3.099 \mathrm{E}-01$ & $3.099 \mathrm{E}-01$ \\
\hline u240 & $1.186 \mathrm{E}-06$ & $1.186 \mathrm{E}-06$ & $1.186 \mathrm{E}-06$ & $1.186 \mathrm{E}-06$ & $1.186 \mathrm{E}-06$ \\
\hline v 49 & $3.718 \mathrm{E}-16$ & $9.763 \mathrm{E}-18$ & $8.031 \mathrm{E}-18$ & $3.729 \mathrm{E}-18$ & $1.732 \mathrm{E}-18$ \\
\hline w181 & $4.155 \mathrm{E}-15$ & $2.062 \mathrm{E}-19$ & $1.211 \mathrm{E}-19$ & $1.502 \mathrm{E}-20$ & $1.862 \mathrm{E}-21$ \\
\hline w185 & $9.219 \mathrm{E}-13$ & $1.043 \mathrm{E}-19$ & $4.419 \mathrm{E}-20$ & $1.521 \mathrm{E}-21$ & $5.238 \mathrm{E}-23$ \\
\hline w188 & $1.708 \mathrm{E}-14$ & $5.708 \mathrm{E}-22$ & $2.266 \mathrm{E}-22$ & $6.035 \mathrm{E}-24$ & $1.607 \mathrm{E}-25$ \\
\hline xe127 & $1.050 \mathrm{E}-03$ & $4.889 \mathrm{E}-18$ & 8.317E-19 & $7.971 \mathrm{E}-22$ & $7.640 \mathrm{E}-25$ \\
\hline xe129m & $2.129 \mathrm{E}-08$ & $0.000 \mathrm{E}+00$ & $0.000 \mathrm{E}+00$ & $0.000 \mathrm{E}+00$ & $0.000 \mathrm{E}+00$ \\
\hline xe131m & $8.390 \mathrm{E}-03$ & $0.000 \mathrm{E}+00$ & $0.000 \mathrm{E}+00$ & $0.000 \mathrm{E}+00$ & $0.000 \mathrm{E}+00$ \\
\hline xe133 & $1.901 \mathrm{E}-09$ & $0.000 \mathrm{E}+00$ & $0.000 \mathrm{E}+00$ & $0.000 \mathrm{E}+00$ & $0.000 \mathrm{E}+00$ \\
\hline y 88 & $3.177 \mathrm{E}-01$ & $4.069 \mathrm{E}-06$ & $2.223 \mathrm{E}-06$ & $2.073 \mathrm{E}-07$ & $1.932 \mathrm{E}-08$ \\
\hline y 89m & $1.524 \mathrm{E}+00$ & $7.221 \mathrm{E}-11$ & $2.016 \mathrm{E}-11$ & $1.349 \mathrm{E}-13$ & $9.027 \mathrm{E}-16$ \\
\hline y 90 & $4.575 \mathrm{E}+04$ & $4.081 \mathrm{E}+04$ & $4.056 \mathrm{E}+04$ & $3.960 \mathrm{E}+04$ & $3.866 \mathrm{E}+04$ \\
\hline y 91 & $3.733 \mathrm{E}+04$ & $4.528 \mathrm{E}-05$ & $1.505 \mathrm{E}-05$ & $1.993 \mathrm{E}-07$ & $2.640 \mathrm{E}-09$ \\
\hline yb169 & $5.711 \mathrm{E}-08$ & $2.904 \mathrm{E}-24$ & $3.879 \mathrm{E}-25$ & $1.436 \mathrm{E}-28$ & $0.000 \mathrm{E}+00$ \\
\hline $\operatorname{zn} 65$ & $7.002 \mathrm{E}-16$ & $5.086 \mathrm{E}-18$ & $3.905 \mathrm{E}-18$ & $1.385 \mathrm{E}-18$ & $4.906 \mathrm{E}-19$ \\
\hline zr 88 & $1.262 \mathrm{E}-07$ & $7.009 \mathrm{E}-14$ & $3.236 \mathrm{E}-14$ & $1.558 \mathrm{E}-15$ & $7.500 \mathrm{E}-17$ \\
\hline $\operatorname{zr} 93$ & $1.562 \mathrm{E}+00$ & $1.562 \mathrm{E}+00$ & $1.562 \mathrm{E}+00$ & $1.562 \mathrm{E}+00$ & $1.562 \mathrm{E}+00$ \\
\hline $\operatorname{zr} 95$ & $8.954 \mathrm{E}+04$ & $6.378 \mathrm{E}-04$ & $2.331 \mathrm{E}-04$ & $4.483 \mathrm{E}-06$ & 8.622E-08 \\
\hline $\operatorname{zr} 96$ & $1.546 \mathrm{E}-13$ & $1.546 \mathrm{E}-13$ & $1.546 \mathrm{E}-13$ & $1.546 \mathrm{E}-13$ & $1.546 \mathrm{E}-13$ \\
\hline Total & $4.3784 \mathrm{E}+06$ & $9.1470 \mathrm{E}+05$ & $8.8857 \mathrm{E}+05$ & $8.0889 \mathrm{E}+05$ & $7.5214 \mathrm{E}+05$ \\
\hline
\end{tabular}

